*aMIS View/Print Document Cover Sheet

This document was retrieved from the Boeing ISEARCH System.

Accession \#: D196069894

Document \#: SD-WM-TRP-233

Title/Desc:

ANALYTICAL \& EXPERIMENTAL EVALUATION OF SOLID WASTE DRUM FIRE PERFORMANCE [VOL 1] [SEC 1 OF 3] 
This document was too large to scan as a whole document, therefore it required breaking into smaller sections.

DOCUMENT NUMBER: WHC-SD-WM-TRP-233 Rew 0 SECTION 1 OF 3

TITLE: Analytical \& Experimental

Evaluation of Solid Waste Drum Fire Performance [vol. 1]

DATE: $10 / 11 / 1995$

ORIGINATOR: Lovie $R L$

CO: WHC

RECIPIENT:

CO:

REFERENCES: EDT-612225 
THIS PAGE INTEENTIONALLY LEFT BLANK 


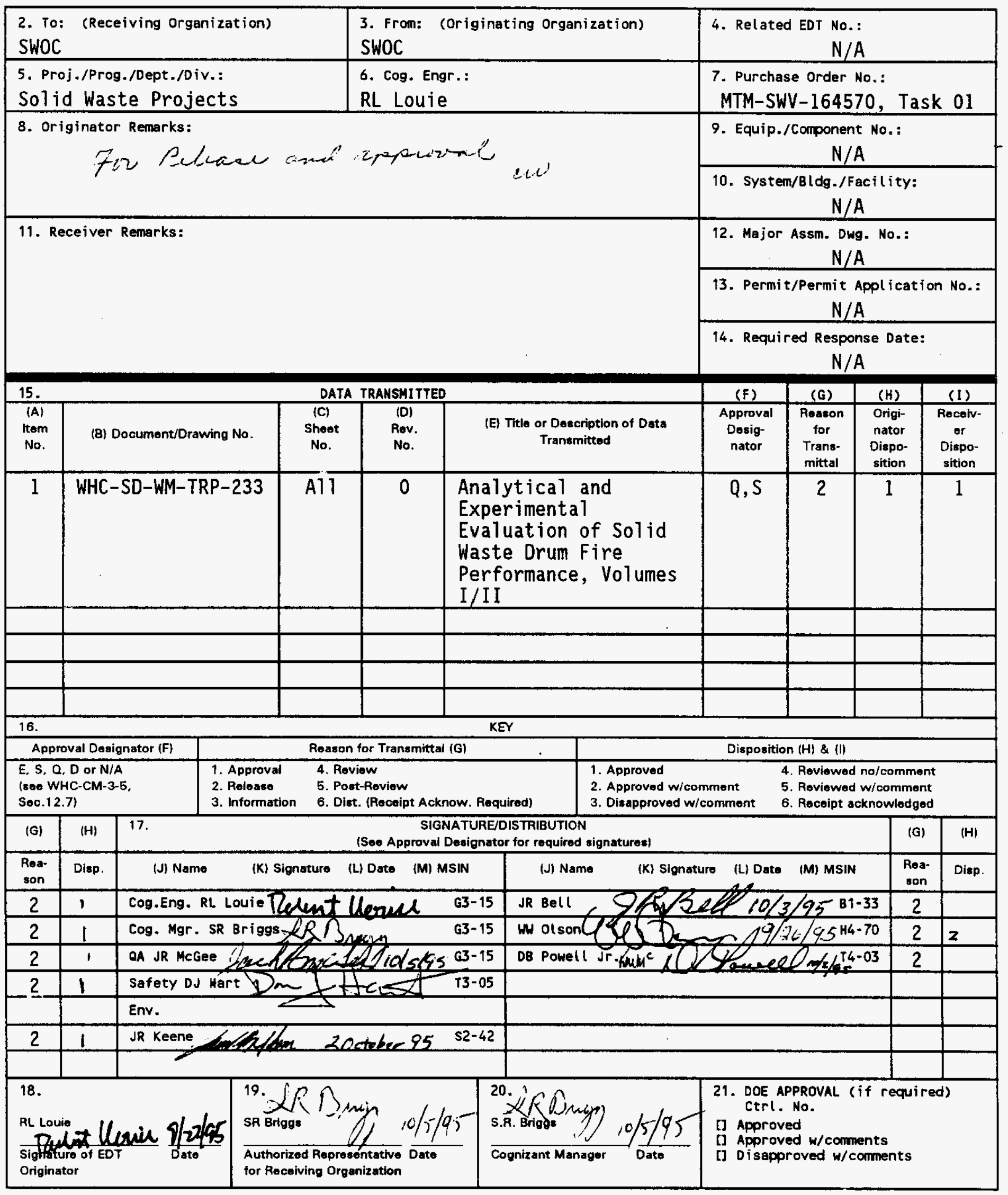


INSTRUCTIONS FOR COMPLETION OF THE ENGINEERING DATA TRANSMITTAL

(USE BLACK INK OR TYPE)

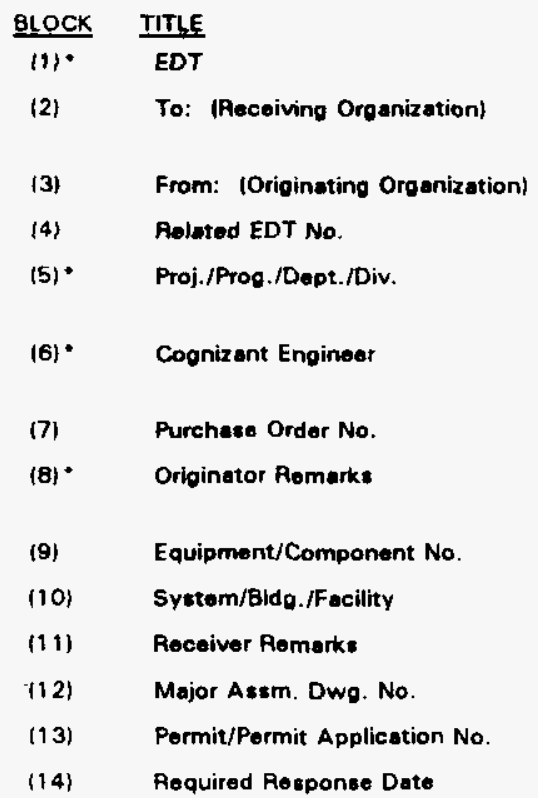

(G) Reason
(H) Disposition
(J) Name
$(\mathrm{K})$ - Signature
$(\mathrm{L})^{*}$ Date
(M) MSIN

(18) Signature of EDT Originator

(19) Authorized Pepresentative for Receiving Orgsnization

(20). Cognizant Manager

(21) * DOE Approval
- Pro-essioned EDT number.

- Enter the individual's name, titlo of the orgenization, or ontity (0.8. Dietribution) that the EDT is being transmittod to.

- Enter the titlo of the organization originating and tranamitting tho EDT.

- Enter EOT numbers which relate to the date being transmitted.

- Enter the Project/Program/Department/Division titlo or Project/Program acronym or Project Number, Work Order Number or Organization Code.

- Enter the name of the individual identified as being responsible for coordinating disposition of the EDT.

- Enter related Purchase Order (P. O.) Number, if available.

- Enter special or additional commente concerning tranemittal, or "Key" retrieval wards may be entered.

- Enter equipment/component number of affected itom, if appropriato.

- Enter applicablo system, building or facility number, if appropriate.

- Enter special or additional comments concerning tranamittal.

- Enter applicable drawing number of major assembly, if appropriato.

- Enter applicable permit or permit applicution number, if appropriate.

- Enter the dato a response is required from individuals identified in Block 17 (Signature/Distribution).

- Enter soquential number, beginning with $t$, of the information listed on EOT.

- Enter the unique identification number assigned to the document or drawing being tranemitted.

- Enter the sheet number of the information being trantmitted. If no shoot number, leave blenk.

- Enter the revision number of the information boing transmitted. If no revieion number, leave blank.

- Enter the title of the document or drawing or a brief description of the eubject if no titlo is identified.

- Enter the appropriate Approval Designetor (Block 15). Also, indicate the appropriote approvaly for each item listed, i.o., SO, ESQ, etc.

- Enter the appropriate code to identify the purpose of the data trenemittel (see Block 16).

- Enter the appropriate disponition code (eee Block 16).

- Enter the appropriate disposition code (see Block 16)

- Number codes used in completion of Blocks 15 (G), (H), and (I), and 17 (G), (H) (Signature/Distribution).

- Enter the code of the reason for transmittal (Block 16).

- Enter the code for the disposition (Block 16).

- Enter the signature of the individual completing the Disposition 17 (H) and the Transmittal.

- Obtain appropriate signature(s).

- Enter date signature is obteined.

- Enter MSIN. Note: If Distribution Sheet is used, show ontire distribution fincluding that indicated on Page 1 of the EDT) on the Distribution Sheet.

- Enter the signature and date of the individual originating the EDT lentered prior to transmittal to Receiving Organization). If the EDT originator is the coonizant enginoer, sign both Blocks 17 and 18 .

- Enter the signature and date of the individual identified by the Receiving Organization as authorized to spprove disposition of the EDT and acceptance of the data transmitted, as applicable.

- Enter the signature and date of the cagnizant manager. (This signature is authorization for release.)

- Enter DOE approval lif required) by signature or control number that tracks the approval to a signature. and indicate DOE action. 


\section{RELEASE AUTHORIZATION}

Document Number: WHC-SD-WM-TRP-233, Rev. 0

Document Title: Analytical and Experimental Evaluation of Solid Waste Drum Fire Performance, Volumes I and II

Release Date: $\quad 10 / 11 / 95$

This document was reviewed following the procedures described in WHC-CM-3-4 and is:

\section{APPROVED FOR PUBLIC RELEASE}

WHC Information Release Administration Specialist:

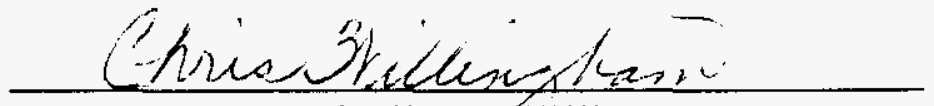

$10 / 11 / 95$

c. WILLINGHAM

TRADEMARK DISCLAIMER. Reference herein to any specific commercial product, process, or service by trade name, trademark, manufacturer, or otherwise, does not necessarily constitute or imply its endorsement, recommendation, or favoring by the United States Government or any agency thereof or its contractors or subcontractors.

This report has been reproduced from the best available copy. Available in paper copy. Printed in the United States of America. To obtain copies of this report, contact:

Westinghouse Hanford Company - Document Control Services

P.O. Box 1970, Mailstop H6-08, Richland, WA 99352

Telephone: (509) 372-2420; Fax: (509) 376-4989 
THIS PAGE INTENTIONALLY LEFT BLANK 
2. Title

ANALYTICAL AND EXPERIMENTAL EVALUATION OF SOLID WASTE DRUM FIRE PERFORMANCE, VOLUMES 1 AND II
5. Key Words
Solid Waste
Drum Fire
Test
Fire Drum Waste
Fire Test
Fire Propagation

3. Number

WHC-SD-WM-TRP-233

4. Rev No.

0

7. Abstract

Fire hazards associated with drum storage of radioactively contaminated wastes are a major concern in DOE facilities design for long term storage of solid wastes in drums. These facilities include drums stored in pallet arrays and in rack storage

6. Author

Name: RL Louje

\section{Teent Uerin}

signature

Organization/Charge code W8KD50/ML4WA

\section{systems. This report details testing in this area.}

8. RELEASE STAMP

OFFICIAL RELEASE

BY WHC

DATE OCT 111995

Station 35 
THIS PAGE INTENTIONALIT LEFT BLANK 


\title{
Analytical and Experimental Evaluation of Solid Waste Drum Fire Performance
}

\author{
Volume I
}

\author{
Prepared for \\ Westinghouse Hanford Company \\ Richland, WA 99352
}

Prepared by

Chris F. Haecker

Los Alamos Technical Associates, Inc.

Kennewick, WA 99306

Brian T. Rhodes, Jesse J. Beitel, Daniel T. Gottuk Craig L. Beyler, and Eric R. Rosenbaum

Hughes Associates, Inc.

Columbia, MD 21045 
THIS PAGE INTENTIONALLY

LEFT BLANK 
ANALYTICAL AND EXPERIMENTAL EVALUATION OF SOLID WASTE DRUM FIRE PERFORMANCE

Prepared By:

Hughes Associates, Inc. and

Los Alamos Technical Associates, Inc,

Technical Peer Review Conducted By:

Dr. James A. Milke

\section{APPROVED :}

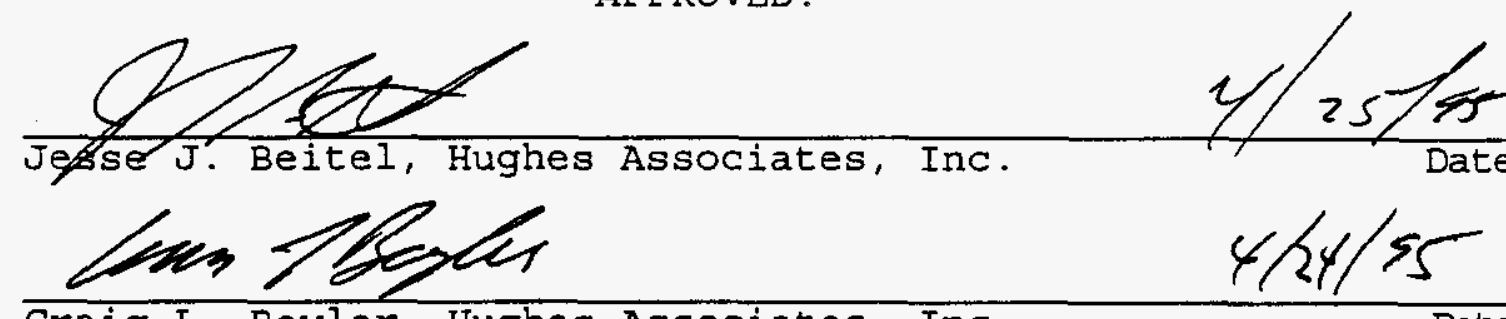

Craig L. Beyler, Hughes Associates, Inc.

Date
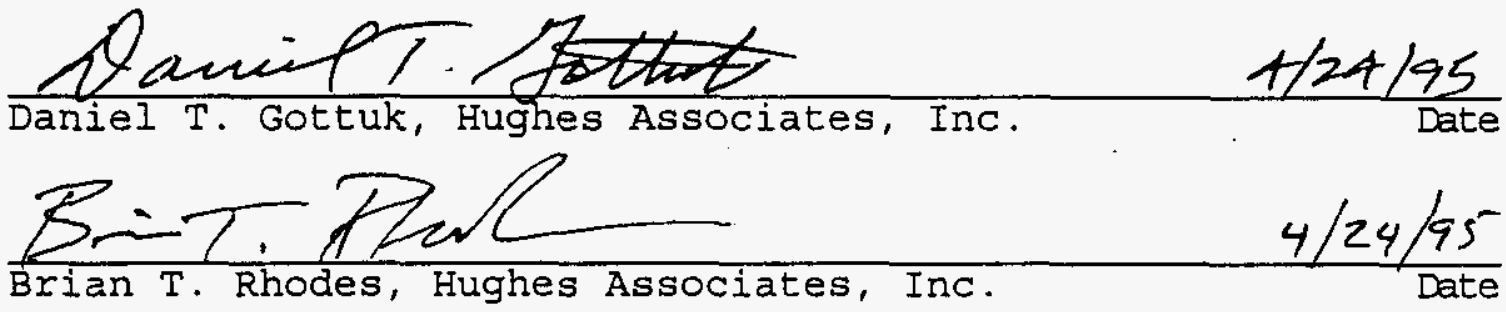

EnR.Rh 4/25/95

Eric R. Rosenbaum, Hughes Associates, Inc. Date

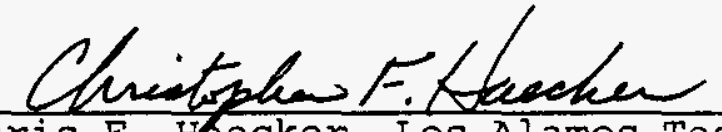

$20 \operatorname{Sen} 95$

Chris F. Hecker, Los Alamos Technical Associates, Inc. Date

jamen $R$. Hetel 23 for 95

James A. Milke, Independent Technical Reviewer i 


\section{THIS PAGE INTENTIONALIY}

LEFT BLANT 


\section{CONTENTS}

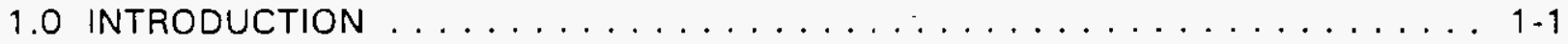

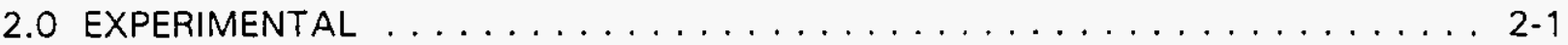

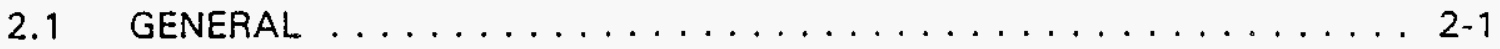

2.1 .1 Test Facility . . . . . . . . . . . . . . . . . 2-2

2.1 .2 Instrumentation . . . . . . . . . . . . . . . . 2

2.1.3 Data Acquisition ..................... 2-3

2.1 .4 Visual Documentation .................. 2-3

2.1 .5 Test Drums . . . . . . . . . . . . . . . . . . 2-3

2.1.6 Combustible Contents .................. 2-6

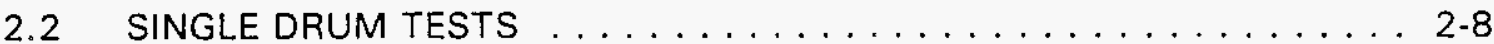

2.2.1 Single Drum Burning Rate Tests . . . . . . . . . . . . . 2-9

2.2.2 Stacked Drum Tests . . . . . . . . . . . . . . 2-12

2.3 DRUM RESPONSE FIRE EXPOSURE TESTS $\ldots \ldots \ldots \ldots \ldots \ldots \ldots$

2.3.1 Adjacent Fire Tests - $0.48 \mathrm{~m}$ Propane Burner . . . . . . . . . 2-18

2.3.2 Adjacent Fire Tests -1.2 m Propane Burner . . . . . . . . 2.23

2.3.3 Adjacent Fire Tests - Trash Fire . . . . . . . . . . . . . 2 2-27

2.3.4 Engulfing Fire Tests $-2.1 \mathrm{~m}$ Pool Fire . . . . . . . 2-30

2.4 GASKET SEAL TESTS .................. 2-30

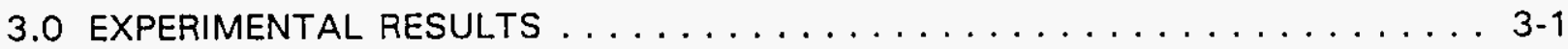

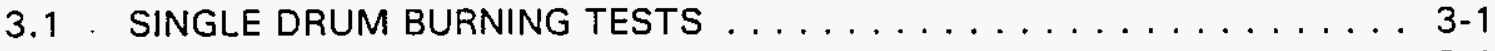

3.1.1 Single Drum Burning Rate Tests . . . . . . . . . . 3 3-1

3.1 .2 Stacked Drum Tests . . . . . . . . . . . . . . . . . 3-7

3.2 DRUM RESPONSE FIRE EXPOSURE TESTS $\ldots \ldots \ldots \ldots \ldots \ldots \ldots \ldots$

3.2.1 Thermal Exposure . . . . . . . . . . . . . . . 3-20

3.2.2 Role of Flammable Liquids . . . . . . . . . . . . . . . 3.22

3.2.3 Drum Seal Failure and Resulting Material Loss . . . . . . . . . 3-23

3.2 .4 Lid Loss . . . . . . . . . . . . . . . . . . . . . . . . . 3.24

3.2 .5 Trash Fire Exposure . . . . . . . . . . . . . . . 3-29

3.2 .6 Vent Filters . . . . . . . . . . . . . . . . . . . . 3-29

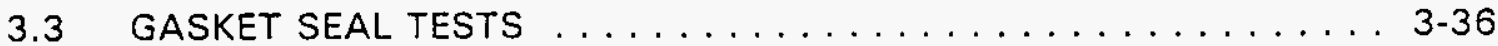

4.0 ANALYTICAL - DRUM RESPONSE MODELS $\ldots \ldots \ldots \ldots \ldots \ldots \ldots$

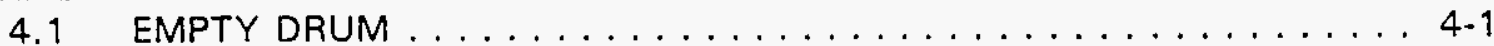

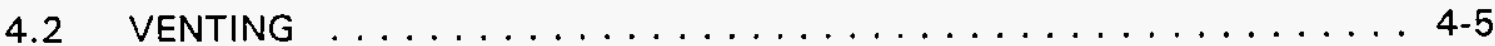

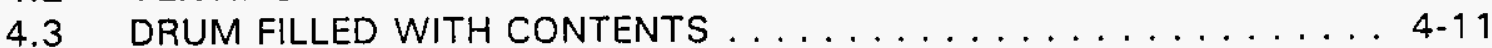

4.3.1 Lumped Mass Contents Analysis . . . . . . . . . . . . . . . 4-11

4.3.2 Semi-infinite Solid Contents Analysis . . . . . . . . . . . . 4 4-16

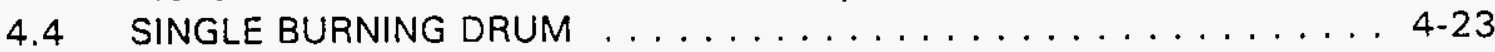

4.4.1 Burning Drum Flame Heat Flux . . . . . . . . . . . . 4 4-26

4.4.2 Radiant Heat Transfer from a Burning Drum . . . . . . . . . . 4 4-28

4.5 DRUM TO DRUM FIRE PROPAGATION ... . . . . . . . . . . 4 .29

4.5.1 Drum to Drum Configuration Analysis ............ . 4-29

4.5.2 Drum to Drum Heat Transfer . . . . . . . . . . . . . . 4. 4-33

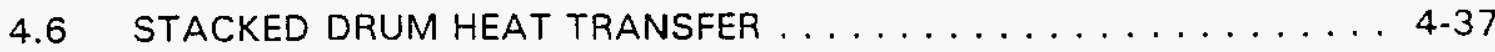

4.7 HEAT TRANSFER TO RACK FRAME $\ldots \ldots \ldots \ldots \ldots \ldots \ldots \ldots \ldots$ 
THIS PAGE INTENTIONALLY LEFT BLANK 
CONTENTS (continued)

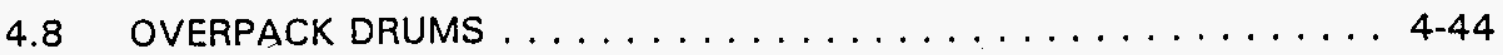

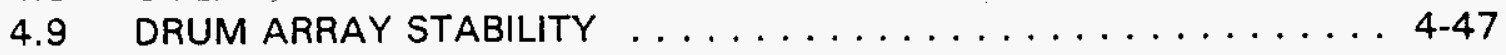

4.9.1 Single Stack Pile Stability Model . . . . . . . . . . . . . . . 4-47 . . . . . . . . . . . . . . .

4.9.2 Multiple Stack Pile Stability Model . . . . . . . . . . . . . . 4-52

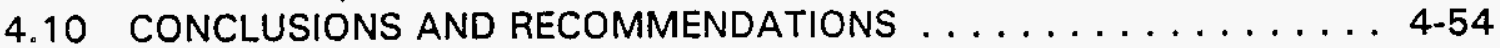

4.11 NOMENCLATURE $\ldots \ldots \ldots \ldots \ldots \ldots \ldots \ldots \ldots \ldots \ldots \ldots$

5.0 REFERENCES $\ldots \ldots \ldots \ldots \ldots \ldots \ldots \ldots \ldots \ldots \ldots \ldots \ldots \ldots \ldots \ldots \ldots$

Appendix A Summary of Fire Test Literature on Solid Waste Drum Storage ..... A-1

Appendix B Test Data $\ldots \ldots \ldots \ldots \ldots \ldots \ldots \ldots \ldots \ldots \ldots \ldots$

Appendix C Proposed Test Plan for Drum Array Fire Test . . . . . . . . . . C-1

Appendices $B$ and $C$ are located in Volume II of this report. 
THIS PAGE INTENTIONALWY LEFT BLANK

4 


\section{LIST OF FIGURES}

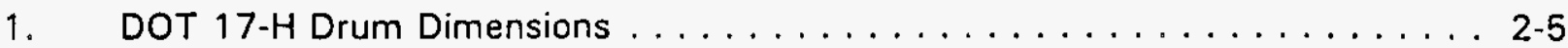

2. Test Arrangement for Single Drum Burning Tests . . . . . . . . . . . 2-10

3. Test Arrangement for Single Drum Burning Tests $\ldots \ldots \ldots \ldots \ldots \ldots \ldots$

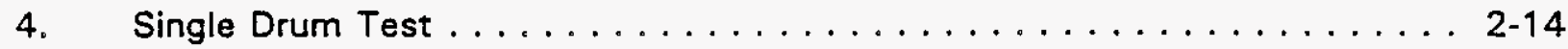

5. Test Arrangement for Stacked Drum Tests . . . . . . . . . . . . 2-15

6. Test Arrangement for Test No. SWD 18, Stacked Drum Test . . . . . . . . . 2-16

7. Vertical Single Drum Test in Progress . . . . . . . . . . . . . 2-17

8. $0.48 \mathrm{~m}$ Burner and Drum Arrangement $\ldots \ldots \ldots \ldots \ldots \ldots \ldots$

9. Experimental Arrangement and Instrumentation for Adjacent Fire Exposure Tests ............................... 2-20

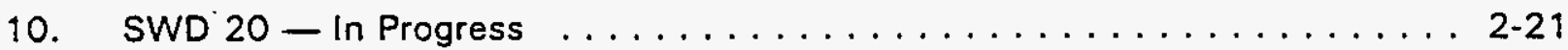

11. $1.2 \mathrm{~m}$ Water-filled Propane Burner Setup . . . . . . . . . . . . . . . . 2.24

12. $1.2 \mathrm{~m}$ Burner and Drum Arrangement $\ldots \ldots \ldots \ldots \ldots \ldots \ldots \ldots$

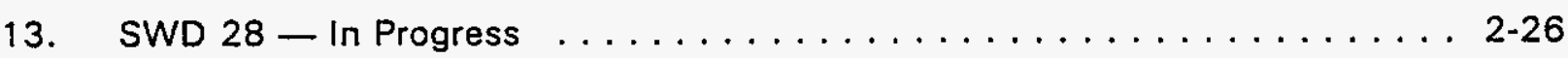

14. Trash Fire Exposure Test Setup ... . . . . . . . . . . . . 2-28

15. Trash Fire Exposure Test ... . . . . . . . . . . . . . . . 2-29

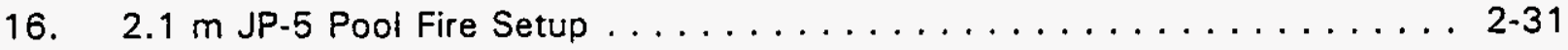

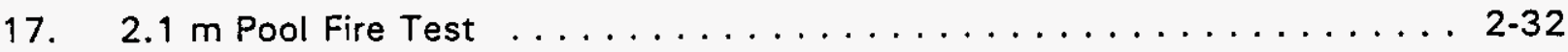

18. Gasket Seal Test ........................ 2-33

19. Drum Temperature and Heat Flux Data for Standard Load Single Drum Burning Test ............................. $3-4$

20. Drum Temperature and Heat Flux Data for $2 / 3$ Standard Load Single Drum Burning Test ..........................

21. Drum Temperature and Heat Flux Data for Kerosene Single Drum Burning Test ............................. 3-6 
THIS PAGE INTENTIONALIY

IEEFT BLANK

4 


\section{LIST OF FIGURES (continued)}

22a. Transducer Heat Flux Data for Kerosene Stacked Drum Test . . . . . . . . . 3-10

22b. Water Calorimeter Heat Flux Data for Kerosene Stacked Drum Test . . . . : 3-11

23a. Transducer Heat Flux Data for $1 / 3$ Standard Load Stacked Drum Test . . . . 3-12

23b. Water Calorimeter Heat Flux Data for $1 / 3$ Standard Load Stacked Drum Test 3-13

24. Water Calorimeter Results for Drum Response Tests . . . . . . . . . . . 3-21

25. Drum Wall Temperature as a Function of Pressure at Time of Failure . . . . 3-26

26. Drum Wall Temperature as a Function of Failure Time . . . . . . . . . 3.27

27. Arrhenius Decomposition Results for Drum Response Tests . . . . . . . . . 3-28

28. Peak Drum Pressure as a Function of Failure Time . . . . . . . . . 3-30

29. Maximum Pressure Cumulative Probability (all tests) . . . . . . . . . . . 3-31

30. Maximum Pressure Cumulative Probability $10.48 \mathrm{~m}$ burner) $\ldots \ldots \ldots 32$

31. Maximum Pressure Cumulative Probability $(1.2 \mathrm{~m}$ test $) \ldots \ldots \ldots$. . . . . 33

32. Maximum Pressure Cumulative Probability $(2.1 \mathrm{~m}$ tests $) \ldots \ldots \ldots$. . . . . 34

33. Lid Loss Pressure Frequency Distribution $\ldots \ldots \ldots \ldots \ldots \ldots \ldots \ldots$

34. Heat Transfer Components for an Empty Drum Exposed to an External Heat Flux ............................ 4.2

35. Empty Drum Configuration Used for Shape Factor Analysis . . . . . . . . . 4-4

36. Pressure Rise for Drum with Various Vent Areas Exposed to a $60 \mathrm{~kW} / \mathrm{m}^{2}$ Full Perimeter Heat Flux ..................... 4-7

37. Internal Pressure for Drum with No Vent Exposed to a $65 \mathrm{~kW} / \mathrm{m}^{2}$ Full Perimeter Heat Flux ......................... 4.8

38. Internal Air Temperature for Drum with No Vent Exposed to a $65 \mathrm{~kW} / \mathrm{m}^{2}$ Full Perimeter Heat Flux ........................ 4-9

39. Hot Wall Temperature for Drum with No Vent Exposed to a $65 \mathrm{~kW} / \mathrm{m}^{2}$ Full Perimeter Heat Flux . . . . . . . . . . . . . . . . . . . 4-10

40. Internal Pressure for Drum With a $1.6 \mathrm{~cm}(5 / 8 \mathrm{in.})$ Diameter Vent Exposed to a $65 \mathrm{~kW} / \mathrm{m}^{2}$ Full Perimeter Heat Flux ................ . . . . 2 
THIS PAGE INTENTIONALLY LEFT BLANK

- 


\section{LIST OF FIGURES (continued)}

41. Internal Air Temperature for Drum with a $1.6 \mathrm{~cm}$ (5/8 in.) Diameter Vent Exposed to a $65 \mathrm{~kW} / \mathrm{m}^{2}$ Full Perimeter Heat Flux . . . . . . . . . . 4-13

42. Hot Wall Temperature for Drum with a $1.6 \mathrm{~cm}(5 / 8 \mathrm{in.})$ Diameter Vent Exposed to a $65 \mathrm{~kW} / \mathrm{m}^{2}$ Full Perimeter Heat Flux . . . . . . . . . . . . . . 4-14

43. Heat Transfer Components for the Lumped Mass Analysis . . . . . . . . . 4-15

44. Heat Transfer Components for the Semi-infinite Solid Analysis . . . . . . . 4-17

45. Drum Wall Temperatures for Drum Containing $2.3 \mathrm{~kg}$ Paper Using Integral Method with $45 \mathrm{~kW} / \mathrm{m}^{2}$ Exposure over one-half Drum Perimeter . . . . . . . 4-19

46. Internal Pressure for Drum Containing $2.3 \mathrm{~kg}$ Paper Using Integral Method with $45 \mathrm{~kW} / \mathrm{m}^{2}$ Exposure Over one-half Drum Perimeter . . . . . . . . . . . .

47. Drum Wall Temperature for Drum Containing $2.3 \mathrm{~kg}$ Paper Using Integral Method with $65 \mathrm{~kW} / \mathrm{m}^{2}$ Full Perimeter Exposure ...............

48. Internal Pressure for Drum Containing $2.3 \mathrm{~kg}$ Paper Using Integral Method with $65 \mathrm{~kW} / \mathrm{m}^{2}$ Full Perimeter Exposure .....................

49. Drum Wall Temperature for Standard Load Drum Using Integral Method with 65 $\mathrm{kW} / \mathrm{m}^{2}$ Full Perimeter Exposure . . . . . . . . . . . . . . .

50. Internal Pressure for Standard Load Drum Using Integral Method with $65 \mathrm{~kW} / \mathrm{m}^{2}$ Full Perimeter Exposure . . . . . . . . . . . . . . . . .

51. Nomenclature and Arrangement for Configuration Factors for Tight Packed

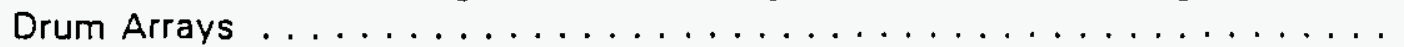

52. Nomenclature and Arrangement for Configuration Factors Used in Drum to Drum Heat Transfer Analysis . . . . . . . . . . . . . . . . . .

53. Nomenclature and Arrangement for Drum to Drum Heat Transfer Analysis for

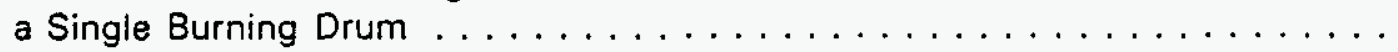

54. Nomenclature and Arrangement for Drum to Drum Heat Transfer Analysis for

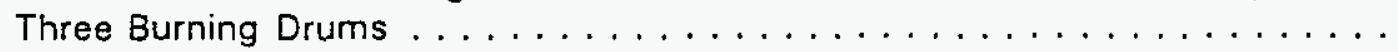

55. Nomenclature and Arrangement for Stacked Drum Heat Transfer Analysis ..

56. Steel Plate Temperature for Stacked Drum Configuration with a $35.2 \mathrm{~kW} / \mathrm{m}^{2}$ Incident Heat Flux . . . . . . . . . . . . . . . . . . . .

57. Internal Air Temperature for Stacked Empty Drum with a $35.2 \mathrm{~kW} / \mathrm{m}^{2}$ incident Heat Flux to the Steel Plate $\ldots \ldots \ldots \ldots \ldots \ldots$ 

LIST OF FIGURES (continued)

58. Internal Drum Pressure for Stacked Drum with a $35.2 \mathrm{~kW} / \mathrm{m}^{2}$ incident Heat Flux to the Steel Plate . . . . . . . . . . . . . . . . . . . . . 4-43

59. Rack Frame Temperatures for Various Flame Heat Fluxes . . . . . . . . . . 4-45

60. 85 Gallon Overpack Drum Containing Empty 55 Gallon Drum . . . . . . . . 4-46

61. Temperature and Pressure for Overpack Drum Storage Filled $1 / 2$ with Diatomaceous Earth ......................... 4-50

62. Single Stack Stability Model Geometry Nomenclature . . . . . . . . . . . . 4-51

63. Multiple Stack Stability Model Geometry Nomenclature . . . . . . . . . . . 4-53 


\section{THIS PAGE INTENTIONALLY \\ LEFT BLANR




\section{LIST OF TABLES}

1. Standard Combustible Loading . . . . . . . . . . . . . 2-7

2. Heats of Combustion $\left(\Delta H_{c}\right)$ used for Heat Release Rate (HRR) Estimation . . . 2-8

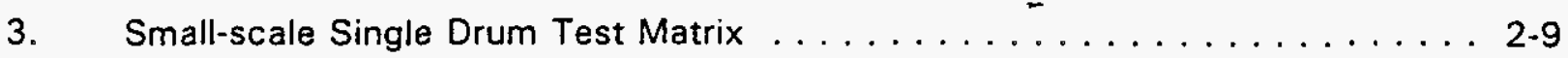

4. Single Drum Tests - Stacked Drum Test Matrix $\ldots \ldots \ldots \ldots \ldots$. . . . . .

5. Adjacent Fire Exposure - 0.48 m Propane Burner - Test Matrix . . . . . 2-22

6. Adjacent Fire Exposure - $1.2 \mathrm{~m}$ Propane Burner - Test Matrix . . . . . . 2-27

7. Engulfing Fire Exposure - Test Matrix $\ldots \ldots \ldots \ldots \ldots \ldots$

8. Gasket Seal Test Matrix . . . . . . . . . . . . . . . . . . 2-34

9. Data Summary - Single Drum Tests . . . . . . . . . . . . . . 3-2

10. Mass Burning Rates and Heat Release Rates for Single Drum Burning Tests . . 3-3

11. Data Summary - Stacked Drum Tests $\ldots \ldots \ldots \ldots \ldots \ldots$

12. Vertical Drum Tests Summary . . . . . . . . . . . . . . . . 3-9

13. Lid Loss Summary of Fire Exposure Tests . . . . . . . . . . . . . . . . . . 3-14

14. Data Summary of Fire Exposure Tests . . . . . . . . . . . . . . 3-16

15. Thermal Exposures for Drum Response Tests . . . . . . . . . . . . . . . . 3-20

16. Flammability Limits and Autoignition Temperature of Flammable Liquids . . . 3-22

17. Summary of Tests with and without Vents - Engulfing Fires . . . . . . 3-36

18. Summary of Gasket Seal Tests $\ldots \ldots \ldots \ldots \ldots \ldots \ldots \ldots$

19. Single Drum Burning Tests - Radiosity . . . . . . . . . . . . . . 4-27

20. Burning Drum and Target Drum Temperature and Heat Flux Summary . . . 4 4-29 
THIS PAGE INTENTIONALLY LEFT BLANK

\section{4}


Analytical and Experimental Evaluation of Solid Waste Drum Fire Performance

\subsection{INTRODUCTION}

Fire hazards associated with drum storage of radioactively contaminated wastes are a major concern in DOE facilities designed for long term storage of solid wastes in drums. These facilities include drums stored in pallet arrays and in rack storage systems. Combustible materials included in the drum include ordinary combustibles and in some cases flammable/combustible liquids either absorbed on ordinary combustibles or in containers. The major concerns with regard to drum storage fires is the potential for releases of radioactive particulates which contaminate the drum contents.

Prior testing, reviewed in Appendix $A$, has shown that drums containing ordinary combustibles exposed to large pool fires can experience lid loss. The loss of the lid, of course, exposes the contents to the atmosphere and with heat input from the exposing pool fire, the drum contents burn. In this report, this will be called lid loss failure. A fraction of the radioactive particulates contained within the drum is lost into the fire plume and is distributed by the plume. In cases where lid loss does not occur, the lid gasket is thermally degraded by the fire and loss of containment occurs as heated air and pyrolysis products escape from the drum at the lid seal. This will be referred to as lid seal failure in this report. In this case, only a small fraction of the material in the drum is lost.

While the prior testing provides definite insights into the response of solid waste drums exposed to pool fires, many issues of importance remain unaddressed and/or unclear. For instance, the fraction of drums exposed to pool fires which experience lid loss varied widely in prior testing. Since the exposure fires were not characterized in these investigations, it is not possible to assess if these variations are related to the heat input to the drum, the contents of the drum, or if the lid loss process is inherently random (or all of the above). Further, since all drums were placed within the pool fire, it is unclear if one-sided flame exposures would have the same impact as a full pool fire exposure. Thus, we have no idea if drums adjacent to a fire source would experience lid loss. Neither does prior testing assess the ability of burning drums to sufficiently heat neighboring drum to cause lid loss, thus allowing the possibility of indefinite fire propagation within drum arrays. Such questions need to be answered to allow determination of the possible extent of fire propagation in drum storage facilities as well as to allow evaluation and design of appropriate fire safety methods and systems.

The work reported here is the first phase of work designed to provide the understanding of waste drum response to fire needed to assess the extent of fire propagation in drum arrays which are currently in use at Hanford or are being designed for future use at Hanford. The work includes full-scale drum fire testing as well as analytical modeling of drum response to fire. Experimental work has included measurements of burning rates of drum fires and heat transfer from these drum fires to neighboring drums. The response of drums to a range of well characterized fire sources has also been studied. These tests include measurements of heat transfer, drum wall temperatures, and drum pressure. Analytical modeling has focused on the heat transfer processes involved in heating a target drum as well as on the stability of drum arrays in which one or more drum lid losses occurs. 
The report first describes the experimental work with the test setup and methods followed by the experimental results. The analytical models are then developed with comparisons of data and predictions included for each model developed. Complete plots of the experimental data and a proposed test plan for Phase II work is included in Volume II. 


\subsection{EXPERIMENTAL}

Hughes Associates, inc. (HAl) carried out an experimental program in order to characterize the response of solid waste drums in the event of a fire. It was identified that the solid waste drums in typical storage facilities could potentially be exposed to several types of fires. These exposures include the following:

- Fire within a laterally adjacent drum or a drum radiating to neighboring drums,

- Fire within a drum below if rack storage is used,

- Fire that is adjacent to but not totally engulfing the $\operatorname{drum}(s)$, and

- Fire/flames which engulf the $\operatorname{drum}(s)$.

Each of these types of exposing fires provides differing heat inputs to the exposed drums, and as such, information of each type of exposure was required. In order to evaluate the various fire scenarios described above, the experimental program was subdivided into various tasks. These tasks were as follows:

- Single drum burning tests,

- Adjacent fire exposure tests, and

- Engulfing fire tests.

In previous tests [Hasegawa et al. 1993; Greenhalgh et al. 1994], data concerning the performance of solid waste drums has been developed. These tests generally consisted of engulfing fires and utilized drum loading that were different than the combustible loadings developed by Westinghouse Hanford Compancy (WHC). Also in these tests, while drum performance was monitored, little information concerning the characterization of the exposure fire was developed. In this program, it was decided that along with monitoring the performance of the drums, the exposing fire would be characterized such that future analyses could be more meaningful.

\subsection{GENERAL}

In the single drum burning tests, it was assumed that a fire had occurred within a drum and that all of the available fuel would burn within the drum with no restriction on the amount of oxygen available. This scenario assumed that the lid was lost but the contents were not expelled. The purpose of these tests was to develop information concerning content burning rates, drum wall temperatures, and heat fluxes to the adjacent drums.

The purpose of the adjacent fire exposure tests was to evaluate the fire performance of the solid waste drums when exposed to a fire adjacent to the drums, but not engulfing the drums. Various fire exposures were performed, and the surface area of the drums exposed to the fire was also varied. The purpose of the total engulfment fire tests was to evaluate the fire performance of the solid waste drums when exposed to a "worst case" fire event.

There are several phenomena which may occur when drums are exposed to fire. The most dramatic is lid failure which results in total lid removal and allows burning of the contents. In this report, this is called lid loss failure. Loss of containment can also occur without the loss of the lid. The lid seal can be destroyed by the fire, creating an escape path 
for gases in the drum. This will be referred to as lid seal failure. Air will not be able to enter the drum with damaged seals at the rates required to support flaming combustion. However, pyrolysis gases generated within the drum can escape.

\subsubsection{Test Facility}

The fire tests were conducted inside the main burn building of the Naval Research Laboratories' Chesapeake Beach Fire Test Facility. The building was $15.2 \mathrm{~m}$ by $15.2 \mathrm{~m}$ by $10.6 \mathrm{~m}$ high ( $50 \mathrm{ft}$ by $50 \mathrm{ft}$ by $35 \mathrm{ft}$ high). The tests were conducted indoors so that ambient weather conditions such as wind and rain would not affect the fire tests.

Combustion air was provided via louvered air vents installed along the walls of the test building, and combustion products were removed via vents in the ceiling area. In some tests, the combustion products were removed via a hood system installed in the upper portion of the building.

\subsubsection{Instrumentation}

In general, the instrumentation used in the tests consisted of measurements for temperatures, heat flux, pressure, and mass loss. The measurements were taken at various locations, and these are described in detail in Sections 2.2 and 2.3.

2.1.2.1 Temperature. All temperature measurements were taken using Type $K$, chromel-alumel thermocouples. Drum wall surface measurements used thermocouples electrically welded to the drum walls while internal temperature measurements utilized inconel sheathed, grounded bead thermocouples placed inside the drums via pressure tight bulkhead fittings.

2.1.2.2 Heat Flux. The heat flux measurements were obtained by total heat gauges. The transducers were calibrated Medtherm Schmidt Boelter gauges with a stated accuracy of \pm 3 percent and identified as follows:

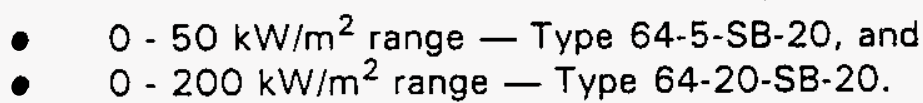

A certified calibration was provided by the manutacturer for each transducer. Typically, the heat flux gauges were installed in a target drum that was receiving the same fire exposure as the other target drums.

2.1.2.3 Water Calorimeter. In some tests, the integrated heat input to the target drums was measured via a water filled drum. In the vertical single drum tests, the vertical target drum was filled with $94 \mathrm{~L}$ ( $25 \mathrm{~g}$ al) of water, and its temperature was measured. The water was mixed using a mixing blade. In the adjacent drum tests and the engulfing fire tests, a completely filled drum was used. Again the water temperature was monitored, and the water was mixed using a submersible pump. 
2.1.2.4 Pressure. The pressure inside selected drums was monitored using Setra Model $280 E$ pressure gauges were with a range of $0-50$ psi absolute. The pressure transducers had a manufacturer's stated accuracy of \pm 0.11 percent full scale. Before and during the test program, the transducers' calibrations were verified and were found to maintain accuracies within 1 percent. The gauges were mounted remote from the drums, and copper tubing was used to penetrate the drum using. pressure tight bulkhead fittings.

2.1.2.5 Mass Loss. Drum mass loss was measured in all tests. In the single drum burning tests, the mass loss was measured by placing the burn drum on a load cell (Weigh-Tronix, model no. 2424-200) device whereby the mass loss could be measured with respect to time. In the adjacent fire tests and the engulfing fire tests, the exposed drums were weighed before and after each test. The $90 \mathrm{~kg}(200 \mathrm{lb})$ load cell had a staed accuracy of $\pm 4.5 \mathrm{~g}$. The calibration was checked at the beginning of each day of testing with no significant drift observed.

\subsubsection{Data Acquisition}

The data acquisition system was located remote from the test setup. All instrumentation was hard-wired to the data acquisition system and a computer monitor and trigger switches, located inside the test building, allowed for remote viewing and operation of the system.

The outputs of the instrumentation were recorded on an IBM compatible 386 computer using a 12-bit data acquisition system consisting of a Metrabyte DAS- 8 analog/digital 1/O board with EXP-16 multiplexer and signal conditioning boards.

LABTECH Notebook software was used to operate the data acquisition system. All data was saved as standard ASCll files and post-test processing was performed using $\mathrm{HAl}$ developed FORTRAN codes.

In general, data were collected at 1 second intervals. In Test Nos. SWD 22, 23, and 25-36, a second data acquisition system was utilized. This system was identical to the system described above, but it was arranged to scan at 20 millisecond intervals. This faster scan rate was used to record the internal drum pressures and several temperature measurements.

\subsubsection{Visual Documentation}

In general, documentation of the tests consisted of $1 / 2 \mathrm{in.}$ VHS video tape and $35 \mathrm{~mm}$ color photographs.

\subsubsection{Test Drums}

The solid waste drums used in this program were identified as $208 \mathrm{~L}(55 \mathrm{gal})$, full-removable-head, steel (double-seam chime) drums (DOT 17-H, manufactured by $V$ an Leer Container). The selection of this type of drum was based on information provided by WHC [Carver, 1993]. The WHC has used both the 17-C and the 17-H type of drum. Since the 
17-H type of drum has a thinner wall and bottom thickness (18 gauge) than the 17-C type of drum (16 gauge), it was felt that the use of the $17-\mathrm{H}$ drum would provide an adequate bounding condition. The lids for both types of drums are 16 gauge.

In general, the $17-\mathrm{H}$ drum is approximately $60.33 \mathrm{~cm}(23.75 \mathrm{in.})$ in diameter and 87.63 $\mathrm{cm}$ (34.5 in.) high. Figure 1 provides a sketch of the $17 . \mathrm{H}$ type drum. All of the test drums had no liners and the exterior faces of the side walls were painted black. In the single drum tests, reconditioned drums were used, and in all other tests, new $17-\mathrm{H}$ (UN1A2/Y1.5/100) drums were employed. Tests drums were not reused except the water filled target drums and the target drum containing the heat flux transducers.

In these tests, two different types of gasket seals were used: sponge rubber gaskets (Van Leer, model no. G488) and EPDM (Ethylene-propylene-diene monomer rubber) gaskets. Sponge rubber gaskets were used prior to 1991 [Carver, 1994], and thereafter, EPDM gaskets were used. Consequently, the majority of drums in storage contain sponge rubber gaskets. The sponge rubber gasket material is permeable and therefore not air tight as the closed-cell EPDM gaskets. Since most WHC drums contain sponge rubber gaskets, the majority of tests conducted used sponge rubber gaskets.

The bolted closing ring was 12 gauge steel, rounded-section type. Two tests were performed using a steel square-section closure ring. Per WHC specifications [Carver, 1993], the closing ring was closed to $54 \mathrm{~J}(40 \mathrm{ft}-\mathrm{lb})$ torque when drums were sealed.

It should be noted that torquing of closure rings may be a significant variable with respect to lid loss of drums exposed to a fire. Depending on the torquing method used, a range of tightness could be achieved with the closure ring while still measuring $54 \mathrm{~J}$ torque. For example, if one tightened the closure bolt with a torque wrench, $54 \mathrm{~J}$ could be achieved with a $5 \mathrm{~cm}$ separation between the ends of the closure ring. However, this torque was typically from the bolt head binding against the closure ring surface rather than the tightness of the ring around the lid and drum. Consequently, when the bolt was backed off and then tightened again, $54 \mathrm{~J}$ was not achieved until the separation between the ends of the closure ring was as small as $0.6 \mathrm{~cm}$. Therefore, depending on the person and method used, a significant range of closure ring tightness can be achieved.

All lids were secured by the same technician using the method of repetitively backing the bolt out and then tightening to $54 \mathrm{~J}$ torque. This procedure assured that the rings were torqued to the same specification. As a result of using this method, it is believed that the drums tested probably were tightened more than the existing drums at WHC.

In one test, vent filters were incorporated into the lids. Each lid contained two threaded bung holes, one $1.9 \mathrm{~cm}(0.75 \mathrm{in}$.) diameter and one $6.4 \mathrm{~cm}(2.5 \mathrm{in.})$ diameter. A Nucfil carbon vent filter (NucFil-013, $1.9 \mathrm{~cm}(0.75 \mathrm{in.}) \cdot 14$ NPT) was placed in the $1.9 \mathrm{~cm}$ $(0.75 \mathrm{in.})$ diameter hole and tightened to one-half turn past hand tight. The $6.4 \mathrm{~cm}(2.5 \mathrm{in}$. hole was closed with a $0.3 \mathrm{~cm}(1 / 8 \mathrm{in.})$ rubber gasket, and a solid steel bung. 


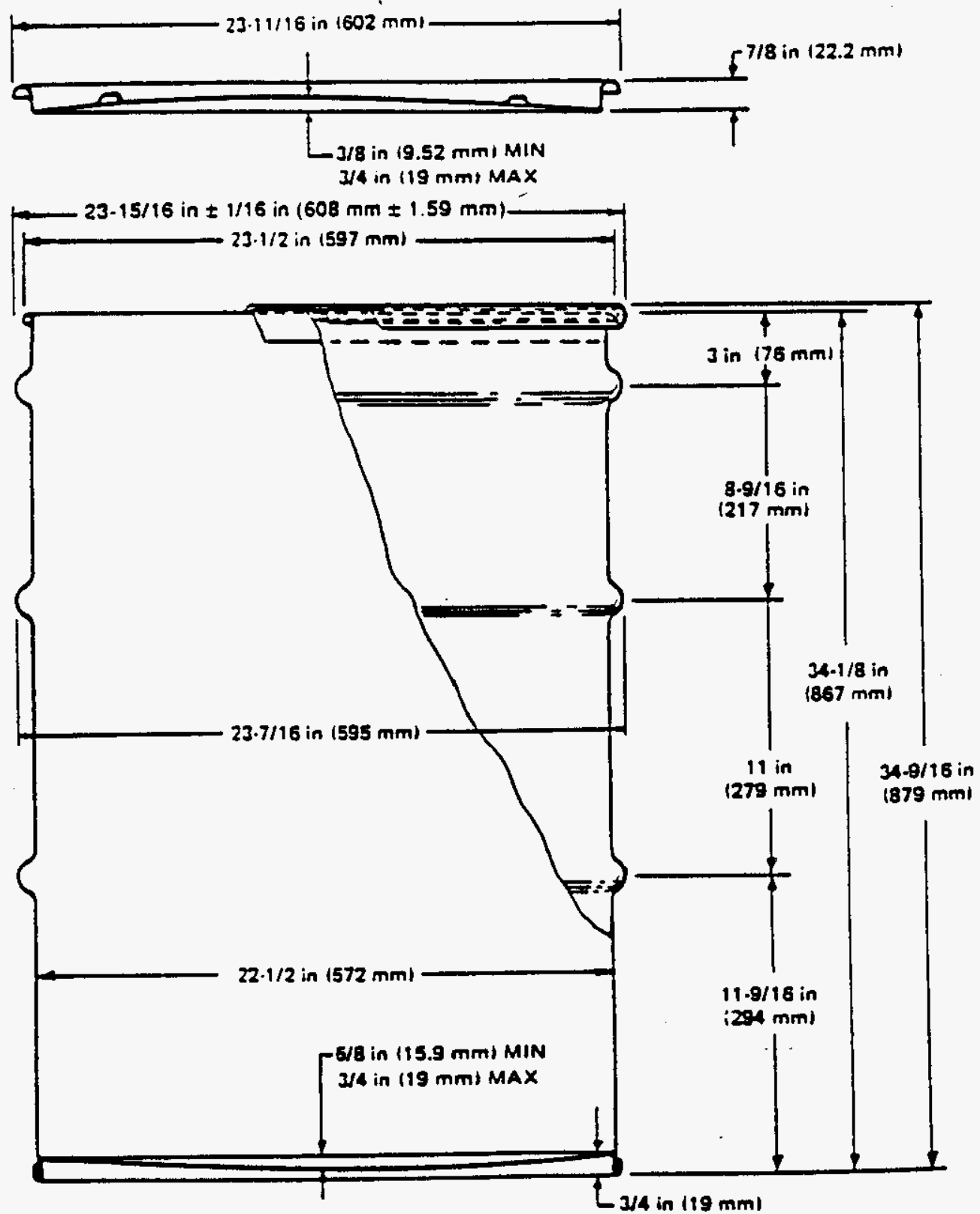

Figure 1. DOT 17-H Drum Dimensions 


\subsubsection{Combustible Contents}

Information provided by WHC [Boothe, 1994] provided a breakdown of the typical combustible loadings in solid waste drums that was used in this program. The average weight of the drum contents was $57.6 \mathrm{~kg}(127 \mathrm{lb})$ with the noncombustible components weighing approximately $31.7 \mathrm{~kg}(70 \mathrm{lb})$ and the combustible components weighing approximately 25.9 $\mathrm{kg}(57 \mathrm{lb})$. Based on these values and the breakdown of the materials, the average drum combustible contents used in this program was composed of the following:

\begin{tabular}{lrl} 
Rubber & $2.7 \mathrm{~kg}(6.0 \mathrm{lb})$ & 10.4 percent \\
Plastic & $13.4 \mathrm{~kg}(29.5 \mathrm{lb})$ & 51.7 percent \\
Paper & $6.6 \mathrm{~kg}(14.5 \mathrm{lb})$ & 25.5 percent \\
Cotton & $3.2 \mathrm{~kg}(7.0 \mathrm{lb})$ & 12.4 percent \\
\hline Total & $25.9 \mathrm{~kg}(57 \mathrm{lb})$ & 100 percent
\end{tabular}

This average combustible content did not include wood which was reported to constitute less than 1 percent of the total combustible content.

Based on the above described average combustible loading, a "standard" combustible load was developed for use in this program. The combustible drum contents consisted of the following commercially available items which simulated the actual drum contents:

- Rubber - Neoprene rubber sheets approximately $0.32 \mathrm{~cm}(0.1 .25 \mathrm{in}$.) thick and approximately $15 \mathrm{~cm}(6 \mathrm{in.}) \times 30 \mathrm{~cm}(12 \mathrm{in.})$.

- Plastic - Fifty percent by weight of 6-mil polyethylene plastic bags and 50 percent by weight of 10 -mil polyethylene sheet.

- Paper - One-third by weight of brown paper sheet $(61 \mathrm{~cm}$ (24 in.) wide rolls), $1 / 3$ by weight of bond paper $(22 \times 28 \mathrm{~cm}(8.5 \times 11 \mathrm{in.}))$, and $1 / 3$ by weight of cut-up bond paper $(11 \times 14 \mathrm{~cm}(4.25 \times 5.5 \mathrm{in})$.$) , which simulated filter paper.$

- Cotton - Fifty-seven percent by weight of cotton fabric and 43 percent by weight of cotton towels.

Table 1 provides a breakdown of the combustiole contents for the "standard" load and the procedure for its loading into the drums. The loading procedure required that materials such as the brown paper, the plastic sheet, and the cotton fabric be unfolded and placed into the drums. This combustible loading did require compression as it was packed into the drums so that the entire load could be placed in the drum. The drums were loaded in the same manner for each test by having the same technician repeat the standard loading procedure. Considering the nature of the tests and that the fuel loading was quite dense, the compression process is considered to have had little impact on the results. 
Table 1. Standard Combustible Loading.

\begin{tabular}{|l|c|}
\hline \multicolumn{2}{|c|}{ Combustible Contents } \\
\hline Material & Standard Loading \\
\hline Plastic bags & $6.7 \mathrm{~kg}(14.8 \mathrm{lb})$ \\
\hline 10 -mil plastic sheet & $6.7 \mathrm{~kg}(14.8 \mathrm{lb})$ \\
\hline Rubber, $95 \times 30 \mathrm{~cm}(6 \times 12 \mathrm{in.})$ & $2.7 \mathrm{~kg}(6.0 \mathrm{lb})$ \\
\hline Brown paper & $2.2 \mathrm{~kg}(4.8 \mathrm{lb})$ \\
\hline Bond paper, $22 \times 28 \mathrm{~cm}(8.5 \times 11 \mathrm{in})$. & $2.2 \mathrm{~kg}(4.8 \mathrm{lb})$ \\
\hline Cut paper & $2.2 \mathrm{~kg}(4.8 \mathrm{lb})$ \\
\hline Cotton towels & $1.4 \mathrm{~kg}(3.0 \mathrm{lb})$ \\
\hline Cotton fabric & $1.8 \mathrm{~kg}(1.8 \mathrm{lb})$ \\
\hline \multicolumn{1}{|c|}{ Total } & $25.9 \mathrm{~kg}(57.0 \mathrm{lb})$ \\
\hline
\end{tabular}

Standard Loading - Into Drum

Layer 1 Starting at bottom

$1 / 3$ of the brown paper

$1 / 3$ of the 10 -mil plastic

$1 / 3$ of the bond paper

$1 / 3$ of the towels

$1 / 3$ of the bags

$1 / 3$ of the cut paper

$1 / 3$ of the fabric

$1 / 3$ of the rubber

Layer 2 Repeat loading of Layer 1

Layer 3 Repeat loading of Layer 1

During the test program, several variations of the standard load were used:

- Standard Load - small pieces - This was the standard load, however, the brown paper, plastic film, and cotton fabric was cut into small pieces $(\sim 0.6 \mathrm{x}$ $0.6 \mathrm{~m}(\sim 2 \times 2 \mathrm{ft}))$, and the load was hand mixed prior to loading into the drums;

- Standard load - bagged - This was the standard load, however, the brown paper, the plastic film, and the cotton fabric was left in their folded configurations, and the entire load was placed in a clear polyethylene bag which was then loaded into the drum. The open end of the bag was twisted and secured with a plastic tie. This configuration filled the drum approximately onehalf full. 
Table 2 provides the heats of combustion, $\Delta H_{c}$, which are used to estimate the heat release rate of the drum content fires. The heat release rate of the standard load is approximated based on the combustible drum content composition:

$\Delta \mathrm{H}_{\mathrm{c}}$ of standard load $\approx(10.4 \%$ rubber $)(24.3 \mathrm{~kJ} / \mathrm{g})+(51.7 \%$ plastic $)(43.2 \mathrm{~kJ} / \mathrm{g})$ $+(25.5 \%$ paper $)(16.3 \mathrm{~kJ} / \mathrm{g})+(12.4 \%$ cotton $)(16.5 \mathrm{~kJ} / \mathrm{g})=31.2 \mathrm{~kJ} / \mathrm{g}$

For several of the fire exposure tests, small amounts of combustible liquids (50-100 g) were used in addition to the standard drum contents. The amount of liquid used in these tests was sufficient to allow the vapor concentration to reach equilibrium saturation levels. Tables 5 , 6 , and 7 in Section 2.3 describe the drum contents and combustible liquids in further detail.

Table 2. Heats of Combustion $\left(\Delta H_{c}\right)$ used for Heat Release Rate (HRR) Estimation.

\begin{tabular}{|l|c|}
\hline \multicolumn{1}{|c|}{ Fuel } & $\begin{array}{c}\Delta \mathrm{H}_{\mathrm{c}}(\mathrm{kJ} / \mathrm{g})[\text { Babrauskas and } \\
\text { Grayson, 1992] }\end{array}$ \\
\hline Kerosene & 43.3 \\
\hline Paper - brown & 16.3 \\
\hline Cotton & 16.5 \\
\hline Neoprene & 24.3 \\
\hline Polyethylene & 43.2 \\
\hline Standard load & 31.2 \\
\hline
\end{tabular}

\subsection{SINGLE DRUM TESTS}

The purpose of these tests was to develop information concerning content burning rates, drum wall temperatures, and heat fluxes to adjacent drums. The single drum tests are used to address the first two fire exposures identified in Section 2.0:

- Fire within a laterally adjacent drum or a drum radiating to neighboring drums, and

- Fire within a drum below if rack storage is used.

The first series of tests (SWD 1-10) entailed a single burning drum containing different fuel loadings. Mass loss, heat flux, and drum wall temperatures were measured for each of the tests. The second set of tests (SWD11-18) were similar except that they included a stacked drum above the burning drum. The vertical distance between the drums was varied as was the burning drum contents. Each of the single drum tests is described in the following sections. 


\subsubsection{Single Drum Burning Rate Tests}

Table 3 provides the test matrix for the single drum burning rate tests. Figures 2 and 3 provide diagrams of the test and instrumentation arrangement used in these tests. The combustible contents used in the burn drum were chosen based on information provided by WHC (Section 2.1.6). Thermocouples were used to measure the wall temperatures of the burn drum (TC Nos. 1-15) and the target drum (TC Nos. 21-24) located $5.1 \mathrm{~cm}(2$ in.) away. Three heat flux transducers (HF Nos. 1-3) were mounted in the target drum flush with the wall closest to the burn drum. Two additional transducers (HF Nos. 4-5) were positioned $61 \mathrm{~cm}$ (24 in.) away from the burn drum at a height of $46 \mathrm{~cm} 118 \mathrm{in.}$ ) and $84 \mathrm{~cm}(33 \mathrm{in.})$ respectively. Five thermocouples (TC Nos. 16-20) were used to measure the centertine temperature of the plume above the burn drum. They were mounted vertically every $15 \mathrm{~cm}$ (6 in.) above the lip of the drum.

Table 3. Small-scale Single Drum Test Matrix.

\begin{tabular}{|c|c|c|c|c|c|l|}
\hline \multirow{2}{*}{$\begin{array}{c}\text { Test } \\
\text { Number }\end{array}$} & $\begin{array}{c}\text { Mass of } \\
\text { Combustible } \\
\text { Content (kg) }\end{array}$ & \multicolumn{3}{|c|}{ Percent of Combustibles } & \multicolumn{2}{|}{ Comments } \\
\cline { 3 - 7 } & Paper & Rubber & Plastic & Cotton & \\
\hline SWD 1 & 25.9 & 25.5 & 10.4 & 51.7 & 12.4 & Top ignition \\
\hline No ID & 25.9 & 25.5 & 10.4 & 51.7 & 12.4 & Bottom ignition \\
\hline SWD 2 & 25.9 & 25.5 & 10.4 & 51.7 & 12.4 & $\begin{array}{l}\text { Steel as chimneys; bottom } \\
\text { ignition with grate }\end{array}$ \\
\hline SWD 3 & 2.3 & 100 & 0 & 0 & 0 & Bottom ignition with grate \\
\hline SWD 4 & 25.9 & 25.5 & 10.4 & 51.7 & 12.4 & Top ignition \\
\hline SWD 5 &.- & 0 & 0 & 0 & 0 & 7.6 L /2 gal) kerosene \\
\hline SWD 6 & 2.3 & 100 & 0 & 0 & 0 & Top ignition \\
\hline SWD 7 & 6.8 & 100 & 0 & 0 & 0 & Top ignition \\
\hline SWD 8 & 2.3 & 0 & 0 & 100 & 0 & Top ignition \\
\hline SWD 9 & 6.8 & 0 & 0 & 100 & 0 & Top ignition \\
\hline SWD & 17.3 & 25.5 & 10.4 & 51.7 & 12.4 & $\begin{array}{l}\text { 2/3 Standard load/small } \\
\text { pieces - top ignition }\end{array}$ \\
\hline 10 & & & & & & \\
\hline
\end{tabular}

Test No. SWD 1 consisted of the standard load placed in a drum and ignited on the top. As an initiator, approximately $20 \mathrm{~mL}$ of heptane was spread over the top of the standard load and ignited.

Test Nos. "No ID" and SWD 2 consisted of attempts to ignite the standard load via bottom ignition in the drum. In the "No ID" test, ignition using an ignitor soaked in heptane and placed in the bottom of the drum was unsuccessful. In SWD 2, a open mesh grate was placed $2.5 \mathrm{~cm}$ (1 in.) above the bottom of the drum and $5.1 \mathrm{~cm}$ by $5.1 \mathrm{~cm}(2 \mathrm{in}$. by 2 in.) pieces of steel angle were placed along the sides of the drum to act as chimneys. Ignition using a ignitor soaked in heptane and placed below the grate was not successful. 


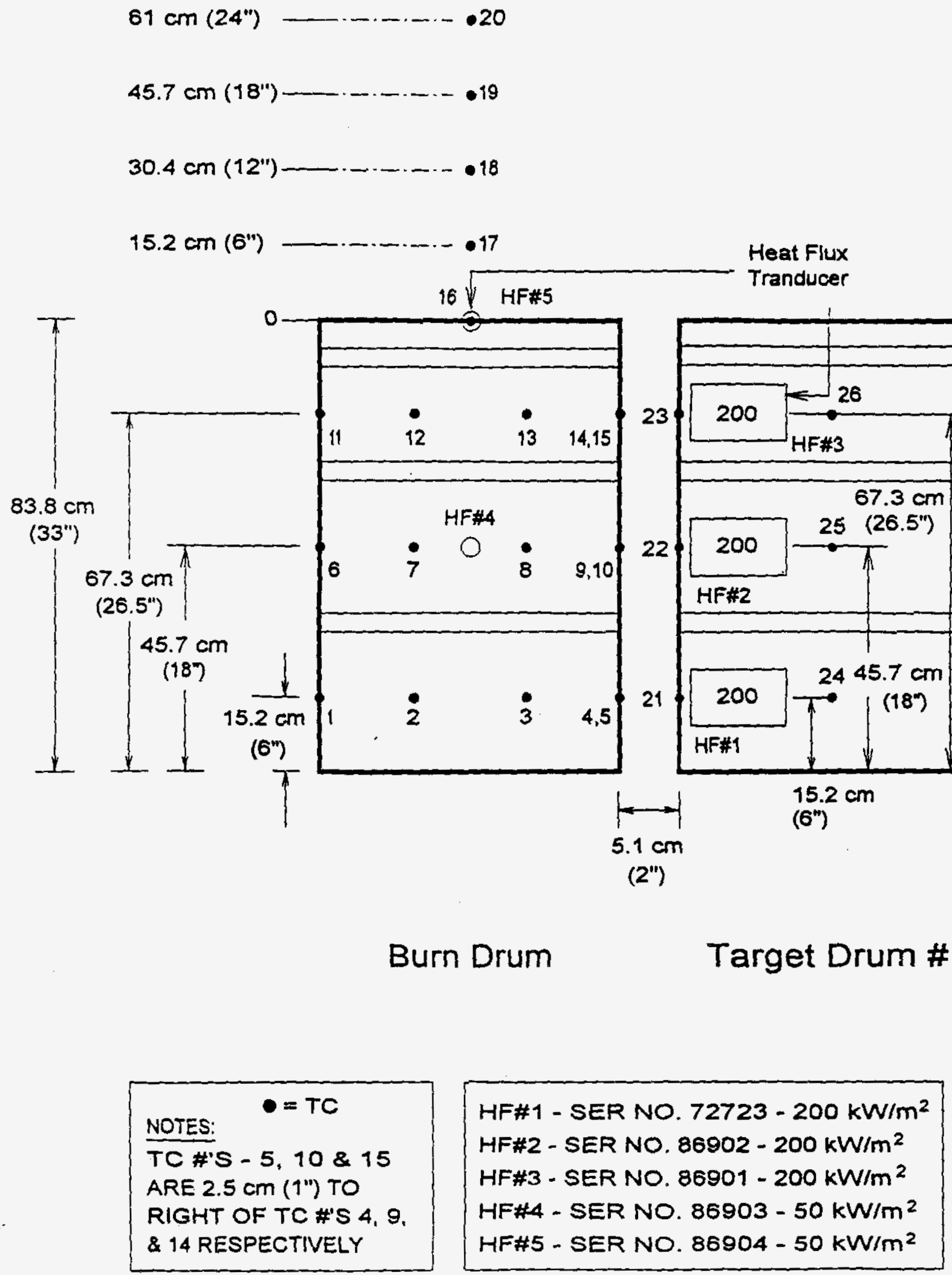

Figure 2. Test Arrangement for Single Drum Burning Tests 


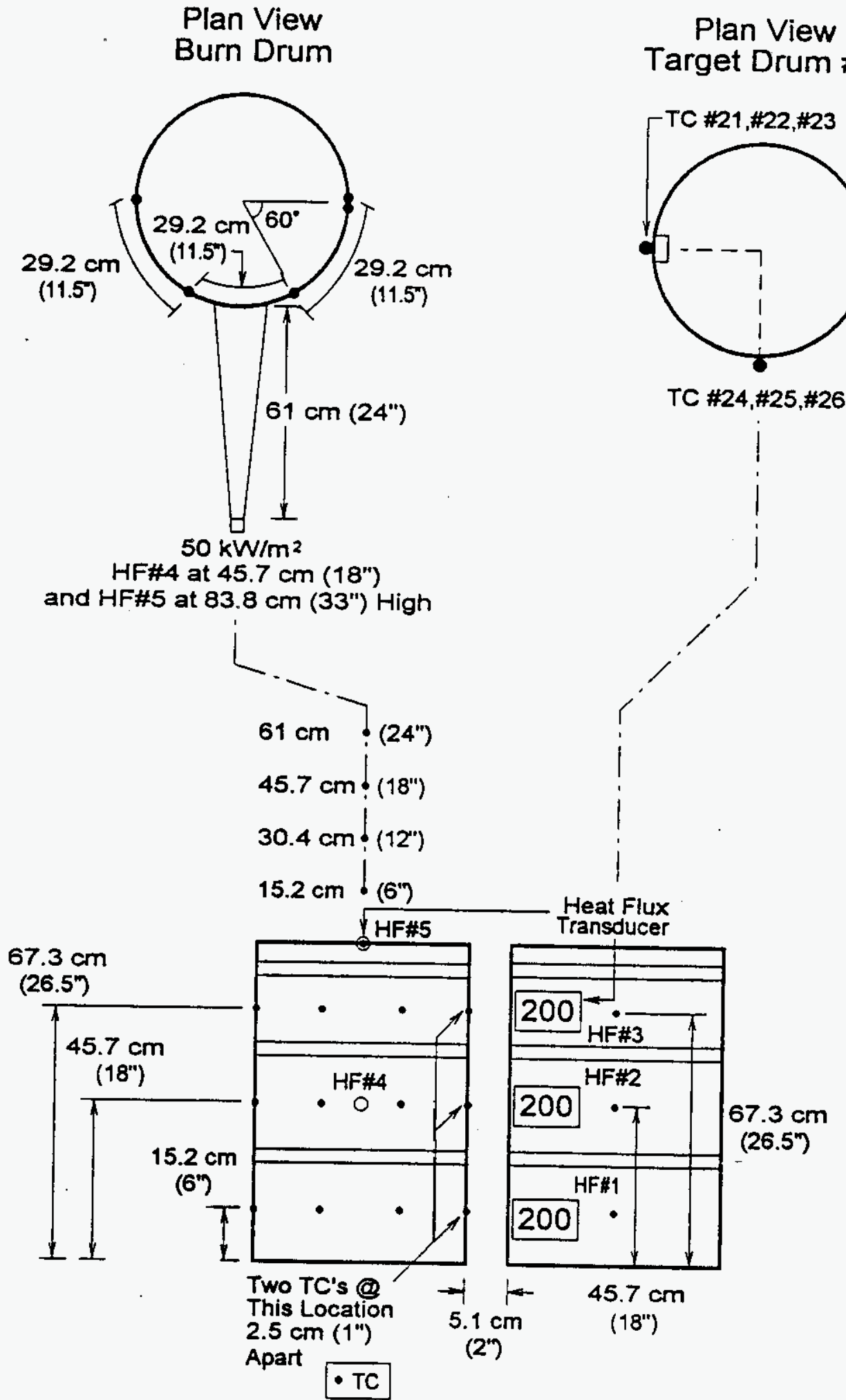

Bum Drum Target Drum \#1

Figure 3. Test Arrangement for Single Drum Burning Tests 
Test No. SWD 3 was $2.3 \mathrm{~kg}$ of paper ignited at the bottom of the drum. In this test, a grate was employed as in SWD 2. In this case, ignition was successful. In both tests (SWD 2 and SWD 3), the drum had a $2.5 \mathrm{~cm}$ (1 in.) diameter hole in the side of the drum near the bottom.

Test No. SWD 4 was a repeat of Test No. SWD 1. Test Nos. SWD $5-10$ were variations utilizing different types and loading densities of fuels. In Test No. SWD 5, kerosene was used to determine a bounding condition. In tests with 100 percent paper or 100 percent plastic, the combustible filled the drum. The $2.3 \mathrm{~kg}$ loading of paper needed little compression to fill the drum. However, the $6.8 \mathrm{~kg}$ loading required heavy compression (i.e., a man standing on top) to fit all of the contents in the drum. Figure 4 provides photographs of the single drum burning rate test arrangement.

\subsubsection{Stacked Drum Tests}

Table 4 provides the test matrix for the vertical single drum tests. Figure 5 provides a sketch of the test and instrumentation arrangement used in these tests. The setup of these tests was the same as the single drum burning rate tests with the exception of the stacked drum. One of the primary goals of the vertical single drum burning tests was to obtain flame heat flux data from a burning drum to a target above. This was accomplished in two ways: (1) heat flux transducers in the target drum, and (2) using the target drum as a water calorimeter. Three heat flux transducers (HF Nos. 6-8) were located in the bottom of the target drum. They were positioned radially such that each transducer covered an equal area of the drum bottom. For the water calorimeter, the target drum was filled with approximately $95 \mathrm{~L}(25 \mathrm{gal})$ of water during the tests. Three thermocouples (TC Nos. 27-29) were used to measure the temperature of the water. The change in water temperature was used to estimate the flame heat input to the drum.

Test Nos. SWD 11 - 14 were used to evaluate the effect of vertical separation between the burn drum and the vertical target drum. In these tests, kerosene was used as it appeared to provide a "worst case" condition based on the single drum burning rate tests and was quite reproducible.

Test Nos. SWD 15 - 17 were used to compare different burn drum loadings/materials while keeping the vertical separation constant.

For Test No.-SWD 18, an empty, sealed drum was placed directly on top of a $0.64 \mathrm{~cm}$ $(0.25$ in.) thick steel plate that was separated from the top of the kerosene burn drum by 15 . $\mathrm{cm}(6 \mathrm{in.})$. Figure 6 shows the drum configuration for this test. Figure 7 provides a photograph of a vertical single drum test in progress.

\subsection{DRUM RESPONSE FIRE EXPOSURE TESTS}

The purpose of these tests was to develop information concerning the response of sealed drums subjected to various fire exposures. Four different fire exposures were used:

(1) $0.48 \mathrm{~m}$ square propane burner,

(2) $1.2 \mathrm{~m}$ square propane burner,

(3) standard load trash fire, and

(4) $2.1 \mathrm{~m}$ square pool fire. 
WHC-SD-WM-TRP-233 REV. 0

Table 4. Single Drum Tests - Stacked Drum Test Matrix.

\begin{tabular}{|c|c|c|c|c|c|c|c|c|}
\hline \multirow[t]{2}{*}{$\begin{array}{c}\text { Test } \\
\text { Number }\end{array}$} & \multirow{2}{*}{$\begin{array}{l}\text { Mass of } \\
\text { Combustible } \\
\text { Content } \\
\text { (kg (lb)) }\end{array}$} & \multicolumn{4}{|c|}{ Percent of Combustibles } & \multicolumn{2}{|c|}{$\begin{array}{l}\text { Spacing } \\
\text { Between } \\
\text { Stacked } \\
\text { Drums }\end{array}$} & \multirow[t]{2}{*}{ Remarks } \\
\hline & & Plastic & Rubber & Paper & Cotton & $(\mathrm{cm})$ & (in.) & \\
\hline SWD 11 & $2.3(5.1)$ & -- & -- & -. & - & 15.2 & 6 & $\begin{array}{l}2.8 \mathrm{~L}(0.75 \mathrm{gal}) \\
\text { of kerosene }\end{array}$ \\
\hline SWD 12 & $1.6(3.5)$ & -- & $\overline{--}$ & -. & - & 30.5 & 12 & $\begin{array}{l}1.9 \mathrm{~L}(0.5 \mathrm{gal}) \\
\text { of kerosene }\end{array}$ \\
\hline SWD 13 & $1.6(3.5)$ & -- & - & - & - & 45.7 & 18 & $\begin{array}{l}1.9 \mathrm{~L}(0.5 \mathrm{gal}) \\
\text { of kerosene }\end{array}$ \\
\hline SWD 14 & $1.6(3.5)$ & -- & - & -- & - & 61.0 & 24 & $\begin{array}{l}1.9 \mathrm{~L}(0.5 \mathrm{gall} \\
\text { of kerosene }\end{array}$ \\
\hline SWD 15 & $2.3(5.1)$ & 100 & - & - & - & 15.2 & 6 & 10 mil plastic \\
\hline SWD 16 & $8.6(18.9)$ & 51.7 & 10.4 & 25.5 & 12.4 & 15.2 & 6 & $\begin{array}{l}1 / 3 \text { of standard } \\
\text { load - small } \\
\text { pieces }\end{array}$ \\
\hline SWD 17 & $25.9(57.0)$ & 51.7 & $\begin{array}{l}10.4 \\
\end{array}$ & 25.5 & 12.4 & 15.2 & 6 & $\begin{array}{l}\text { standard load } \\
\text { - bagged }\end{array}$ \\
\hline SWD 18 & $2.3(5.1)$ & -- & -- & - & - & 15.2 & $\overline{6}$ & $\begin{array}{l}2.8 \mathrm{~L}(0.75 \mathrm{gal}) \\
\text { of kerosene }\end{array}$ \\
\hline
\end{tabular}




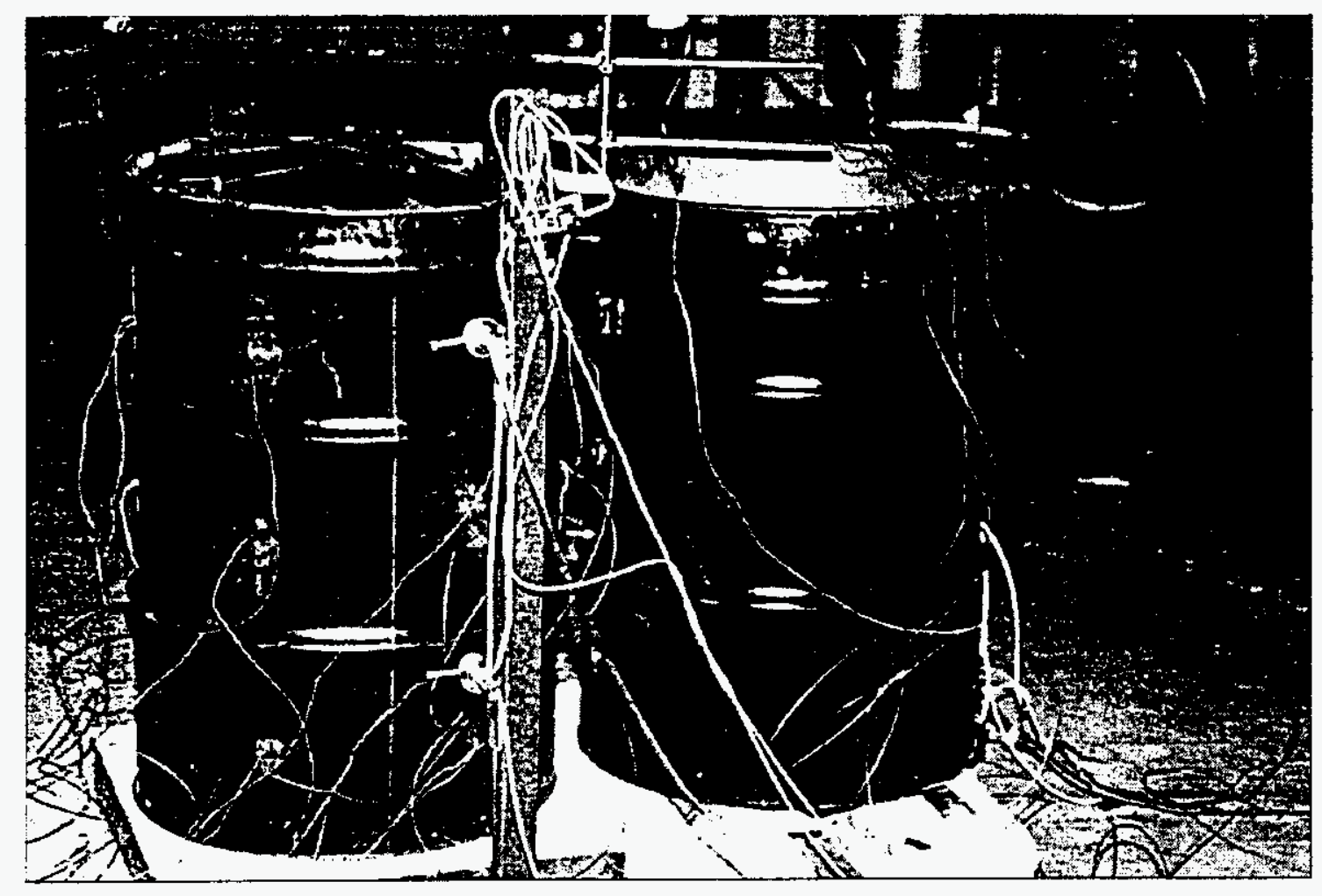

Test Arrangement

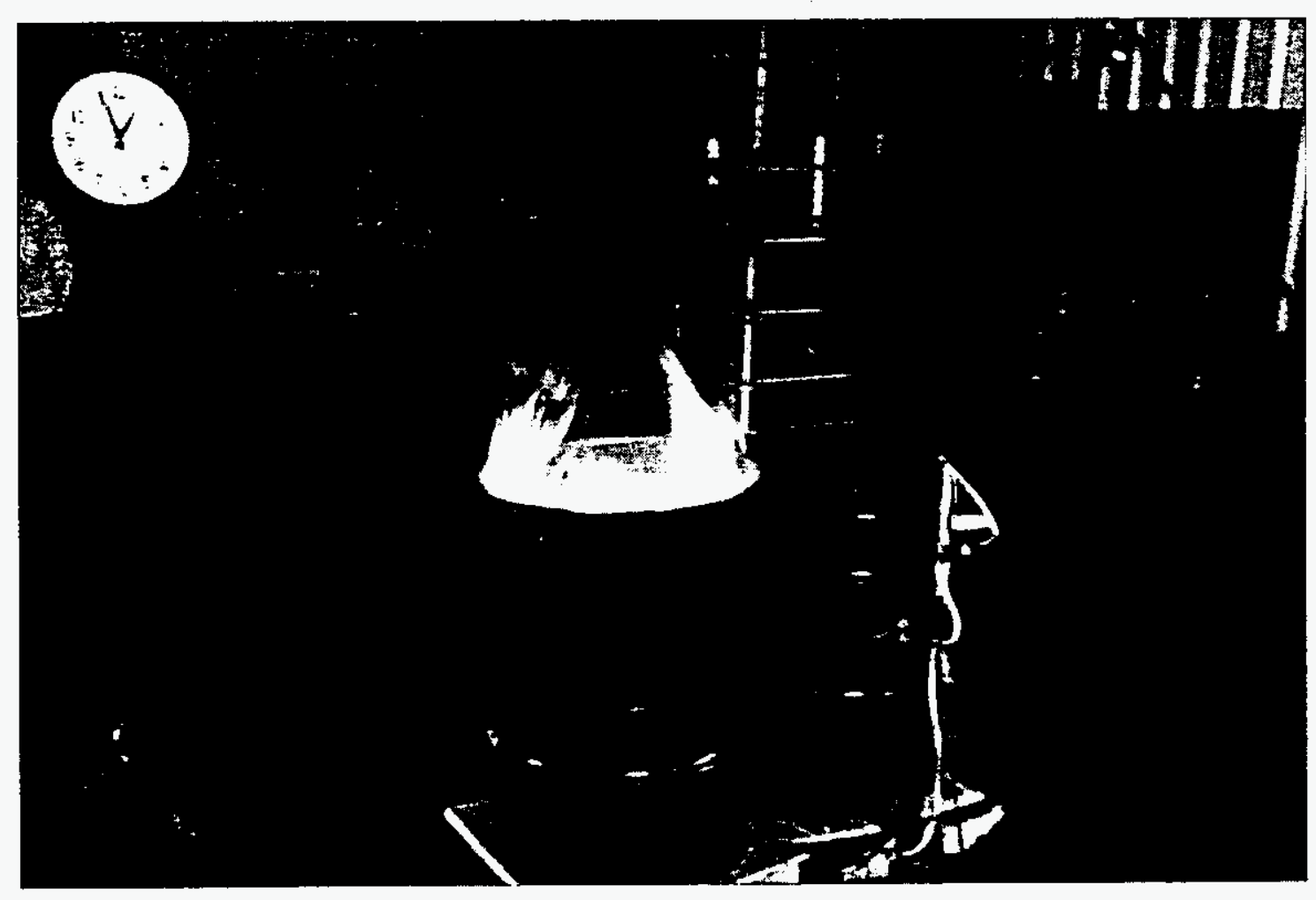

Test in Progress 

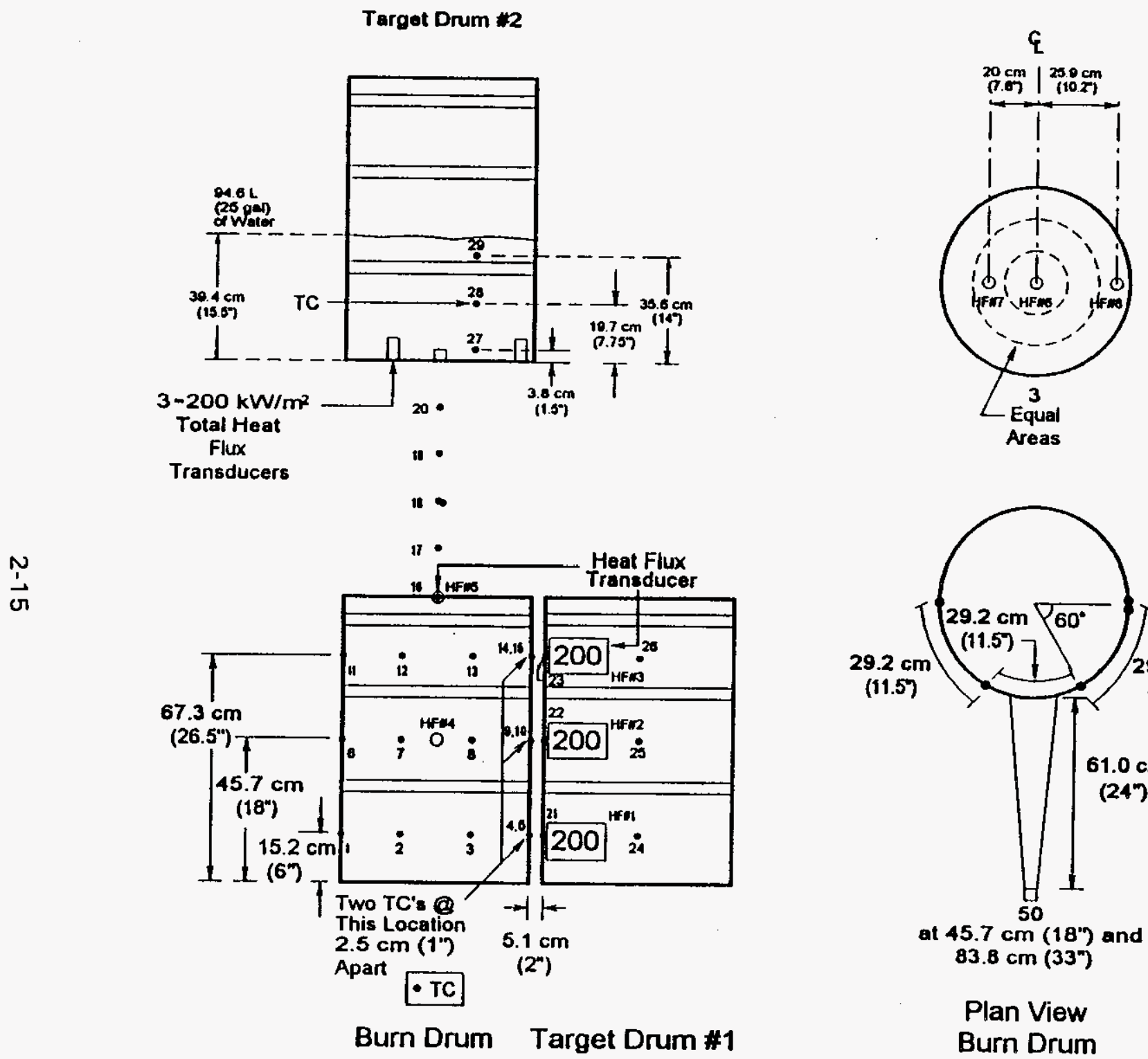

\section{Plan View \\ Target \\ \#2 Drum}

HF*1 - Not Used

HF\#2 - Ser. No. 72723

HF\#3 - Ser. No. 86901

HF\#4 - Ser. No. 86903

HF\#5 - Ser. No. 86904

HF $\$ 6$ - Ser. No. 72727

HF\#7 - Ser. No. 72724

HF\#8 - Ser. No. 86902

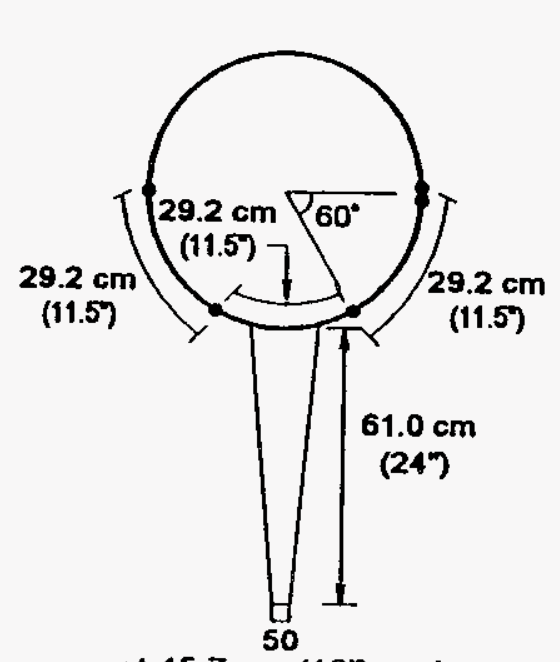

TC *21, $\# 22, \# 23$

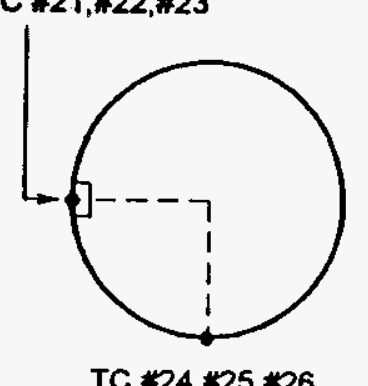

Plan View

Target

\#1 Drum

Plan View

Burn Drum

Figure 5. Test Arrangement for Stacked Drum Tests 


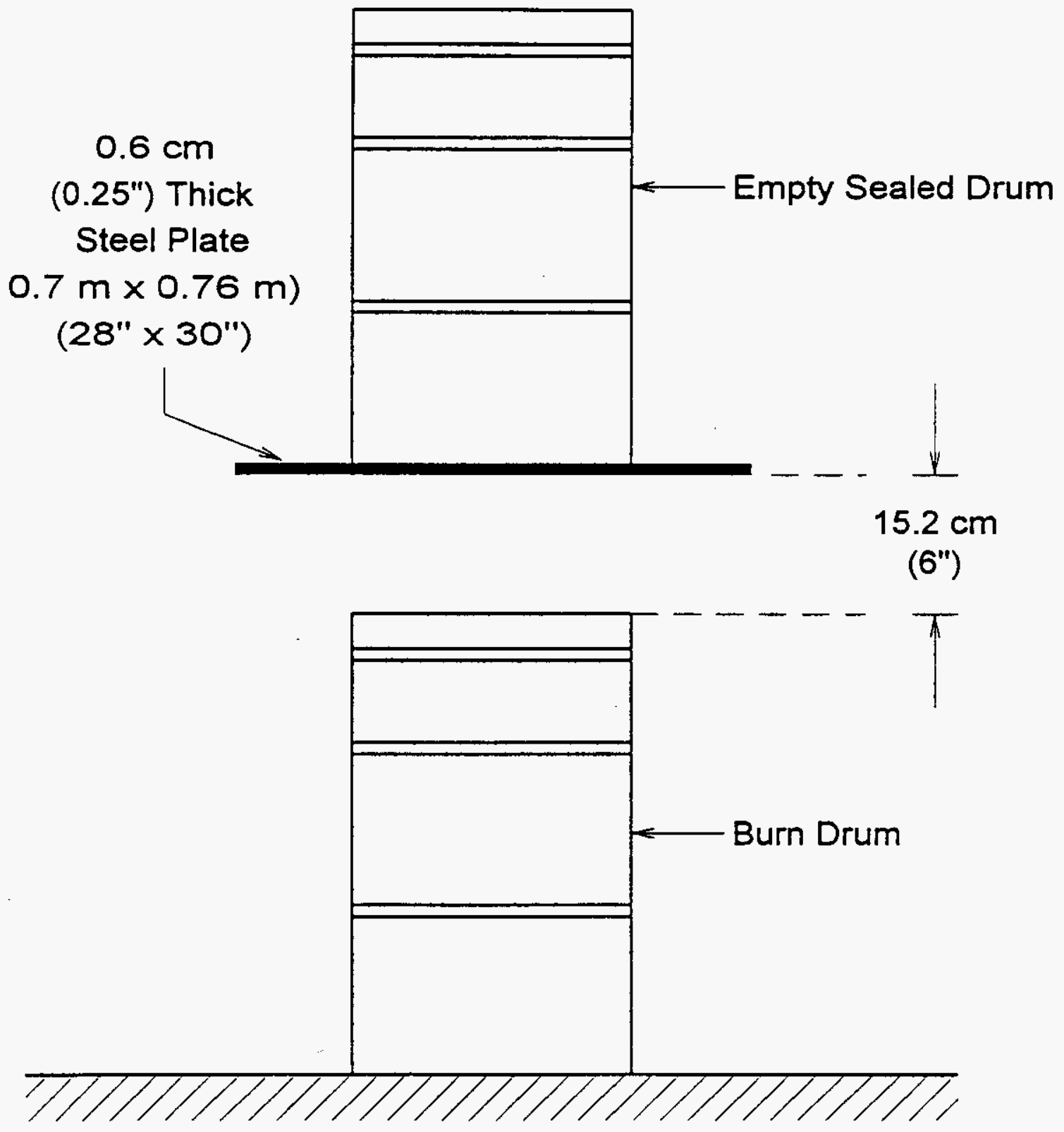

Figure 6. Test Arrangement for Test No. SWD 18, Stacked Drum Test 


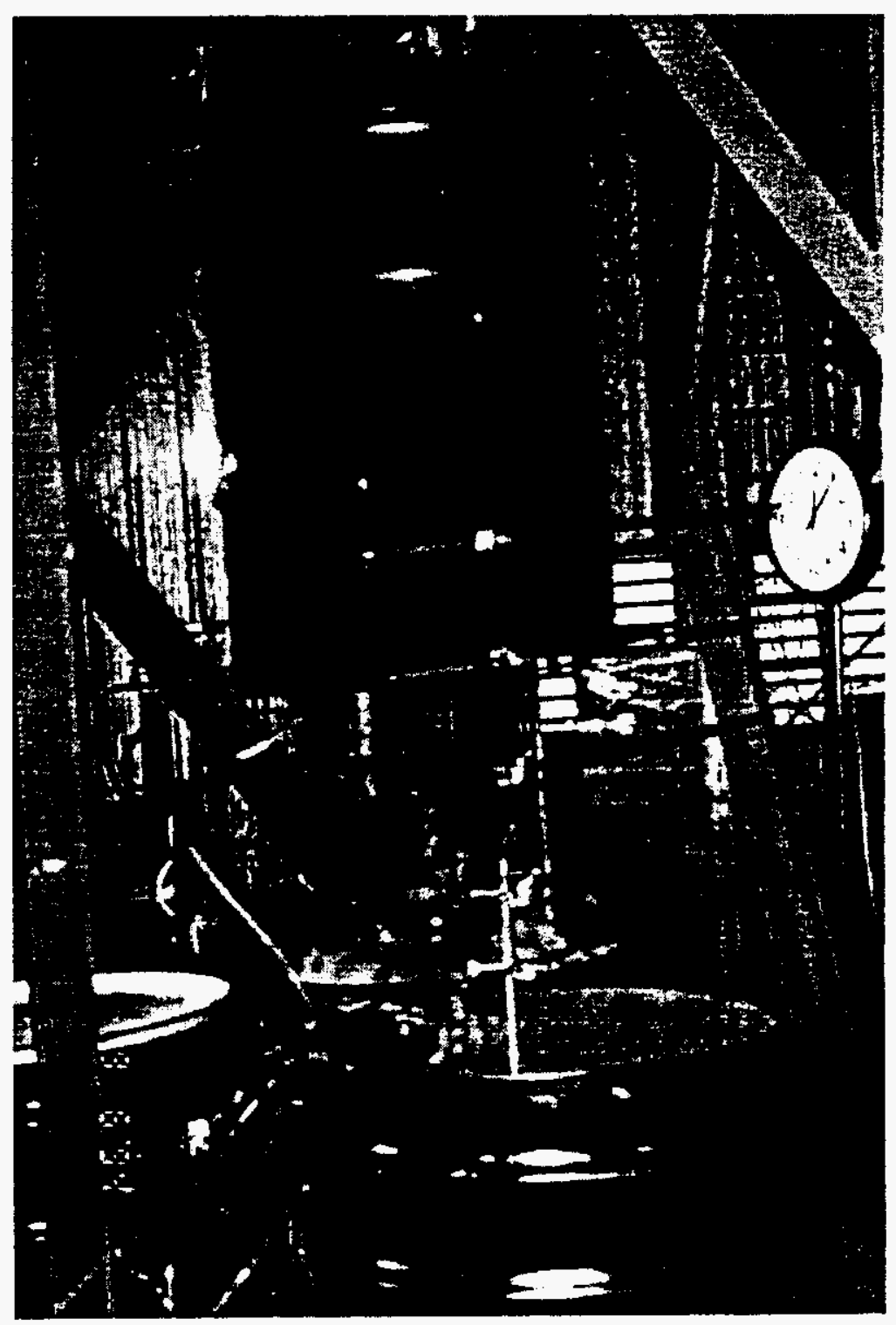

Figure 7. Vertical Single Drum Test in Progress 
The fire exposures were constructed such that each provided a different level of heat input to the exposed drums. This was accomplished by not only varying the size of the fire, but also changing the exposed surface area of the drums. The configuration and test descriptions for each of the different fire exposures are described in the following sections.

\subsubsection{Adjacent Fire Tests - $0.48 \mathrm{~m}$ Propane Burner}

The first exposure fire consisted of a 0.48 by $0.48 \mathrm{~m}$ ( 19 by 19 in.) sand-filled propane fired gas burner. The burner was fired at a fuel flow rate of $0.354 \mathrm{~m}^{3} / \mathrm{min}$. $\left(12.5 \mathrm{ft}^{3} / \mathrm{min}\right.$. $)$. For a heat of combustion and density of $46.5 \mathrm{~kJ} / \mathrm{g}$ and $1.8 \mathrm{~kg} / \mathrm{m}^{3}$, this provided a theoretical heat output of approximately $500 \mathrm{~kW}$.

The four drums were arranged as shown in Figure 8. Drum No. 1 and Drum No. 4 were the test drums. Drum No. 2 was the target drum that contained the heat flux transducers, and Drum No. 3 was the water filled drum that was used to measure the integrated heat input to the drums. Figure 9 provides a diagram of the instrumentation arrangement used in these tests.

For all of the drum response tests, a pressure transducer and thermocouple were used to measure the inside drum pressure and temperature. The pressure and inside thermocouple tap location for tests SWD 19 and SWD 24 was located in the center on the bottom of the drum. The internal pressure port was formed from a u-shaped piece of copper tubing positioned so the open end faced the bottom of the drum. This orientation prevented the drum contents from blocking the opening. The inside thermocouple was located in the center of the drum. The pressure and inside thermocouple tap location for tests SWD 25 and SWD 37 was located on the side of the drum $70 \mathrm{~cm}$ from the base. The change in location was needed in order to allow the drums to be placed on the larger fire exposure test apparatus. All drums were pressure checked to $34.5 \mathrm{kPa}(5 \mathrm{psig})$ and allowed to equilibrate to atmospheric pressure just prior to ignition of the fire. This procedure ensured seal integrity of the drums.

For the $0.48 \mathrm{~m}$ tests, the drums were arranged such that they were touching the sides of the sand burner. In essence, approximately $1 / 4$ or less of the surface area of the drum walls was directly exposed to the fire source.

Table 5 provides the test matrix for the $0.48 \mathrm{~m}$ square propane burner tests. Several drum loadings were tested as to determine the effect contents have on drum response. Test Nos. SWD 19 and 20 were performed to provide a range of potential contents to the fire. Test Nos. SWD 21,22, 23, 26 and 27 were various fuel loads that also incorporated small amounts of flammable liquids. These tests were performed to determine a potentially "worst case" for lid loss. Test Nos. SWD 24, 25, 26, and 27 were performed with the EPDM gasket seals instead of the sponge rubber seals. Also, Test Nos. SWD 25 and 26 had square shaped closure rings instead of the usual rounded closure ring. Figure 10 provides a photograph of Test No. SWD 20 in progress. 
WHC-SD-WM-TRP-233 REV. O

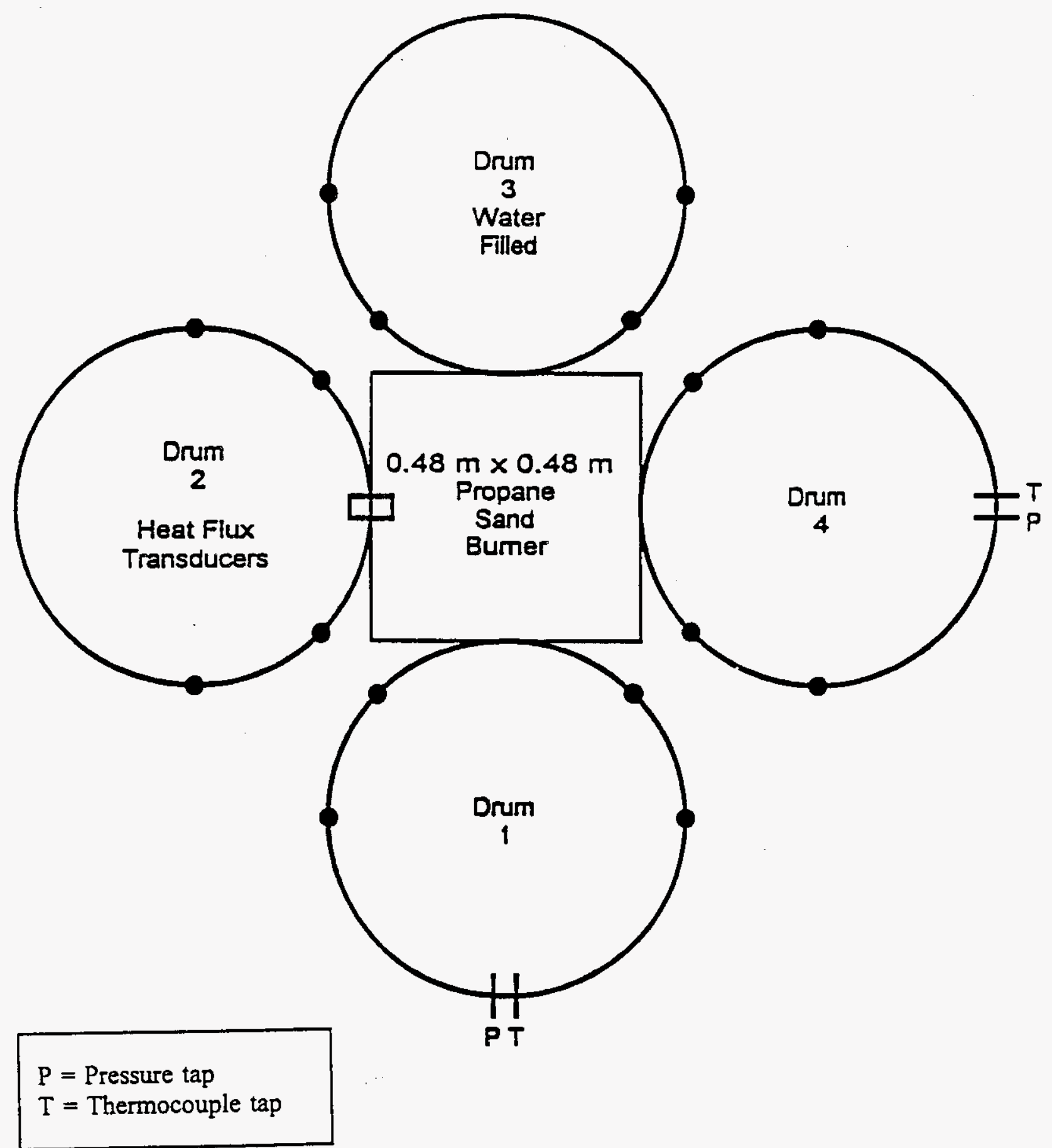

Figure 8. $0.48 \mathrm{~m}$ Burner and Drum Arrangement 
WHC-SD-WM-TRP-233 REV. O
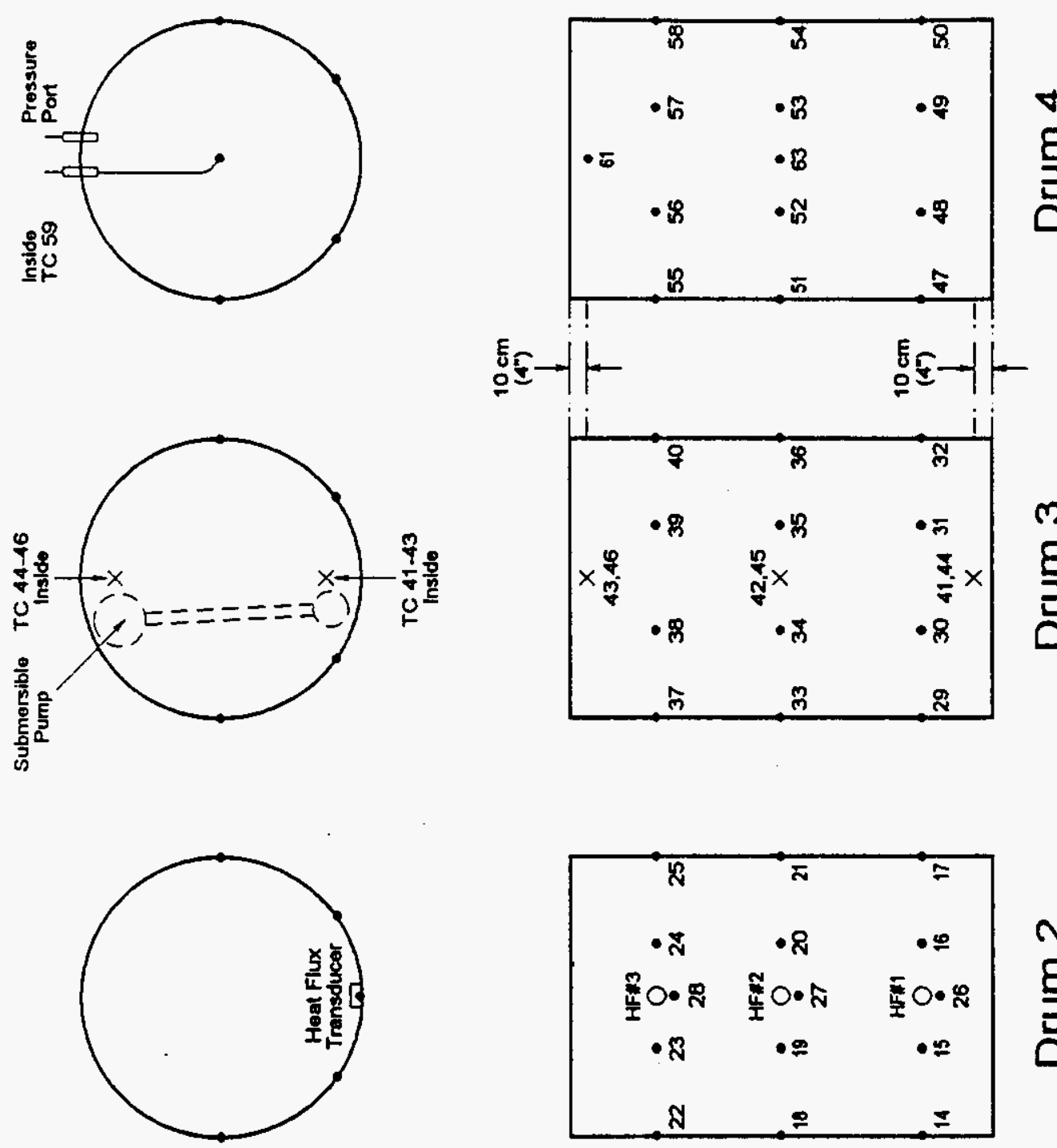

동

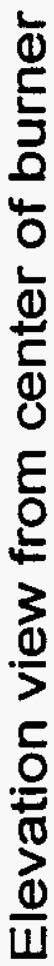
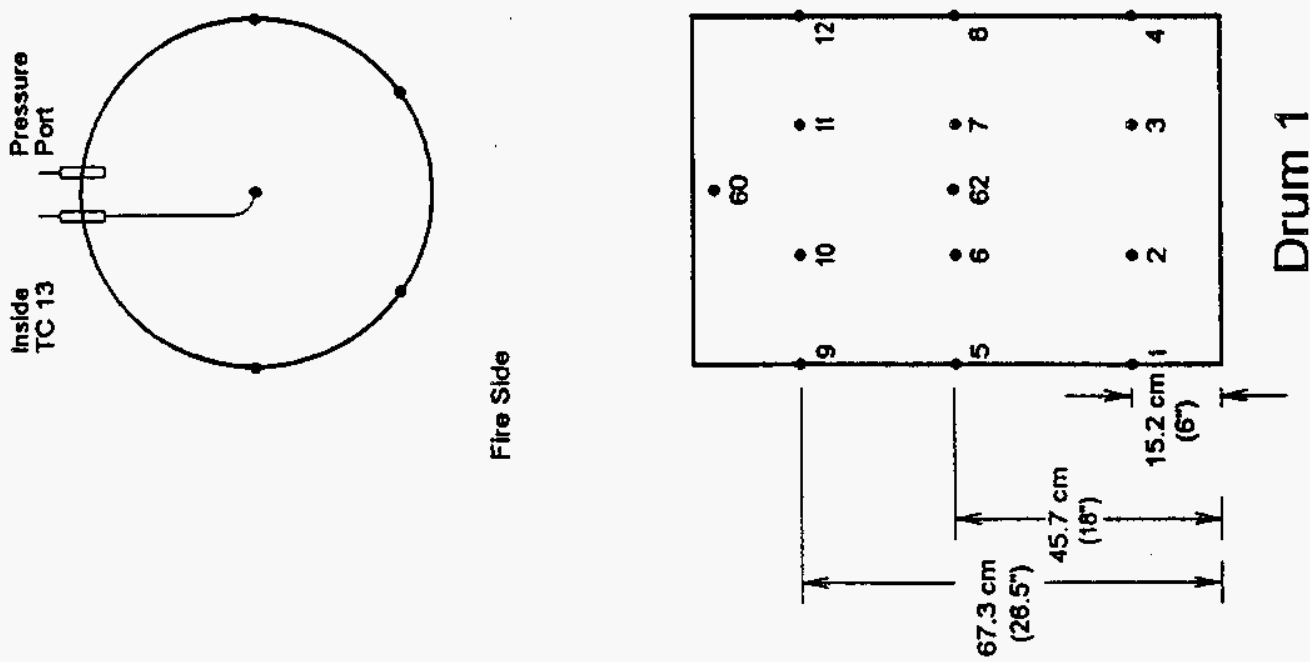

옹

$\frac{0}{5}$
$\frac{0}{14}$ 
WHC-SD-WM-TRP-233 REV. O

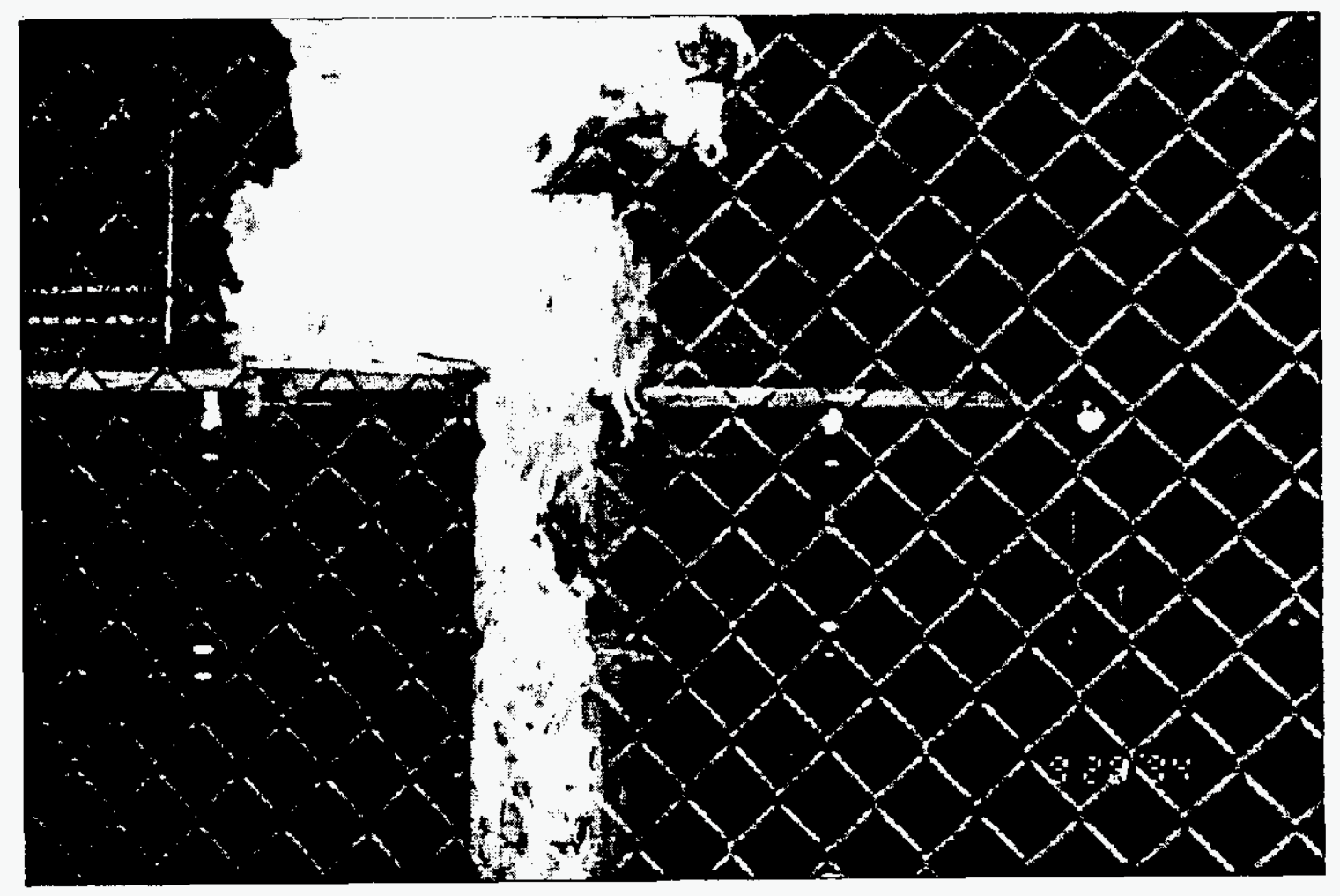

BEST AVAILABLE COPY

Figure 10. SWD 20 - In Progress 
Table 5. Adjacent Fire Exposure - $0.48 \mathrm{~m}$ Propane Burner - Test Matrix $(2$ sheets).

\begin{tabular}{|c|c|c|c|c|}
\hline Test ID & $\begin{array}{l}\text { Drum } \\
\text { ID }\end{array}$ & Drum Contents & Exposure & Comments \\
\hline SWD 19 & $\begin{array}{l}1 \\
4\end{array}$ & $\begin{array}{l}\text { Standard load } \\
\text { Empty }\end{array}$ & $0.48 \mathrm{~m}$ burner & \\
\hline SWD 20 & $\begin{array}{l}1 \\
4\end{array}$ & $\begin{array}{l}2.3 \mathrm{~kg}(5 \mathrm{lb}) \text { brown paper } \\
2.3 \mathrm{~kg}(5 \mathrm{lb}) 10 \text { mil plastic }\end{array}$ & $0.48 \mathrm{~m}$ burner & \\
\hline SWD 21 & 4 & $\begin{array}{l}2.3 \mathrm{~kg}(5 \mathrm{lb}) \text { brown paper and } \\
0.2 \mathrm{~kg}(0.5 \mathrm{lb}) \text { of cotton towels } \\
\text { soaked with } 50 \mathrm{~g} \text { of heptane; } \\
\text { towels placed mid-height next to } \\
\text { fire exposed side } \\
\text { Empty }\end{array}$ & $0.48 \mathrm{~m}$ burner & \\
\hline SWD 22 & $\begin{array}{l}1 \\
4\end{array}$ & $\begin{array}{l}\text { Standard load - bagged with } 50 \\
\mathrm{~g} \text { of acetone poured on rags } \\
\text { placed on top of the load } \\
2.3 \mathrm{~kg}(5 \mathrm{lb}) \text { brown paper and } \\
0.14 \mathrm{~kg}(0.3 \mathrm{lb}) \text { of cotton towels } \\
\text { soaked with } 50 \mathrm{~g} \text { of acetone; } \\
\text { towels place near bottom of } \\
\text { drum and against the side away } \\
\text { from the fire }\end{array}$ & $0.48 \mathrm{~m}$ burner & \\
\hline SWD 23 & 4 & $\begin{array}{l}2.3 \mathrm{~kg}(5 \mathrm{lb}) \text { brown paper and } \\
0.14 \mathrm{~kg}(0.3 \mathrm{lb}) \text { of cotton towels } \\
\text { soaked with } 50 \mathrm{~g} \text { of heptane; } \\
\text { towels placed near bottom of } \\
\text { drum and against the side away } \\
\text { from the fire } \\
2.3 \mathrm{~kg}(5 \mathrm{lb}) \text { brown paper and } \\
0.18 \mathrm{~kg}(0.4 \mathrm{lb}) \text { of cotton towels } \\
\text { soaked with } 50 \mathrm{~g} \text { of acetone; } \\
\text { towels placed mid-height next to } \\
\text { fire exposed side }\end{array}$ & $0.48 \mathrm{~m}$ burner & \\
\hline SWD 24 & $\begin{array}{l}1 \\
4 \\
\end{array}$ & $\begin{array}{l}\text { Standard load } \\
\text { Empty }\end{array}$ & $0.48 \mathrm{~m}$ burner & $\begin{array}{l}\text { with EPDM gasket seal } \\
\text { with EPDM gasket seal }\end{array}$ \\
\hline SWD 25 & 1 & $\begin{array}{l}2.3 \mathrm{~kg}(5 \mathrm{lb}) \text { brown paper } \\
2.3 \mathrm{~kg}(5 \mathrm{lb}) 10 \text { mil plastic }\end{array}$ & $0.48 \mathrm{~m}$ burner & $\begin{array}{l}\text { square backed closure } \\
\text { ring and EPDM gasket } \\
\text { seals } \\
\text { square backed closure } \\
\text { ring and EPDM gasket } \\
\text { seals }\end{array}$ \\
\hline
\end{tabular}


Table 5. Adjacent Fire Exposure - $0.48 \mathrm{~m}$ Propane Burner - Test Matrix (2 sheets).

\begin{tabular}{|c|c|c|c|c|}
\hline Test ID & $\begin{array}{c}\text { Drum } \\
\text { ID }\end{array}$ & Drum Contents & Exposure & Comments \\
\hline SWD 26 & 1 & $\begin{array}{l}2.3 \mathrm{~kg}(5 \mathrm{lb}) \text { brown paper and } \\
0.18 \mathrm{~kg}(0.4 \mathrm{lb}) \text { of cotton towels } \\
\text { soaked with } 100 \mathrm{~g} \text { of toluene; } \\
\text { towels placed mid-height next to } \\
\text { fire exposed side } \\
\text { Standard load - bagged with } \\
100 \mathrm{~g} \text { of toluene poured over the } \\
\text { load }\end{array}$ & $0.48 \mathrm{~m}$ burner & $\begin{array}{l}\text { square backed closure } \\
\text { ring and EPDM.gasket } \\
\text { seals } \\
\text { square backed closure } \\
\text { ring and EPDM gasket } \\
\text { seals }\end{array}$ \\
\hline SWD 27 & 1 & $\begin{array}{l}2.3 \mathrm{~kg}(5 \mathrm{lb}) \text { brown paper and } \\
0.18 \mathrm{~kg}(0.4 \mathrm{lb}) \text { of cotton towels } \\
\text { soaked with } 100 \mathrm{~g} \text { of toluene; } \\
\text { towels placed mid-height next to } \\
\text { fire exposed side } \\
\text { Standard load - bagged with } \\
100 \mathrm{~g} \text { of toluene poured over the } \\
\text { load }\end{array}$ & $0.48 \mathrm{~m}$ burner & $\begin{array}{l}\text { with EPDM gasket seal } \\
\text { with EPDM gasket seal }\end{array}$ \\
\hline
\end{tabular}

\subsubsection{Adjacent Fire Tests - 1.2 m Propane Burner}

In Test Nos. SWD 28, and $33-36$, the exposing fire was a 1.2 by $1.2 \mathrm{~m}$ ( 4 by $4 \mathrm{ft}$ ) water-filled propane fired burner. Figure 11 provides a sketch of this burner. Target drums were placed on top of the burner such that the fuel flowed up and around the sides of the drums. The burner was fired at a flow rate of $0.708 \mathrm{~m}^{3} / \mathrm{min}\left(25 \mathrm{ft}^{3} / \mathrm{min}\right)$. For a heat of combustion and density of $46.5 \mathrm{~kJ} / \mathrm{g}$ and $1.8 \mathrm{~kg} / \mathrm{m}^{3}$, this provided a theoretical heat output of approximately $1 \mathrm{MW}$.

Figure 12 shows the drum arrangement for the $1.2 \mathrm{~m}$ propane burner fire exposure tests. Test SWD 28 differed from this arrangement in that the drums were positioned at the sides of the burner (i.e., rotated $45^{\circ}$ from Fig. 12 arrangement). The change in this arrangement increased the fire exposed area of the drums. In this series of tests, the drums were positioned over the burner such that a greater amount of wall surface area would be exposed to the fire compared to the $0.48 \mathrm{~m}$ tests. In general, approximately $1 / 2$ of each of the target drum's surface area was exposed to the fire. The instrumentation arrangement was the same as for the $0.48 \mathrm{~m}$ propane burner tests (Figure 9). Table 6 provides the test matrix for the $1.2 \mathrm{~m}$ square propane burner tests.

Test Nos. SWD 28 and 33 were evaluations of a range of potential drum loadings. Test Nos. SWD 34 - 36 were various fuel loads that, in some cases, also incorporated small amounts of flammable liquids. These tests were performed to determine a potentially "worst case" for lid loss. Figure 13 provides a photograph of Test No. SWD 28 in progress. 
WHC-SD-WM-TRP-233 REV. 0
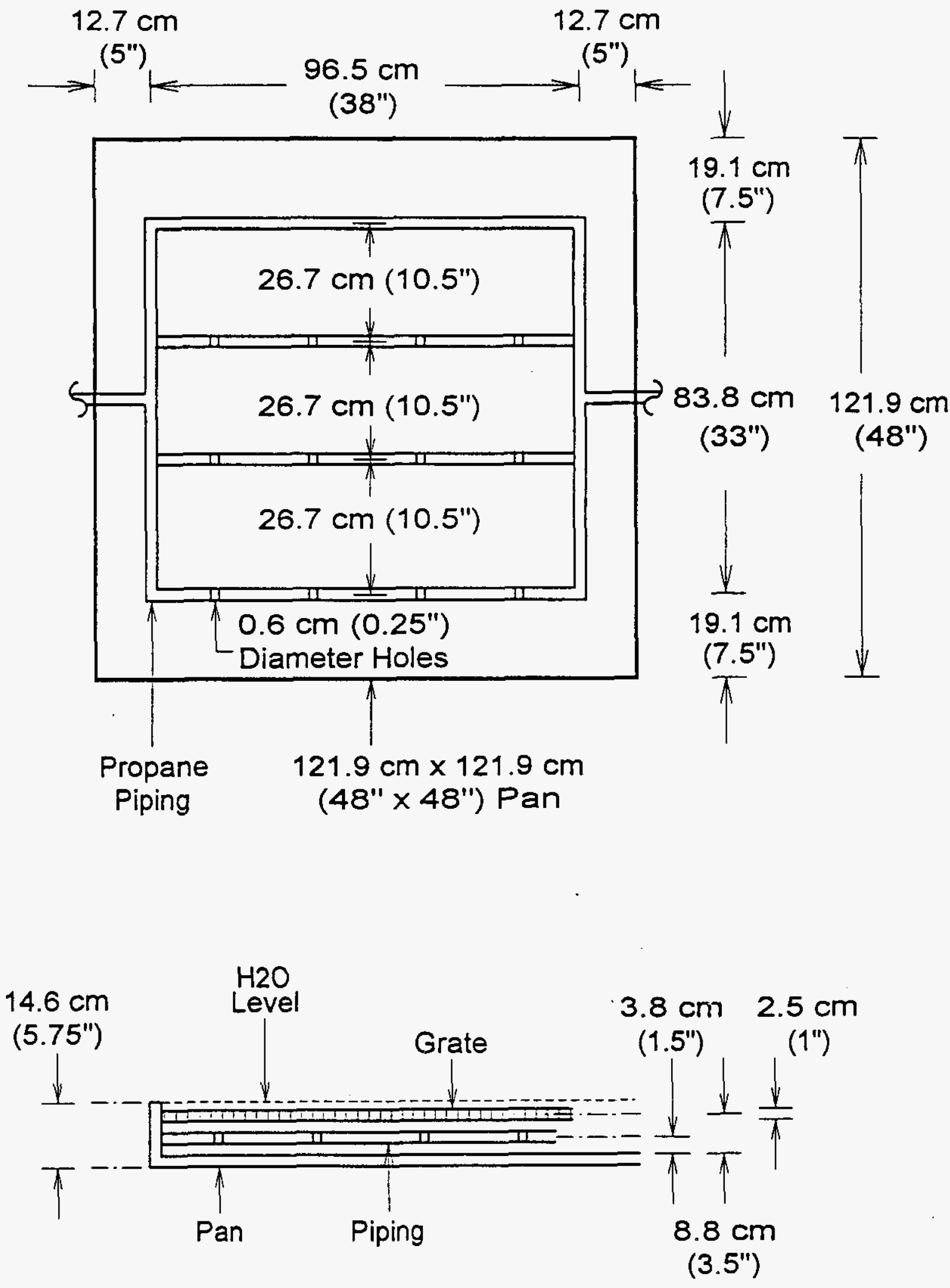

Figure 11. $1.2 \mathrm{~m}$ Water-filled Propane Burner Setup 


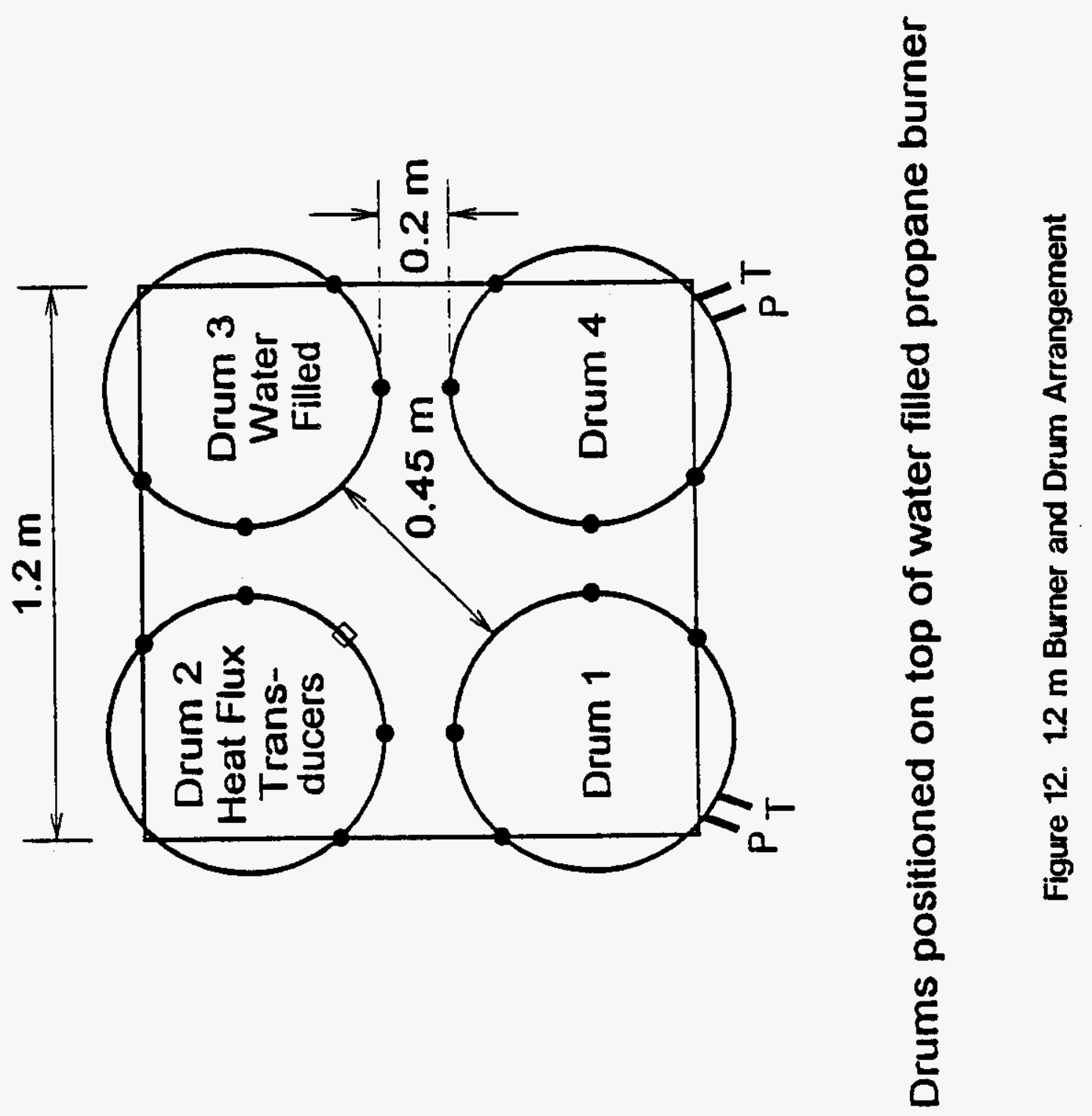


WHC-SD-WM-TRP-233 REV. 0

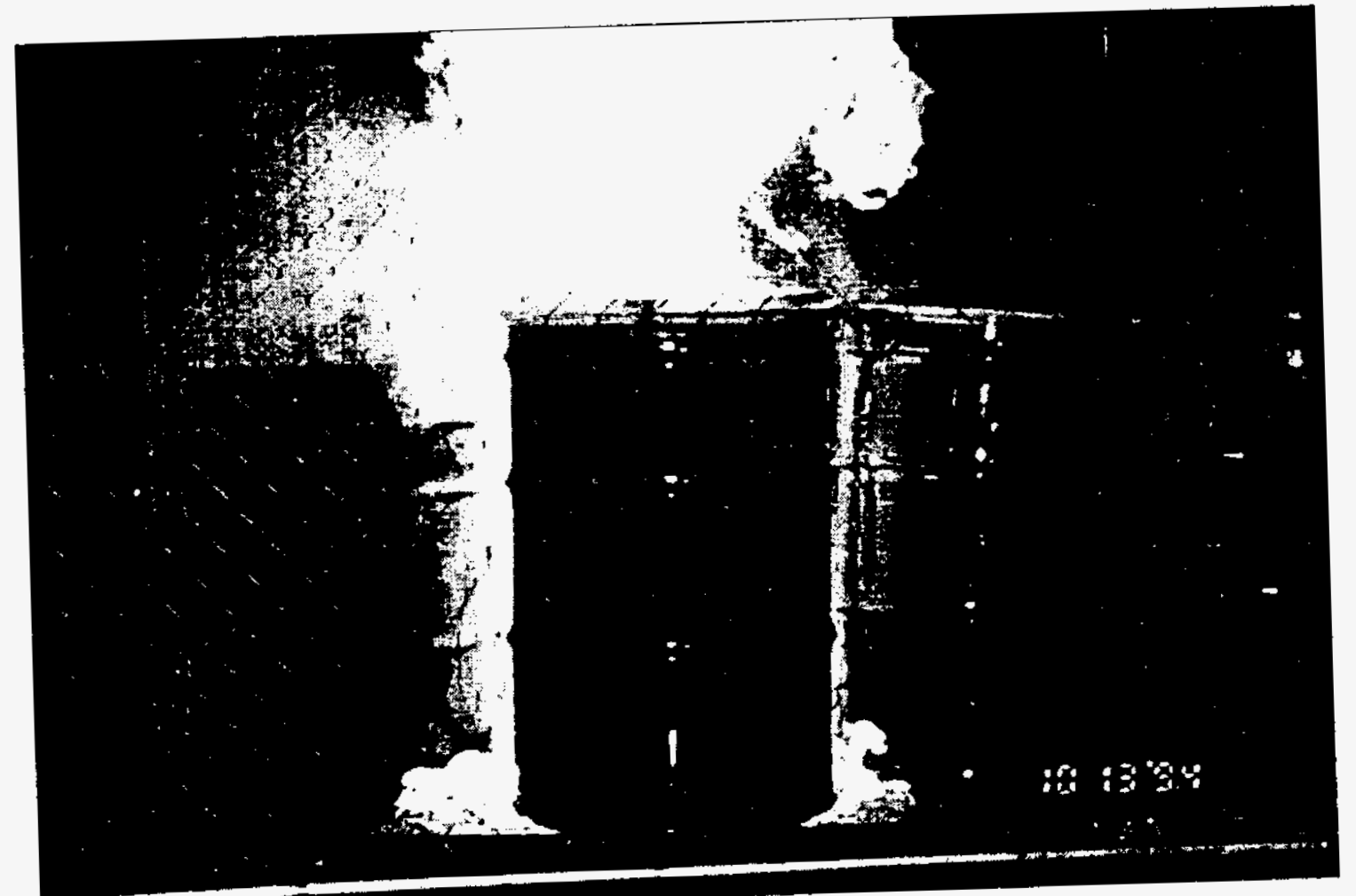

\section{BEST AVAILABLE COPY}

Figure 13. SWD 28 - In Progress 
Table 6. Adjacent Fire Exposure - $1.2 \mathrm{~m}$ Propane Burner - Test Matrix.

\begin{tabular}{|c|c|c|c|c|}
\hline Test ID & Drum ID & Drum Contents & Exposure & Comments \\
\hline SWD 28 & $\begin{array}{l}1 \\
4\end{array}$ & $\begin{array}{l}2.3 \mathrm{~kg}(5 \mathrm{lb}) \text { brown paper } \\
2.3 \mathrm{~kg}(5 \mathrm{lb}) 10 \mathrm{mil} \text { plastic }\end{array}$ & $1.2 \mathrm{~m}$ burner & \\
\hline SWD 33 & $\begin{array}{l}1 \\
4\end{array}$ & $\begin{array}{l}2.3 \mathrm{~kg}(5 \mathrm{lb}) \text { brown paper } \\
2.3 \mathrm{~kg}(5 \mathrm{lb}) 10 \text { mil plastic }\end{array}$ & $1.2 \mathrm{~m}$ burner & \\
\hline SWD 34 & 4 & $\begin{array}{l}2.3 \mathrm{~kg}(5 \mathrm{lb}) \text { brown paper and } \\
0.18 \mathrm{~kg}(0.4 \mathrm{lb}) \text { of cotton } \\
\text { towels soaked with } 50 \mathrm{~g} \text { of } \\
\text { heptane; towels placed mid- } \\
\text { height next to fire exposed side } \\
\text { Empty }\end{array}$ & $1.2 \mathrm{~m}$ burner & \\
\hline SWD 35 & 4 & $\begin{array}{l}2.3 \mathrm{~kg}(5 \mathrm{lb}) \text { brown paper and } \\
0.14(0.3 \mathrm{lb}) \text { of cotton towels } \\
\text { soaked with } 50 \mathrm{~g} \text { of heptane; } \\
\text { towels placed on top and next } \\
\text { to fire exposed side } \\
\text { Standard load }\end{array}$ & $1.2 \mathrm{~m}$ burner & $\cdot$ \\
\hline SWD 36 & 1 & $\begin{array}{l}2.3 \mathrm{~kg}(5 \mathrm{lb}) \text { brown paper and } \\
0.18 \mathrm{~kg}(0.4 \mathrm{lb}) \text { of cotton } \\
\text { towels soaked with } 100 \mathrm{~g} \text { of } \\
\text { toluene; towels placed mid- } \\
\text { height next to fire exposed side } \\
\text { Standard load - bagged with } \\
100 \mathrm{~g} \text { of toluene poured over } \\
\text { the load }\end{array}$ & $1.2 \mathrm{~m}$ burner & \\
\hline SWD 37 & $\begin{array}{l}1 \\
4\end{array}$ & $\begin{array}{l}2.3 \mathrm{~kg}(5 \mathrm{lb}) \text { brown paper } \\
2.3 \mathrm{~kg}(5 \mathrm{lb}) \text { brown paper }\end{array}$ & trash fire & \\
\hline
\end{tabular}

\subsubsection{Adjacent Fire Tests - Trash Fire}

The third type of exposure fire that was used in this series was a trash fire. Figure 14 provides a sketch of the test arrangement used in Test No. SWD 37 . In this test, approximately 1.5 standard loads were spread between the drums. The use of 1.5 standard loads was chosen as the fuel source as it approximately represents the case of the four surrounding drums (i.e., surrounding target drums 1 and 4) having ejected one-third of their contents (i.e., the maximum amount observed to have ejected from a standard load drum experiencing lid loss failurel. The test materials were ignited via a small amount of heptane poured over the simulated trash. Figure 15 provides photographs of Test No. SWD 37. 


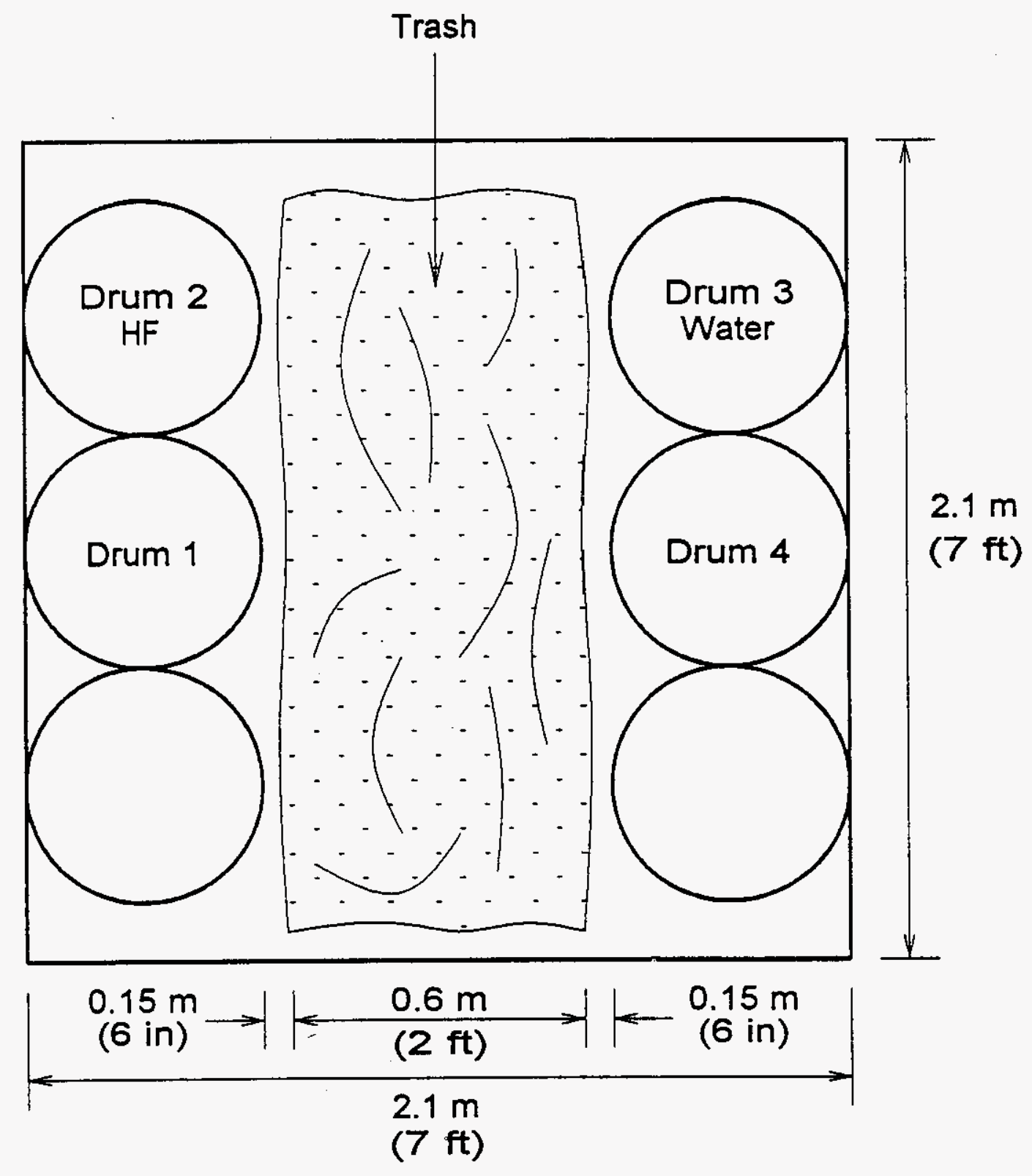

Figure 14. Trash Fire Exposure Test Setup 
WHC-SO-WM-TRP-233 REV.O

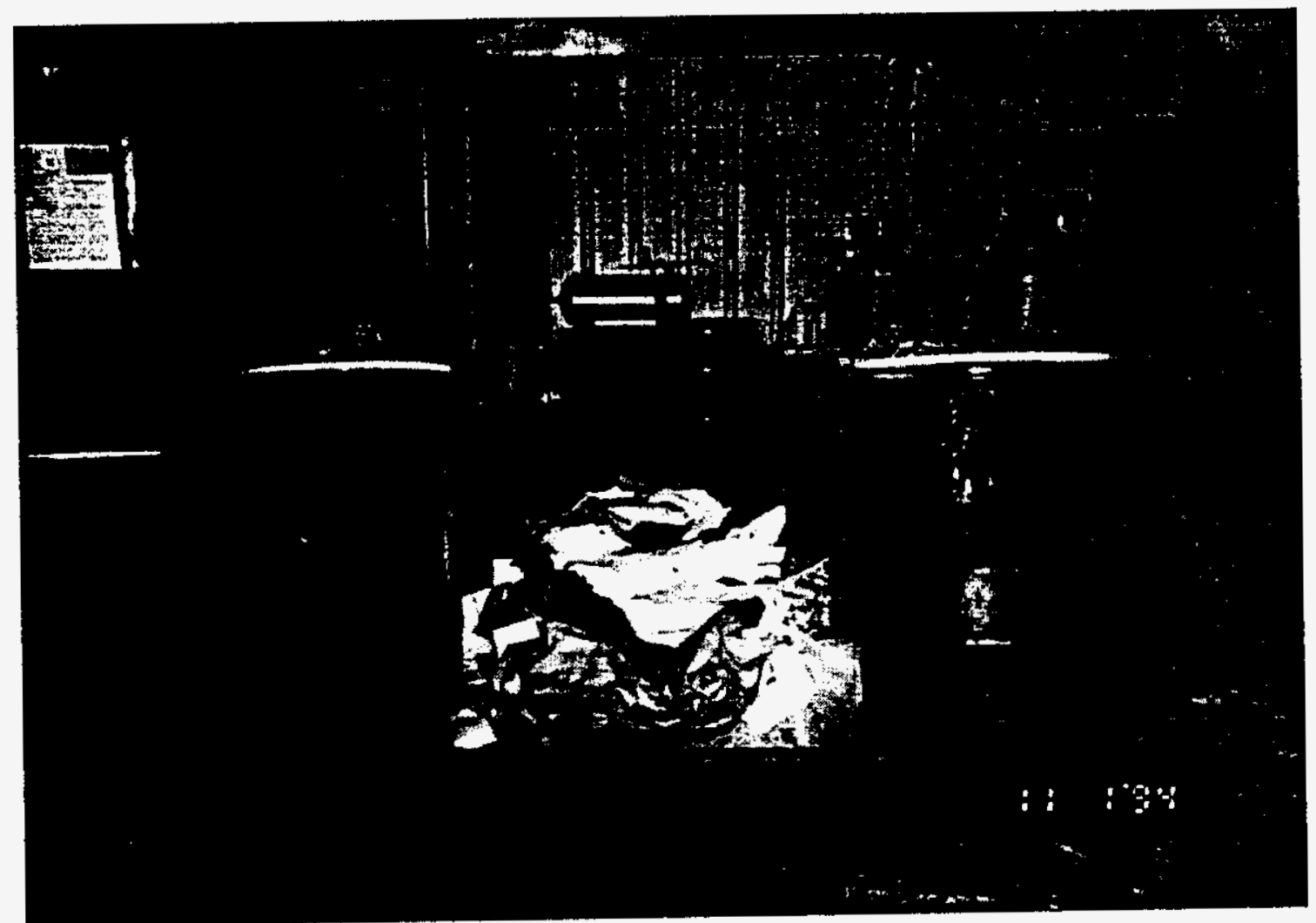

\section{SWD 37 - Pre-Test Set-Up}

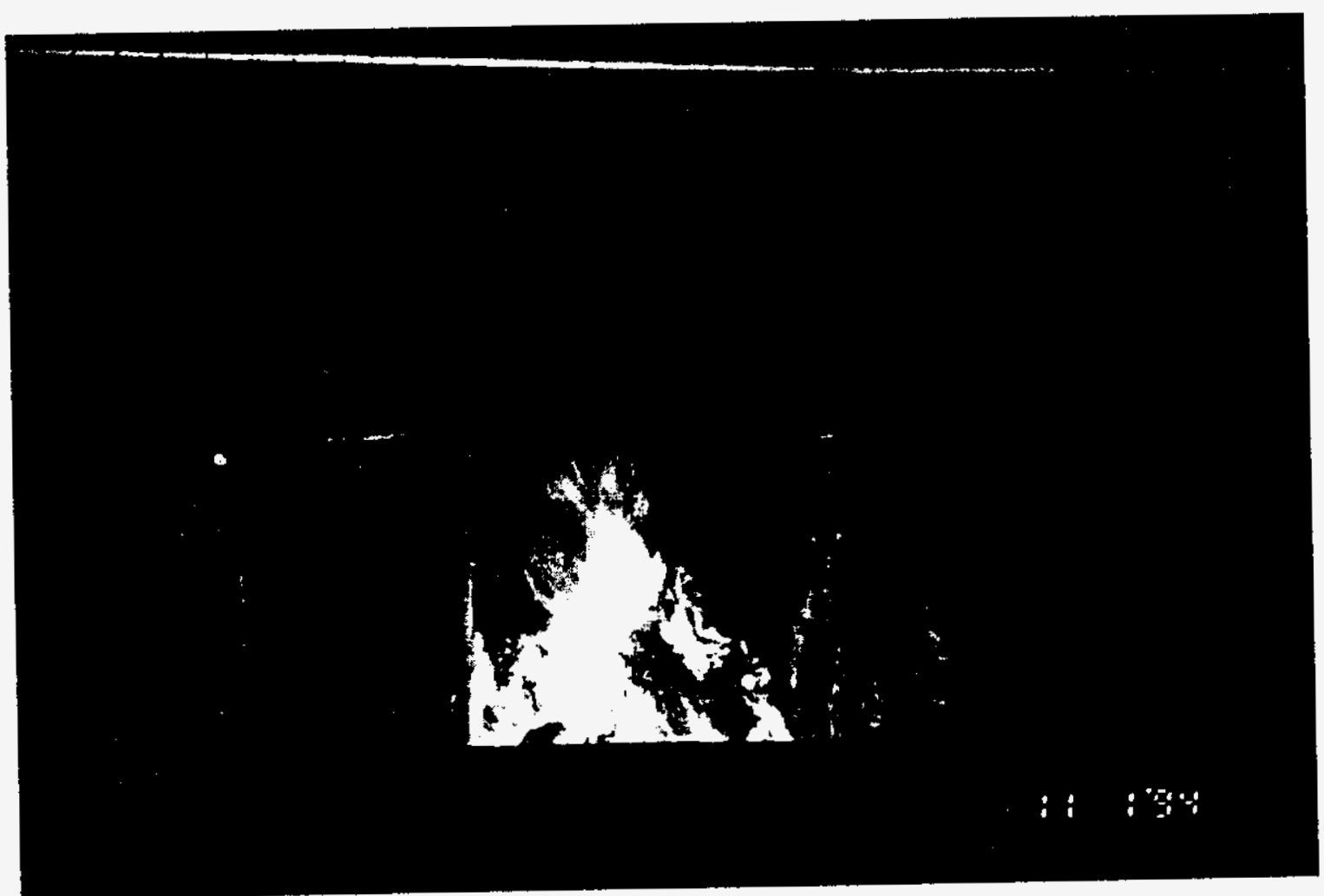

SWD 37 - Test in Progress

Figure 15. Trash Fire Exposure Test 


\subsubsection{Engulfing Fire Tests $-2.1 \mathrm{~m}$ Pool Fire}

In this series of tests, the target drums were positioned inside a 2.1 by $2.1 \mathrm{~m}$ (7 by 7 ft) pan. Figure 16 provides a diagram of the test arrangement, and Figure 9 provides the general instrumentation arrangement used in these tests, except that the exterior drum wall thermocouple were positioned around the entire periphery of the drum. This thermocouple arrangement is shown in Figure 16. Thermocouples 60 and 62 on Drum No. 1 and Thermocouples No. 61 arid 63 on Drum No. 4 were positioned in line with the center of the burner. After the drums were positioned, the pan was filled with $75.8 \mathrm{~L}(20 \mathrm{gall}$ of JP-5 fuel which floated on top of $1.3 \mathrm{~cm}(0.5 \mathrm{in}$.) of water. JP-5 was chosen because of its similarity to diesel fuel. The mass loss and heat release rates of the two fuels do not differ significantly. Subtracting the area of the four drums $\left(0.292 \mathrm{~m}^{2}\right.$ each) yields a burn area of $3.38 \mathrm{~m}^{2}$. Using a burning rate of $0.039 \mathrm{~kg} / \mathrm{m}^{2}$-s and a heat of combustion of $40 \mathrm{MJ} / \mathrm{kg}$ [Babrauskas, 1988] for the fuel results in a theoretical heat output of approximately $5.3 \mathrm{MW}$. Table 7 provides the test matrix for the engulfing fire exposure tests.

Table 7. Engulfing Fire Exposure - Test Matrix.

\begin{tabular}{|c|c|l|l|l|}
\hline Test ID & Drum ID & \multicolumn{1}{|c|}{ Drum Contents } & Exposure & Comments \\
\hline SWD 29 & 1 & $\begin{array}{l}2.3 \mathrm{~kg}(5 \mathrm{lb}) \text { brown paper and 0.18 } \\
\mathrm{kg}(0.4 \mathrm{lb}) \text { of cotton towels soaked } \\
\text { with } 100 \mathrm{~g} \text { of toluene; towels placed } \\
\text { on top of load } \\
\text { Standard load - bagged with } 100 \mathrm{~g} \\
\text { of toluene poured on rags placed on } \\
\text { top of the load }\end{array}$ & Pool fire & \\
\hline SWD 30 & 1 & $\begin{array}{l}2.3 \mathrm{~kg}(5 \mathrm{lb}) \text { brown paper } \\
\text { Empty }\end{array}$ & Pool fire & \\
\hline SWD 31 & 1 & $\begin{array}{l}2.3 \mathrm{~kg}(5 \mathrm{lb}) 10 \text { mil plastic } \\
\text { Standard load }\end{array}$ & Pool fire & \\
\hline SWD 32 & 1 & $\begin{array}{l}2.3(5 \mathrm{lb}) \text { brown paper } \\
2.3(5 \mathrm{lb}) \text { plastic }\end{array}$ & Pool fire & $\begin{array}{l}\text { with vent } \\
\text { filter }\end{array}$ \\
\hline
\end{tabular}

Test Nos. SWD $29-32$ were evaluations of a range of potential drum loadings. Test No. SWD 29 incorporated small amounts of flammable liquids. This test was performed to determine a potentially "worst case" for lid loss. Test No. SWD 32 incorporated the use of the vent filters on the two combustible filled target drums. Figure 17 provides photographs of Test No. SWD 29.

\subsection{GASKET SEAL TESTS}

A series of tests was also conducted to evaluate the performance of the gasket seals with respect to various temperatures and pressures. A single $17-\mathrm{H}$ drum was used in each test. Figure 18 provides a photograph of the test arrangement. 


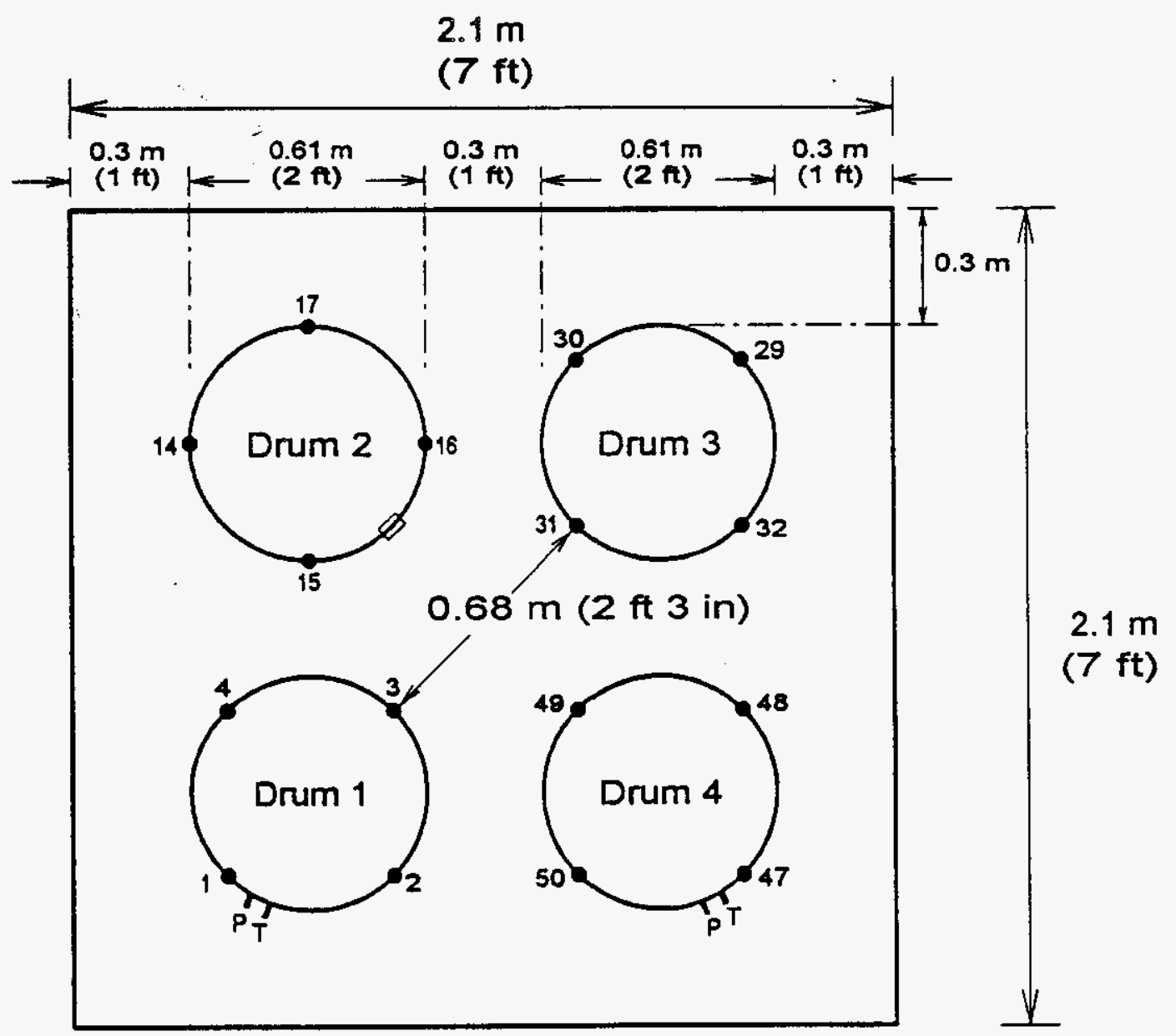

Drums in $2.1 \mathrm{~m}\left(84^{\prime \prime}\right) \times 2.1 \mathrm{~m}$ pool fire of JP-5 (Bottom row of TC's are shown for orientation)

Figure 16. $2.1 \mathrm{~m}$ JP-5 Pool Fire Setup 


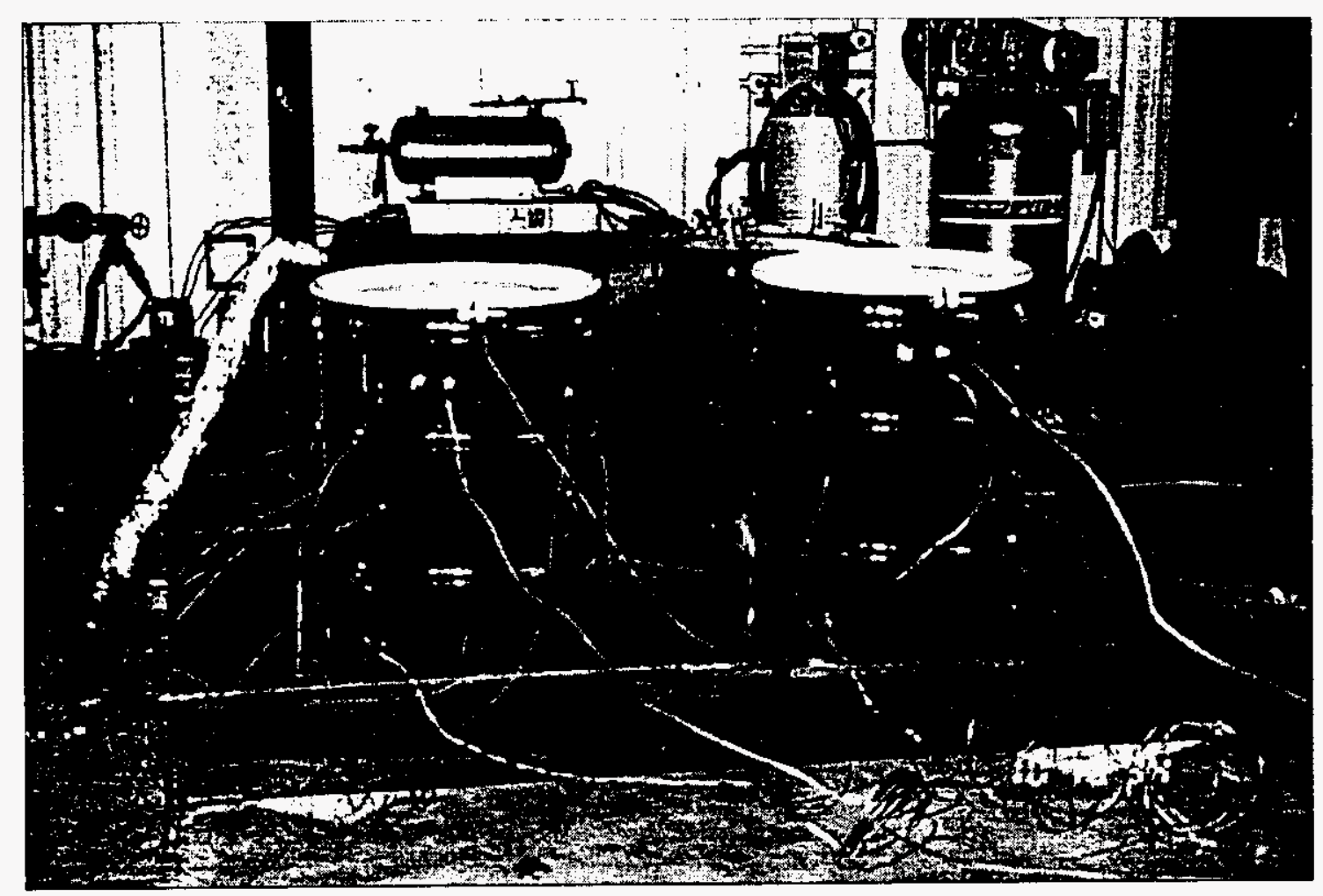

SWD 29 - Pre-Test

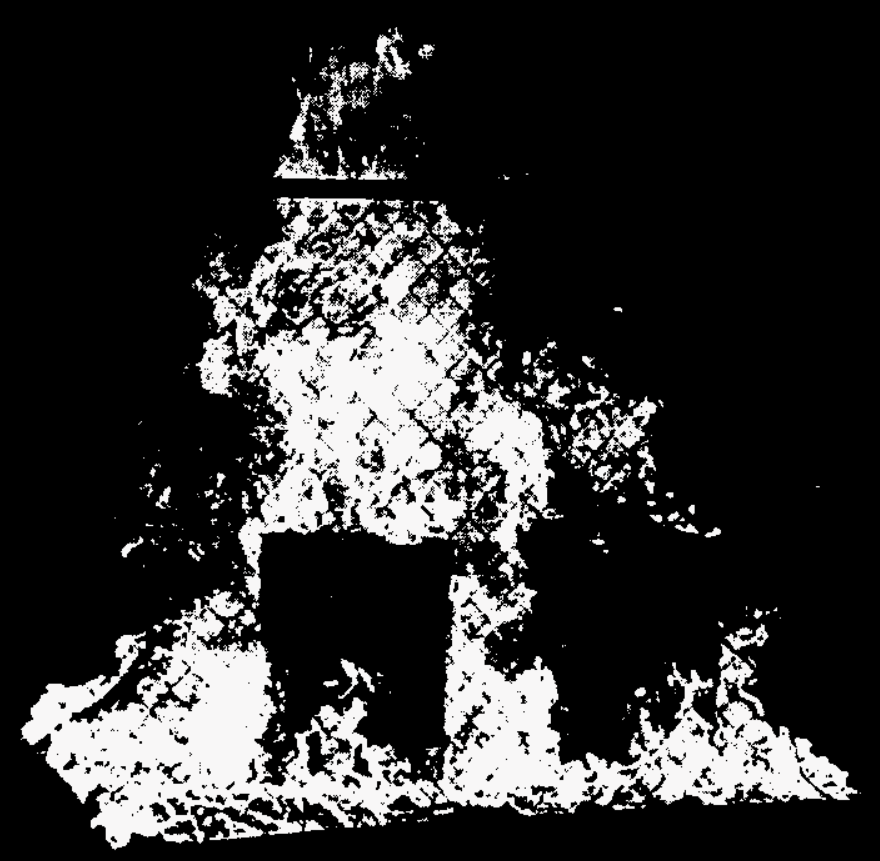

if ig.g.8

\section{SWD 29 - Test in Progress}

Figure 17. $2.1 \mathrm{~m}$ Pool Fire Test 


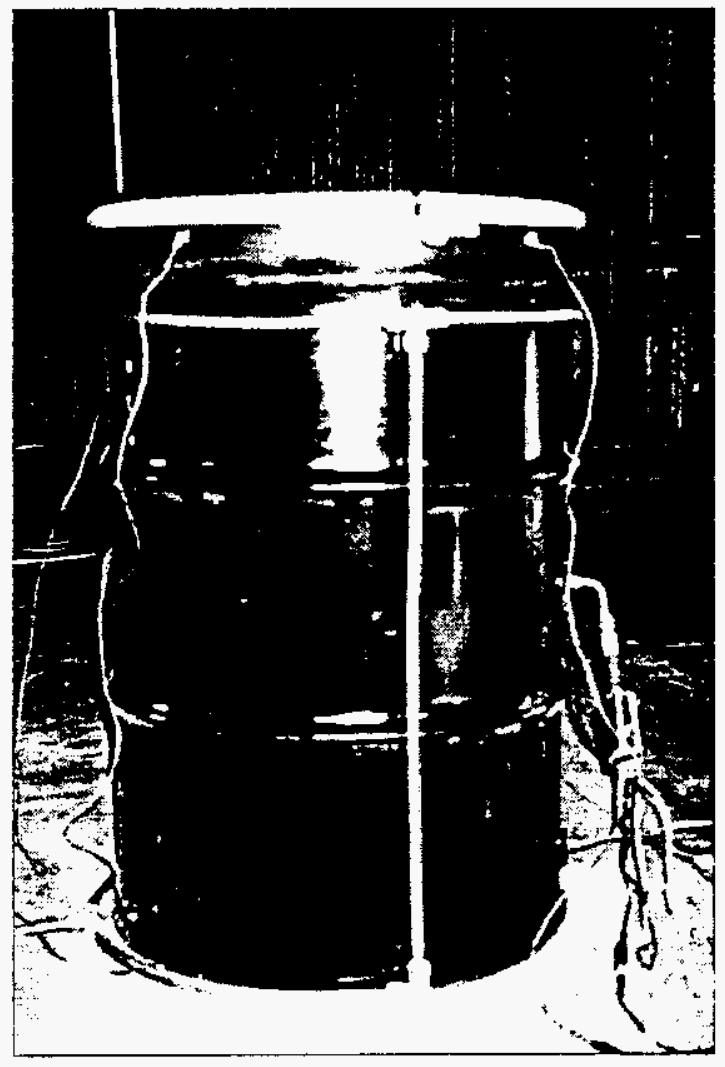

Test Arrangement

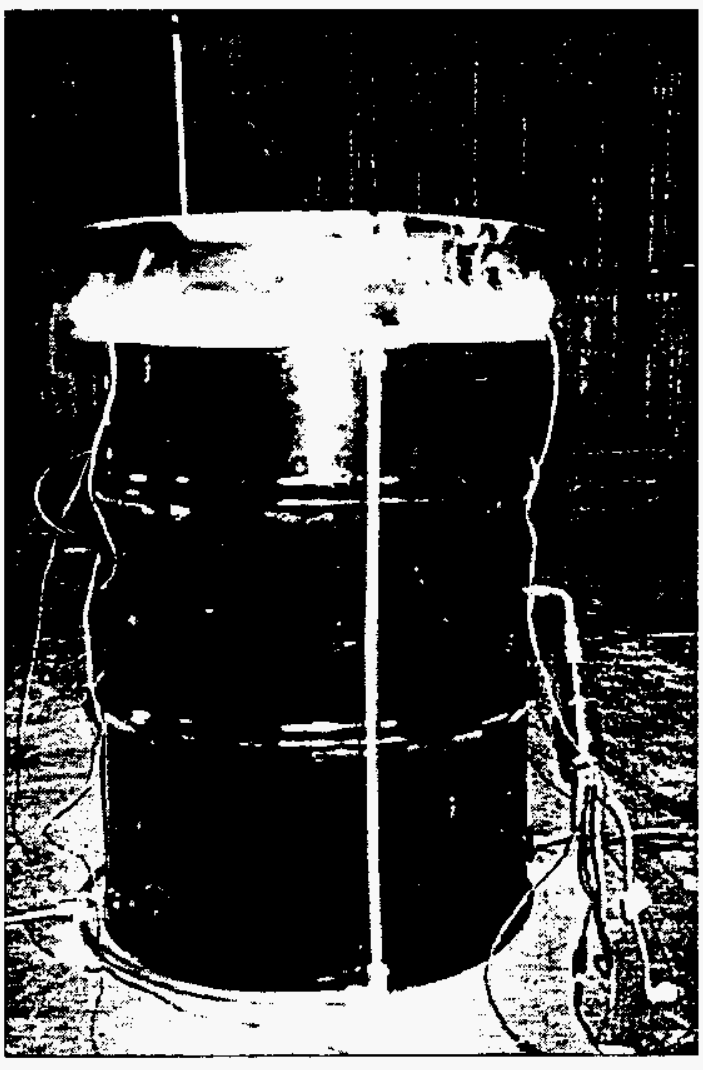

Test in Progress

Figure 18. Gasket Seal Test 
The tests consisted of an empty drum with a sponge rubber gasket seal (see Section 2.1.5). Temperatures of the drum wall surface, near the lid, were measured at four locations. The drum was pressurized to a specific overpressure, and a ring burner was then ignited. The ring burner consisted of $1.3 \mathrm{~cm}(0.5 \mathrm{in}$.) diameter copper tubing with $0.3 \mathrm{~cm}(1 / 8 \mathrm{in}$.$) holes$ spaced approximately $1.3 \mathrm{~cm}(0.5 \mathrm{in.})$ apart. The burner was fueled with propane gas. Table 8 provides the test matrix for the gasket seal tests.

Table 8. Gasket Seal Test Matrix.

\begin{tabular}{|c|c|c|c|}
\hline Test No. & Overpressure (psi) & Fuel Flow (Lpm) & Gasket Material \\
\hline GS-1 & +5 & 5.43 & Sponge rubber \\
\hline GS-2 & +5 & 7.59 & Sponge rubber \\
\hline GS-3 & +10 & 5.43 & Sponge rubber \\
\hline GS-4 & +10 & 7.59 & Sponge rubber \\
\hline
\end{tabular}




\subsection{EXPERIMENTAL RESULTS}

\subsection{SINGLE DRUM BURNING TESTS}

In this program, it was desired that the experimental results could be used in conjunction with an analytical analysis in order to characterize drum performance under fire exposures. Although the goal of this work was to assess the hazard of fire propagation through drum arrays, it was first necessary to gain an understanding of how individual burning drums behaved. This was the purpose of the single drum tests. Since the interaction between drums plays a major role in fire propagation through a storage array, measurements of drum wall temperatures, radiative heat fluxes, and mass burning rates were essential for these tests. The following sections present the experimental results for the single drum tests.

\subsubsection{Single Drum Burning Rate Tests}

Table 9 provides a summary of the data obtained from the single drum burning rate tests. Figures 2 and 3 (Section 2.2.1) show the configuration of the heat flux transducers and thermocouples. Graphical data outputs for these tests are provided in Volume II-Appendix $\mathrm{B}$ under the appropriate test number. The table provides a listing of the following:

- The maximum heat fluxes received by the target drum (HF 1, HF 2, and HF 3);

- The maximum heat flux received by the target calorimeters, $0.6 \mathrm{~m}(2 \mathrm{ft})$ from the drum (HF 4 and HF 5 r;

- Maximum average temperatures at each horizontal set on the burning drum; and

- Maximum temperature of drum wall surface on target drum (TC 21, 22, 23) at each horizontal location.

Figures 19,20 , and 21 provide examples of the burning drum surface temperatures and heat fluxes attained by the target calorimeters. Figure 19 is the data from SWD 4 which involved the standard load. Figure 20 is the data from SWD 10 which involved two-thirds of the standard load cut into small pieces. Figure 21 provides the data from SWD 5 which involved $7.6 \mathrm{~L}$ ( 2 gal) of kerosene.

In general, the highest heat fluxes on the target drum (HF 1-3) and the calorimeters at

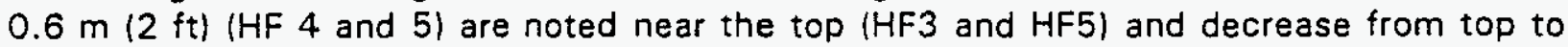
bottom. The temperatures on the surface of the burning drum also follow the same trends as the heat flux. This is due to the fuel burning from top to bottom. For fuels such as kerosene and plastic (which melts into a pool), this trend does not hold.

Table 10 provides a summary of the average mass burning rate and the estimated peak and average heat release rates which were developed using the weight loss measurements and published heats of combustion (see Table 2) for the materials involved. The average mass burning rate and subsequently the average heat release rate were calculated over the time intervais noted in Table 10. These time intervals correspond to the quasi-steady state burning period of interest for each fuel package. The times chosen bracket the most obvious period of near constant mass loss rate. For Tests SWD 8 and SWD 9, which used plastic sheet for the fuel, the time interval corresponds to the time after the plastic melted and became a pool fire. 
Table 9. Data Summary - Single Drum Tests.

\begin{tabular}{|c|c|c|c|c|c|c|c|c|c|c|c|c|}
\hline Test No. & Materials & $\begin{array}{c}\operatorname{Max} \\
H F 1 \\
(\mathrm{~kW} / \mathrm{m} \\
2)\end{array}$ & $\begin{array}{c}\operatorname{Max} H F \\
2 \\
\left(\mathrm{~kW} / \mathrm{m}^{2}\right. \\
)\end{array}$ & $\begin{array}{c}\operatorname{Max} H F \\
3 \\
\left(\mathrm{~kW} / \mathrm{m}^{2}\right. \\
)\end{array}$ & $\begin{array}{c}\text { Max HF } \\
4 \\
\left(k W / m^{2}\right. \\
)\end{array}$ & $\begin{array}{c}\text { Max HF } \\
5 \\
\left(\mathrm{~kW} / \mathrm{m}^{2}\right. \\
)\end{array}$ & $\begin{array}{c}\operatorname{Max} \bar{X} \\
\operatorname{TC} 1-5 \\
\left({ }^{\circ} \mathrm{C}\right)\end{array}$ & $\begin{array}{c}\operatorname{Max} \bar{X} \\
\operatorname{TC} 6- \\
10\left({ }^{\circ} \mathrm{Cl}\right.\end{array}$ & $\begin{array}{l}\operatorname{Max} \bar{X} \\
\operatorname{TC} 11- \\
15\left({ }^{\circ} \mathrm{C}\right)\end{array}$ & $\begin{array}{c}\operatorname{Max} \bar{X} \\
\operatorname{TC} 21 \\
\left.{ }^{\circ} \mathrm{C}\right)\end{array}$ & $\begin{array}{c}\operatorname{Max} \bar{X} \\
\text { TC } 22 \\
\left.I^{\circ} \mathrm{C}\right)\end{array}$ & $\begin{array}{c}\operatorname{Max} \\
X \text { TC } \\
23 \\
\left({ }^{\circ} \mathrm{C}\right)\end{array}$ \\
\hline SWD 1 & Std Load - top & 0.5 & 6.7 & 13.7 & 1.5 & 2.1 & 147 & 258 & 459 & NM & NM & NM \\
\hline SWD 2 & Std Load - bottom & 0.7 & 0.8 & 0.7 & 0.1 & 0.2 & 44 & 27 & 24 & NM & NM & $\overrightarrow{N M}$ \\
\hline SWD 3 & Paper $(2.3 \mathrm{~kg})$ - bottom & 4.0 & 7.4 & 8.7 & 1.6 & 1.2 & 260 & 376 & 328 & NM & NM & NM \\
\hline SWD 4 & Std Load - top & 0.45 & 4.3 & 11.3 & 1.7 & 2.4 & 40 & 303 & 456 & NM & NM & NM \\
\hline SWD 5 & Kerosene & 15.7 & 23.9 & 23.9 & 3.9 & 6.0 & 498 & 524 & 535 & 231 & 273 & 287 \\
\hline SWD 6 & Paper $(2.3 \mathrm{~kg})-$ top & 4.1 & 10.0 & 11.3 & 0.8 & 1.3 & 281 & 316 & 407 & 93 & 124 & 147 \\
\hline SWD 7 & Paper $(6.8 \mathrm{~kg})-$ top & 0.7 & 1.1 & 3.8 & 0.2 & 0.4 & 28 & 64 & 190 & 28 & 35 & 84 \\
\hline SWD 8 & Plastic $(2.3 \mathrm{~kg})-$ top & 18.7 & 17.9 & 15.4 & 4.0 & 3.8 & 545 & 508 & 478 & 256 & 238 & 229 \\
\hline SWD 9 & Plastic $(6.8 \mathrm{~kg})$ - top & 19.3 & 17.8 & 14.9 & 3.7 & 3.3 & 538 & 490 & 454 & 251 & 244 & 230 \\
\hline SWD 10 & $2 / 3 \mathrm{Std}$ Load - mixed - top & 10.6 & 12.7 & 7.2 & 2.4 & 2.0 & 371 & 435 & 348 & 206 & 205 & 146 \\
\hline
\end{tabular}

Note: $N M=$ Not measured for these tests 
Table 10. Mass Burning Rates and Heat Release Rates for Single Drum Burning Tests.

\begin{tabular}{|l|l|c|c|c|c|}
\hline Test No. & \multicolumn{1}{|c|}{ Materials } & $\begin{array}{c}\text { Time Interval } \\
\text { for Calculating } \\
\text { Mass Burning } \\
\text { Rate }(\mathbf{s})\end{array}$ & $\begin{array}{c}\text { Mass } \\
\text { Burning } \\
\text { Rate (g/s) }\end{array}$ & $\begin{array}{c}\text { Est. } \bar{X}^{*} \\
\text { HRR } \\
(\mathrm{kW})\end{array}$ & $\begin{array}{c}\text { Estimated* } \\
\text { Peak HRR } \\
(\mathrm{kW})\end{array}$ \\
\hline SWD 1 & Std Load - top & $747 \cdot 3860$ & 1.45 & 45 & 129 \\
\hline SWD 2 & Std Load - bottom & NM & NM & NM & NM \\
\hline SWD 3 & Paper $(2.3 \mathrm{~kg})$ - bottom & $62-1260$ & 1.87 & 31 & 128 \\
\hline SWD 4 & Std Load - top & $70-7260$ & 1.05 & 33 & 49 \\
\hline SWD 5 & Kerosene & $171-2994$ & 2.2 & 95 & 188 \\
\hline SWD 6 & Paper $(2.3 \mathrm{~kg})-$ top & $62-500$ & 3.14 & 51 & 69 \\
\hline SWD 7 & Paper $(6.8 \mathrm{~kg})-$ top & $63-1200$ & 1.29 & 21 & 43 \\
\hline SWD 8 & Plastic $(2.3 \mathrm{~kg})-$ top & $1100-2265$ & 1.68 & 73 & 160 \\
\hline SWD 9 & Plastic $(6.8 \mathrm{~kg})-$ top & $1600-5500$ & 1.57 & 68 & 157 \\
\hline SWD 10 & $2 / 3$ Std Load - mixed - top & $70-22000$ & 0.58 & 18 & 47 \\
\hline
\end{tabular}

Note: $=\dot{m}_{f} \cdot \Delta H_{c}$ where $\dot{m}_{f}$ is the mass burning rate and $\Delta H_{c}$ is the heat of combustion. $N M=$ Not measured for these tests. 
WHC-SD-WM-TRP-233 REV. O

SWD4 - Standard Load
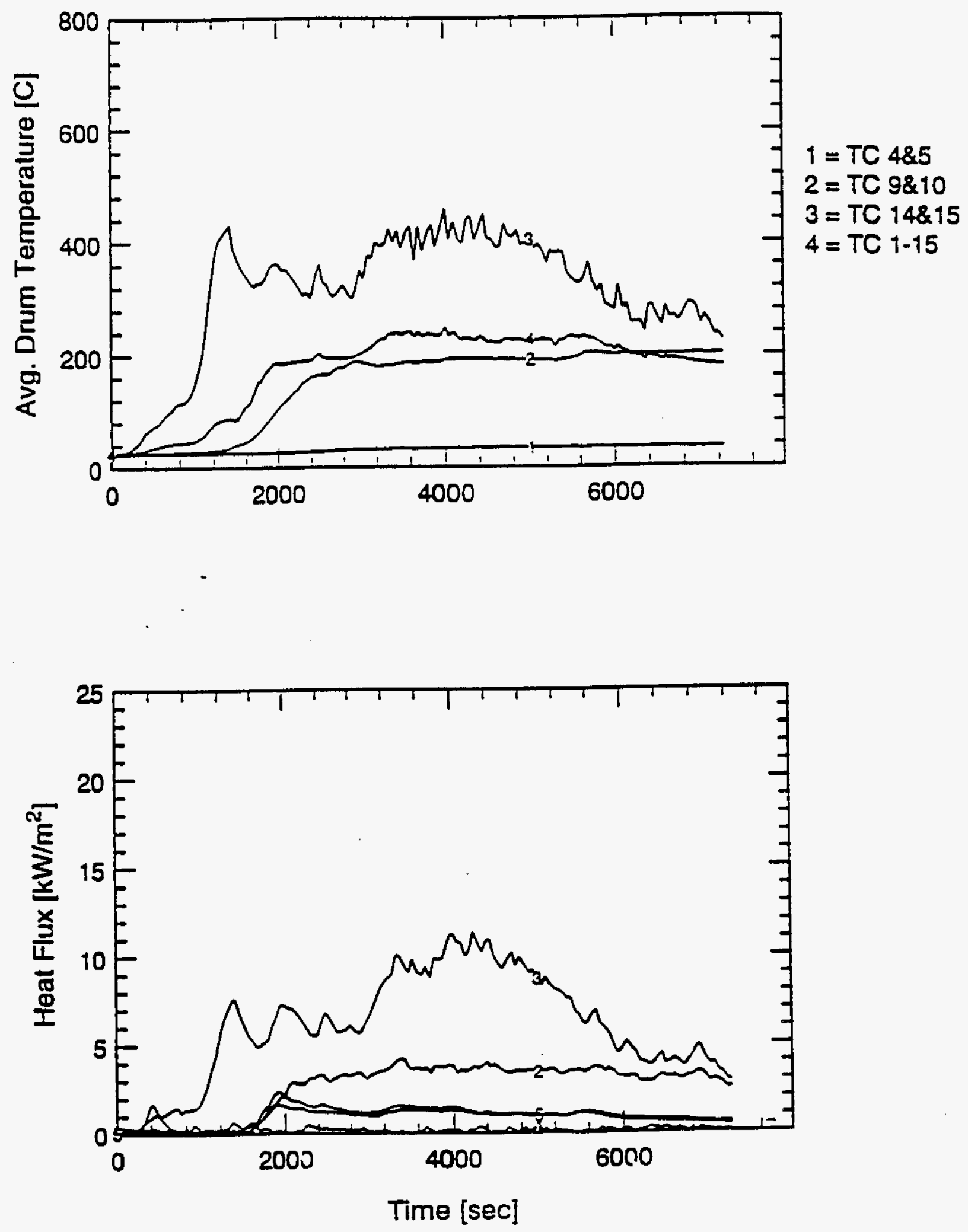

Figure 19. Drum Temperature and Heat Flux Data for Standard Load Single Drum Burning Test 
WHC-SD-WM-TRP-233 REV. O

SWD10 - 2/3 of Mixed Standard Load
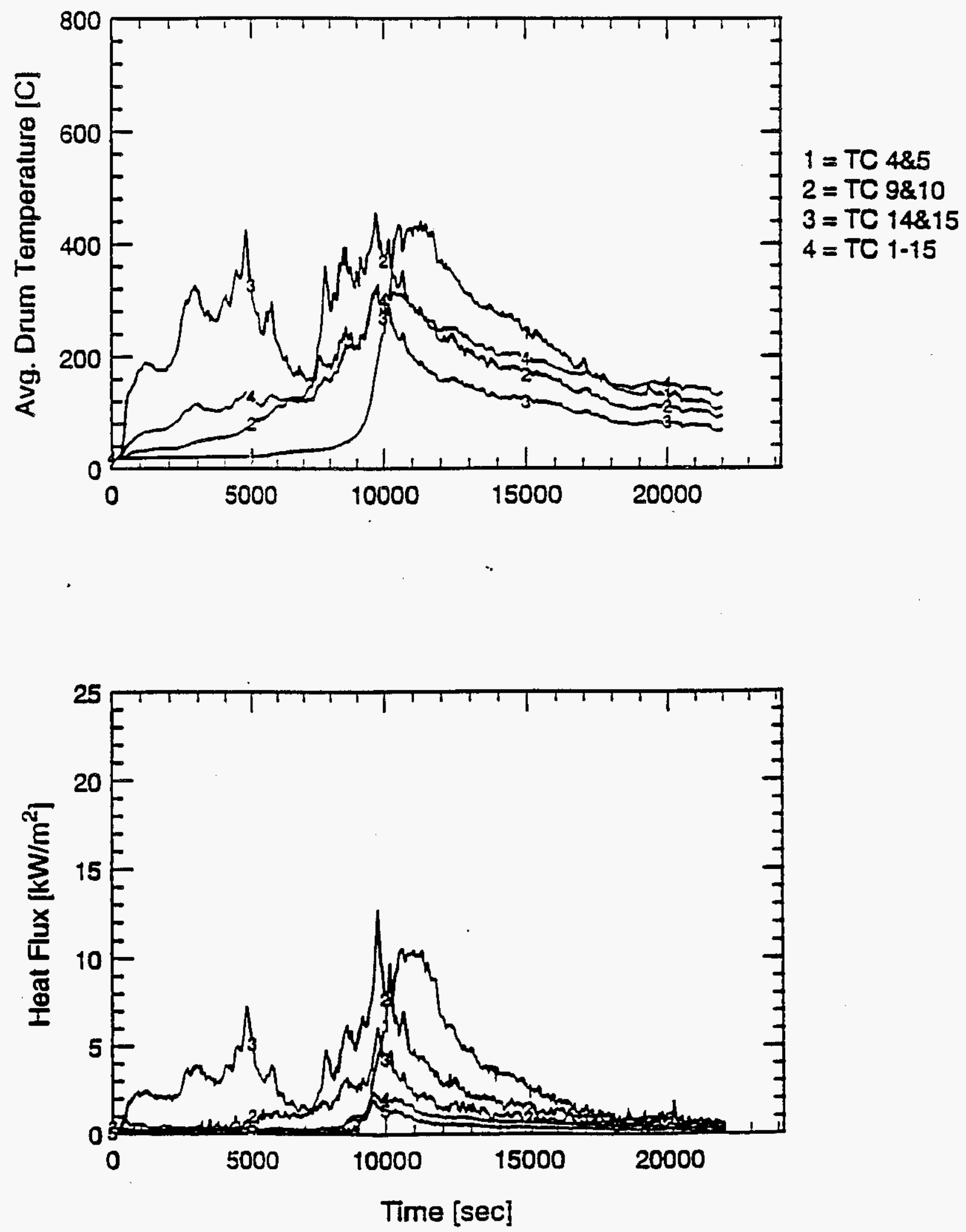

Figure 20. Drum Temperature and Heat Flux Data for $2 / 3$ Standard Load Single Drum Burning Test 
WHC-SD-WM-TRP-233 REV. 0

SWD5 - Kerosene
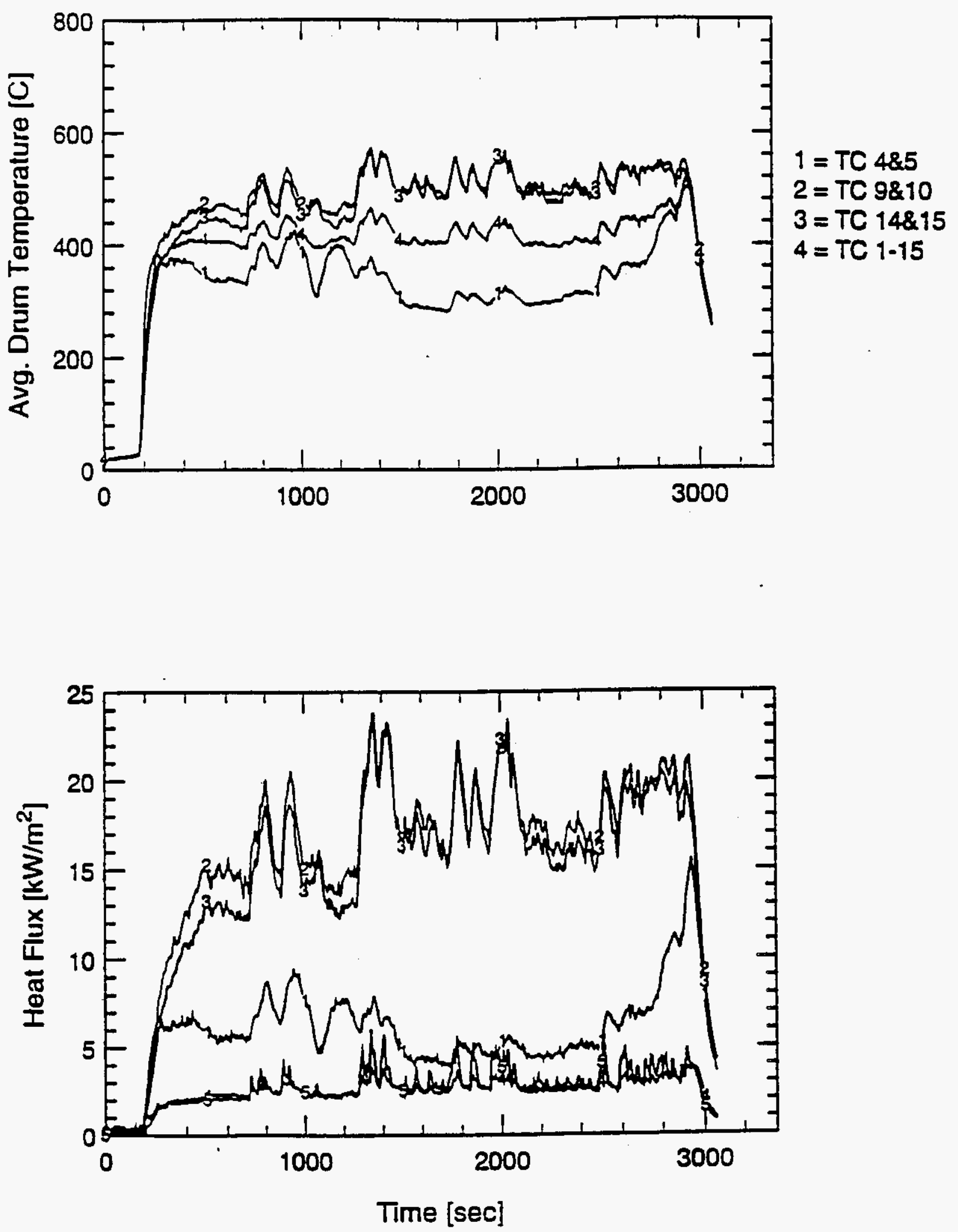

Figure 21. Drum Temperature and Heat Flux Data for Kerosene Single Drum Burning Test 
The tests showed that burning of materials inside the drums was rather unremarkable. Generaliy, the materials burned with flame heights approximately $0.3-0.6 \mathrm{~m}(1-2 \mathrm{ft})$ above the top of the drum. In the tests with kerosene or plastic sheet as the fuel, the flames would periodically flash to a height of $1.5 \mathrm{~m}(5 \mathrm{ft})$ above the drum.

Based on the heat release data, several observations can be made:

- Kerosene provided the highest peak and average heat release rates;

- The peak and average HRR were higher for the lower density paper loading 12.3 $\mathrm{kg})$ than for the higher density paper loading $(6.8 \mathrm{~kg})$;

- Bottom ignition of paper produced a higher peak HRR, but the average HRR was approximately the same. The higher peak HRR occurred at the time when the fire burned through the paper and flames extended up the entire face of the drum;

- The HRRs for the standard load are less than those for paper alone or plastic alone. This is primarily due to the higher loading density and use of other fuels that burn slower, i.e., cotton fabric; and

- The loading density of plastic does not have the same trend as paper since it first melts and forms a pool as it burns. This is evidenced by the close average and peak HRRs for the $2.3 \mathrm{~kg}$ loading and the $6.8 \mathrm{~kg}$ loading.

\subsubsection{Stacked Drum Tests}

Table 11 provides a summary of the data obtained from the vertical single drum burning tests. Figures 2,3 , and 5 show the configuration of the heat flux gauges and thermocouples. Graphical data outputs for these tests are provided in Volume II-Appendix B under the appropriate test number.

The table provides a listing of the following:

- The maximum heat fluxes received by the lateral target drum (HF 2, HF 3);

- The maximum heat flux received by the target calorimeters (HF 4, HF 5), 0.6 $\mathrm{m}(2 \mathrm{ft})$ from the drum;

- The maximum heat flux received by the vertical target drum (HF 6, HF 7, and HF 8);

- Maximum average temperatures at each horizontal set on the burning drum; and

- Maximum temperature of drum wall surface on lateral target drum (TC 21, 22, 23) at each horizontal location. 
WHC-SD-WM-TRP-233 REV. 0

Table 11. Data Summary - Stacked Drum Tests.

\begin{tabular}{|c|c|c|c|c|c|c|c|c|c|c|c|c|c|c|c|}
\hline $\begin{array}{l}\text { Test } \\
\text { No. }\end{array}$ & Materiais & $\begin{array}{c}\text { Vertical } \\
\text { Distanc } \\
\text { e (in.) }\end{array}$ & $\begin{array}{c}\text { Max } \\
\text { HF2 } \\
(\mathrm{kW} / \mathrm{m} \\
2,\end{array}$ & $\begin{array}{c}\text { Max } \\
\text { HF3 } \\
\left(\mathrm{kW} / \mathrm{m}^{2}\right. \\
1\end{array}$ & $\begin{array}{c}\text { Max } \\
\text { HF4 } \\
\text { (kW/m } \\
1\end{array}$ & $\begin{array}{c}\text { Max } \\
\text { HF5 } \\
\left(\mathrm{kW} / \mathrm{m}^{2}\right. \\
1\end{array}$ & $\begin{array}{c}\text { Max } \\
\text { HF6 } \\
\text { (kW/m } / \mathrm{m}^{2} \\
)\end{array}$ & $\begin{array}{c}\text { Max } \\
\text { HF7 } \\
\text { (kW/m }{ }^{2} \\
\text { ) }\end{array}$ & $\begin{array}{c}\text { Max } \\
\text { HF8 } \\
(\mathrm{kW} / \mathrm{m} \\
\left.2_{1}\right)\end{array}$ & $\begin{array}{l}\operatorname{Max} X \\
\operatorname{TC} 1- \\
5\left({ }^{\circ} \mathrm{C}\right)\end{array}$ & $\begin{array}{c}\operatorname{Max} X \\
\operatorname{TC} 6-10 \\
f^{\circ} \mathrm{Cl}\end{array}$ & $\begin{array}{l}\text { Max X } \\
\operatorname{TC~} 11- \\
\left.151^{\circ} \mathrm{C}\right)\end{array}$ & $\begin{array}{c}\text { Max } \\
X \\
\text { TC } \\
21 \\
1^{\circ} \mathrm{Cl}\end{array}$ & $\begin{array}{c}\operatorname{Max} \\
\times \mathrm{TC} \\
22 \\
\left({ }^{\circ} \mathrm{C}\right)\end{array}$ & $\begin{array}{l}\operatorname{Max} X \\
\text { TC } 23 \\
\left({ }^{\circ} \mathrm{C}\right)\end{array}$ \\
\hline $\begin{array}{c}\text { SWD } \\
11\end{array}$ & Kerosene & 6 & 11.5 & 12.3 & 2.4 & 2.5 & 57.3 & 53.9 & 35.6 & 350 & 480 & 432 & 159 & 212 & 209 \\
\hline $\begin{array}{c}\text { SWD } \\
12\end{array}$ & Kerosene & 12 & 16.2 & 15.3 & 3.4 & 3.5 & 56.6 & 59.4 & 37.2 & 430 & 492 & 474 & 192 & 255 & 238 \\
\hline $\begin{array}{c}\text { SWD } \\
13 \\
\end{array}$ & Kerosene & 18 & 16.2 & 15.0 & 3.1 & 3.3 & 46.5 & 43.2 & 25.9 & 420 & 496 & 470 & 190 & 252 & 235 \\
\hline $\begin{array}{c}\text { SWD } \\
14\end{array}$ & Kerosene & 24 & 14.9 & 13.9 & 2.8 & 3.3 & 34.2 & 36.2 & 16.7 & 424 & 487 & 461 & 196 & 242 & 230 \\
\hline $\begin{array}{c}\text { SWD } \\
15\end{array}$ & $\begin{array}{l}2.3 \mathrm{~kg} \\
\text { plastic }\end{array}$ & 6 & 12.2 & 11.6 & 3.0 & 2.6 & 53.4 & 55.9 & 37.7 & 483 & 443 & 418 & 232 & 143 & 192 \\
\hline $\begin{array}{c}\text { SWD } \\
16\end{array}$ & $\begin{array}{l}1 / 3 \text { standard } \\
\text { load - small } \\
\text { pieces }\end{array}$ & 6 & 10.0 & 11.6 & 1.7 & 2.7 & 63.8 & 79.1 & 44.6 & 41.3 & 389 & 500 & 241 & 202 & 155 \\
\hline $\begin{array}{c}\text { SWD } \\
17\end{array}$ & $\begin{array}{l}\text { standard } \\
\text { load - } \\
\text { bagged }\end{array}$ & 6 & 15.4 & 9.8 & 1.3 & 1.1 & 33.5 & 36.7 & 24.2 & 242 & 411 & 345 & 194 & 249 & 188 \\
\hline
\end{tabular}


Based on the single drum burning tests, kerosene was used as the primary fuel in the vertical spacing tests so as to provide the maximum HRR and exposure to the vertical drum. Table 12 provides a summary of vertical drum tests with respect to heat flux incident to the vertical water-filled target drum. The average time periods were subjectively chosen by determining the times which best bracketed the near steadystate maximum heat flux. The data show that, as expected, increasing the vertical distance decreases the incident heat flux to the vertical target drum.

Table 12. Vertical Drum Tests Summary.

\begin{tabular}{|l|l|c|c|c|c|}
\hline Test No. & \multicolumn{1}{|c|}{ Materials } & $\begin{array}{c}\text { Vertical } \\
\text { Separati } \\
\text { on }(\mathbf{c m})\end{array}$ & $\begin{array}{c}\text { Average } \\
\text { Transducer } \\
\text { Heat Flux } \\
\left(\mathbf{k W} / \mathbf{m}^{2}\right)\end{array}$ & $\begin{array}{c}\text { Average } \\
\mathrm{H}_{2} \text { O Cal* } \\
\text { Heat Flux } \\
\left(\mathbf{k W} / \mathrm{m}^{2}\right)\end{array}$ & $\begin{array}{c}\text { Time Span** } \\
\text { (s) }\end{array}$ \\
\hline SWD11 & Kerosene & 15.2 & 35.2 & 35.2 & $500-1500$ \\
\hline SWD12 & Kerosene & 30.5 & 33.9 & 37.5 & $100-800$ \\
\hline SWD13 & Kerosene & 45.7 & 20.2 & 18.8 & $100-800$ \\
\hline SWD14 & Kerosene & 61.0 & 15.9 & 14.9 & $100-1000$ \\
\hline SWD15 & 2.3 kg plastic & 15.2 & 34.6 & 33.4 & $1000-2000$ \\
\hline SWD16 & $\begin{array}{l}1 / 3 \text { standard load - } \\
\text { small pieces }\end{array}$ & 15.2 & 45.6 & 40.9 & $400-600$ \\
\hline SWD17 & standard load - bagged & 15.2 & 22.8 & 17.2 & $3000-4000$ \\
\hline
\end{tabular}

- Assumes the heat flux is incident to the bottom surface area of the drum only.

* * Corresponds to the maximum steady flame heat flux.

Heat flux to the bottom of the vertical target drum was quantified both by the three heat flux transducers and the water calorimeter (see Section 2.2.2). Figures 22 and 23 show the results for Tests SWD11 and SWD16, respectively. The heat flux profiles for Transducers HF6, HF7, and HF8 show over a 50 percent reduction in incident heat flux from the center to the edge of the drum (i.e., HF6 to HF8). The bold line in Figures 22a and 23a represents the average of the HF6, HF7, and HF8 data.

This average has been compared to the heat flux measurement obtained from the water calorimeters in Figures 22b and 23b for Tests SWD 11 and SWD16, respectively. The water calorimeter results assume that the flame heat flux is incident to the bottom surface area of the target drum only. As can be seen, there is very good agreement between the two measurements. The water calorimeter provides a good measurement of the overall heat transfer to the drum whereas the heat flux transducers are best for providing local heat flux data.

Although the one-third standard load in small pieces (SWD16) produced the greatest incident heat flux $\left(-43 \mathrm{~kW} / \mathrm{m}^{2}\right)$ to the vertical target drum, the kerosene fire with a $15.2 \mathrm{~cm}$ (6 in.) spacing (SWD 1 1) resulted in nearly the same flux $\left(-37 \mathrm{~kW} / \mathrm{m}^{2}\right)$ but at a much earlier time and for a longer duration. This result can be seen by comparing the heat flux profiles in Figures $22 \mathrm{~b}$ and $23 \mathrm{~b}$. 
SWD11 - Kerosene - Stacked Drums $15 \mathrm{~cm}$ ( 6 in.) apart

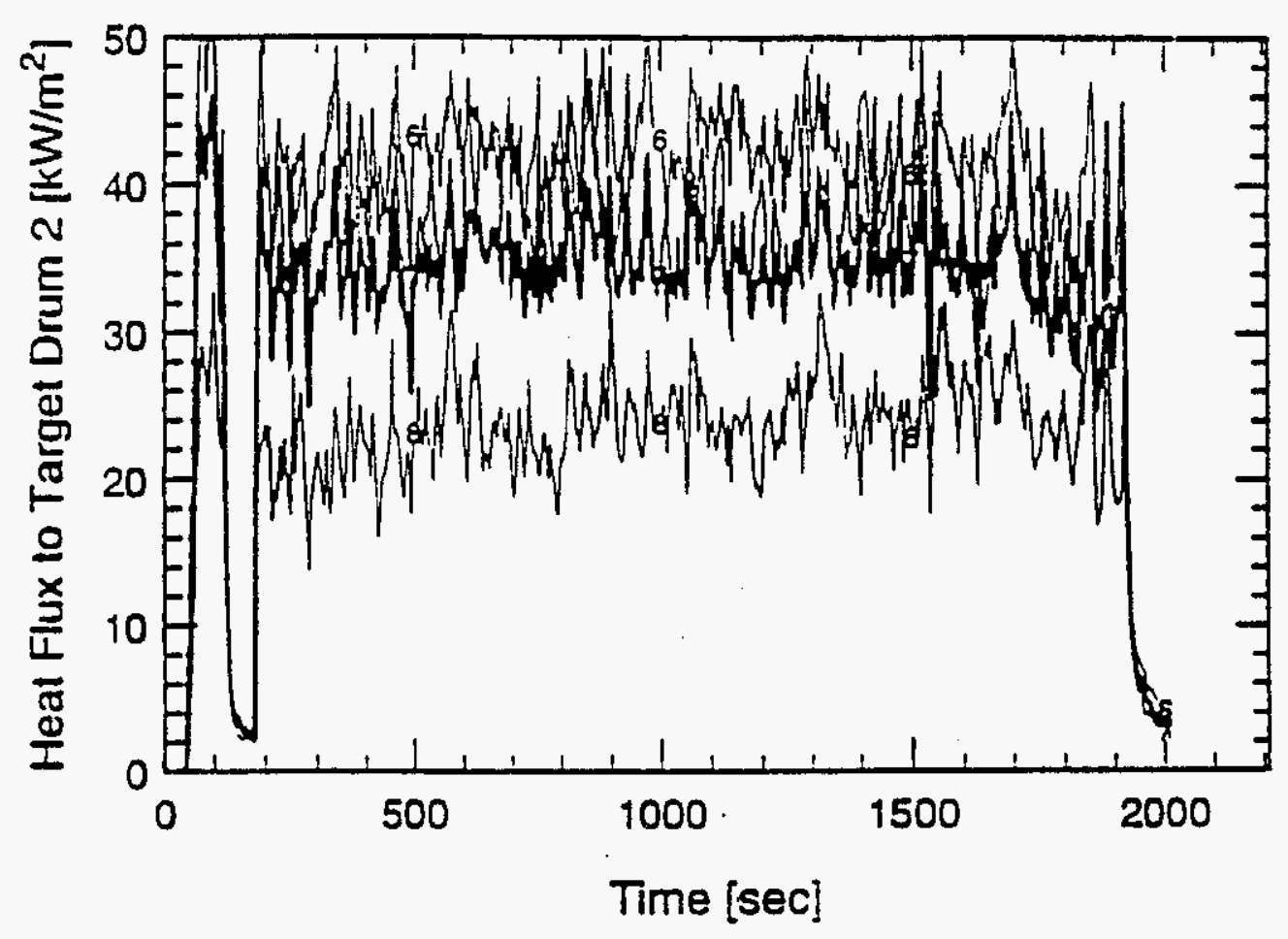

Figure 22a. Transducer Heat Flux Data for Kerosene Stacked Drum Test. 
SWD11 - Kerosene - Stacked Drums $15 \mathrm{~cm}$ (6 in.) apart

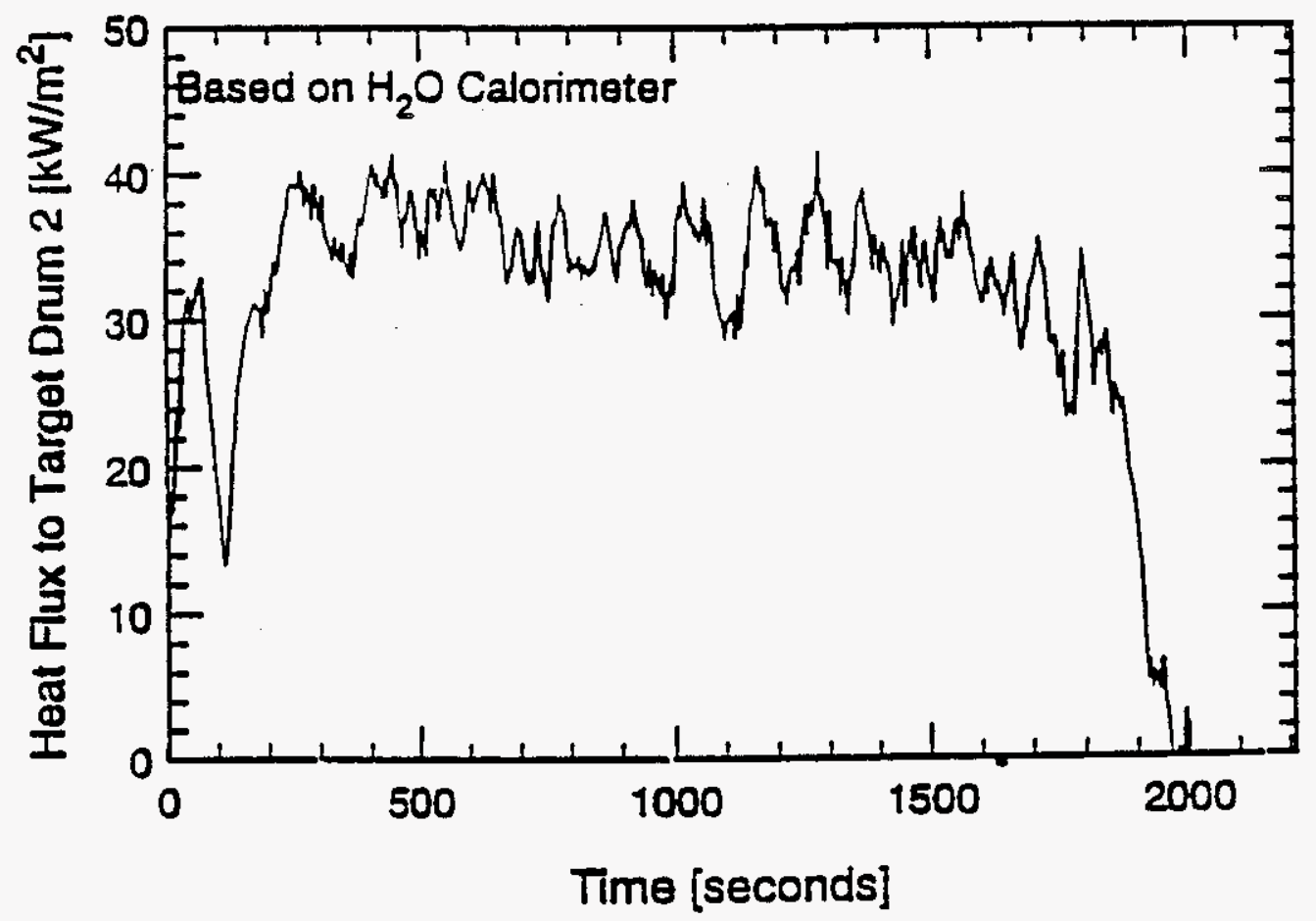

Figure 22b. Water Calorimeter Heat Flux Data for Kerosene Stacked Drum Test. 
SWD16 - 1/3 of Mixed Standard Load - Stacked Drums $15 \mathrm{~cm}$ (6 in.) apart

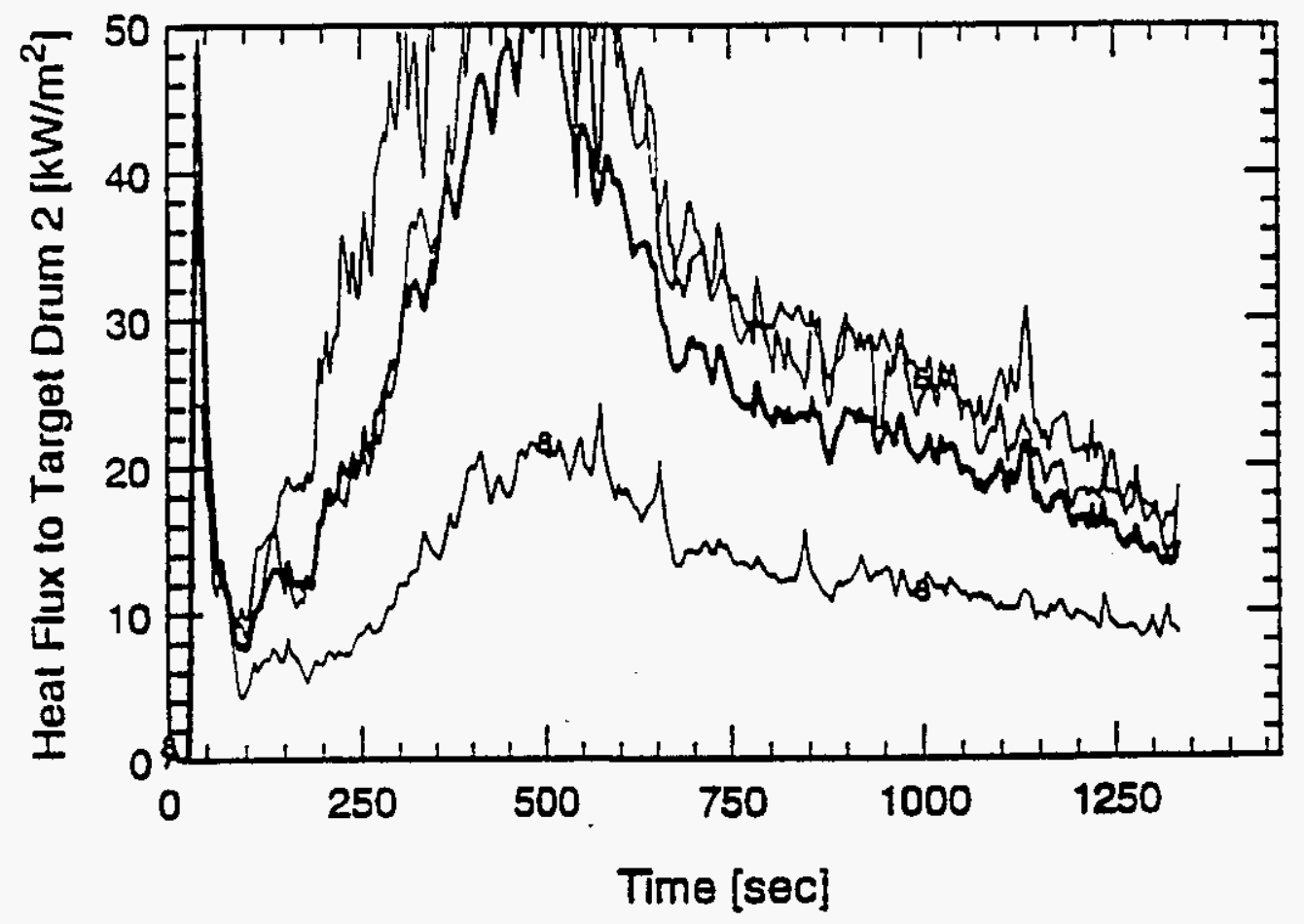

Figure 23a. Transducer Heat Flux Data for $1 / 3$ Standard Load Stacked Drum Test. 
SWD16 - $1 / 3$ of Mixed Standard Load - Stacked Drums $15 \mathrm{~cm}$ (6 in.) apart

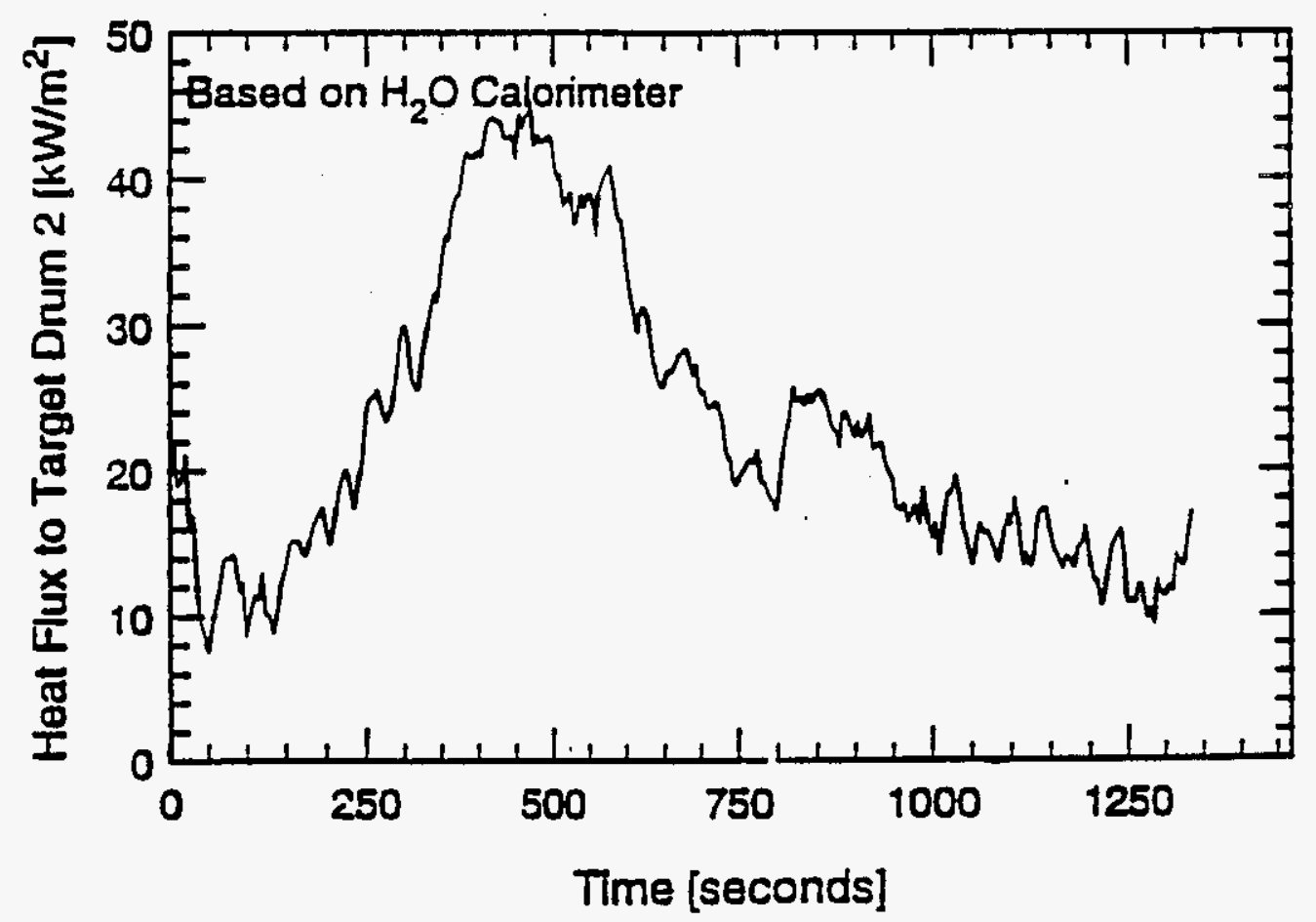

Figure 23b. Water Calorimeter Heat Flux Data for 1/3 Standard Load Stacked Drum Test. 


\subsection{DRUM RESPONSE FIRE EXPOSURE TESTS}

The most significant potential result of drum heating by an exposure fire is the loss of the lid. Lid loss results in the burning of drum contents and the distibution of any hazardous materials which might be contained in the drum. Lid seal failure is a less severe drum failure mode. Lid seal failure results in loss of containment, but also results in drum pressure relief which can prevent lid loss. By preventing lid loss, lid seal failure prevents burning of the contents and thus markedly reduces material releases. Table 13 provides a lid loss summary and Table 14 provides a summary of the data obtained from the drum response tests. Graphical data output for these tests are provided in Volume Il-Appendix B under the appropriate test number. In some of the tests, venting was observed prior to lid loss failure. However, in these cases, the degree of lid seal failure was not sufficient to reduce the pressure in the drum. In all tests where lid loss was not observed, lid seal failure occurred. For these tests, a critical venting time was determined as when the rate of pressure rise becomes negative, thus indicating a pressure reduction in the drum. This did not usually correspond to the fire when venting was first visually observed.

Table 13. Lid Loss Summary of Fire Exposure Tests (2 sheets).

\begin{tabular}{|c|c|c|}
\hline Test/Drum & Contents & Lid Loss \\
\hline \multicolumn{3}{|c|}{$0.48 \mathrm{~m}$ Burner } \\
\hline 19-D1 & Standard load & $\mathrm{N}$ \\
\hline $19-D 4$ & Empty & $\mathrm{N}$ \\
\hline 20.D1 & $2.3 \mathrm{~kg}(5 \mathrm{lb})$ paper & $\mathrm{N}$ \\
\hline 20.D4 & $2.3 \mathrm{~kg}(5 \mathrm{lb})$ plastic & $N$ \\
\hline 21-D1 & $2.3 \mathrm{~kg}(5 \mathrm{lb})$ paper - heptane & $\mathrm{Y}(\mathrm{Al})$ \\
\hline 21-D4 & Empty & $N$ \\
\hline 22-D1 & Bagged standard load - acetone & $N$ \\
\hline 22-D4 & $2.3 \mathrm{~kg}(5 \mathrm{lb})$ paper - acetone & $\mathrm{N}$ \\
\hline 23.D1 & $2.3 \mathrm{~kg}(5 \mathrm{lb})$ paper - heptane & $\mathrm{N}$ \\
\hline 23-D4 & $2.3 \mathrm{~kg}(5 \mathrm{lb})$ paper - acetone & $\mathrm{N}$ \\
\hline 24-D1 & Standard load - EPDM & $\mathrm{N}$ \\
\hline 24-D4 & Empty - EPDM & $N$ \\
\hline 25-D1 & $2.3 \mathrm{~kg}(5 \mathrm{lb})$ paper - EPDM & $\mathrm{N}$ \\
\hline 25-D4 & $2.3 \mathrm{~kg}(5 \mathrm{lb})$ plastic - EPDM & $\mathrm{N}$ \\
\hline 26-D1 & $2.3 \mathrm{~kg}(5 \mathrm{lb})$ paper - toluene - EPDM & $\mathbf{N}$ \\
\hline 26-D4 & Bagged standard load - toluene - EPDM & $\mathrm{N}$ \\
\hline 27-D1 & $2.3 \mathrm{~kg}(5 \mathrm{lb})$ paper - toluene - EPDM & $\mathrm{N}$ \\
\hline 27-D4 & Bagged standard load - toluene - EPDM & $\mathrm{N}$ \\
\hline \multicolumn{2}{|r|}{ Drums resulting in lid loss } & 1 of 18 \\
\hline Notes: & $\mathrm{Al}=$ Autoignition & \\
\hline
\end{tabular}


Table 13. Lid Loss Summary of Fire Exposure Tests (2 sheets).

\begin{tabular}{|c|c|c|}
\hline Test/Drum & Contents & Lid Loss \\
\hline \multicolumn{3}{|c|}{$1.2 \mathrm{~m}$ Burner } \\
\hline 28-D1 & $2.3(5 \mathrm{lb})$ paper & $\bar{N}$ \\
\hline 28-D4 & $2.3 \mathrm{~kg}(5 \mathrm{lb})$ plastic & $N$ \\
\hline 33-D1 & $2.3 \mathrm{~kg}(5 \mathrm{lb})$ paper & $Y$ \\
\hline 33-D4 & $2.3 \mathrm{~kg}(5 \mathrm{lb})$ plastic & $N$ \\
\hline 34-D1 & 2.3 (5 lb) paper - heptane & $N$ \\
\hline 34-D4 & Empty & $N$ \\
\hline 35-D1 & $2.3(5 \mathrm{lb})$ paper - heptane & Y \\
\hline 35-D4 & Standard load & $\mathrm{N}$ \\
\hline 36-D1 & $2.3 \mathrm{~kg}(5 \mathrm{lb})$ paper - toluene & $Y(A I)$ \\
\hline 36-D4 & Bagged standard load - toluene & $\mathrm{N}$ \\
\hline \multicolumn{2}{|r|}{ Drums resulting in lid loss } & 3 of 10 \\
\hline \multicolumn{3}{|c|}{$2.1 \mathrm{~m} \mathrm{JP}-5$ Pool Fires } \\
\hline $29-01$ & $2.3 \mathrm{~kg}(5 \mathrm{lb})$ paper - toluene & $Y$ \\
\hline 29-D4 & Bagged standard load - toluene & Y \\
\hline 30-D1 & $2.3 \mathrm{~kg}(5 \mathrm{lb})$ paper & Y \\
\hline $30-04$ & Empty & $\mathrm{N}$ \\
\hline $31-01$ & $2.3 \mathrm{~kg}(5 \mathrm{lb})$ plastic & $Y$ \\
\hline 31-D4 & Standard load & $Y$ \\
\hline 32-D1 & $2.3 \mathrm{~kg}(5 \mathrm{lb})$ paper & $Y$ \\
\hline 32-D4 & $2.3 \mathrm{~kg}(5 \mathrm{lb})$ plastic & $Y$ \\
\hline \multicolumn{2}{|r|}{ Drums resulting in lid loss } & 7 of 8 \\
\hline \multicolumn{3}{|c|}{ Trash Fire } \\
\hline 37-D1 & $2.3 \mathrm{~kg}(5 \mathrm{lb})$ paper & $N$ \\
\hline 37-D2 & $2.3 \mathrm{~kg}(5 \mathrm{lb})$ paper & $\mathrm{N}$ \\
\hline \multicolumn{2}{|r|}{ Drums resulting in lid loss } & 0 of 2 \\
\hline
\end{tabular}

Notes: $\mathrm{Al}=$ Autoignition 
Table 14. Data Summary of Fire Exposure Tests $(4$ sheets).

\begin{tabular}{|c|c|c|c|c|c|c|c|c|c|c|}
\hline $\begin{array}{l}\text { Test ID/ } \\
\text { Drum ID }\end{array}$ & Contents & $\begin{array}{c}\text { Time to } \\
\text { Vent (sec) } \\
\text { (measured) }\end{array}$ & $\begin{array}{c}\text { Time to } \\
\text { Lid Loss } \\
\text { (sec) }\end{array}$ & $\begin{array}{l}\text { Maximum Wall } \\
\text { Temperature at } \\
\text { Vent/Loss }\left(^{\circ} \mathrm{C}\right)\end{array}$ & $\begin{array}{c}\text { Average Wall } \\
\text { Temperature at } \\
\text { Vent Loss } \\
\left({ }^{\circ} \mathrm{C}\right)^{2}\end{array}$ & $\begin{array}{c}\text { Maximum } \\
\text { Internal } \\
\text { Pressure } \\
\text { (psig) }\end{array}$ & $\begin{array}{c}\text { Average } \\
\text { Internal Air } \\
\text { Temperature } \\
\left({ }^{\circ} \mathrm{C}\right)\end{array}$ & $\begin{array}{c}\text { Average } \\
\mathrm{dP} / \mathrm{dt}^{4} \\
|\mathrm{psig} / \mathrm{s}|\end{array}$ & $\begin{array}{c}\text { Average } \\
\text { Burn Rate } \\
\text { of } \\
\text { Contents } \\
(\mathrm{g} / \mathrm{s}) \\
\end{array}$ & Comments \\
\hline \multicolumn{11}{|c|}{$0.48 \mathrm{~m}$ Burner Exposures } \\
\hline 19-D1 & Standard load & 340 & .. & 660 & 528 & 4.9 & 22 & 0.017 & -- & \begin{tabular}{|l|} 
gasket \\
completely \\
burned out
\end{tabular} \\
\hline 19-D4 & Empty & 120 & .. & 450 & 317 & 6.1 & 114 & 0.109 & -. & $\begin{array}{l}71 \mathrm{~cm}(28 \mathrm{in} .) \\
\text { of gasket } \\
\text { burned out }\end{array}$ \\
\hline 20-D1 & $2.3 \mathrm{~kg}$ paper & 140 & -- & 521 & 386 & 12.4 & 84 & 0.115 & 0.5 & $\begin{array}{l}58 \mathrm{~cm}(23 \mathrm{in} .) \\
\text { of gasket } \\
\text { burned out }\end{array}$ \\
\hline 20-D4 & $2.3 \mathrm{~kg}$ plastic & 300 & $-\cdot$ & 479 & 378 & 5.8 & 252 & 0.195 & 0.07 & $\begin{array}{l}76 \mathrm{~cm}(30 \mathrm{in} .) \\
\text { of gasket } \\
\text { burned out }\end{array}$ \\
\hline $21 \cdot D 1$ & $\begin{array}{l}2.3 \mathrm{~kg} \text { paper - } \\
\text { heptane } \\
\end{array}$ & -. & 79 & 468 & 293 & Al & 70 & Al & $\cdots$ & \begin{tabular}{|l} 
Contents \\
expelled
\end{tabular} \\
\hline 21-D4 & Empty & 210 & -- & 478 & 357 & 8.0 & 205 & 0.039 & -- & \\
\hline 22-D1 & $\begin{array}{l}\text { Standard load } \\
- \text { bagged - } \\
\text { acetone }\end{array}$ & 270 &.- & 625 & NM & 7.5 & 307 & 0.027 & 0.09 & \begin{tabular}{|l} 
Gasket \\
completely \\
burned out
\end{tabular} \\
\hline 22-D4 & $\begin{array}{l}2.3 \mathrm{~kg} \text { paper - } \\
\text { acetone }\end{array}$ & 295 & $\cdots$ & 665 & NM & 16.0 & 134 & 0.063 & 0.72 & \\
\hline 23-D1 & $\begin{array}{l}2.3 \mathrm{~kg} \text { paper }- \\
\text { heptane }\end{array}$ & 120 & -- & 680 & NM & 11.4 & 63 & 0.13 & 0.32 & $\begin{array}{l}\text { Pressure tap } \\
\text { blown off; } \\
\text { entire gasket } \\
\text { burned out }\end{array}$ \\
\hline 23-D4 & $\begin{array}{l}2.3 \mathrm{~kg} \text { paper }- \\
\text { acetone }\end{array}$ & 290 & - & 600 & NM & 16.1 & 85 & 0.061 & 0.21 & $\begin{array}{l}133 \mathrm{~cm} 152.5 \\
\text { in.) of gasket } \\
\text { burned out }\end{array}$ \\
\hline 24-D1 & $\begin{array}{l}\text { Standard load } \\
\text { - EPDM }\end{array}$ & 340 & -- & 750 & 554 & 6.7 & 13 & 0.022 & 0.65 & $\begin{array}{l}57 \mathrm{~cm}(22.5 \\
\text { in.) of gasket } \\
\text { burned out }\end{array}$ \\
\hline
\end{tabular}


Table 14. Data Summary of Fire Exposure Tests (4 sheets).

\begin{tabular}{|c|c|c|c|c|c|c|c|c|c|c|}
\hline $\begin{array}{l}\text { Test ID/ } \\
\text { Drum ID }\end{array}$ & Contents & $\begin{array}{c}\text { Time to } \\
\text { Vent (sec) } \\
\text { (measured) }{ }^{1}\end{array}$ & $\begin{array}{c}\text { Time to } \\
\text { Lid Loss } \\
\text { (sec) }\end{array}$ & $\begin{array}{l}\text { Maximum Wall } \\
\text { Temperature at } \\
\text { Vent/Loss }\left({ }^{\circ} \mathrm{C}\right)\end{array}$ & $\begin{array}{c}\text { Average Wall } \\
\text { Temperature at } \\
\text { Vent Loss } \\
\left({ }^{\circ} \mathrm{C}\right)^{2}\end{array}$ & $\begin{array}{c}\text { Maximum } \\
\text { Internal } \\
\text { Pressure } \\
\text { (psig) }\end{array}$ & $\begin{array}{c}\text { Average } \\
\text { Internal Air } \\
\text { Temperature }^{3} \\
\left(^{\circ} \mathrm{C}\right)\end{array}$ & $\begin{array}{l}\text { Average } \\
\mathrm{dP} / \mathrm{dt}^{4} \\
\text { (psig/s) }\end{array}$ & $\begin{array}{c}\text { Average } \\
\text { Burn Rate } \\
\text { of } \\
\text { Contents } \\
(g / s)\end{array}$ & Comments \\
\hline 24-D4 & Empty - EPDM & 330 & -. & 480 & 330 & 10.1 & 276 & 0.029 & -. & $\begin{array}{l}51 \mathrm{~cm} \text { (20 in.) } \\
\text { of gasket } \\
\text { burned out }\end{array}$ \\
\hline 25-D1 & $\begin{array}{l}2.3 \mathrm{~kg} \text { paper - } \\
\text { EPDM }\end{array}$ & 275 & -. & 630 & 514 & 17.9 & 193 & 0.093 & 0.31 & $\begin{array}{l}72 \mathrm{~cm}(28.5 \\
\text { in.) of gasket } \\
\text { burned out }\end{array}$ \\
\hline $25 \cdot \mathrm{D4}$ & $\begin{array}{l}2.3 \mathrm{~kg} \text { plastic }- \\
\text { EPDM }\end{array}$ & 225 & $\cdots$ & 465 & 407 & 6.4 & 260 & 0.034 & 0.01 & $\begin{array}{l}56 \mathrm{~cm}(22 \mathrm{in} .) \\
\text { of gasket } \\
\text { burned out }\end{array}$ \\
\hline $26 . \mathrm{D1}$ & $\begin{array}{l}2.3 \mathrm{~kg} \text { paper - } \\
\text { toluene - } \\
\text { EPDM }\end{array}$ & 380 & -- & 630 & 498 & 21.9 & 340 & 0.097 & 0.22 & $\begin{array}{l}86 \mathrm{~cm} \mathrm{(34} \mathrm{in.)} \\
\text { of gasket } \\
\text { burned out }\end{array}$ \\
\hline 26-D4 & $\begin{array}{l}\text { Standard load } \\
\text { - toluene - } \\
\text { EPDM }\end{array}$ & 120 & -- & 660 & 398 & 10.5 & 189 & 0.083 & 0.63 & $\begin{array}{l}64 \mathrm{~cm} \text { ( } 25 \mathrm{in} .) \\
\text { of gasket } \\
\text { burned out }\end{array}$ \\
\hline 27.D1 & $\begin{array}{l}2.3 \mathrm{~kg} \text { paper - } \\
\text { toluene - } \\
\text { EPDM } \\
\end{array}$ & 330 & -- & 765 & 447 & 12.9 & 186 & 0.045 & 0.17 & \\
\hline 27.04 & $\begin{array}{l}\text { Standard load } \\
\text { - bagged - } \\
\text { toluene - } \\
\text { EPDM }\end{array}$ & 140 & -- & 495 & 398 & 9.2 & 180 & 0.061 & 0.25 & $\begin{array}{l}124 \mathrm{~cm} \mathrm{(49} \mathrm{in.)} \\
\text { of gasket } \\
\text { burned out }\end{array}$ \\
\hline
\end{tabular}


Table 14. Data Summary of Fire Exposure Tests (4 sheets).

\begin{tabular}{|c|c|c|c|c|c|c|c|c|c|c|}
\hline $\begin{array}{l}\text { Test ID/ } \\
\text { Drum ID }\end{array}$ & Contents & $\begin{array}{c}\text { Time to } \\
\text { Vent (sec) } \\
\text { (measured) }^{1}\end{array}$ & $\begin{array}{c}\text { Time to } \\
\text { Lid Loss } \\
\text { (sec) }\end{array}$ & $\begin{array}{l}\text { Maximum Wall } \\
\text { Temperature at } \\
\text { Vent/Loss }\left({ }^{\circ} \mathrm{C}\right)\end{array}$ & $\begin{array}{c}\text { Average Wall } \\
\text { Temperature at } \\
\text { Vent Loss } \\
\left({ }^{\circ} \mathrm{C}\right)^{2}\end{array}$ & $\begin{array}{c}\text { Maximum } \\
\text { Internal } \\
\text { Pressure } \\
\text { (psig) }\end{array}$ & $\begin{array}{c}\text { Average } \\
\text { Internal Air } \\
\text { Temperature } \\
\left({ }^{\circ} \mathrm{C}\right)\end{array}$ & $\begin{array}{l}\text { Average } \\
\mathrm{dP} / \mathrm{dt}^{4} \\
(\mathrm{psig} / \mathrm{s})\end{array}$ & $\begin{array}{c}\text { Average } \\
\text { Burn Rate } \\
\text { of } \\
\text { Contents } \\
|\mathrm{g} / \mathrm{s}|\end{array}$ & Comments \\
\hline \multicolumn{11}{|c|}{$1.2 \mathrm{~m}$ Burner Exposure } \\
\hline 28-D1 & $2.3 \mathrm{~kg}$ paper & 130 & -. & 616 & 573 & 22.1 & 111 & 0.2 & 0.79 & $\begin{array}{l}\text { Gasket } \\
\text { completely } \\
\text { burned out }\end{array}$ \\
\hline $28-D 4$ & $2.3 \mathrm{~kg}$ plastic & 160 & -- & 606 & 564 & 11.4 & 80 & 0.076 & 0.06 & $\begin{array}{l}81 \mathrm{~cm}(32 \mathrm{in.}) \\
\text { of gasket } \\
\text { burned out }\end{array}$ \\
\hline 33-D1 & $2.3 \mathrm{~kg}$ paper & - & 144 & 690 & 637 & 23.7 & 171 & 0.09 & $\overline{--}$ & $\begin{array}{l}109 \mathrm{~cm} \mathrm{(43} \mathrm{in.)} \\
\text { of gasket } \\
\text { burned out }\end{array}$ \\
\hline 33-D4 & $2.3 \mathrm{~kg}$ plastic & -- & -. & 610 & 394 & 10.7 & 434 & $-\cdot$ & 0.93 & $\begin{array}{l}\text { Knocked over } \\
\text { by 33-D1 }\end{array}$ \\
\hline 34-D1 & $\begin{array}{l}2.3 \mathrm{~kg} \text { paper - } \\
\text { heptane }\end{array}$ & 111 & .. & 646 & 640 & 24.3 & 107 & 0.27 & 1.64 & No gasket left \\
\hline 34-D4 & Empty & 142 & -. & 694 & 399 & 12.1 & 402 & 0.08 & -- & $\begin{array}{l}1 / 2 \text { of gasket } \\
\text { burned out }\end{array}$ \\
\hline 35-D1 & $\begin{array}{l}2.3 \mathrm{~kg} \text { paper - } \\
\text { heptane }\end{array}$ & $\cdots$ & 145 & 655 & 640 & 20.8 & 106 & 0.076 & -- & $\begin{array}{l}74 \mathrm{~cm}(29 \mathrm{in} .) \\
\text { of gasket } \\
\text { burned out }\end{array}$ \\
\hline 35-D4 & Standard load & 300 & -- & 655 & 581 & 8.5 & 22 & 0.026 & 1.35 & \\
\hline 36-D1 & $\begin{array}{l}2.3 \mathrm{~kg} \text { paper - } \\
\text { toluene }\end{array}$ & -- & 56 & 494 & 358 & $\mathrm{Al}$ & 21 & $\mathrm{Al}$ & -. & $\begin{array}{l}\text { Gasket fully } \\
\text { intact }\end{array}$ \\
\hline 36-D4 & $\begin{array}{l}\text { Standard load } \\
\text { - bagged - } \\
\text { toluene }\end{array}$ & 165 & -- & 712 & 616 & 13.6 & 408 & 0.069 & 0.75 & \\
\hline
\end{tabular}


Table 14. Data Summary of Fire Exposure Tests (4 sheets).

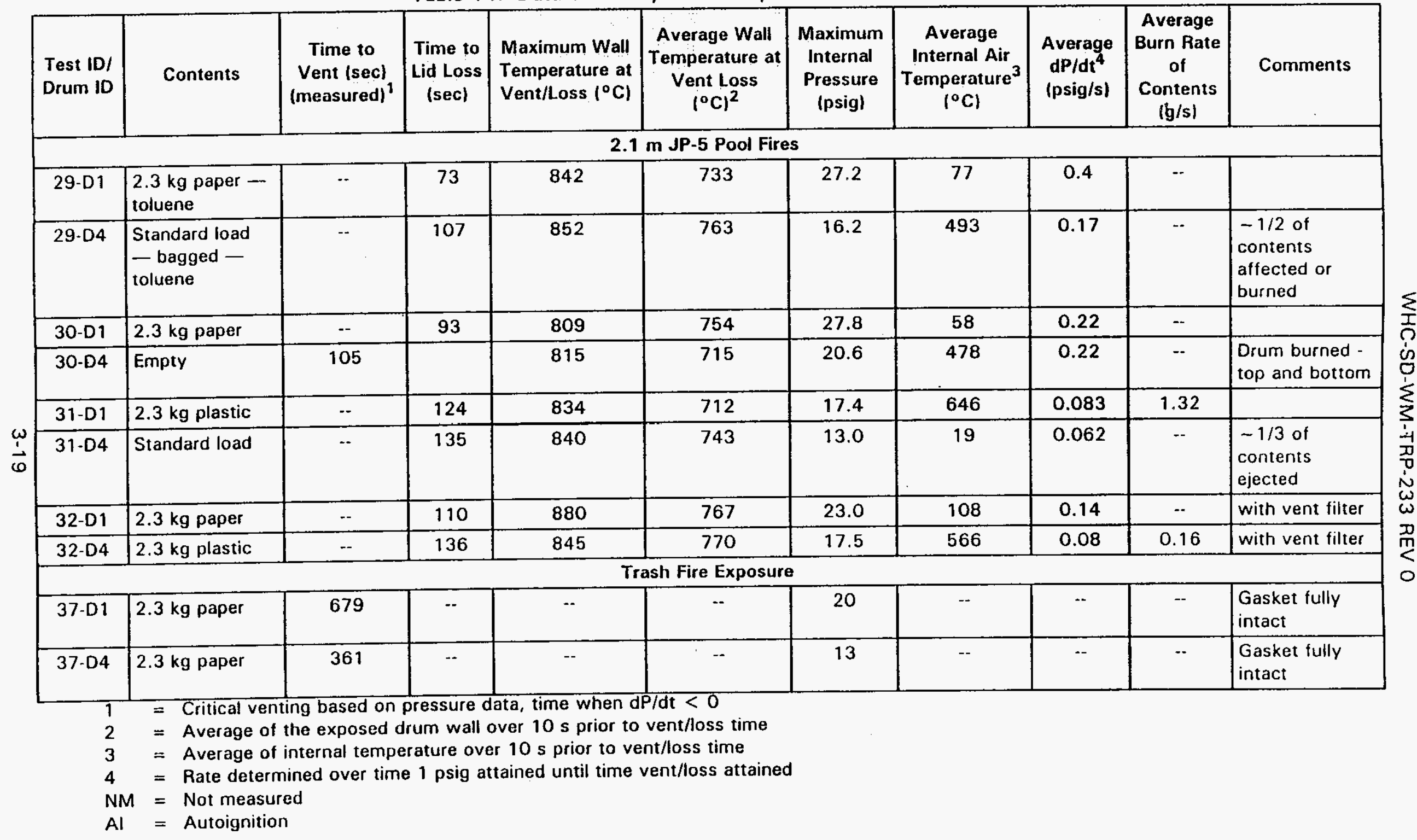




\subsubsection{Thermal Exposure}

The thermal exposure achieved is the major important characteristic of the three experimental configurations examined in this work. The thermal exposure was characterized by both total heat flux transducers placed in the wall of an empty target drum and by the use of a water filled drum which acted as a drum calorimeter. The point measurements with heat flux transducers were placed to characterize the peak local heat fluxes. Conversely, the drum calorimeter provided a measure of the total heat incident upon a drum. A summary of the measured thermal exposure is provided in Table 15 and Figure 24. Also included in Table 15 is the average heat flux to the drum using the area of the drum exposed to the flame as determined visually in the testing. These average heat fluxes will be used in the thermal modeling of these experiments.

Table 15. Thermal Exposures for Drum Response Tests.

\begin{tabular}{|l|c|c|c|c|}
\hline \multicolumn{1}{|c|}{ Fire Exposure } & $\begin{array}{c}\text { Peak Heat Flux } \\
\left(\mathrm{kW} / \mathrm{m}^{2}\right)\end{array}$ & $\begin{array}{c}\text { Heat Input } \\
(\mathrm{kW})\end{array}$ & $\begin{array}{c}\text { Exposed Drum } \\
\text { Area }\left(\mathrm{m}^{2}\right)\end{array}$ & $\begin{array}{c}\text { Average Heat } \\
\text { Flux }\left(\mathrm{kW} / \mathrm{m}^{2}\right)\end{array}$ \\
\hline Trash Fire & 50 & 10 & 0.4 & 25 \\
\hline $0.48 \mathrm{~m}$ Burner & 78 & 18 & 0.4 & 45 \\
\hline $1.2 \mathrm{~m}$ Burner & 100 & 35 & 0.8 & 44 \\
\hline $2.1 \mathrm{~m}$ Pool & 140 & 104 & 1.6 & 65 \\
\hline
\end{tabular}

During Test SWD 30, the remaining contents of drum SWD 29-D4 ignited and started to burn. Drum SWD 29-D4 had been moved from the previous test to a location $2.7 \mathrm{~m}(9 \mathrm{ft}$ ) away from the pan. The contents which filled approximately $30 \mathrm{~cm}(12$ in.) of the drum were charred and had been extinguished with water two days earlier. The pool fire of Test SWD 30 radiated to ignite Drum SWD 29-D4 which burned with flames reaching about $46 \mathrm{~cm}$ $\left(18 \mathrm{in}\right.$.) above the top of the drum. As a result of this incident, two $50 \mathrm{~kW} / \mathrm{m}^{2}$ heat flux transducers were placed near the base of Drum SWD 29-D4 during Tests SWD 31 and SWD 32. The measured peak heat flux was about $12 \mathrm{~kW} / \mathrm{m}^{2}$. The data are plotted in Volume 11-Appendix B for each test. 


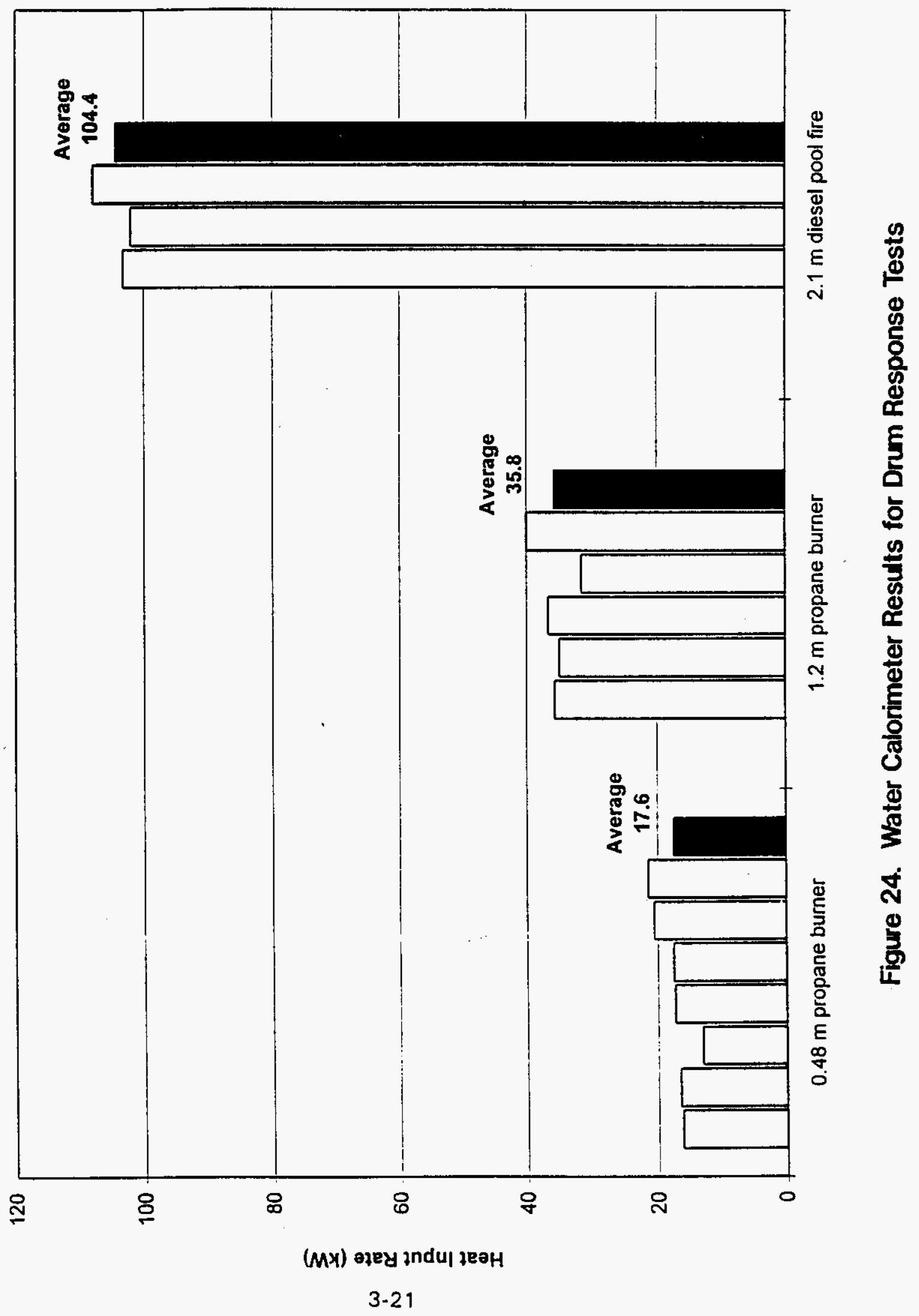




\subsubsection{Role of Flammable Liquids}

In some storage facilities there may be free flammable or combustible liquids included within the drum. Such free liquids could contribute to lid loss failure through evaporation to increase drum pressure or through autoignition of the vapors. Due to these possible contributions, 16 of the tests performed included flammable liquids. Liquids used included heptane, acetone, and toluene. Acetone and toluene have been documented in WHC drum inventories (Boothe, WHC-SD-W306-TI-001 Rev. 1), but no heptane has been documented. Heptane was selected because hydrocarbons have autoignition temperatures which are about $200^{\circ} \mathrm{C}$ less than most other vapors [Beyler, 1988]. Table 16 gives the flammable limits and autoignition temperatures (AIT) of these flammable liquids. Also included are the temperatures at which the equilibrium vapor pressure corresponds to the lower and upper flammable limits. The table clearly shows that heptane and toluene can be within the flammable limits at ambient temperatures, but acetone would be expected to be above the upper flammable limit. Further, a comparison of the published autoignition temperatures (AIT) with drum wall temperatures indicates that autoignition is a reasonable concern. It should be noted that AIT's are scale dependent and geometry dependent so that published values should only be considered as a guide to the suseptibility of vapors to autoignite. Of the 16 drums containing free flammable liquids, only two autoignited (one heptane and one toluene). Autoignition was determined by the extreme rapidity of the pressure rise and the low recorded lid loss pressures which result from large pressure increases within a data collection cycle.

Table 16. Flammability Limits and Autoignition Temperature of Flammable Liquids.

\begin{tabular}{|l|c|c|c|c|c|}
\hline \multicolumn{1}{|c|}{ Fuel } & LFL $(\%)$ & UFL $(\%)$ & $\mathrm{T}_{\text {LFL }}\left({ }^{\circ} \mathrm{C}\right)$ & $\mathrm{T}_{\text {UFL }}\left({ }^{\circ} \mathrm{C}\right)$ & AIT $\left({ }^{\circ} \mathrm{C}\right)$ \\
\hline Acetone & 2.6 & 13.0 & -20 & 8 & 465 \\
\hline Toluene & 1.2 & 7.1 & 4 & 39 & 480 \\
\hline Xylene & 1.1 & 6.6 & 25 & 61 & $465-530$ \\
\hline Heptane & 1.05 & 6.7 & -4 & 27 & 215 \\
\hline
\end{tabular}

One drum (SWD 21-D1) which autoignited contained $2.3 \mathrm{~kg}$ of paper and $0.2 \mathrm{~kg}$ of cotton towels soaked with $50 \mathrm{~g}$ of heptane and was exposed to the $0.48 \mathrm{~m}$ burner fire. The maximum pressure measured in the test was a mere $1.2 \mathrm{psig}$. At the next $1 \mathrm{~s}$ scan, the pressure was ambient. Clearly, the drum lid did not fail due to a 1.2 psig pressure rise. As noted in the data summary in Table 14, no normal drum lid loss occurred at less than 13 psig. The lid loss and recorded maximum pressure of 1.2 psig can only be explained by autoignition of the heptane with a rapid pressure rise and lid loss within the one second scan time. The maximum measured drum wall temperature measured at ignition was $440^{\circ} \mathrm{C}$, well in excess of the autoignition temperature. Four other drums with similar or higher drum wall temperatures did not ignite. The phenomena of autoignition could not be repeated for this fire exposure. Test No. SWD 23-D1, which was a repeat of the heptane autoignition drum, did not experience lid loss failure.

The second drum which autoignited (SWD 36-D1) contained $2.3 \mathrm{~kg}$ of paper and 0.2 $\mathrm{kg}$ of cotton towels soaked with $100 \mathrm{~g}$ of toluene which was exposed to the $1.2 \mathrm{~m}$ burner fire. The maximum pressure measured in the test was $6.8 \mathrm{psig}$. Based on this low value of pressure and the extremely rapid rate of pressure rise, this drum was judged to have 
autoignited. The maximum measured drum wall temperature measured at ignition was $494^{\circ} \mathrm{C}$, just in excess of the autoignition temperature. Seven other drums with similar or higher drum wall temperatures did not ignite.

These test results suggest that autoignition can occur in solid waste drums if free flammable liquids are present. However, the apparent liklihood is of autoignition is only 2 of 16 or 13 percent. While both autoignited drums contained $2.3 \mathrm{~kg}$ of paper with flammable liquid, there were also seven drums containing $2.3 \mathrm{~kg}$ of paper and flammable liquid which did not ignite. Further, the temperature and pressure data show no systematic trends which might explain why some drums autoignite and others do not. There does not seem to be any systematic nature to the results. We know from the data in Table 16 that upon heating the liquid soaked towels, it is possible to develop a rich mixture outside the flammable limits. Thus, autoignition may require that the drum wall temperatures reach high enough values without heating the towels sufficiently to cause excess vapor formation. The two drums which did ignite had the flammable liquid next to the exposed drum wall. However, several tests with the flammable liquid next to the exposed drum wall did not autoignite, and tests where the liquid was remote from the exposed wall did not ignite. As is often the case with autoignition, the process seems very random even when the vapor concentrations and wall temperatures are within the ranges where autoignition is normally expected.

It is significant that with the exception of the two tests believed to have autoignited, the presence of flammable liquids had no effect on the pressure, temperature, or time of lid loss or lid seal failure. Thus, the role of free flammable liquids in the response of drums is limited to its potential to cause autoignition, which as we have already seen occurred in only $13 \%$ of the flammable liquid containing drums tested. As such, the effect of flammable liquids is less than might be imagined. Unfortunately, due to the extremely rapid pressure rise in the autoignition events, it was not possible to measure the peak pressure at lid loss for autoignition induced lid loss. This value is needed for stability analyses for autoignited drums in arrays.

\subsubsection{Drum Seal Failure and Resulting Material Loss}

The drums with lid seal failure typically failed at the drum lid clamp tightening bolt area of the lid with gases jeting from the leakage location. This jet sometimes ignited, resulting in a jet flame of up to about $0.5 \mathrm{~m}$ in length. In no case was the jet flame of any consequence relative to the exposure fire. Table 14 includes the drum mass loss rates for for drums which failed by lid seal failure (venting). Mass loss rates range from 0.01 to $1.64 \mathrm{~g} / \mathrm{s}$ during the fire exposure. There was no relationship between drum contents and the mass lost except that plastic contents tended to result in very low mass losses. This is expected since significant pyrolysis of plastics does not occur until the full plastic load is melted into a pool in the bottom of the drum. 
Excluding the plastic only drums and empty drums, it is rational to correlate the mass loss using an apparent heat of gasification such that

$$
\dot{m}=\frac{\dot{Q}}{L}
$$

where $\dot{m}$ is the mass loss rate, $\dot{Q}$ is the heat input rate, and $L$ is the apparent heat of gasification. Using the mass loss rate data in Table 14 and the respective heat input rate values in Table 15 for all of the tests except empty and plastics only drums yields an average heat of gasification of about $40 \mathrm{~kJ} / \mathrm{g}$. The variations in the apparent heat of gasification between tests are about a factor of two. A conservative value of $20 \mathrm{~kJ} / \mathrm{g}$ overpredicts all the available data and may be useful for bounding analyses. These apparent heats of gasification are unusually large. This results from the use of an incident flux for the determination and the unusually large heat losses associated with combustibles within a drum.

\subsubsection{Lid Loss}

In the $0.48 \mathrm{~m}$ propane burner tests, 18 drums were exposed, and only one drum experienced lid loss. This drum included $50 \mathrm{~g}$ of heptane which autoignited, resulting in lid loss (see Section 3.2.2). In the $1.2 \mathrm{~m}$ propane burner exposure fire tests, ten drums were exposed, and only three drums experienced lid loss. One of the lid losses was due to autoignition of toluene contained in the drum while the remaining two drums lost their lids due to heat induced overpressures of 23.7 and $20.8 \mathrm{psig}$. In the trash fire test, two drums were exposed, and neither experienced lid loss failure. In the $2.1 \mathrm{~m}$ pool fire exposure tests, eight drums were exposed, and seven of the drums experienced lid loss. The only drum which did not lose its lid was an empty drum. The experiments effectively span the relevant exposures expected in the drum storage facilities. Excluding the autoignition lid losses, the presence of flammable liquids was found to have no significant effect on the performance of a drum (see Section 3.2.2). Therefore, while the two autoignition lid loss drums are treated as unique events, no further distinction is made between drums with and without flammable liquids. In the $0.48 \mathrm{~m}$ burner and trash fire tests, 0 percent of the drums experience lid loss; in the 1.2 $\mathrm{m}$ burner tests, 22 percent of the drums experience lid loss; and in the $2.1 \mathrm{~m}$ pool tests, 87 percent of the drums experience lid loss.

A probabilistic approach is taken because of the nonrepeatability of the drum tests. While the heat input rate to the drums did not significantly change for a given exposure (see Figure 24l, the failure mode of similar drums varied. Table 14 indicates several tests in which drums with identical contents were exposed to the same fire exposure and in one case experienced lid loss failure and in another only lid seal failure. This was especially true for the $1.2 \mathrm{~m}$ burner experiments.

Lid loss failure and lid seal failure can be regarded as competing failure mechanisms. If the lid seal degradation provides sufficient vent area prior to the failure of the lid clamp, then pressures will be relieved, and subsequent weakening of the clamp will not cause lid loss. Since clamp failure is a structural failure, it is rational to expect it to be a function of the temperature of the steel and the pressure in the drum. Typically, structural failure of steel can be cast in terms of a critical temperature with critical temperatures ranging from 500 to $700^{\circ} \mathrm{C}$. This narrow range results from the fairly rapid loss of strength above $500^{\circ} \mathrm{C}$ such 
that significant changes in loading have only a small effect on the critical temperature. Figure 25 shows a plot of the average exposed drum wall temperature at the time of lid loss or seal failure as a function of the drum pressure at failure. This plot indicates that there is no systematic trend in the temperature with pressure. Figure 26 shows a plot of the average exposed drum wall temperature as a function of the time. Lid loss failures occur at wall temperatures in the range $600-800^{\circ} \mathrm{C}$. Based on this information, a critical temperture in the range of $600-700^{\circ} \mathrm{C}$ would be a reasonable criterion for lid loss failure, with the precise value depending upon the degree of conservatism required.

This approach is simple and satisfactorily represents the available data. However, the criterion does not deal with gasket decomposition. If the temperatures are raised slowly, the gasket could decompose and vent before the critical temperature is reached, and lid loss would be predicted and would not occur. Based on an examination of Figure 26, it is clear that a correlation to represent the lid seal failure time is not possible. Lid seal failures occurred over a range of 2-6 minutes at temperatures of 300 to $600^{\circ} \mathrm{C}$, reflecting a great deal of random variation. However, an approach can be formulated which would allow lid seal failure to be estimated.

Since lid seal failure results from thermal decomposition of the gasket seal material, it is reasonable to attempt to model the process using a one step Ahrrenius decomposition law. The extent of decomposition, D, follows the integral of the Arrhenius rate equation,

$$
D=\int_{0}^{t} \exp ^{-E / R T} d t
$$

where $t$ is the exposure time, $T$ is the drum wall temperature, and $E$ is an activation energy for the decomposition process. The extent of decomposition parameter, $D$, is unitless and has no direct physical meaning. Its value is related to the effect of the thermal exposure, and a critical value of $D$ is expected to define lid seal failure if the correlation is successful. The free parameter in this expression is the activation energy. D was calculated for all experiments conducted using a range of trial activation energies, $E$, to find that value of $E$ which minimized the variation in $D$ at lid seal failure and which provided the best descrimination between lid seal failures and lid loss failures. Figure 27 shows the calculated $D$ at lid loss and/or lid seal failure for all the available experiments using $E / R=800 \mathrm{~K}$ as a function of the failure time. The goal in such a plot is to define a $D_{c r}$ which will not be reached in tests where lid loss occurs, but are approximately reached in all tests in where lid scal failure occurs. Figure 27 shows that the proposed correlation is not a viable method for representing the lid seal failure results. A successful correlation would have all the lid loss points below a critical value of $D$, and all the lid seal failures would cluster about a single value of $D$. While a $D$ value less than about 50 includes all of the lid loss drums, it also includes a similar number of lid seal failure tests. This is not an acceptable criteria for the failure mode of a drum. A range of values of $E / R$ were explored without success. Both Figures 26 and 27 indicate that lid seal failure is a highly random process. 


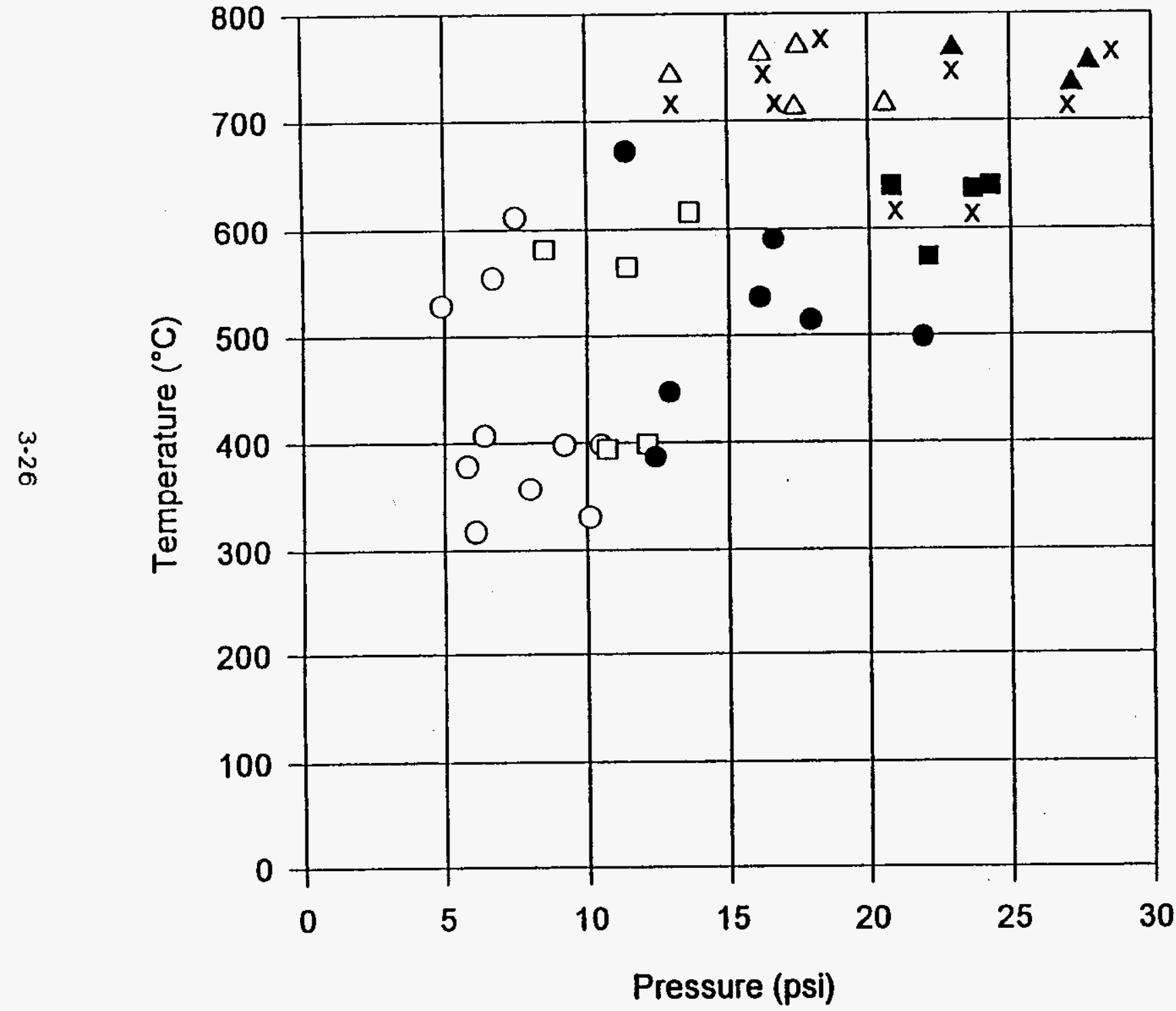

O $0.48 \mathrm{~m}$ Burner

口 $1.2 \mathrm{~m}$ Burner

$\triangle 2.1 \mathrm{~m}$ Pool Fire

- $0.48 \mathrm{~m}$ Burner

- $\quad 1.2 \mathrm{~m}$ Burner

- $\quad 2.1 \mathrm{~m}$ Pool Fire

Solid Symbols - $2.3 \mathrm{~kg}$ Brown Paper With and Without Toluene, Heptane, and Acetone

Open Symbols - Standard Load With and Without Toluene, $2.3 \mathrm{~kg}$ Plastic, and Empty Drum

$x$ - Lid Loss

Figure 25. Drum Wall Temperature as a Function of Pressure at Time of Failure 


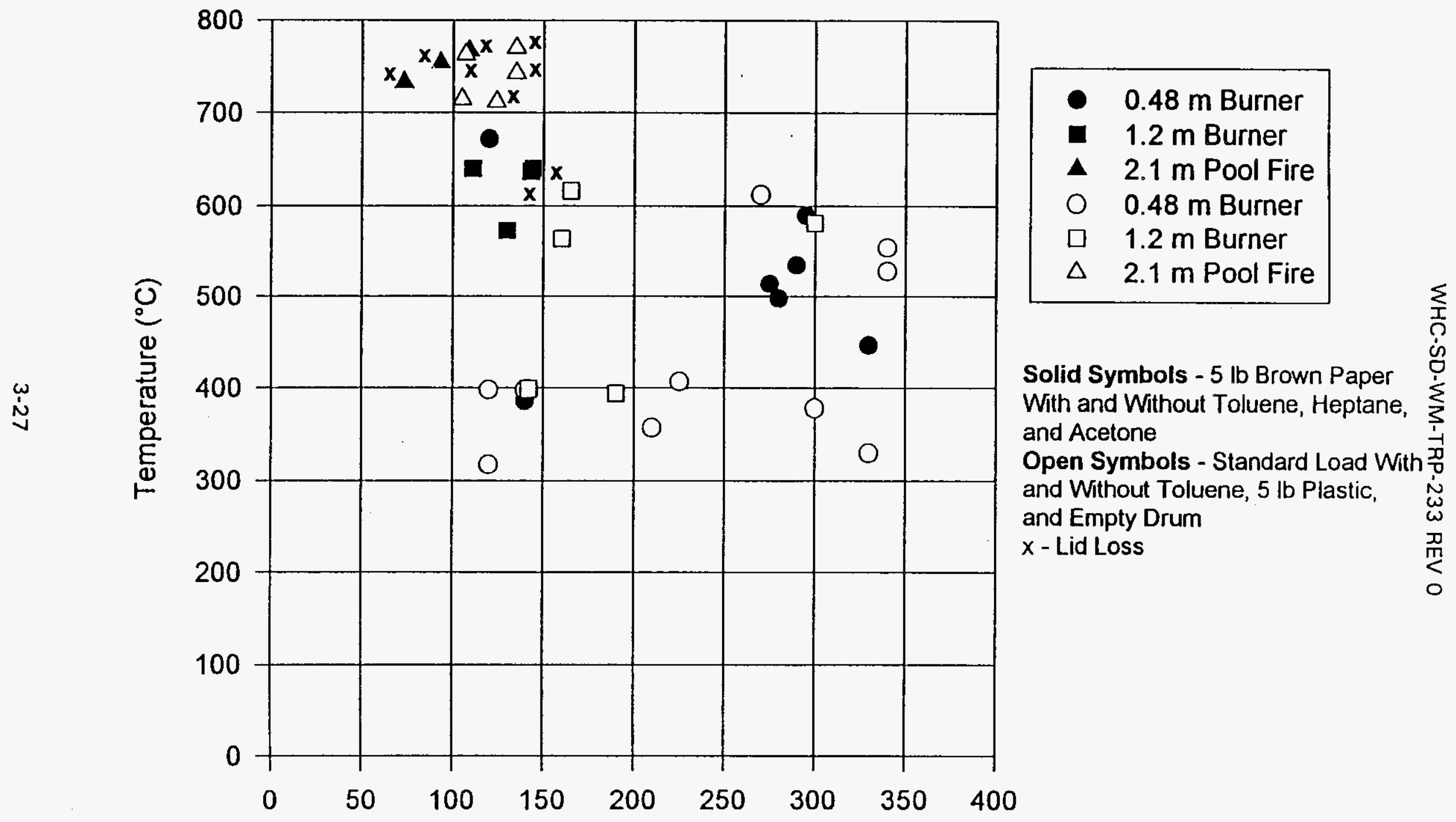

Time (sec)

Figure 26. Drum Wall Temperature as a Function of Failure Time 


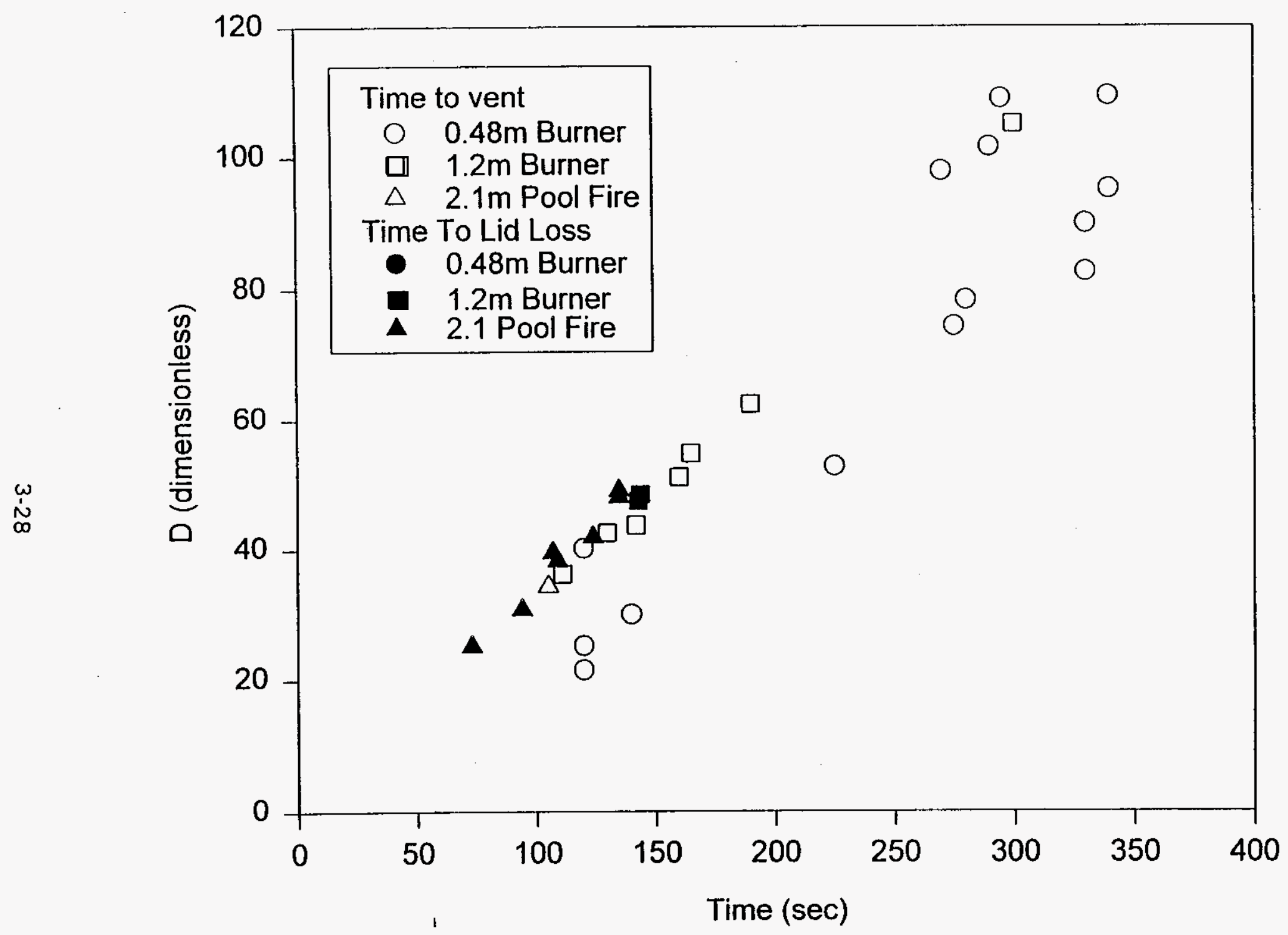

Figure 27. Arrhenius Decomposition Results for Drum Response Tests 
The drum pressure at lid loss is important to the stability of a drum array, if the lid loss drum is not in the top tier. Figure 28 shows a plot of the peak drum pressure as a function of the time to lid or lid seal failure. While there is a wide range of peak pressures observed, there seem to be systematic differences in the peak pressure with the type of contents in the drum. The $2.3 \mathrm{~kg}$ paper loads systematically yield higher peak pressures at lid loss than the remaining load types. The standard load drums had the lowest observed pressures at lid loss. This systematic difference was not noted in the drum wall temperatures. The observed systematic differences in peak pressure with load type cannot be explained by any measurements made in this test program. Since the most realistic drum loads yield the lowest lid loss pressures, this inability to explain the results is quite significant. As the array stability modeling (Section 4.10) will show, the differences in lid loss pressures can mean the difference between a drum array which is stable with regard to a lid loss in the array and an array which is not stable.

For purposes of defining a lid loss pressure for use in array stability analyses, it is useful to examine the probability of lid loss as a function of pressure. Figure 29 is a cummulative probability plot for all the data, and Figures $30-32$ are similar plots for each fire exposure separately. Figure 33 shows a frequency plot for lid loss pressures. These plots allow one to select a lid release pressure for a stability analysis which reflects the appropriate level of conservatism.

\subsubsection{Trash Fire Exposure}

Only a single test was conducted for the trash fire exposure. In this test, the two target drums each contained $2.3 \mathrm{~kg}$ of paper. Neither of the drums experienced lid loss failure, and they vented at a maximum pressure of 20 and 13 psig. As seen in Table 15, the thermal exposure from the trash fire was almost half that of the $0.48 \mathrm{~m}$ propane burner. During the test, no visible venting occurred, and after the test, the gasket seals were found to be undamaged. Unlike the burner and pool fire tests, the drum seals in the trash fire test were not in direct contact with the flame. As such, lid seal degradation occurred much slower than for the other exposures.

Drums which experience lid loss failure can expel the contents from the drum and lead to contents burning adjacent to other drums. Since lid loss failure did not occur for this test nor did it occur for the $0.48 \mathrm{~m}$ burner tests, it has been concluded that trash fire exposures will not lead to lid loss failure. However, drum contents fire are an important area of study which requires further investigation to confirm that fire propagation in drum arrays by expelled contents fires cannot occur.

\subsubsection{Vent Filters}

Two of the $2.1 \mathrm{~m}$ full engulfment tests had vent filters installed. Table 17 provides a comparison of several parameters between drums of like fuel loading but with and without vents. 


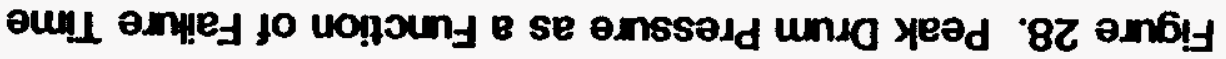

(วəs) əu!!

OOt OSE OOE OSZ OOZ OSL OOL OS 0

ssoา $p ! 7-x$

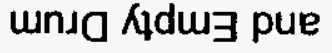
'o!nseld 6x है 2 'auanjo! inou!!M pue पi!M pe07 pjepueis - sjoquKs uado әuoləơ pue

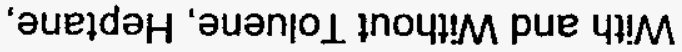

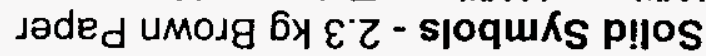

\begin{tabular}{|c|c|}
\hline & $\nabla$ \\
\hline 」əu」ng ய 乙゙l & $\square$ \\
\hline Jəuנng & O \\
\hline 2$\rfloor ! \jmath 100 d u l z$ & $\boldsymbol{\nabla}$ \\
\hline 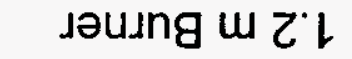 & \\
\hline 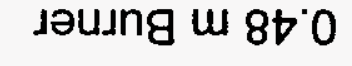 & \\
\hline
\end{tabular}

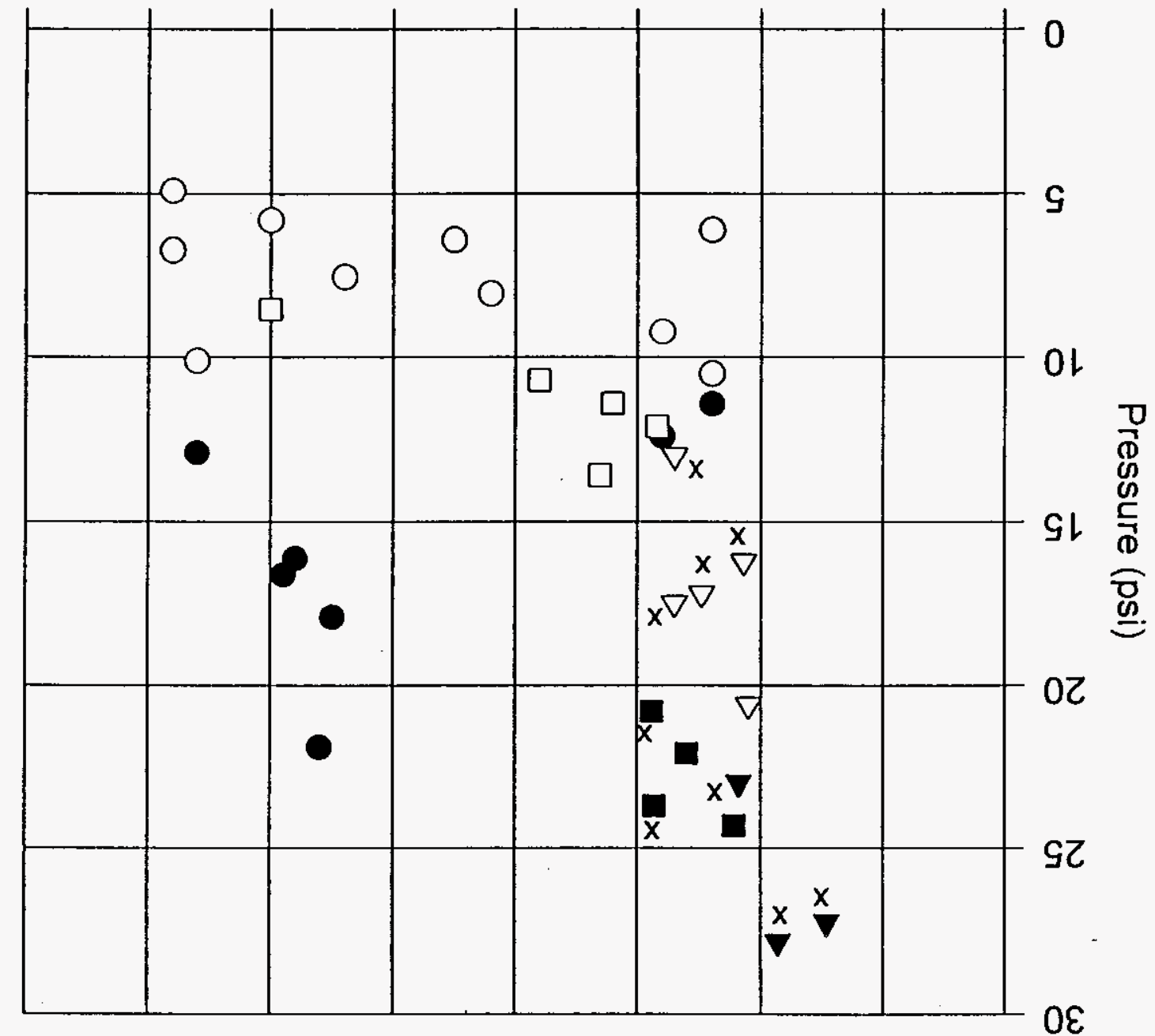


WHC-SD-WM-TRP-233 REV O

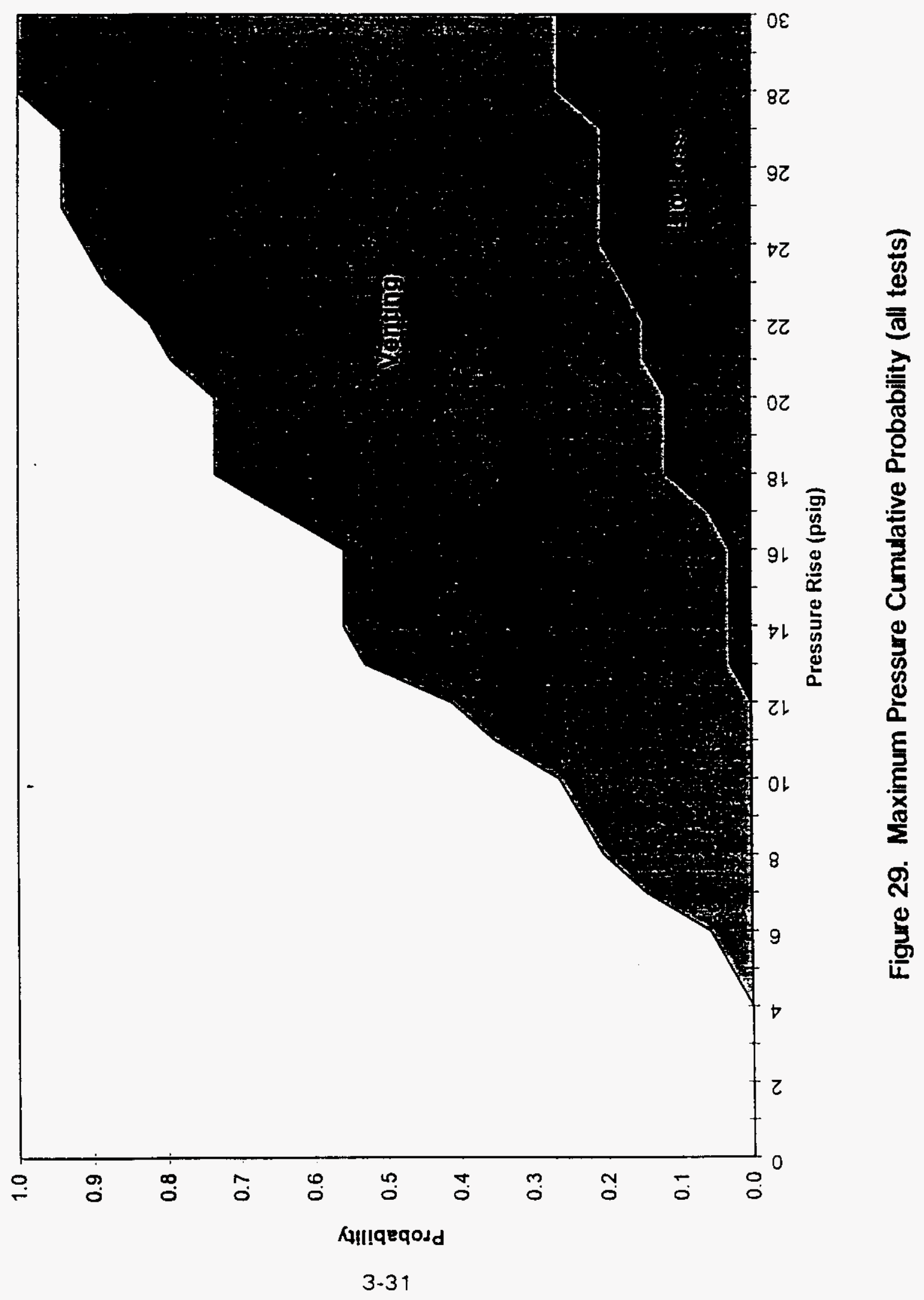




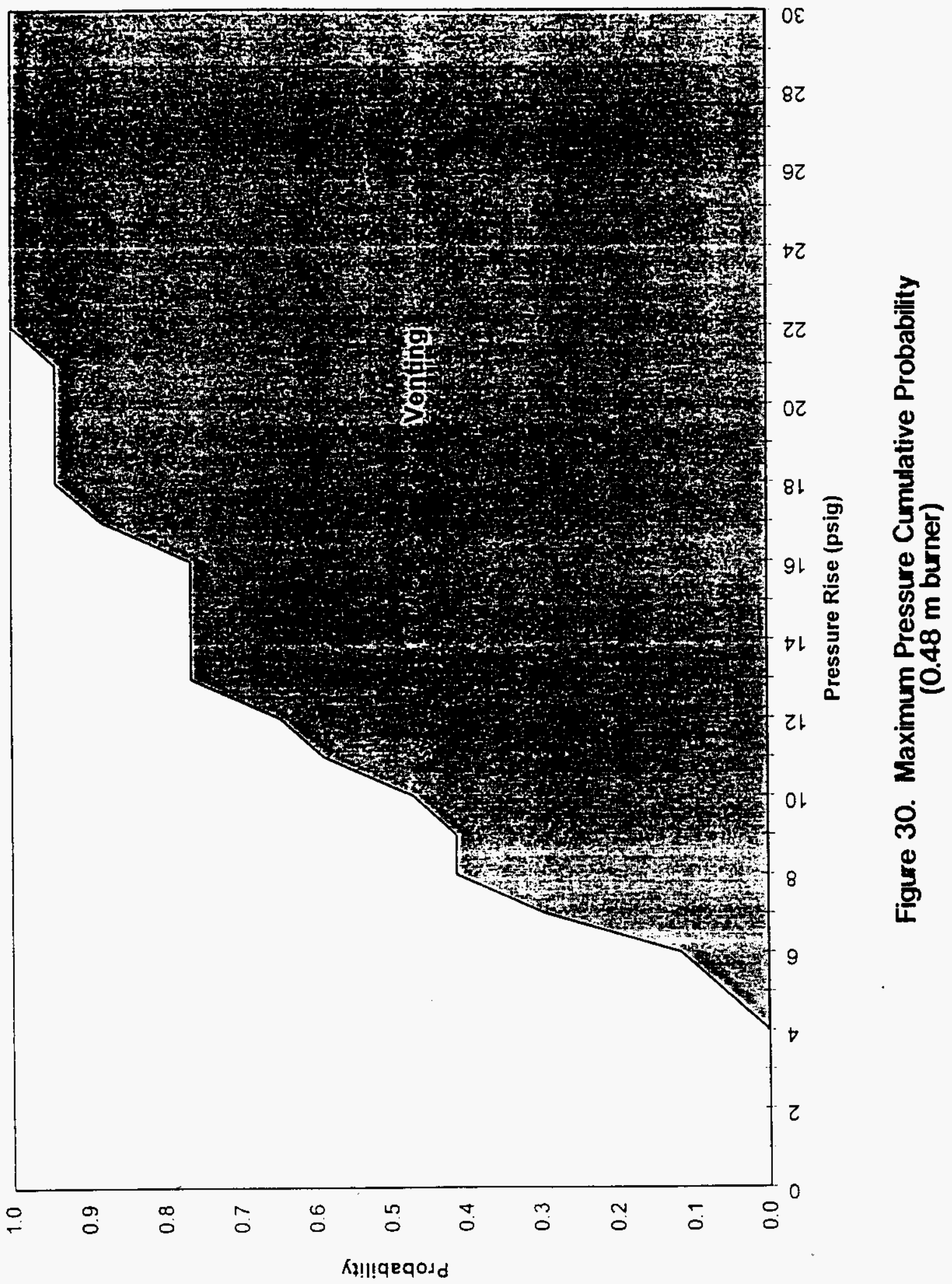




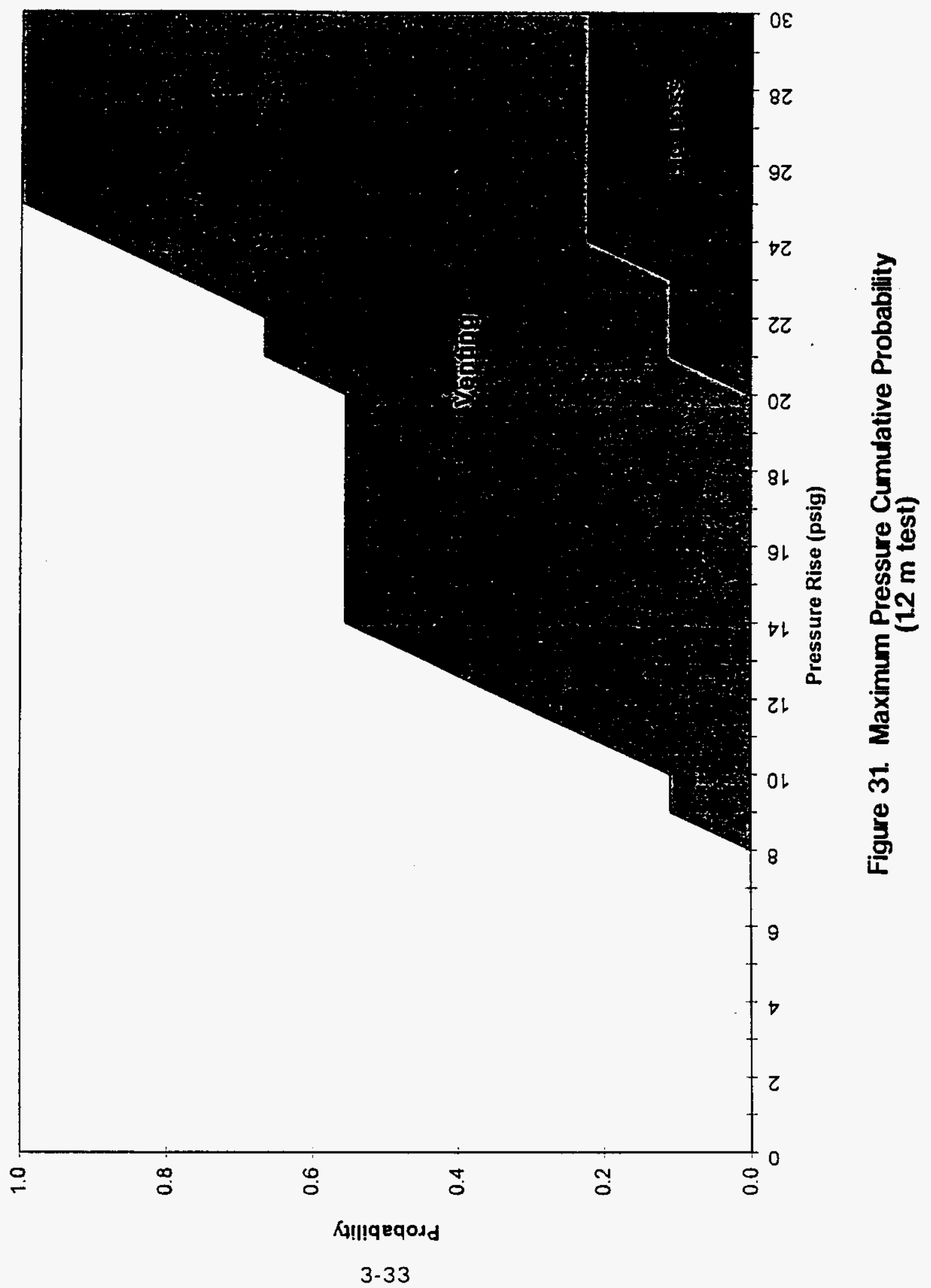


WHC-SD-WM-TRP-233 REV O

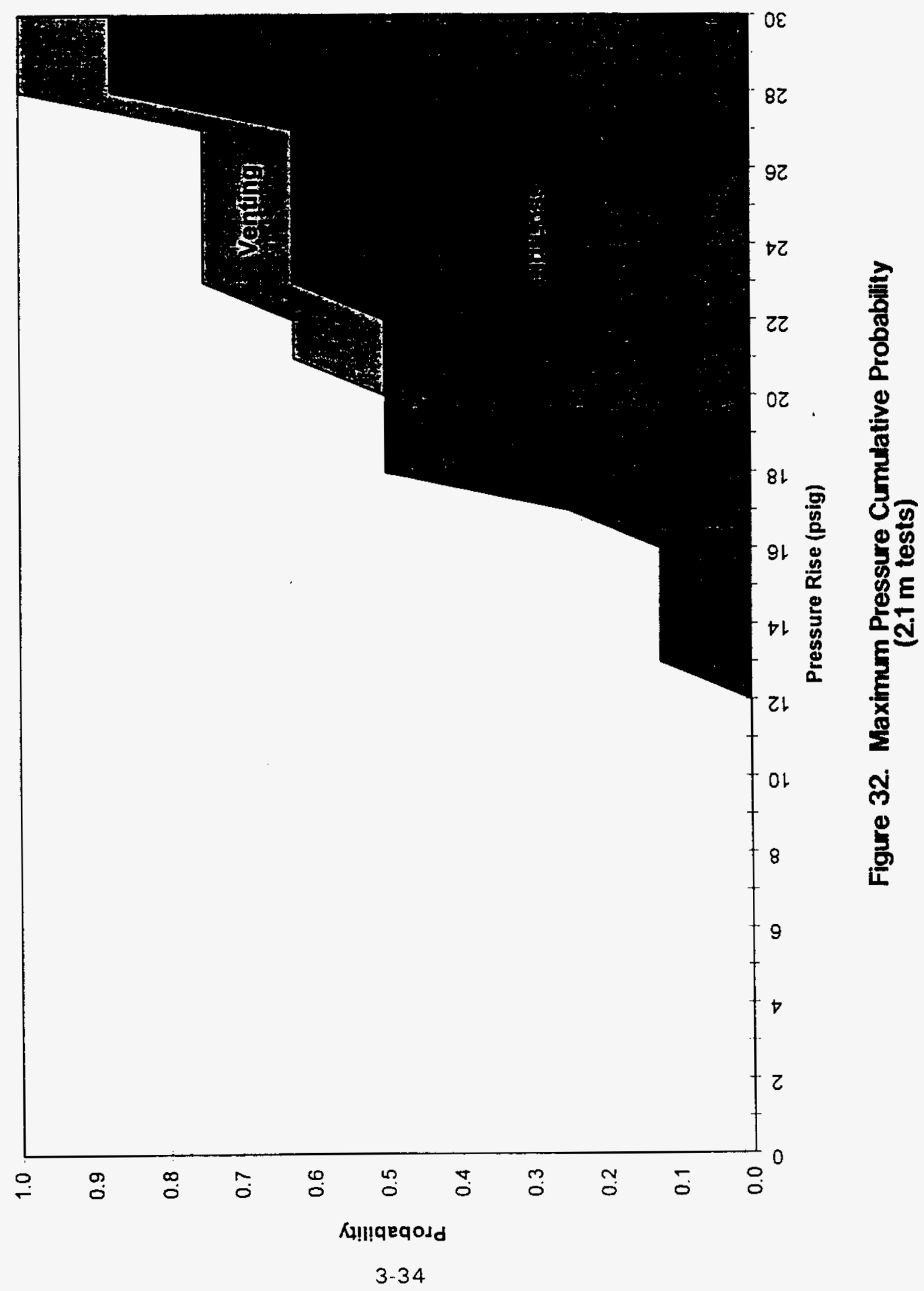




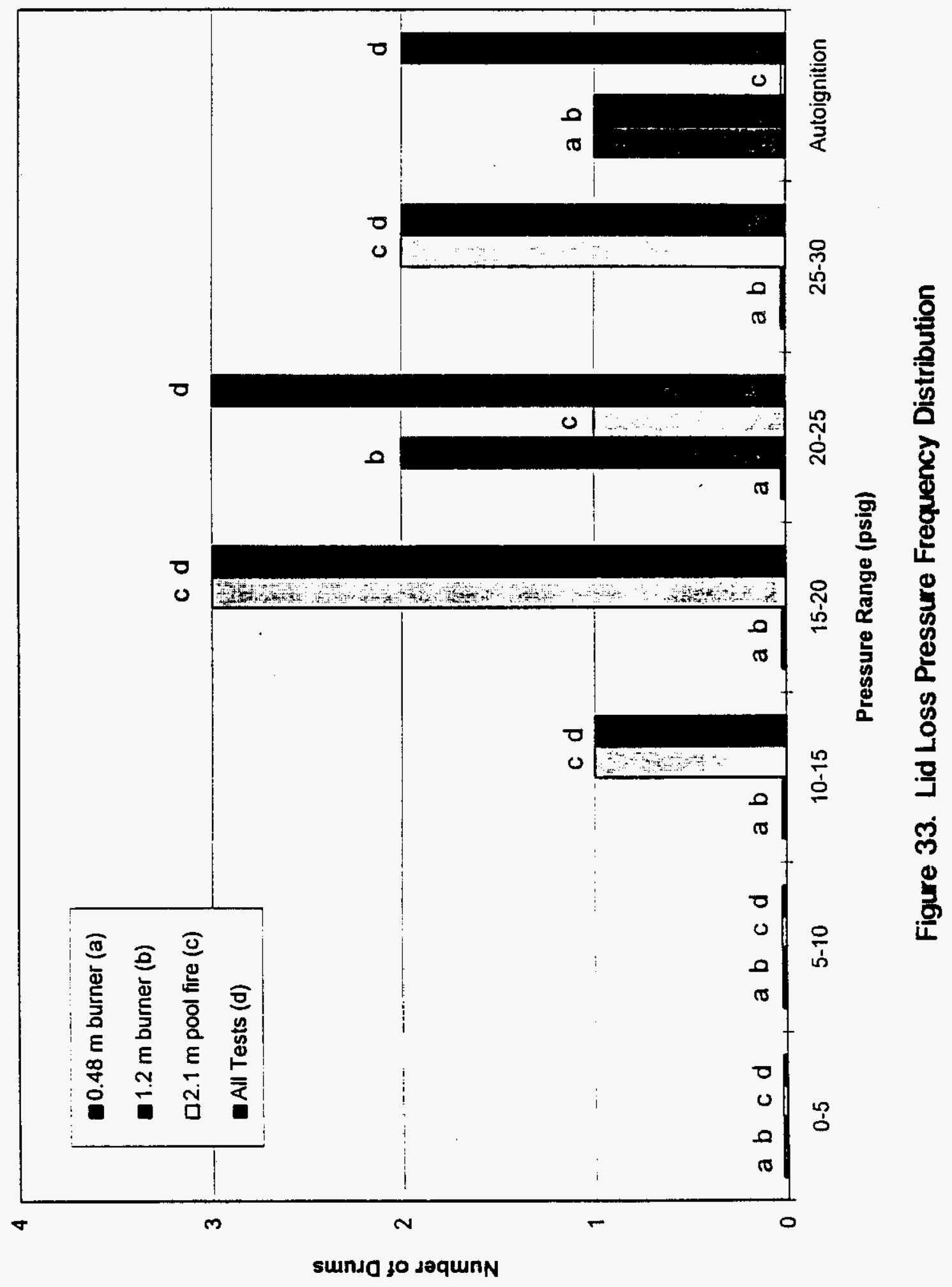


Table 17. Summary of Tests with and without Vents - Engulfing Fires.

\begin{tabular}{|c|c|c|c|c|}
\hline Test ID/Drum ID & Contents & $\begin{array}{c}\text { Time to Lid Loss } \\
\text { (s) }\end{array}$ & $\begin{array}{c}\text { Vents } \\
\text { (nstalled }\end{array}$ & $\begin{array}{c}\text { Maximum Internal } \\
\text { Pressure (psig) }\end{array}$ \\
\hline SWD 30-D1 & $2.3 \mathrm{~kg}$ paper & 93 & No & 27.8 \\
\hline SWD 32-D1 & $2.3 \mathrm{~kg}$ paper & 110 & Yes & 23.0 \\
\hline SWD 31-D1 & $2.3 \mathrm{~kg}$ paper & 124 & No & 17.4 \\
\hline SWD 32-D4 & $2.3 \mathrm{~kg}$ paper & 136 & Yes & 17.5 \\
\hline
\end{tabular}

As shown in Table 17, the use of the vent filter does not result in a significant difference in time to lid loss or maximum pressure inside the drums. Based on these results, it can be seen that small vent areas will have little impact on the overall drum response.

\subsection{GASKET SEAL TESTS}

Table 18 provides a summary of the gasket seal tests. Graphical data outputs for these tests are provided in Appendix B under the appropriate test number. The data tends to show that at the lower fuel settings, the gaskets lose integrity at a lower temperature regardless of the pressure.

Table 18. Summary of Gasket Seal Tests.

\begin{tabular}{|c|c|c|c|c|}
\hline Test No. & Overpressure (psi) & Fuel Flow (Lpm) & $\begin{array}{c}\text { Temperature at } \\
\text { Venting }\left({ }^{\circ} \mathrm{C}\right)\end{array}$ & $\begin{array}{c}\text { Time to } \\
\text { Vent (s) }\end{array}$ \\
\hline GS-1 & +5 & 5.43 & 258 & 330 \\
\hline GS-2 & +5 & 7.59 & 340 & 280 \\
\hline GS-3 & +10 & 5.43 & 271 & 358 \\
\hline GS-4 & +10 & 7.59 & 318 & 432 \\
\hline
\end{tabular}

Drum wall time-temperature curves are similar to the drum response testing described earlier in that the drum wall temperatures rise over a period of about 2-3 minutes before reaching nearly steady values. As such, the results of the gasket seal tests are similar to the low wall temperature at lid seal failure tests using the $0.48 \mathrm{~m}$ burner fire exposure. 


\subsection{ANALYTICAL — DRUM RESPONSE MODELS}

In addition to the HAl drum testing, a heat transfer analysis was conducted for various drum loading and storage configurations. Developing an analytical methodology to model the response of storage drums to fire exposures is desirable so that fire propagation through drum storage facilities can be assessed in the absence of specific fire testing.

For this analysis, drum dimensions are taken as those of a hollow cylinder $0.88 \mathrm{~m}$ (35 in.) in height, $0.59 \mathrm{~m}$ (23 in.) diameter, and $1.21 \mathrm{~mm}$ (18 gauge) wall thickness. Except where otherwise noted, the top and bottom of the drum are assumed to be adiabatic. This is a conservative approach because it does not allow heat losses through the top and bottom of the drum. In cases where this is not a conservative assumption, heat transfer to the top and bottom of the drum is considered. Analytical results are compared to HAl test data in order to assess the utility of such theoretical models. The following sections address the various drum configurations included in this analysis.

\subsection{EMPTY DRUM}

Analytically, an empty drum configuration is the simplest to model. Since there are no drum contents, heat is transferred through only two mediums: the steel drum wall, and the air inside the drum. The thermal properties for both the steel and the air are well known and are easily found in the literature. Figure 34 shows a cross sectional view of a single empty drum where a section of the wall, bounded by the angle $\theta$, is exposed to an external heat flux. The temperature of the exposed and unexposed sections (also referred to as the hot wall and cold wall) are assumed to be uniform along the perimeter of the drum. Although actual local drum wall and gas temperatures will vary, the internal pressure will only be dependant upon the average internal gas temperature. Since the wall thickness to surface area ratio is very small, the temperature gradient across the drum wall is assumed to be zero. This assumption is supported by the HAl drum tests where wall temperatures were measured at points on both the inside and outside of the drum for selected tests and were found not to differ by more than a few degrees Celsius. As such, the one dimensional unsteady heat transfer equations for the hot wall, cold wall, internal air temperature, and pressure are given by

Hot Wall

$$
c_{s} \rho_{s} d \frac{d T_{1}}{d t}=\dot{q}_{e x t}^{\prime \prime}+F_{1-1} \sigma T_{1}^{4}+\left(\frac{A_{2}}{A_{1}}\right) F_{2-1} \sigma T_{2}^{4}-h_{c}\left(T_{1}-T_{\infty}\right)-h_{c}\left(T_{1}-T_{a}\right)-\sigma\left(2 T_{1}^{4}-T_{-}^{4}\right)
$$


THIS PAGE INTENTIONALLY LEFT BLANK 


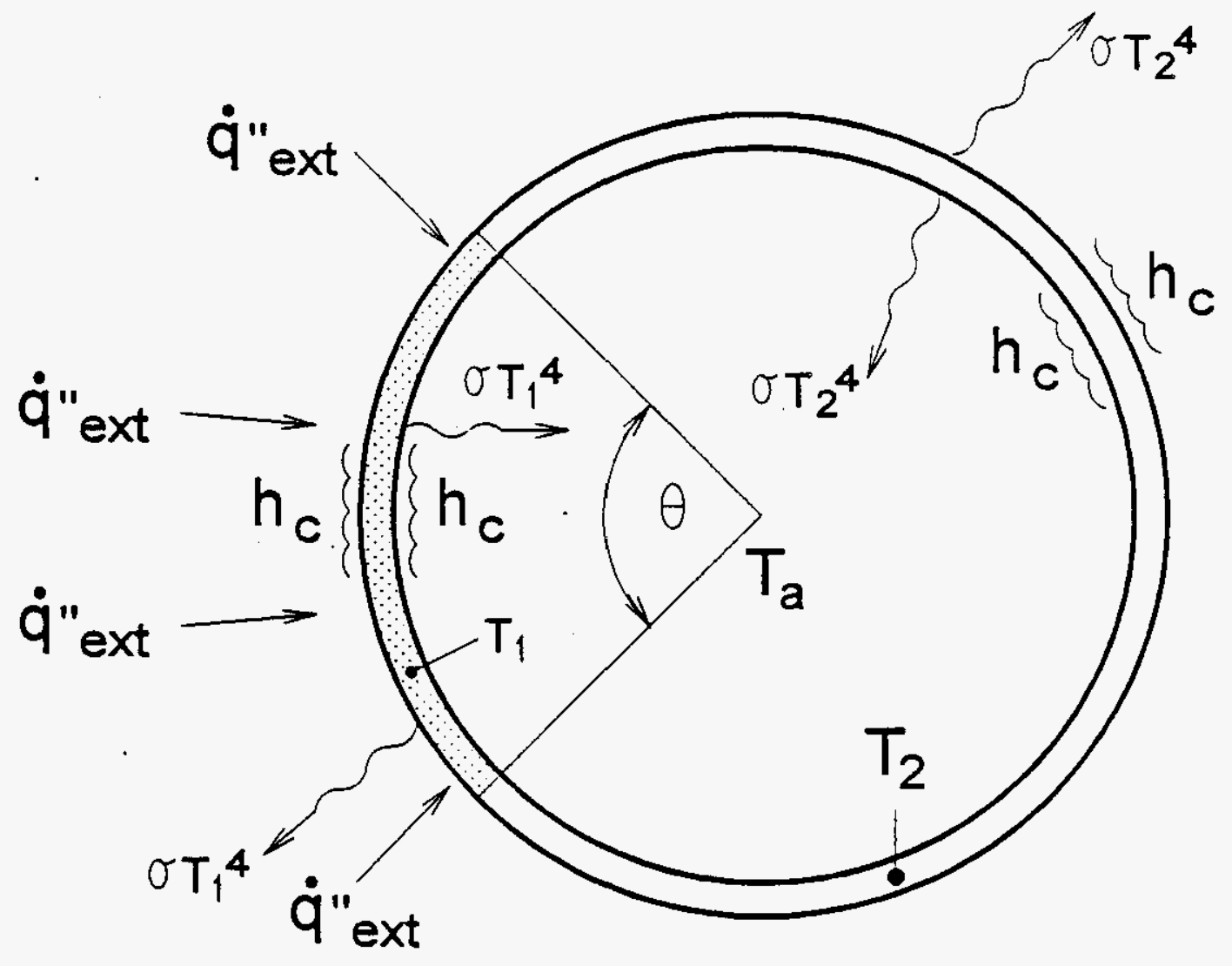

Figure 34. Heat Transfer Components for an Empty Drum Exposed to an External Heat Flux 
Cold Wall

$$
c_{s} P_{s} d \frac{d T_{2}}{d t}=\left(\frac{A_{1}}{A_{2}}\right) F_{1-2} \sigma T_{1}^{4}+F_{2-2} \sigma T_{2}^{4}+h_{c}\left(T_{a}-T_{2}\right)-h_{c}\left(T_{2}-T_{-}\right)-\sigma\left(2 T_{2}^{4}-T_{-}^{4}\right)
$$

Internal Gas Temperature

$$
c_{v} \rho_{o} V \frac{d T_{a}}{d t}=A_{1} h_{c}\left(T_{1}-T_{a}\right)-A_{2} h_{c}\left(T_{a}-T_{2}\right)
$$

Drum Pressure

$$
p=p_{o}\left(\frac{T_{a}}{T_{a, o}}\right)
$$

An important mechanism of heat transfer within the drum is the radiant energy exchange between the hot and cold drum walls. The thermal radiation to the drum wall is dependant upon the radiosity of the hot and cold walls in addition to the shape (configuration, view) factor between the walls. Whereas, the radiosity is a function of the wall temperature and may be determined from the heat transfer energy equations, the shape factor is solely a function of the drum geometry. The shape factor is effectively the fraction of energy leaving one surface which reaches a second surface. The top and bottom of the drum are taken as adiabatic in this model. The drum is taken as a cylinder of infinite height such that simple and conservative two dimensional shape factors may be determined. For all of the shape factor notations $\left(\mathrm{F}_{\mathrm{a}-\mathrm{b}}\right)$ used in this analysis, the first subscript indicates the surface which energy is leaving, and the second subscript is the surface which the energy is incident upon. For the empty drum configuration, there are four different shape factors within the drum: (1) the hot wall radiates to itself $\left(F_{1-1}\right),(2)$ the hot wall radiates to the cold wall $\left(F_{1-2}\right),(3)$ the cold wall radiates to itself $\left(F_{2-2}\right)$, and $(4)$ the cold wall radiates to the hot wall $\left(F_{2-1}\right)$. Figure 35 shows the layout of an exposed drum. The segment $x$ joins the points where the hot and cold wall meet. Using standard configuration analysis techniques,

$F_{x-1}=1$,

$F_{1-x} A_{1}=F_{x-1} A_{x}$.

and $F_{1-x}=A_{x} / A_{1}$.

Any radiant energy from the hot wall which does not pass through the segment $x$ will expose itself. Therefore,

$F_{1-1}=1-F_{1-x}$

or $F_{1-1}=1-A_{x} / A_{1}$.

Similarly, any radiant energy from the hot wall which passes through the segment $x$ will expose the cold wall. Therefore,

$F_{1-2}=F_{1-x}$

or $F_{1-2}=A_{x} / A_{1}$. 


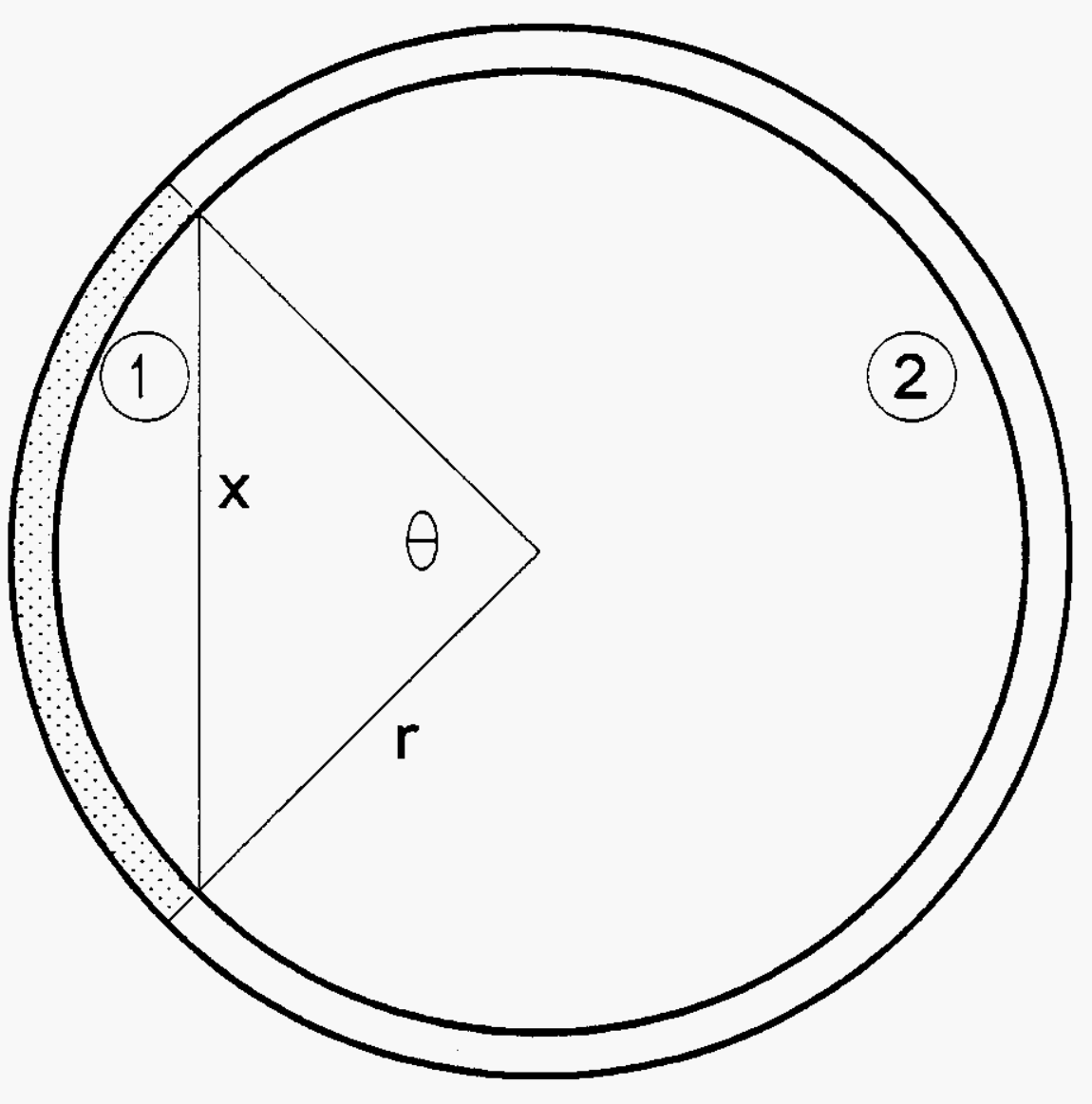

Figure 35. Empty Drum Configuration used for Shape Factor Analysis 
Likewise for the cold wall,

$F_{2 \cdot 2}=1-A_{x} / A_{2}$

and $F_{2 \cdot 1}=A_{x} / A_{2}$.

Since $A_{1}=\theta \mathrm{rh}$

$A_{2}=(2 \pi-\theta) r h$

and $A_{x}=2 r h \sin (\theta / 2)$

where, $r$ is the drum radius and $h$ is the drum height,

$$
\begin{array}{ll}
F_{1-1}=1-\left(\frac{2}{\theta}\right) \sin \left(\frac{\theta}{2}\right) & F_{2-2}=1-\left[\frac{2}{(2 \pi-\theta)}\right] \sin \left(\frac{\theta}{2}\right) \\
F_{1-2}=\left(\frac{2}{\theta}\right) \sin \left(\frac{\theta}{2}\right) & F_{2-1}=\left[\frac{2}{(2 \pi-\theta)}\right] \sin \left(\frac{\theta}{2}\right)
\end{array}
$$

For a given angle $\theta$, the shape factors between the walls may be determined.

\subsection{VENTING}

Equations 2 through 6 apply to an empty, air tight drum. If the drum is not air tight, as is often the case during a fire exposure, venting of the drum will occur. When exposed to an external heat source, drum venting may occur in several ways: (1) fitters or vent clips, (2) lid seal degradation, and (3) violent lid loss. Filters and vent clips allow a drum to maintain atmospheric pressure when subjected to normal temperature fluctuations. They are not designed, however, to prevent a rapid increase in pressure as might be expected during a fire exposure. As a drum is heated, the gasket providing the seal between the drum lip and lid will start to decompose. As the pressure builds in the drum, gases will vent at the weakest points of the lid seal. As the seal continues to degrade, the venting area will increase allowing more gases to escape. If this mode of failure continues, the drum will eventually reach ambient pressure. If the pressure and temperature in the drum increase rapidly, there may be insufficient time to vent the drum before violent lid loss occurs. In any case, the venting rate of the drum is a function of the internal pressure and the vent area. The venting rate is given by the compressible venting equation [Emmons, 1988]:

$$
\dot{m}_{v e n t}=\min \left\{\begin{array}{c}
C A_{v} \sqrt{\frac{m_{a}}{V} P_{a}}\left[\gamma\left(\frac{2}{\gamma+1}\right)^{\frac{\gamma+1}{\gamma-1}}\right]^{\frac{1}{2}} \\
C A_{v} \sqrt{\frac{m_{a}}{V} P_{a}}\left\{\frac{\gamma}{\gamma-1}\left(\frac{P_{a m b}}{P_{a}}\right)^{\frac{2}{\gamma}}\left[1-\left(\frac{P_{a m b}}{P_{a}}\right)^{\frac{\gamma-1}{\gamma}}\right]\right\}^{\frac{1}{2}}
\end{array}\right\}
$$

where the first expression is for choked-(sonic) flow. The vent area and internal pressure may change with time as is the case for a venting drum. If drum venting is to be accounted for, the venting rate and heat loss due to venting must be included in the empty drum energy equations. Whereas the drum wall temperature equations will remain the same, the internal temperature and pressure equations will change. As such, the unsteady heat transfer equations for an empty drum with venting are given as 
Hot Wall

$$
c_{s} \rho_{s} d \frac{d T_{1}}{d t}=\dot{q}_{\text {ext }}^{\prime \prime}+F_{1-1} \sigma T_{1}^{4}+\left(\frac{A_{2}}{A_{1}}\right) F_{2-1} \sigma T_{2}^{4}-h_{c}\left(T_{1}-T_{-}\right)-h_{c}\left(T_{1}-T_{a}\right)-\sigma\left(2 T_{1}^{4}-T_{a}^{4}\right)
$$

Cold Wall

$$
c_{s} \rho_{s} d \frac{d T_{2}}{d t}=\left(\frac{A_{1}}{A_{2}}\right) F_{1-2} \sigma T_{1}^{4}+F_{2-2} \sigma T_{2}^{4}+h_{c}\left(T_{a}-T_{2}\right)-h_{c}\left(T_{2}-T_{-}\right)-\sigma\left(2 T_{2}^{4}-T_{-\infty}^{4}\right)
$$

Internal Gas Temperature

$$
c_{v} \frac{d\left(m_{a} T_{a}\right)}{d t}=A_{1} h_{c}\left(T_{1}-T_{a}\right)-A_{2} h_{c}\left(T_{a}-T_{2}\right)-c_{p} T_{a} \dot{m}_{v e n t}
$$

where

$$
\frac{d m_{a}}{d t}=-\dot{m}_{v e n t}
$$

Since the mass of air in the drum will not be constant, the internal pressure is given by

$$
p=\frac{\bar{R} m_{a} T_{a}}{V}
$$

Figure 36 shows the pressure rise for an empty drum with various vent areas. The drum is fully exposed to a $60 \mathrm{~kW} / \mathrm{m}^{2}$ external heat flux. Initially, venting has little effect on the pressure rise in the drum. Ultimately, the larger vent areas result in a lower maximum drum pressure and a more rapid venting rate.

In order to assess the application of these equations, the calculated pressure and temperatures are compared to $\mathrm{HAl}$ drum response test results. Specifically, an empty drum was fully exposed to a $2.1 \mathrm{~m}(7 \mathrm{ft}$ ) square pool fire (SWD30-D4, Section 3.2).

Using a water calorimeter to measure the heating rate of the drums in the pool fire yielded an average heat flux of $65 \mathrm{~kW} / \mathrm{m}^{2}$ to the drum wall (Section 3.2). The external flux is assumed to evenly heat the entire perimeter of the drum wall. Solving Equations 3-6 for an empty drum with no vent, Figures 37,38 , and 39 show a comparison of the calculated and measured pressure rise, air temperature, and hot wall temperature, respectively. Since the drum is fully exposed to the fire, there is no cold wall temperature. Initially, the results compare rather well. It should be noted that the time lag in the measured results is due to the growth period of the pool fire. The analytical results assume that the incident heat flux is 65 $\mathrm{kW} / \mathrm{m}^{2}$ at time zero, whereas this is not the case for the pool fire experiment. At about 80 $s$, the measured pressure is seen to drop. This is due to lid seal degradation and subsequent venting. As a result, the calculated pressure and temperatures over estimate the experimental measurements once venting begins. 
WHC-SD-WM-TRP-233 REV 0

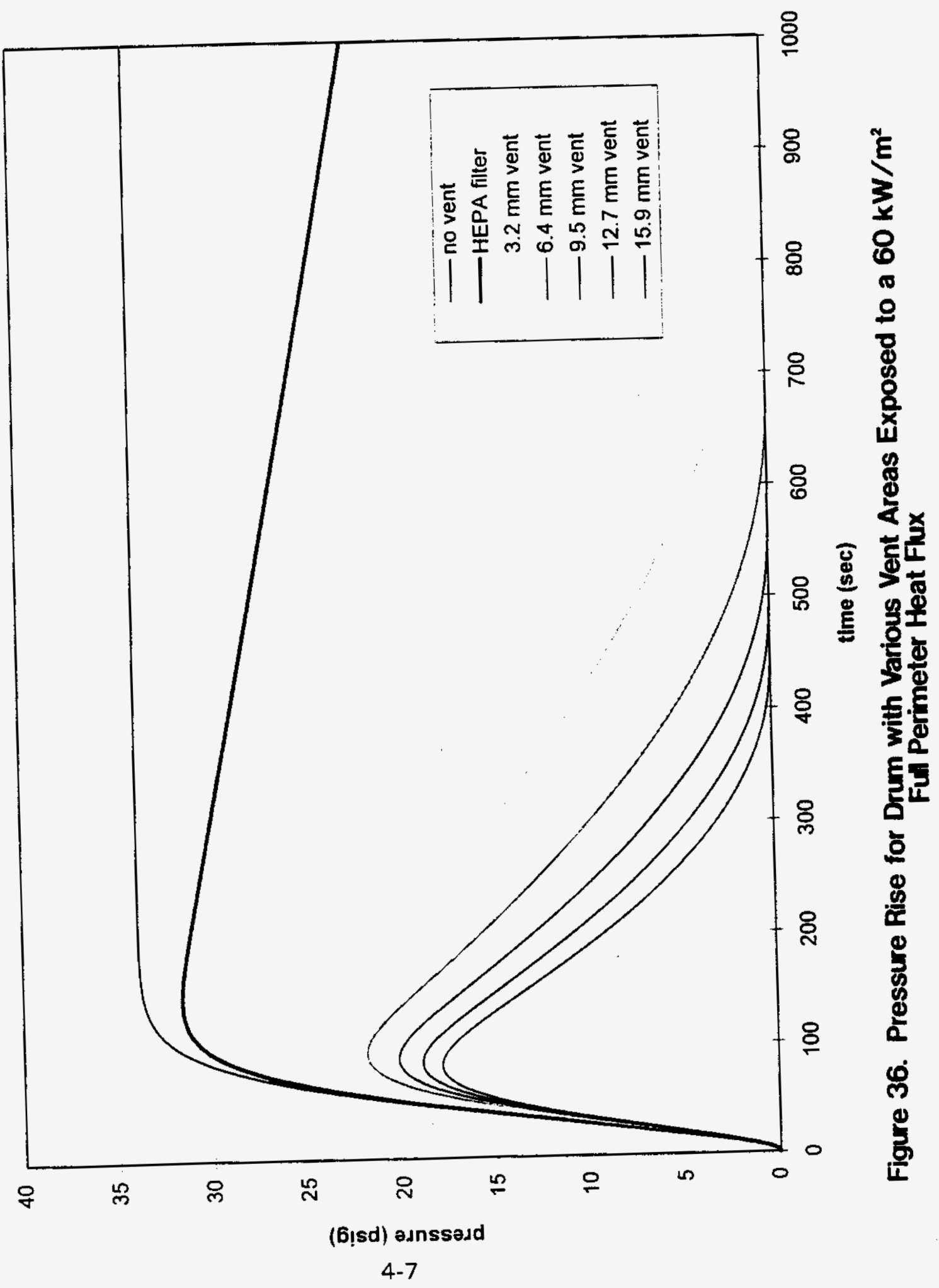


WHC-SD-WM-TRP-233 REV 0

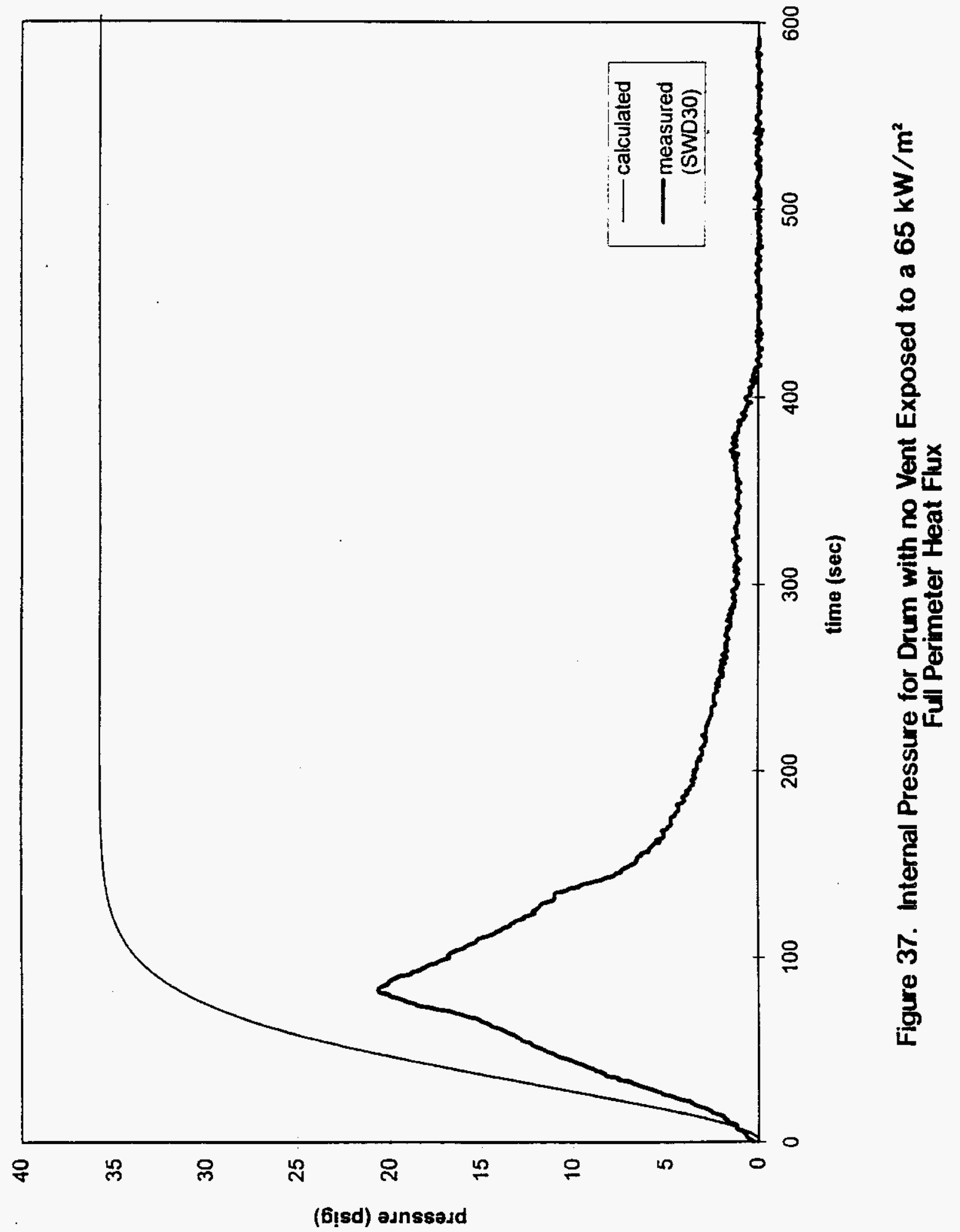

4-8 


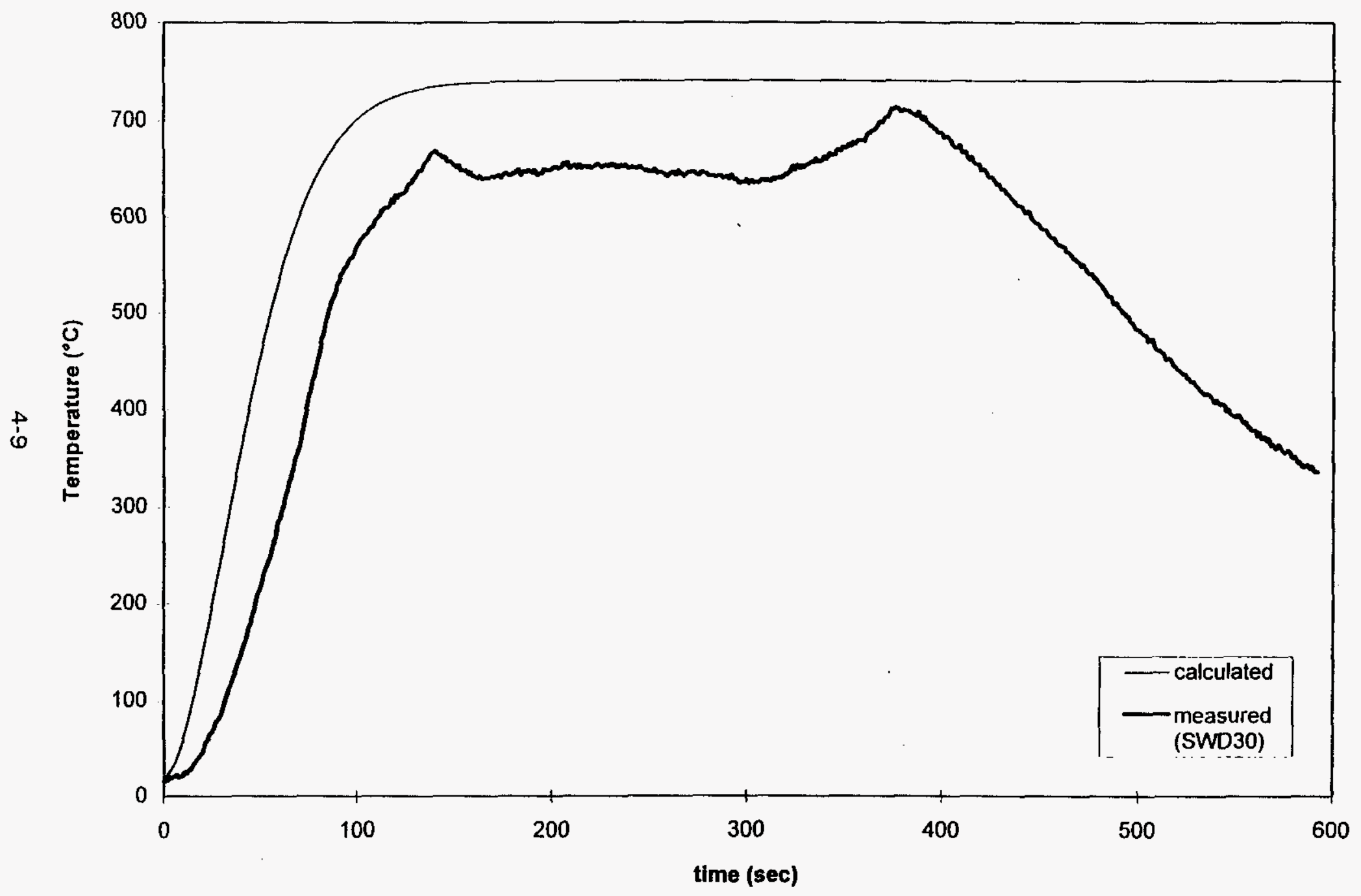

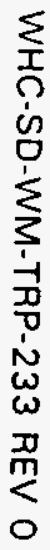

Figure 38. Internal Air Temperature for Drum with no Vent Exposed to a $65 \mathrm{~kW} / \mathrm{m}^{2}$ Full Perimeter Heat Flux 


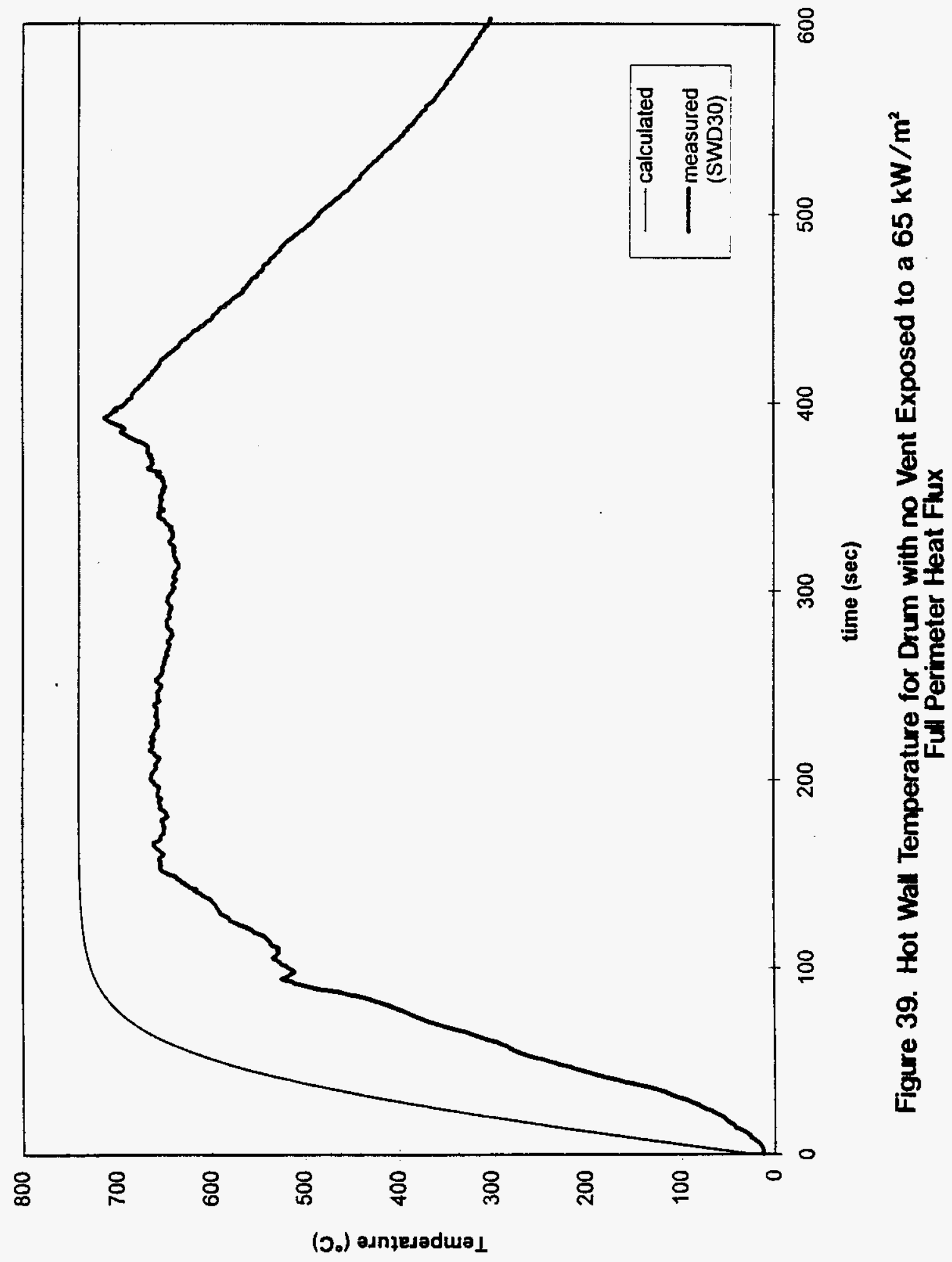


The venting equations can be used to account for the effects of lid seal failure for this test case. Solving Equations $13-18$ for the above drum exposure, Figures 40,41 , and 42 show the calculated pressure, air temperature, and hot wall temperature for a drum with a $16 \mathrm{~mm}$ (5/8 in.) diameter vent which is open at time zero. The vent area is assumed not to vary during the test. The predicted drum pressure (Figure 40) does not exactly correspond to the measured values because the vent area does not actually remain constant during the experiment. Although the actual vent area is unknown and changes with time during the test, this comparison illustrates the effect of venting via lid seal failure. Note that the vent has little effect on the initial pressure rise and the decay in pressure is slow once the drum begins to vent. Because the vent area can not accurately be predicted as a function of time, it is difficult to analytically account for venting due to lid seal degradation. If venting is ignored, calculated pressure and temperature results will be conservative compared to actual conditions.

\subsection{DRUM FILLED WITH CONTENTS}

Under normal storage conditions, the waste containers will not be empty. The drums are used to store a variety of materials ranging from noncombustible chemicals and metals to combustable loadings such as paper, plastic and flammable liquids. Under fire conditions, the contents will affect the heating rate and pressure rise in the drum. For low external exposures, the contents will act as a heat sink thus absorbing much of the energy transferred to the drum. This will result in a slower temperature and pressure rise in the drum. For this, a lumped mass analysis is used to model the drum exposure. For larger exposures, such as those involving direct flame impingement, a lumped mass analysis is not suitable. In these cases, the contents will be treated as a semi-infinite solid within the drum. The utility of each of the heat transfer analyses are assessed in the following sections.

\subsubsection{Lumped Mass Contents Analysis}

Figure 43 shows a cross sectional view of a single drum filled with a homogeneous substance where, again, a section of the wall is exposed to an external heat source. As with the empty drum configuration (and all other configurations), the hot wall, cold wall, and internal air temperatures are assumed to be uniform. As a first approximation, the temperature of the drum contents is also assumed to be uniform. This is known as a lumped mass analysis. In addition, the contents are assumed to completely fill the drum, thus absorbing all of the radiant energy from the interior drum walls. Therefore, there is no radiant heat transfer between the drum walls. Although the contents may be combustible, it is assumed that no pyrolysis occurs within the drum. If pyrolysis is included, the pressure in the drum is very dependant upon the molecular weight of the combustion products. Since the molecular weights of the products can vary as much as ten fold, it is inappropriate to include pyrolysis effects given the limitations of this analysis. However, by the time the combustible surface reaches a temperature at which pyrolysis can occur, the drum temperatures and pressure are already high enough to expect drum failure by either lid seal degradation or lid loss. Given the time frame in question, it seems acceptable to assume that pyrolysis does not contribute significantly to drum lid loss failures. In the HAl drum response tests, there were not added effects observed of pyrolysis influencing drum lid loss or seal failure. The one dimensional unsteady heat transfer equations for the hot wall, cold wall, drum contents, and internal air temperature are given by 


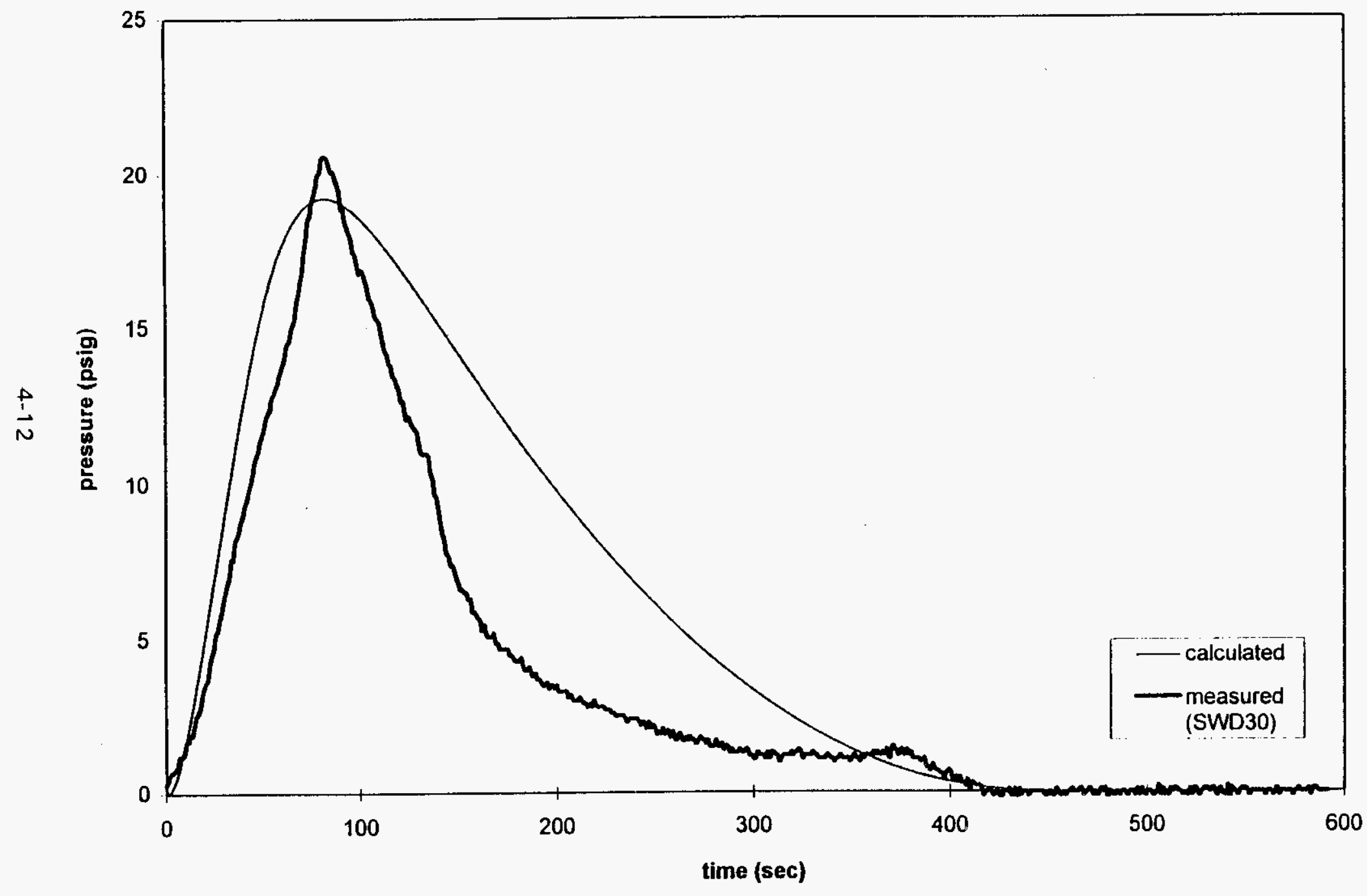

Figure 40. Internal Pressure for Drum with a $1.6 \mathrm{~cm}$ (5/8 in.) Diameter Vent Exposed to a $65 \mathrm{~kW} / \mathrm{m}^{2}$ Full Perimeter Heat Flux 


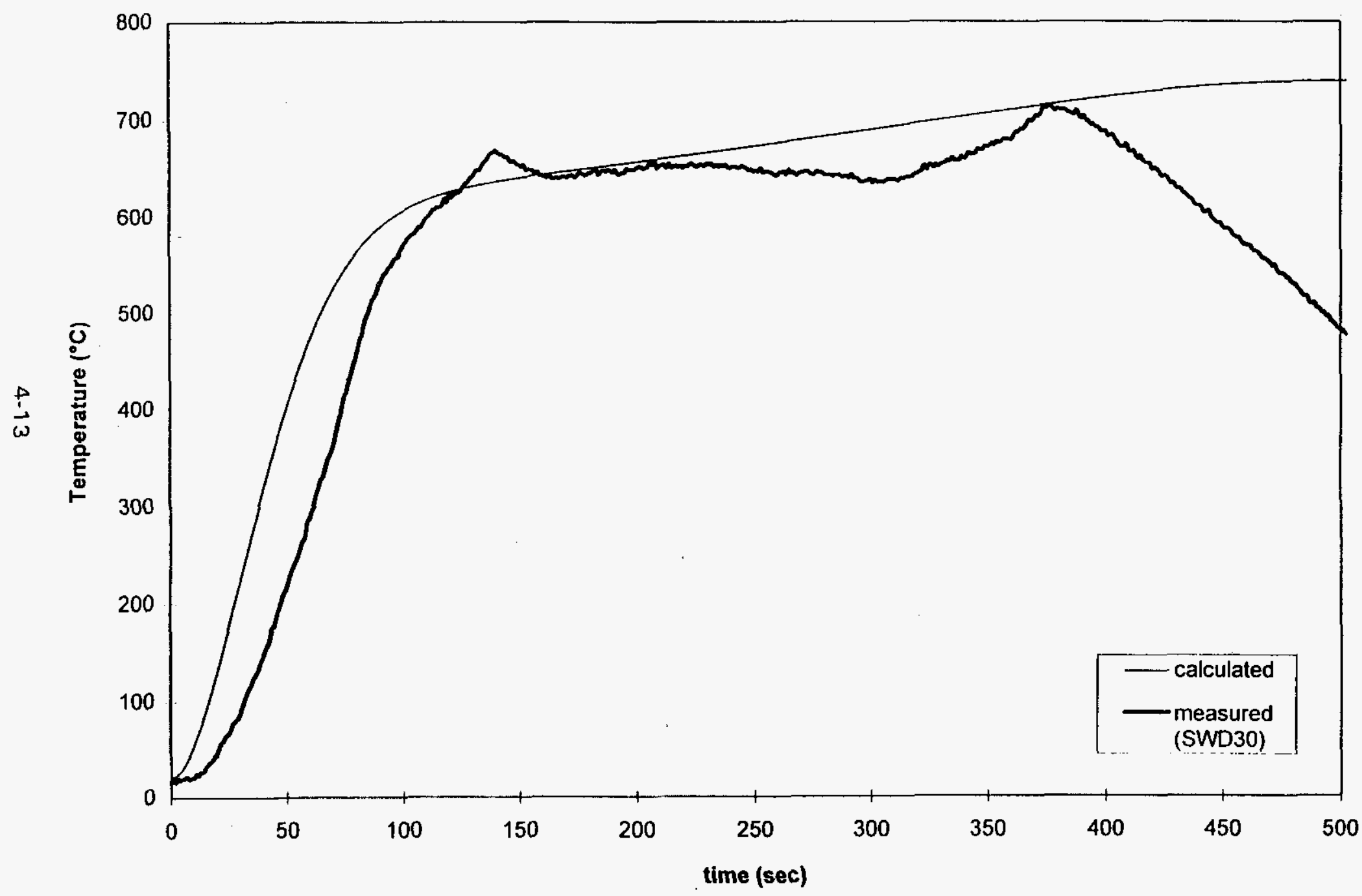

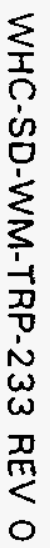

Figure 41. Internal Air Temperature for a Drum with a $1.6 \mathrm{~cm}(5 / 8 \mathrm{in})$ Diameter Vent Exposed to a $65 \mathrm{~kW} / \mathrm{m}^{2}$ Full Perimeter Heat Flux 


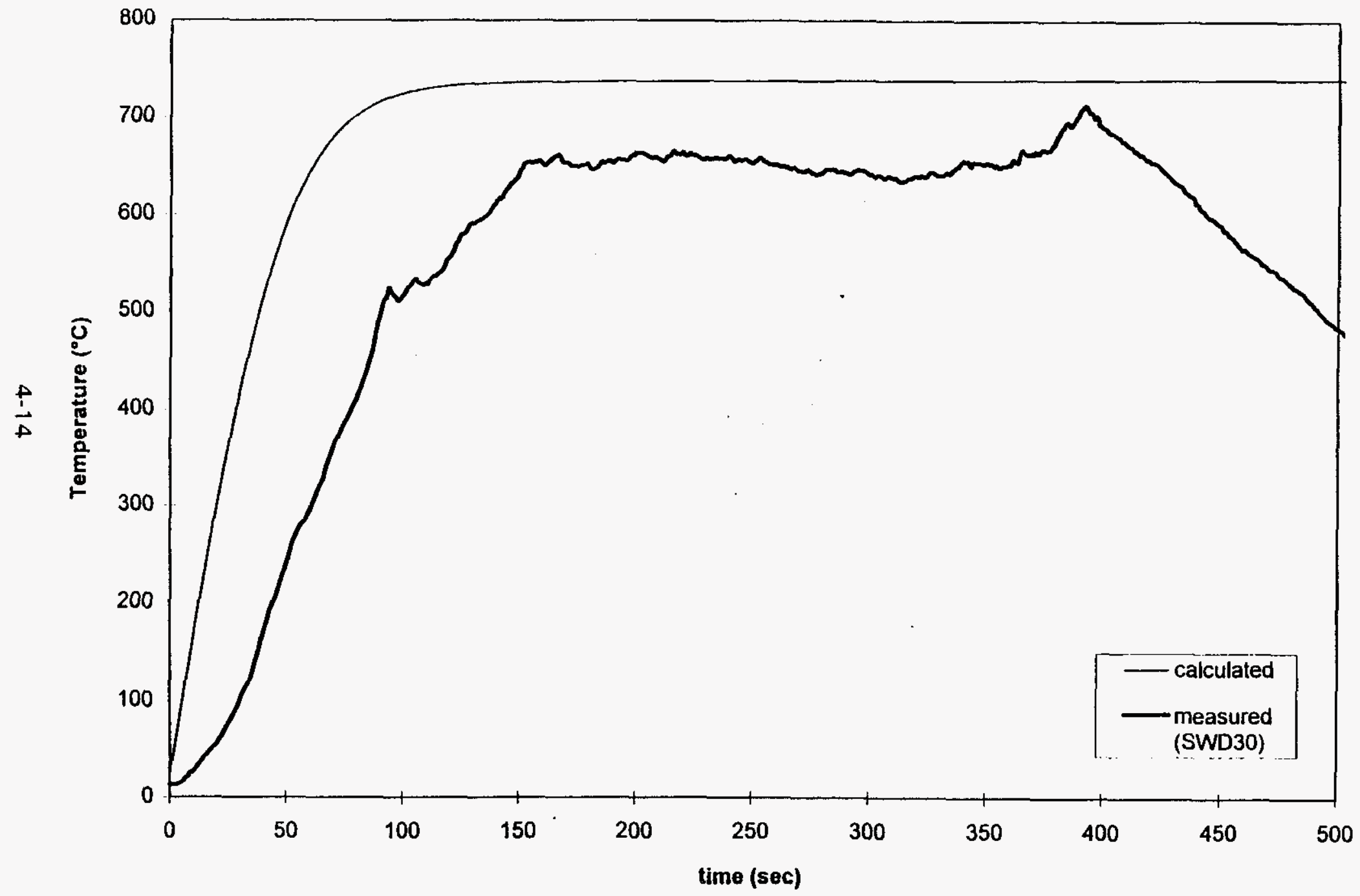

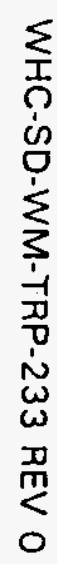

Figure 42. Hot Wall Temperature for a Drum with a $1.6 \mathrm{~cm}$ (5/8 in) Diameter Vent Exposed to a $65 \mathrm{~kW} / \mathrm{m}^{2}$ Full Perimeter Heat Flux 


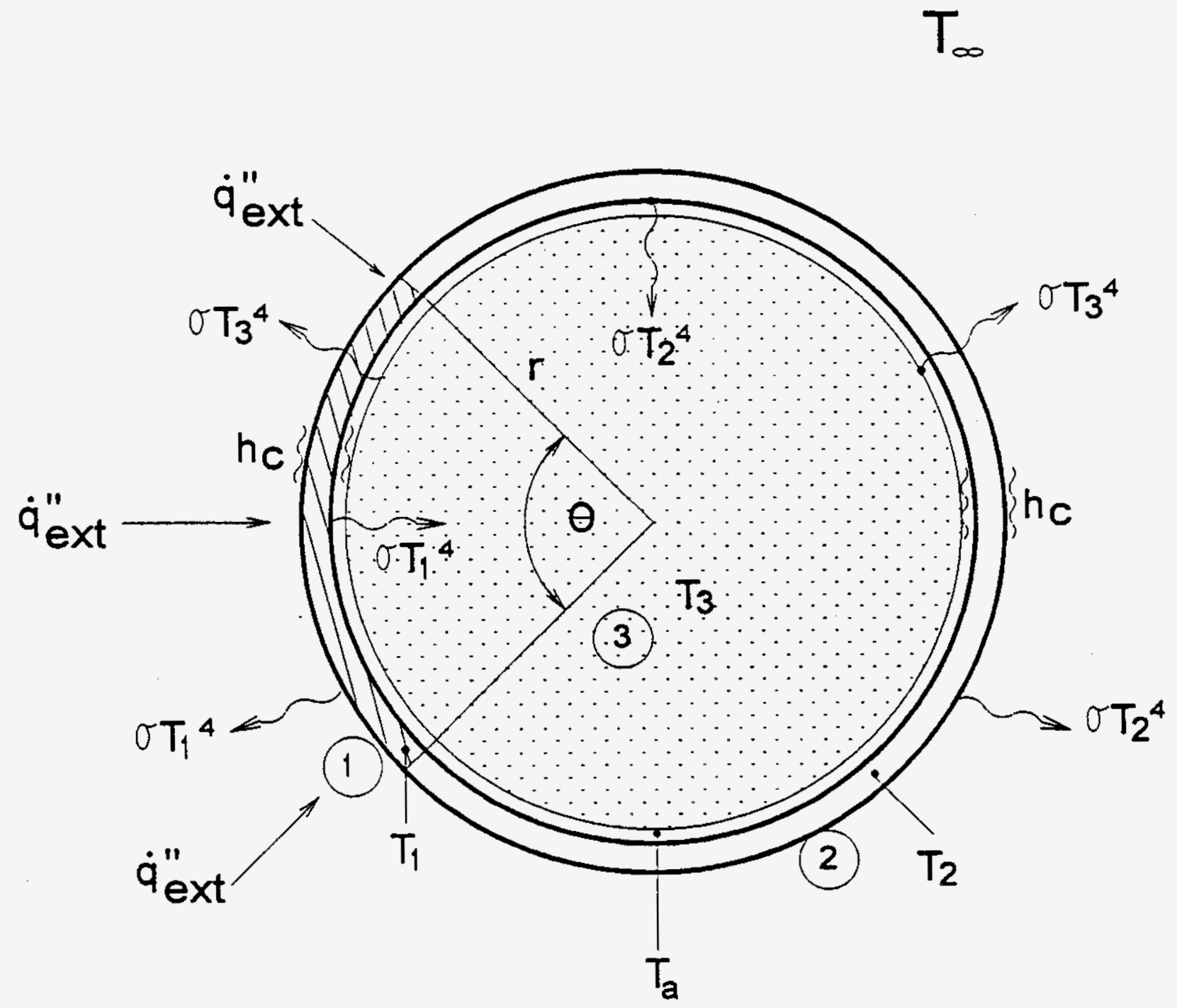

Figure 43. Heat Transfer Components for the Lumped Mass Analysis 
Hot Wall

$$
c_{s} \rho_{s} d \frac{d T_{1}}{d t}=\dot{q}_{e \mathrm{I}}^{\prime \prime}+\sigma T_{3}^{4}-h_{\mathrm{c}}\left(T_{1}-T_{\omega}\right)-h_{c}\left(T_{1}-T_{a}\right)-\sigma\left(2 T_{l}^{4}-T_{*}^{4}\right)
$$

Cold Wall

$$
c_{s} P_{s} d \frac{d T_{2}}{d t}=\sigma T_{3}^{4}+h_{c}\left(T_{a}-T_{2}\right)-h_{c}\left(T_{2}-T_{\sigma}\right)-\sigma\left(2 T_{2}^{4}-T_{*}^{4}\right)
$$

Drum Contents

$$
c_{3} \rho_{3} V \frac{d T_{3}}{d t}=A_{1} \sigma T_{1}^{4}+A_{2} \sigma T_{2}^{4}+A_{3} h_{c}\left(T_{a}-T_{3}\right)-A_{3} \sigma T_{3}^{4}
$$

Internal Gas Temperature

$$
c_{\nu} \rho_{0} V \frac{d T_{a}}{d t}=A_{1} h_{c}\left(T_{1}-T_{a}\right)-A_{3} h_{c}\left(T_{a}-T_{3}\right)-A_{2} h_{c}\left(T_{a}-T_{2}\right)
$$

Drum Pressure

$$
p=p_{o}\left(\frac{T_{a}}{T_{a, o}}\right)
$$

This lumped mass analysis of the drum contents is expected to be valid at very low heat fluxes and after long exposure times such that the temperature gradient in the contents will be small. At higher irradiances and shorter exposure times, however, the surface temperature of the contents will be much higher than the temperature at the center. As such a more detailed analysis is considered.

\subsubsection{Semi-infinite Solid Contents Analysis}

In order to account for the temperature distribution in the drum contents, an integral model for a semi-infinite solid is employed. As seen in Figure 44, the maximum temperature of the contents is at the surface and decreases according to an assumed cubical profile within the solid. This is a common approximation used to model the temperature distribution in thermaily thick solids exposed to an external heat flux at the surface. As with the lumped mass analysis, pyrolysis is assumed not to occur within the drum. The unsteady energy equations are essentially the same as those for the lumped mass analysis except the equation for the contents. Using an integral model with the cubical temperature profile, the surface temperature of the drum contents is given as [Eckert and Drake, 1972]: 


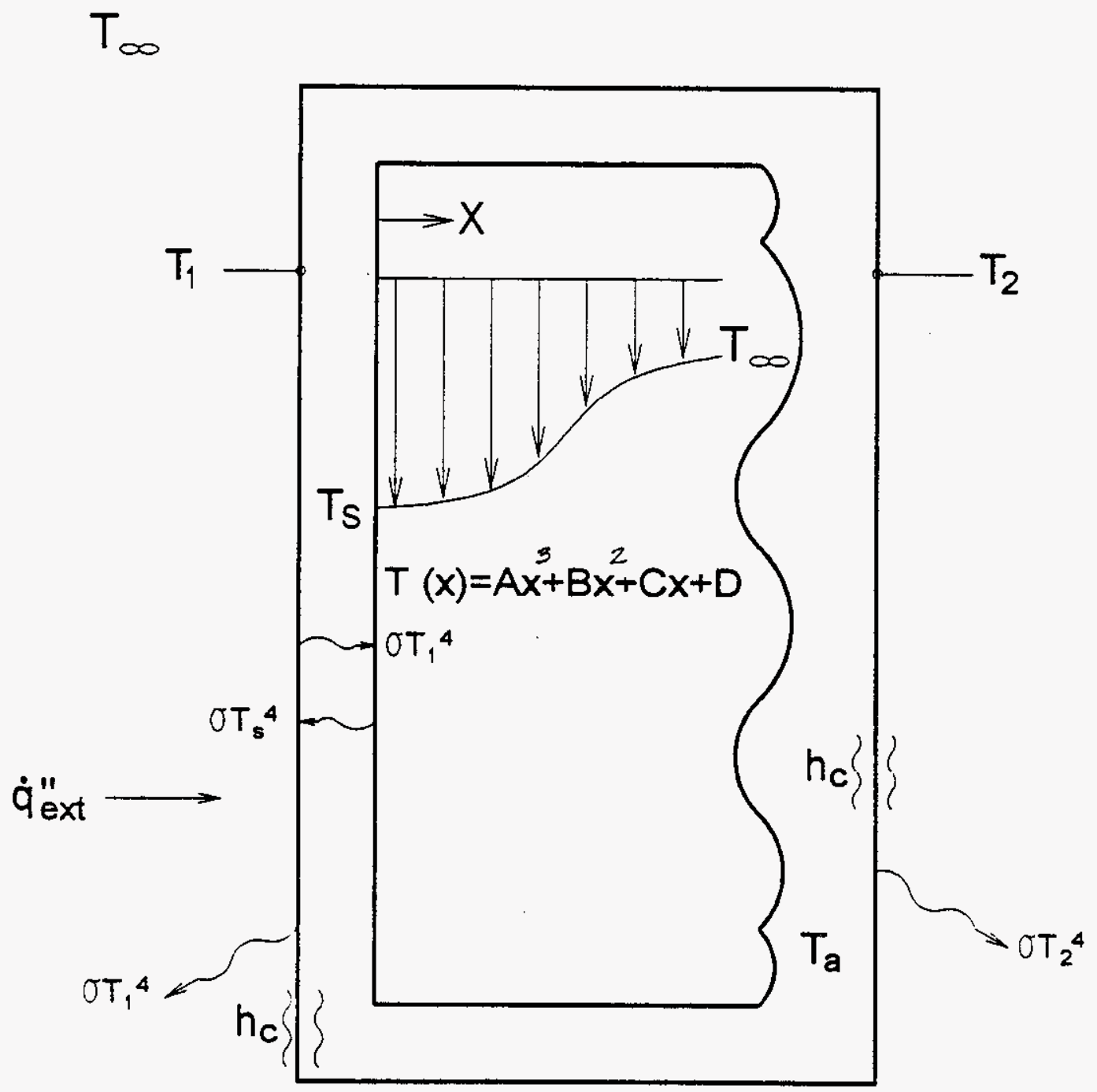

Figure 44. Heat Transfer Components for the Semi-Infinite Solid Analysis 


$$
\frac{d}{d t}\left[\frac{\left(T_{3 \text { surf }}-T_{\omega}\right)^{2}}{\dot{q}^{\prime \prime}}\right]=\frac{4}{3} \frac{\dot{q}^{\prime \prime}}{k \rho c}
$$

where

$$
\dot{q}^{\prime \prime}=\sigma\left(T_{1}^{4}-T_{3 \text { surf }}^{4}\right)+h_{c}\left(T_{a}-T_{3 \text { surt }}\right.
$$

and the temperature distribution in the solid is given as

$$
T(x, t)-T_{0}=\left(T_{3 \text { surf }}-T_{0}\right)\left(1-\frac{\dot{q}^{\prime \prime}}{3 k\left(T_{3 \text { surf }}-T_{0}\right)} x\right)^{3}
$$

One of the difficulties in using the semi-infinite solid equations is characterizing the packing contents. Although the model assumes the drum contents to be a uniform solid, they are actually packed such that many air gaps exist. Further, for mixed contents, the type of material, and thermal properties, will not be uniform throughout the drum. As seen in Equation 24 , the surface temperature is a function of the thermal inertia $(k \rho c)$ of the contents.

Since the content's actual thermal properties cannot be used because of the nonuniform storage configuration, the thermal inertia is based on materials having densities similar to the bulk packing density of the drum contents. In general, materials with similar densities have approximately the same thermal inertia.

Figures 45 and 46 illustrate the predictive results of the semi-infinite solid analysis for one particular configuration. For this HAl drum response test, a 55 gallon drum containing 2.3 $\mathrm{kg}(5 \mathrm{lb})$ of paper was exposed to a $1.2 \mathrm{~m}(4 \mathrm{ft})$ square gas burner over approximately one-half its perimeter (SWD28-D1, Section 3.2). Water calorimeter results for this burner setup yielded an average flame heat flux of $45 \mathrm{~kW} / \mathrm{m}^{2}$ over one-half the drum perimeter. The $2.3 \mathrm{~kg}$ of paper is assumed to evenly fill the drum and has a packing density of $21 \mathrm{~kg} / \mathrm{m}^{3}$. In order to estimate the thermal properties of the paper contents, properties for polyurethane foam, which has a density similar to the packing density of the paper contents $\left(\rho=20 \mathrm{~kg} / \mathrm{m}^{3}\right)$ used. As such, in Equations 24-26, $\rho=20 \mathrm{~kg} / \mathrm{m}^{3}, \mathrm{~K}=3.4 \times 10^{-5} \mathrm{~kW} / \mathrm{m}-\mathrm{K}, \mathrm{c}=1.4 \mathrm{~kJ} / \mathrm{kg}-\mathrm{K}$, and $\mathrm{k} \rho \mathrm{c}$ $=9.5 \times 10^{-4} \mathrm{~kW}^{2}-\mathrm{s} / \mathrm{m}^{4}-\mathrm{K}^{2}$. As seen in Figure 45, the calculated hot wall temperature corresponds well with the measured values. In the experiment, the cold wall temperature was not measured. Rather, the temperature at the intersection of the assumed hot and cold wall was measured (see Section 2.3.21. The temperatures along this intersection were averaged and provide an upper bound for the cold wall temperature. As a result, the calculated cold wall temperature is lower than the measured vaiues, as would be expected. The calculated and measured internal drum pressures (Figure 46) seem to agree up until the time of lid seal failure $(-130 \mathrm{~s})$. The pressure decay is typical of a venting drum as shown in Figure 36.

Figures 47 and 48 compare results for a different test configuration. Again the drum contains $2.3 \mathrm{~kg}$ of paper, but, this time is fully exposed to a $2.1 \mathrm{~m}$ pool fire (SWD30-D1, Section 3.21. The incident flame heat flux for this configuration was found to be $65 \mathrm{~kW} / \mathrm{m}^{2}$ over the entire drum perimeter. The time for the pool fire to become fully involved is evident (0-30 s), particularly in Figure 48. This results in somewhat of a lag time in each of the two graphs. Even so, the temperature and pressure results seem to agree fairly well. For this test, the drum failed due to lid loss at a peak pressure of 28 psig. 


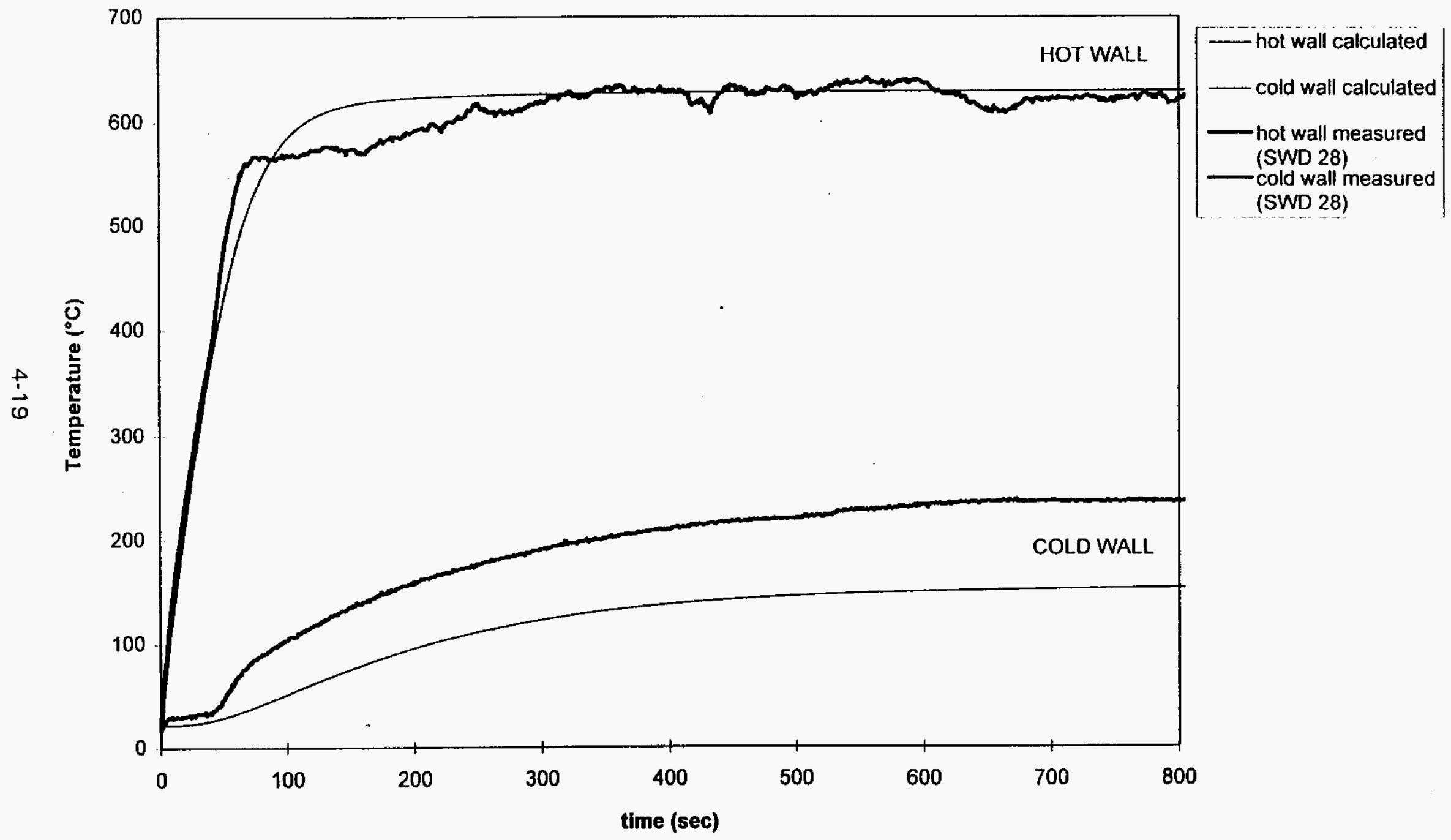

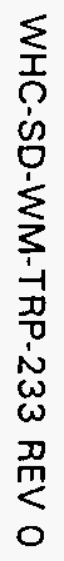

Figure 45. Drum Wall Temperatures for Drum Containing $2.3 \mathrm{~kg}$ Paper Using integral Method with $45 \mathrm{~kW} / \mathrm{m}^{2}$ Exposure over one-half Drum Perimeter 


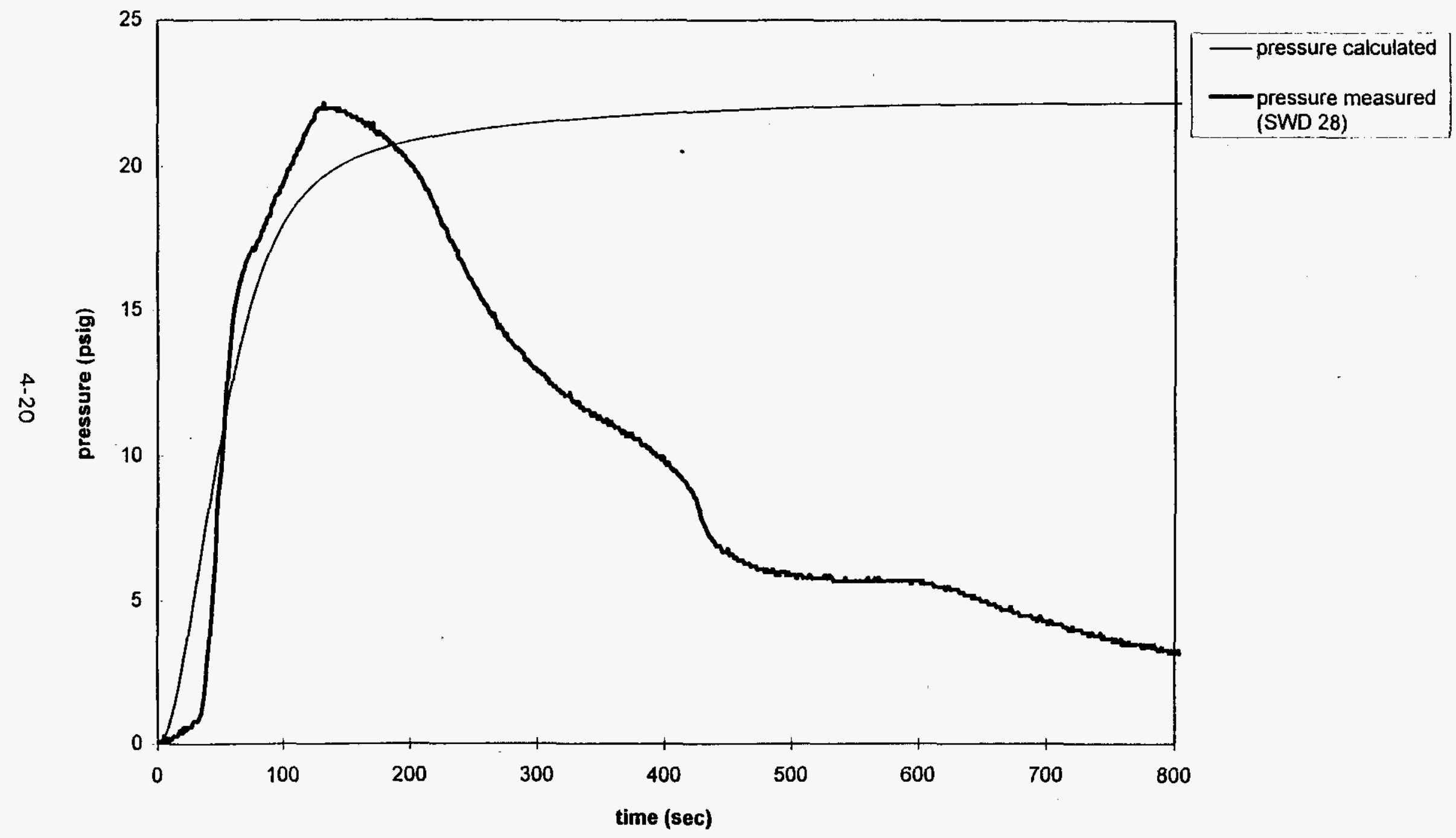

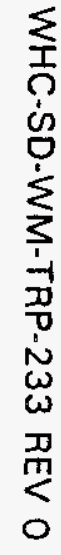

Figure 46. Internal Pressure for Drum Containing $2.3 \mathrm{~kg}$ Paper Using Integral Method with $45 \mathrm{~kW} / \mathrm{m}^{2}$ Exposure over one-half Drum Perimeter 


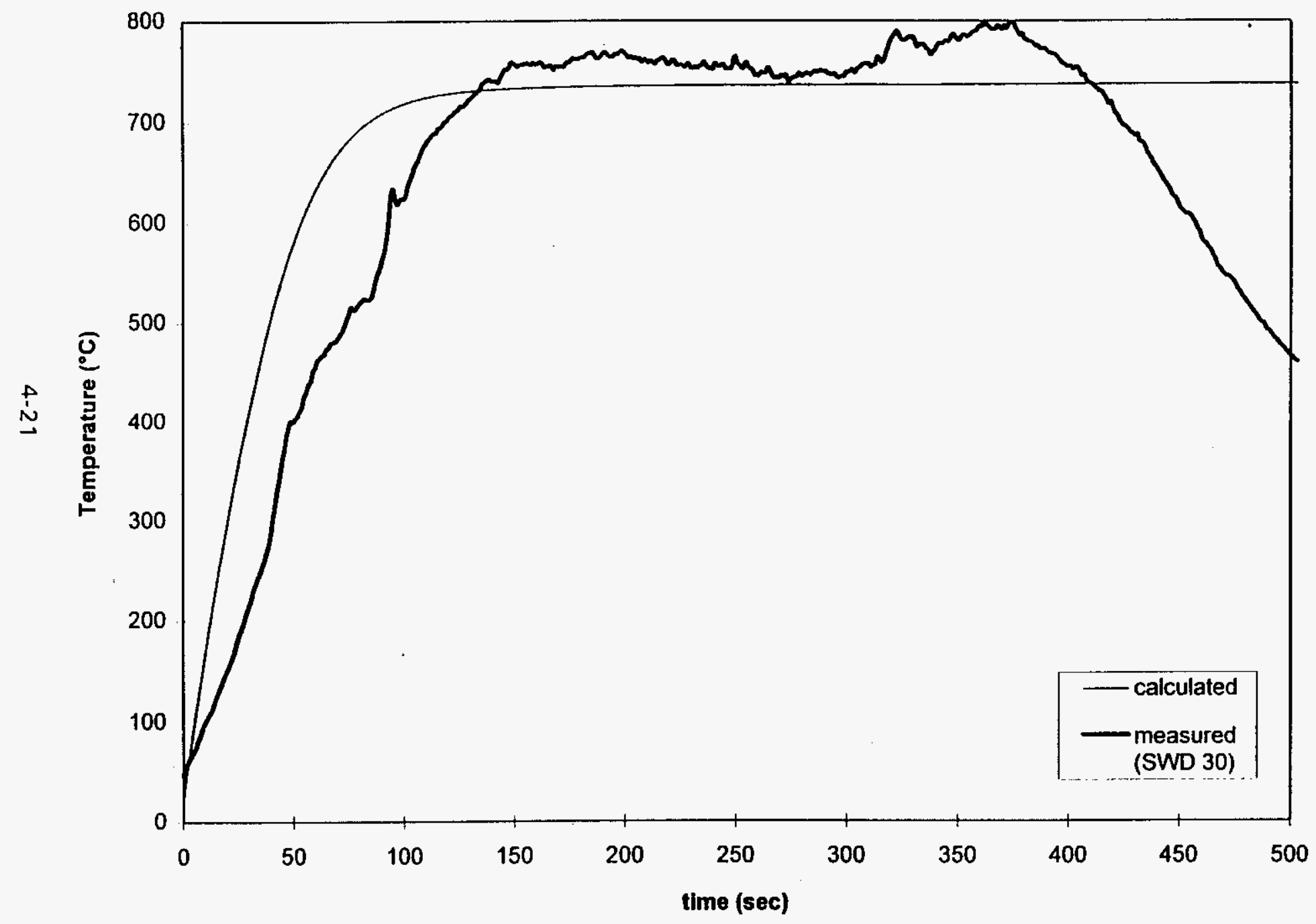

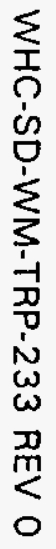

Figure 47. Drum Wall Temperature for Drum Containing $2.3 \mathrm{~kg}$ Paper Using htegral Method with $65 \mathrm{~kW} / \mathrm{m}^{2}$ Full Perimeter Exposure 


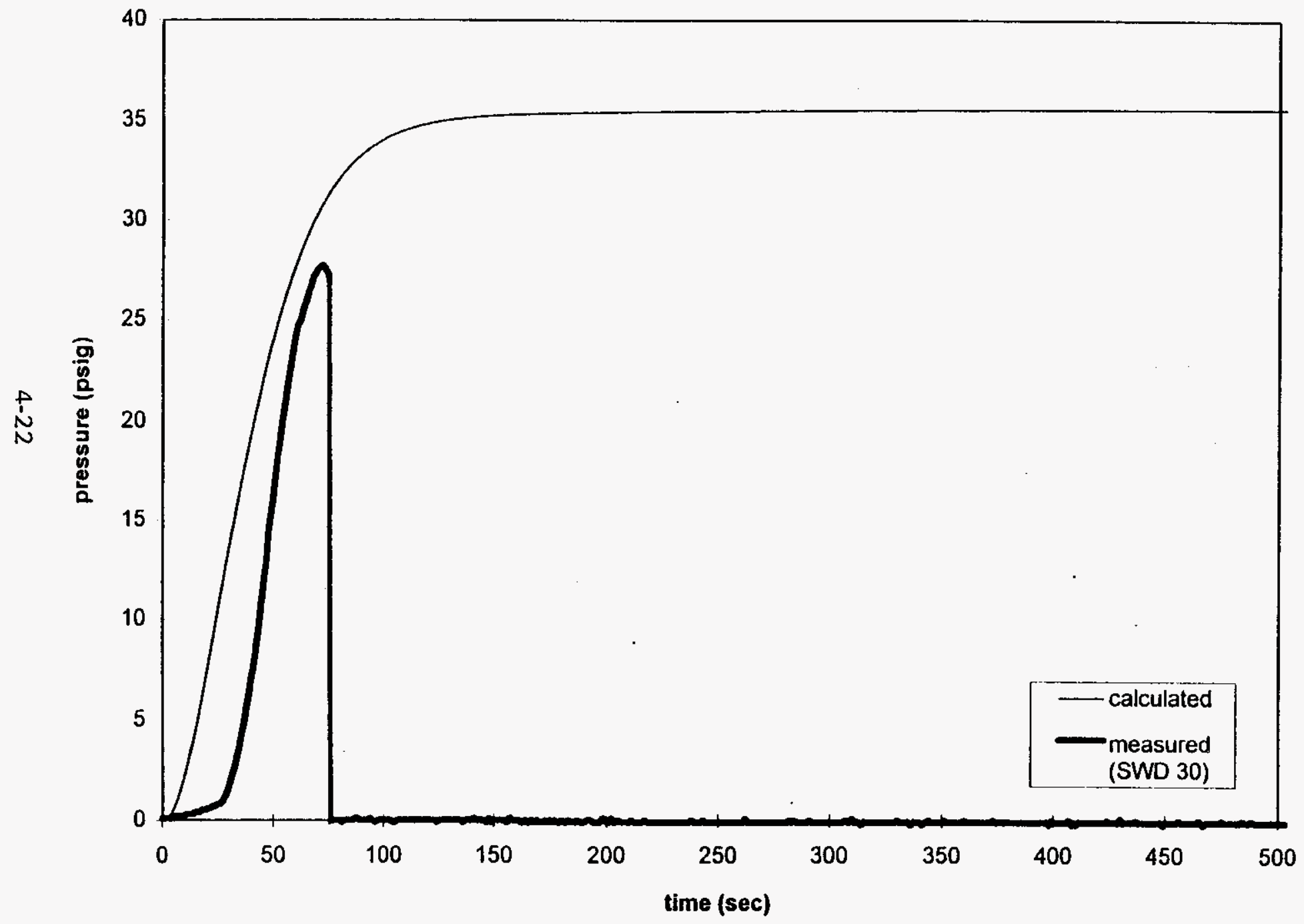

Figure 48. Internal Pressure for Drum Containing $2.3 \mathrm{~kg}$ Paper Using Integral Method with $65 \mathrm{~kW} / \mathrm{m}^{2}$ Full Perimeter Exposure 
Figures 49 and 50 compare results for a standard load drum exposed to the $2.1 \mathrm{~m}$ pool fire (SWD31-D4, Section 3.2). Again, the incident heat flux for this exposure was found to be $65 \mathrm{~kW} / \mathrm{m}^{2}$. For this test, although the temperature prediction is close to the measured value, the predicted pressure rise is significantly different. It is unclear why the standard load drums resulted in such a slow pressure rise and why they consistently failed at a lower pressure than drums with lighter loads. Even after the pool fire becomes fully involved, the pressure rise is quite different than that for the $2.3 \mathrm{~kg}$ paper load seen in Figure 48 . For this test, the standard load drum experienced lid loss failure at a peak pressure of 13 psig.

One of the criteria used earlier to discriminate between lid loss and lid seal failures was a critical temperature. For the drum response tests, all of the drums which experienced lid loss failure had exposed drum wall temperatures greater than $625^{\circ} \mathrm{C}$. Although not all drums which achieved this temperature experienced lid loss, it seems to serve as a lower limit for the lid loss failures. Figures 47 and 49 show that for the $2.1 \mathrm{~m}$ pool fire, the predicted exposed drum wall temperatures are greater than $700^{\circ} \mathrm{C}$. For both these tests, the drums experienced lid loss failure. For the $1.2 \mathrm{~m}$ burner comparison (Figure 45), the predicted exposed drum wall temperature is slightly greater than $600^{\circ} \mathrm{C}$. This drum did not experience lid loss failure. For the $0.48 \mathrm{~m}$ tests in which none of the drums experienced lid loss, the predicted exposed drum wall temperatures range from $540^{\circ} \mathrm{C}$ to $600^{\circ} \mathrm{C}$ depending upon the drum contents. These results are consistent with the drum response tests in which lid loss failures did not occur at temperatures below $625^{\circ} \mathrm{C}$. The calculated temperature results can be used together with the experimental results to predict if lid loss will occur for fire exposures which may occur in drum storage facilities.

\subsection{SINGLE BURNING DRUM}

When a drum experiences lid loss or is breached in some other manner, if the contents are not completely ejected they may begin to burn within the drum. Depending on the amount and type of combustible in the drum, the fire size will vary significantly. HAl burning rate experiments found heat release rates to range from $20 \mathrm{~kW}$ to $100 \mathrm{~kW}$ for different fuel loadings (SWD1-10, Section 3.1). The lower heat release rates were characteristic of the ordinary combustibles such as brown paper or the standard load (Section 2.1.6) packing. Whereas, the higher heat release rates were for the flammable liquid fires or plastic contents which melted into a pool fire. In general, it was difficult to obtain a large fire for contents burning in the drum. A large part of this was due to the limited air flow to fuel on the bottom of the drum. Additionally, as some of the contents burned and formed a char layer, they tended to insulate the remaining fuel beneath. 


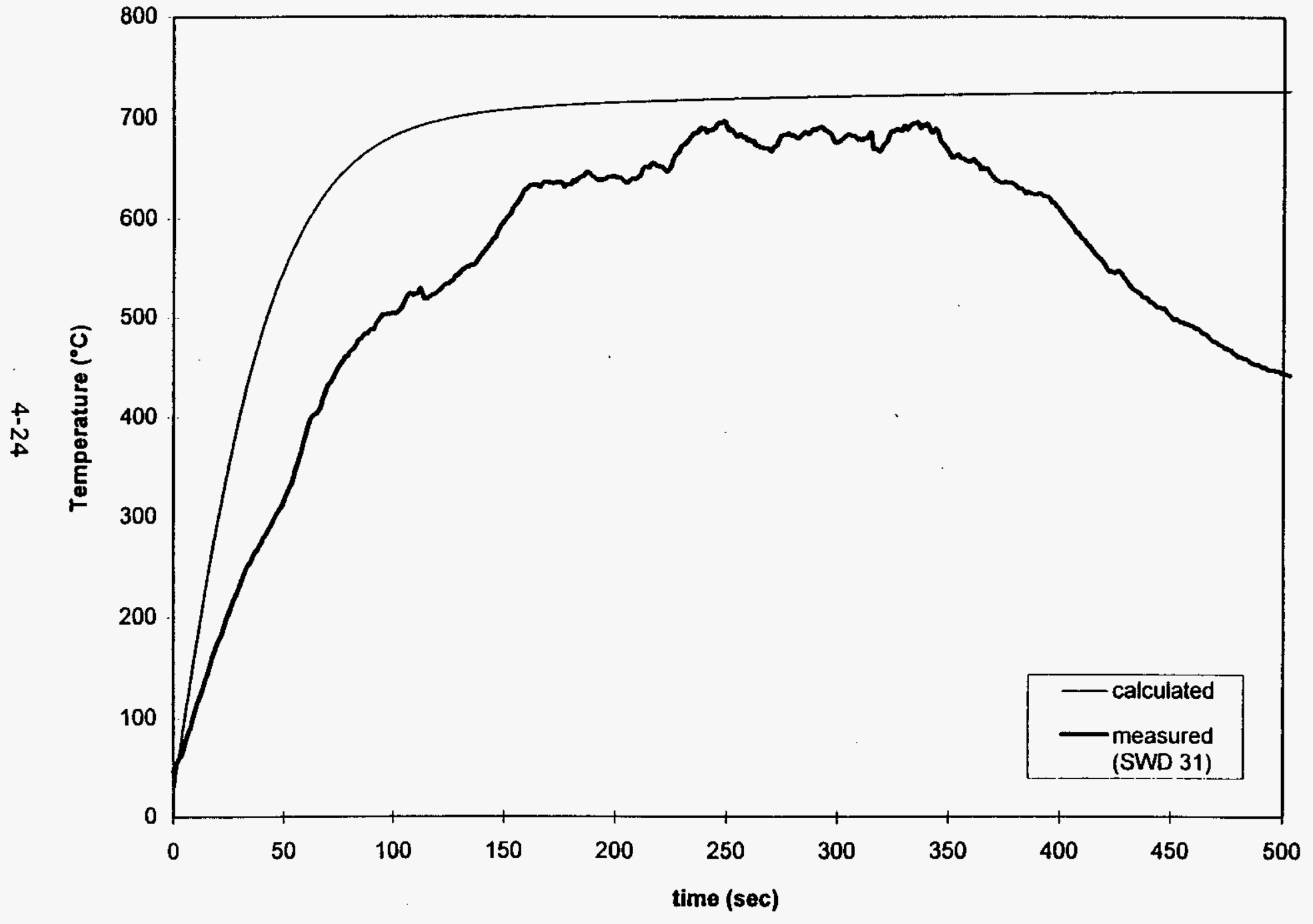

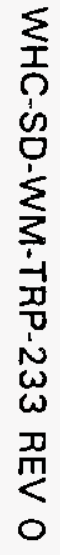

Figure 49. Drum Wall Temperature for Standard Load Drum Using Integral Method with $65 \mathrm{~kW} / \mathrm{m}^{2}$ Full Perimeter Exposure 


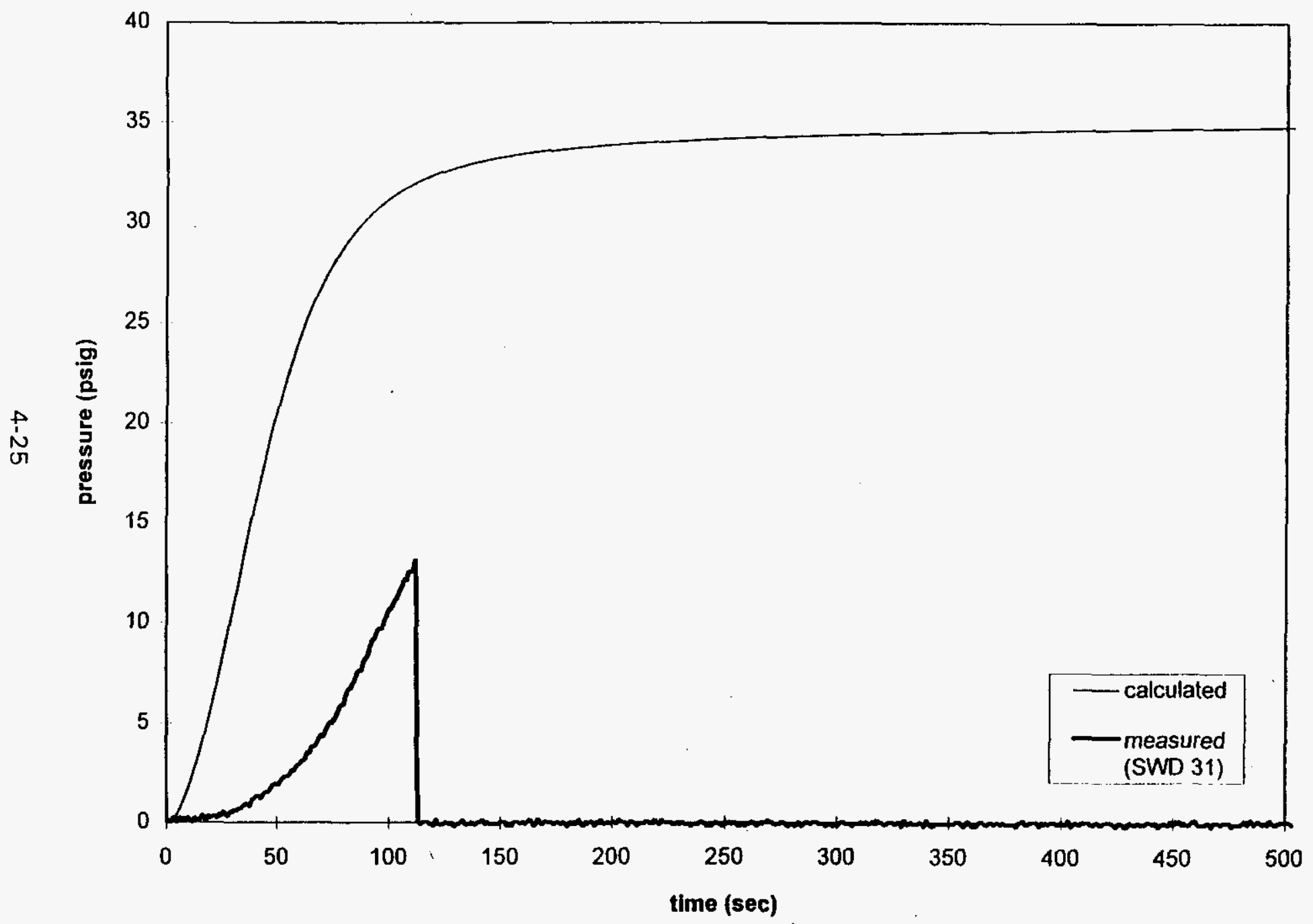

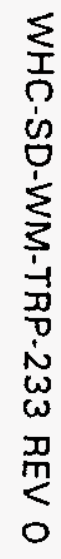

Figure 50. Internal Pressure for Standard Load Drum Using integral Method with $65 \mathrm{~kW} / \mathrm{m}^{2}$ Full Perimeter Exposure 


\subsubsection{Burning Drum Flame Heat Flux}

One of the hazards of a burning drum is that it will radiate to neighboring drums, thus providing a possible means of fire propagation. The radiosity of the burning drum is a function of the steel wall temperature. If the burning drum is taken as a cylinder of infinite height, the heat flux to a target, some distance away, is given by

$$
\dot{q}_{t}^{\prime \prime}=\varepsilon \quad \sigma T_{s}^{4}\left(\frac{r_{1}}{r_{2}}\right)
$$

where $r_{1}$ is the drum radius, $r_{2}$ is the distance from the center of the burning drum to the target, and $T_{s}$ is the burning drum wall temperature. The temperature of the burning drum wall may be determined as it was for the external heat source exposures. For this case, the flame heat flux from the burning drum contents controls the wall temperature. As such, the unsteady drum wall temperature (thickness $\delta$ ) of the burning drum, $T_{s}$, is given by

$$
c_{s} \rho_{s} \delta \frac{d T_{s}}{d t}=\dot{q}_{f l}^{\prime \prime}-h_{c}\left(T_{s}-T_{-\infty}\right)-\sigma\left(2 T_{s}^{4}-T_{-}^{4}\right)
$$

The flame flux acts over the interior of the burning drum. The interior wall reradiates, and the exterior wall cools both convectively and radiatively. Radiation from the drum contents to the interior wall is included in the flame heat flux term. The radiosity of the burning drum is seen to be primarily a function of the flame heat flux from the burning contents. Although flame heat fluxes from the burning contents were not directly measured for the HAl drum burning tests (Section 3.1), they can be calculated from the measured drum wall temperatures using Equation 28.

Table 19 shows the peak average burning drum wall temperature for each of the HAI single drum tests (Section 3.1). The flame heat fluxes in Table 17 were calculated using Equation 28 and represent the peak average flame heat flux from the burning contents to the inside surface of the drum wall. With the exception of the kerosene (SWD 5) and the plastic "pool" fire (SWD 8,9) tests, the peak average flame heat flux ranged from $2 \mathrm{~kW} / \mathrm{m}^{2}$ to 16.2 $\mathrm{kW} / \mathrm{m}^{2}$. This is the range of flame heat fluxes which would be expected from a burning standard load drum. For the tests involving only $2.3 \mathrm{~kg}(5 \mathrm{lb})$ of paper, these would be representative of a drum which expelled most of its contents leaving only a small amount of combustibles in the drum to burn. The peak average flame heat flux does not appear to vary significantly based on the amount of combustibles in the drum. 
Table 19. Single Drum Burning Tests - Radiosity.

\begin{tabular}{|c|l|c|c|}
\hline Test No. & \multicolumn{1}{|c|}{$\begin{array}{c}\text { Materials - } \\
\text { Ignition Location }\end{array}$} & $\begin{array}{c}\text { Peak } \overline{\mathbf{X}} \text { Wall } \\
\left.\text { Temperature } \text { ( }^{\circ} \mathrm{C}\right)\end{array}$ & $\begin{array}{c}\text { Peak } \overline{\mathbf{X}} \text { Flame Heat } \\
\text { Flux }\left(\mathbf{k W} / \mathbf{m}^{2}\right)^{*}\end{array}$ \\
\hline SWD 1 & Standard Load - top & 237 & 9.0 \\
\hline SWD 2 & Standard Load - bottom & no ignition & $\cdots$ \\
\hline SWD 3 & Paper $(2.3 \mathrm{~kg})$ - bottom & 304 & 14.4 \\
\hline SWD 4 & Standard Load - top & 250 & 9.9 \\
\hline SWD 5 & Kerosene & 466 & 36.9 \\
\hline SWD 6 & Paper $(2.3 \mathrm{~kg})-$ top & 238 & 9.0 \\
\hline SWD 7 & Paper $(6.8 \mathrm{~kg})-$ top & 86 & 2.0 \\
\hline SWD 8 & Plastic $(2.3 \mathrm{~kg})-$ top & 503 & 44.5 \\
\hline SWD 9 & Plastic $(6.8 \mathrm{~kg})-$ top & 475 & 38.7 \\
\hline SWD 10 & 2/3 Standard Load - mixed - & 322 & 16.2 \\
\hline
\end{tabular}

*calculated from Equation 28 with $T_{s}$ from Table 19

$\mathrm{T}_{\infty}=295 \mathrm{~K}, \mathrm{c}_{\mathrm{s}}=0.47 \mathrm{~kJ} / \mathrm{kg}-\mathrm{K}, \rho_{\mathrm{s}}=7800 \mathrm{~kg} / \mathrm{m}^{3}, \delta=1.21 \mathrm{~mm}, \mathrm{~h}_{\mathrm{c}}=8 \mathrm{~W} / \mathrm{m}^{2}-\mathrm{K}$ [Holman, 1990]

For these tests, the burning drum wall temperature and the flame heat flux varied depending on the contents and was not always uniform over the height of the drum. This was especially true for the standard load drums where the burning occured at the top and slowly regressed as the contents were consumed. Although it is possible to account for non-uniform heat flux distributions, it is simpler and more conservative to bound the flame heat flux and assume it to be uniform over the entire interior drum wall.

In order to assess the validity of using an average heat flux rather than a distributed flux over the height of the drum, a comparison between the two was made using a computational fluid dynamics model (CFD). The CFD model was used to simulate a burning drum exposing a sealed empty drum adjacent to it. The burning drum contents were assumed to yield three different flame heat fluxes; one for the top third, one for the middle, and one for the bottom third of the drum. Flame heat fluxes were determined from HAl burning rate tests in which the wall temperatures at these locations were used to calculate the flame heat flux using Equation 28. As a result, the burning drum imposed three distinct fluxes to the target drum rather than a single average value. When compared to the results using an average flame heat flux based on the average wall temperature of the HAl burning drum, internal pressures and average internal air temperatures agreed within 5 percent. It was found that the target drum maximum and minimum wall temperatures for the two models varied by up to 15 percent, however, the average wall temperatures also agreed within 5 percent. This was done for both a standard load test and a $6.8 \mathrm{~kg}(15 \mathrm{lb})$ plastic load test. The standard load flame heat fluxes ranged from $1 \mathrm{~kW} / \mathrm{m}^{2}$ at the bottom of the drum to $29 \mathrm{~kW} / \mathrm{m}^{2}$ at the top. For the plastic contents, the flame heat fluxes used in the CFD model ranged from $32 \mathrm{~kW} / \mathrm{m}^{2}$ at the bottom to $44 \mathrm{~kW} / \mathrm{m}^{2}$ at the top. Since the range of flame heat fluxes from burning drums was covered by these two drum loadings, a single uniform value for the flame heat flux will be used in the burning drum scenarios considered. 
In addition to the HAl testing, other analogies may be used to determine a suitable bounding flame heat flux. Two sources of data are available for this assessment; burning wall heat transfer measurements, and flame heat flux measurements between parallel walls. The wall fire heat fluxes to the burning surface were measured by Quintiere and Harkleroad [1985] and Hasemi [1984] who found heat fluxes to the burning surface of up to $20-30 \mathrm{~kW} / \mathrm{m}^{2}$. An opposing wall would be expected torexperience similar fluxes via flame contact as well as some radiation from the burning surface which would be expected to be about $15 \mathrm{~kW} / \mathrm{m}^{2}$ (consistent with a black fuel surface at $450^{\circ} \mathrm{C}$ ). The sum of these two fluxes is about 40 $\mathrm{kW} / \mathrm{m}^{2}$. Fires burning between closely spaced parallel plates has recently been studied by Foley and Drysdale [1994]. They measured fluxes to the wall yielding an average value of 37 $\mathrm{kW} / \mathrm{m}^{2}$. Thus, the two methods of estimation are remarkably consistent though, originating from different sources. (Note that these are very close to the kerosene (SWD5) and plastic (SWD8,9) peak average flame heat fluxes calculated in Table 19).

These fluxes represent bounding heat fluxes in regions of direct flame contact because they assume that a gap always exists between the fuel and the drum wall to allow a flame over the interior of the drum wall. In fact, with $26 \mathrm{~kg}$ of combustibles and similar amounts of noncombustibles (as found in the standard load), this is not generally expected. Similarly, it assumes that all the air gap is used to support flame. This is also not possible since at least half of the gap areas need to be used to supply air to allow burning in the fuel/drum wall gap. As such, assuming an average flux of half the above noted levels is a bounding average heat flux. Thus, an average flame heat flux of $20 \mathrm{~kW} / \mathrm{m}^{2}$ will be used as the bounding flame heat flux for the burning drum contents. (Note that this is higher than any of the peak average flame heat fluxes for the standard load drums in Table 19.1

\subsubsection{Radiant Heat Transfer from a Burning Drum}

Since drum wall temperatures were measured for the HAl burning rate tests, it is possible assess the utility of Equation 27 without solving Equation 28 or knowing the actual flame heat flux. During the tests, a target drum was positioned $5 \mathrm{~cm}(2 \mathrm{in}$.) away from the burning drum and the wall temperature of the target drum and the incident heat flux were measured (Section 2.2.1). Using the measured temperature of the burning drum, the incident heat flux to the target drum is calculated using Equation 27. With this, the steady wall temperature of the target drum, $T_{w}$, is calculated by

$$
\dot{q}_{i n c}^{\prime \prime}-2 h_{c}\left(T_{w}-T_{-}\right)-2 \sigma\left(T_{w}^{4}-T_{-}^{4}\right)=0
$$

where the heat transfer coefficient, $h_{c}$, was determined from a natural convection correlation for a cylinder [Holman, 1990] and is taken as $8 \mathrm{~W} / \mathrm{m}^{2}-\mathrm{K}$. Table 20 shows the results for the calculated and measured temperature and incident heat flux for a variety of drum loadings. The measured burning drum temperature is a local temperature taken at mid-height on the side facing the target drum. Two thermocouples (TC\#9 and TC\#10, SWD1-10, Section 2.2) are averaged to obtain a single value for the burning drum wall temperature. The measured incident heat flux and the target drum temperature (HF\#2 and TC\#22, SWD 1-10, Section 2.2) are also local values taken at a point directly opposite the location of thermocouples 9 and 10 on the burning drum. All measurements are averaged over a 10 to 30 minute period depending upon the test. The results for the calculated incident heat flux are in good agreement with the measured values, especially for the kerosene and plastic "pool fire" tests. 
Table 20. Burning Drum and Target Drum Temperature and Heat Flux Summary.

\begin{tabular}{|c|c|c|c|c|c|c|c|c|}
\hline \multirow{2}{*}{$\begin{array}{c}\text { Test No. } \\
\text { (time of data } \\
\text { sample, sec) }\end{array}$} & \multirow{2}{*}{$\begin{array}{l}\text { Drum } \\
\text { Contents }\end{array}$} & \multirow{2}{*}{$\begin{array}{l}\text { Measured } \\
\text { Burning } \\
\text { Drum Temp }\end{array}$} & \multicolumn{3}{|c|}{ Target Drum Incident Heat Flux } & \multicolumn{3}{|c|}{$\begin{array}{c}\text { Target Drum Temperature } \\
\text { Rise }\end{array}$} \\
\hline & & & $\begin{array}{c}\text { Calculated } \\
\left(\mathrm{kW} / \mathrm{m}^{2}\right)\end{array}$ & $\begin{array}{l}\text { Measured } \\
\left(\mathrm{kW} / \mathrm{m}^{2}\right)\end{array}$ & $\begin{array}{c}\text { Error } \\
\left(k W / m^{2}\right. \\
(\%)\}\end{array}$ & $\begin{array}{c}\text { Calculated } \\
\left({ }^{\circ} \mathrm{C}\right)\end{array}$ & $\begin{array}{l}\text { Measured } \\
\left({ }^{\circ} \mathrm{C}\right)\end{array}$ & $\begin{array}{l}\text { Error } \\
1^{\circ} \mathrm{C} \\
(\%)\end{array}$ \\
\hline $\begin{array}{c}\text { SWD5 } \\
(600 \cdot 1200)\end{array}$ & Kerosene & $\begin{array}{l}480^{\circ} \mathrm{C} \\
(753 \mathrm{~K})\end{array}$ & 15.5 & 15.9 & $\begin{array}{c}0.4 \\
(2.5)\end{array}$ & 268 & 206 & $\begin{array}{c}62 \\
(30)\end{array}$ \\
\hline $\begin{array}{c}\text { SWD9 } \\
(2000-2600)\end{array}$ & $\begin{array}{l}6.8 \mathrm{~kg}(15 \\
\text { ib) Plastic }\end{array}$ & $\begin{array}{l}449^{\circ} \mathrm{C} \\
(722 \mathrm{~K})\end{array}$ & 13.1 & 13.1 & $\begin{array}{l}0.0 \\
(0.0)\end{array}$ & 241 & 180 & $\begin{array}{c}61 \\
(34)\end{array}$ \\
\hline $\begin{array}{c}\text { SWD 10 } \\
(9000-11000)\end{array}$ & $\begin{array}{l}\text { 2/3 Standard } \\
\text { Load }\end{array}$ & $\begin{array}{l}336^{\circ} \mathrm{C} \\
(609 \mathrm{~K})\end{array}$ & 6.7 & 7.3 & $\begin{array}{c}0.6 \\
(8.2)\end{array}$ & 148 & 104 & $\begin{array}{c}44 \\
(42)\end{array}$ \\
\hline
\end{tabular}

The measured incident heat flux data for the standard load test was very scattered and was difficult to obtain a useable average. The calculated wall temperatures of the target drum tend to overestimate the measured values. This is likely because Equation 29 does not consider conduction losses along the drum perimeter. However, for this analysis, it is conservative, and allows a simple one-dimensional model, to ignore the conductive losses.

\subsection{DRUM TO DRUM FIRE PROPAGATION}

The heat transfer between drums stored in an array configuration is of particular concern. Whereas an initiating event such as a pool fire will cause exposed drums to fail and burn, the spread of fire through the drum array is limited by the ability of the burning drums to heat neighboring drums via radiation to allow fire propagation. In this section, it is assumed that one or more drums are burning and radiating to neighboring drums. In order to' determine the incident heat flux to the exposed drums, both the radiosity of the burning drums and the shape factors between drums must be determined.

\subsubsection{Drum to Drum Configuration Analysis}

In order to determine the shape factors between a burning drum and its neighbors, a $3 \times 3$ array of tightly packed drums is considered. Figure 51 shows such an array where the cylinders are assumed to be of infinite height and are touching each other. As such, all of the radiation from the burning drum (drum 1) is absorbed by the surrounding drums (drums 2 and 3). This is conservative because it does not allow any heat to be lost to the surroundings. The shape factors for the individual drums in Figure 51 are reported as [Howell, 1982]:

$$
\begin{aligned}
& F_{1-2}=0.18 \\
& F_{1 \cdot 3}=0.07 .
\end{aligned}
$$

Since all of the radiation from the burning drum is absorbed by the surrounding drums, the sum of the individual shape factors must equal 1 ,

$$
4 F_{1-2}+4 F_{1-3}=1 \text {. }
$$




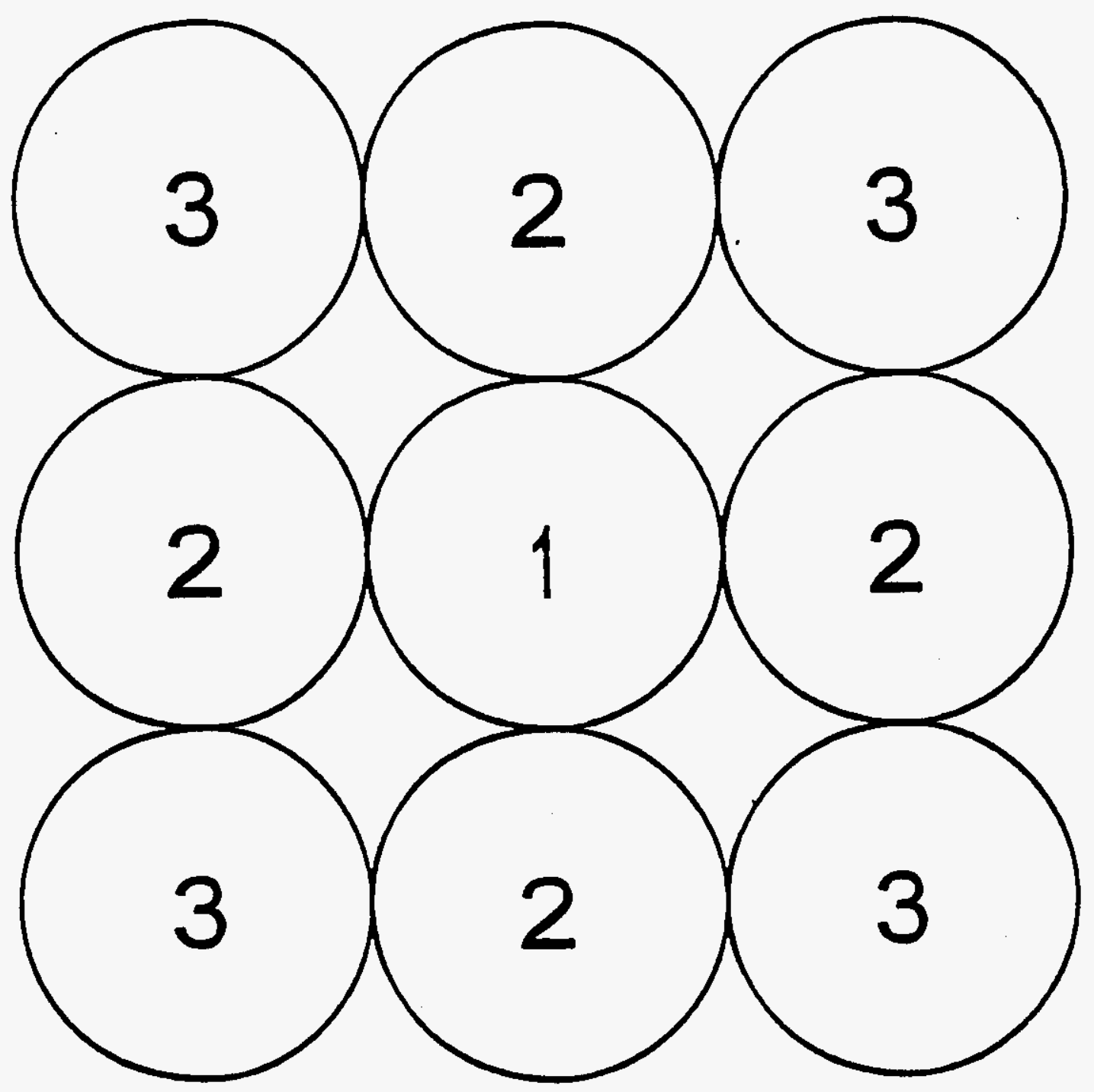

Figure 51. Nomenclature and Arrangement for Configuration Factors for Tight Packed Drum Arrays 
In the drum to drum heat transfer analysis, only part of the array is considered in order to reduce the number of equations which need to be solved. Since the $3 \times 3$ array is symmetrical, one-quarter of the array can be taken as representative of the entire array. Figure 52 shows this configuration where the surface area of the burning drum is divided into 4 equal areas such that

$$
A_{1}=A_{a}+A_{b}+A_{c}+A_{d}
$$

and

$$
A_{a}=A_{b}=A_{c}=A_{d}
$$

Again, since the drums are touching each other and are of infinite height, an enclosure is formed between the 4 cylinders in Figure 52. The heat transfer within this enclosure is the basis for the drum to drum analysis. Therefore, the shape factors, within the enclosure, are needed for each of the drums. For this configuration, there are two shape factors: (1) between opposite drums facing one another $\left(F_{o p 1-o p 2}\right)$, and $(2)$ between adjacent drums next to one another $\left(F_{\mathrm{ad} 1-\mathrm{ad} 2}\right)$. These shape factors are different from those reported by Howell because the surface area of the drum walls are different. In this analysis, only 1/4 of the surface area is considered since only $1 / 4$ of the drum wall is within the enclosure in Figure 52. Since the values reported by Howell consider the entire cylinder surface area, it can be shown that

$$
A_{1} F_{1-3}=A_{c} F_{a-3}+A_{b} F_{b-3}+A_{c} F_{c-3}+A_{d} F_{d-3}
$$

Since the drums are touching each other, no radiation from the back side of drum 1 can reach drum 3. Therefore,

$$
F_{b-3}=F_{c-3}=F_{d-3}=0
$$

Substituting into Equation 33 yields,

$$
F_{a-3}=A_{1} / A_{a} F_{1-3} .
$$

or,

$$
F_{a-3}=4 F_{1-3} \text {. }
$$

This is the shape factor between opposite drums facing one another $\left(F_{o p 1-o p 2}\right)$. Since the four drums form an enclosure, any radiation from the burning drum not absorbed by drum 3 will be absorbed by the two adjacent drums. Therefore,

$$
F_{a-3}+2 F_{a-2}=1
$$

Substituting $\mathrm{F}_{\mathrm{a}-3}=4 \mathrm{~F}_{1-3}$ and using Equation 30 , it can be shown that

$$
F_{a-2}=2 F_{1-2}
$$




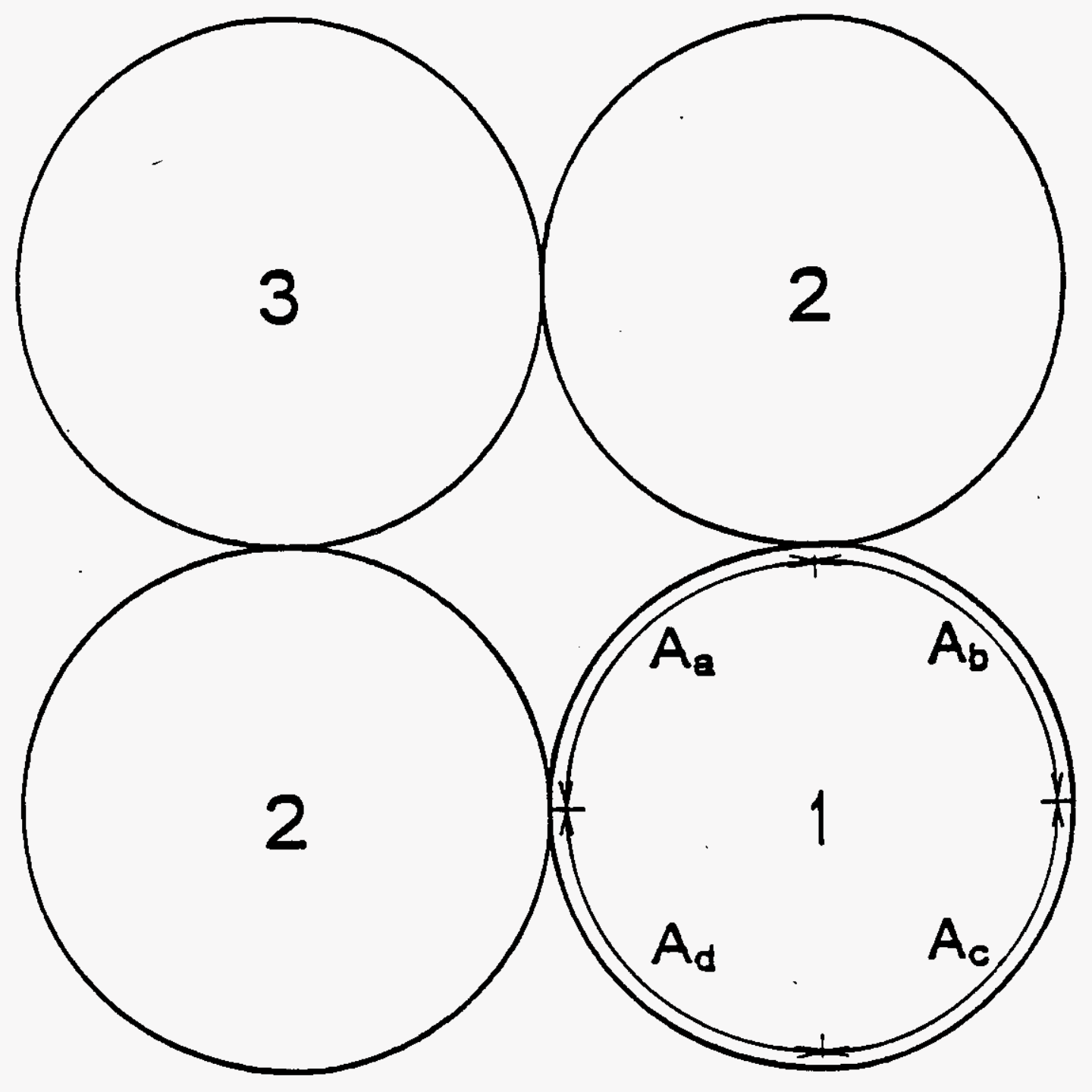

Figure 52. Nomenclature and Arrangement for Configuration Factors Used in Drum to Drum Heat Transfer Analysis 
This is the shape factor between adjacent drums facing one another $\left(F_{\text {ad1-ad2 }}\right)$. Substituting the values for $F_{1.2}$ and $F_{1-3}$ reported by Howell, the shape factors required for the drum to drum heat transfer analysis are as foliows:

$F_{\mathrm{op} 1.0 \mathrm{p} 2}=0.28$

and $F_{\text {ad1.ad } 2}=0.36$.

\subsubsection{Drum to Drum Heat Transfer}

A heat transfer analysis is considered for a tightly packed single tier array of 55 gallon storage drums. A $3 \times 2$ drum configuration in which one or several drums are burning, is taken as representative of the array. Figure 53 shows the layout for one drum burning in a row radiating to neighboring drums. The center drum in the second row is taken as the primary target since it will receive the maximum exposure. The target drum is assumed to be empty since this is the bounding worst case scenario in terms of temperature and pressure rise. The source drum is assumed to have contents burning providing the bounding flame heat flux of $20 \mathrm{~kW} / \mathrm{m}^{2}$ to the inside drum surface (Section 4.4.1). The remaining non-burning drums are assumed to be adiabatic in order to maximize radiative feedback to the target drum. The heat transfer coefficient, $h_{c}$, for the drum walls was determined from a natural convection correlation for a cylinder [Holman, 1990] and is taken as $8 \mathrm{~W} / \mathrm{m}^{2}-\mathrm{K}$. Using the configuration factors derived in Section 4.5.1 and assuming all of the exposed surfaces to have equal area, the steady heat transfer equations for the system are given as follows:

Burning Drum Wall

$$
\dot{q}_{f l}^{\prime \prime}+\sigma\left(F_{a d l-a d 2} T_{1}^{4}+F_{o p 1-o p 2} T_{3}^{4}+F_{a d l-a d 2} T_{4}^{4}-2 T_{s}^{4}\right)-h_{c}\left(T_{s}-T_{\infty}\right)=0
$$

Target Drum Hot Wall

$$
\begin{aligned}
\sigma\left(F_{a d l-a d 2} T_{s}^{4}+F_{a d l-a d 2} T_{3}^{4}+F_{o p l-o p 2} T_{4}^{4}+F_{2-1}\right. & \left.T_{2}^{4}+F_{1-1} T_{1}^{4}-2 T_{1}^{4}\right)- \\
& h_{c}\left(T_{1}-T_{-}\right)-h_{c}\left(T_{1}-T_{a}\right)=0
\end{aligned}
$$

Target Drum Cold Wall

$$
\sigma\left(F_{1-2} T_{1}^{4}+F_{2-2} T_{2}^{4}+T_{-}^{4}-2 T_{2}^{4}\right)+h_{c}\left(T_{a}-T_{2}\right)-h_{c}\left(T_{2}-T_{-}\right)=0
$$

Air in Target Drum

$$
h_{c}\left(T_{1}-T_{a}\right)-h_{c}\left(T_{a}-T_{2}\right)=0
$$

Target Drum Pressure

$$
p=p_{o}\left(\frac{T_{a}}{T_{a, o}}\right)
$$




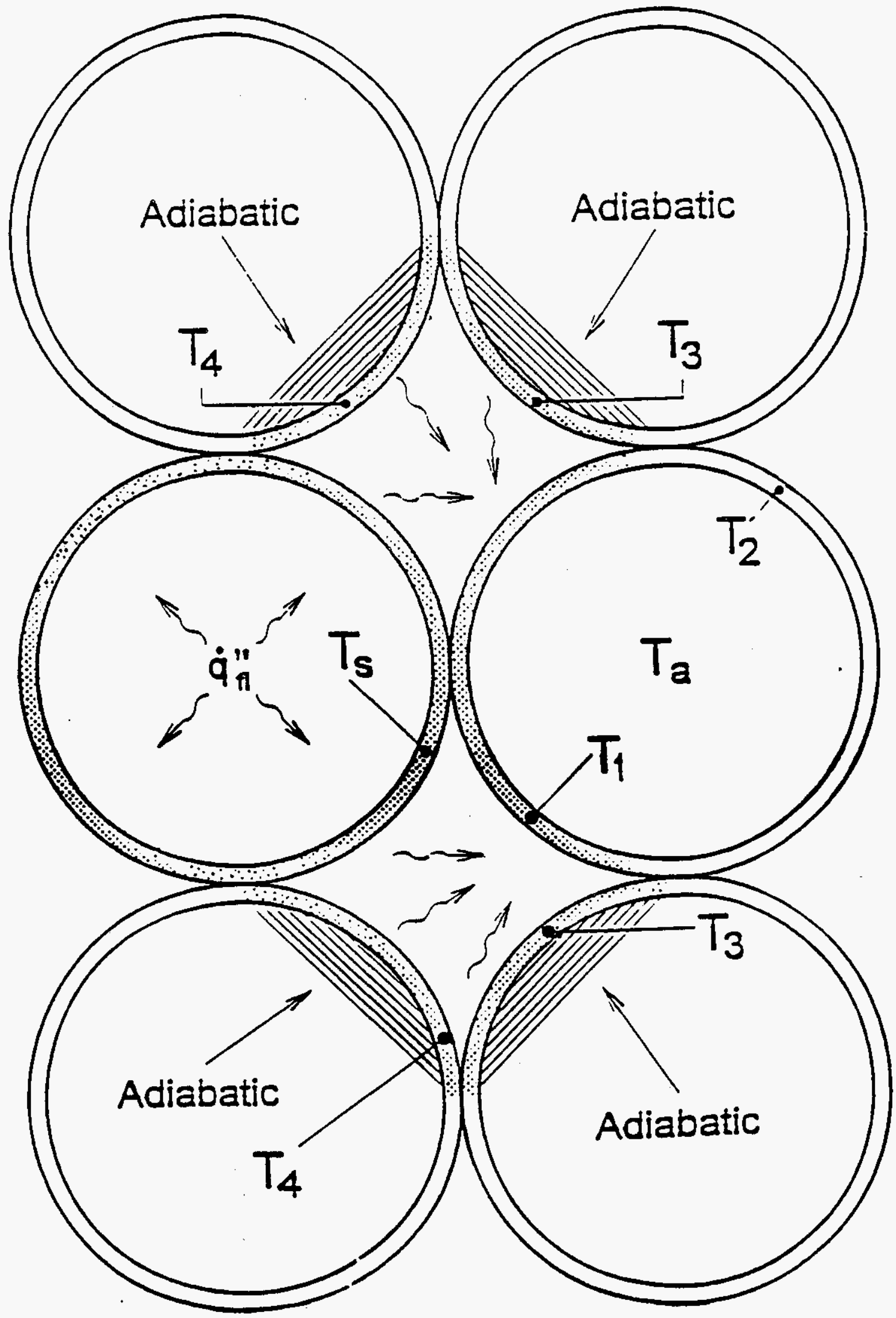

Figure 53. Nomenclature and Arrangement for Drum to Drum Heat Transfer Analysis for a Single Burning Drum 
Adjacent Adiabatic Drum Wall

$$
\sigma\left(F_{o p 1-o p 2} T_{s}^{4}+F_{a d 1-a d 2} T_{1}^{4}+F_{o d I-a d 2} T_{4}^{4}-T_{3}^{4}\right)-h_{c}\left(T_{3}-T_{-}\right)=0
$$

Opposite Adiabatic Drum Wall

$$
\circ\left(F_{\text {odl-odz }} T_{s}^{4}+F_{\text {opl-op } 2} T_{1}^{4}+F_{\text {adl }- \text { adz }} T_{3}^{4}-T_{4}^{4}\right)-h_{c}\left(T_{4}-T_{-4}\right)=0
$$

Equation 39 is for the burning drum, Equations $40,41,42$ and 43 are for the target drum, and Equations 44 and 45 are for the adiabatic drums. Solving Equations $39-45$ yields a maximum pressure rise of approximately $6.9 \mathrm{psig}$. The exposed target drum temperature is $220^{\circ} \mathrm{C}(490 \mathrm{~K})$, and the air temperature in the drum is $167^{\circ} \mathrm{C}(440 \mathrm{~K})$.

As a more severe exposure, consider all three drums in the first row to be burning and radiating to the target drum in the next row. Figure 54 shows such a configuration. The steady equations for this scenario are the same as for the single drum burning except Equation 45 , for the adiabatic drum opposite to the target drum, will include a flame heat flux term and will not be adiabatic,

$$
\dot{q}_{f}^{\prime \prime}+\sigma\left\langle F_{o d l-a d 2} T_{s}^{4}+F_{o p l-o p 2} T_{1}^{4}+F_{a d l-a d 2} T_{3}^{4}-2 T_{4}^{4}\right)-h_{c}\left(T_{4}-T_{-}\right)=0
$$

Again, the flame heat flux for the burning drums is taken as $20 \mathrm{~kW} / \mathrm{m}^{2}$. Solving these equations yields a maximum pressure rise of approximately $10.3 \mathrm{psig}$. The exposed target drum temperature is $299^{\circ} \mathrm{C}(572 \mathrm{~K})$, and the air temperature in the drum is $236^{\circ} \mathrm{C}(509 \mathrm{~K})$.

Since there have been no reported test data involving fire propagation through a drum array, it is desireable to use the available HAl experimental results (Section 3.2 ) to bound the drum array exposure. The incident heat flux to the target drum is simply the sum of the radiative fluxes from each of the neighboring drums. As such, the radiative heating rate of the target drum is given as follows:

$$
\dot{Q}_{\text {net }}=A_{1} \sigma\left(F_{a d l-a d z} T_{s}^{4}+F_{a d l-a d 2} T_{3}^{4}+F_{o p l-o p 2} T_{4}^{4}\right)
$$

where $A_{t}$ is the exposed area of the target drum $10.8 \mathrm{~m}^{2} \mathrm{i}$. For the three drum burning case, $Q_{\text {net }}$ is found to be $9.0 \mathrm{~kW}$. For the HAl $0.48 \mathrm{~m}(19 \mathrm{in}$.) drum response tests, the average heating rate of the drums was $17.6 \mathrm{~kW}$ (Section 3.2). As such, the HAI $0.48 \mathrm{~m}$ tests bound the drum to drum heat transfer. In these tests, only 1 of 18 drums failed due to lid loss. However, the single drum which failed contained heptane and is believed to have autoignited (Section 3.2). Therefore, in the absence of heptane, this exposure is not expected to cause lid loss. Rather, the drum will experience only lid seal failure. The calculated temperatures in the exposed target drum will not lead to pyrolysis gas generation. Therefore, a drum to drum exposure will not cause lid loss failure. Likely, the drums next to the fully burning drums would experience lid seal failure and vent to the surroundings. 


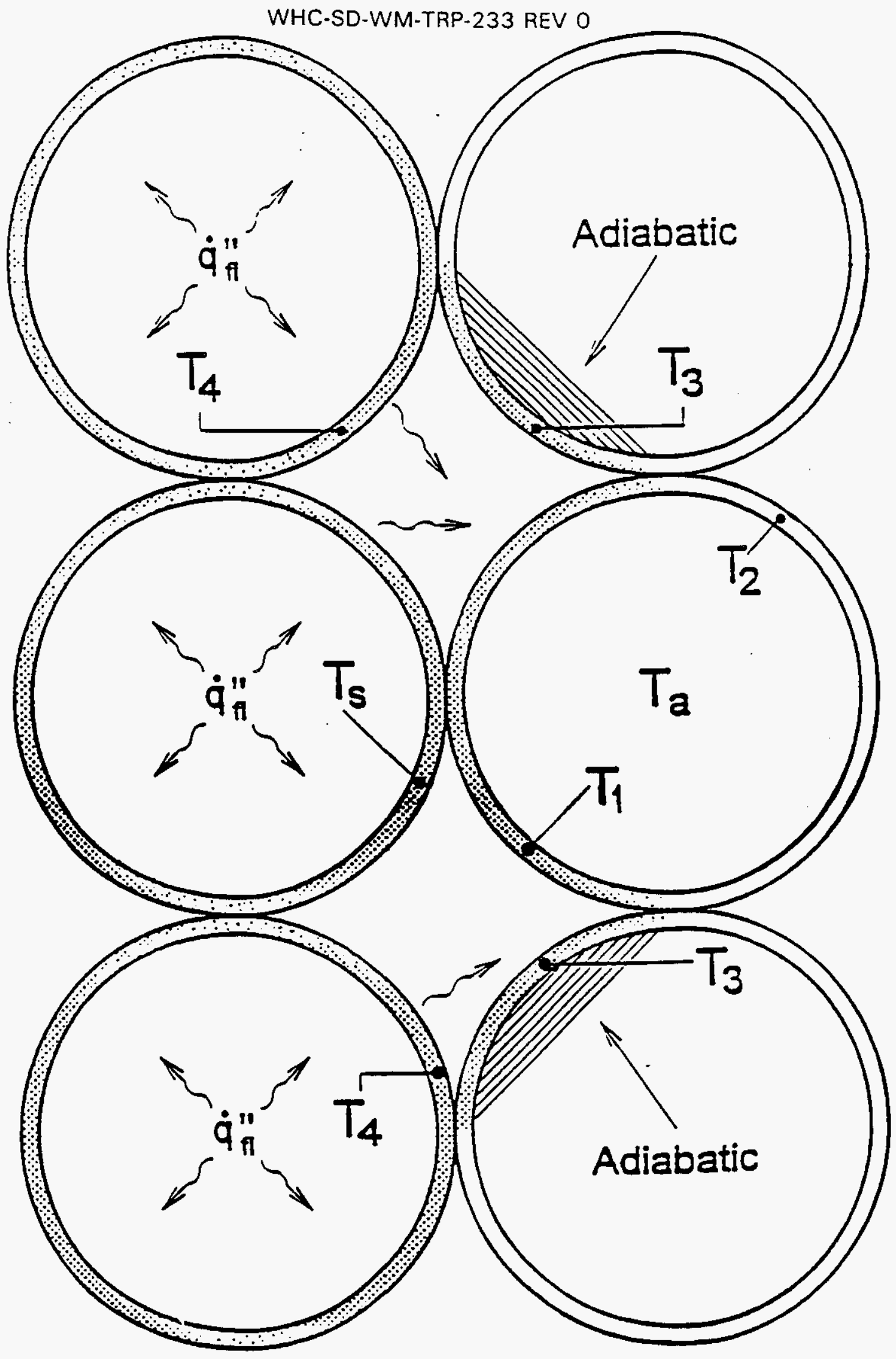

Figure 54. Nomenclature and Arrangement for Drum to Drum Heat Transfer Analysis for Three Burning Drums 
The above analysis indicates that fire will not propagate through a tightly packed array via radiation from the burning drums. The heat transfer analysis is considered very conservative in that it assumes the flame heat flux to be the bounding value $\left(20 \mathrm{~kW} / \mathrm{m}^{2}\right)$ and no heat is lost in the radiant energy exchange between the drums. Even so, the HAl $0.48 \mathrm{~m}$ burner tests yielded a heat input rate of almost twice that found for the drum to drum exposure. Since these tests are not expected to cause lid loss failure, the radiant exposure from a burning drum(s) will not cause lid loss failure either. Although lid seal failure may occur as a result of the burning drum exposures, the fire will not spread laterally beyond the initial drums which are burning.

\subsection{STACKED DRUM HEAT TRANSFER}

So far, all of the analytical methods which have been addressed included only lateral heat transfer to the storage drums. But, when a drum loses its lid and the contents begin to burn within the container, the fire has the potential to propagate not only to adjacent drums but also to drums stored above. This is true for rack storage configurations where there is sufficient space between rack tiers to allow a drum to lose its lid and burn in the rack. In this case, the primary heat transfer to the exposed container is not through the drum wall but rather through the drum bottom. Therefore, the top and bottom of the drums can not be assumed to be adiabatic for this configuration.

Figure 55 shows the layout for an empty drum on a steel plate exposed to an external heat flux below. Analytically, an empty drum provides the worst case temperature and pressure rise and is also the simplest configuration to model. The external irradiance is provided by a burning drum and will vary depending upon the type of contents burning and the distance between the steel plate and the burning contents.

As the steel plate is heated, it radiates to the bottom of the drum above. Likewise, the bottom of the drum will convect to the air in the drum and radiate to the drum wall and lid. Since the top and bottom of the drum are included in this analysis, the shape factors within the drum will be different than those for the empty drum presented earlier. In this case, there are three different shape factors: (1) the bottom radiates to the drum wall (including the lid) $\left(F_{b-d}\right),(2)$ the wall radiates to the bottom $\left(F_{d-b}\right)$, and $(3)$ the wall radiates to itself $\left(F_{d-d}\right)$. Since the bottom can not see itself and the drum forms an enclosure,

$$
\begin{aligned}
& F_{b-d}=1 \\
& A_{b} F_{b-d}=A_{d} F_{d-b} \\
& F_{d-b}=A_{b} / A_{d} \\
& \text { and, } \\
& F_{d-d}=1-F_{d-b} \\
& F_{d-d}=1-A_{b} / A_{d}
\end{aligned}
$$


WHC-SD-WM-TRP-233 REV 0

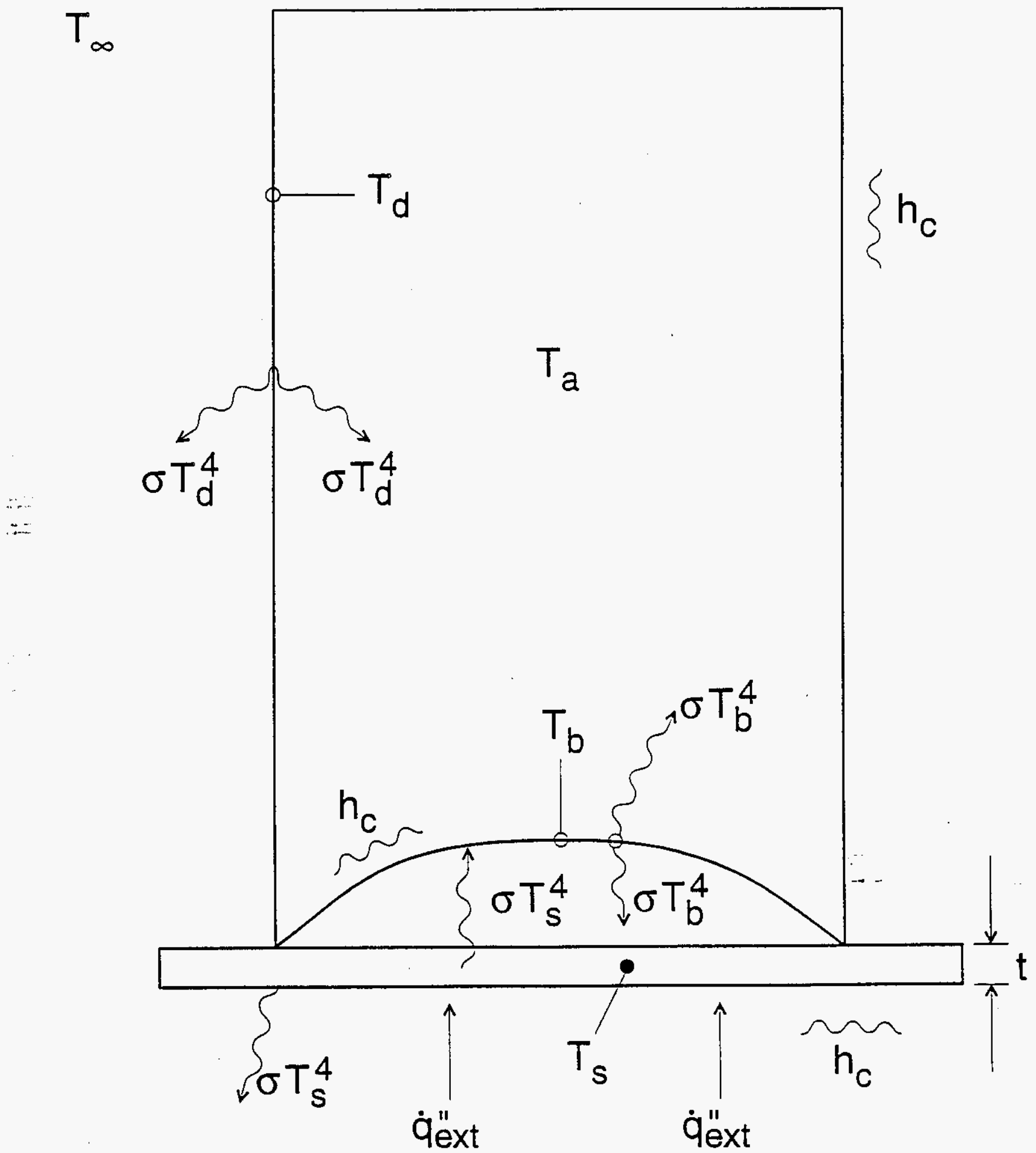

Figure 55. Nomenclature and Arrangement for Stacked Drum Heat Transfer Analysis 
The unsteady heat transfer equations for the stacked drum configuration are given as,

Steel Plate

$$
c_{s} \rho_{s} t \frac{d T_{s}}{d t}=\dot{q}_{c t}^{\prime \prime}-\sigma\left(2 T_{s}^{4}-T_{b}^{4}-T_{-}^{4}\right)
$$

Stacked Drum Bottom

$$
c_{s} \rho_{s} d \frac{d T_{b}}{d t}=\sigma\left(T_{s}^{4}-2 T_{b}^{4}+F_{d-b}\left(\frac{A_{d}}{A_{b}}\right) T_{d}^{4}\right)-h_{c}\left(T_{b}-T_{o}\right)
$$

Stacked Drum Wall and Lid

$$
c_{s} \rho_{s} d \frac{d T_{b}}{d t}=\sigma\left(F_{b-d}\left(\frac{A_{b}}{A_{d}}\right) T_{b}^{4}-2 T_{d}^{4}+F_{d-d} T_{d}^{4}+T_{-}^{4}\right)-h_{c}\left(T_{d}-T_{-}\right)+h_{c}\left(T_{0}-T_{d}\right)
$$

\section{Air in Stacked Drum}

$$
c_{a} P_{a} V_{a} \frac{d T_{a}}{d t}=A_{b} h_{c}\left(T_{b}-T_{a}\right)-A_{d} h_{c}\left(T_{a}-T_{d}\right)
$$

Drum Pressure

$$
p=p_{0}\left(\frac{T_{a}}{T_{a, 0}}\right)
$$

As indicated by these equations, the response of the steel plate and the stacked drum are driven by the heat flux from below, $\dot{q} "$ ext, which was studied experimentally (see Section $3.1 .2)$ to provide suitable inputs to the model. As found in Section 3.1.2, the heat fluxes decrease with increasing separation between the burning and target drums.

HAl performed a test of the stacked drum configuration in which a sealed empty drum was supported on a $6 \mathrm{~mm}(0.25$ in.) steel plate (SWD18, Section 2.2.2). The steel plate was positioned $15 \mathrm{~cm}$ ( 6 in.) above an open kerosene burning drum. The burning drum contained $2.8 \mathrm{~L}(0.75 \mathrm{gal})$ of kerosene which filled the bottom of the drum approximately $1.2 \mathrm{~cm}(0.5$ in.). The spacing and fuel were chosen as worst case storage conditions for the rack configuration based on the previous HAl stacked drum experiments (SWD11.17, Section 3.1). The stacked drum did not lose its lid but did vent during the test. The stacked drum equations are solved and compared to the measured data in order to assess the utility of this model.

The external heat flux to the rack in Equation 50 was determined from additional $\mathrm{HAl}$ testing (SWD11-17, Section 3.1). The heat flux to the bottom of stacked drums was measured using 3 heat flux transducers and a water calorimeter. The spacing between drums and the fuel type was varied to obtain a range of incident fluxes. For a $15 \mathrm{~cm}$ spacing between drums and kerosene fuel burning, both the average of the three heat flux transducers and the water calorimeter yielded an incident heat flux of $35.2 \mathrm{~kW} / \mathrm{m}^{2}$ (SWD11, Table 12). The measurements were averaged between 500 and $1500 \mathrm{~s}$ during the test. The water calorimeter results assumed that the heat flux is incident to the bottom area of the drum only. 
It is remarkable how well these two independent means of measurement agree for this test case.

In addition to heat flux, local gas temperature measurements along the side of the drum were measured for this test. Three thermocouples, one $15 \mathrm{~cm}(6 \mathrm{in.})$ from the bottom of the exposed drum, one $30 \mathrm{~cm}(12 \mathrm{in.})$, and one $46 \mathrm{~cm}$ (18 in.) were positioned $2.5 \mathrm{~cm}(1 \mathrm{in}$.) away from the stacked drum wall. Air temperatures during the test ranged from $200^{\circ} \mathrm{C}$ at the lowest thermocouple to $100^{\circ} \mathrm{C}$ at the highest thermocouple (approximately half-way up the drum). Although temperatures above this point of the drum were not measured, they would at least be higher than the ambient room temperature. If the temperature at the top of the drum is assumed to be ambient, this yields an average gas temperature of $107^{\circ} \mathrm{C}$ surrounding the drum. Since the temperature toward the top of the drum will be higher than ambient conditions, this is expected to be a lower bound value. Likewise, the temperature at the bottom of the drum may be as high as $300^{\circ} \mathrm{C}$ and at the top of the drum $50^{\circ} \mathrm{C}$, this yields an upper bound average temperature of $150^{\circ} \mathrm{C}$. As such, the average gas temperature around the drum would be expected to be approximately $110^{\circ} \mathrm{C}$ to $150^{\circ} \mathrm{C}$. For this test simulation, the average temperature surrounding the drum is taken as $125^{\circ} \mathrm{C}$.

Figures 56-58 show the measured and calculated values for the steel plate temperature, internal air temperature, and drum pressure for the stacked drum test (SWD18). The drum bottom and wall temperatures of the stacked drum were not measured during the test. The measured steel plate temperature is an average of the top and bottom plate temperature, though the temperature difference between the top and bottom of the plate did not vary by more than $55^{\circ} \mathrm{C}$. During the test, the kerosene fire went out several times due to lack of air to the fuel. This is evident in Figure 56 between 200 and $400 \mathrm{~s}$ where the temperature becomes jagged. As such, there is a time lag between the calculated and measured plate temperature. The calculated and measured internal air temperatures seem to agree well in Figure 57 . For the range of expected average gas temperatures surrounding the drum 1110 $150^{\circ} \mathrm{Cl}$, the calculated internal air temperature results may vary up to \pm 15 percent from that shown in Figure 57. Even so, the results appear to correlate well. The measured internal drum pressure shown in Figure 58 does not agree with the calculated results. This is likely due to the drum venting during the experiment. However, lid seal failure would not be expected to occur so quickly for this type of exposure since the top of the drum was the coolest part. Although the drum wall and seal temperatures were not measured in this test, the seal temperature can be estimated based on the internal air temperature. The temperature of the seal would be close to the internal air temperature in this test since the top of the drum is not exposed to the fire and the ambient air surrounding the top of the drum is relatively cool. As seen in Figure 53, the air temperature was less than $50^{\circ} \mathrm{C}$ at the time venting begins to occur $(-250 \mathrm{~s})$. Since lid seal degradation usually occurs at temperatures in the range of $250^{\circ} \mathrm{C}$ to $350^{\circ} \mathrm{C}$, it is unlikely that the drum vented due to lid seal failure. Rather, it is likely that the stacked drum was poorly sealed from the start of the test. If the drum did not vent, the maximum pressure in the drum would have been approximately 7.8 psig based on the maximum measured internal air temperature. Based on available drum response test results (Sections 3.2), this pressure is not expected to result in lid loss. Since the lid seal temperature would remain relatively cool during the exposure, it is unclear whether lid seal failure would occur. Because this test was a bounding scenario of both the fuel source and the tier spacing, fire propagation due to vertical heat transfer is not expected for the stacked drum configurations. 


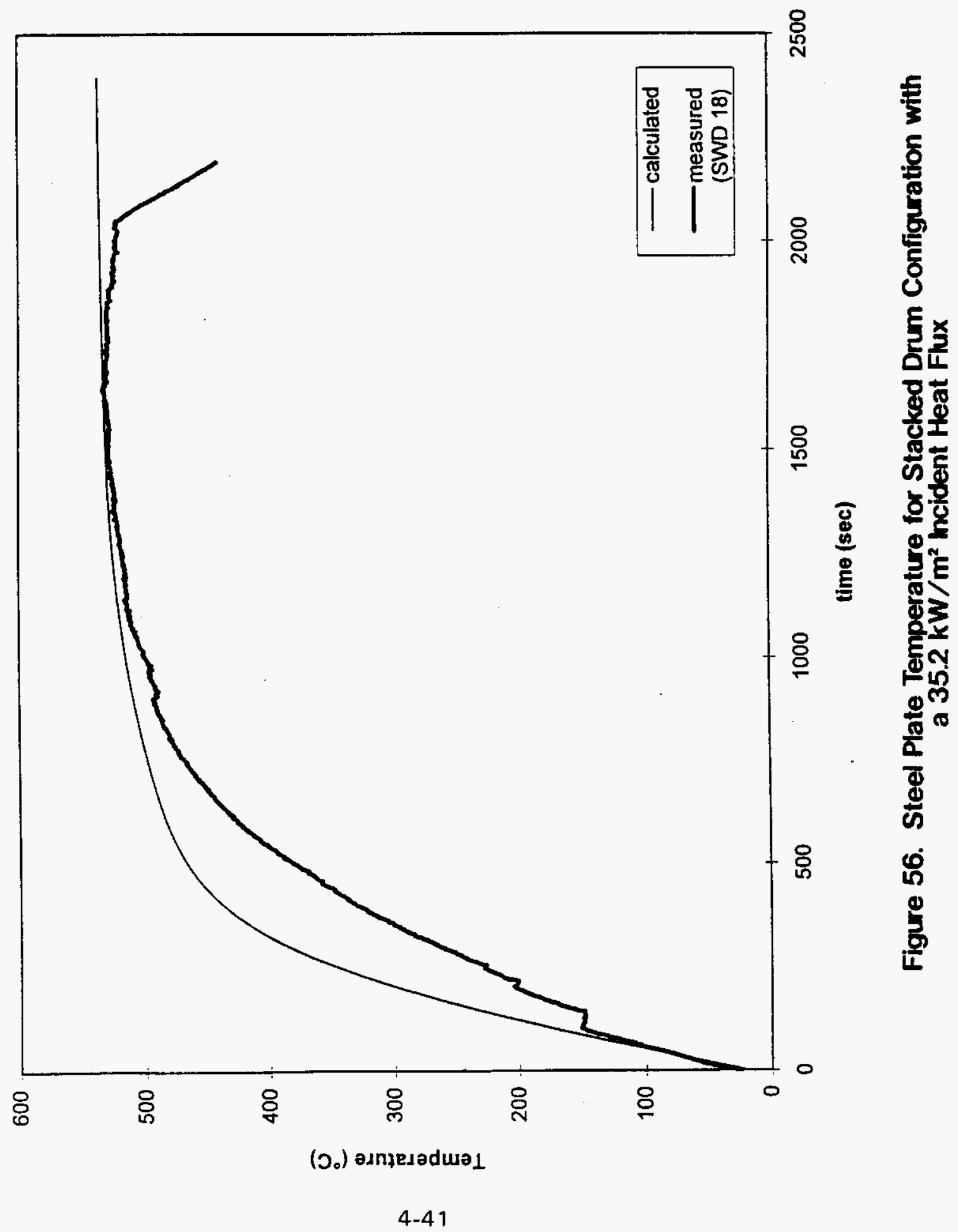




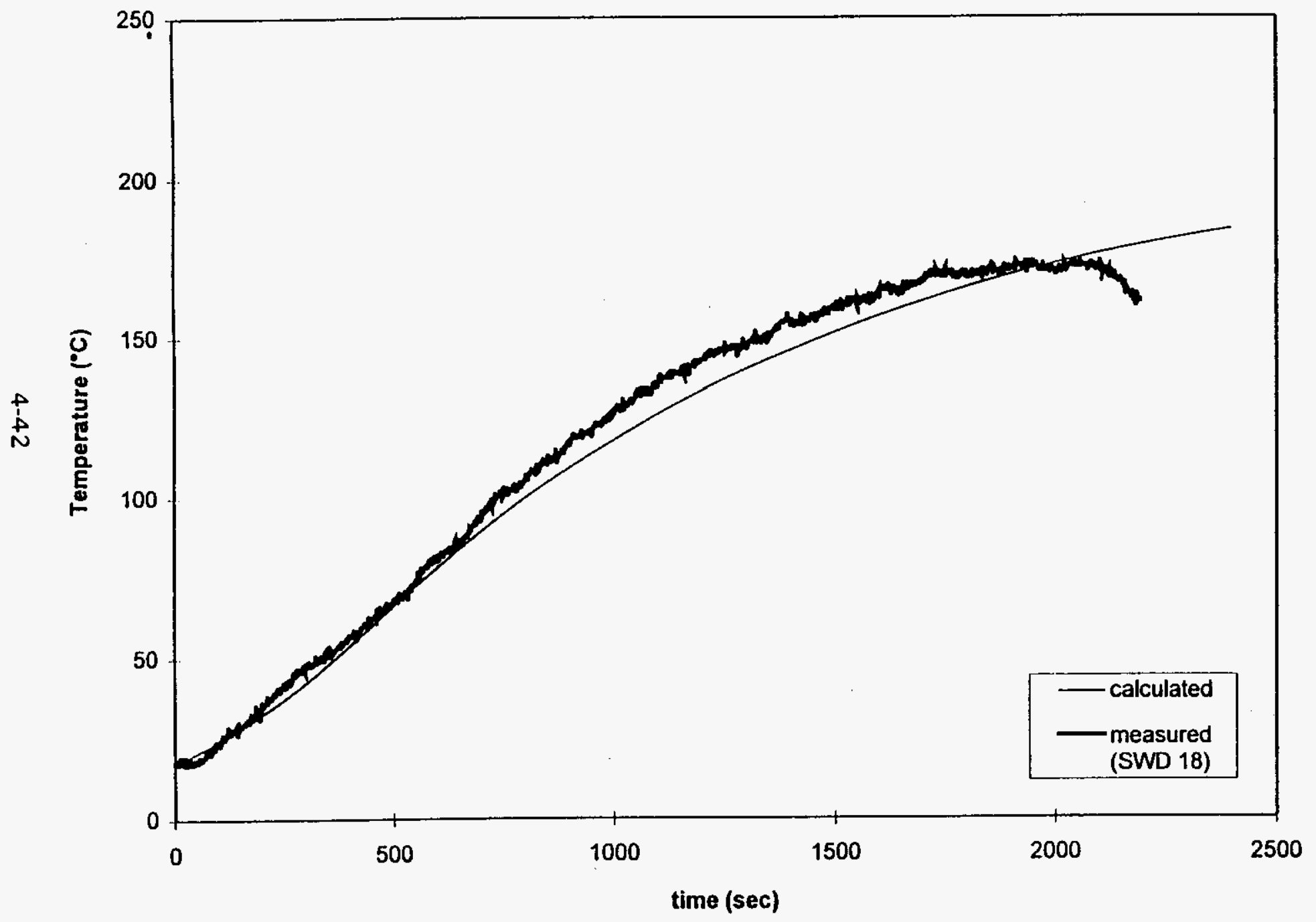

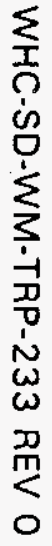

Figure 57. Internal Air Temperature for Stacked Drum Configuration with a $35.2 \mathrm{~kW} / \mathrm{m}^{2}$ Incident Heat Flux to the Steel Plate 
WHC-SD-WM-TRP-233 REV 0

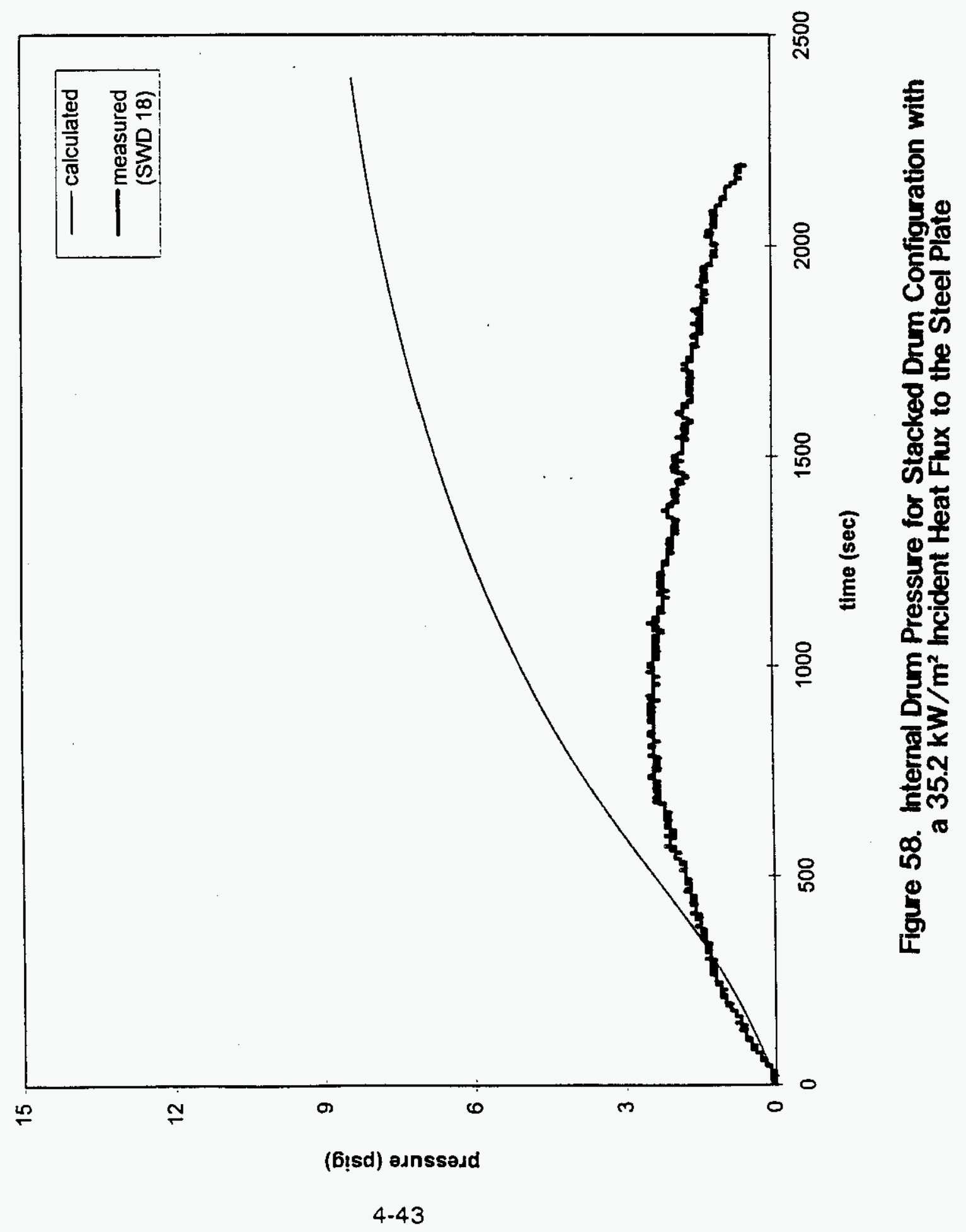




\subsection{HEAT TRANSFER TO RACK FRAME}

The rack structure supporting the stacked drums will also be exposed during a fire. For direct flame impingement, local heat fluxes as high as $120 \mathrm{~kW} / \mathrm{m}^{2}$ can be expected. The unsteady temperature for a square tubular column of thickness $\delta$ completely exposed to an external heat flux is given as

$$
\rho_{s} c_{s} \delta \frac{d T_{s}}{d t}=\dot{q}_{e x}^{\prime \prime}-\sigma\left(T_{s}^{d}-T_{-}^{4}\right)-h_{c}\left(T_{s}-T_{s}\right)
$$

For $6 \mathrm{~mm}(0.25 \mathrm{in}$. $)$ thick steel, taking $T_{\infty}=295 \mathrm{~K}, c_{\mathrm{s}}=0.47 \mathrm{~kJ} / \mathrm{kg}-\mathrm{K}, \rho_{\mathrm{s}}=1800 \mathrm{~kg} / \mathrm{m}^{3}$ and $h_{c}=8 \mathrm{~W} / \mathrm{m}^{2}-\mathrm{K}$, the temperature is shown in Figure 59 for a range of external irradiances. The steel columns will start to lose their structural integrity at temperatures exceeding $500^{\circ} \mathrm{C}$. Direct flame contact $\left\langle\dot{q}_{f l} "=120 \mathrm{~kW} / \mathrm{m}^{2}\right\rangle$ for $2-3$ minutes would be expected to cause the rack structure to collapse unless it was supported by some means other than the exposed columns.

\subsection{OVERPACK DRUMS}

An overpack drum is typically an 85 gallon drum enclosing a 55 gallon waste container with the space between the drums either fully or partially filled with diatomaceous earth. Overpack drums completely filled with diatomaceous earth will have a very long thermal penetration time compared to those only partially filled. This is because if the interior drum is not completely insulated, it will be partially exposed to the heated 85 gaflon drum. Figure 60 shows an overpack drum which is half filled with diatomaceous earth. The 85 gallon drum is completely exposed to an external heat source and the interior drum is considered to be empty. The unsteady temperature equations for the overpack system are given by

\section{Gallon Drum Wall}

$$
\rho_{s} c_{s} d_{z} \frac{d T_{s 2}}{d t}=\dot{q}_{e x \pi}^{\prime \prime}-\sigma\left(2 T_{s 2}^{4}-T_{-}^{4}-T_{s i}^{4}-F_{2-2} T_{s 2}^{4}\right)-h_{c}\left(T_{s 2}-T_{\infty}\right)-h_{c}\left(T_{s 2}-T_{a 2}\right)
$$

Air in 85 Gallon Drum

$$
\rho_{a} c_{p} V_{a 2} \frac{d T_{a 2}}{d t}=A_{2} h_{c}\left(T_{s 2}-T_{a 2}\right)-A_{1} h_{c}\left(T_{a 2}-T_{s l}\right)
$$

55 Gallon Drum Wall

$$
\rho_{s} c_{s} d_{1} \frac{d T_{s l}}{d t}=\sigma\left(F_{2-1} T_{s 2}^{4}-T_{s l}^{4}\right)+h_{c}\left(T_{a 2}-T_{s l}\right)-h_{c}\left(T_{s l}-T_{a l}\right)
$$




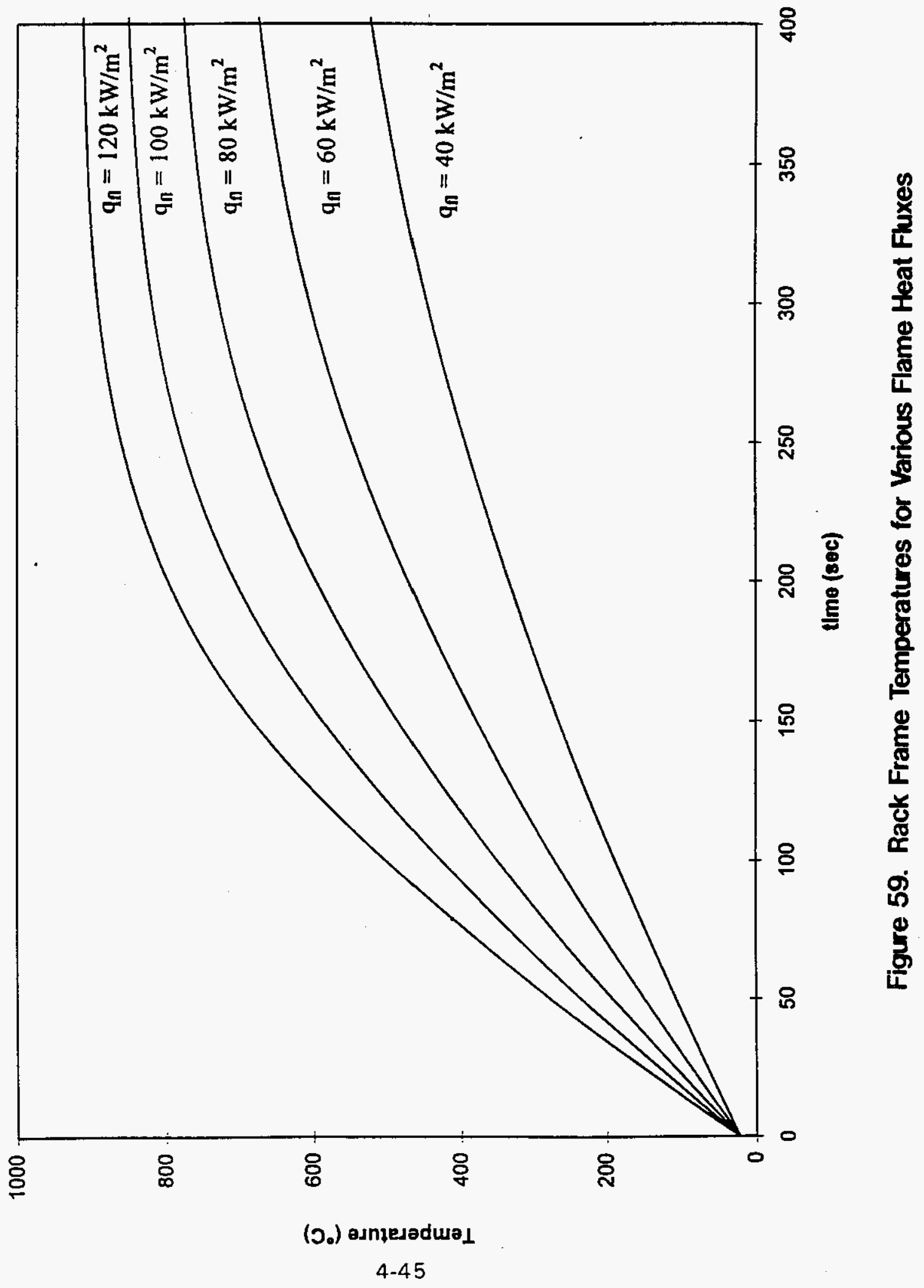


WHC-SD-WM-TRP-233 REV O

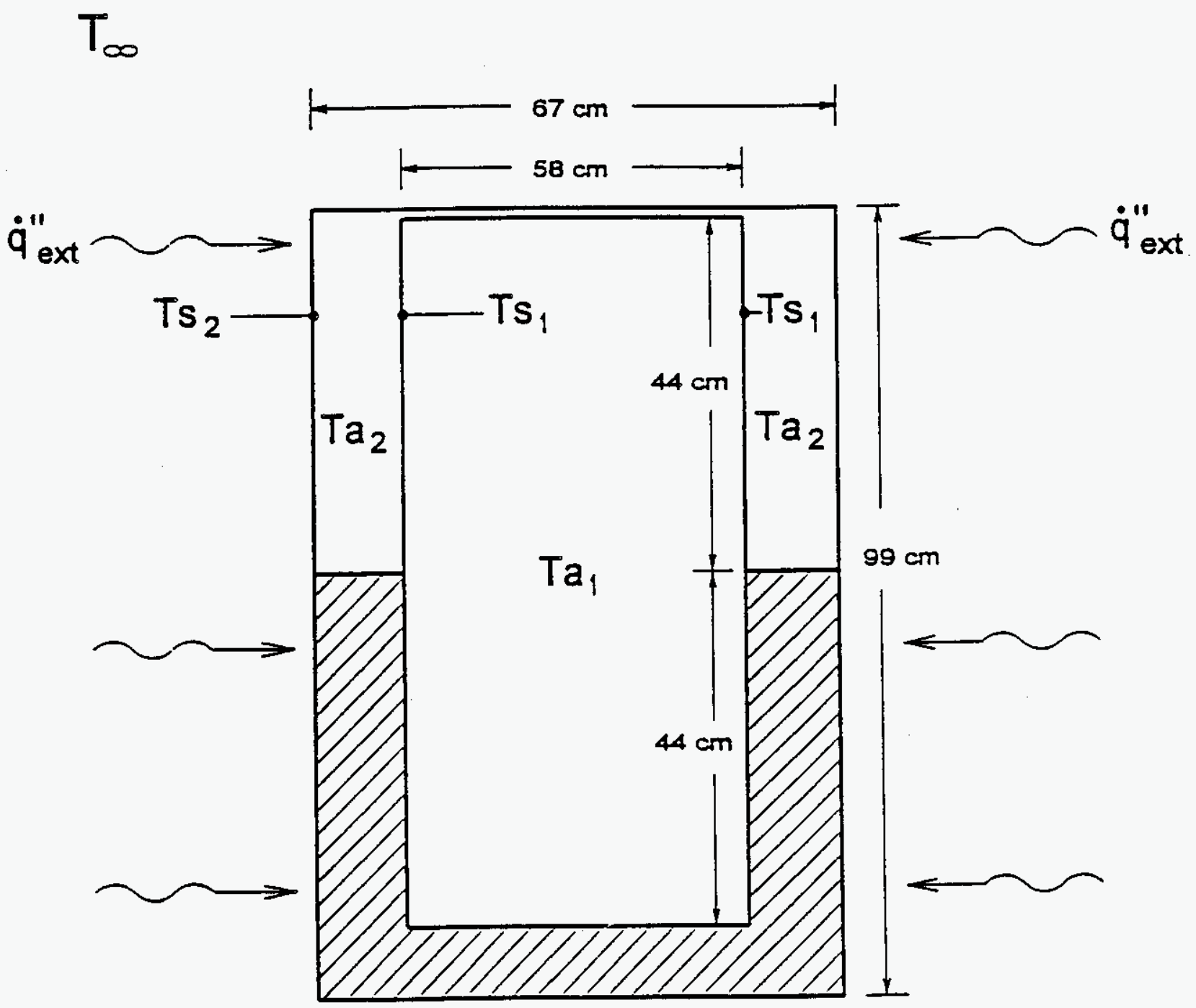

Figure 60. 85 Gallon Overpack Drum Containing Empty 55 Gallon Drum 
Air in 55 Gallon Drum

$$
\rho_{a} c_{p} V_{a l} \frac{d T_{a l}}{d t}=A_{1} h_{c}\left(T_{s l}-T_{a l}\right)
$$

Because conduction through the diatomaceous earth is very small compared to the radiative and convective heat transfer through the air, the insulation is assumed to be adiabatic. Therefore, heat enters the system through the top half of the drums only. Practically speaking, the heat transfer to the interior drum is the same as for the empty drum configuration presented earlier (Section 4.1). However, in this case, the drum is heated convectively and radiatively rather than from a single external heat source. Additionally, the effective volume of the interior drum is doubled since heat is transferred to only half the drum.

Although most of the radiant energy from the 85 gallon drum wall reaches the interior shell $\left(F_{2-1}\right)$, some of it radiates back to itself $\left(F_{2-2}\right)$. The two shape factors are determined from standard configuration analysis, assuming the top and bottom to be adiabatic as before, and are given as,

$F_{2 \cdot 1}=A_{1} / A_{2}$ and,

$F_{2-2}=1-A_{1} / A_{2}(60)$

where, $A_{1}$ and $A_{2}$ are the exposed areas of the interior and exterior drum walls respectively. For the configuration shown in Figure 60,

$F_{2 \cdot 1}=0.87$ and,

$F_{2.2}=0.13$.

Solving Equations 56-60, Figure 61 shows the temperature and pressure rise for the 85 gallon and 55 gallon drum when the overpack is exposed to a $60 \mathrm{~kW} / \mathrm{m}^{2}$ external heat flux. There is approximately a 1 minute time lag before the temperature and pressure begin to rise in the interior drum. However, after this initial delay, the temperature and pressure increases rapidly as it did for the 85 gallon drum. The interior drum reaches typical failure temperatures and pressures within several minutes after the initial exposure. Therefore, in order to significantly increase the failure time, overpack drums should be completely filled with diatomaceous earth. Testing with overpack drums should be pursued in the Phase II drum testing.

\subsection{DRUM ARRAY STABILITY}

\subsubsection{Single Stack Pile Stability Model}

Loss of pile stability due to lid release from the relief of internal pressure buildup can be modeled using simple conservation of angular momentum principles. We conservatively assume that the lid loaded by drums above rotates about one lid edge in response to the lid release. This rotational response maximizes the contribution of the pressure release to pile 
instability. The analysis assumes that the lid has sufficient stiffness to remain straight. This is conservative. If the lid deforms or buckles, additional energy is expended for bending or buckling and an equivalent vent area can be provided with a lesser amount of rotation. The equation of motion of the loaded lid is,

$$
\begin{gathered}
M=I \dot{w} \\
M=\left(P_{1}-P_{a m b}\right) A_{1} R_{1}-L_{1} R_{1}^{\prime} g
\end{gathered}
$$

where

$M=$ moment about the lid edge,

$P_{1}=$ drum pressure,

$A_{1}=$ drum lid area,

$\mathrm{R}_{1}=$ drum radius,

$L_{1}=$ lid load (other drums above),

$H_{C G}=$ height of the center of gravity of the lid load,

$R_{1}^{\prime}=$ distance from edge to $C G$ of loaded drum,

$I=$ moment of inertia,

$\dot{w}=$ rate of change of angular velocity, and

$\mathrm{g}=$ gravitational acceleration.

The first term is the moment applied by the drum overpressure, and the second term is the gravitational restoring moment of the lid load. $R_{1}^{\prime}$ varies as the drum stack rotates as indicated in Figure 62. From basic geometry, we find,

$$
\frac{R_{1}}{R_{1}^{\prime}+R_{1}^{\prime \prime}}=\cos \theta
$$

Combining these yields,

$$
\begin{aligned}
& R_{1}^{\prime \prime}=\left(R_{1} \tan \theta+H_{C G}\right) \sin \theta \\
& R_{1}^{\prime}=\frac{R_{1}}{\cos \theta}-\left(R_{1} \tan \theta+H_{C G}\right) \sin \theta
\end{aligned}
$$

The moment of inertia about the edge of rotation is [Housner and Hudson, 1959],

$$
I=C \frac{5}{4} \pi R_{1}^{4}=L_{1} \frac{5}{4} R_{1}^{2}
$$

where $C$ is the load per unit area of drum lid.

In practice, the pressure quickly decreases due to venting. The venting rate is given by the compressible venting equation [Emmons, 1988],

where the first expression in for choked (sonic) flow. In this problem, the initial pressure in the drum will likely lead to choked flow, but later the flow will cease to be choked. The vent area changes with time and is yet to be determined. 


$$
\dot{m}_{v e n t 1}=\min \left\{\begin{array}{l}
C A_{1} \sqrt{\rho_{1} P_{1}}\left[\gamma\left(\frac{2}{\gamma+1}\right)^{\frac{\gamma+1}{\gamma-1}}\right]^{1 / 2} \\
C A_{1} \sqrt{\rho_{1} P_{1}}\left\{\frac{\gamma}{\gamma-1}\left(\frac{P_{\text {amb }}}{P_{1}}\right)^{2 / \gamma}\left[1-\left(\frac{P_{\text {amb }}}{P_{1}}\right)^{\frac{\gamma-1}{\gamma}}\right]\right\}^{1 / 2}
\end{array}\right\}
$$

We will consider that heating rates and volitization rates which give rise to drum overpressuring are slow relative to the venting rate and can be ignored during venting. The drum will be taken to be substantially empty $\left(V=0.2 \mathrm{~m}^{3}\right)$. Conservation of mass for the drum yields,

$$
\frac{d m_{d}}{d t}=-\dot{m}_{\text {vent }}
$$

Since venting is rapid, we will take the process to be adiabatic [Wark, 1988].

$$
m_{d}(t)=m_{d}(0)\left(\frac{P_{1}(t)}{P_{1}(0)}\right)^{1 / \gamma}
$$

Taking derivatives of both sides of Equation 69 with respect to time yields,

$$
\frac{d m_{d}}{d t}=\frac{m_{d}(0)}{P_{1}(0)^{1 / \gamma}} \frac{d}{d t}\left(P_{1}^{1 / \gamma}\right)=\frac{m_{d}(0)}{p_{1}(0)^{1 / \gamma}} \frac{1}{\gamma} P_{1}^{\frac{1}{\gamma}-1} \frac{d P}{d t}
$$

Equating Equation 68 to Equation 70 and solving for $d p / d t$ yields,

$$
\frac{d P_{1}}{d t}=-\dot{m}_{\text {vent }} \frac{\gamma P_{1}(0)^{1 / \gamma}}{m_{d}(0)} P_{1}^{\frac{r-1}{r}}
$$

where, $m_{d}(0)=V_{d r u m} \rho_{0}$, the initial contents of the drum.

Equation 71 needs to be integrated along with the following equations to deduce the rotational dynamics of the loaded lid. It should be noted that if in the course of the drum release, $R_{1}{ }^{\prime}$ becomes negative the drum stack will fall, whereas if it remains positive the lid will fall back onto the drum.

$$
\begin{array}{ll}
w=\int_{0}^{t} \dot{w} d t & \dot{w}(0)=\text { known from } E q .61 \\
\theta=\int_{0}^{t} w d t & w(0)=\theta(0)=0
\end{array}
$$

It remains to determine the vent area as a function of the lid opening angle, $\theta$. 

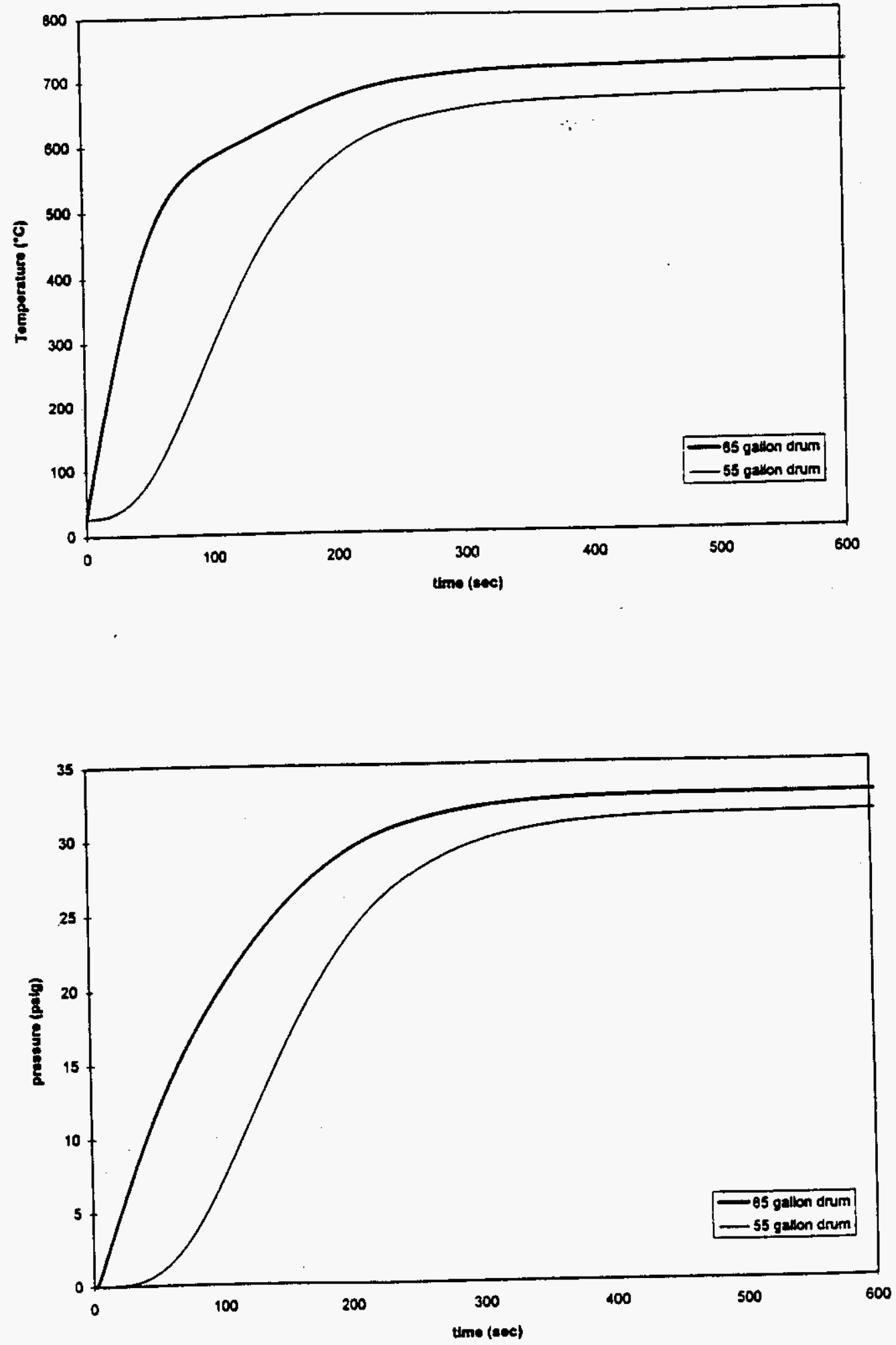

Figure 61. Temperature and Pressure for Overpack Drum Storage Filled $1 / 2$ with Diatomaceous Earth

4-50 
WHC-SD-WM-TRP-233 REV O
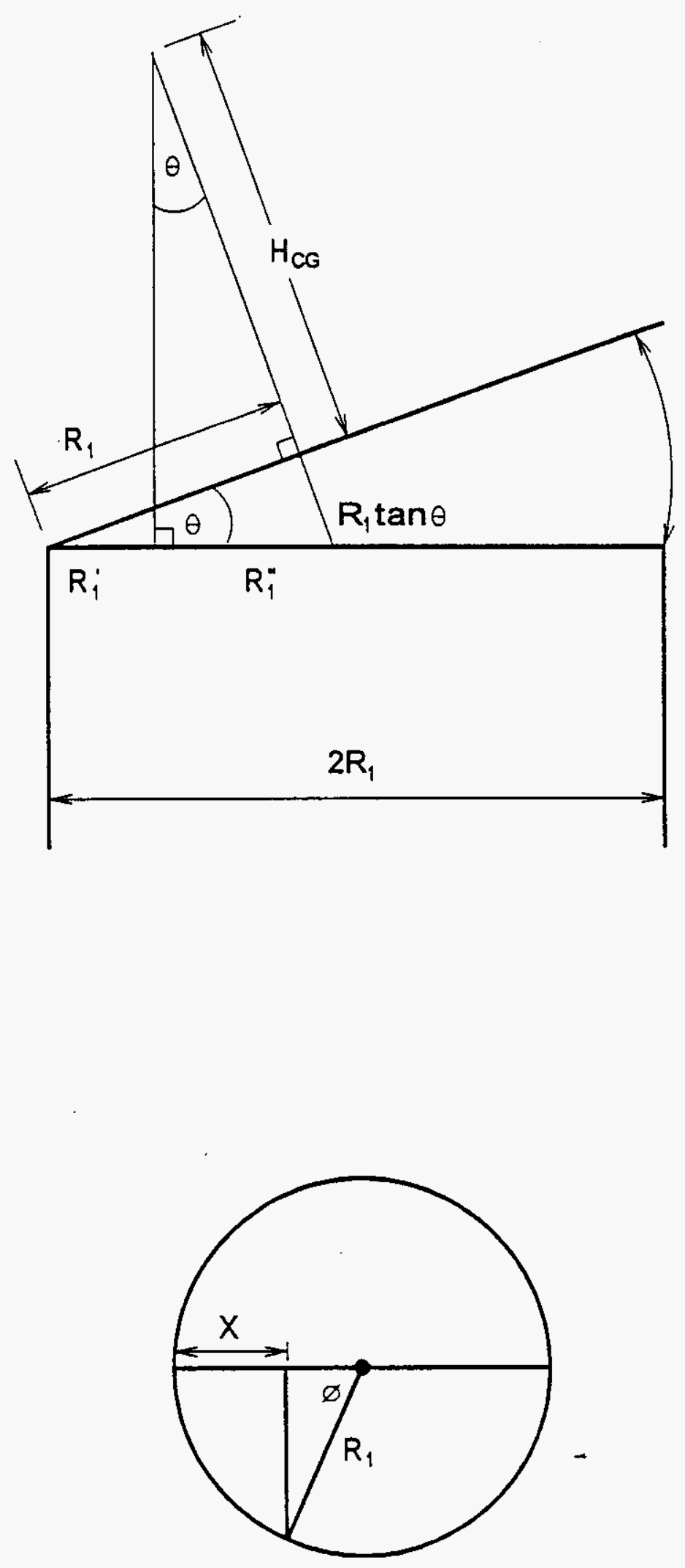

Figure 62. Single Stack Stability Model Geometry Nomenclature 
The vent/leak area, $A_{v}$, is dependent on the lid angle, $\theta$, and can be determined by integration around drum perimeter (see Figure 62),

$$
A_{v}=2 \int_{0}^{\pi} h A_{1} d \phi
$$

where

$h$ is the height of the lid above the drum,

$R_{1}$ is the radius of the drum, and

$\phi$ is the angle in the drum top plane about the drum centerline.

The height, $h$, is given by the distance from the rotational edge, $X$, and the rotation angle, $\theta$, as

$$
h=X \tan \theta
$$

$X$ can be related to the angle $\phi$ by geometry,

$$
\begin{aligned}
& \frac{R_{1}-X}{R_{9}}=\cos \phi \\
& \text { or } X=R_{1}(1-\cos \phi)
\end{aligned}
$$

Substituting into the $A_{y}$ equation yields,

$$
\begin{aligned}
& A_{v}=2 \int_{0}^{\pi} R_{1}(1-\cos \phi) \tan \theta R_{1} d \phi \\
& A_{v}=2 R_{1}^{2} \tan \theta \int_{0}^{\pi}(1-\cos \phi) d \phi=2 \pi R_{1}^{2} \tan \theta
\end{aligned}
$$

The equations to be solved simultaneously are Equations 71 and 73 with Equations 61,62 , $64,65,66,67$ and 76 as subsidiary equations.

\subsubsection{Multiple Stack Pile Stability Model}

The multiple stack model as developed in this section is intended to partially account for the effect of the plywood or pallet in stabilizing the drum array. This model deals only with the multiple drum stack where all of the interacting drums are piled on the same sheets of plywood or pallets (see Figure 63). As such, the calculation is conservative in that it ignores array interconnections which exist in the arrays. Rotation is assumed to be about the minor axis of the plywood or pallet.

The model follows the basic approach of the single stack model with the addition of several generalizations of that model. As such, this section will include only additions and modifications to the single stack model.

The most important difference between the single and multiple stack model is the addition of a second stack/center of gravity. Figure 63 illustrates the situation. Within this context, up to eight drums per tier can be treated. An important assumption in this model is that the plywood sheet or pallet is assumed to remain plane and the multiple drum lids rotate about the same axis. This is again a conservative assumption in that the right drum lid which rotates $2 \theta$ for a rotation of $\theta$ of the other drum could lift rather than rotate and as such would vent more rapidly. 

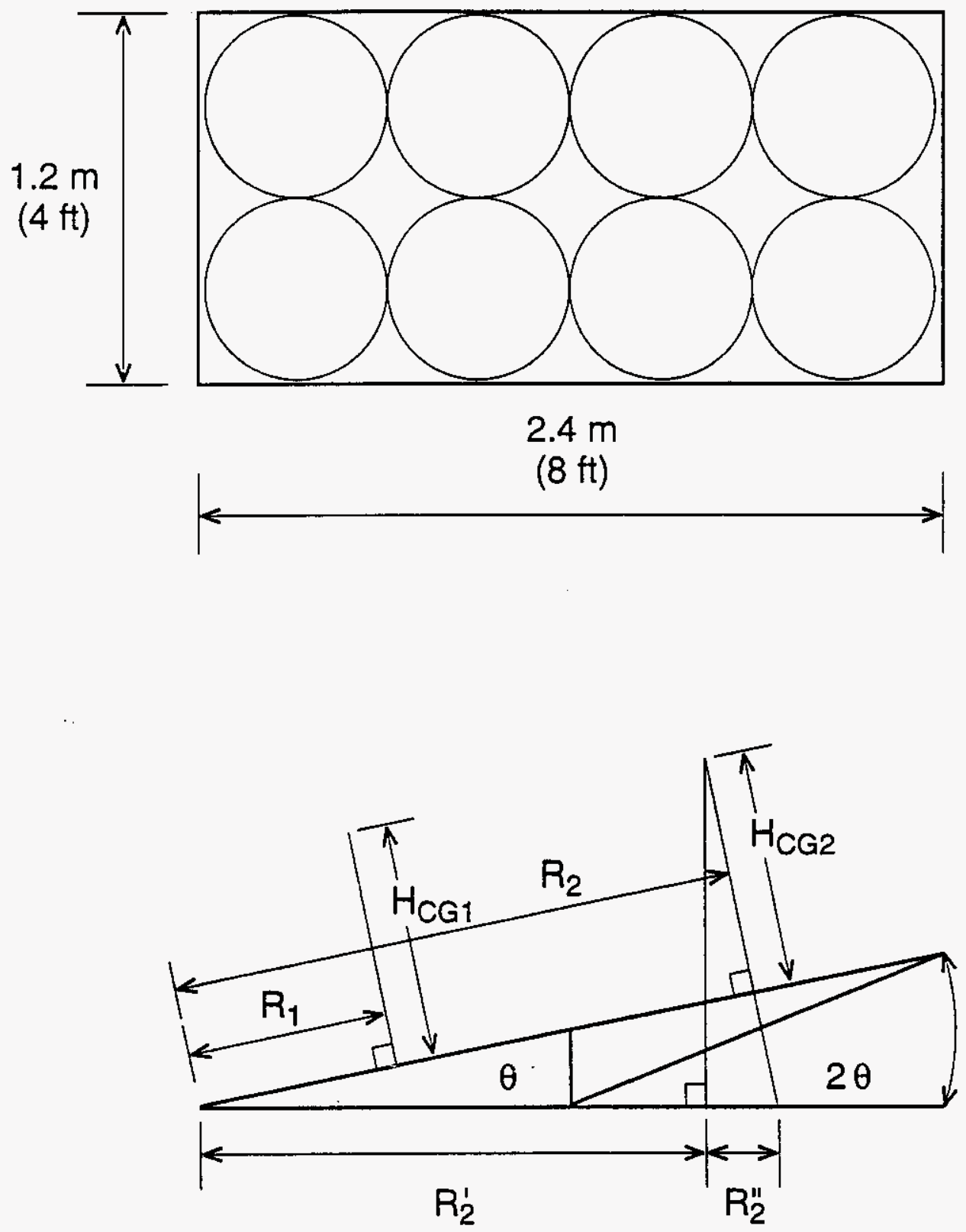

Figure 63. Multiple Stack Stability Model Geometry Nomenclature 
The moment equation for the situation pictured in Figure 63 is includes a pressure and gravity term for each of the drum rows. The moment is given by,

$$
M=n p_{1}\left(P_{1}-P_{s m b}\right) A_{1} R_{1}-n_{1} L_{1} R_{1}^{\prime} g+n p_{2}\left(P_{2}-P_{a m b}\right) A_{2} R_{2}-n_{2} L_{2} R_{2}^{\prime} g
$$

The index no $p_{1}$ is the number of drums in this row that release simultaneously, and $n_{1}$ is the total number of drums in that row. Similarly, $\mathrm{np}_{2}$ is the number of drums in row two that release simultaneously, and $n_{2}$ is the total number of drums in row two. The values of $n_{1}$ and $n_{2}$ can be as high as four (one full plywood sheet), and $n p_{1}$ and $n p_{2}$ may take on any value up to $n_{1}$ and $n_{2}$ respectively. $R_{2}^{\prime}$ can be taken to be equal to $3 R_{1}$, ignoring the small gap between drums. An expression for $R_{2}$ is derived analagous to Equation 65 for the single stack model.

In this case, $3 R_{1}$ is substituted for $R_{1}$. This yields

$$
R_{2}^{\prime}=\frac{3 R_{1}}{\cos \theta}-\left(3 R_{1} \tan \theta+H_{C G 2}\right) \sin \theta
$$

The venting equation for the second drum row is the same as for the single drum case except That the rotation angle is $2 \theta$ as shown in Figure 31.

$$
A_{v 2}=2 \pi R^{2} \tan (2 \theta)
$$

Finally, the moment of inertia for the drums about the axis of rotation is given by,

$$
I=n_{1} \frac{5}{4} L_{1} R_{1}^{2}+n_{2} \frac{37}{4} L_{2} R_{1}^{2}
$$

The second term dominates due to the $3 R_{1}$ lever arm.

This results in four simultaneous equations to be solved; two pressure equations, the angular velocity equation, and the angle equation.

\subsection{CONCLUSIONS AND RECOMMENDATIONS}

The Phase I work of this project has provided new insights into the performance of solid waste drums exposed to fire. The measurements and models provide the basis for the evaluation of fire propagation in both pallet arrays and rack storage configurations.

The burning of drum contents has been found to be extremely slow with average heat release rates in the range of $12.73 \mathrm{~kW}$. Similarly, heat fluxes to neighboring drums were found to be quite low with maximum heat fluxes less than $20 \mathrm{~kW} / \mathrm{m}^{2}$ The worst case time average heat flux to the next tier of a rack storage system was $41 \mathrm{~kW} / \mathrm{m}^{2}$ (for a $15 \mathrm{~cm}$ spacing). It was found through a combination of experimental and analytical methods that horizontal drum to drum fire propagation based on the burning of drum contents within a drum is not expected. A similar analysis for the rack configuration showed that drum burning cannot support upward fire propagation in a rack configuration.

Drum response fire testing has provided data for ordinary combustibles contents drums exposed to characterized fire sources where the response ranges from no lid losses for the smallest source to 87 percent lid loss for the most severe source. The large pool fire results, 
in which 87 percent of drums fail by lid loss, is a higher percentage than any observed in any prior test series. The drum pressures measured at lid loss ranged from 13.28 psig with an average of 20.8 psig. The highest pressures were noted for very lightly loaded drums. The average pressure at lid loss is higher than the maximum pressure observed by previous investigators. Lid loss times were in the range of 70-145 s. All these lid loss times were more rapid than the fastest lid loss previously measured. In prior individual drum testing studies reviewed in Appendix A, lid losses were observed up to $420 \mathrm{~s}$, whereas in this study any drum which did not experience lid loss by $150 \mathrm{~s}$, failed by lid seal faiture in $340 \mathrm{~s}$ or less.

Drum response testing and modeling have provided a means of assessing the response of a drum exposed to initiating sources such as fuel spill pool fires. The measurements obtained in the testing indicate that an exposed drum wall temperature of $625^{\circ} \mathrm{C}$ is a good criterion for lid loss failure. Efforts to predict or correlate lid seal failure were not successful. The drum heat transfer models developed provide very good predictions of exposed drum wall temperatures. Thus, the drum heat transfer models coupled with the lid loss criterion developed from the testing can be used to predict if lid loss will occur for fire exposures which may occur in drum storage facilities. The exposed drum wall temperature criterion of $625^{\circ} \mathrm{C}$ was developed solely on the basis of testing performed in this study and has not been validated for a broader range of potential fires.

Drum response testing included some tests with free flammable liquids within the drum. These tests showed that autoignition of the fuel vapor/air mixture within the drum is possible, though it occurred in only 13 percent of the drums containing flammable liquids. The pressure rise in the two autoignited drums was so rapid that lid loss pressures were not recordable.

Drum array stability modeling has shown the critical importance of the use of plywood or pallets to improve array stability against lid loss failures in the array. Individual drum stacks are calculated to be very unstable and the use of plywood or pallets is shown to greatly improve the array stability.

While great progress has been made in Phase I of this work, there are several areas where additional work is required. First and foremost. Phase I work did not include any testing with actual storage arrays. The experimental results and models of Phase I are sufficient to make predictions of the performance of drums in arrays, but the methods need to be validated against actual drum array tests. This needs to be done for both pallet storage and rack storage configurations. These tests will provide validation for the Phase I results and will provide assurances that new phenomena not anticipated by Phase I testing and analysis do not play an important role in drum array fire propagation. The drum array stability model developed in Phase I has not been compared to any experimental data. Such data can only be obtained through drum array testing proposed for Phase II.

Several areas which require additional investigation have been identified in the course of the Phase I work. Perhaps the most important area is the study of drum contents fire exposures. The drum response testing has shown that the expulsion of contents from the drum can lead to contents burning adjacent to other drums. This exposure was not initially included in the scope of Phase I work and only one exploratory test was performed in Phase 1. This test indicated that contents fires adjacent to drums may not pose a fire exposure which would be capable of causing lid loss failure. However, there is very little data in the literature regarding burning rates and heat fluxes from trash fires and further work is needed to confirm that fire propagation in drum arrays by expelled contents fires cannot occur. 
Other areas of investigation identified in the Phase I work include the effect of drum corrosion on drum response to fire and the effect steel overpack drums on fire performance. These issues can be addressed through testing of the type used in Phase I. A model of overpack drums has been developed in Phase I, but there are no data available for comparison. This testing should include the half filled overpack currently used and completely filled overpacks. The use of overpacks fully filled with diatamatious earth has the potential for providing excellent passive fire resistance to all drums. This could improve fire performance greatly and reduce the need for other levels of fire protection. All drums tested in Phase I were in good structural condition and no drum wall failures were observed. The behavior of corroded drum walls may introduce failure modes not previously investigated. These areas should be investigated in Phase II.

Finally, the results of this work provide the tools required to evaluate the fire performance of solid waste drum storage arrays. In order to facilitate the use of these tools, it is important to formulate an analysis methodology which formalizes the procedures for using these tools to allow appropriate use of the results of this work for storage sites at Hanford or elsewhere at DOE facilities.

\subsection{NOMENCLATURE}

$\begin{array}{lll}A & \text { area } \\ & \mathrm{C} & \text { specific heat } \\ \mathrm{F} & \text { shape factor } \\ \mathrm{h} & \text { heat transfer coefficient } \\ \mathrm{k} & \text { thermal conductivity } \\ \mathrm{m} & \text { mass } \\ \mathrm{p} & \text { pressure } \\ \mathrm{q} & \text { heat } \\ \mathrm{r} & \text { radius } \\ \mathrm{R} & \text { universal gas constant } \\ \mathrm{t} & \text { time } \\ \mathrm{T} & \text { temperature } \\ \mathrm{V} & \text { volume } \\ \mathrm{X} & \text { depth } \\ \delta & \text { thickness } \\ \varepsilon & \text { emissivity } \\ r & \text { ratio of specific heats } \\ \rho & \text { density } \\ \sigma & \text { Stefan Boltzmann constant } \\ \theta, \Phi & \text { angle }\end{array}$




\section{Subscripts}

$\begin{array}{ll}1,2,3,4 & \text { surface designation } \\ a, b, c, d & \text { surface designation } \\ a & \text { air } \\ \text { ad } & \text { adjacent } \\ \text { amb } & \text { ambient } \\ b & \text { bottom } \\ c & \text { convective } \\ d & \text { drum } \\ \text { ext } & \text { external } \\ \text { fl } & \text { flame } \\ \text { inc } & \text { incident } \\ o & \text { initial } \\ \text { op } & \text { opposite } \\ s & \text { steel } \\ \text { surf } & \text { surface } \\ t & \text { target } \\ v & \text { constant volume } \\ v & \text { vent } \\ w & \text { wall } \\ x & \text { line segment (Figure 3) } \\ \infty & \text { ambient }\end{array}$




\subsection{REFERENCES}

Babrauskas, V. and S.J. Grayson (ed.), Heat Release in Fires, Elsevier Applied Science, London, 1992.

Beyler, C.L., "Flammability Limits of Premixed and Diffusion Flames," Section 1/Chapter 17. The SFPE Handbook of Fire Protection Engineering, P.J. DiNenno, Ed., National Fire Protection Association, Quincy, MA, 1988.

Boothe, G. F., "Radiological and Chemical Source Terms for Solid Waste Operations Complex", WHC-SD-W306-Ti-001 REV 1, 1994.

Carver, M.R. (1993), "Drums, DOT UN1A2, Solid Material, 114/208/322 Liter Salvage, Galvanized, Steel," HS-V-PO010, Impact Level3-SQ, Westinghouse Hanford Company, January 20, 1993.

Carver, M.R. (1994), Westinghouse Hanford Company, private communication, September 29, 1994.

Eckert, E. and Drake, R., Analysis of Heat and Mass Transfer, McGraw-Hill Book Company, New York, 1972.

Emmons, H.W., "Vent Flows", Section 1/Chapter 8, The SFPE Handbook of Fire Protection Engineering, P. DiNenno, Editor-in-Chief, Society of Fire Protection Engineers, Boston, MA, 1988.

Foley, M. and Drysdale, D., "Heat Transfer from Flames Between Vertical Paralle! Walls", University of Edinburgh, Unit of Fire Safety Engineering.

Greenhalgh, W.O., J.A. Demiter, and W.W. (Bill) Olson (1994), "Exposure of Drummed Solid Waste to Fire - Draft Report," WHC-SD-WM-DTR-032 REV O, May 1994.

Hasegawa, H.K., K.J. Staggs, and S.M. Doughty (1993), "Fire Testing of 55 Gallon Metal Waste Drums for Dry Waste Storage," CCRL-CR-115037, Fire Research Discipline, Special Projects Division, Hazards Control Department, Lawrence Livermore Nationa! Laboratory, July 1993.

Hasemi, Y., "Experimental Wall Flame Heat Transfer Correlations for the Analysis of Upward Wall Flame Spread", Fire Science and Technology, 4 (2), December 30, 1984.

Holman, J.P., Heat Transfer, Seventh Edition, McGraw-Hill Book Company, New York, 1990.

Housner, G.W. and Hudson, D.E., Applied Mechanics Dynamics, Second Edition, Van Nostrand Company, Inc., New York, 1959.

Howell, J.R., A Catalog of Radiation Configuration Factors, McGraw-Hill Book Company, New York, 1982. 
Quintiere, J.G. and Harkleroad, M., "Wall Flames and Implications for Upward Flame Spread", AIAA-85-0456, AIAA 23rd Aerospace Sciences Meeting, Reno, NV, January 14-17, 1985.

Wark, K. Jr., Thermodynamics, Fifth Edition, McGraw-Hill Book Company, New York, 1988. 
WHC-SD-WM-TRP-233 REV O

Appendix A

Summary of Fire Test Literature on Solid Waste Drum Storage 


\section{Summary}

A limited number of tests have been conducted on the response of $17 \mathrm{C}$ drums to pool fire exposures and internal explosions $(A 1-A 6)$. The general results of this testing indicate that drums exposed to pool fires may vent at the lid seal, in some cases throwing the lid some distance and scattering some of the contents. In other cases, the failure of the drum seal results in gas or flame jetting only. Testing involving multi-tiered arrays of drums indicates that, while lower tier drums may vent violently, the pile of drums is not disrupted and the contents of the array remain in the drums. The 0.25 -in. plywood between tiers acts as a thermal/flame barrier until it burns through. Internal hydrogen explosion tests show that drum failure is by drum lid seal failure just as in the pool fire tests. Internal explosions can lead to loss of the lid and some of the contents. The pool fire tests and the explosion tests indicate that drum lid seal failures occur over a range of pressure of 1 to 20 psi.

While failure of the drum via drum seam failure is possible, none have been observed in testing. As such, drum failures seem to occur by lid loss via drum overpressure. In many cases lid seals can fail without lid loss. While materials can be lost via pyrolsis without lid loss, active burning of contents requires lid loss.

\section{Fire Exposure Tests}

SANL(A 1):Tests of $17 \mathrm{C}$ solid waste drums exposed to pool fires were conducted by Sandia Laboratories (A1) in support of waste acceptance criteria and hazard analysis for the WIPP site. Two types of tests were conducted. The first test type twelve drums were placed in a 10-ft diameter salt-filled pan, and 50 gallons of diesel fuel was added and ignited. Some of the drums were filled with simulated combustible wastes. Some had plastic liners while others did not. Some of the drums had $1 / 8$-inch vent holes, and others were sealed. Of the twelve drums tested, one vented unlined drum failed by violently blowing the lid and some contents out of the drum. Two other lined drums vented by flaring, and one lined drum ruptured at the base of the drum late in the test. Eight other drums did not vent or fail. Wind effects may have affected the uniformity of the pool fire exposure. Venting of the drums with $1 / 8$-inch hole did not affect drum behavior.

In a second type of pool fire test, one plastic lined and one unlined drums were exposed to pool fires (no salt bed) with durations of 6.5-8 minutes. In one test, the drums were filled with combustibles while in the other test the drums were empty. The unlined drums failed via lid loss with the unfilled drum failing in 3 minutes (internal pressure of 20 psig) and the filled drum failing in 5 minutes (internal pressure of 14 psig). The plastic lined drums did not fail during the tests.

Of the 16 drums tested, three violently blew their lids. All the drums which failed by lid loss were unlined drums. Of the 5 unlined drums tested, three failed by lid loss. The most rapid lid loss was 3 minutes for the unfilled drum. The other two lid failures were filled drums and occurred in 5-7 minutes. This indicates that the contents of the drums is acting as a heat sink, slowing the venting process. Further, the plastic drum lining appeared to prevent fid loss failures. Drum venting failures for lined drums were all flare/jet failures and did not result in lid loss. The largest jet flame was 4 feet long. 
INEL(A2): Two tests were performed with DOT $17 \mathrm{C}$ drums and DOT 7A boxes. The first test included five $17 \mathrm{C}$ drums in a 6 feet square salt filled pan with 15 gallons of diesel fuel. The drums were plastic lined and the lids were not sealed. All five drum lids popped in the first 3-5 minutes of the intense exposure.

In the second test, 16 drums were stacked in four tiers with $3 / 8$-inch plywood between the tiers. The stacking arrangement is that of interest for the SWBG. Again, the drums were not sealed in this test, and plastic drum liners were used. In this test, no salt was present in the burn pan. Venting of the drums began at 3 minutes into the exposure, and the pan fire burned for 15 minutes. While the drums vented, the four tier stack was not disrupted. Some lids were dislodged during venting so that flame jet issued from the lid.

In these tests, plastic lined drums did vent, but the drums were not sealed. Based on the SANL tests and other tests on 17C drums, it is not clear if these drums would have vented if sealed. While the unsealed drums in the 4 tier array did vent and did not affect the stability of the array, it is not clear if sealing the drums would have resulted in sufficient venting force to topple the array. The $3 / 8$ inch thick plywood between the first and second tier of drums was entirely destroyed except where protected by the drums, and the plywood between the second and third tiers was severely damaged as judged from a report photo. There was no evidence of serious damage to the plywood between tiers three and four.

LLNL(A4): Five pool fire experiments were conducted using a total of 14 single solid waste drums and four salvage drum assemblies. The pool fires were 6 feet in diameter with the drums occupying less than half that area. As such, the flames were more severe than would be expected in a tight drum array. The fuel used for the tests was isopropanol, a luminous but low soot fuel, chosen to facilitate visual observation throughout the test duration.

Of the 14 single drums tested, three failed by complete lid loss. Times to lid loss ranged from 180 to $270 \mathrm{~s}$. Peak pressure at lid loss was 8.6 to $13.2 \mathrm{psi}$. Lid seal failures in the remaining 11 drums occurred less violently with a maximum pressure in the range 1 to 14 psi and times to lid seal failure in the range 180 to $345 \mathrm{~s}$.

For this test series, the worst case drum was a violent lid loss at $180 \mathrm{~s}$ with a release pressure of $13 \mathrm{psi}$. The drums which lost their lids, the contents were substantially consumed $18-9 \mathrm{~kg}$ of $10 \mathrm{~kg}$ were consumed) while other drums lost less than $1.2 \mathrm{~kg}$ of their $10 \mathrm{~kg}$ load.

Hydrogen Explosion Tests (INEL) (A3): Based on the possible presence of hydrogen in solid waste storage drums, tests were conducted with 14 and 30 percent hydrogen concentrations in $17 \mathrm{C}$ drums lined with $90-\mathrm{mil}$ plastic. On ignition, only the 30 percent hydrogen (stoichiometric) drums failed by loss of the lid. Typically the lid was thrown 100 feet, and drum contents were partially lost. The fraction of material lost from the drum ranged from 0.41 percent. In one test, two drums were filled with stoichiometric hydrogen/air mixtures with one drum stacked on the other. Ignition of the lower drum resulted in autoignition of the upper drum. The lid of the lower drum was not blown off. Pretest pressure testing of the drums yielded venting at about 10 psi. 
Facility Fire Test (A5): Four 55-gallon drums were placed in a wooden building loaded with ordinary combustibles. The entire building was burned to demonstrate the response of the drums during an actual facility fire. The drums were on a bi-level steel rack, 2 drums per tier. The building burned for approximately 2 hours, although, the drums were exposed to the fire for only 30 to 60 minutes.

Of the 4 drums tested, none experienced violent lid loss, however, they all vented at the lid seal. As the drums vented, the gases ignited in a torchlike jet. The rack structure remained in place during the test.

Wood Pallet Test $(A 6)$ : 55-gallon drums, filled with noncombustible waste material, were placed on wooden pallets stacked three tiers high. The ignition source consisted of a wooden crib, wood shavings, and ethyl alcohol. The test demonstrated that the wooden pallets did not significantly ignite and basically self extinguished once the ignition source was consumed.

\section{Appendix A References}

A1. Hunter, T., "Summary of Research and Development Activities in Support of Waste Acceptance Criteria for WIPP," Sandia Laboratories, Albuquerque, NM, SAND79-1305, 1979.

A2. Brown, R., "DOT 7A FRP Box Fire Test at the INEL," EG\&G Idaho, Inc., TREE-1367, 1979.

A3. Beers, R., "Gas Generation Studies: Year-end Report, FY 1983," EG\&G Idaho, Inc., RHB-387-83, 1983.

A4. Hasegawa, H.K., Staggs, K.J., Doughty, S.M., "Fire Testing of 55 Gallon Metal Waste Drums for Dry Waste Storage," Lawrence Livermore National Laboratory, UCRL-CR$115037,1993$.

A5. Greenhalgh, W. O., Demiter, J. A., Olson, W. W., "Exposure of Drummed Solid Waste to Fire", WHC-SD-WM-DTR-032 REV 0, 1994.

A6. Snook, B., "Pallet Fire Test for Steel Drum Storage on Wooden Pallets", Martin Marietta, Portsmouth Gaseous Diffusion Plant, 1992. 
T.able A1. Summary of Fire Test Literature on Solid Waste Drum Storage (4 sheets).

\begin{tabular}{|c|c|c|c|c|c|}
\hline TEST NAME & DRUM SPEC & CONTENTS & EXPOSURE & EXP TIME & RESULTS \\
\hline $\begin{array}{l}\text { Sandia Laboratories } \\
\text { Series A }\end{array}$ & DOT-17C 55-gallon & & $\begin{array}{l}12 \text { drums arranged in a } 10 \text { feet } \\
\text { diameter pan containing } 50 \text { gallon } \\
\text { diesel fuel pool fire. Tank bottom } \\
\text { was covered with } 3 \text { inches of } \\
\text { granulated salt. }\end{array}$ & & \\
\hline Drum 1 & $\begin{array}{l}\text { polyethylene lining, } \\
\text { unvented }\end{array}$ & $\begin{array}{l}\text { simulated } \\
\text { combustible waste }\end{array}$ & 12 drum in 10 foot pan & 45 minutes & drum vented with mild flaring. \\
\hline Drum 2 & $\begin{array}{l}\text { polyethylene lining, } \\
1 / 8 \text { inch vent }\end{array}$ & $\begin{array}{l}\text { simulated } \\
\text { combustible waste }\end{array}$ & 12 drum in 10 foot pan & 45 minutes & $\begin{array}{l}\text { drum vented resulting in a } \\
\text { strong torchlike jet extending } \\
\text { up to } 4 \text { feet long. }\end{array}$ \\
\hline Drum 3 & unlined, unvented & $\begin{array}{l}\text { simulated } \\
\text { combustible waste }\end{array}$ & 12 drum in 10 foot pan & 45 minutes & $\begin{array}{l}\text { drum blew its lid at about } 7 \\
\text { minutes and scattered burning } \\
\text { debri about the area. }\end{array}$ \\
\hline Drum 4 & $\begin{array}{l}\text { polyethylene lining, } \\
\text { unvented }\end{array}$ & $\begin{array}{l}\text { simulated } \\
\text { combustible waste }\end{array}$ & 12 drum in 10 foot pan & 45 minutes & $\begin{array}{l}\text { bottom seam of drum ruptured } \\
\text { with violent release of flaming } \\
\text { gas. }\end{array}$ \\
\hline Drums $5-12$ & 55-gallon varied & $\begin{array}{l}\text { simulated } \\
\text { combustible waste }\end{array}$ & $\begin{array}{l}\text { wind conditions prevented spread } \\
\text { of the fire beyond drums } 1-4\end{array}$ & 45 minutes & $\begin{array}{l}\text { drum } 7 \text { had a badly bulged lid } \\
\text { but remained intact and held } \\
\text { its seal. drum } 8 \text { emitted a } \\
\text { smoke plume from its vent } \\
\text { hole. Nothing reported for } \\
\text { other drums in Series A. }\end{array}$ \\
\hline $\begin{array}{l}\text { Sandia Laboratories } \\
\text { Series B }\end{array}$ & $\begin{array}{l}\text { DOT-17C } \\
55 \cdot \text { gallon }\end{array}$ & & $\begin{array}{l}\text { two drums in shallow pan } \\
\text { containing } 5 \text { gallons of diesel fuel } \\
\text { floated on water }\end{array}$ & & \\
\hline Test 1 - Drum 1 & polyethylene liner & $\begin{array}{l}\text { simulated } \\
\text { combustible waste }\end{array}$ & two drum in shallow pan & 8 minutes & $\begin{array}{l}\text { drum held its seal at a } \\
\text { maximum internal pressure } \\
\text { and temperature of } 6 \text { psig and } \\
66^{\circ} \mathrm{C} \text {. }\end{array}$ \\
\hline Drum 2 & unlined & $\begin{array}{l}\text { simulated } \\
\text { combustible waste }\end{array}$ & two drum in shallow pan & 8 minutes & $\begin{array}{l}\text { drum seal failed at a maximum } \\
\text { internal pressure and } \\
\text { temperature of } 14 \text { psig and } \\
121^{\circ} \mathrm{C} \text {. }\end{array}$ \\
\hline
\end{tabular}


Table A1. Summary of Fire Test Literature on Solid Waste Drum Storage (4 sheets).

\begin{tabular}{|c|c|c|c|c|c|}
\hline TEST NAME & DRUM SPEC & CONTENTS & EXPOSURE & EXP TIME & RESULTS \\
\hline Test 2 - Drum 1 & polyethylene liner & empty & $\begin{array}{l}\text { two drum in shallow pan - flames } \\
\text { were blown toward unlined drum }\end{array}$ & 6.5 minutes & $\begin{array}{l}\text { drum held its scal at a peak } \\
\text { internal pressure of } 15 \text { psig. } \\
\text { Peak temperature inside drum } \\
\text { reached } 300^{\circ} \mathrm{C} \text {. }\end{array}$ \\
\hline Drum 2 & unlined & empty & $\begin{array}{l}\text { two drum in shallow pan - flames } \\
\text { were blown toward unlined drum }\end{array}$ & 6.5 minutes & $\begin{array}{l}\text { drum blew its lid at about } 3 \\
\text { minutes at an internal pressure } \\
\text { of } 20 \text { psig. Peak temperature } \\
\text { inside drum reached } 638^{\circ} \mathrm{C} \text {. }\end{array}$ \\
\hline \multicolumn{6}{|l|}{$\begin{array}{l}\text { Lawrence Livermore } \\
\text { National Laboratory }\end{array}$} \\
\hline WDE119A & $\begin{array}{l}\text { DOT-17C } \\
\text { 55-gallon }\end{array}$ & empty & $\begin{array}{l}\text { ASTM E-119 time temperature } \\
\text { curve }\end{array}$ & 12 minutes & $\begin{array}{l}\text { drum vented with a peak } \\
\text { internal pressure of } 16 \text { psig. }\end{array}$ \\
\hline WDPAN1 - Drum 1 & $\begin{array}{l}\text { DOT-17C } \\
55 \text {-gallon }\end{array}$ & $\begin{array}{l}10 \mathrm{~kg} \text { Class A } \\
\text { combustibles }\end{array}$ & $\begin{array}{l}4 \text { drums in a } 6 \text { foot diamter pan } \\
\text { containing } 10 \text { gallons of } \\
\text { Isopropanol }\end{array}$ & 10 minutes & $\begin{array}{l}\text { drum vented with a peak } \\
\text { internal pressure of } 8 \text { psig. }\end{array}$ \\
\hline Drum 2 & $\begin{array}{l}\text { DOT-17C } \\
\text { 55-gallon }\end{array}$ & $\begin{array}{l}10 \mathrm{~kg} \text { Class } \mathrm{A} \\
\text { combustibles } \\
\end{array}$ & 4 drums in 6 foot pan & 10 minutes & $\begin{array}{l}\text { drum vented with a peak } \\
\text { internal pressure of } 10.5 \mathrm{psig.}\end{array}$ \\
\hline Drum 3 & $\begin{array}{l}\text { DOT-17C } \\
55-\text { gallon }\end{array}$ & $\begin{array}{l}10 \mathrm{~kg} \text { Class } \mathrm{A} \\
\text { combustibles }\end{array}$ & 4 drums in 6 foot pan & 10 minutes & $\begin{array}{l}\text { drum vented with a peak } \\
\text { internal pressure of } 12.8 \mathrm{psig} .\end{array}$ \\
\hline Drum 4 & \begin{tabular}{|l|} 
DOT-17C \\
55-gallon
\end{tabular} & $\begin{array}{l}10 \mathrm{~kg} \text { Class } \mathrm{A} \\
\text { combustibles }\end{array}$ & 4 drums in 6 foot pan & 10 minutes & $\begin{array}{l}\text { drum vented with a peak } \\
\text { internal pressure of } 12.4 \text { psig. }\end{array}$ \\
\hline WDPAN2 - Drum 1 & $\begin{array}{l}\text { DOT-17C } \\
55 \text {-gallon }\end{array}$ & $\begin{array}{l}10 \mathrm{~kg} \text { Class } A \\
\text { combustibles }\end{array}$ & $\begin{array}{l}4 \text { drumis in a } 6 \text { foot diamter pan } \\
\text { containing } 10 \text { gallons of } \\
\text { Isopropanol }\end{array}$ & 16 minutes & $\begin{array}{l}\text { drum blew its lid ejecting } \\
\text { contents at about } 4.5 \text { minutes } \\
\text { at an internal pressure of } 8.6 \\
\text { psig. Peak temperature inside } \\
\text { drum reached } 480^{\circ} \mathrm{C} \text {. } \\
\end{array}$ \\
\hline Drum 2 & \begin{tabular}{|l|} 
DOT-17C \\
55-gallon \\
\end{tabular} & \begin{tabular}{|l|}
$10 \mathrm{~kg}$ Class $\mathrm{A}$ \\
combustibles
\end{tabular} & 4 drums in 6 foot pan & 16 minutes & $\begin{array}{l}\text { drum vented with a peak } \\
\text { internal pressure of } 11.4 \text { psig. }\end{array}$ \\
\hline Drum 3 & \begin{tabular}{|l|}
$\begin{array}{l}\text { Over pack or } \\
\text { salvage }\end{array}$ \\
\end{tabular} & Drum 5 & 4 drums in 6 foot pan & 16 minutes & \begin{tabular}{|l|} 
drum vented with a peak \\
internal pressure of 7 psig.
\end{tabular} \\
\hline Drum 4 & Transuranium (TRU) & $\begin{array}{l}10 \mathrm{~kg} \text { Class } \mathrm{A} \\
\text { combustibles }\end{array}$ & 4 drums in 6 foot pan & 16 minutes & $\begin{array}{l}\text { pressure oscillated between } 0 \\
\text { and } 1 \text { psig. Pressure was } \\
\text { constantly being relieved } \\
\text { through fitter in lid. }\end{array}$ \\
\hline
\end{tabular}


Table A1. Summary of Fire Test Literature on Solid Waste Drum Storage (4 sheets).

\begin{tabular}{|c|c|c|c|c|c|}
\hline TEST NAME & DRUM SPEC & CONTENTS & EXPOSURE & EXP TIME & RESULTS \\
\hline Drum 5 & $\begin{array}{l}\text { DOT-17C } \\
55 \text {-gallon }\end{array}$ & $\begin{array}{l}10 \mathrm{~kg} \text { Class } \mathrm{A} \\
\text { combustibles }\end{array}$ & inside of Drum 3 & 16 minutes & $\begin{array}{l}\text { drum vented with a peak } \\
\text { internal pressure of } 10.8 \mathrm{psig} .\end{array}$ \\
\hline WDPAN3 - Drum 1 & $\begin{array}{l}\text { DOT-17C } \\
55-\text { gallon }\end{array}$ & $\begin{array}{l}10 \mathrm{~kg} \text { Class } \mathrm{A} \\
\text { combustibles }\end{array}$ & $\begin{array}{l}4 \text { drums in a } 6 \text { foot diamter pan } \\
\text { containing } 10 \text { gallons of } \\
\text { Isopropanol }\end{array}$ & 16 minutes & $\begin{array}{l}\text { drum blew its lid ejecting } \\
\text { contents at about } 4 \text { minutes at } \\
\text { an internal pressure of } 12.5 \\
\text { psig. Peak temperature inside } \\
\text { drum reached } 450^{\circ} \mathrm{C} \text {. }\end{array}$ \\
\hline Drum 2 & \begin{tabular}{|l|} 
DOT-17C \\
55 -gallon \\
\end{tabular} & $\begin{array}{l}10 \mathrm{~kg} \text { Class } \mathrm{A} \\
\text { combustibles }\end{array}$ & 4 drums in 6 toot pan & 16 minutes & \begin{tabular}{|l|} 
drum vented with a peak \\
internal pressure of $12.5 \mathrm{psig}$.
\end{tabular} \\
\hline Drum 3 & $\begin{array}{l}\text { Over pack or } \\
\text { salvage }\end{array}$ & Drum 5 & 4 drums in 6 foot pan & 16 minutes & $\begin{array}{l}\text { drum vented with a peak } \\
\text { internal pressure of } 5 \text { psig. }\end{array}$ \\
\hline Drum 4 & Transuranium (TRU) & $\begin{array}{l}10 \mathrm{~kg} \text { Class } A \\
\text { combustibles }\end{array}$ & 4 drums in 6 foot pan & 16 minutes & $\begin{array}{l}\text { A peak pressure of } 2.75 \mathrm{psig} \\
\text { was reached but decreased } \\
\text { steadily to zero by the end of } \\
\text { the test. }\end{array}$ \\
\hline Drum 5 & $\begin{array}{l}\text { DOT-17C } \\
55 \text {-gallon }\end{array}$ & $\begin{array}{l}10 \mathrm{~kg} \text { Class } \mathrm{A} \\
\text { combustibles }\end{array}$ & inside of Drum 3 & 16 minutes & $\begin{array}{l}\text { drum vented with a peak } \\
\text { internal pressure of } 5 \text { psig. }\end{array}$ \\
\hline WDPAN4 - Drum 1 & $\begin{array}{l}\text { DOT- } 17 \mathrm{C} \\
55 \text {-gallon with } \\
\text { polyethylene liner }\end{array}$ & $\begin{array}{l}10 \mathrm{~kg} \text { Class } \mathrm{A} \\
\text { combustibles }\end{array}$ & $\begin{array}{l}3 \text { drums in a } 6 \text { foot diamter pan } \\
\text { containing } 12 \text { gallons of } \\
\text { Isopropanol }\end{array}$ & 17 minutes & $\begin{array}{l}\text { drum vented with a peak } \\
\text { internal pressure of } 14 \text { psig. } \\
\text { flames from the lid seal } \\
\text { continued for } 9 \text { minutes after } \\
\text { the pool fire went out. }\end{array}$ \\
\hline Drum 2 & $\begin{array}{l}\text { DOT- 17C } \\
55 \text {-gallon with PE } \\
\text { liner }\end{array}$ & $\begin{array}{l}10 \mathrm{~kg} \text { Class } \mathrm{A} \\
\text { combustibles }\end{array}$ & 3 drums in 6 foot pan & 17 minutes & $\begin{array}{l}\text { drum vented with a peak } \\
\text { internal pressure of } 12.7 \text { psig. }\end{array}$ \\
\hline Drum 3 & $\begin{array}{l}\text { Over pack or } \\
\text { salvage with PE liner }\end{array}$ & Drum 4 & 3 drums in 6 foot pan & 17 minutes & $\begin{array}{l}\text { drum vented with a peak } \\
\text { internal pressure of } 7 \text { psig. }\end{array}$ \\
\hline Drum 4 & $\begin{array}{l}\text { DOT- } 17 \mathrm{C} \\
55 \text {-gallon with PE } \\
\text { liner }\end{array}$ & $\begin{array}{l}10 \mathrm{~kg} \text { Class } \mathrm{A} \\
\text { combustibles }\end{array}$ & inside of Drum 3 & 17 minutes & $\begin{array}{l}\text { drum vented with a peak } \\
\text { internal pressure of } 15.5 \text { psig. }\end{array}$ \\
\hline WDPAN5 - Drum 1 & $\begin{array}{l}\text { DOT- } 17 C \\
55 \text {-gallon with PE } \\
\text { liner }\end{array}$ & $\begin{array}{l}10 \mathrm{~kg} \text { Class } A \\
\text { combustibles }\end{array}$ & $\begin{array}{l}3 \text { drums in a } 6 \text { foot diamter pan } \\
\text { containing } 12 \text { gallons of } \\
\text { Isopropanol }\end{array}$ & 13 minutes & $\begin{array}{l}\text { drum vented with a peak } \\
\text { internal pressure of } 11.8 \text { psig. }\end{array}$ \\
\hline
\end{tabular}


Table A1. Summary of Fire Test Literature on Solid Waste Drum Storage (4 sheets).

\begin{tabular}{|c|c|c|c|c|c|}
\hline TEST NAME & DRUM SPEC & CONTENTS & EXPOSURE & EXP TIME & RESULTS \\
\hline Drum 2 & $\begin{array}{l}\text { DOT- } 17 \mathrm{C} \\
55 \text {-gallon with PE } \\
\text { liner }\end{array}$ & $\begin{array}{l}10 \mathrm{~kg} \text { Class } \mathrm{A} \\
\text { combustibles }\end{array}$ & 3 drums in 6 foot pan & 13 minutes & $\begin{array}{l}\text { drum blew its lid ejecting } \\
\text { some contents at about } 3.5 \\
\text { minutes at an internal pressure } \\
\text { of } 13.2 \text { psig. Peak } \\
\text { temperature inside drum } \\
\text { reached } 450+{ }^{\circ} \mathrm{C} \text {. }\end{array}$ \\
\hline Drum 3 & $\begin{array}{l}\text { Over pack or } \\
\text { salvage with PE liner }\end{array}$ & Drum 4 & 3 drums in 6 foot pan & 13 minutes & $\begin{array}{l}\text { drum vented with a peak } \\
\text { internal pressure of } 14 \mathrm{psig.}\end{array}$ \\
\hline Drum 4 & $\begin{array}{l}\text { DOT-17C } \\
55 \text {-gallon with PE } \\
\text { liner }\end{array}$ & $\begin{array}{l}10 \mathrm{~kg} \text { Class } \mathrm{A} \\
\text { combustibles }\end{array}$ & inside of Drum 3 & 13 minutes & $\begin{array}{l}\text { drum vented with a peak } \\
\text { internal pressure of } 13.2 \text { psig. }\end{array}$ \\
\hline Westinghouse & & $\begin{array}{l}\text { metal, wood, dirt, } \\
\text { rubber, plastic, } \\
\text { paper, glass, cloth }\end{array}$ & $\begin{array}{l}\text { drums on a steel rack are located } \\
\text { in wooden building to be burned. } \\
\text { medium fire load consisting of } \\
\text { wooden pallets and ordinary } \\
\text { building materials. }\end{array}$ & $\begin{array}{l}\text { building burned } \\
\text { for about } 2 \\
\text { hours; drums } \\
\text { were exposed } \\
\text { for } 30-60 \\
\text { minutes }\end{array}$ & \\
\hline Drum FT-1 & $\begin{array}{l}\text { DOT - 17C } \\
55 \text {-gallion with PE } \\
\text { liner }\end{array}$ & $\begin{array}{l}59 \mathrm{~kg} \text { with } 22.5 \mathrm{~kg} \\
\text { Class A } \\
\text { combustibles }\end{array}$ & bottom rack & $30-60$ minutes & $\begin{array}{l}\text { drum vented with the gases } \\
\text { igniting in a torchlike jet. }\end{array}$ \\
\hline Drum FT-2 & $\begin{array}{l}\text { DOT - 17C } \\
55 \text {-gallon with PE } \\
\text { liner }\end{array}$ & $\begin{array}{l}62 \mathrm{~kg} \text { with } 25 \mathrm{~kg} \\
\text { Class A } \\
\text { combustibles }\end{array}$ & top rack above FT-1 & $30-60$ minutes & $\begin{array}{l}\text { drum vented with the gases } \\
\text { igniting in a torchlike jet. lid } \\
\text { was severely deformed after } \\
\text { test. }\end{array}$ \\
\hline Drum FT-3 & $\begin{array}{l}\text { DOT - } 17 \mathrm{C} \\
55 \text {-gallion with PE } \\
\text { liner }\end{array}$ & $\begin{array}{l}79 \mathrm{~kg} \text { with } 43 \mathrm{~kg} \\
\text { Class A } \\
\text { combustibles }\end{array}$ & bottom rack & 30-60 minutes & $\begin{array}{l}\text { drum vented with the gases } \\
\text { igniting in a torchlike jet. }\end{array}$ \\
\hline Drum FT-4 & $\begin{array}{l}\text { DOT - } 17 \mathrm{C} \\
55 \text {-galllon with PE } \\
\text { liner }\end{array}$ & $\begin{array}{l}79 \mathrm{~kg} \text { with } 43 \mathrm{~kg} \\
\text { Class A } \\
\text { combustibles }\end{array}$ & top rack above FT-3 & $30-60$ minutes & $\begin{array}{l}\text { drum vented with the gases } \\
\text { igniting in a torchlike jet. }\end{array}$ \\
\hline
\end{tabular}


FMIS View/Print Document Cover Sheet

This document was retrieved from the Boeing ISEARCH System.

Accession \#: D196069895

Document \#: SD-WM-TRP-233

Title/Desc:

ANALYTICAL \& EXPERIMENTAL EVALUATION OF SOLID WASTE DRUM FIRE PERFORMANCE [VOL 2] [SEC 2 OF 3] 
This document was too large to scan as a whole document, therefore it required breaking into smaller sections.

DOCUMENT NUMBER: WHC-SD-WM-TRP-233 RWO

SECTION 2 OF 3

TITLE: Analytical \& Experimental

Evaluation of Solid Waste Drum Zire

Performance [ vol 2]

DATE: $10 / 1 / / 1995$

ORIGINATOR: Lovie, $R L$

CO: WHC

RECIPIENT:

CO:

REFERENCES: EDT-612225 
THIS PAGE INTENTIONALLY LEFT BLANK

\section{4}




\title{
Analytical and Experimental Evaluation of Solid Waste Drum Fire Performance
}

\author{
Volume II \\ Appendix B. Test Data \\ Appendix C. Project Plan for Phase 2 - Drum Array \\ Fire Tests of Solid Waste Drums
}

\author{
Prepared for \\ Westinghouse Hanford Company \\ Richland, WA 99352 \\ Prepared by \\ Chris F. Haecker \\ Los Alamos Technical Associates, Inc. \\ Kennewick, WA 99306 \\ Brian T. Rhodes, Jesse J. Beitel, Daniel T. Gottuk \\ Craig L. Beyler, and Eric R. Rosenbaum \\ Hughes Associates, Inc. \\ Columbia, MD 21045
}



WHC-SD-WM-TRP-233 REV. 0

Appendix B

Test Data 
WHC-SD-WM-TRP-233 REV. 0

SWD 1 - DATA

B-2 
WHC-SD-WM-TRP-233 REV. 0

SWD1 - Standard Load
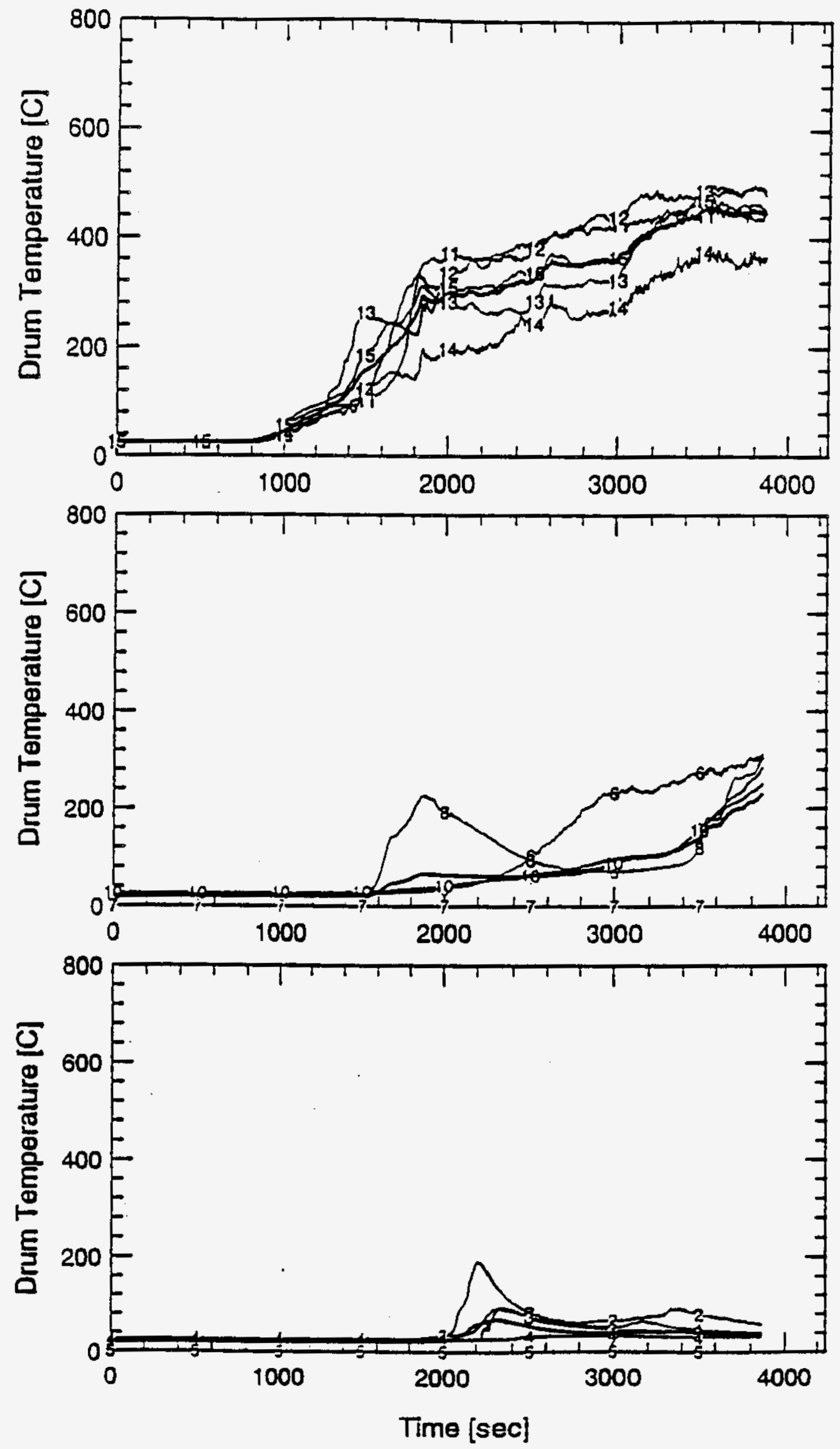

B-3 
SWD1 - Standard Load
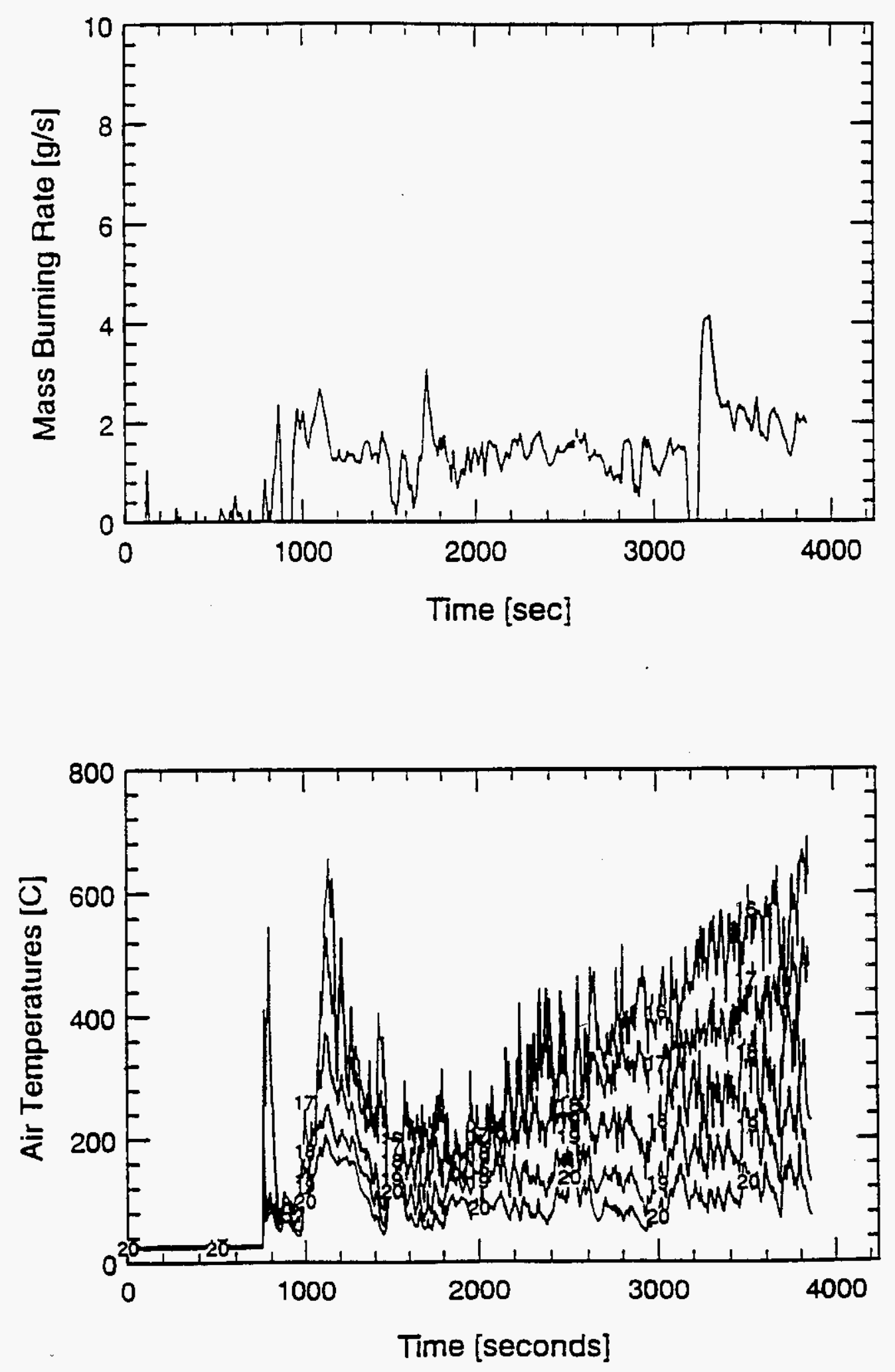
WHC-SD-WM-TRP-233 REV. 0

SWD1 - Standard Load
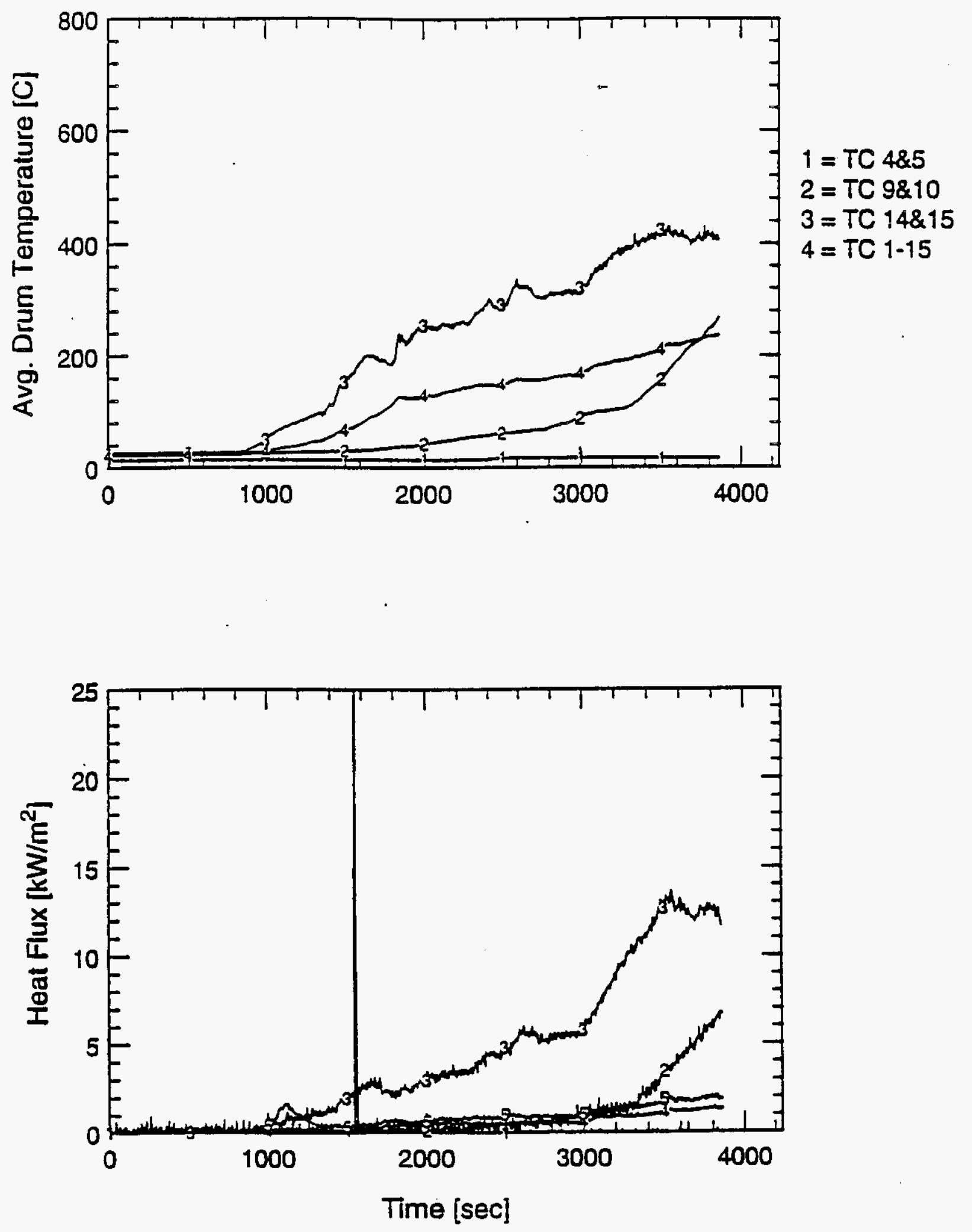

B-5 
WHC-SD-WM-TRP-233 REV. 0

\section{SWD1 - Standard Load}
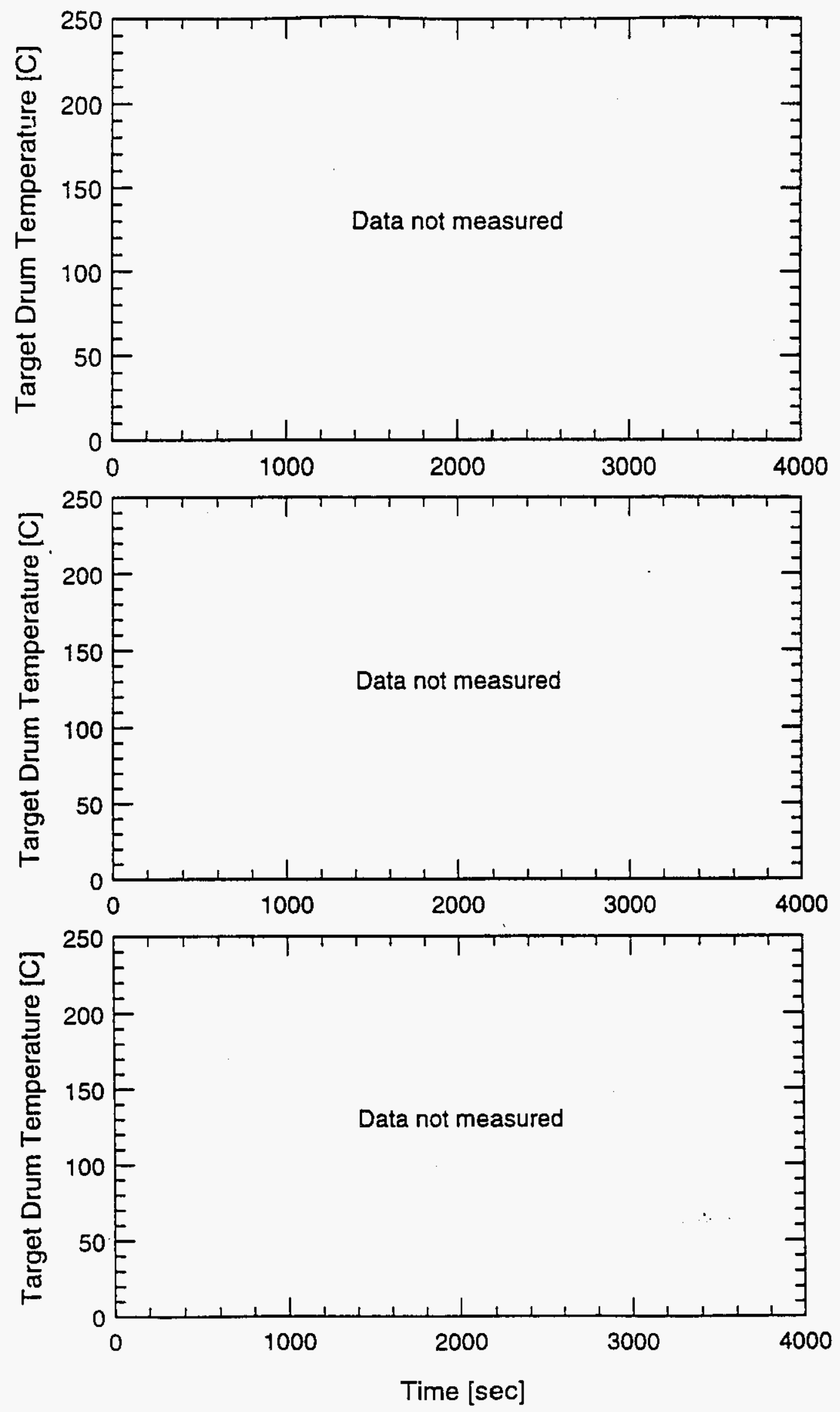
SWD1 - Standard Load
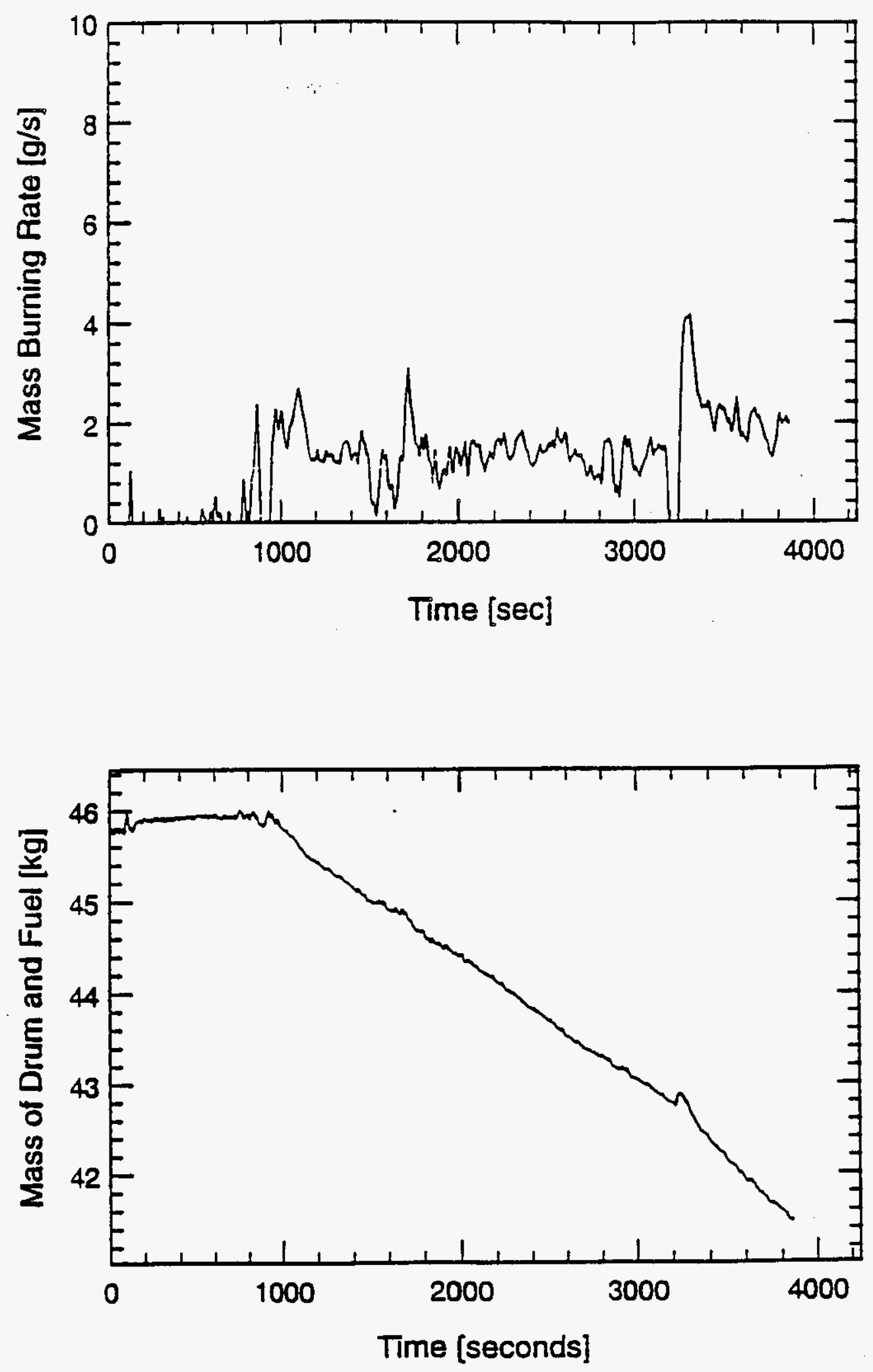
WHC-SD-WM-TRP-233 REV. 0

SWD 2 - DATA

B-8 
WHC-SD-WM-TRP-233 REV. 0

SWD2 - Standard Load with Grate and Angle
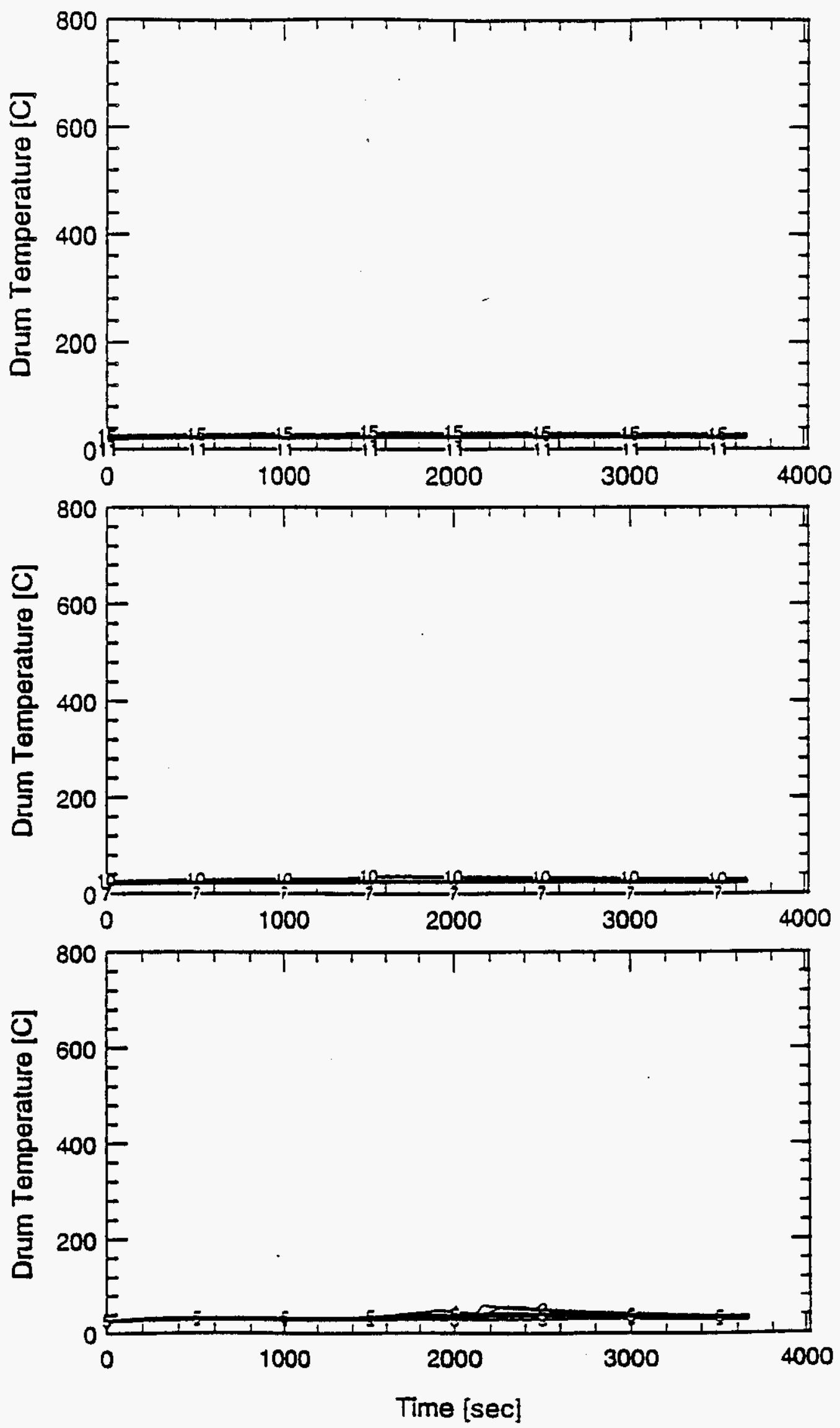

B-9 


\section{SWD2 - Standard Load with Grate and Angle}
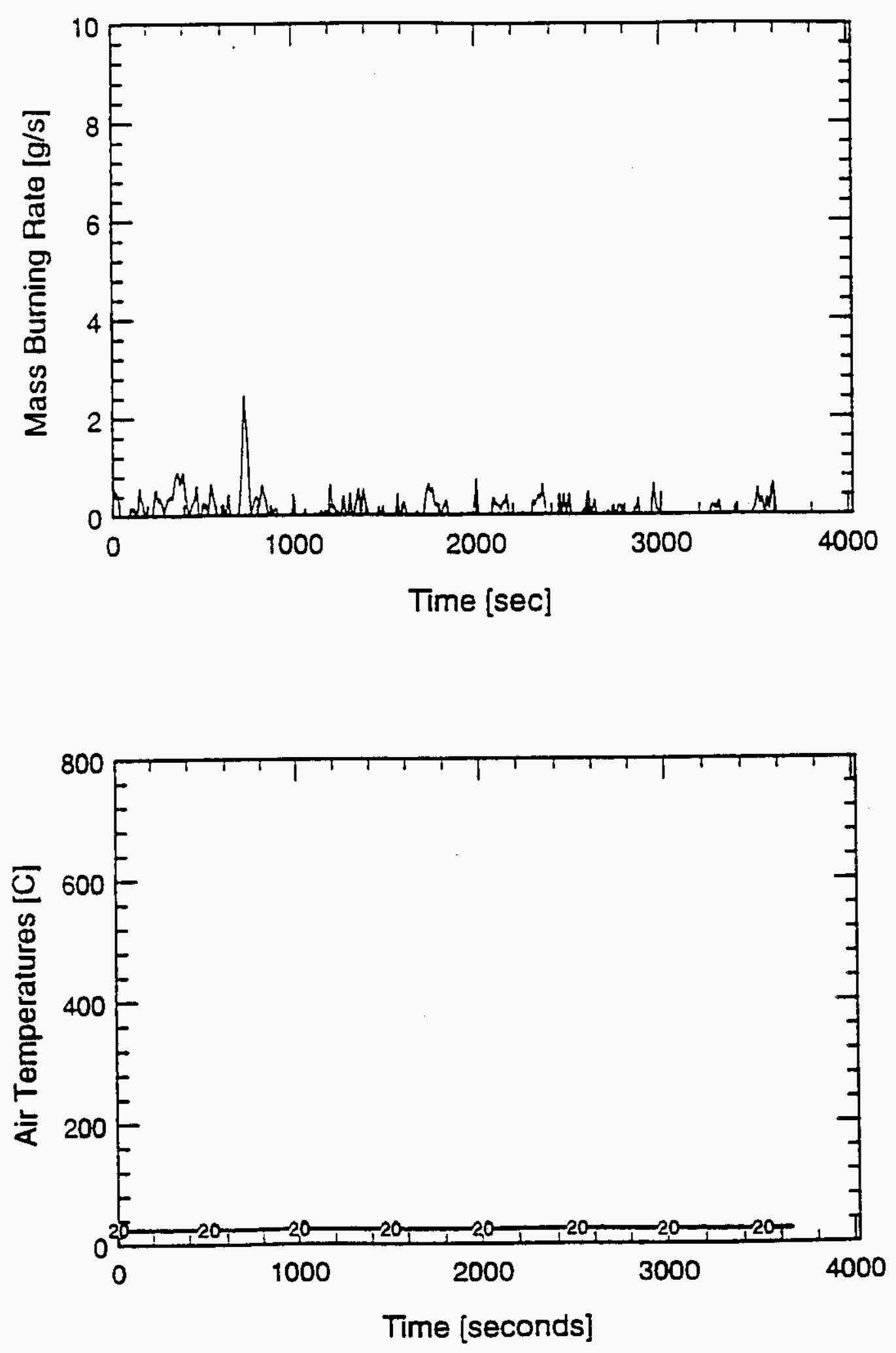


\section{SWD2 - Standard Load with Grate and Angle}
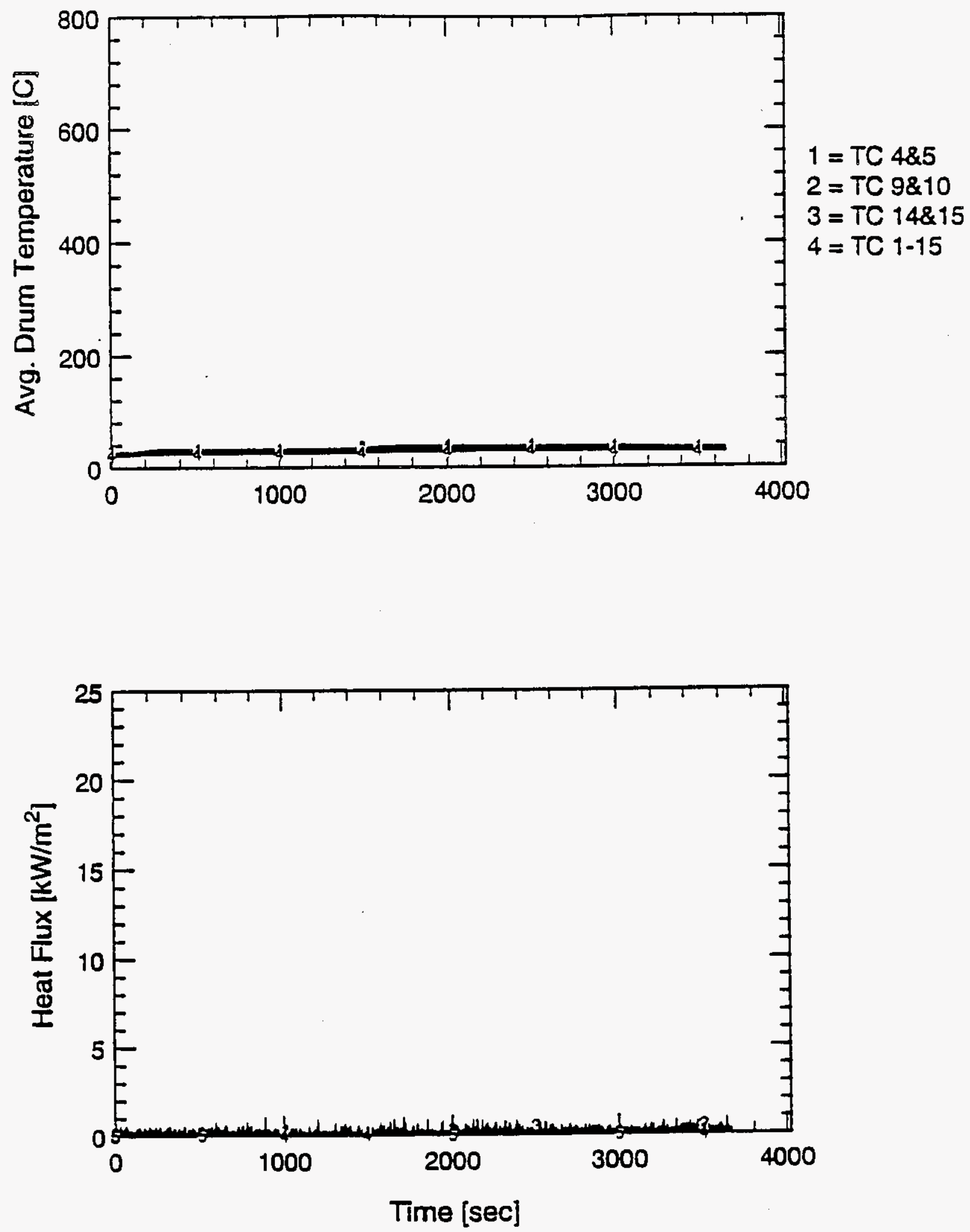
WHC-SD-WM-TRP-233 REV. 0

SWD2 - Standard Load with Grate and Angle
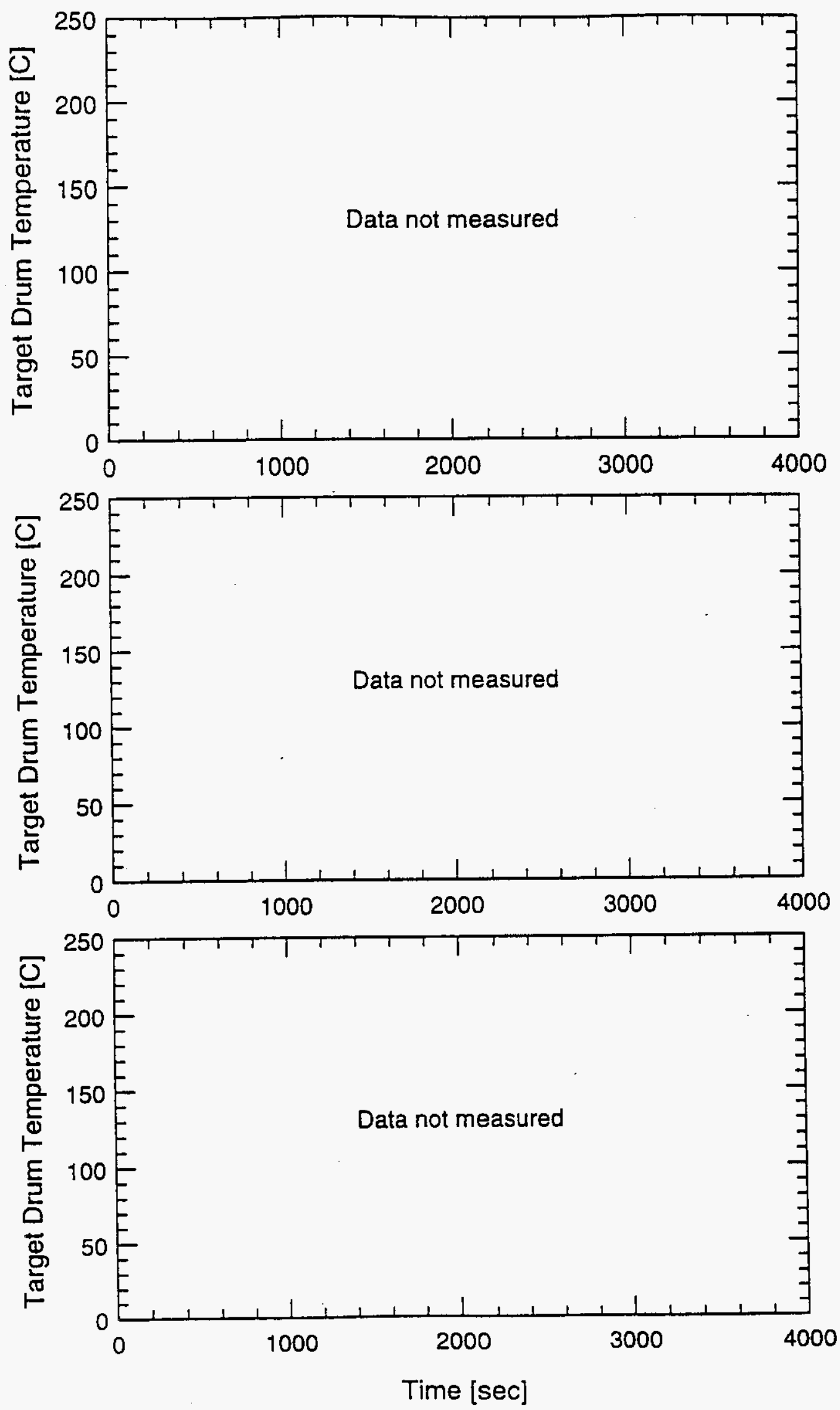

B-12 
WHC-SD-WM-TRP-233 REV. 0

SWD2 - Standard Load with Grate and Angle
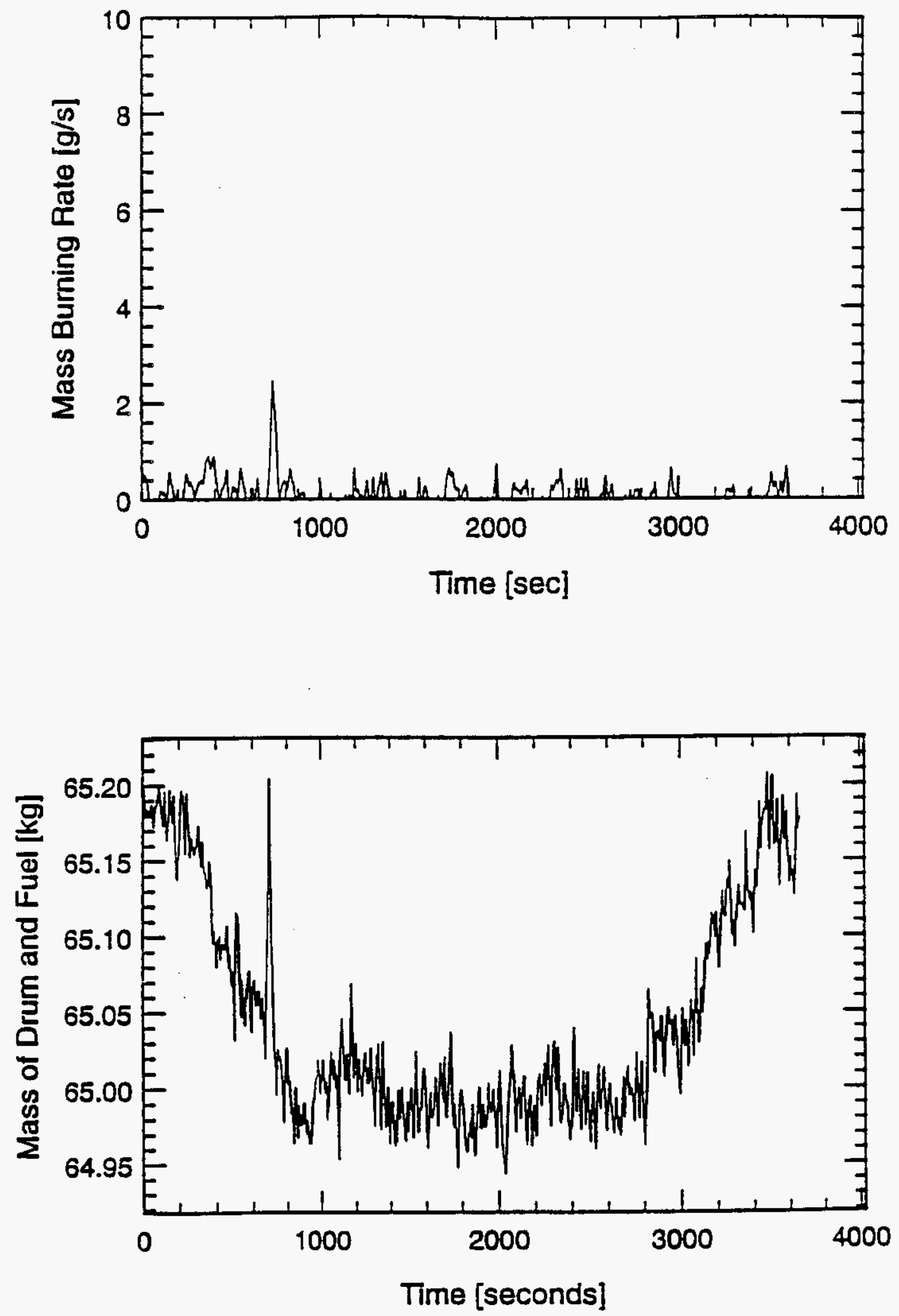
WHC-SD-WM-TRP-233 REV. 0

SWD 3 - DATA 
WHC-SD-WM-TRP-233 REV. 0

SWD3 - 5lb of Brown Paper - Bottom ignition
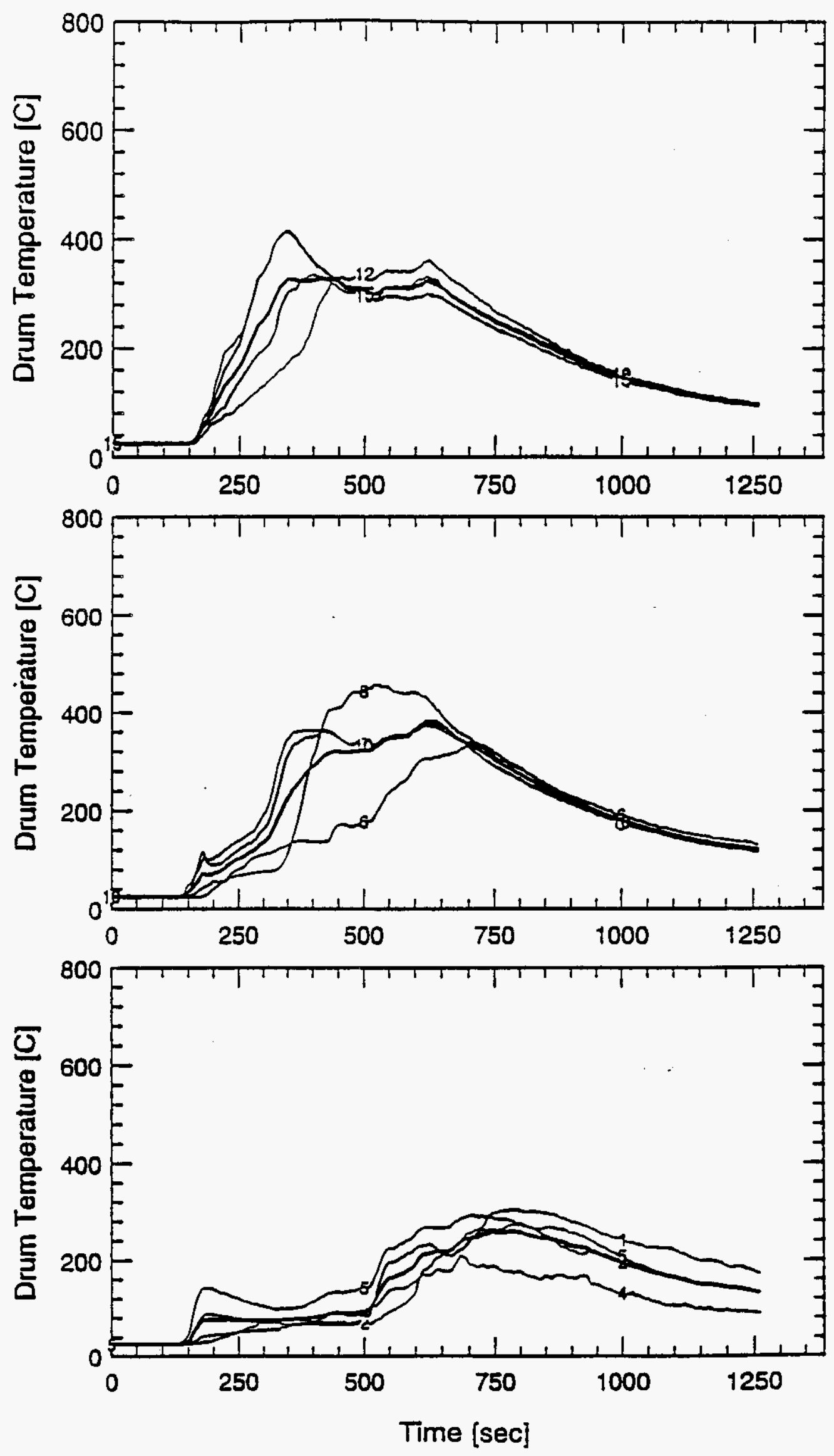

B-15 
WHC-SD-WM-TRP-233 REV. 0

SWD3 - 5lb of Brown Paper - Bottom ignition
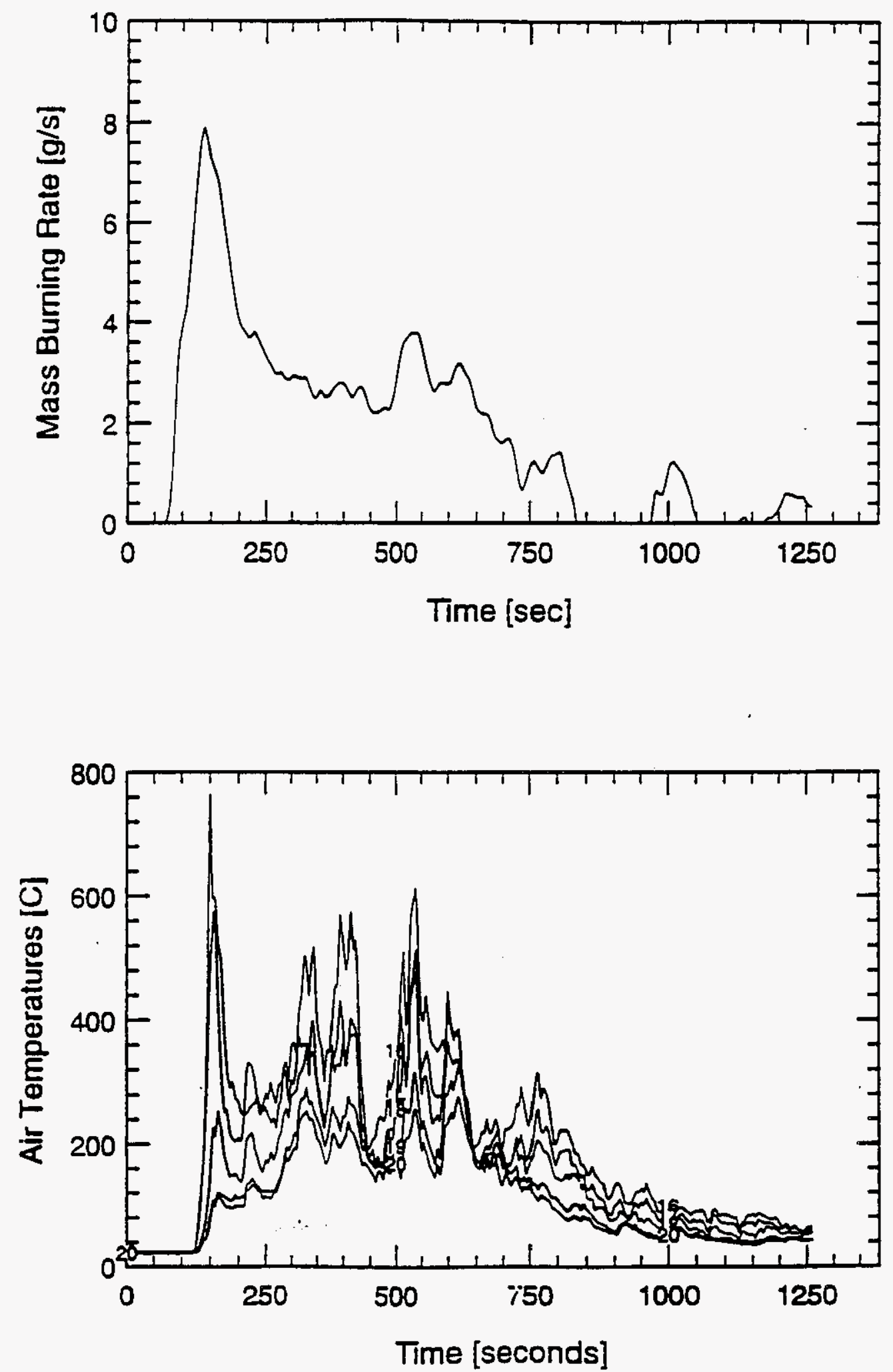

B-16 
SWD3 - 5lb of Brown Paper - Bottom ignition
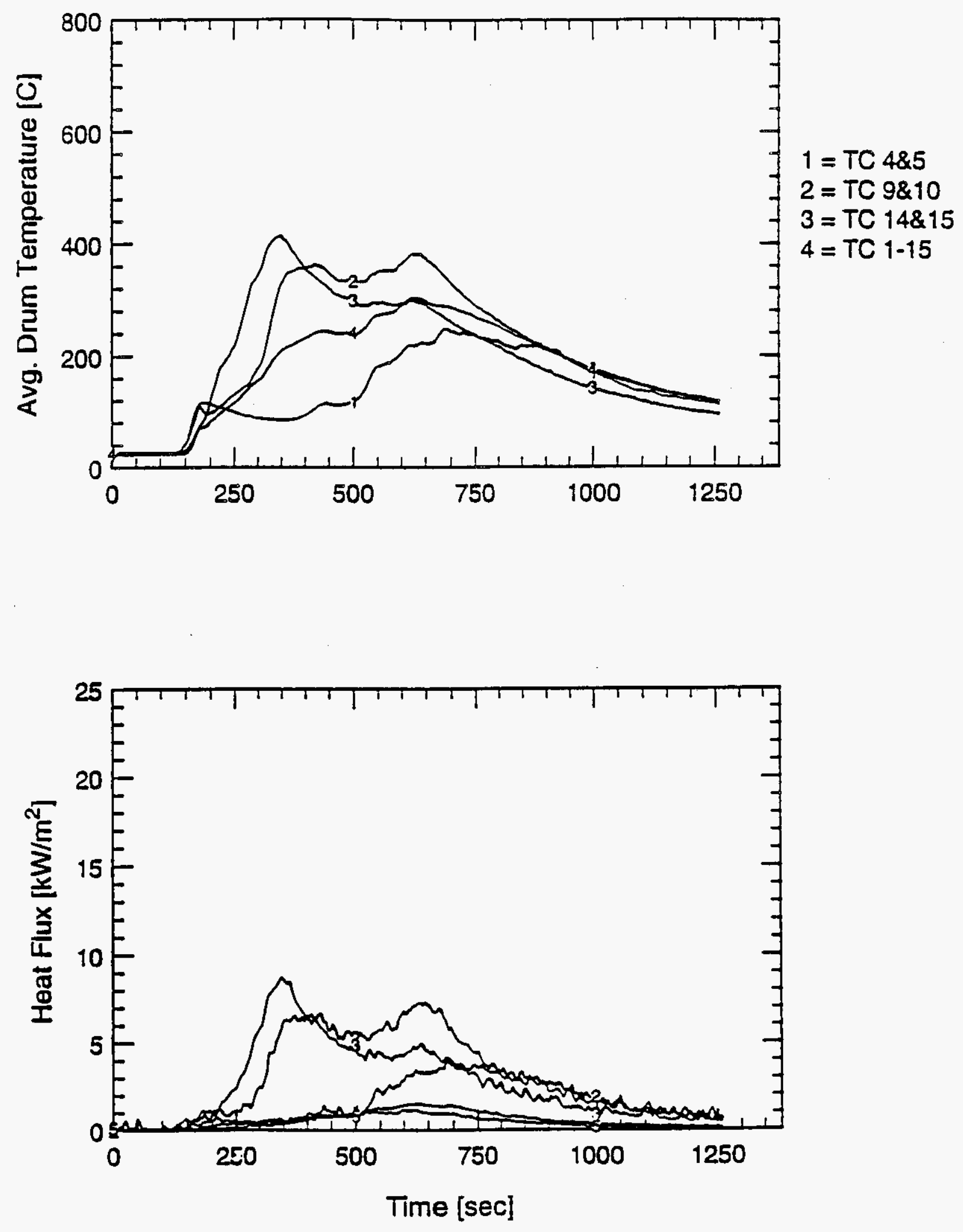
WHC-SD-WM-TRP-233 REV. 0

SWD3 - 5lb of Brown Paper - Bottom ignition
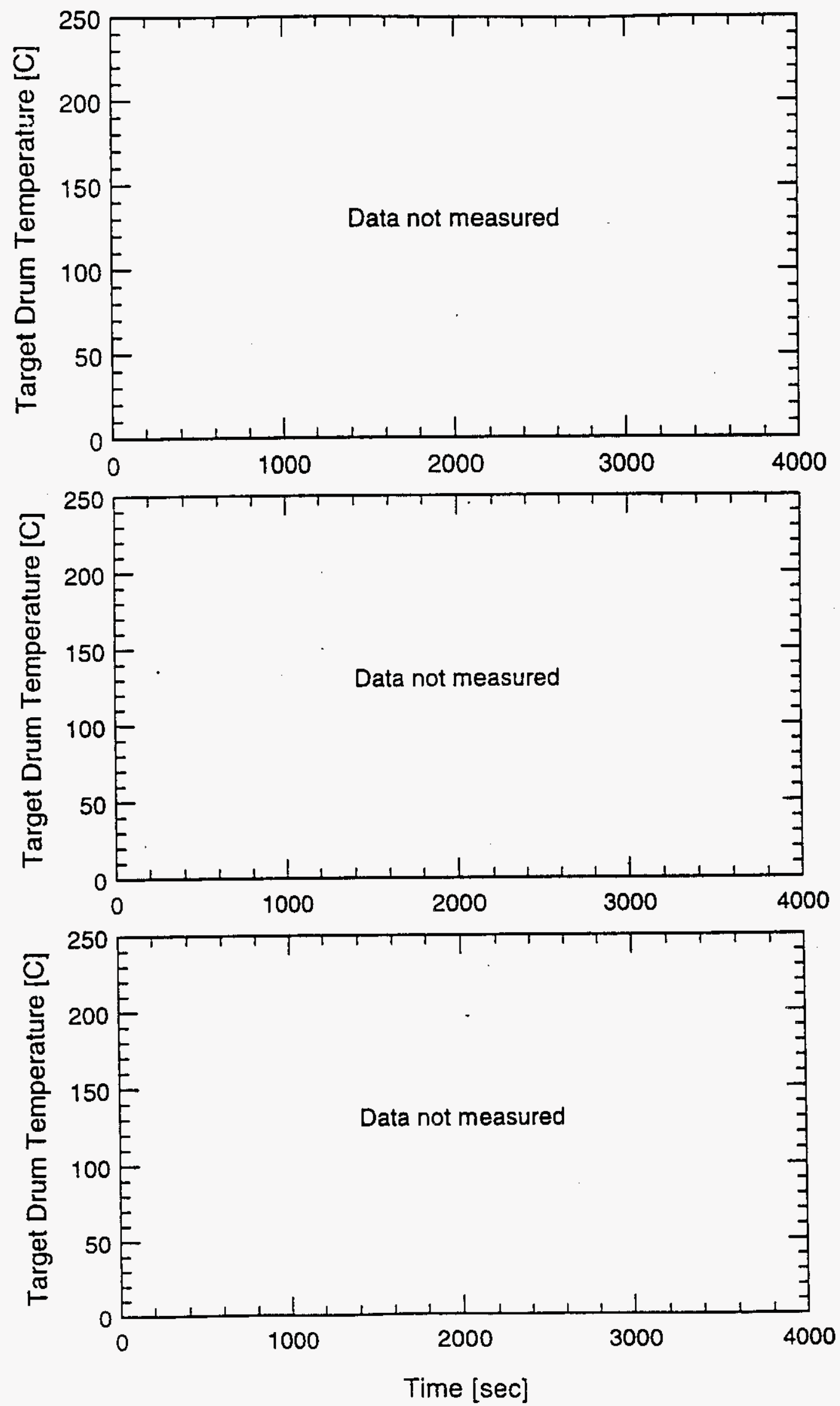

B-18 
SWD3 - 5lb of Brown Paper - Bottom ignition
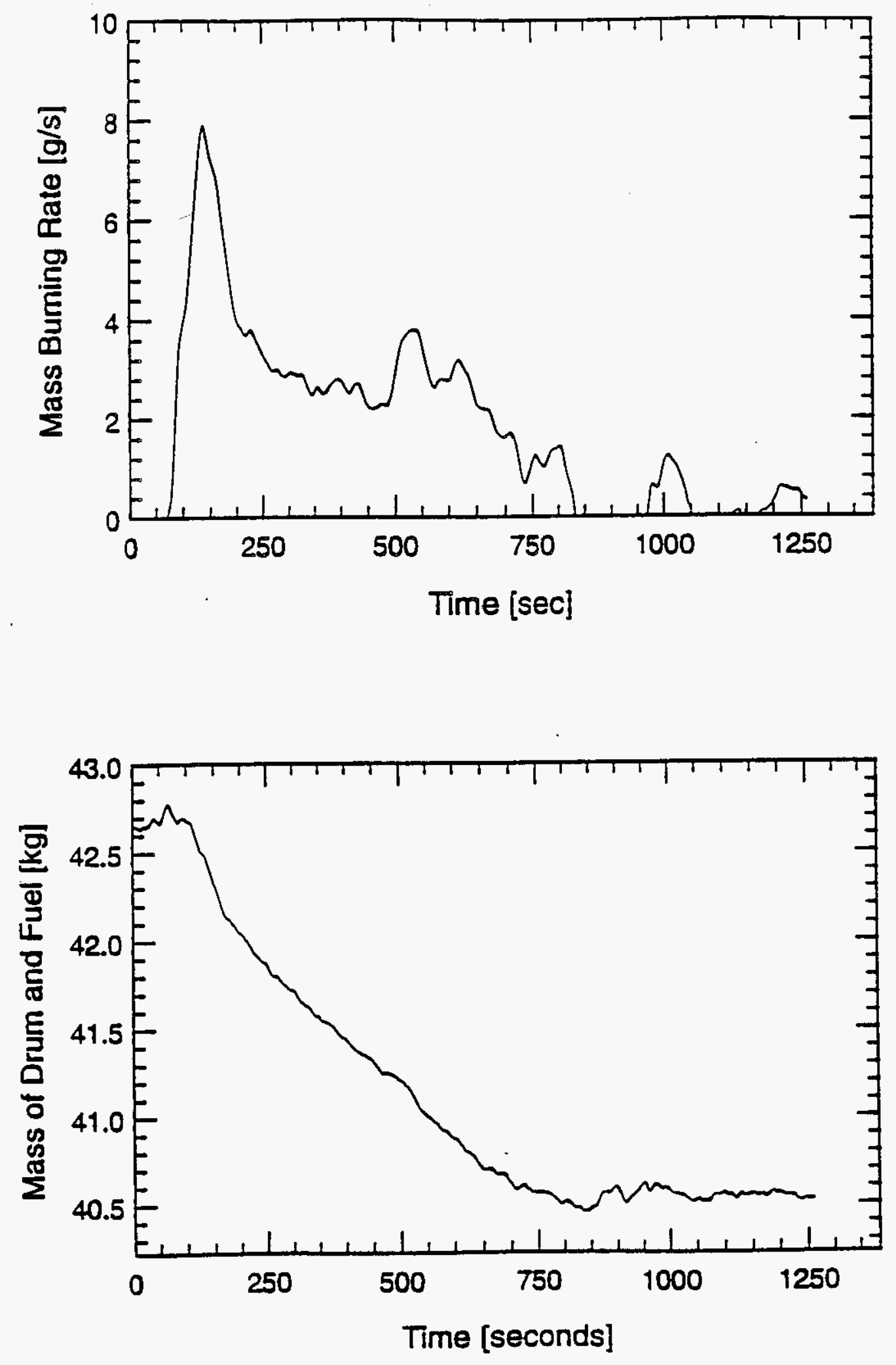
WHC-SD-WM-TRP-233 REV. 0

SWD 4 - DATA

B-20 
WHC-SD-WM-TRP-233 REV. O

SWD4 - Standard Load
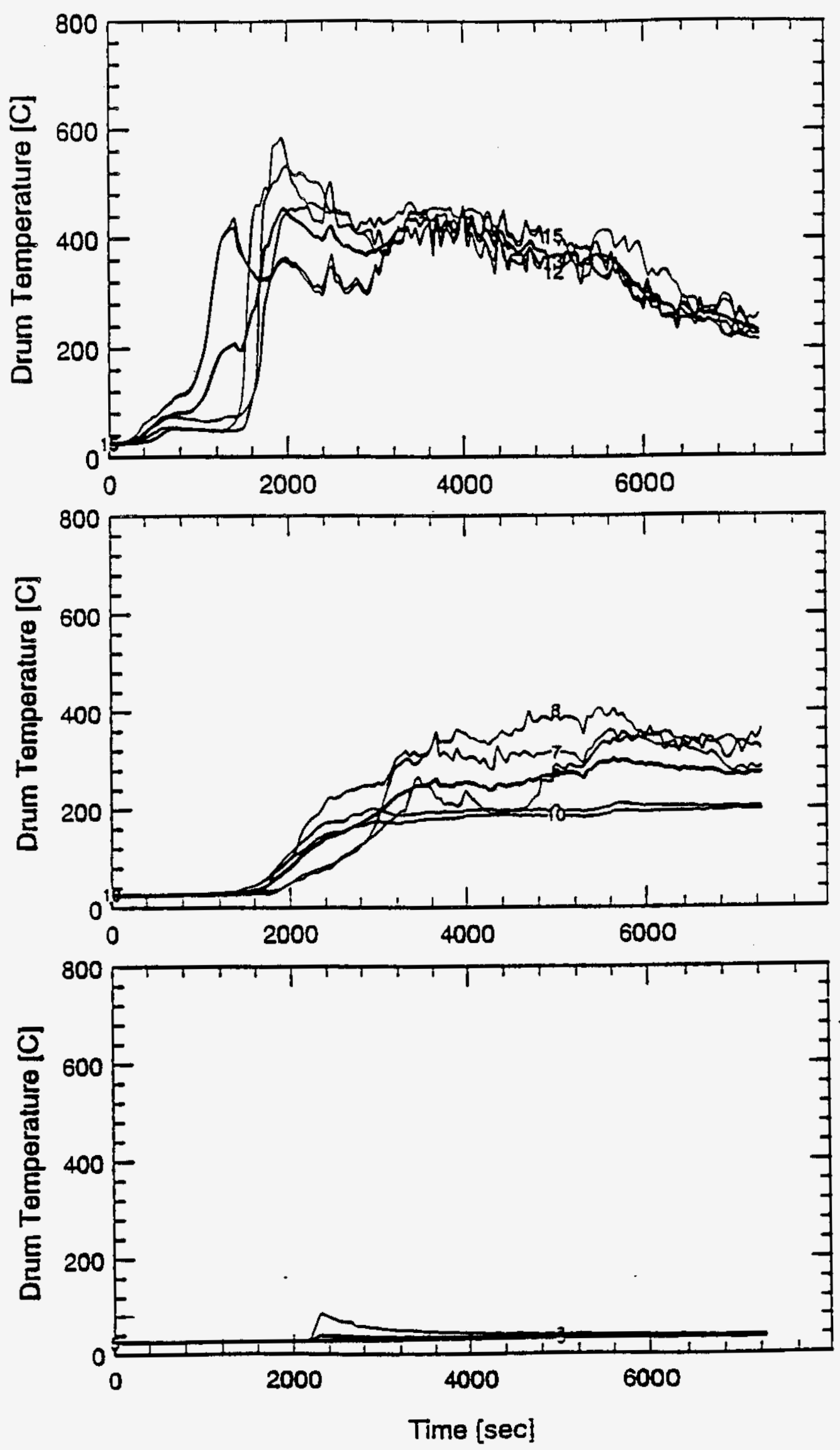
WHC-SD-WM-TRP-233 REV. 0

SWD4 - Standard Load
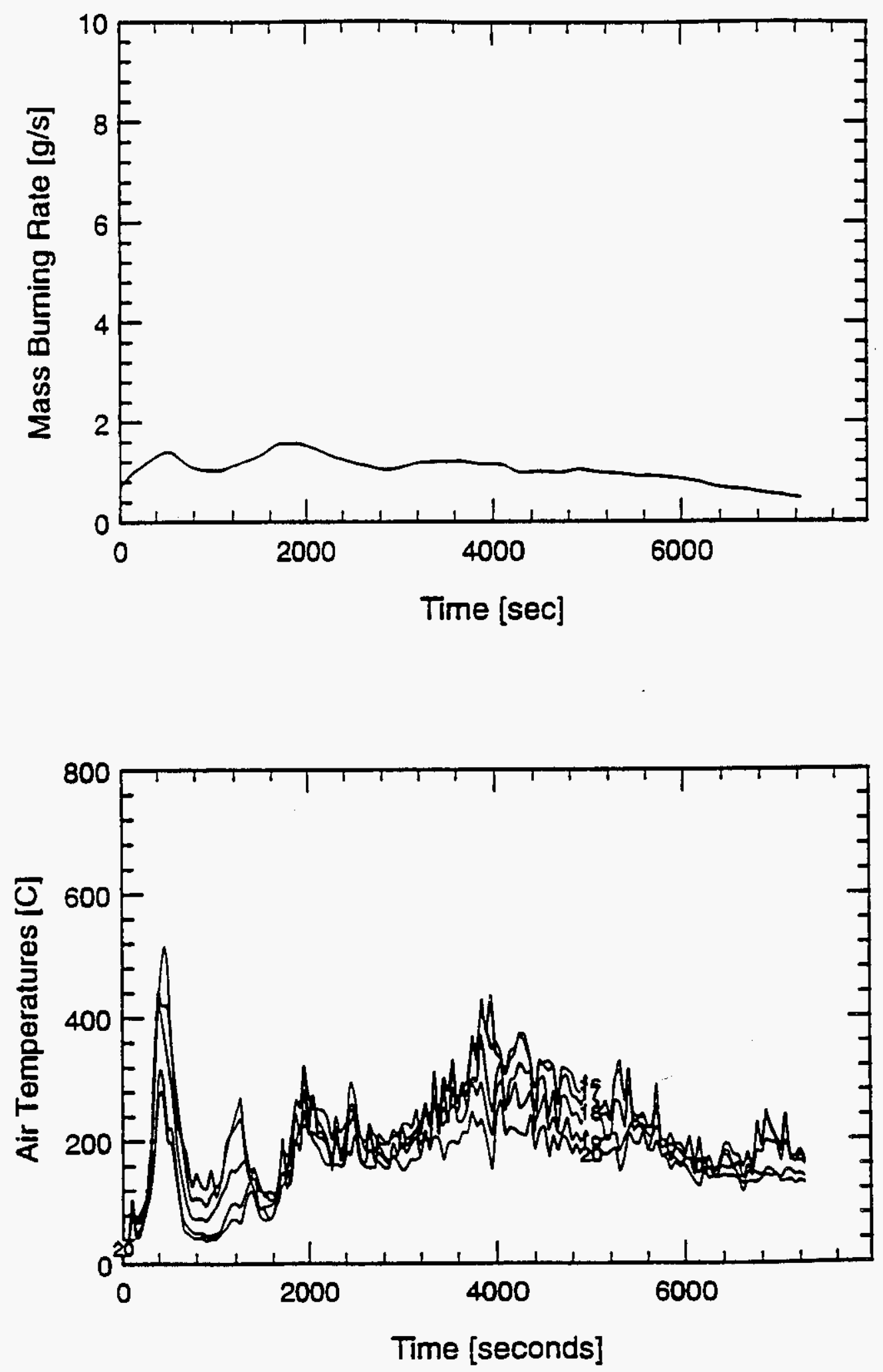
WHC-SD-WM-TRP-233 REV. 0

\section{SWD4 - Standard Load}
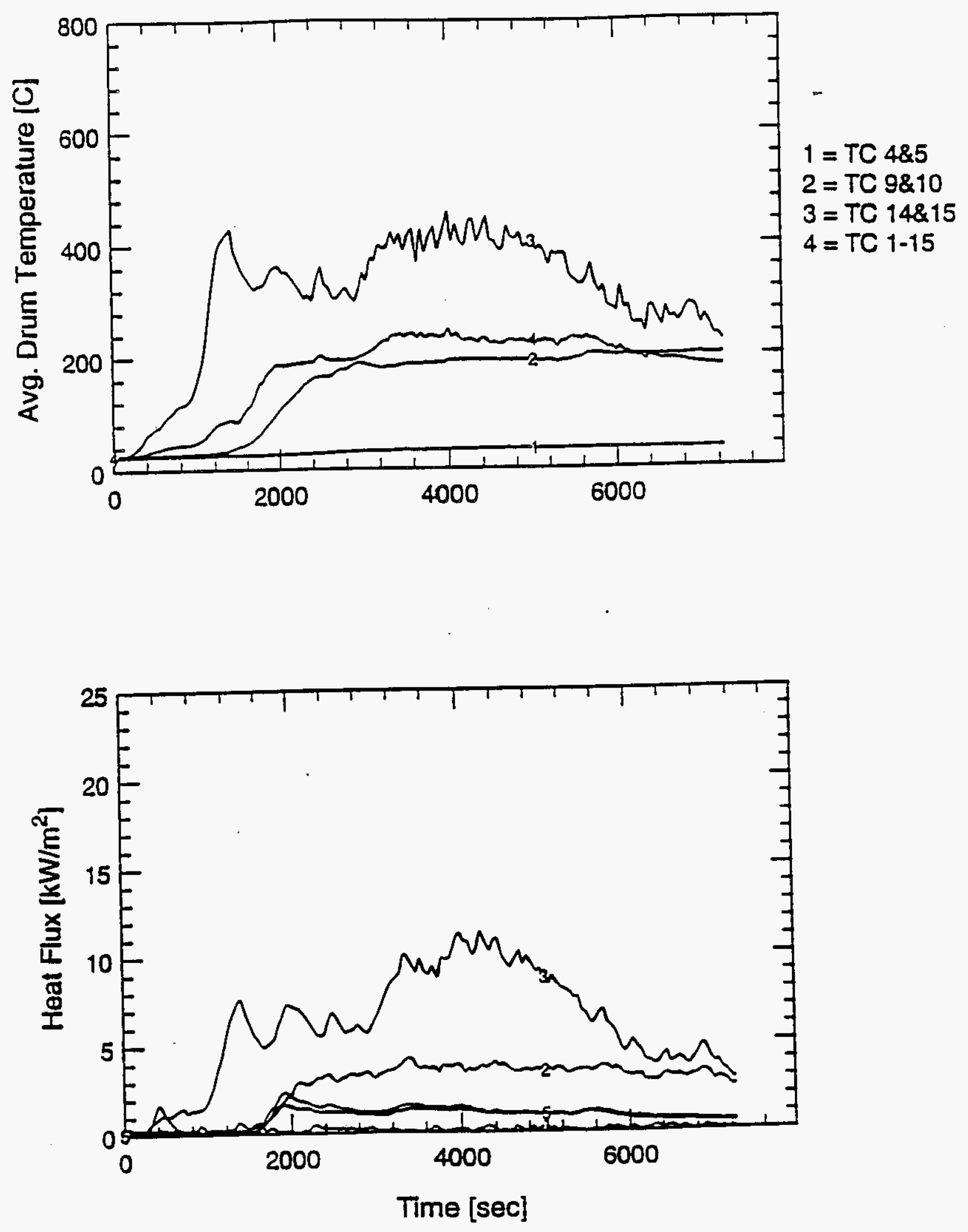
WHC-SD-WM-TRP-233 REV. 0

SWD4 - Standard Load
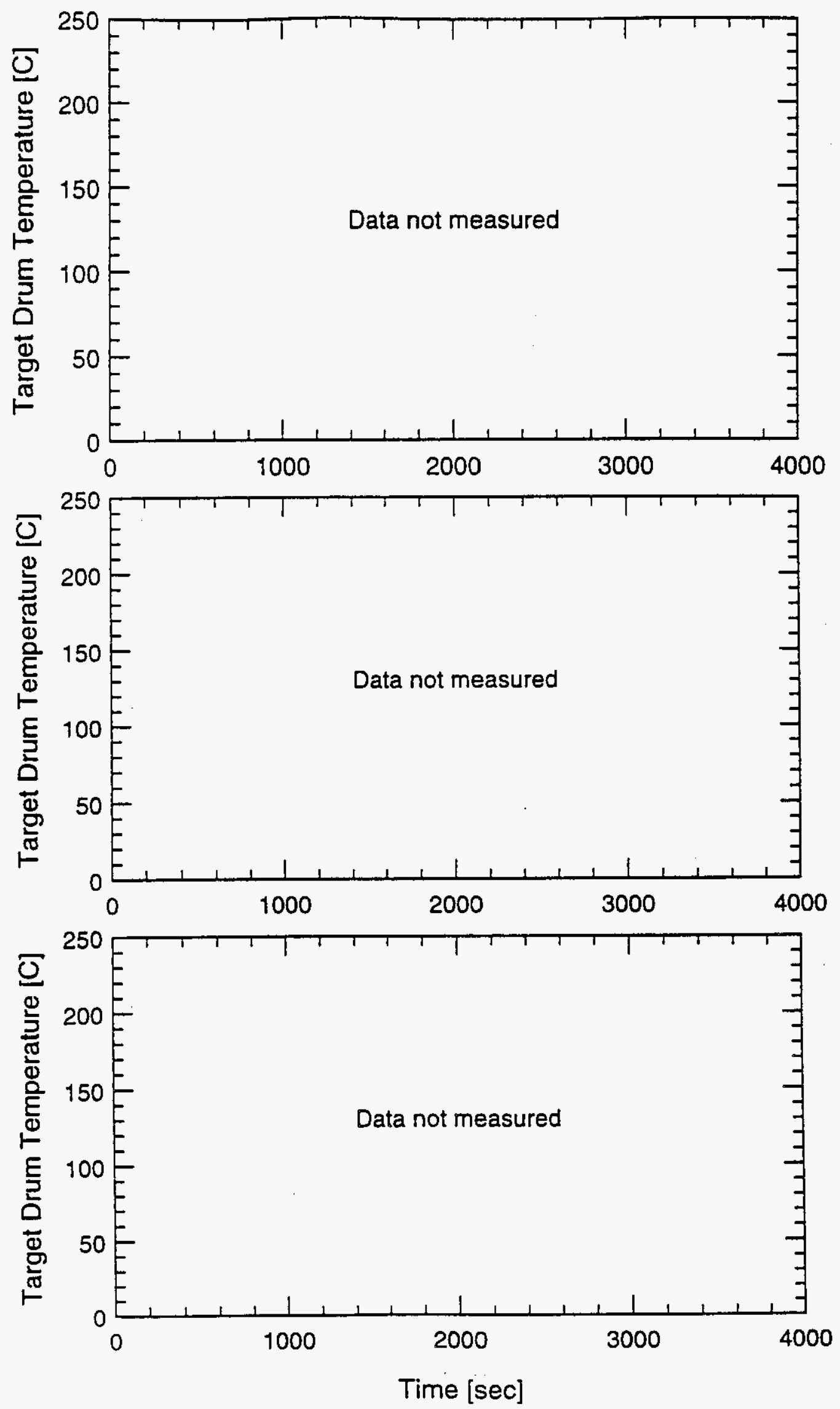
WHC-SD-WM-TRP-233 REV. 0

SWD4 - Standard Load
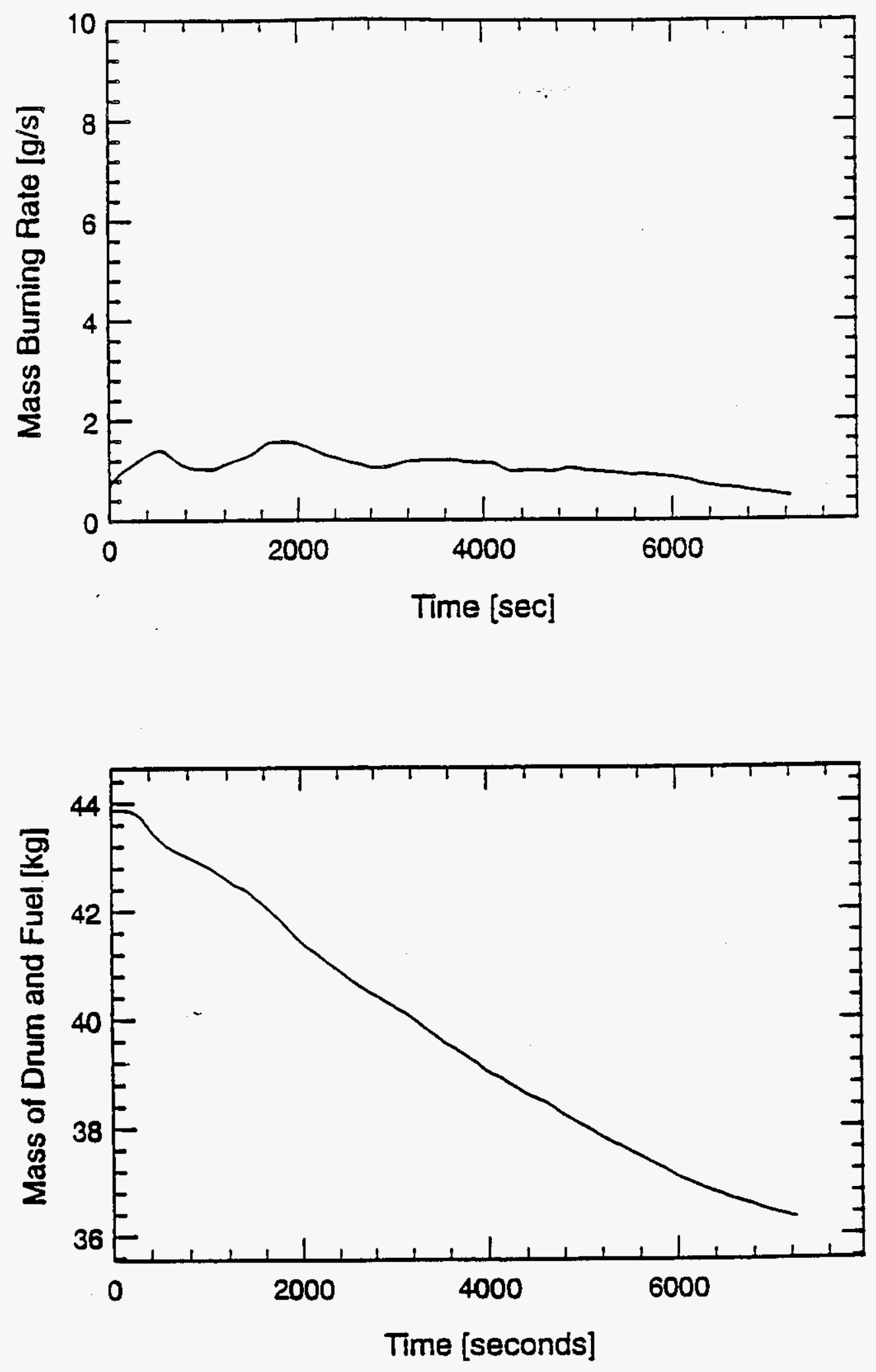

B-25 
WHC-SD-WM-TRP-233 REV. 0

SWD 5 - DATA 
WHC-SD-WM-TRP-233 REV. 0

SWD5 - Kerosene
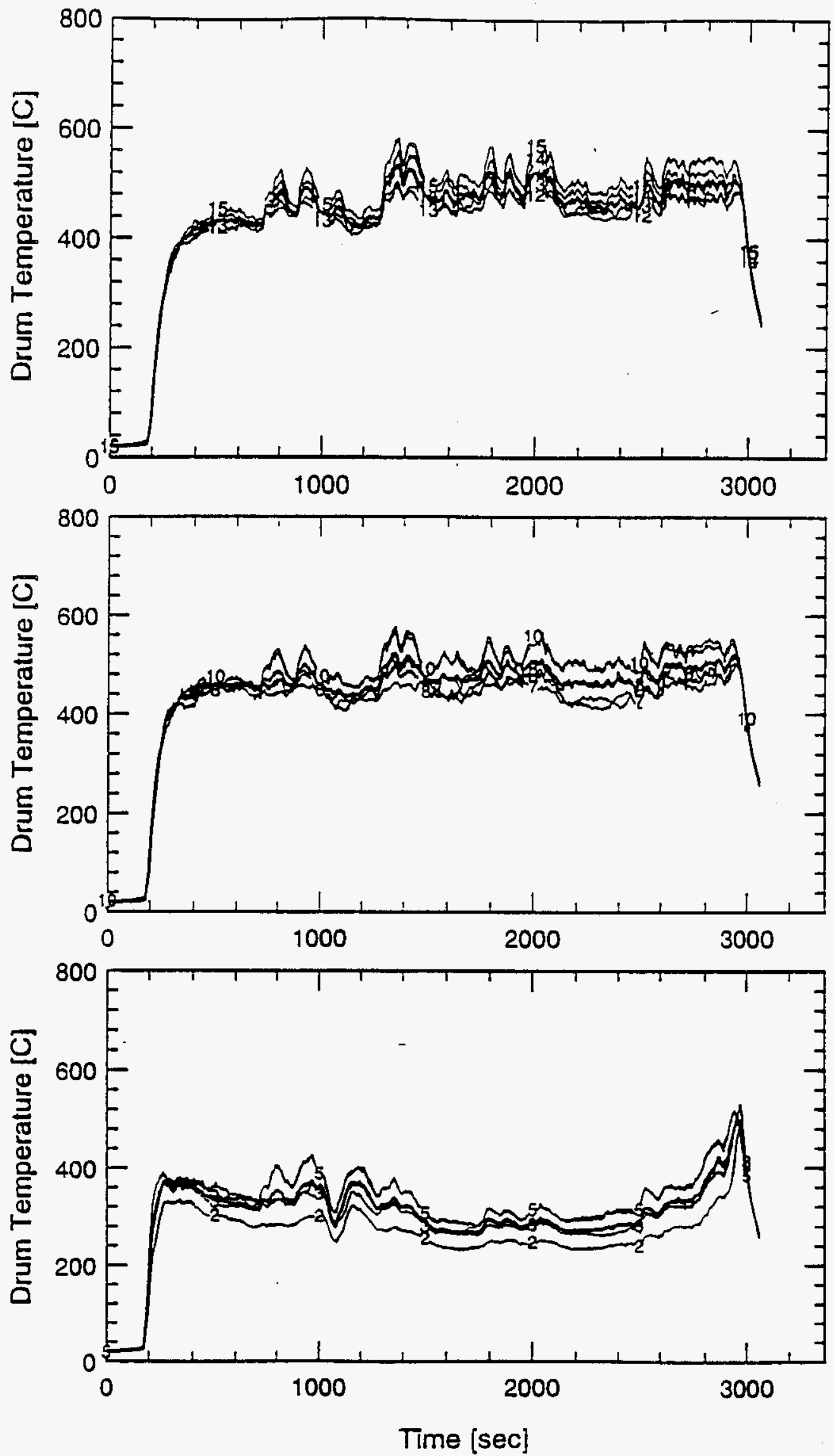
WHC-SD-WM-TRP-233 REV. 0

SWD5 - Kerosene
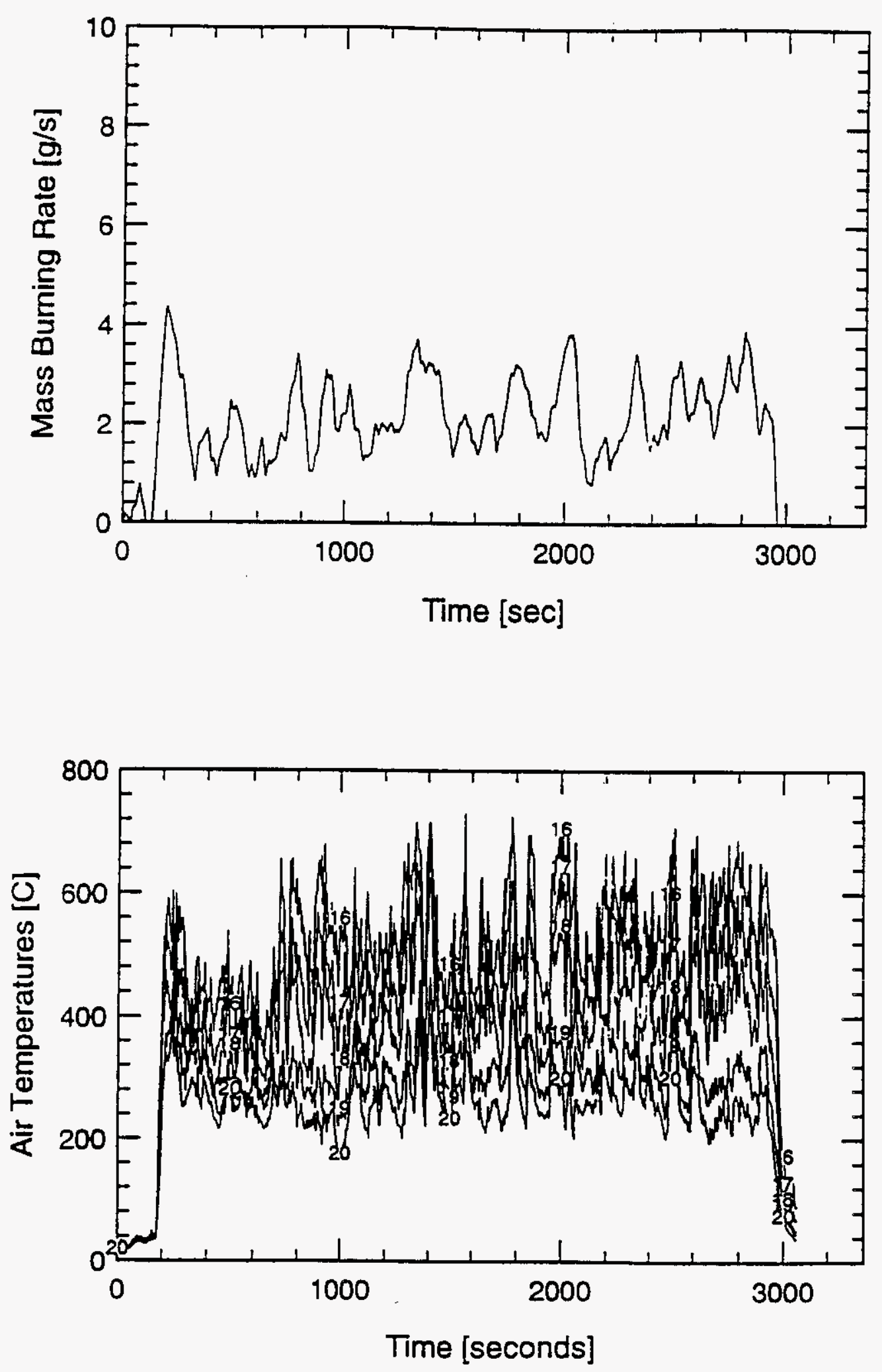
WHC-SD-WM-TRP-233 REV. 0

SWD5 - Kerosene
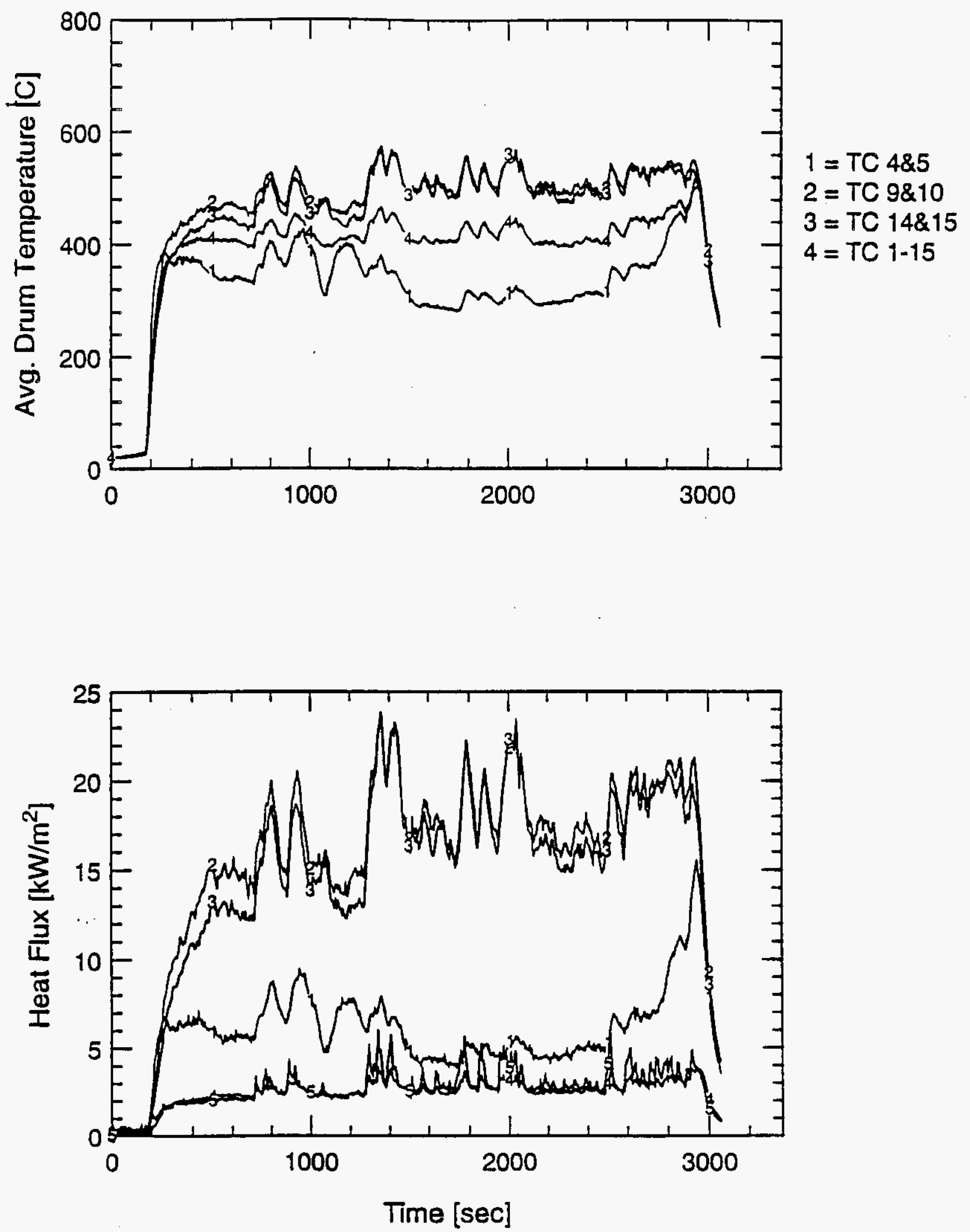
WHC-SD-WM-TRP-233 REV. 0

SWD5 - Kerosene
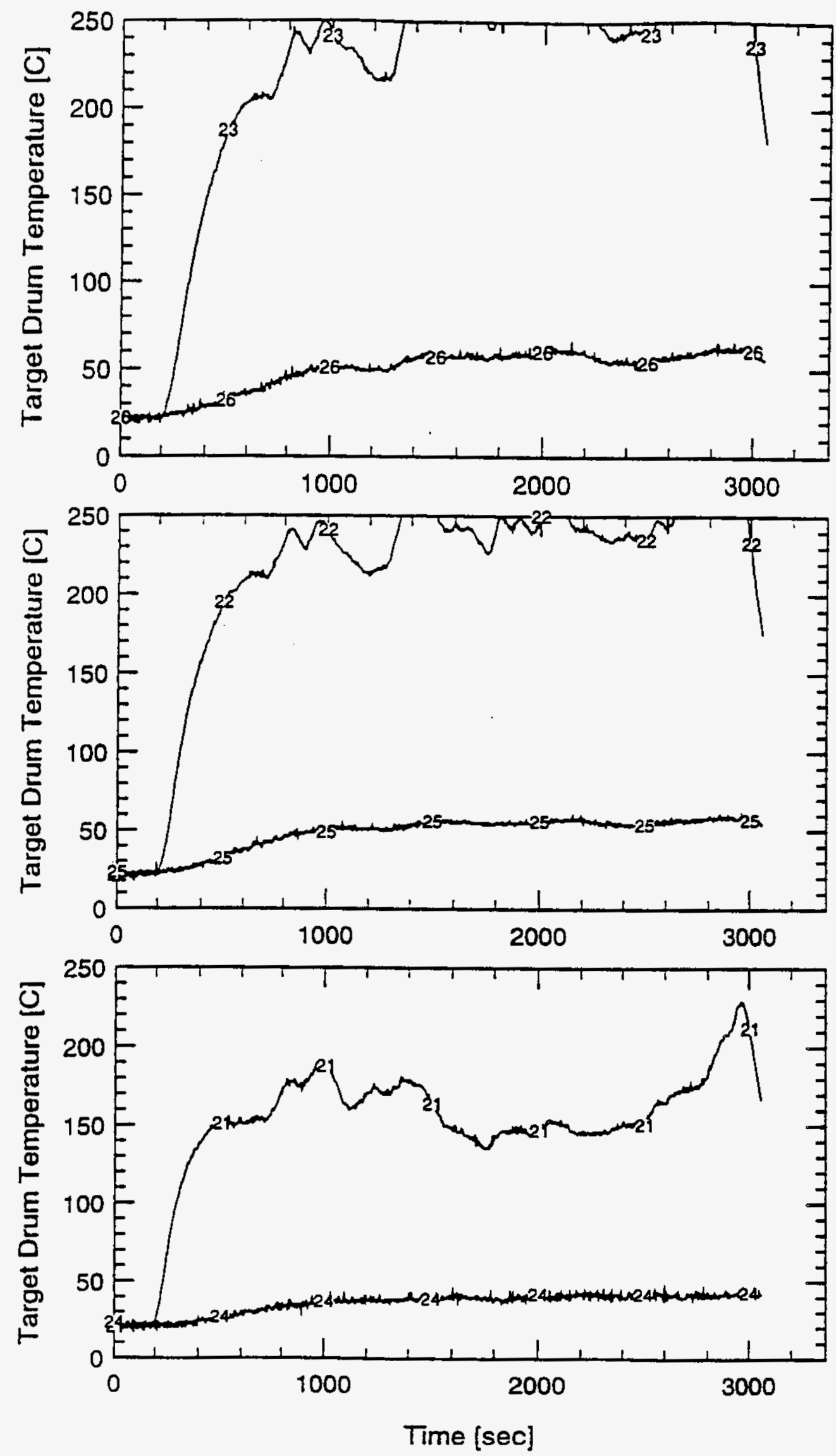

B-30 
WHC-SD-WM-TRP-233 REV. 0

SWD5 - Kerosene
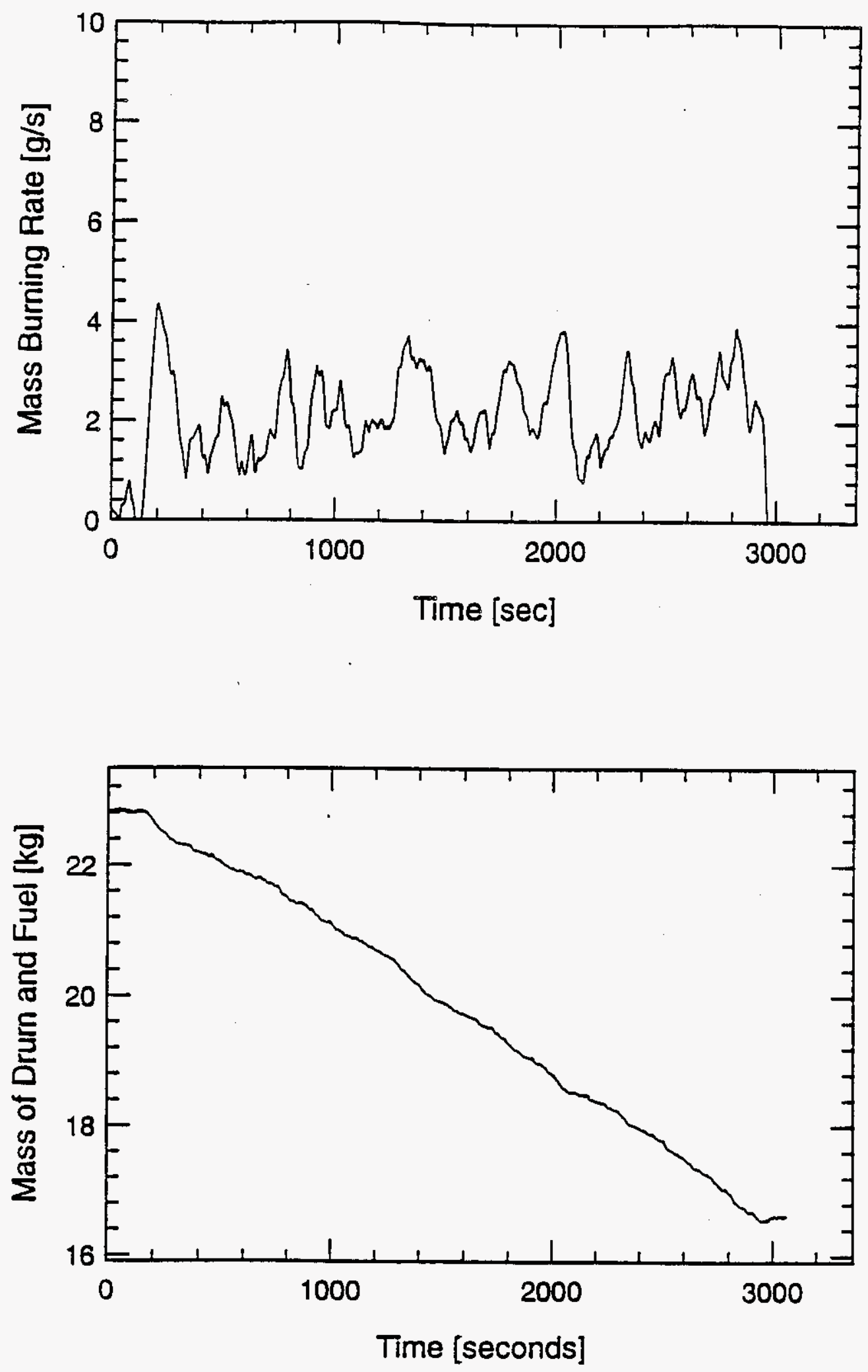
WHC-SD-WM-TRP-233 REV. 0

SWD 6-DATA

B-32 

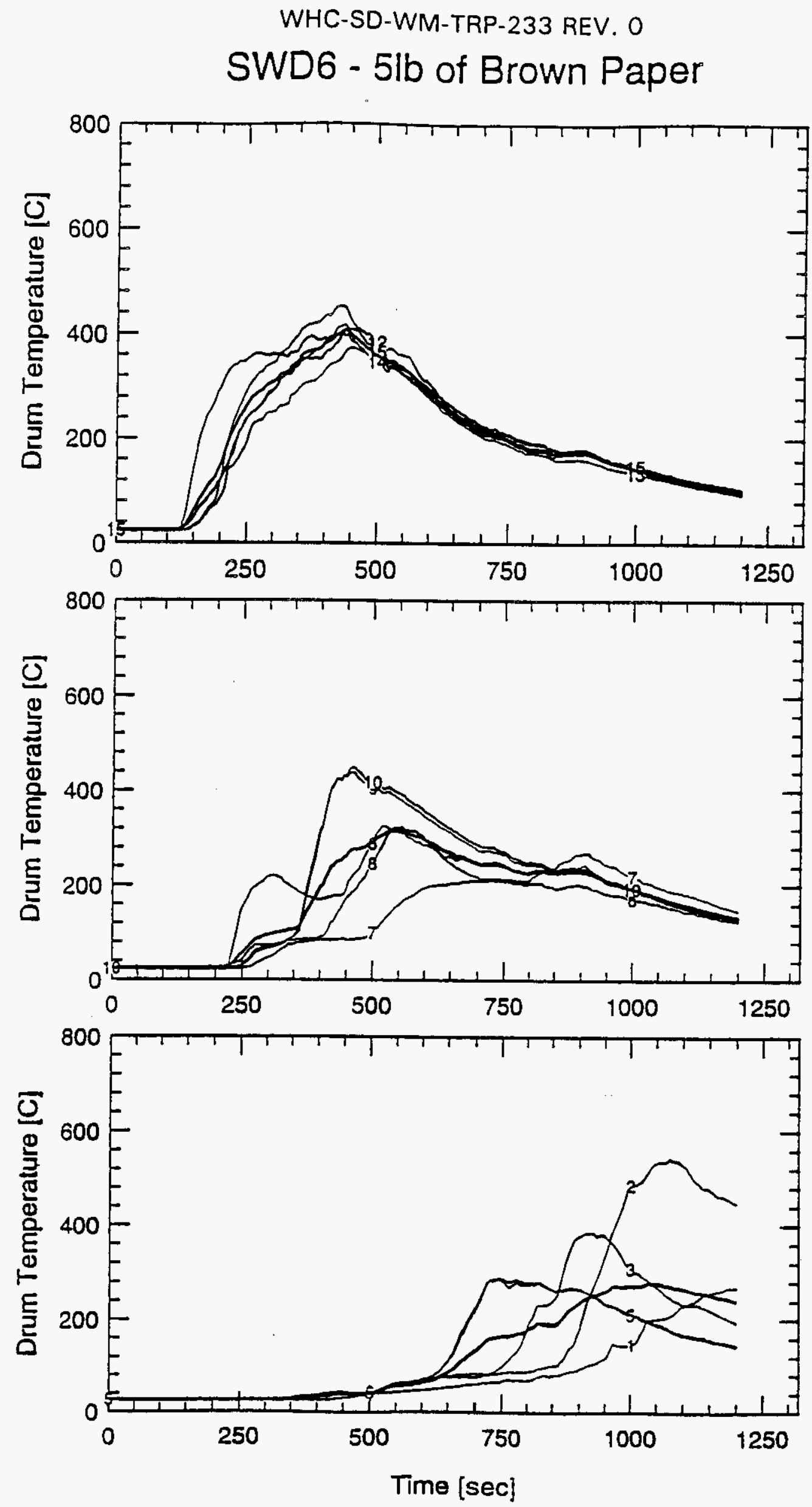

B-33 
WHC-SD-WM-TRP-233 REV. 0

\section{SWD6 - 5lb of Brown Paper}
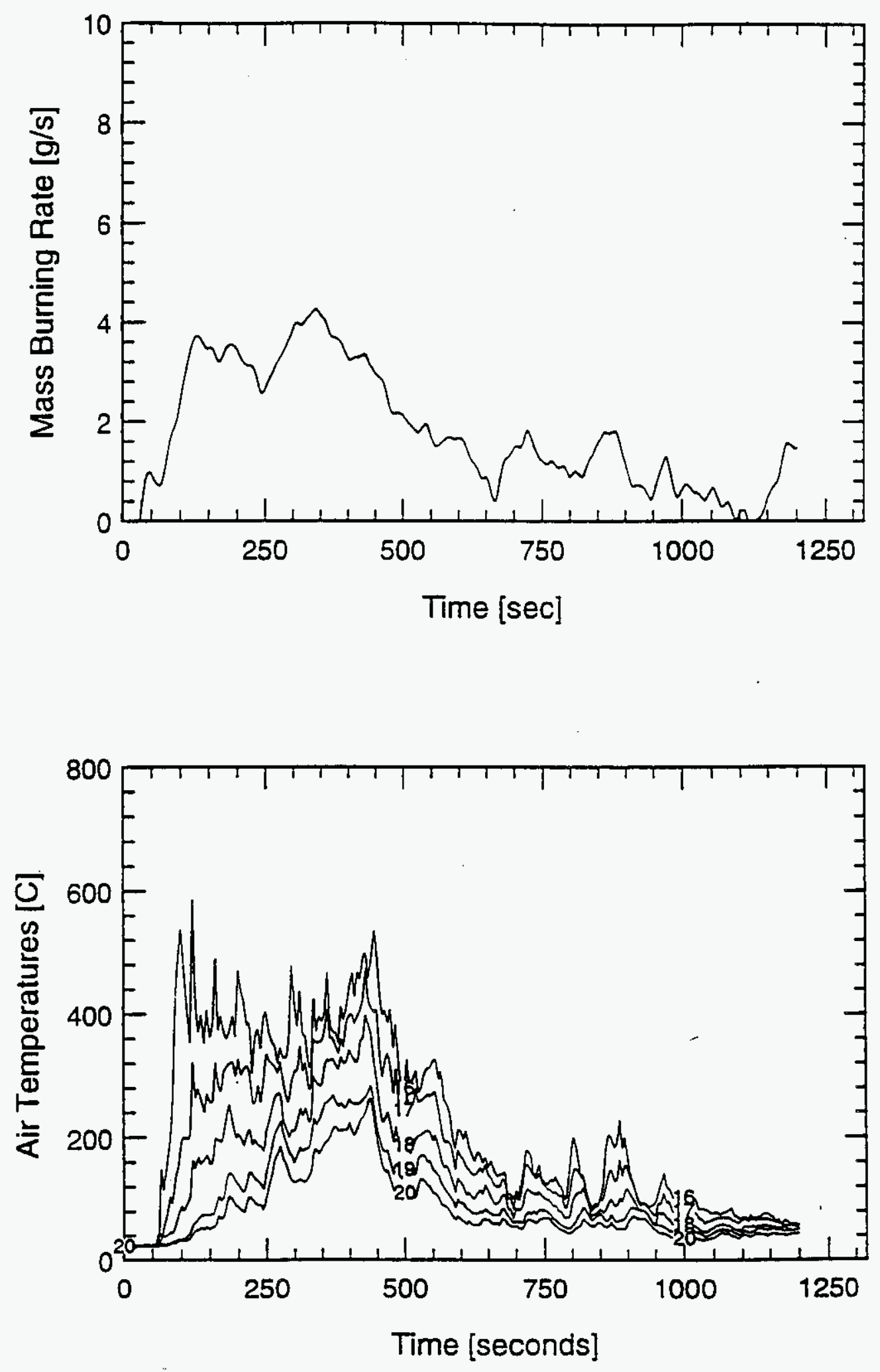


\section{SWD6 - 5lb of Brown Paper}
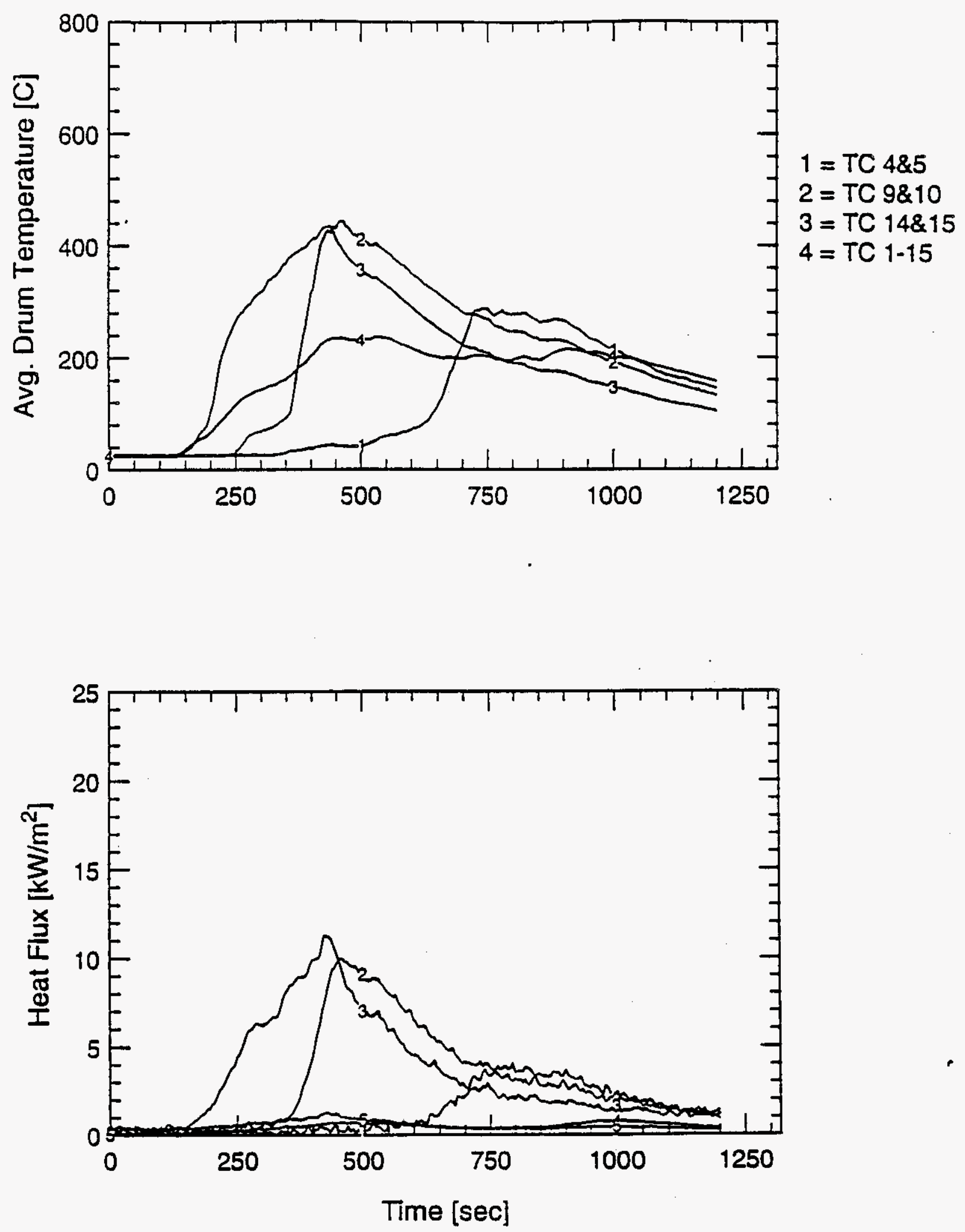
WHC-SD-WM-TRP-233 REV. 0

\section{SWD6 - 5lb of Brown Paper}
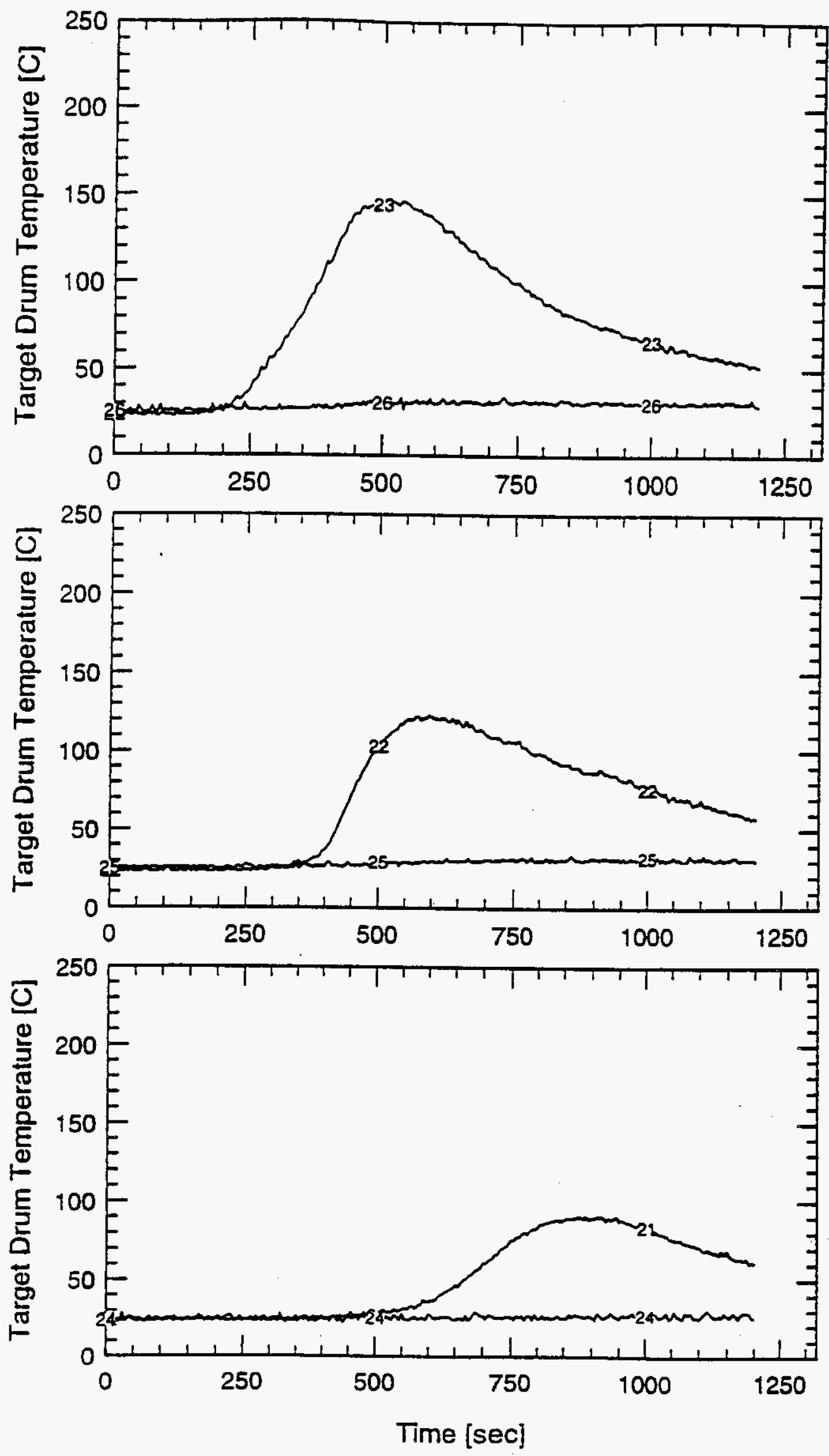
WHC-SD-WM-TRP-233 REV. 0
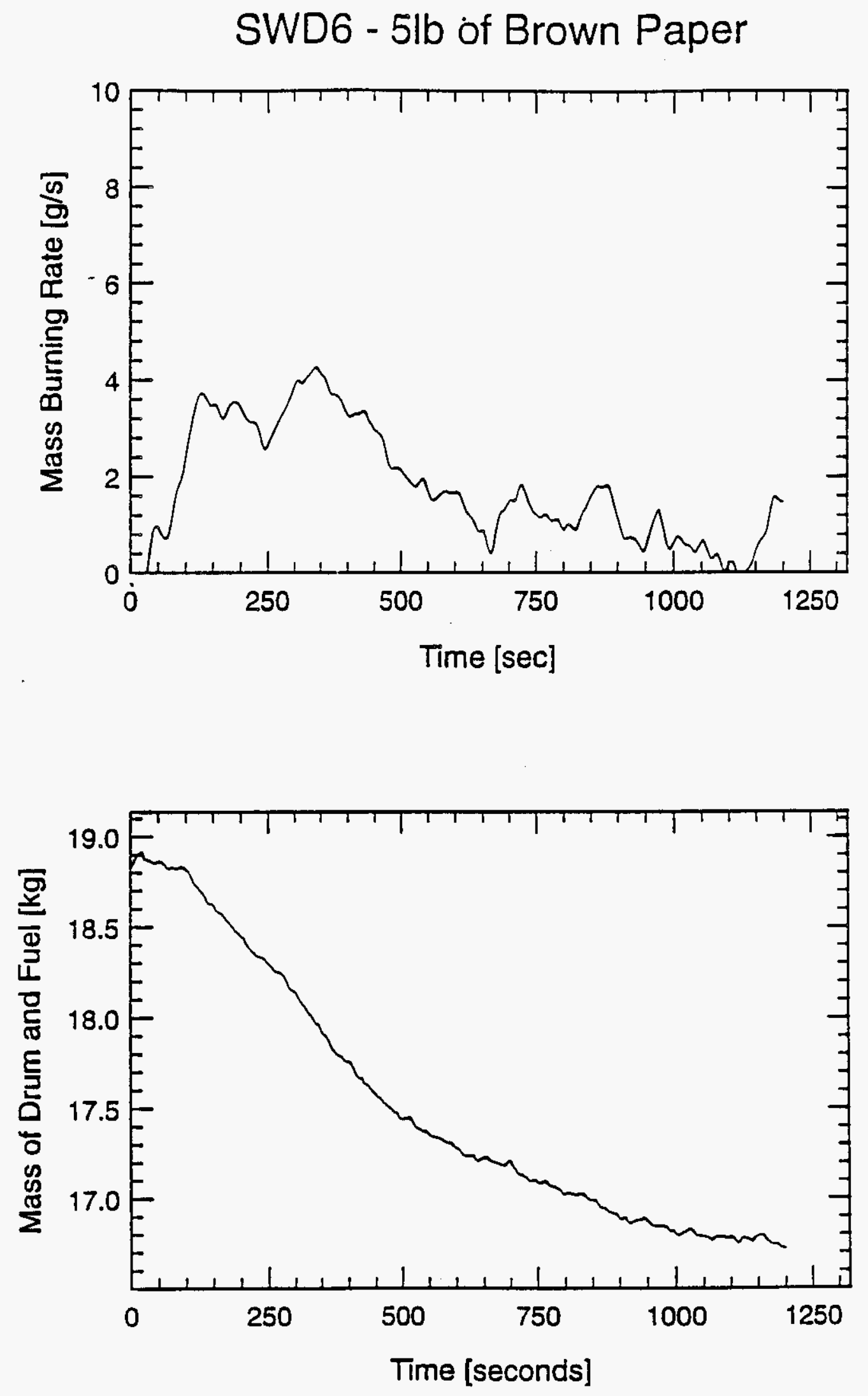
WHC-SD-WM-TRP-233 REV. 0

SWD 7 - DATA

B-38 
WHC-SD-WM-TRP-233 REV. 0

SWD7 - 15lb of Brown Paper
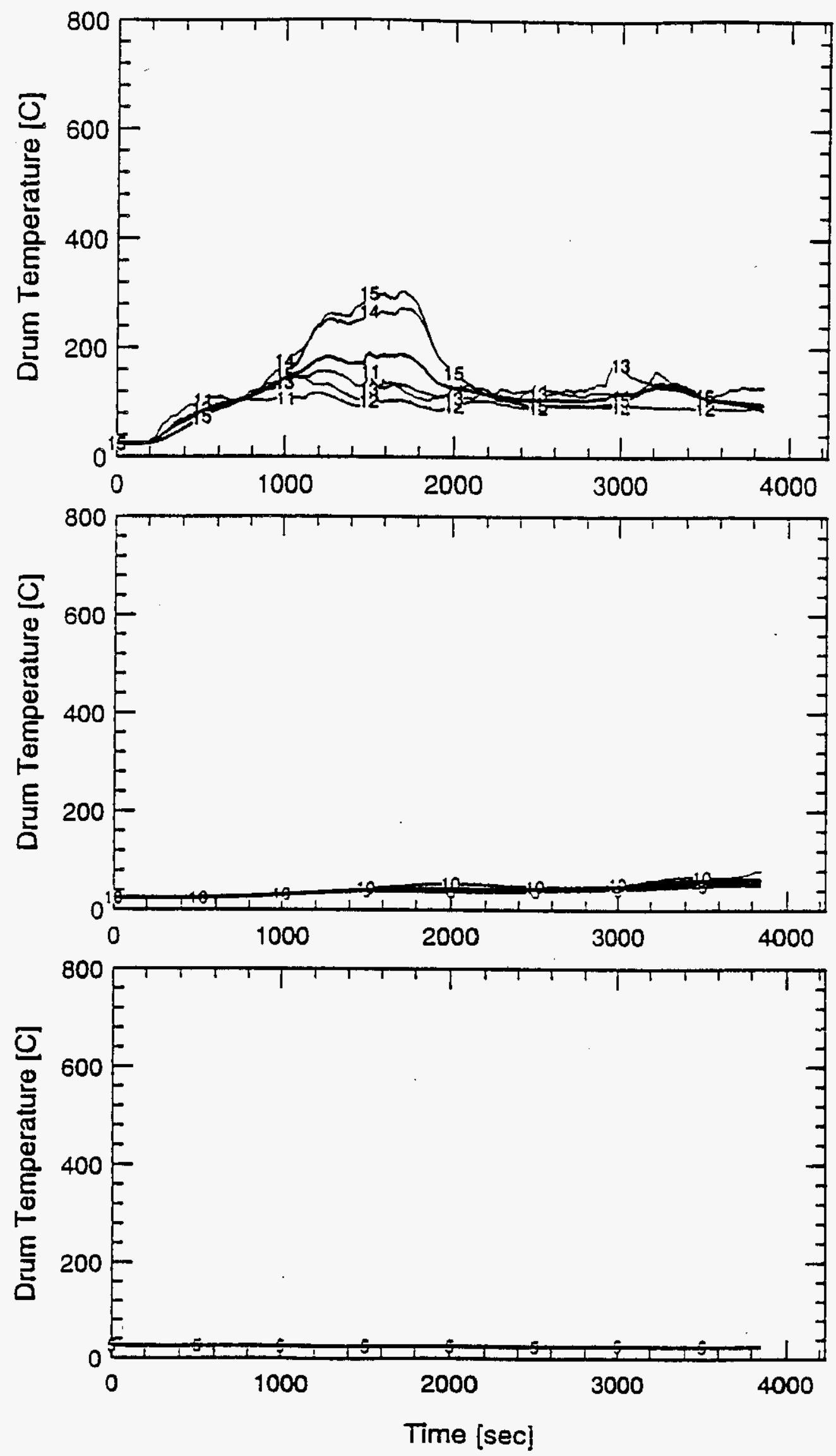


\section{SWD7 - 15lb of Brown Paper}
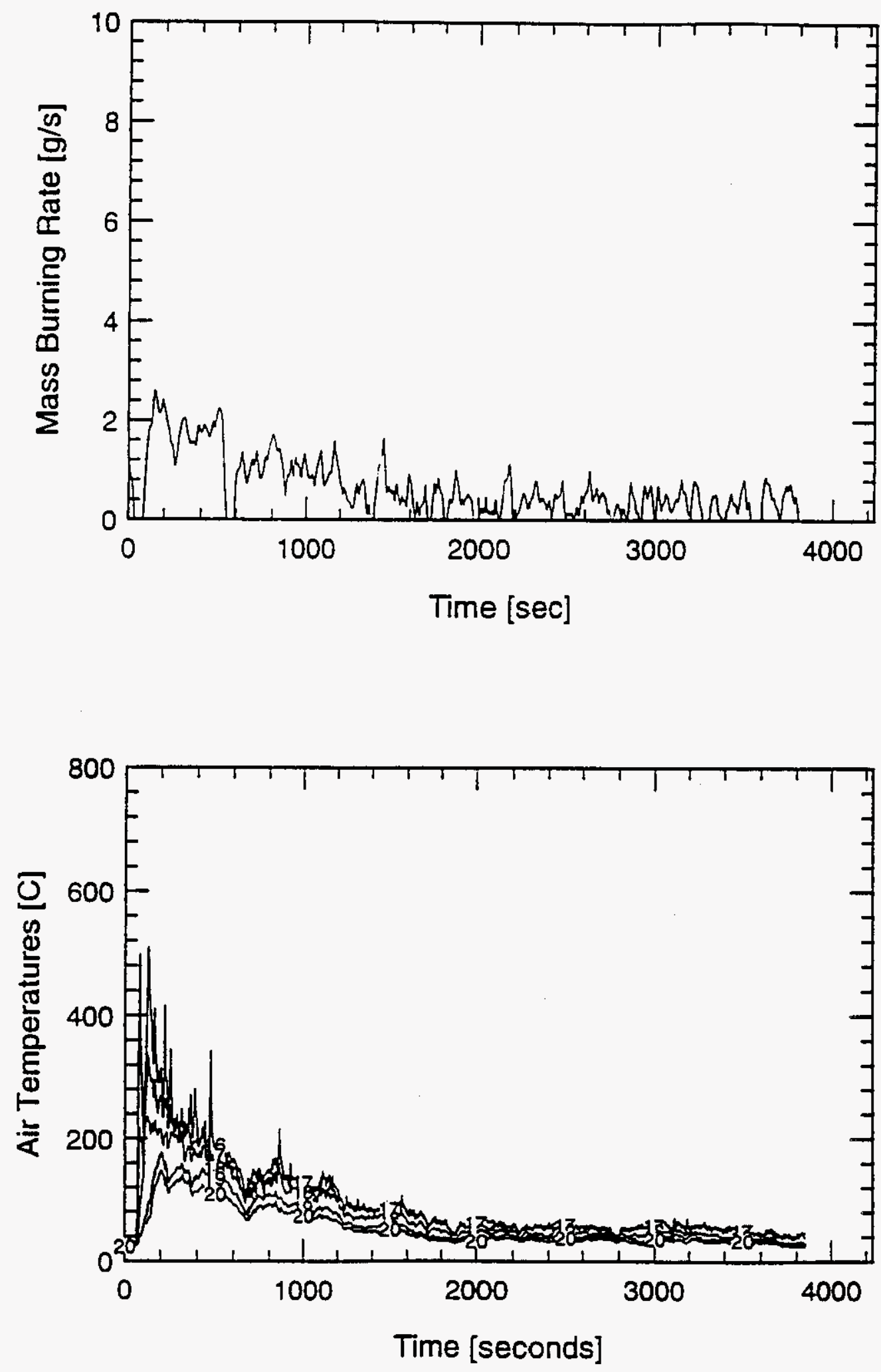
SWD7 - 15lb of Brown Paper
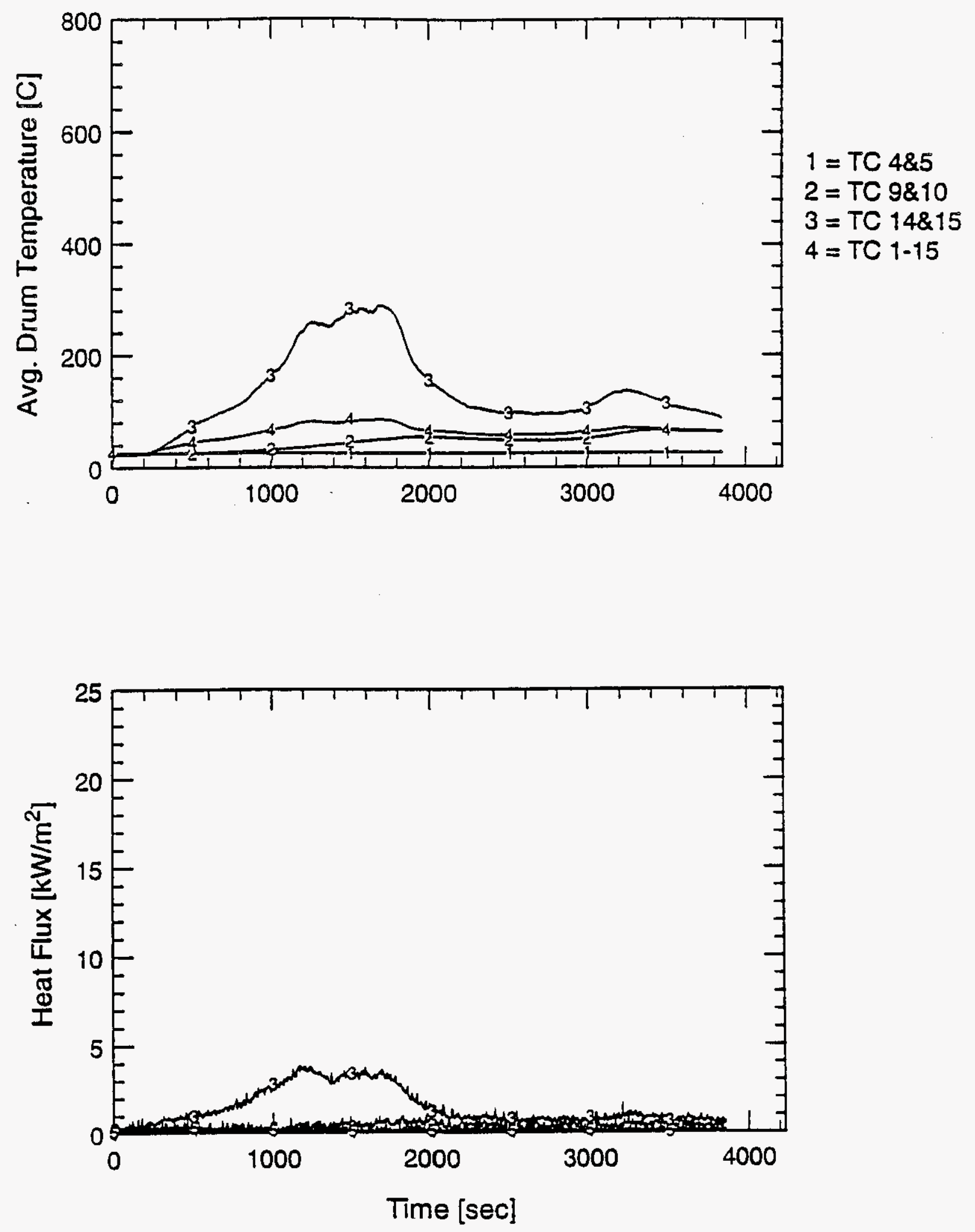
WHC-SD-WM-TRP-233 REV. 0

SWD7 - 15lb of Brown Paper
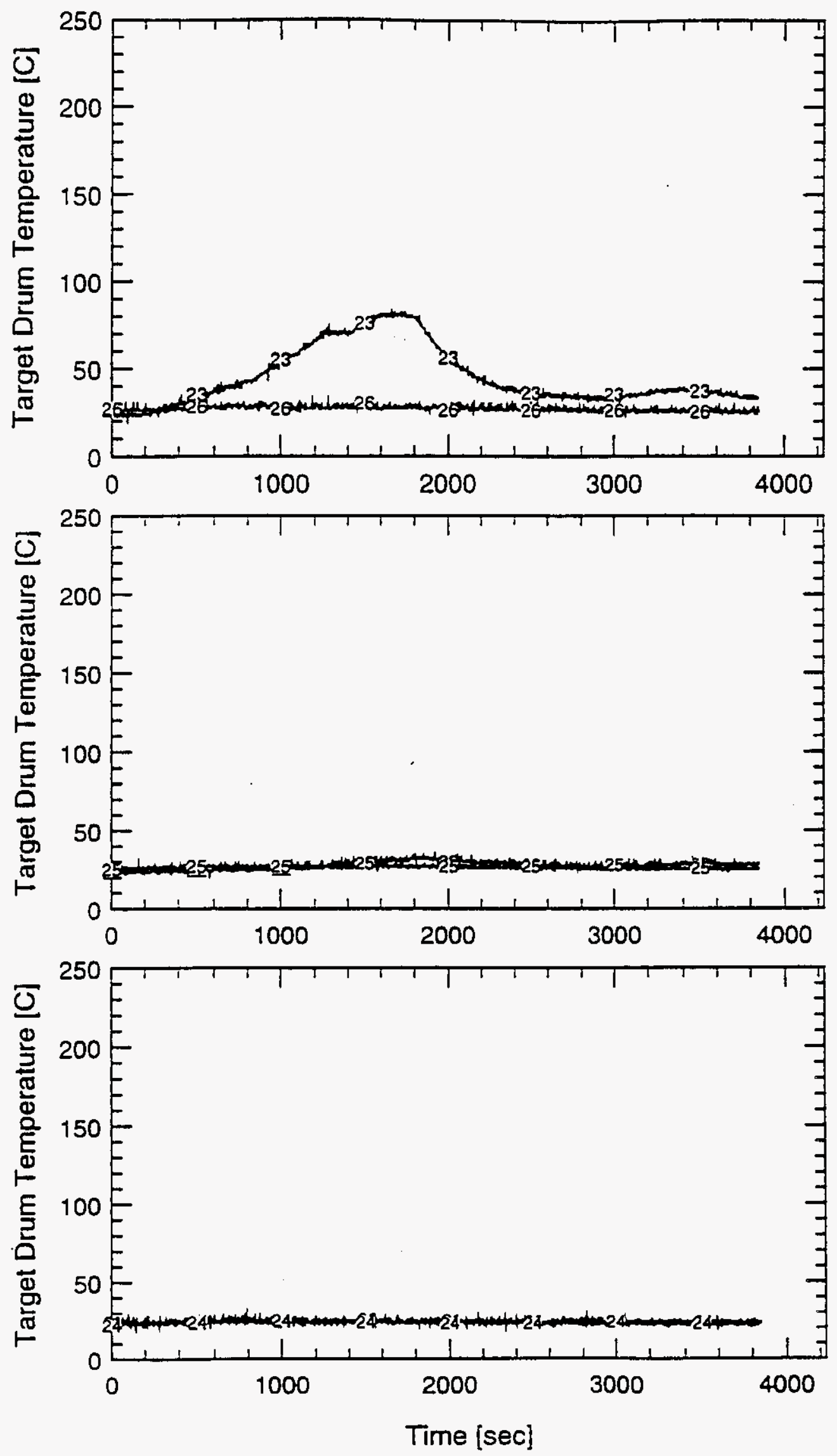

B-42 
WHC-SD-WM-TRP-233 REV. 0

SWD7 - 15lb of Brown Paper
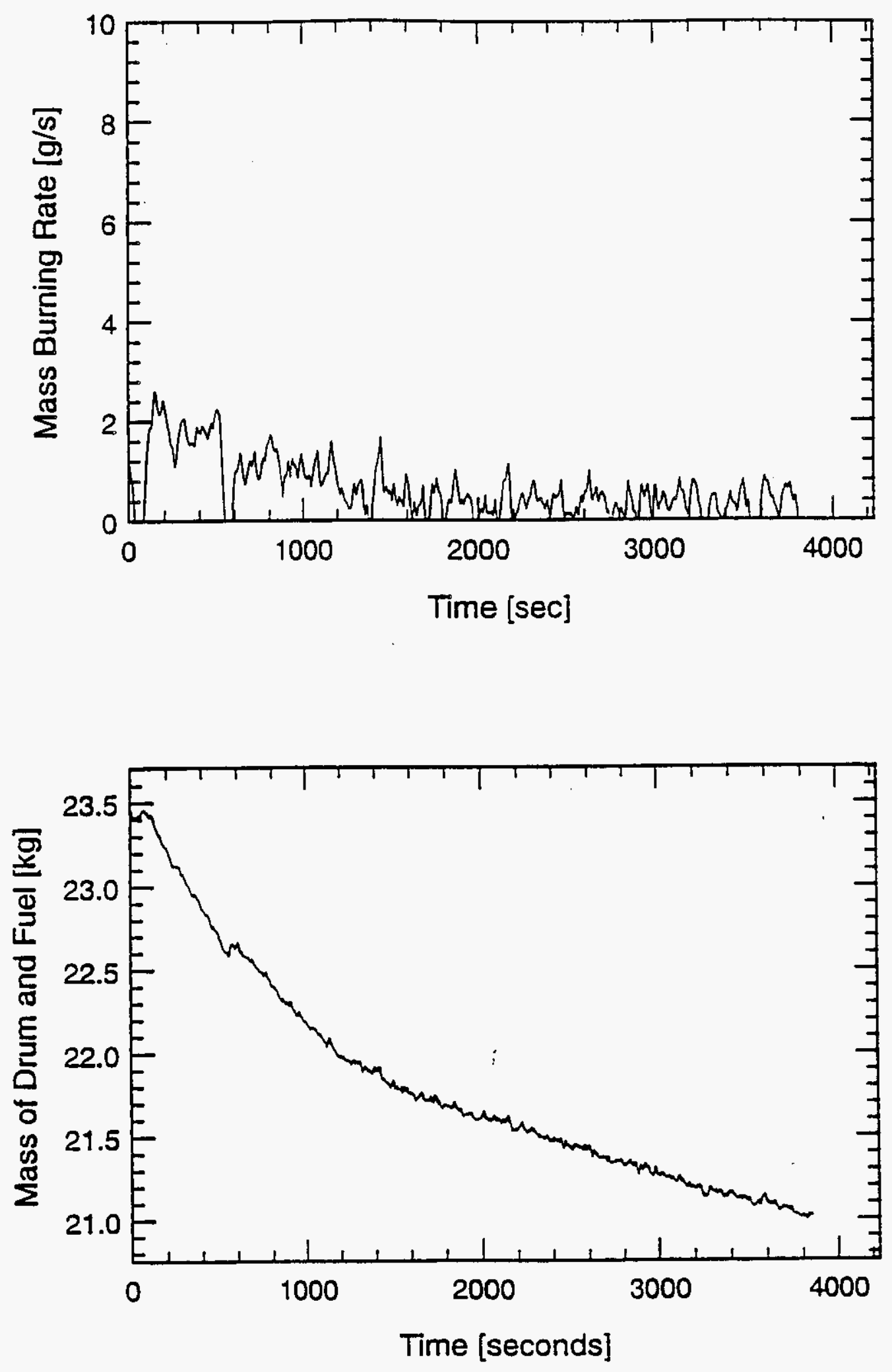
WHC-SD-WM-TRP-233 REV. 0

SWD 8 - DATA 
WHC-SD-WM-TRP-233 REV. O

SWD8 - 5lb of Plastic
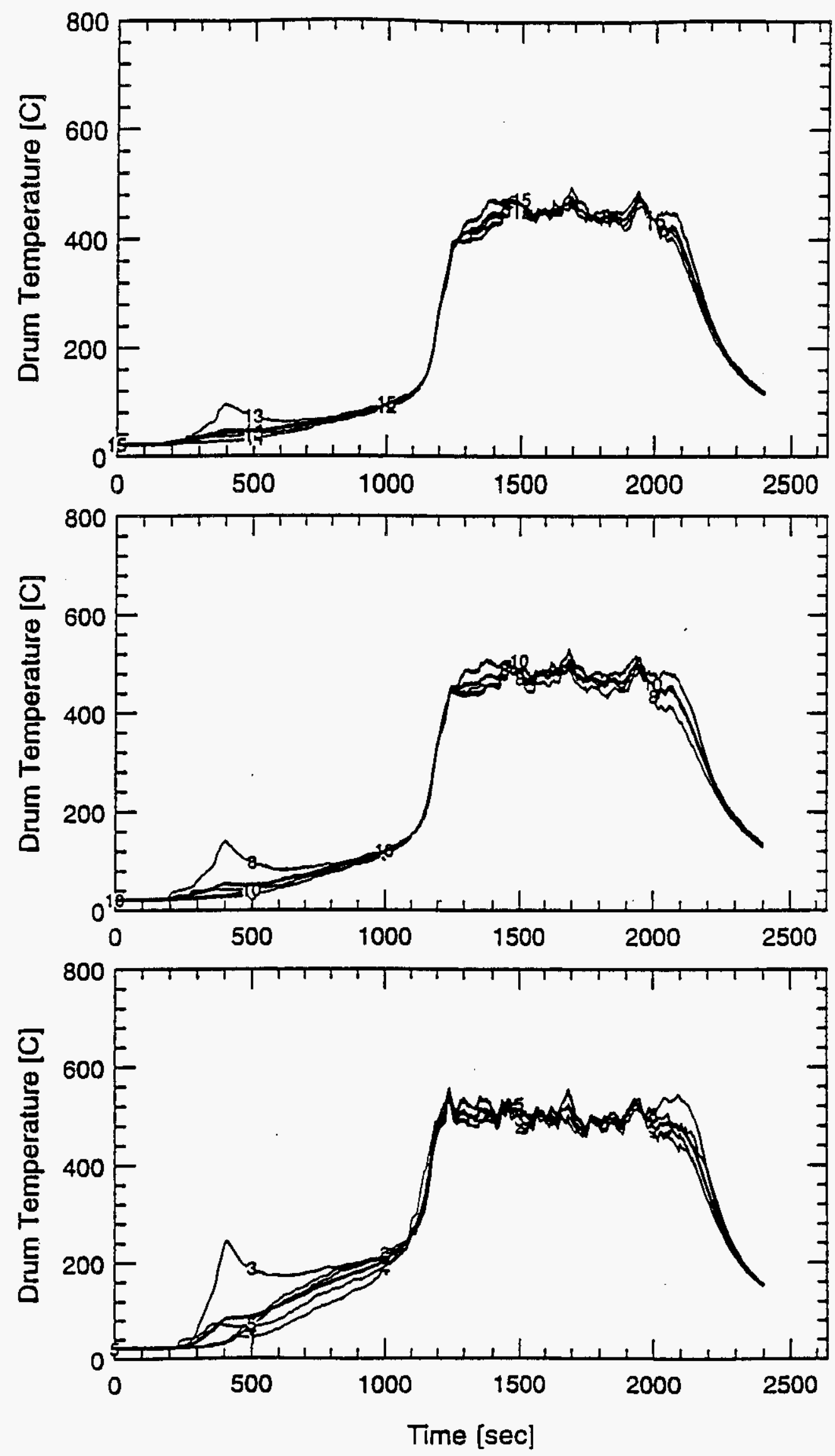
WHC-SD-WM-TRP-233 REV. 0

\section{SWD8 - 5lb of Plastic}
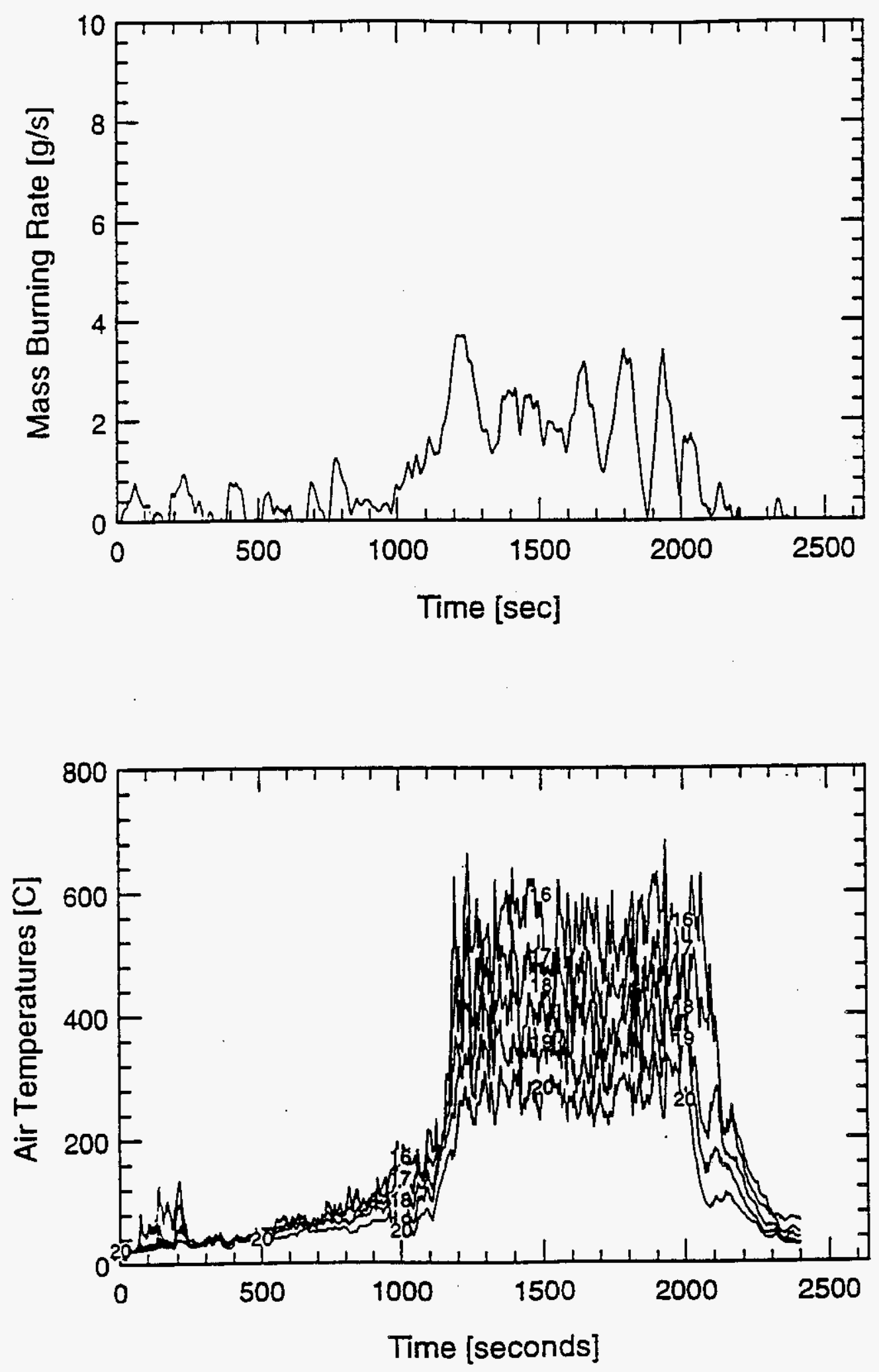
WHC-SD-WM-TRP-233 REV. 0

SWD8 - 5lb of Plastic
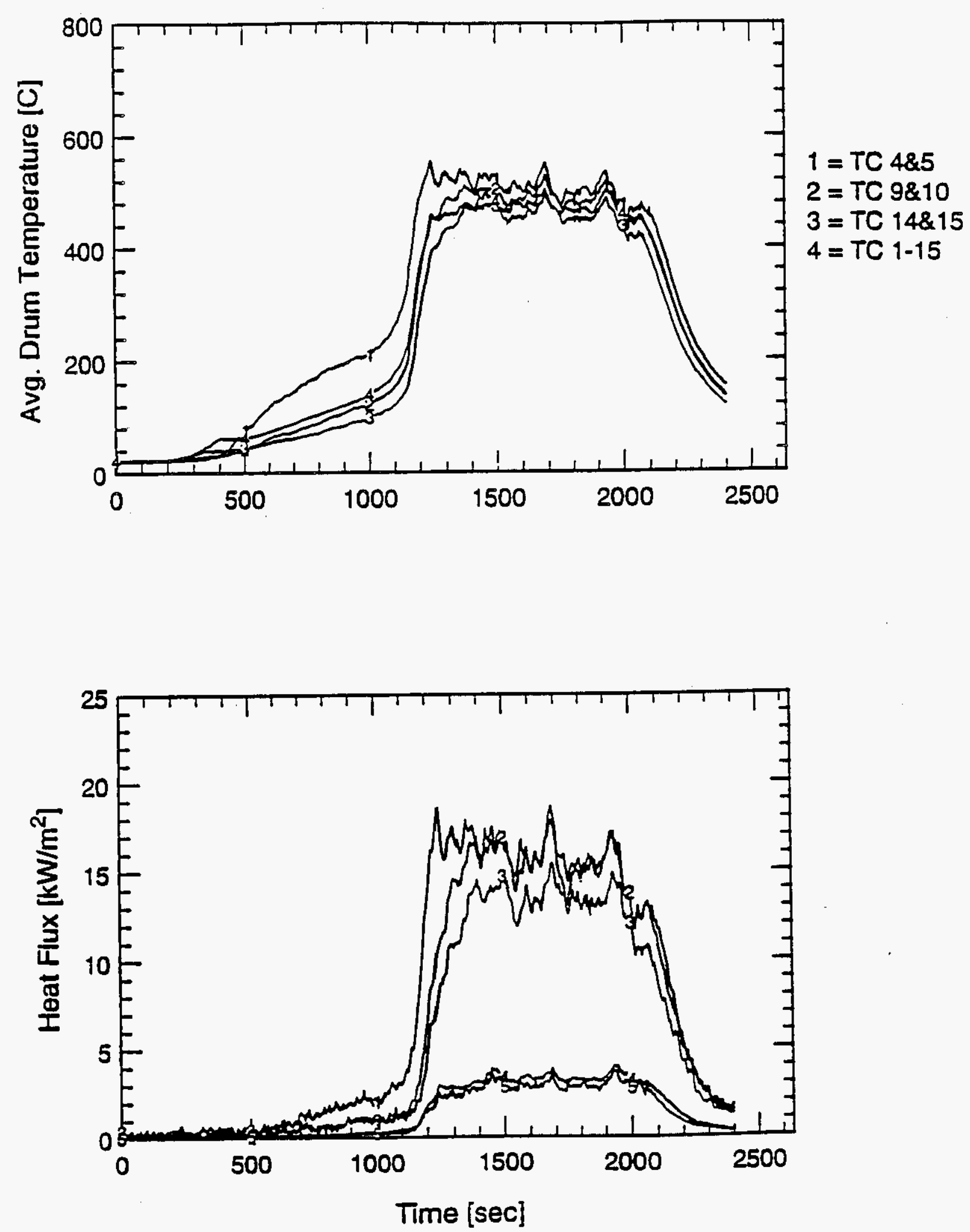
WHC-SD-WM-TRP-233 REV. 0

SWD8 - 5lb of Plastic
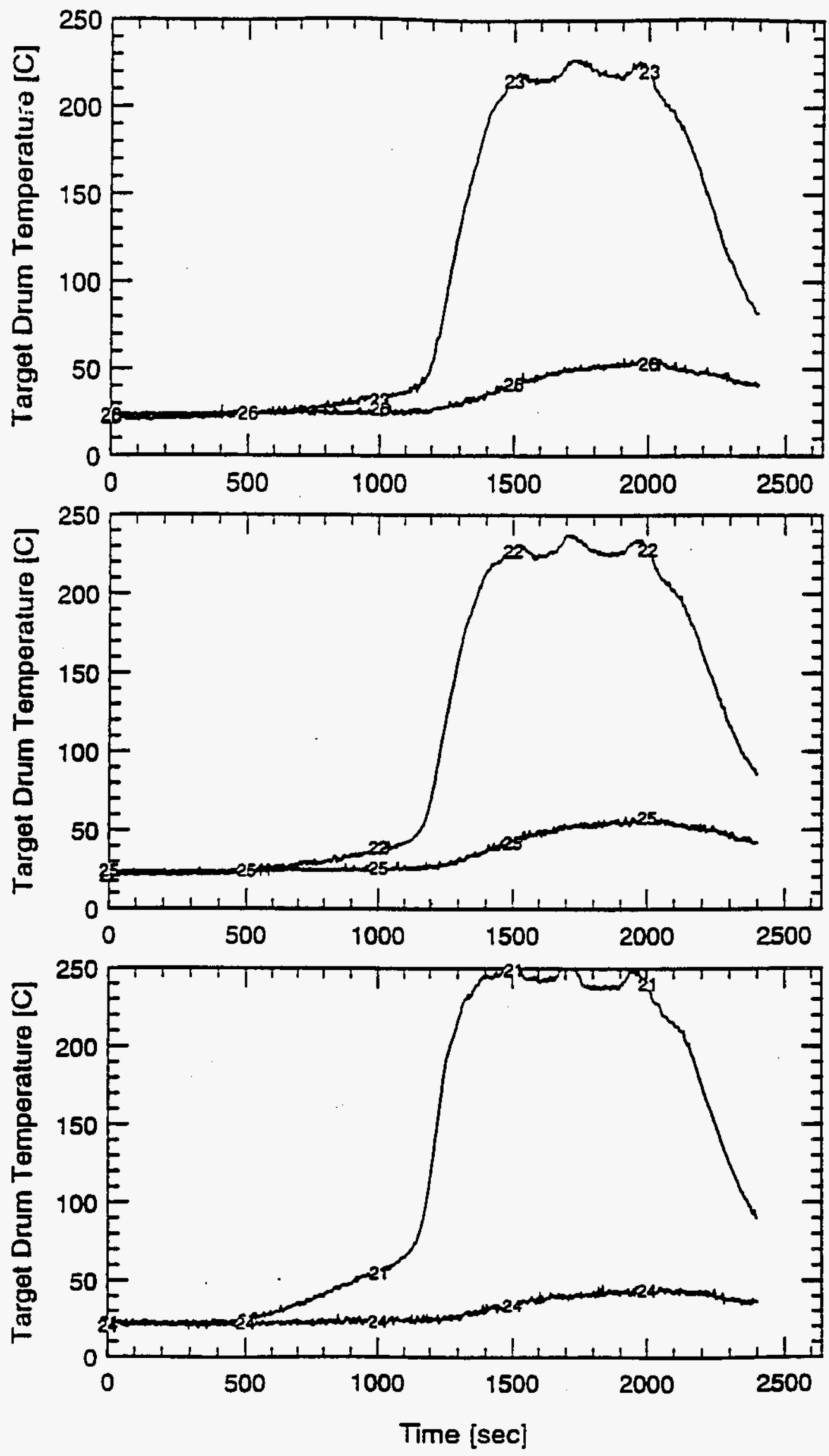
WHC-SD-WM-TRP-233 REV. 0

SWD8 - 5ib of Plastic
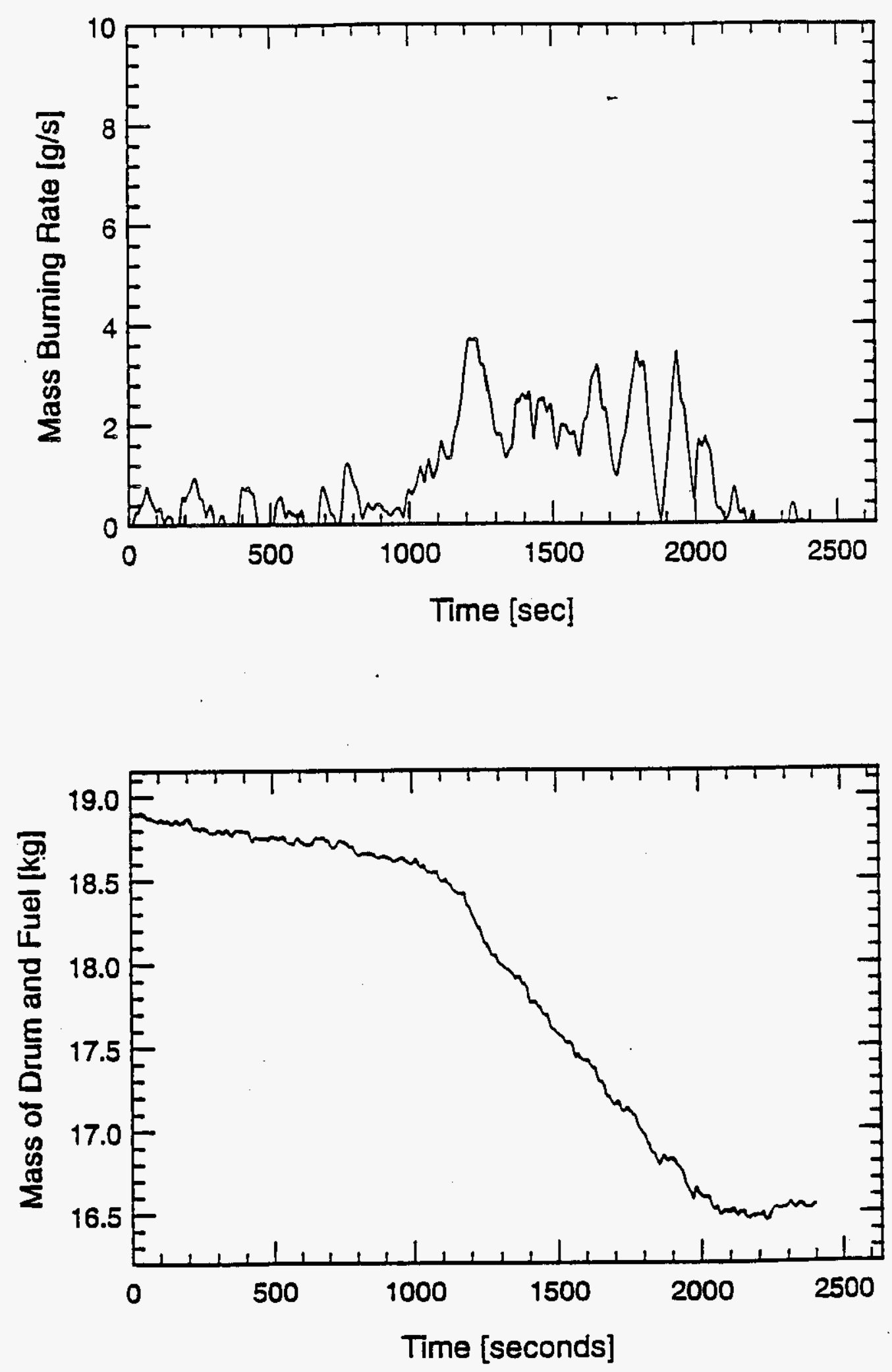
WHC-SD-WM-TRP-233 REV. 0

SWD 9 - DATA

B -50 
WHC-SD-WM-TRP-233 REV. 0

\section{SWD9 - 15ib of Plastic}
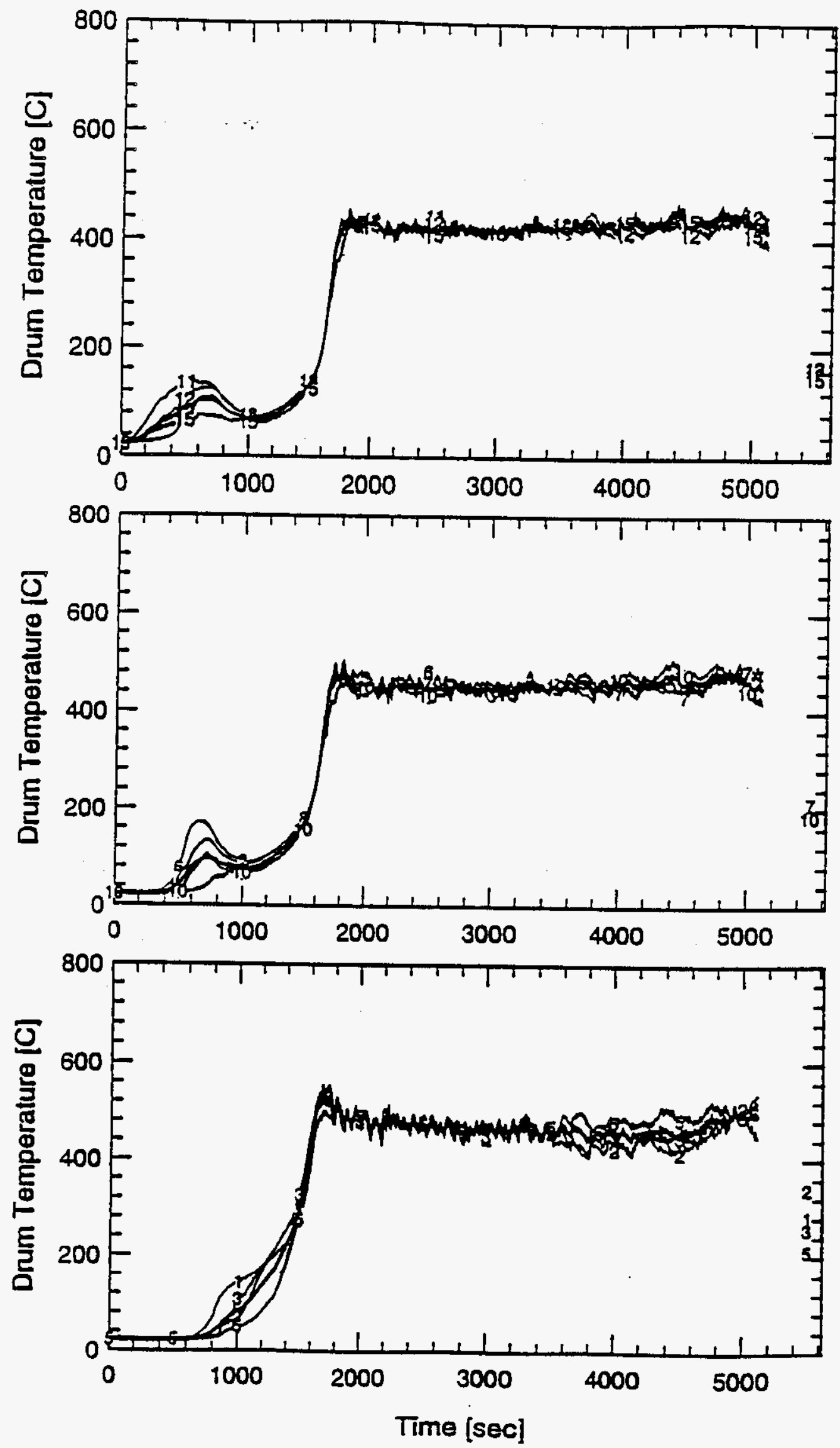
WHC-SD-WM-TRP-233 REV. 0

SWD9 - 15ib of Plastic
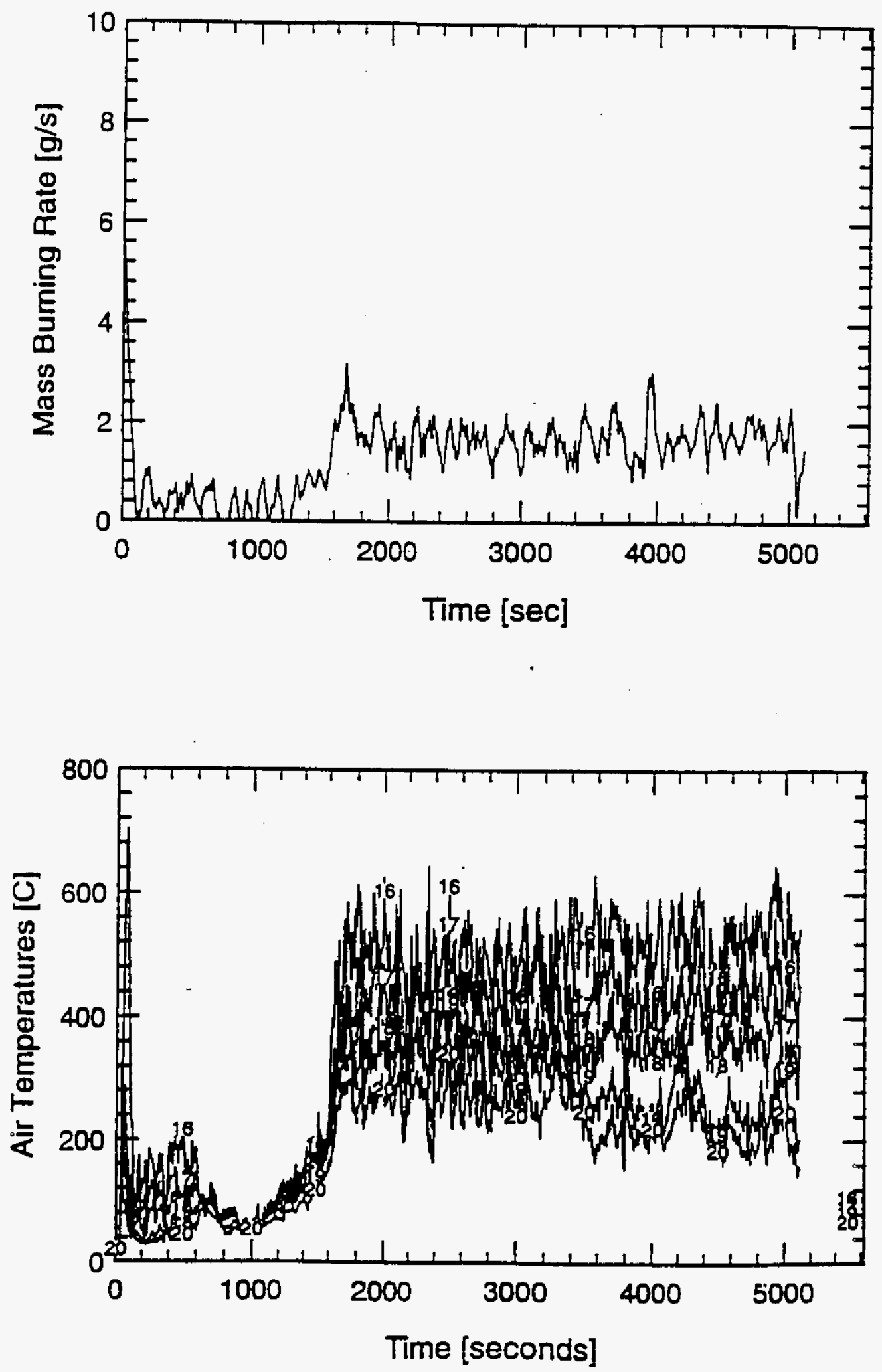
WHC-SD-WM-TRP-233 REV. 0

SWD9 - 15lb of Plastic
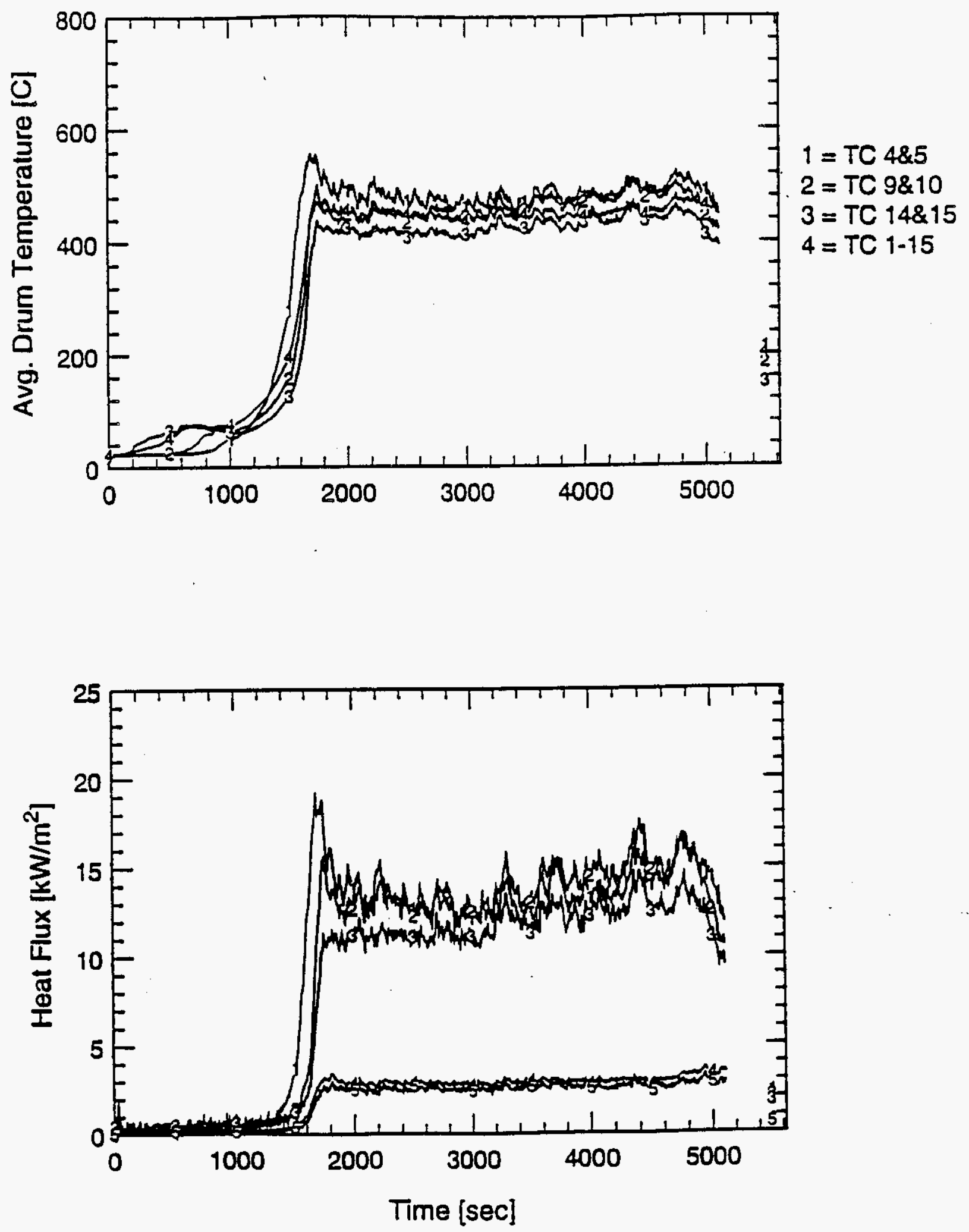
WHC-SD-WM-TRP-233 REV. 0

SWD9 - 15lb of Plastic
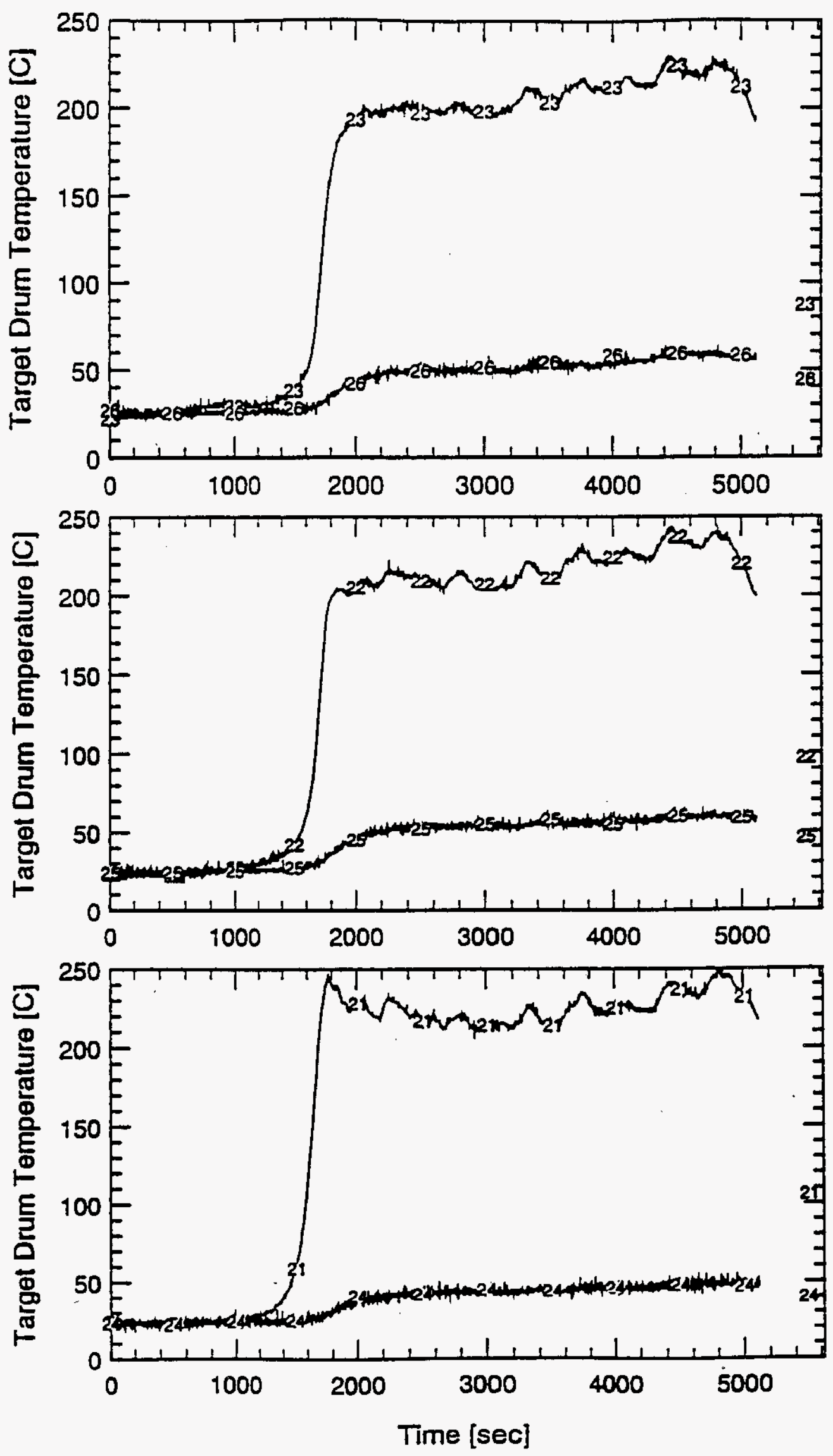
WHC-SD-WM-TRP-233 REV. 0

SWD9 - 15ib of Plastic
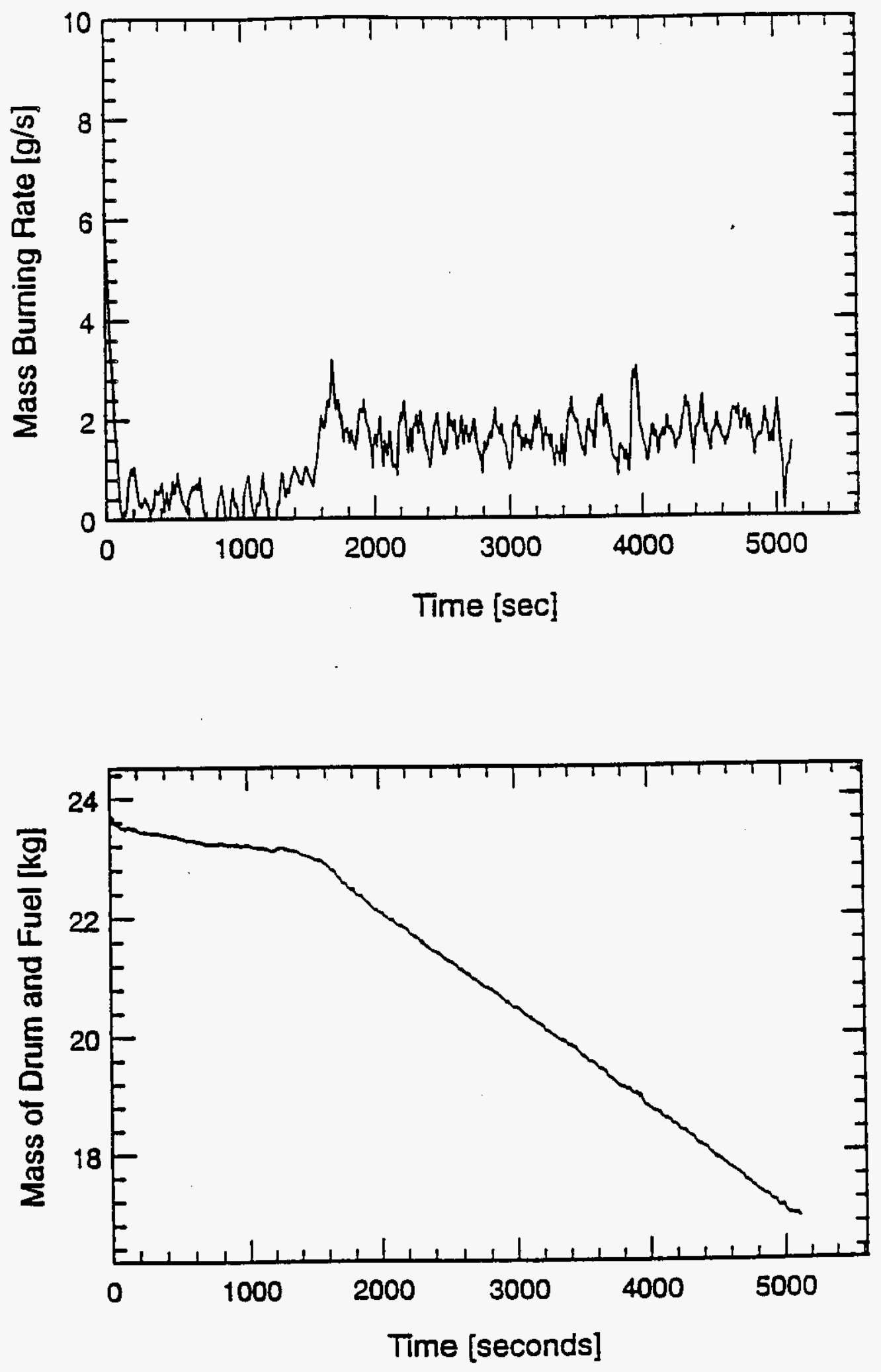
WHC-SD-WM-TRP-233 REV. 0

SWD 10 - DATA 
WHC-SD-WM-TRP-233 REV. 0

SWD10 $-2 / 3$ of Mixed Standard Load
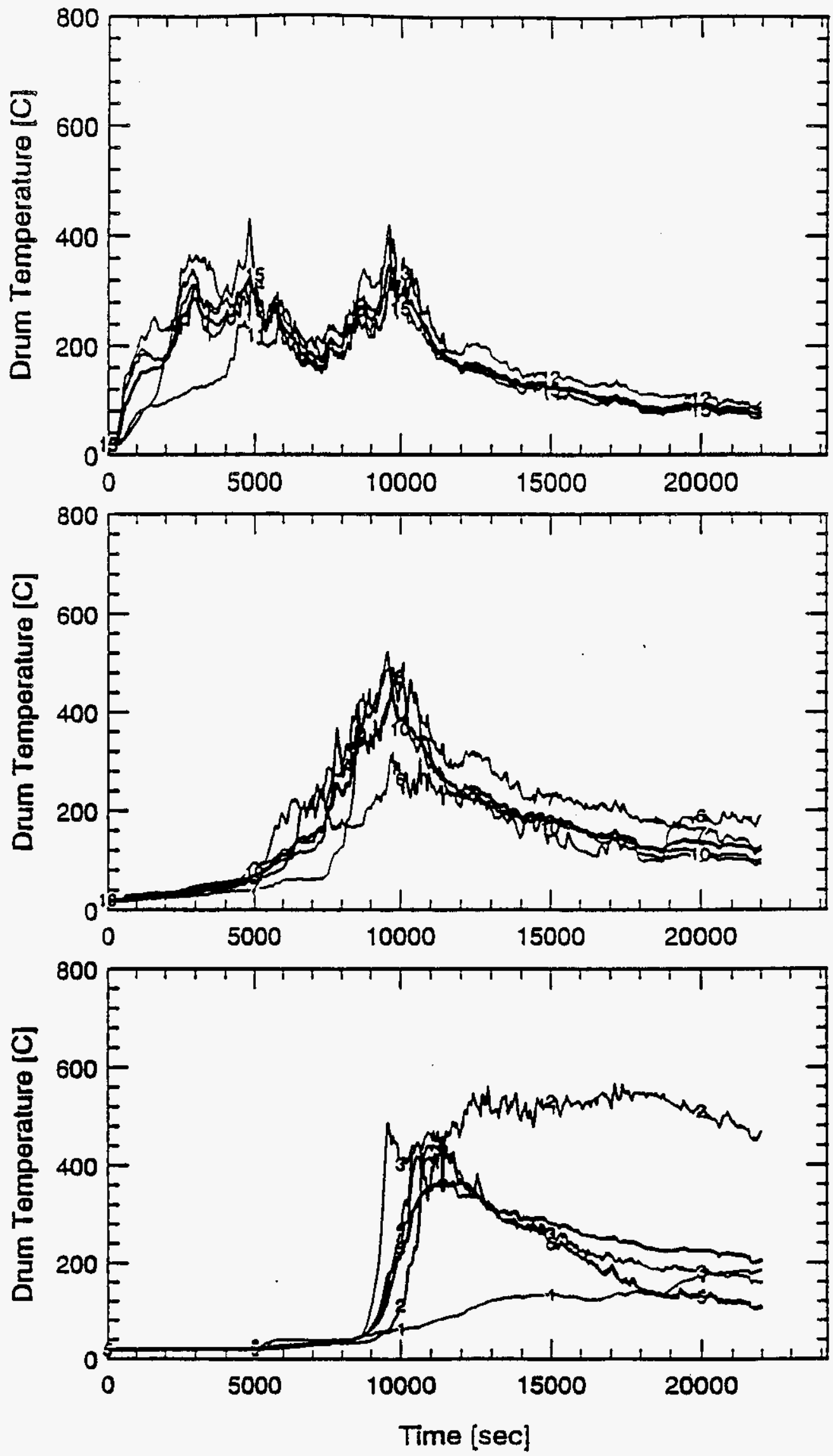


\section{SWD10 - 2/3 of Mixed Standard Load}
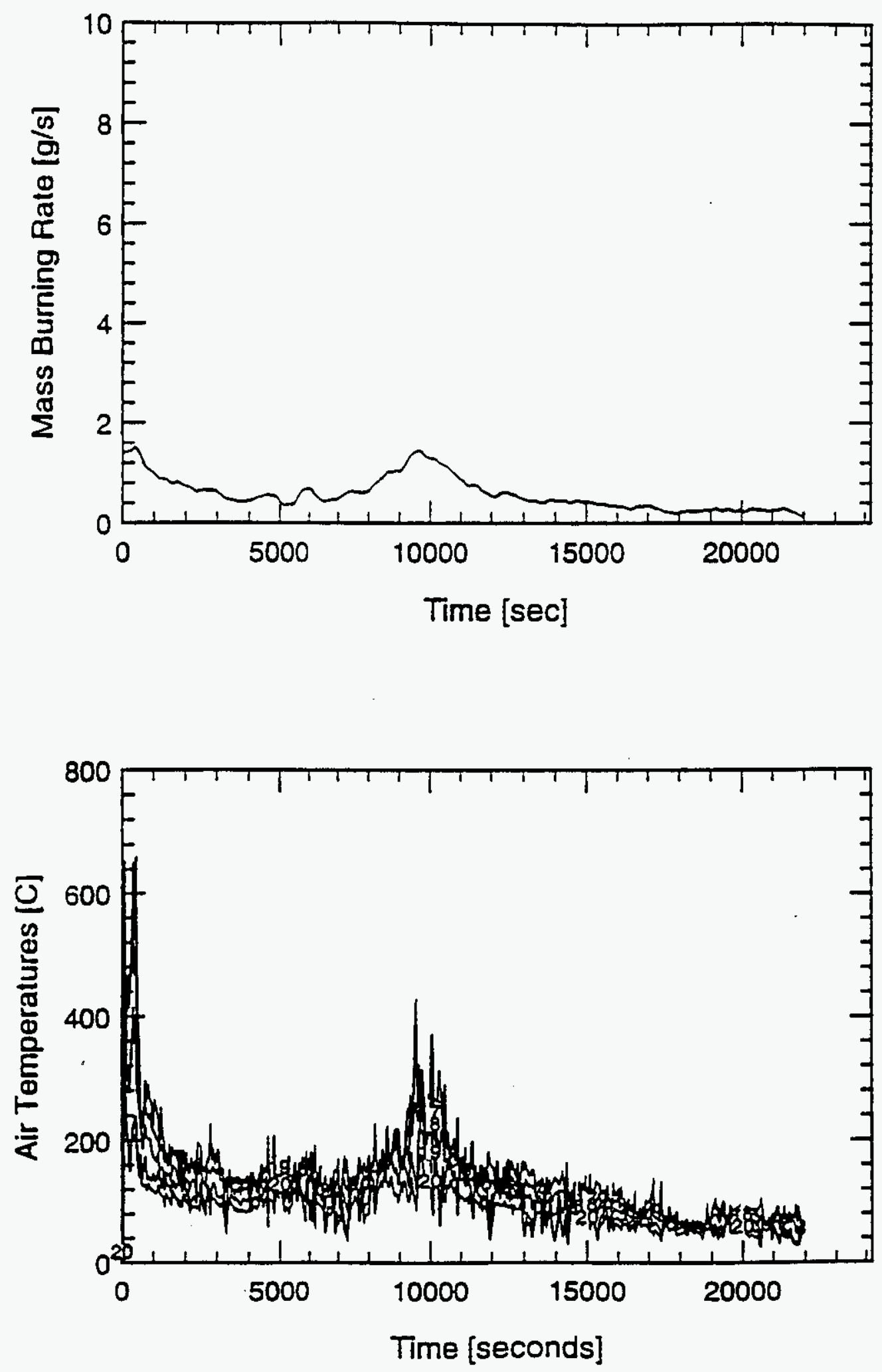


\section{SWD10 $-2 / 3$ of Mixed Standard Load}
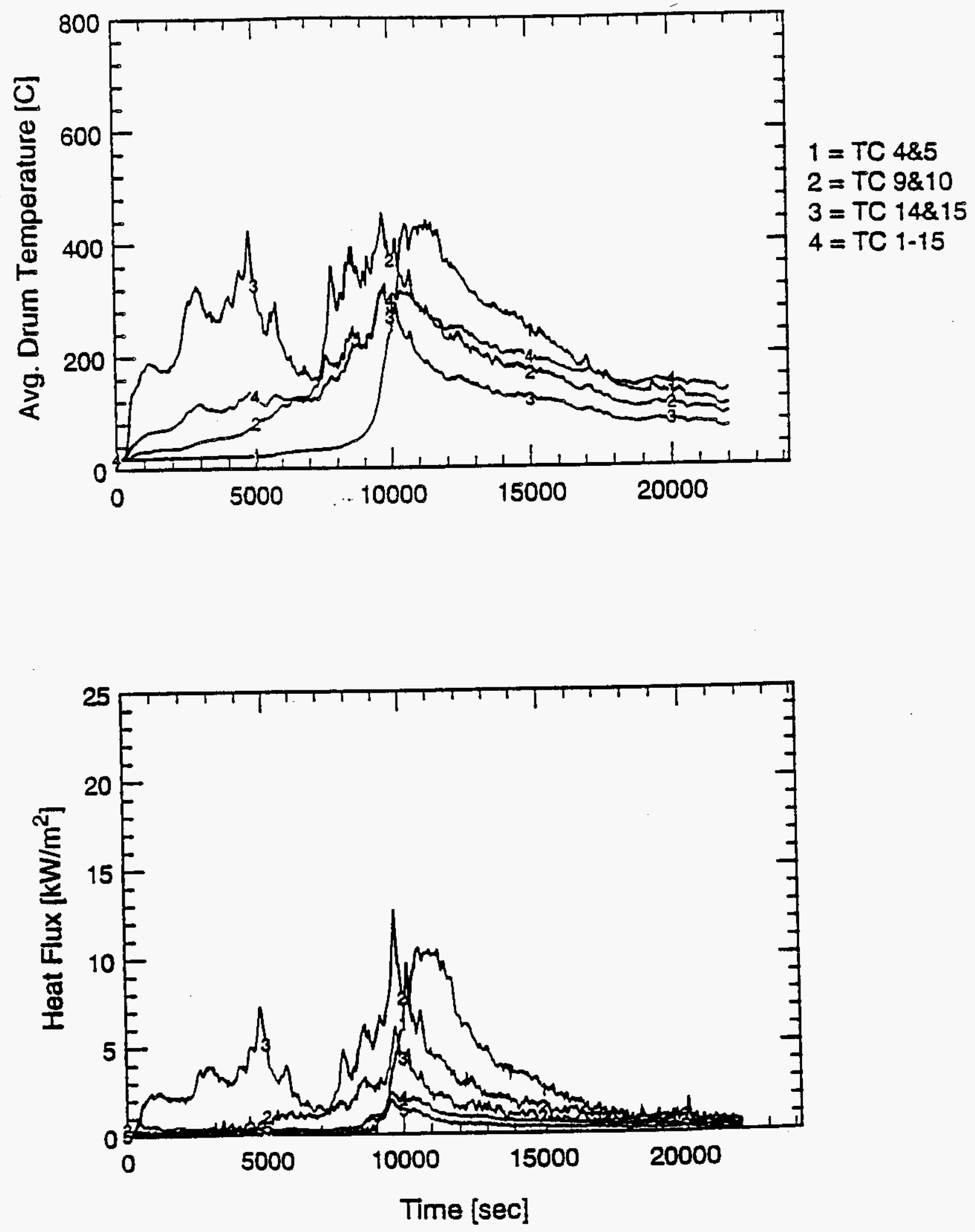
WHC-SD-WM-TRP-233 REV. O

SWD10 $-2 / 3$ of Mixed Standard Load
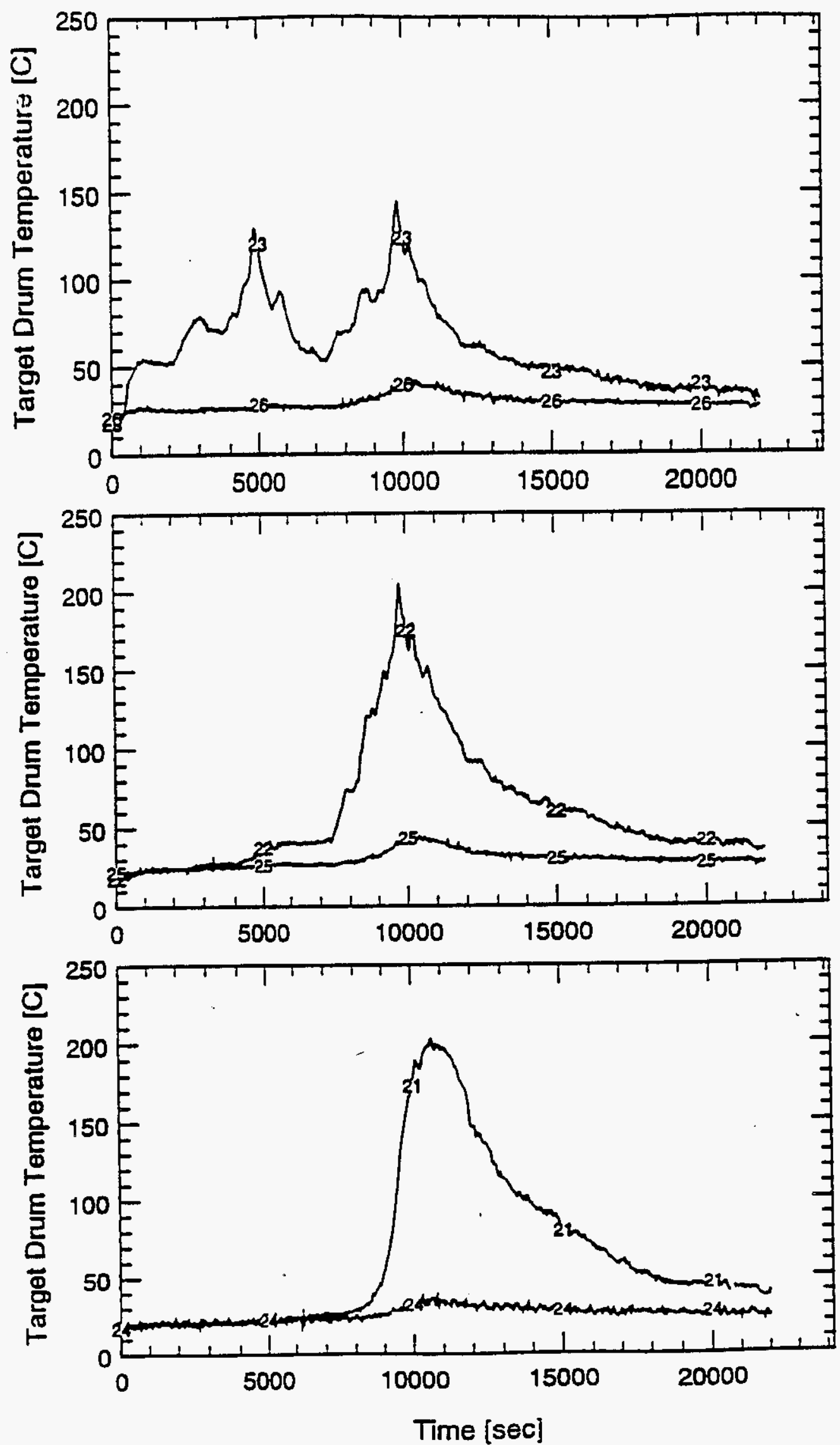
WHC-SD-WM-TRP-233 REV. 0

SWD10 $-2 / 3$ of Mixed Standard Load
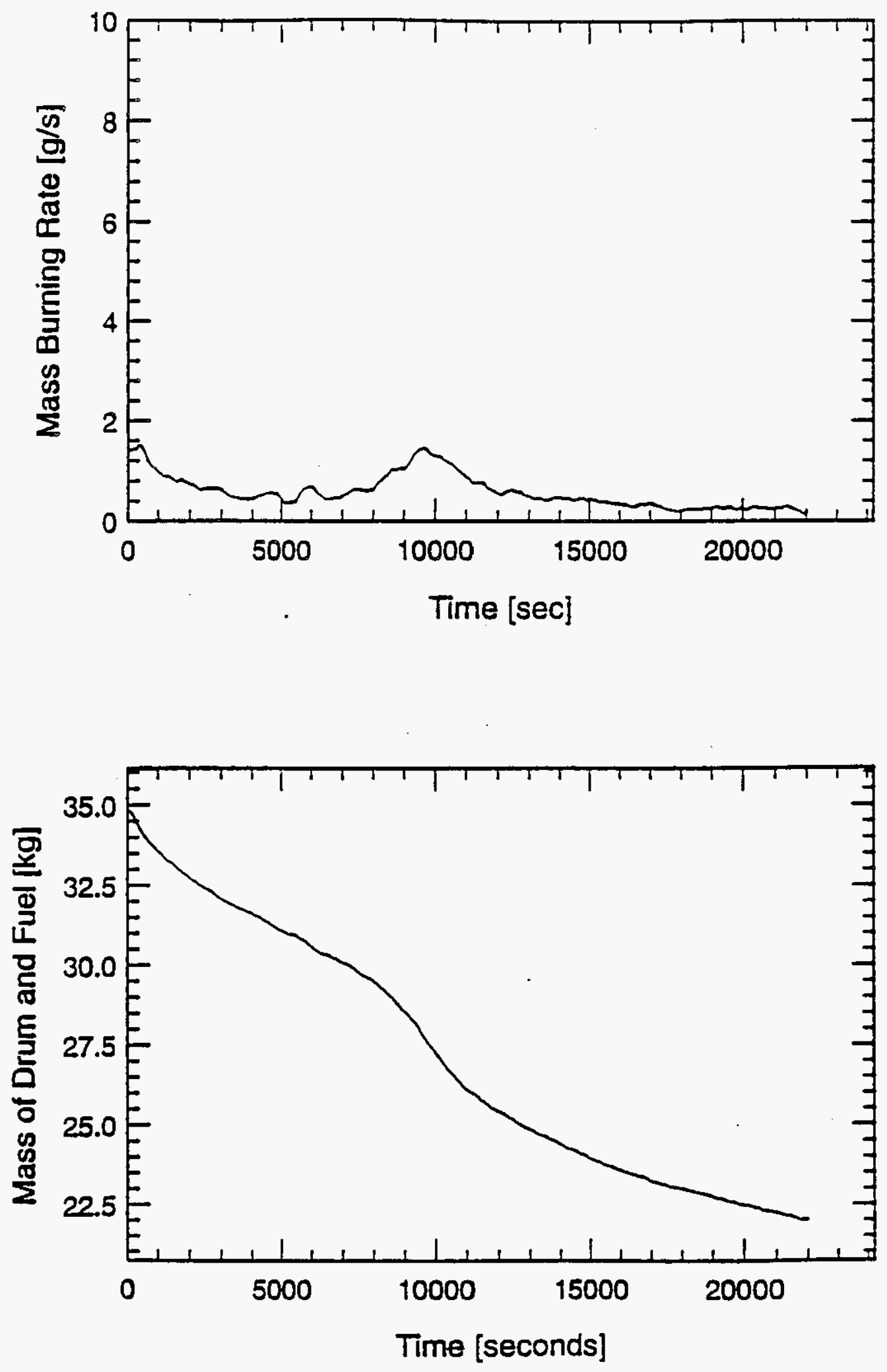

B-61 
WHC-SD-WM-TRP-233 REV. 0

SWD 11 - DATA

B-62 

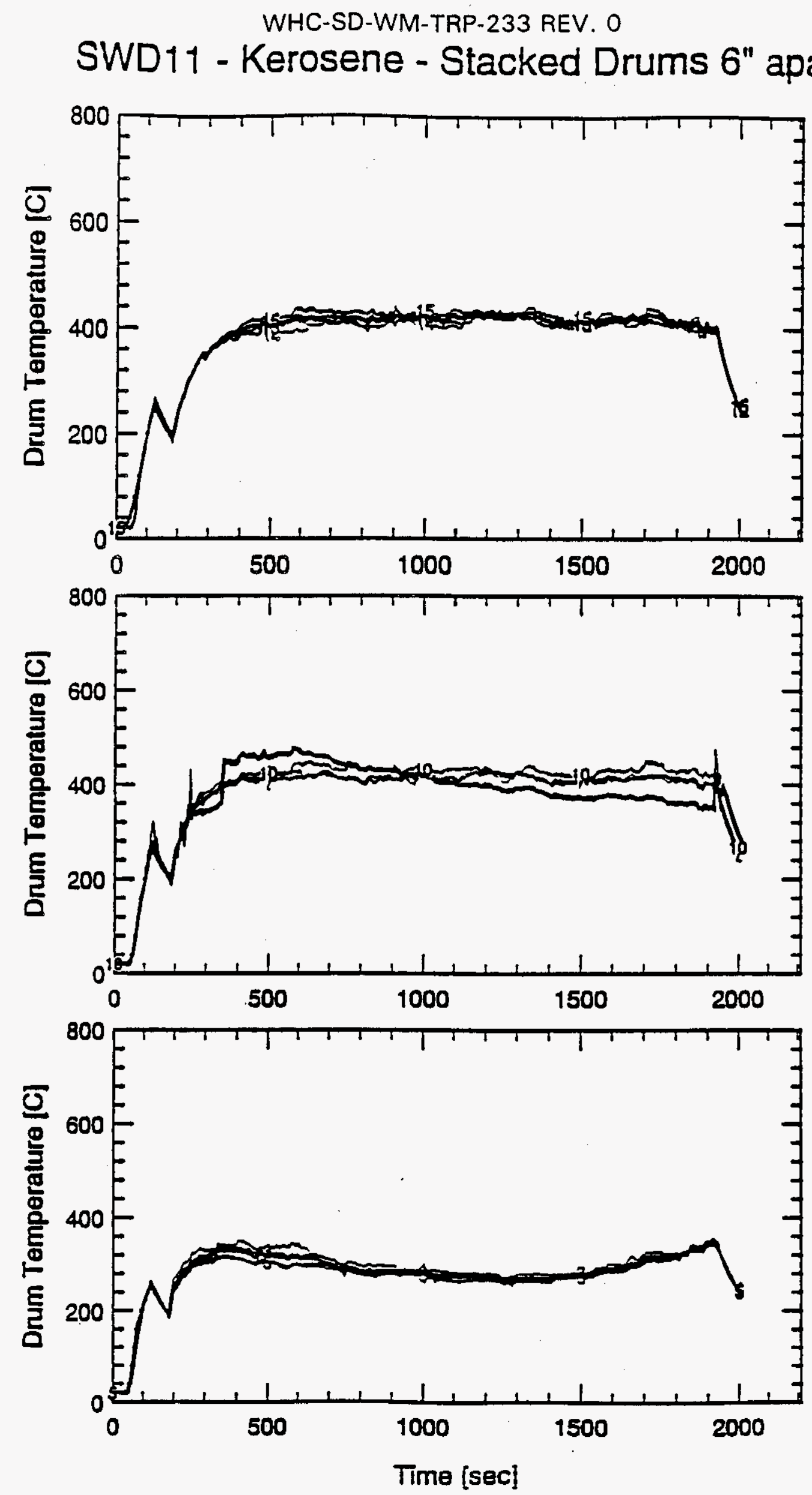
SWD11 - Kerosene - Stacked Drums 6" apart
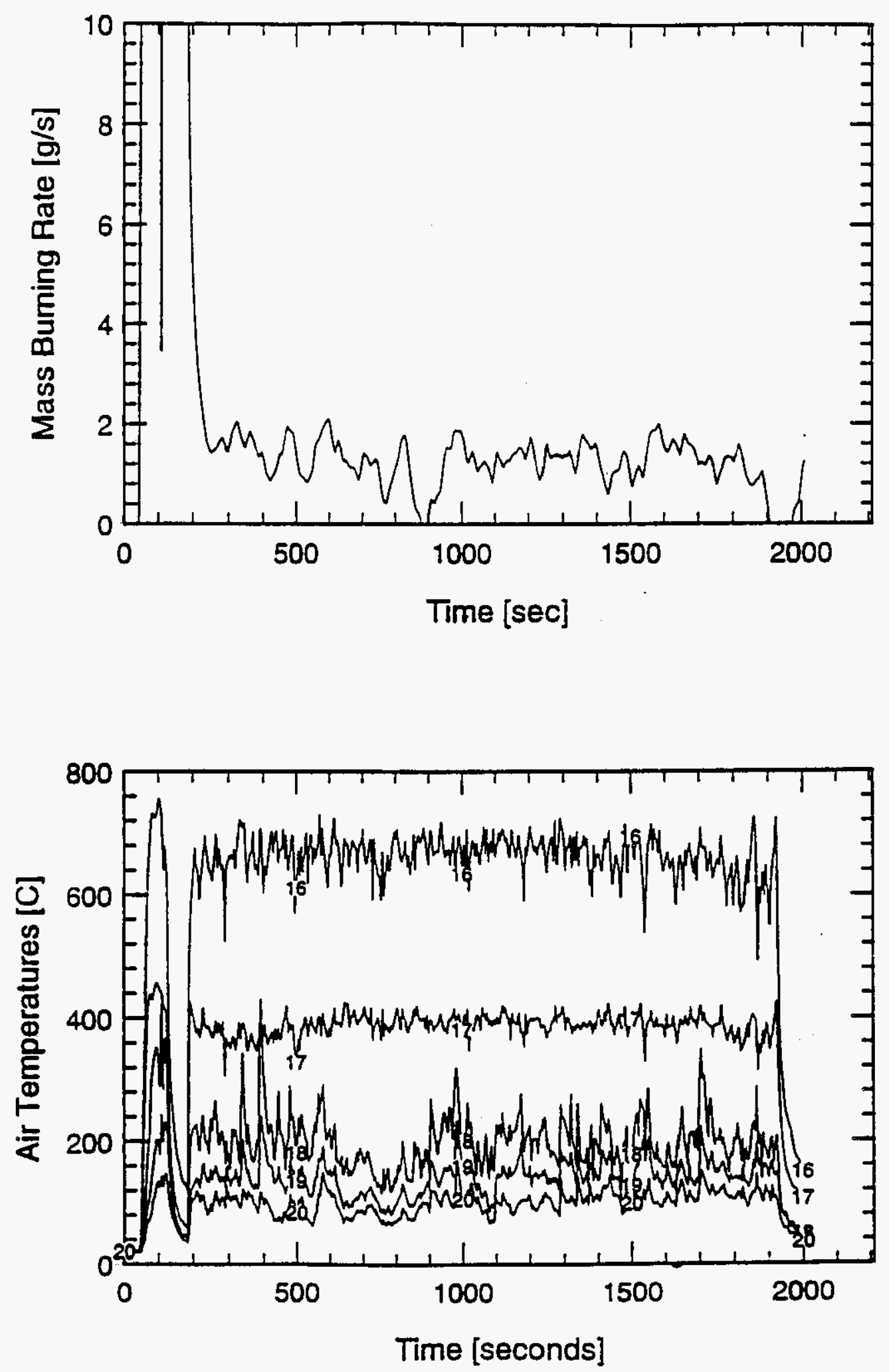
SWD11 - Kerosene - Stacked Drums 6" apart
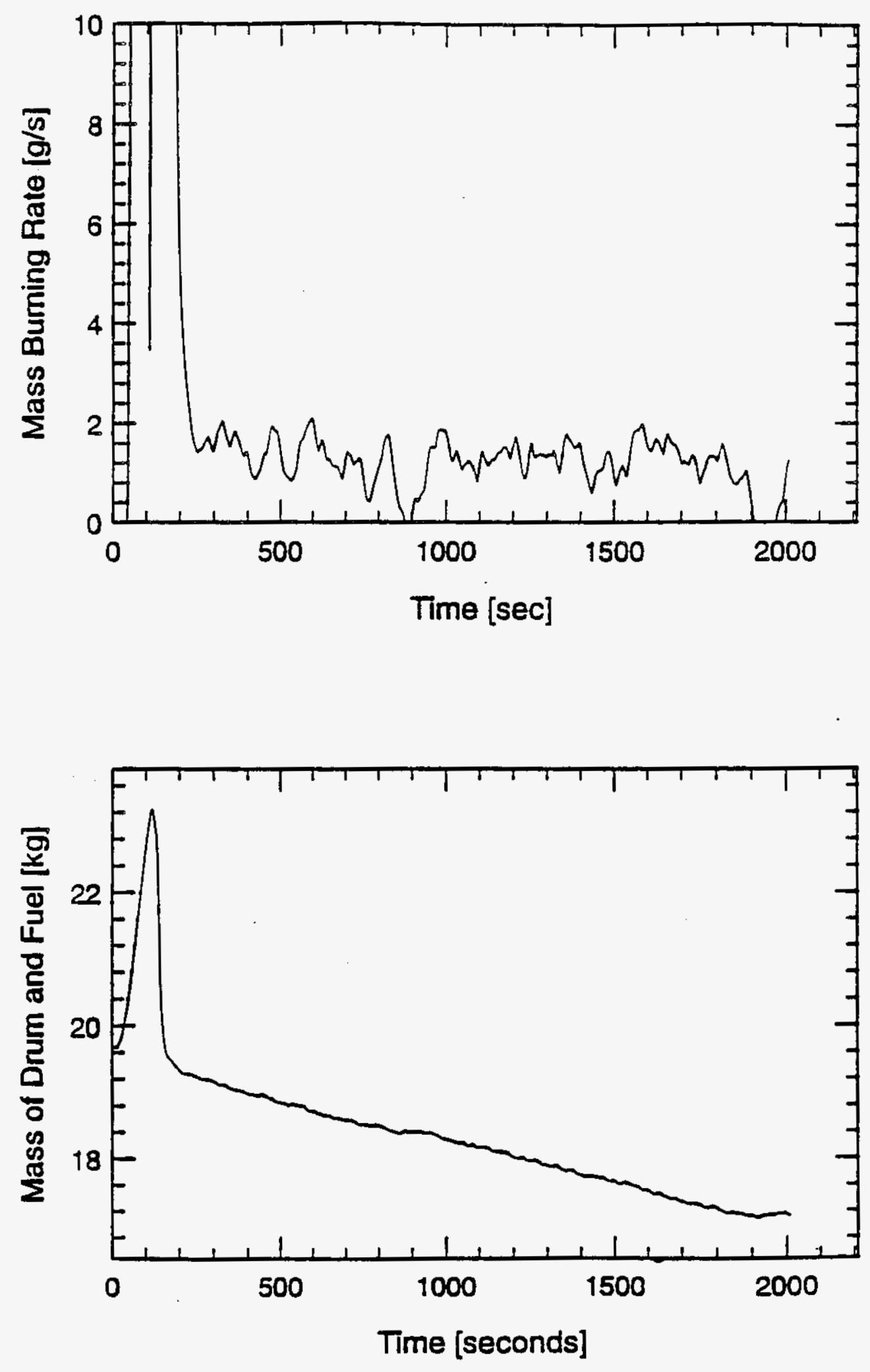
SWD11 - Kerosene - Stacked Drums 6" apart
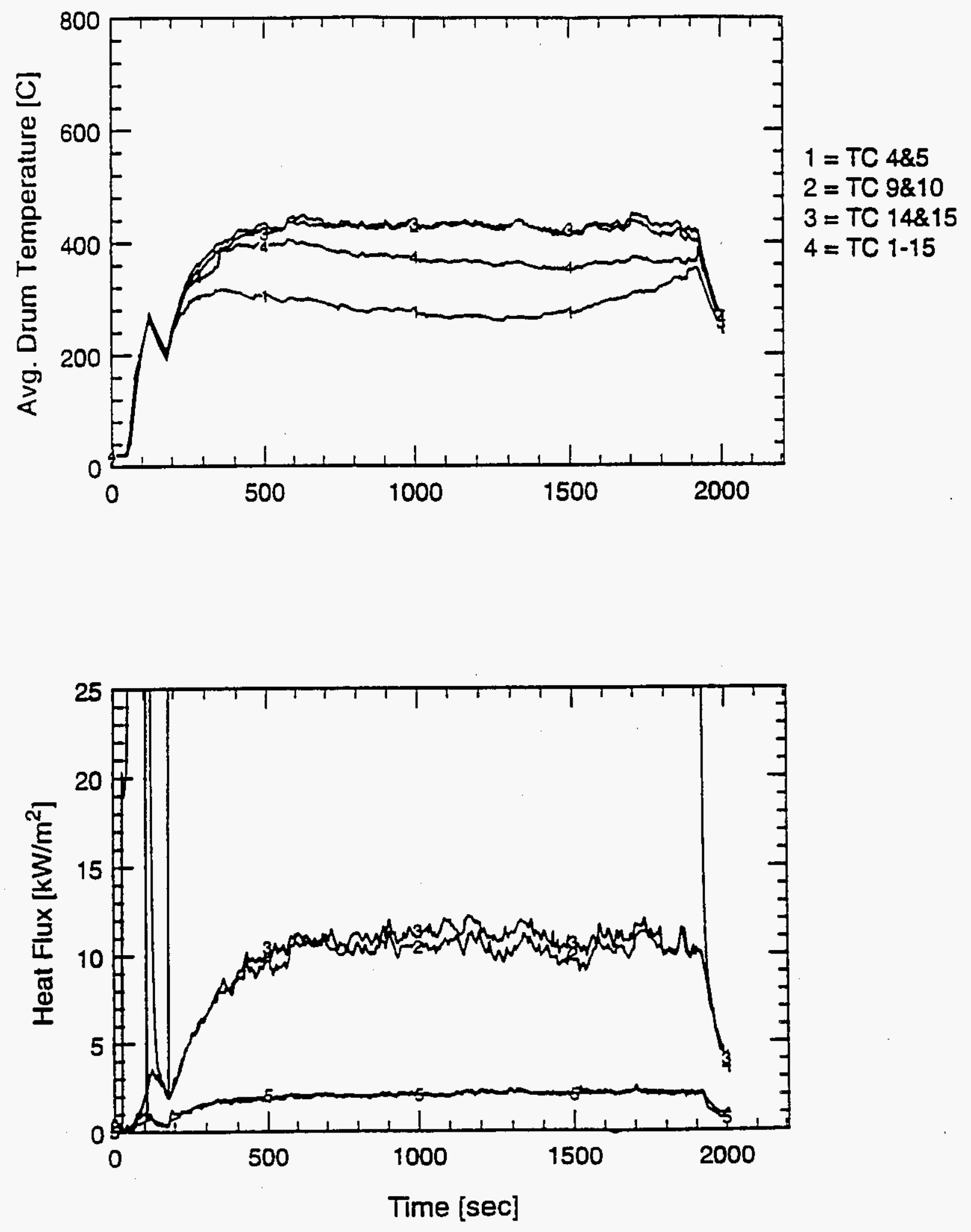
WHC-SD-WM-TRP-233 REV. 0

SWD11 - Kerosene - Stacked Drums 6" apart
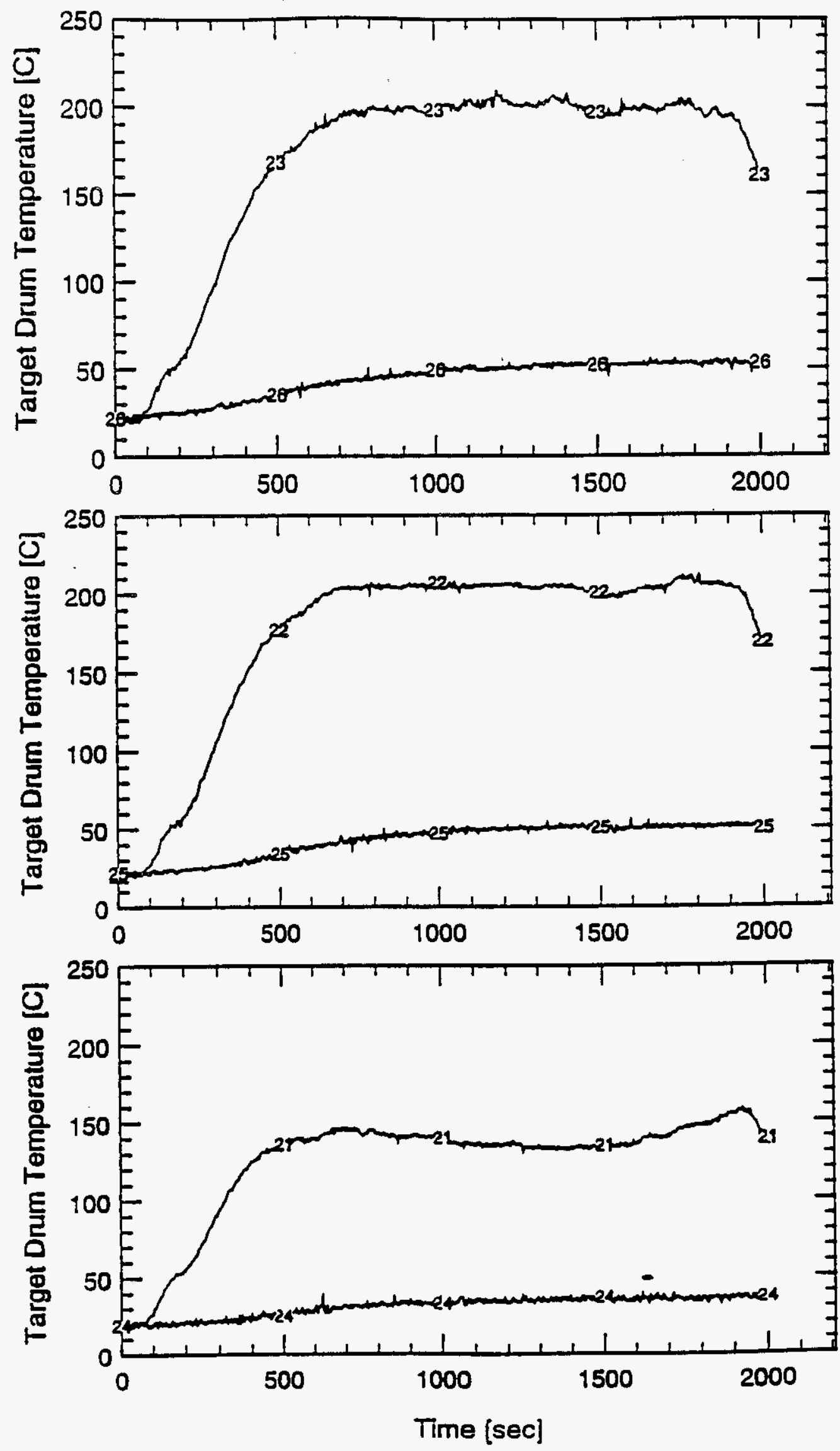

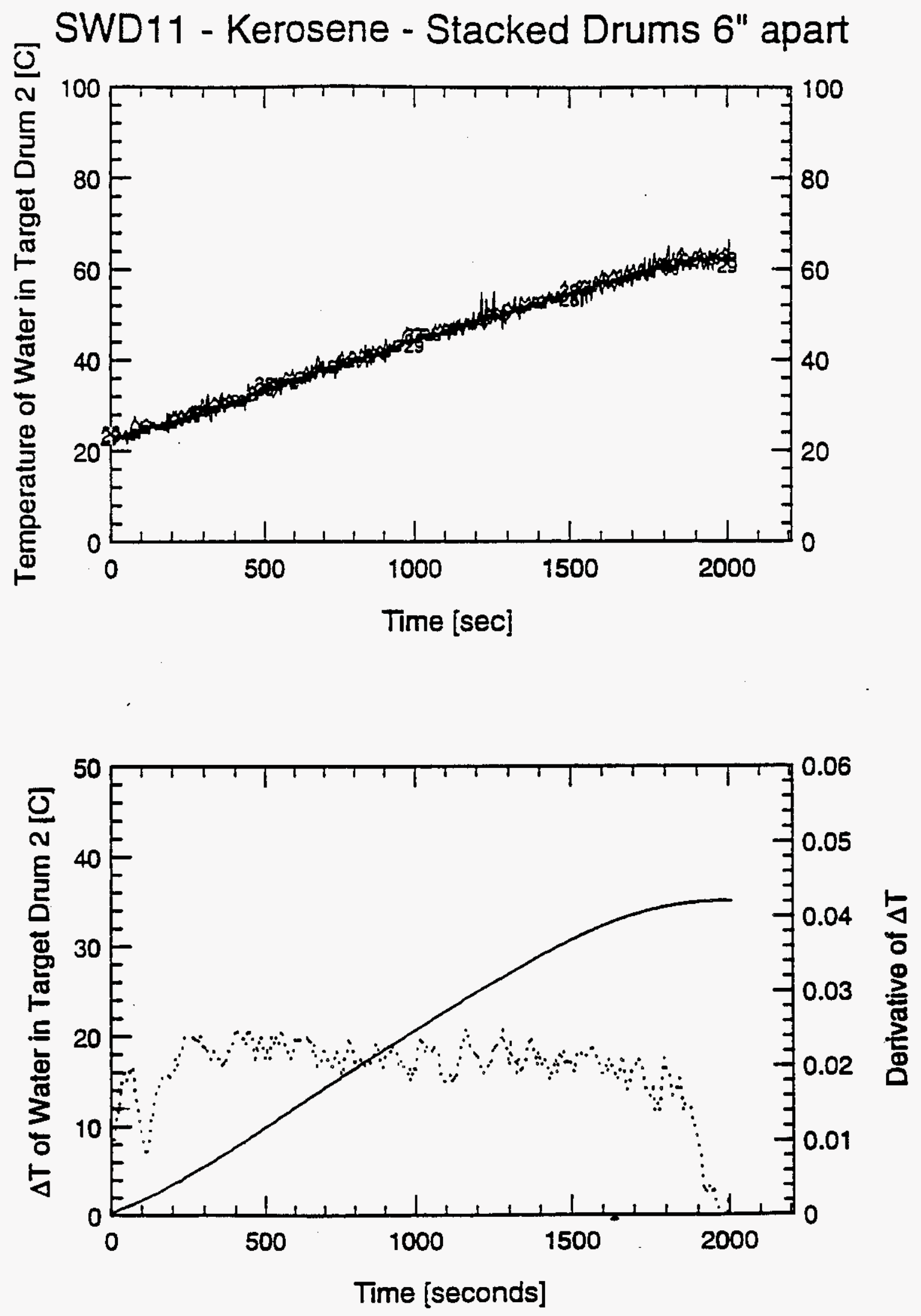
SWD11 - Kerosene - Stacked Drums 6" apart
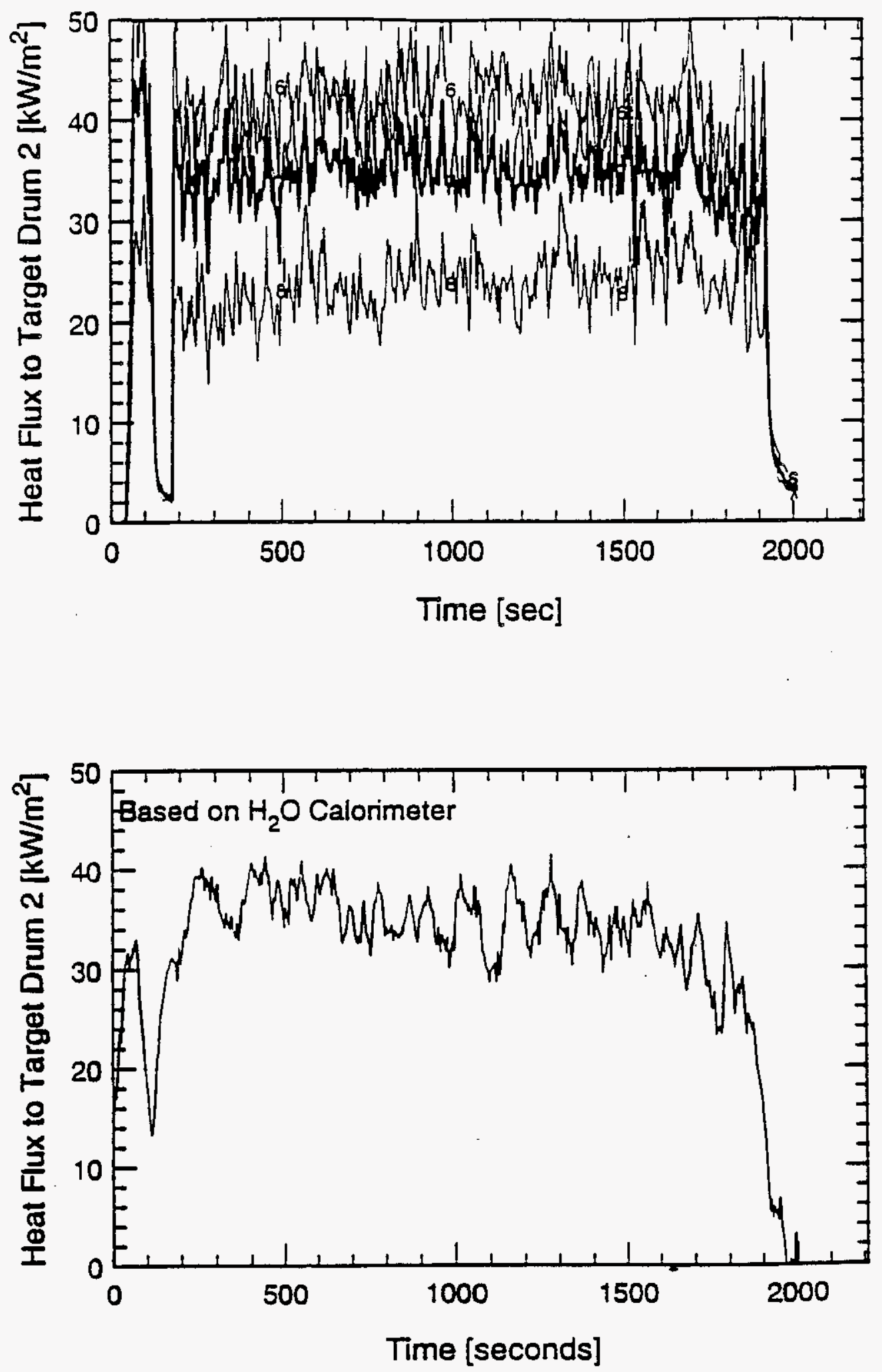
WHC-SD-WM-TRP-233 REV. 0

SWD 12 - DATA 


\section{SWD12 - Kerosene - Stacked Drums 12" apart}
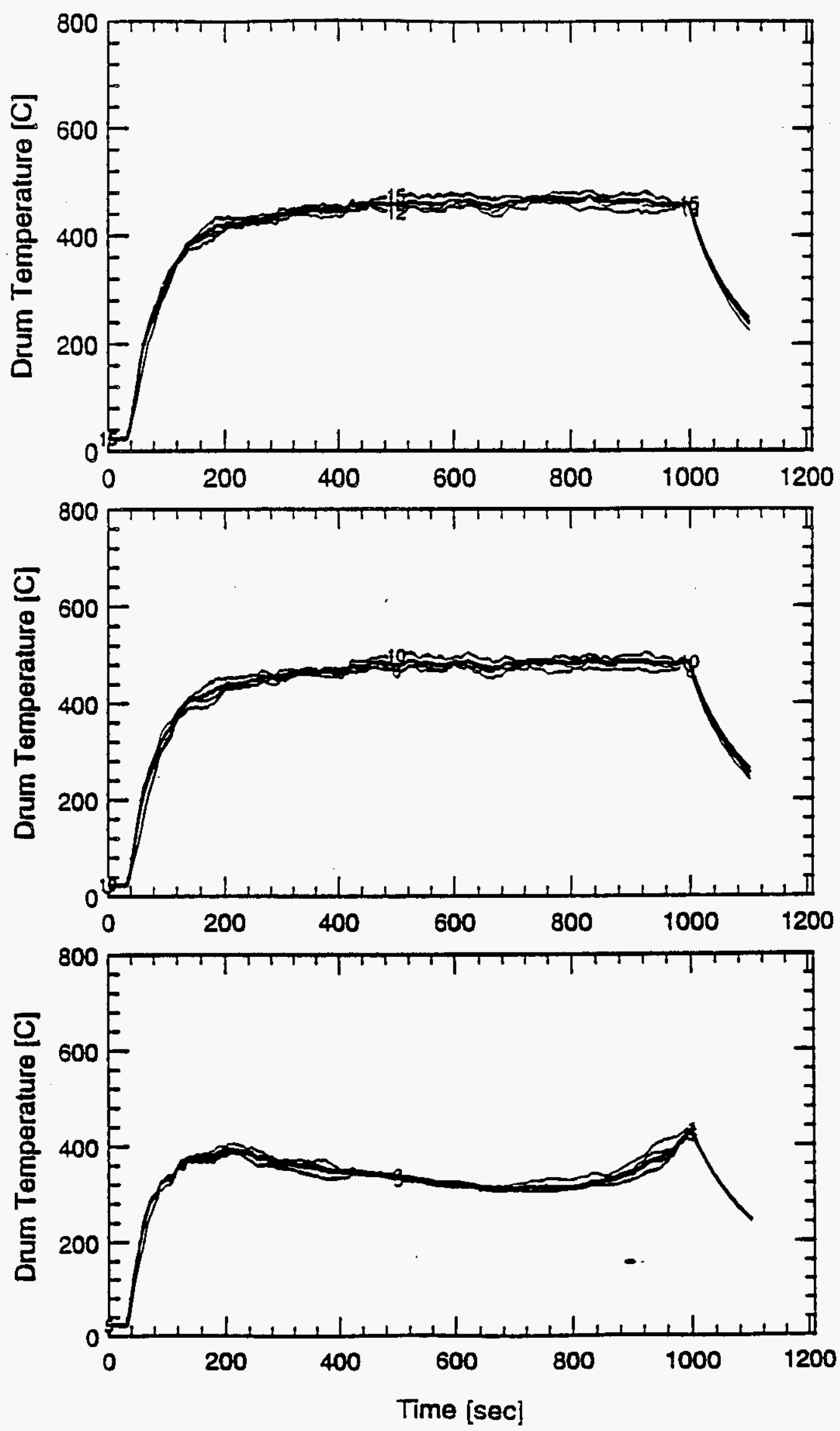
SWD12 - Kerosene - Stacked Drums 12" apart
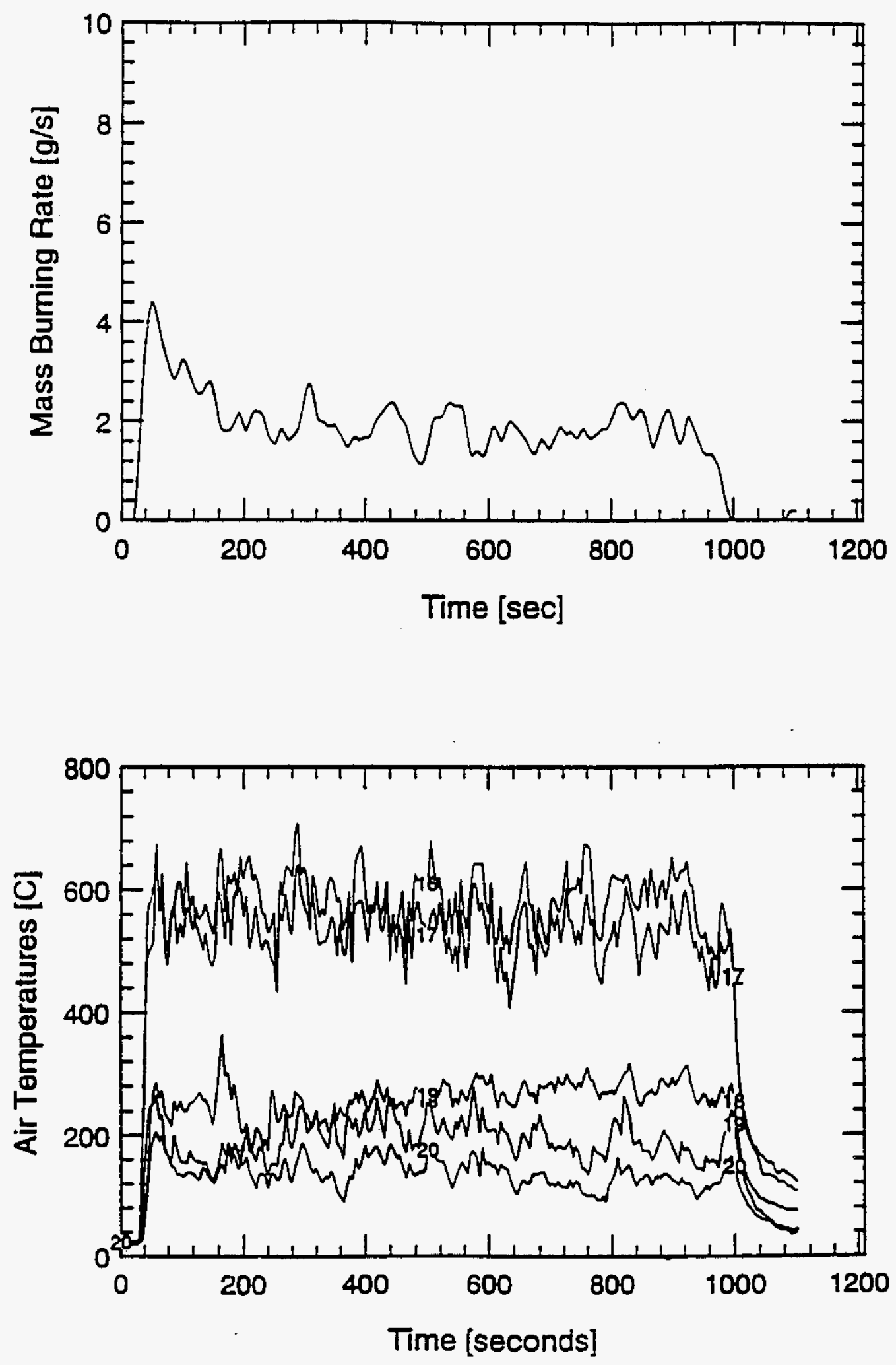
SWD12 - Kerosene - Stacked Drums 12" apart
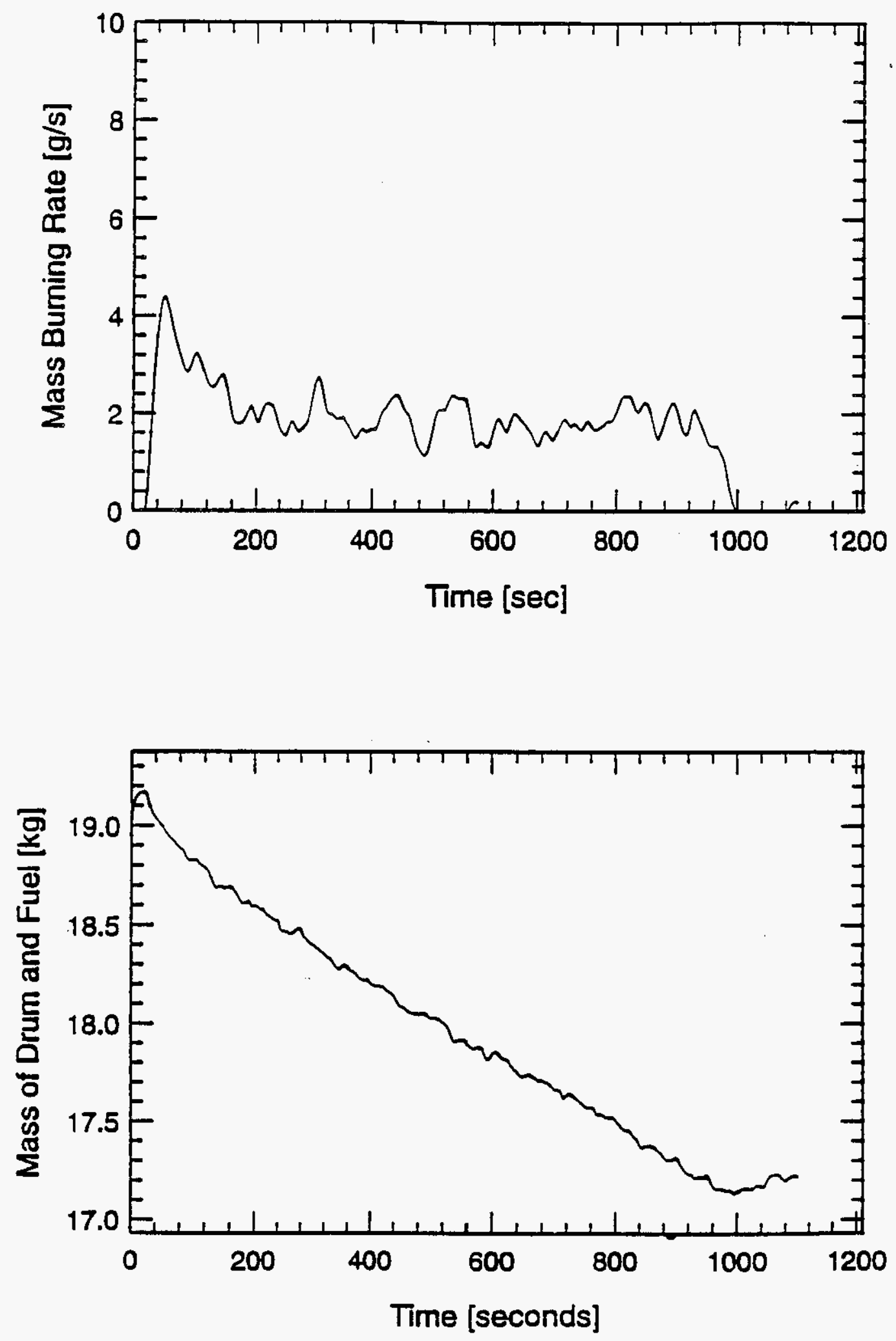
SWD12 - Kerosene - Stacked Drums 12" apart
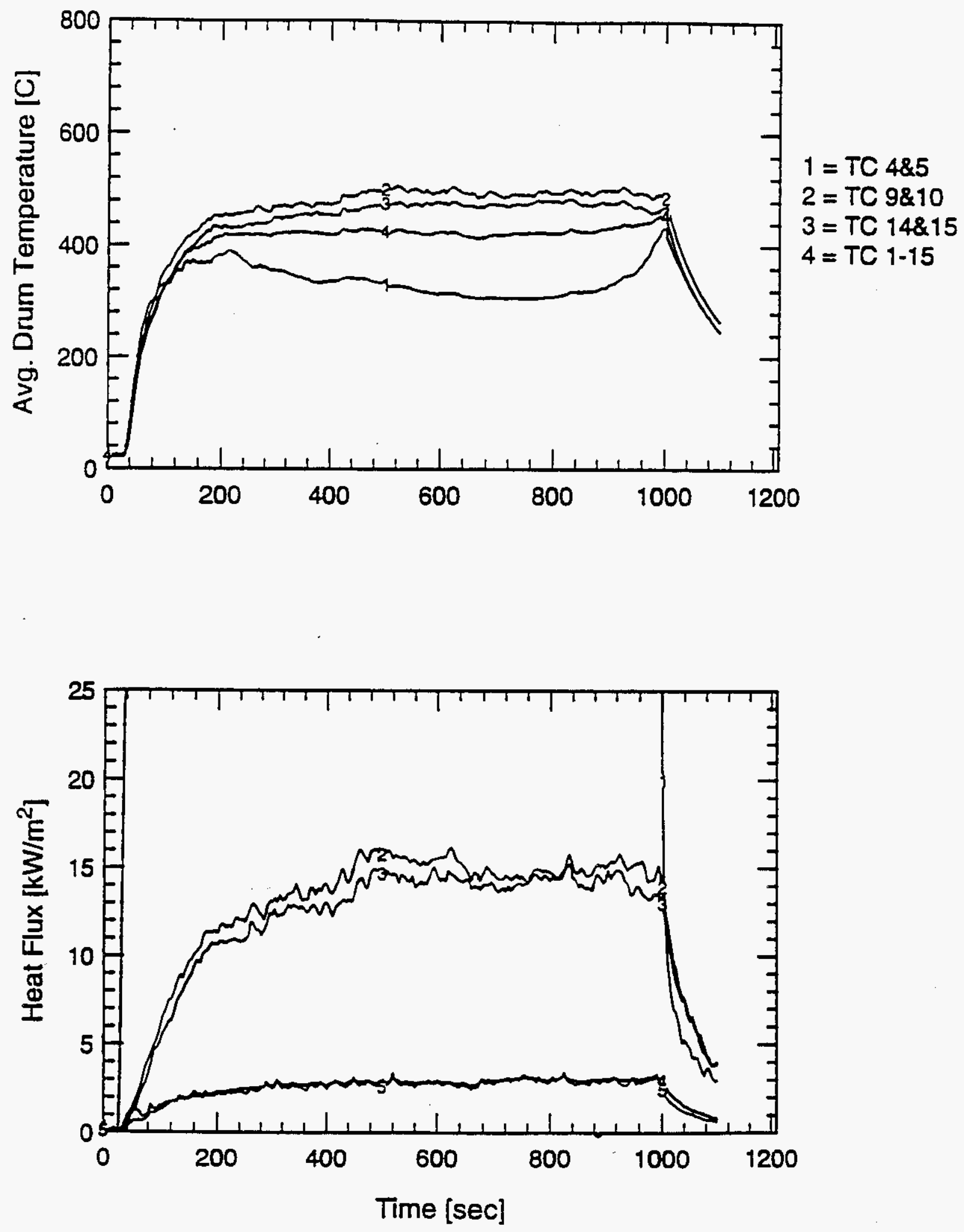
SWD12 - Kerosene - Stacked Drums 12" apart
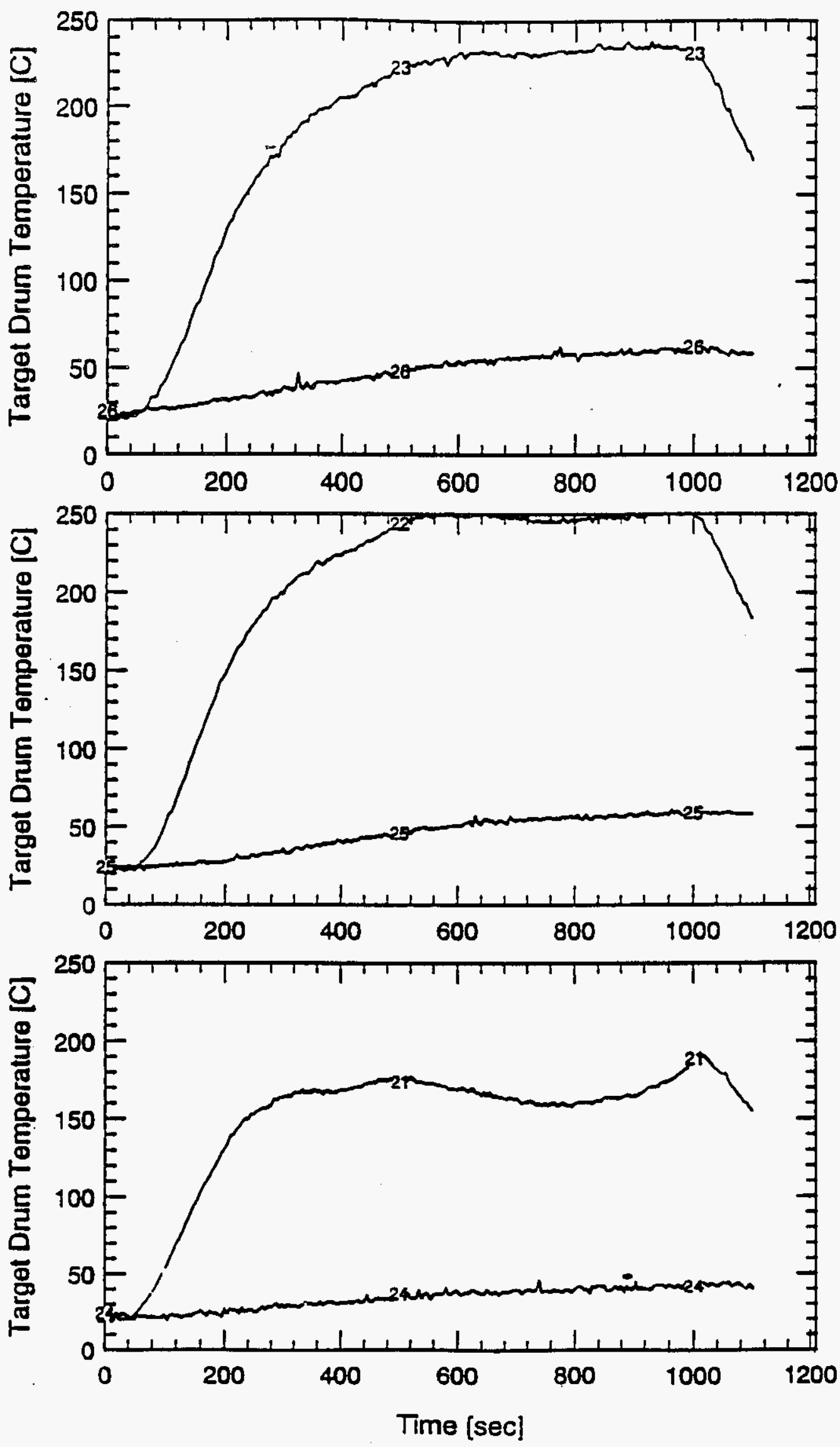

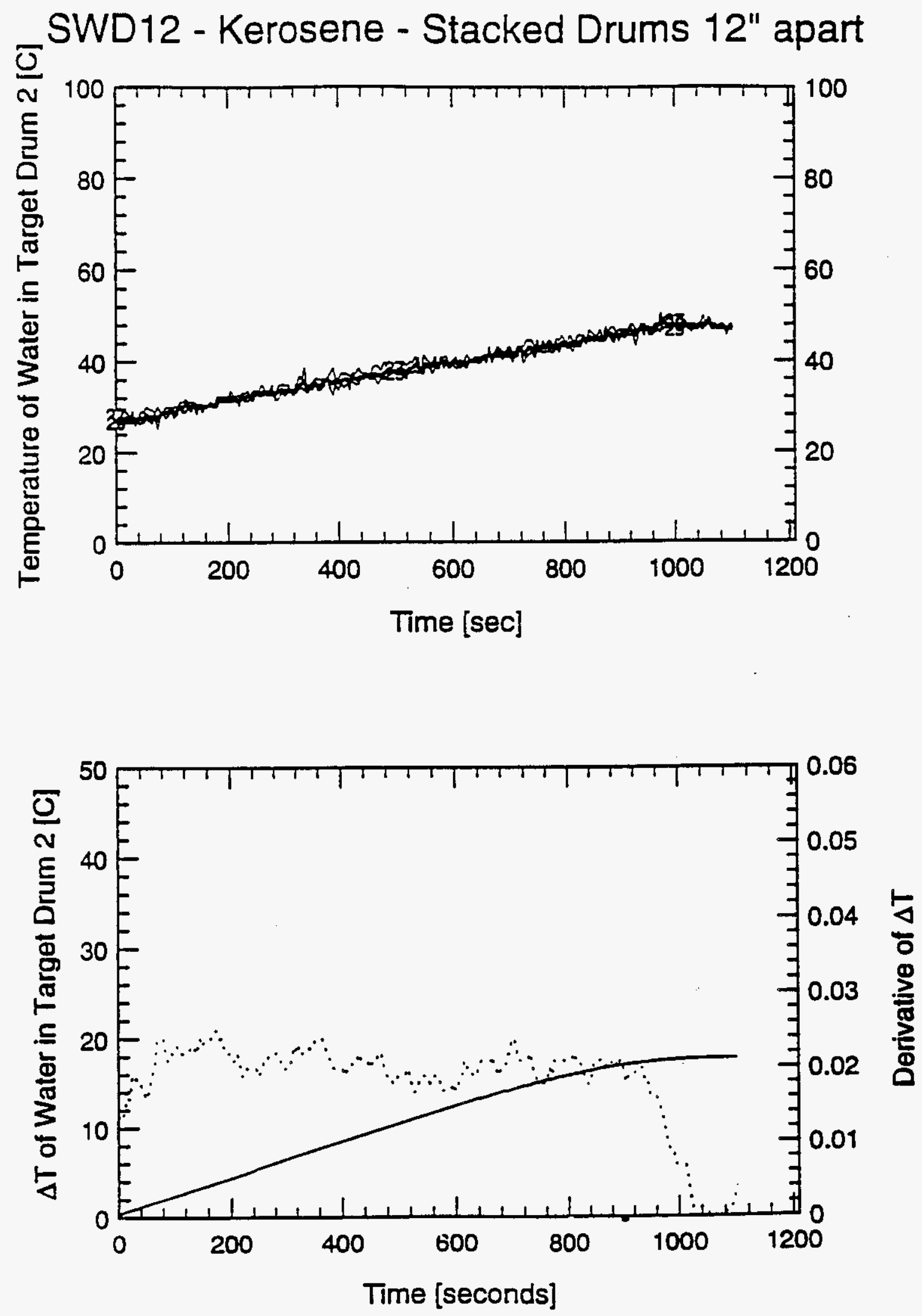
SWD12 - Kerosene - Stacked Drums 12" apart
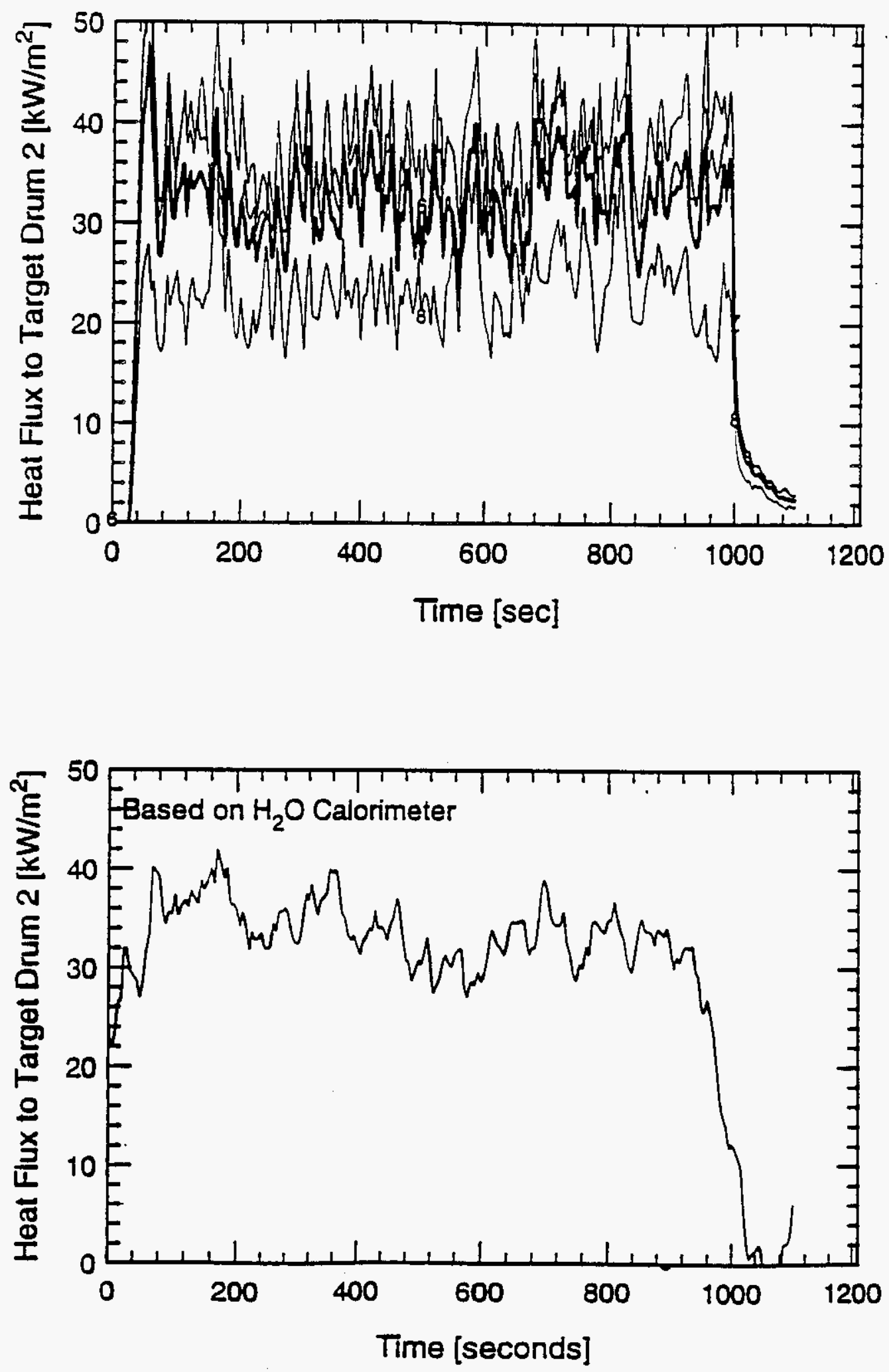
WHC-SD-WM-TRP-233 REV. 0

SWD 13 - DATA

B-78 
SWD13 - Kerosene - Stacked Drums 18" apart
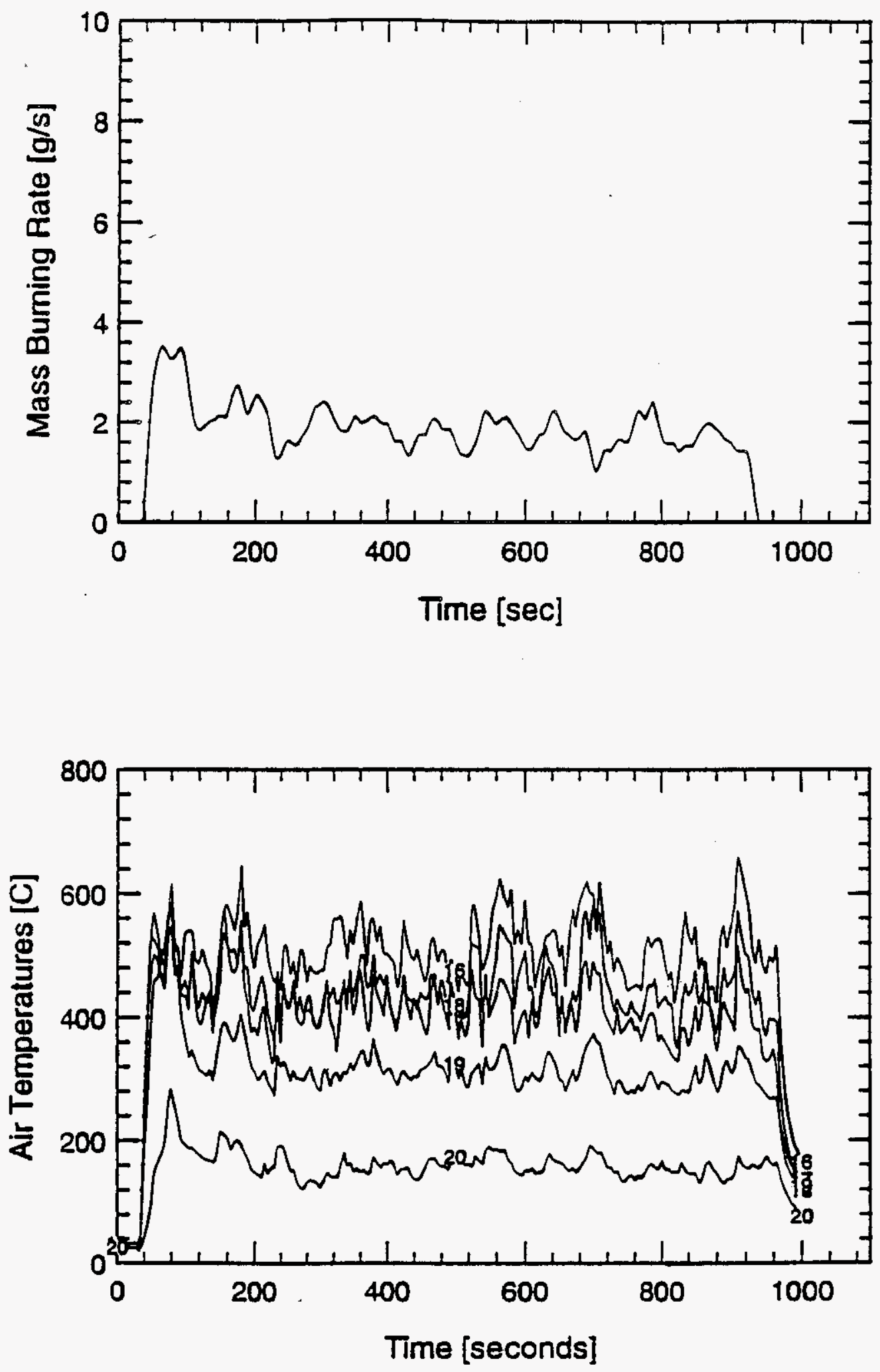
WHC-SD-WM-TRP-233 REV. 0

\section{SWD13 - Kerosene - Stacked Drums 18" apart}
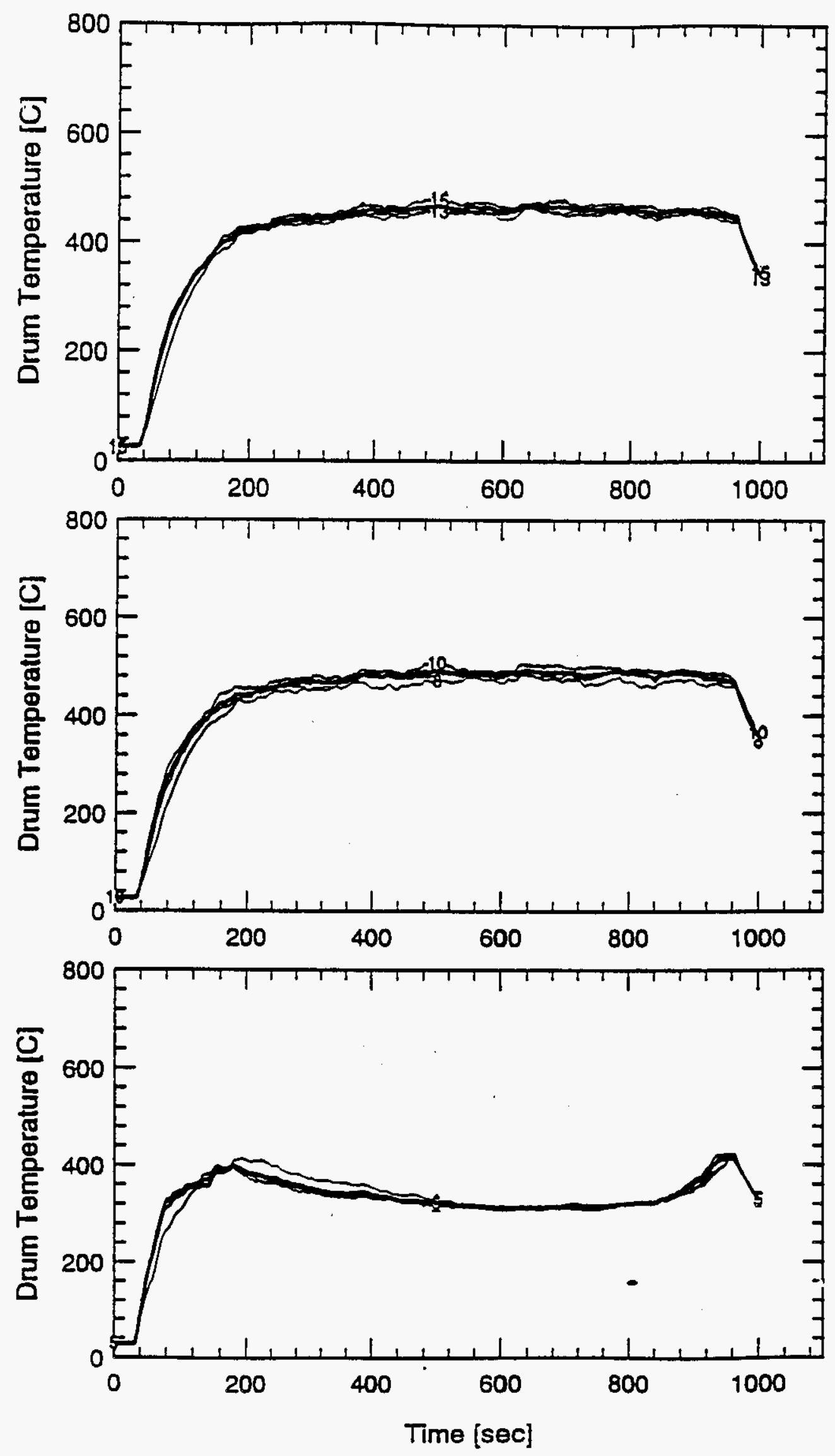

B-80 


\section{SWD13 - Kerosene - Stacked Drums 18" apart}
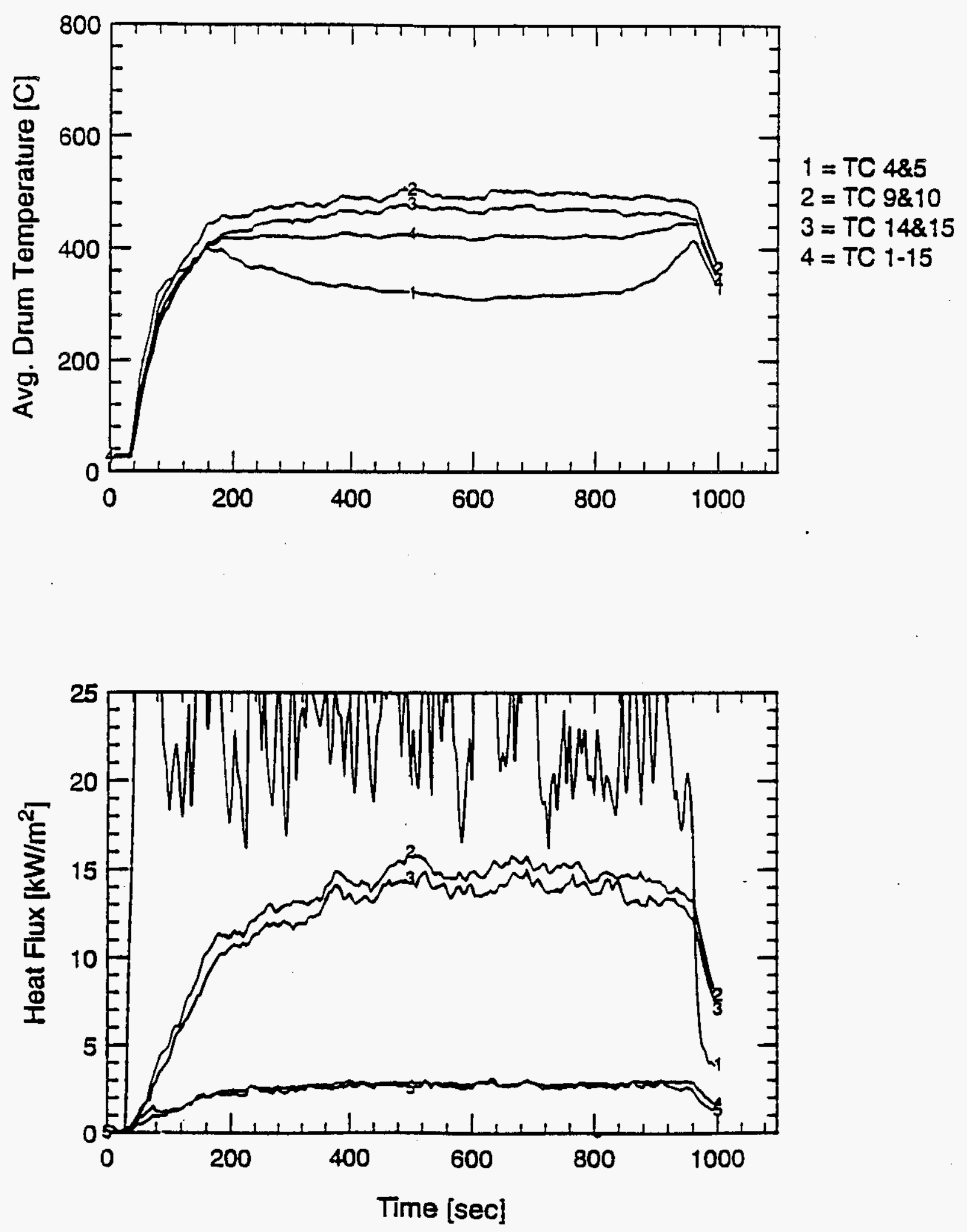
SWD13 - Kerosene - Stacked Drums 18" apart
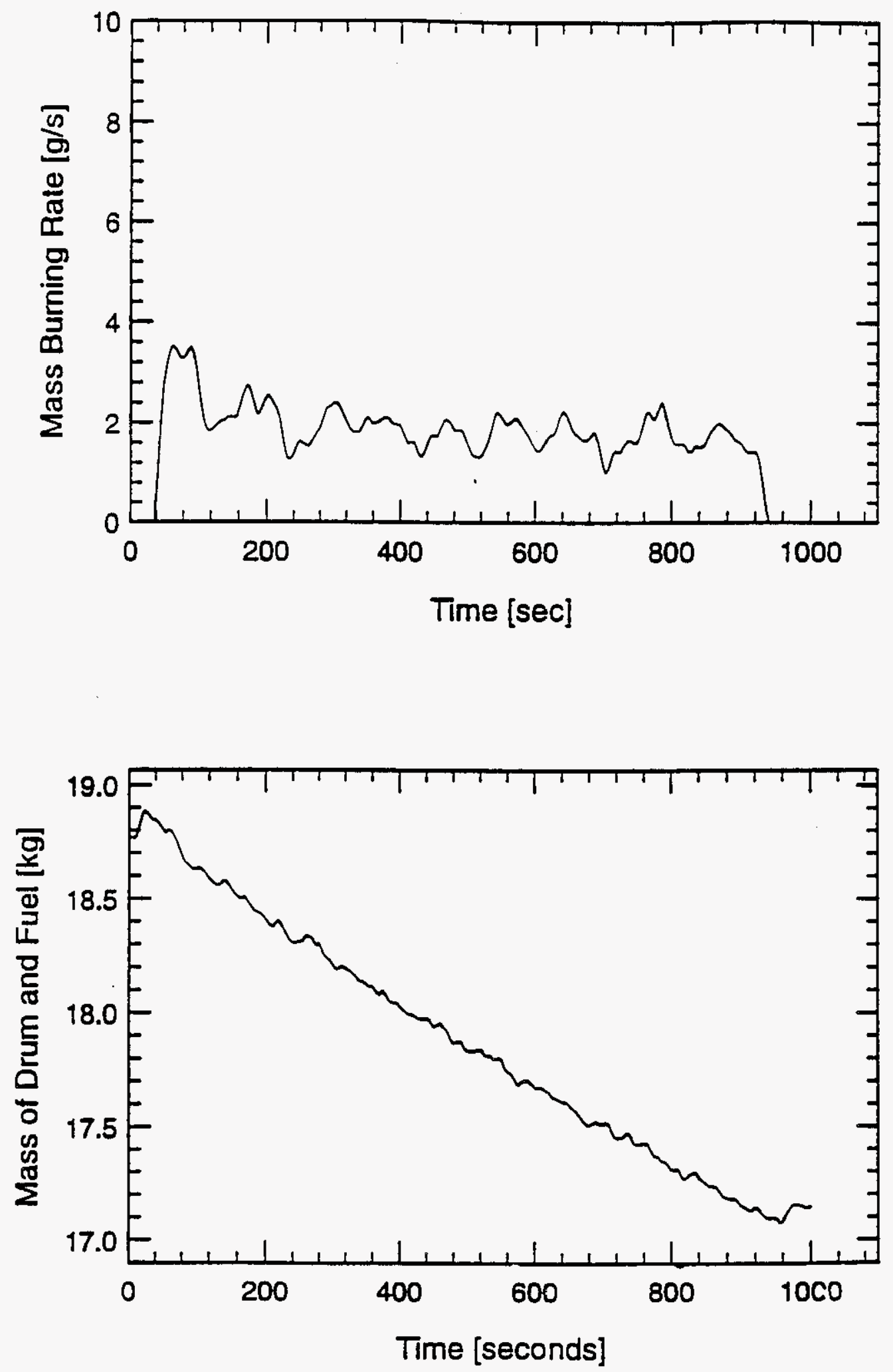

B-82 


\section{SWD13 - Kerosene - Stacked Drums 18" apart}
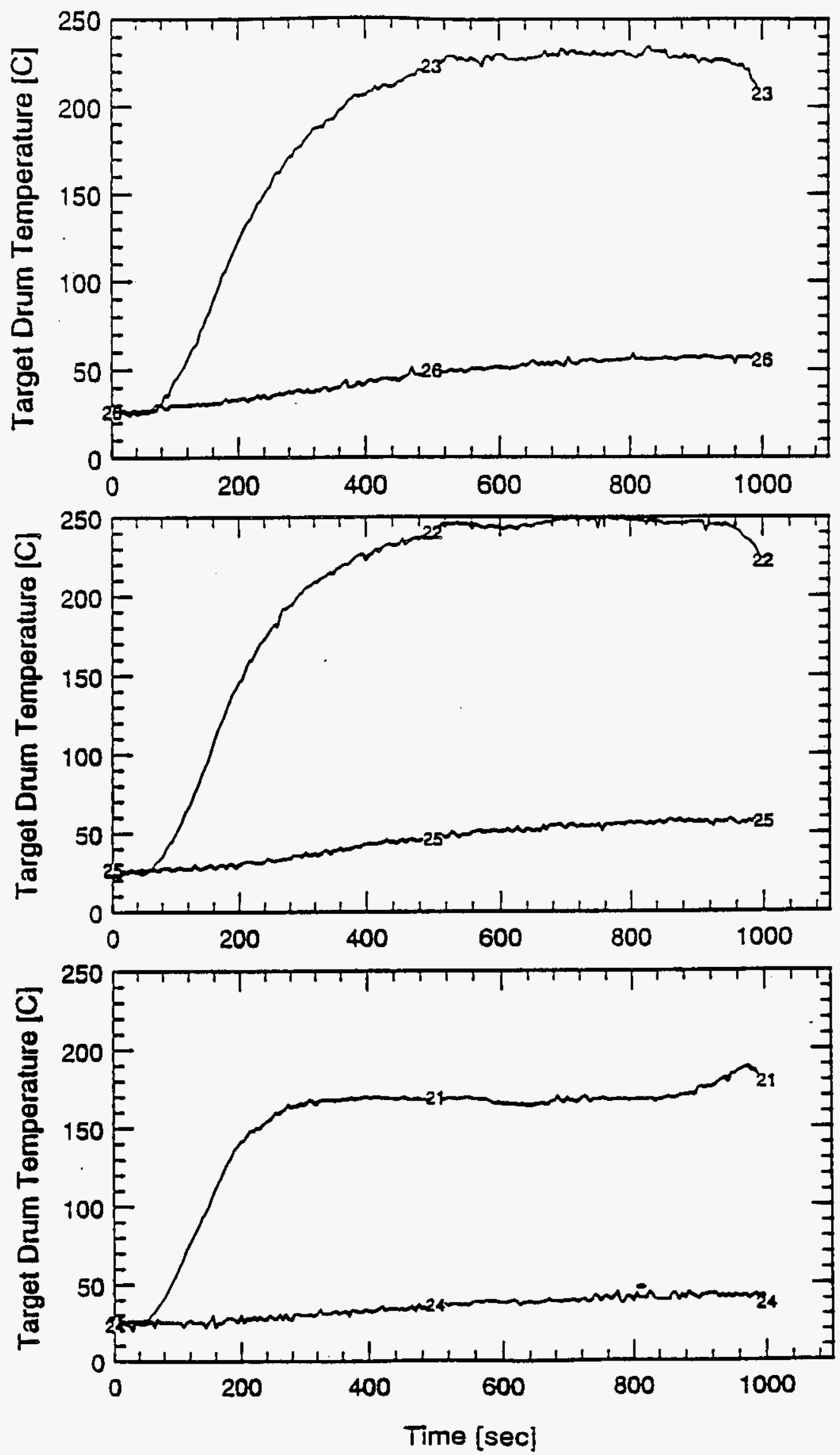

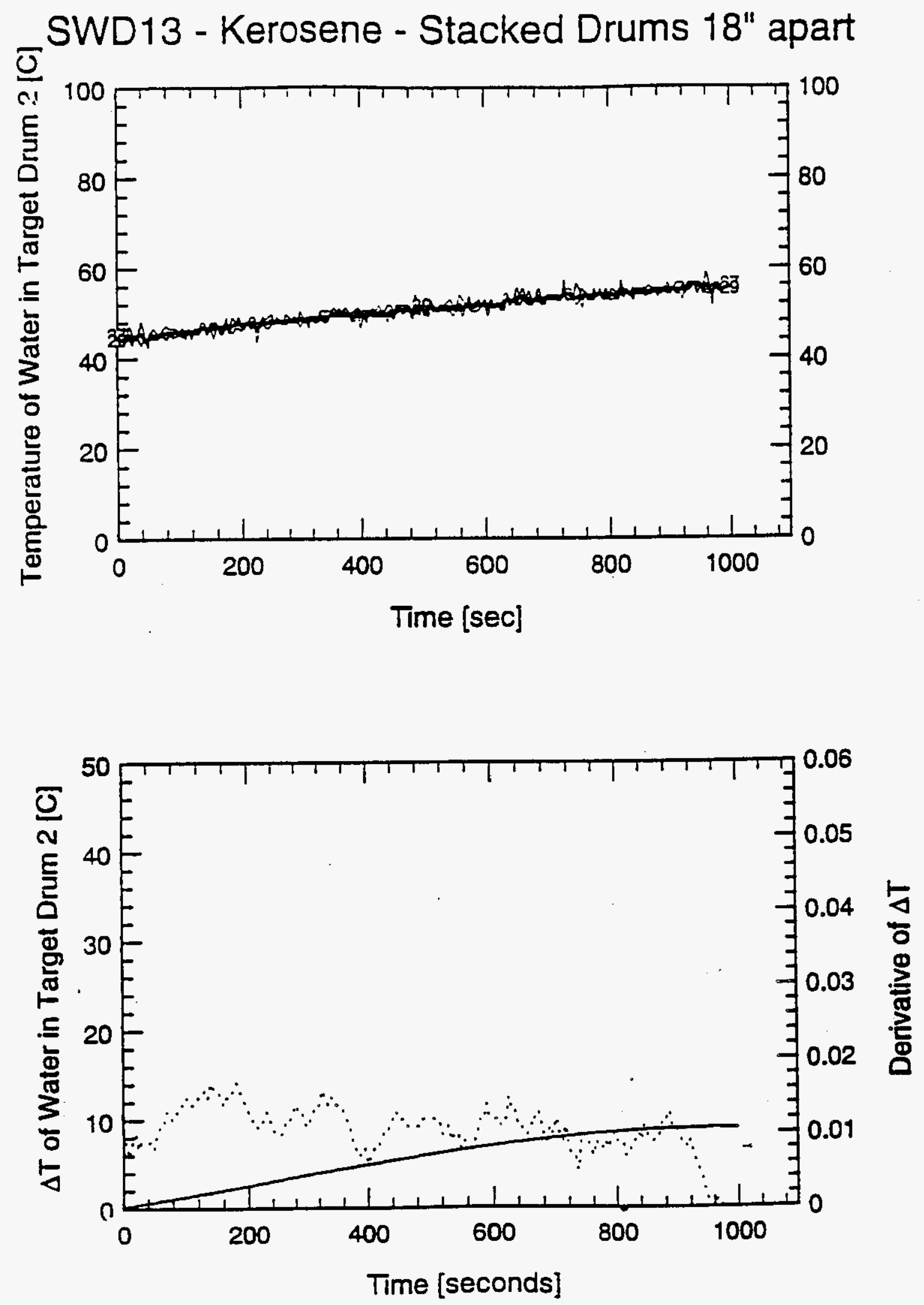


\section{SWD13 - Kerosene - Stacked Drums 18" apart}
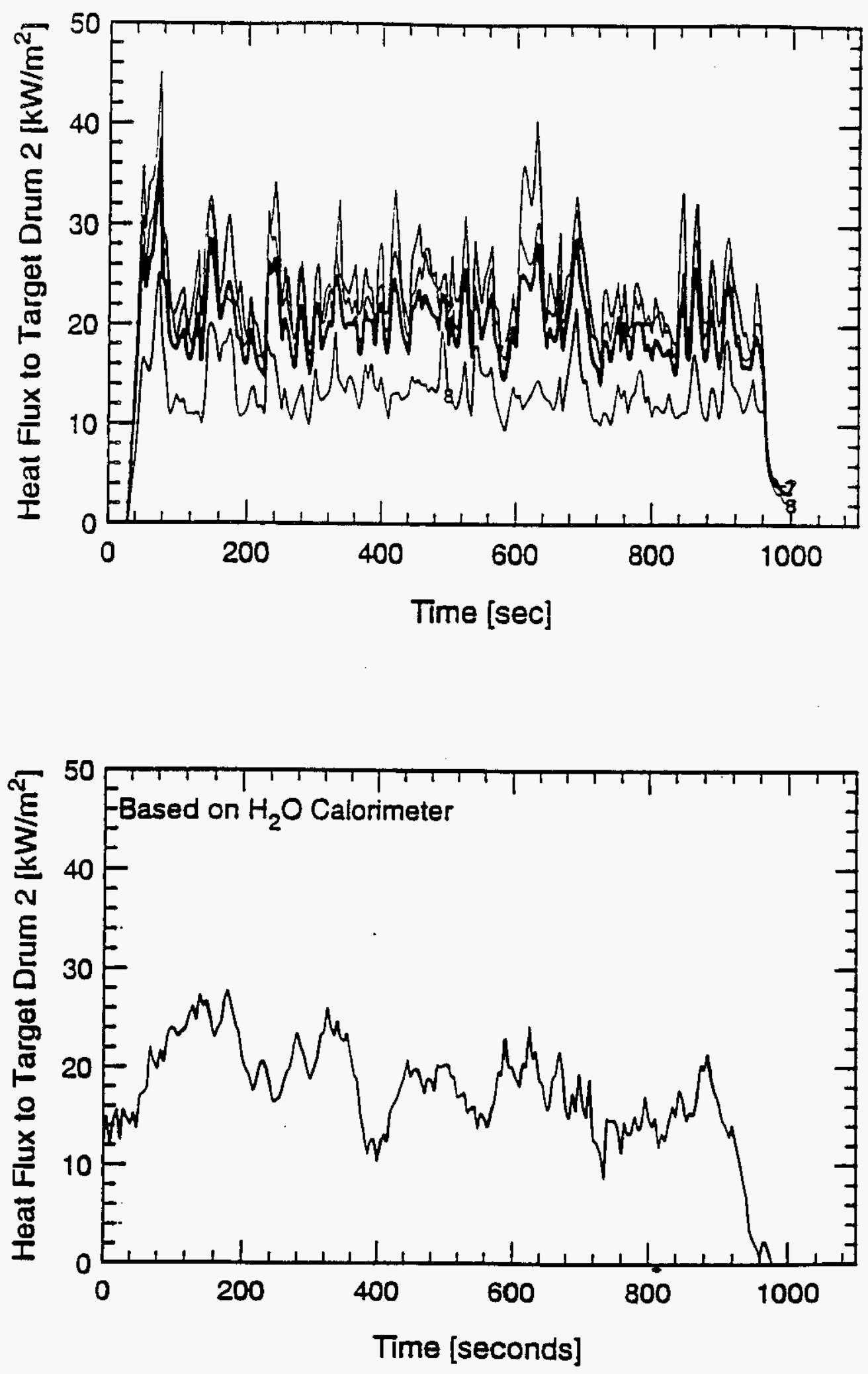
WHC-SD-WM-TRP-233 REV. 0

SWD 14 - DATA

B-86 
WHC-SD-WM-TRP-233 REV. 0

SWD14 - Kerosene - Stacked Drums 24" apart
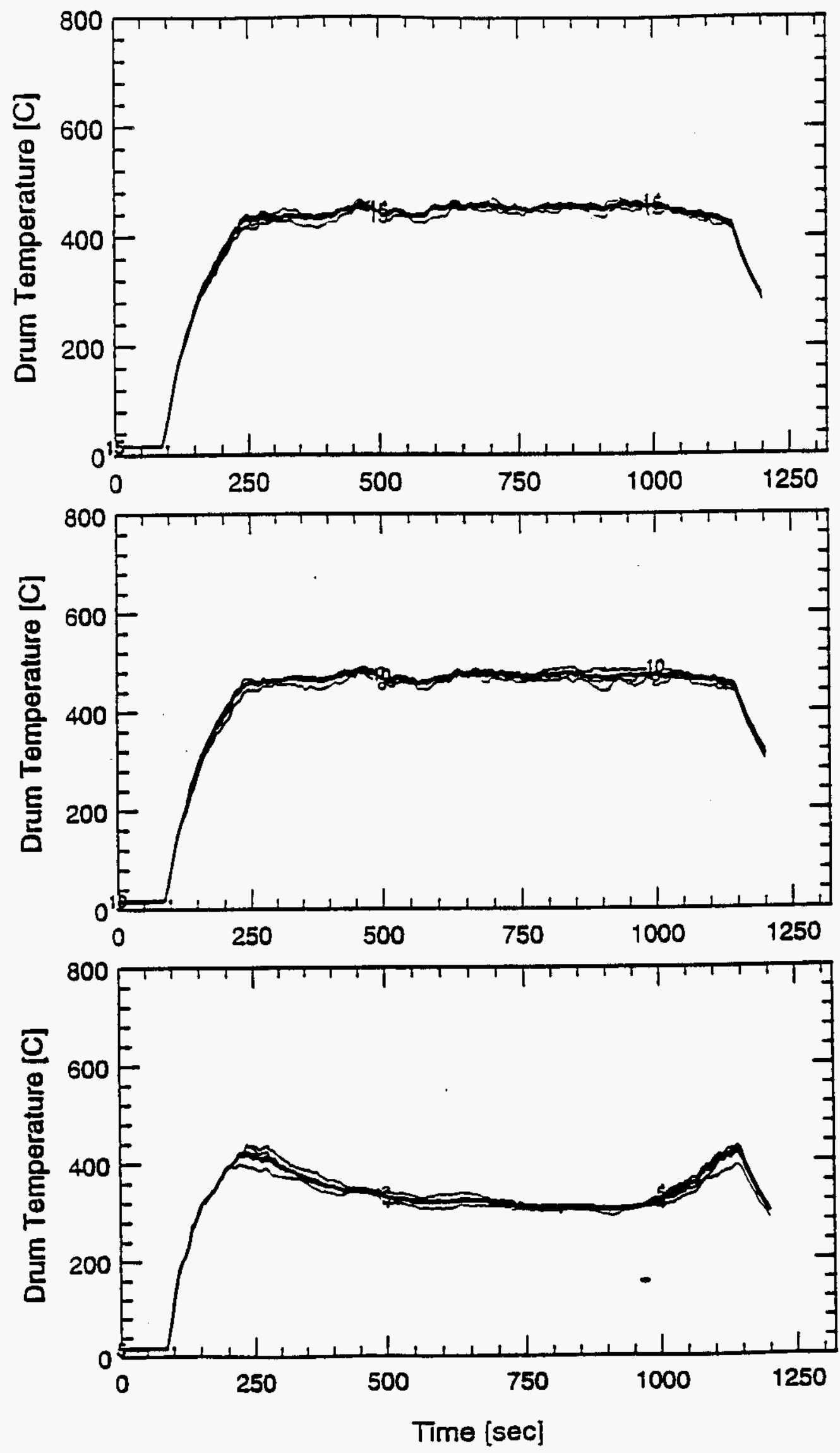
WHC-SD-WM-TRP-233 REV. 0

SWD14 - Kerosene - Stacked Drums 24" apart
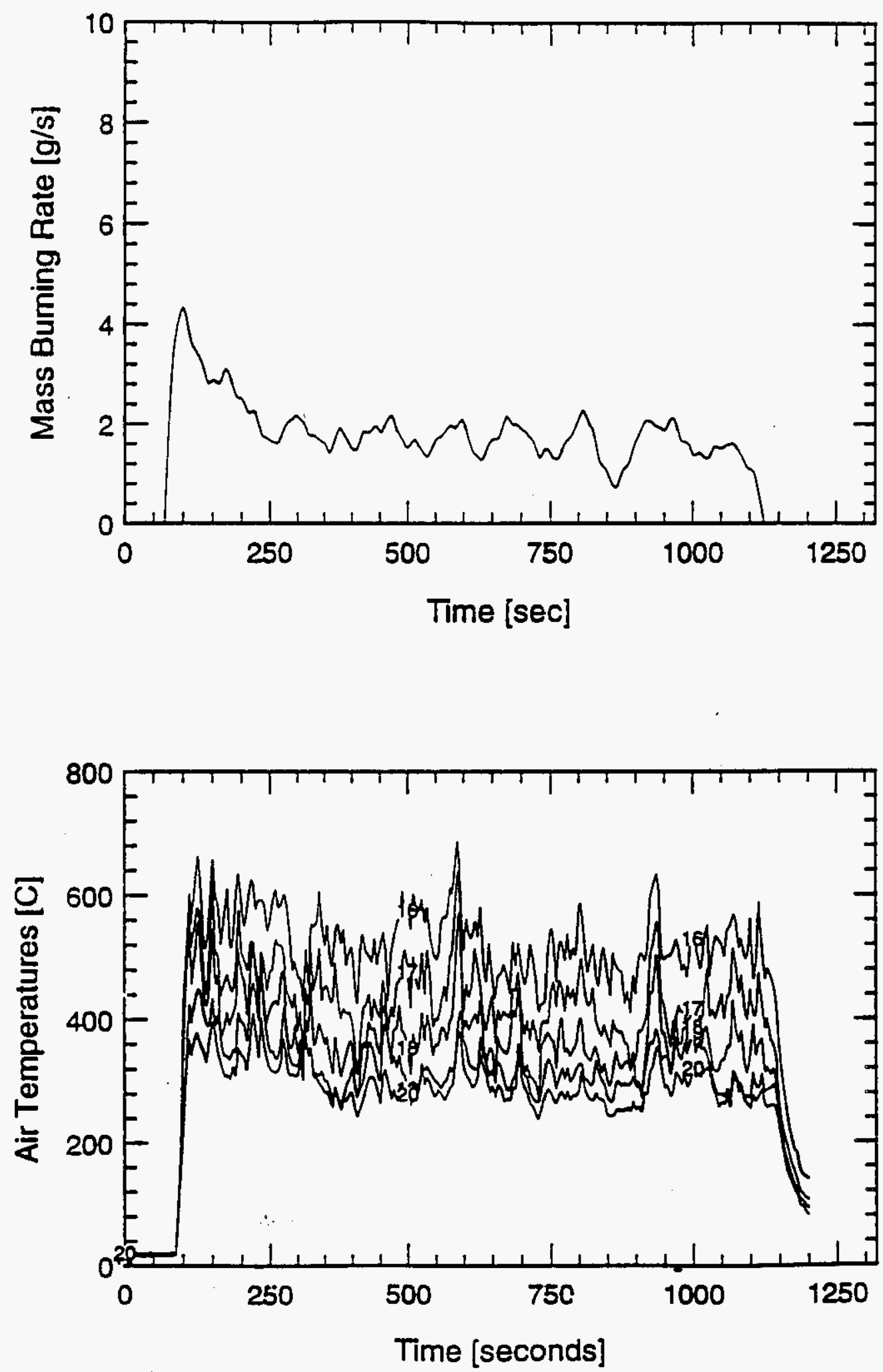
SWD14 - Kerosene - Stacked Drums 24" apart
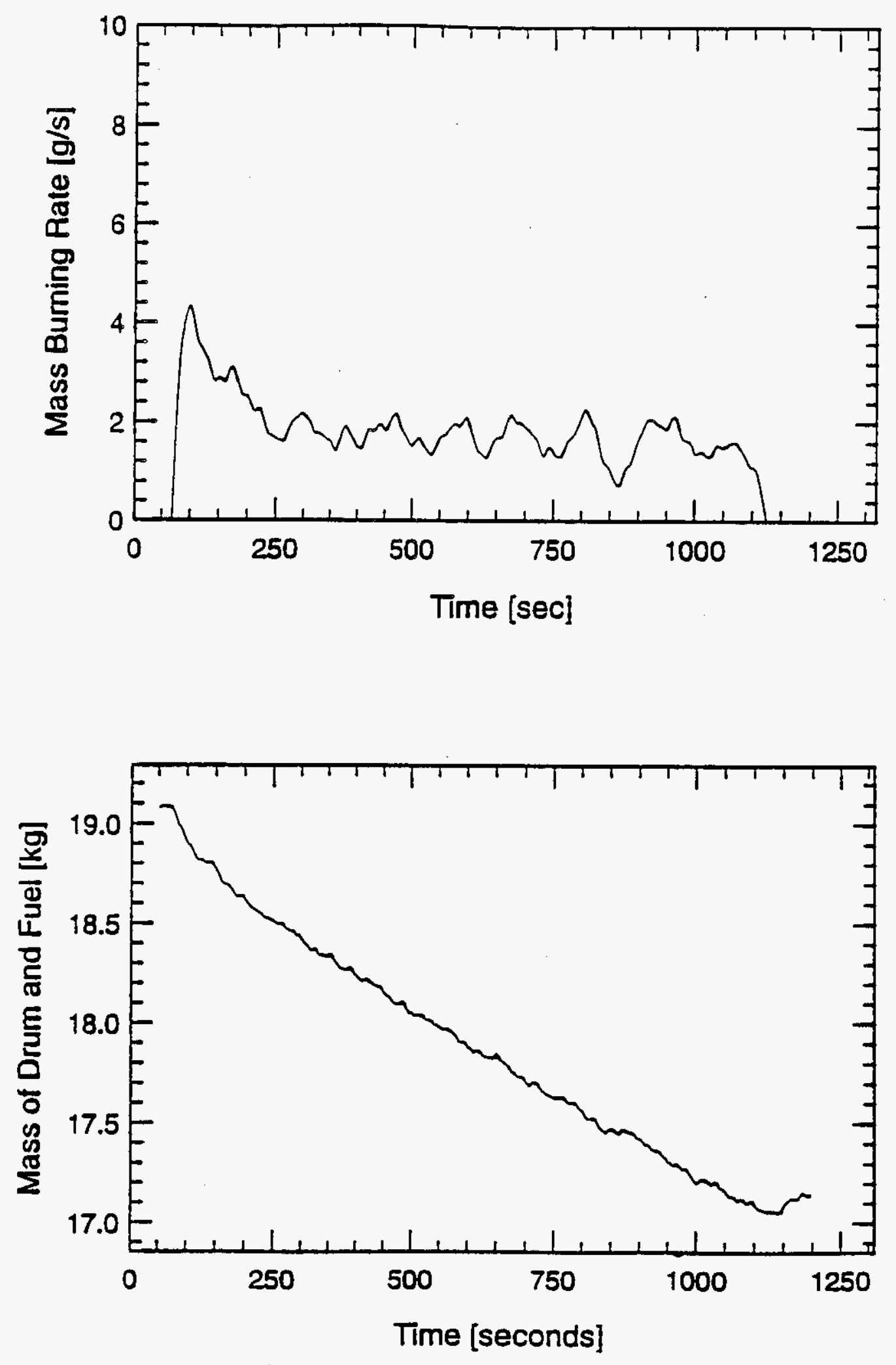
WHC-SD-WM-TRP-233 REV. 0

SWD14 - Kerosene - Stacked Drums 24" apart
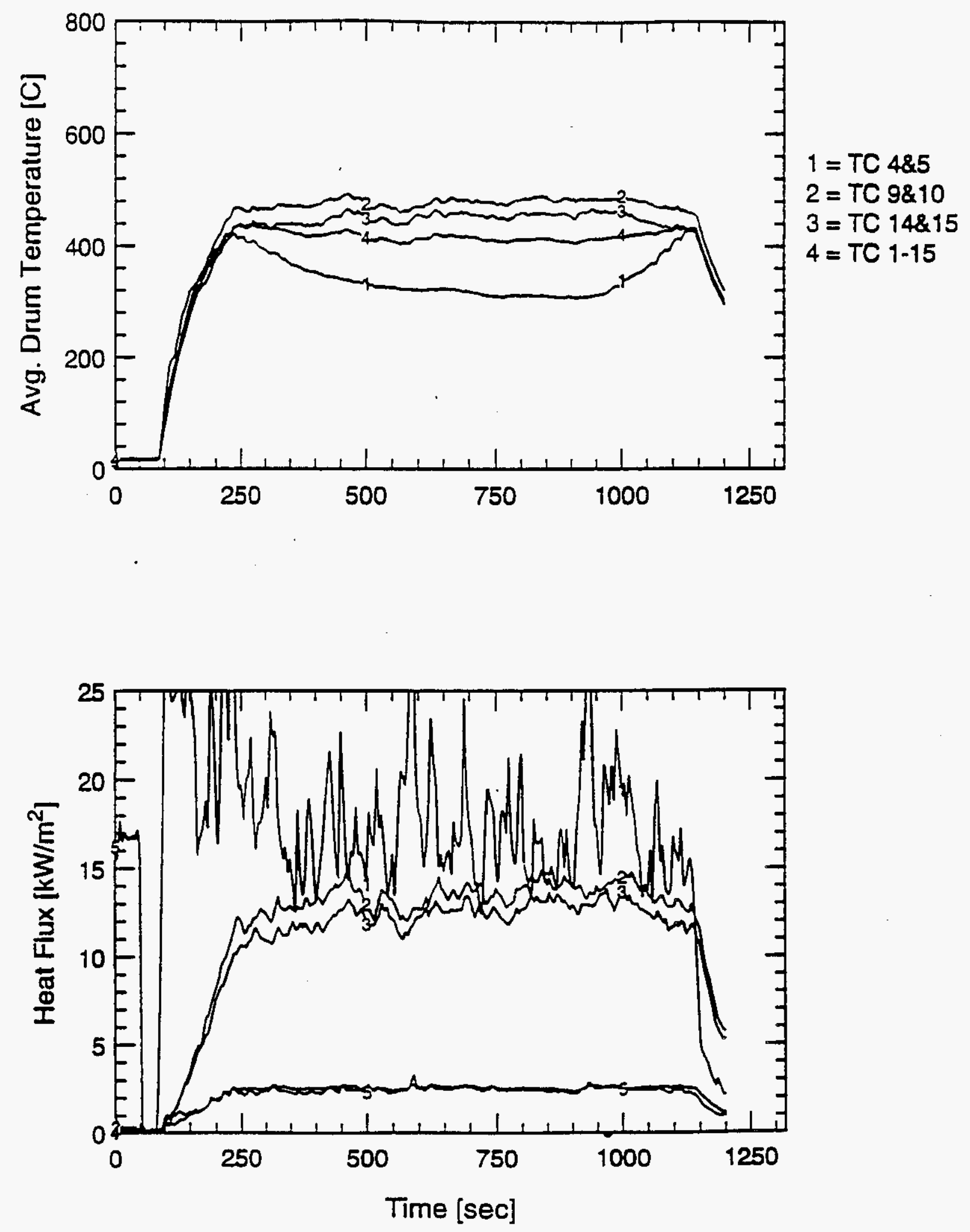
WHC-SD-WM-TRP-233 REV. 0

SWD14 - Kerosene - Stacked Drums 24" apart
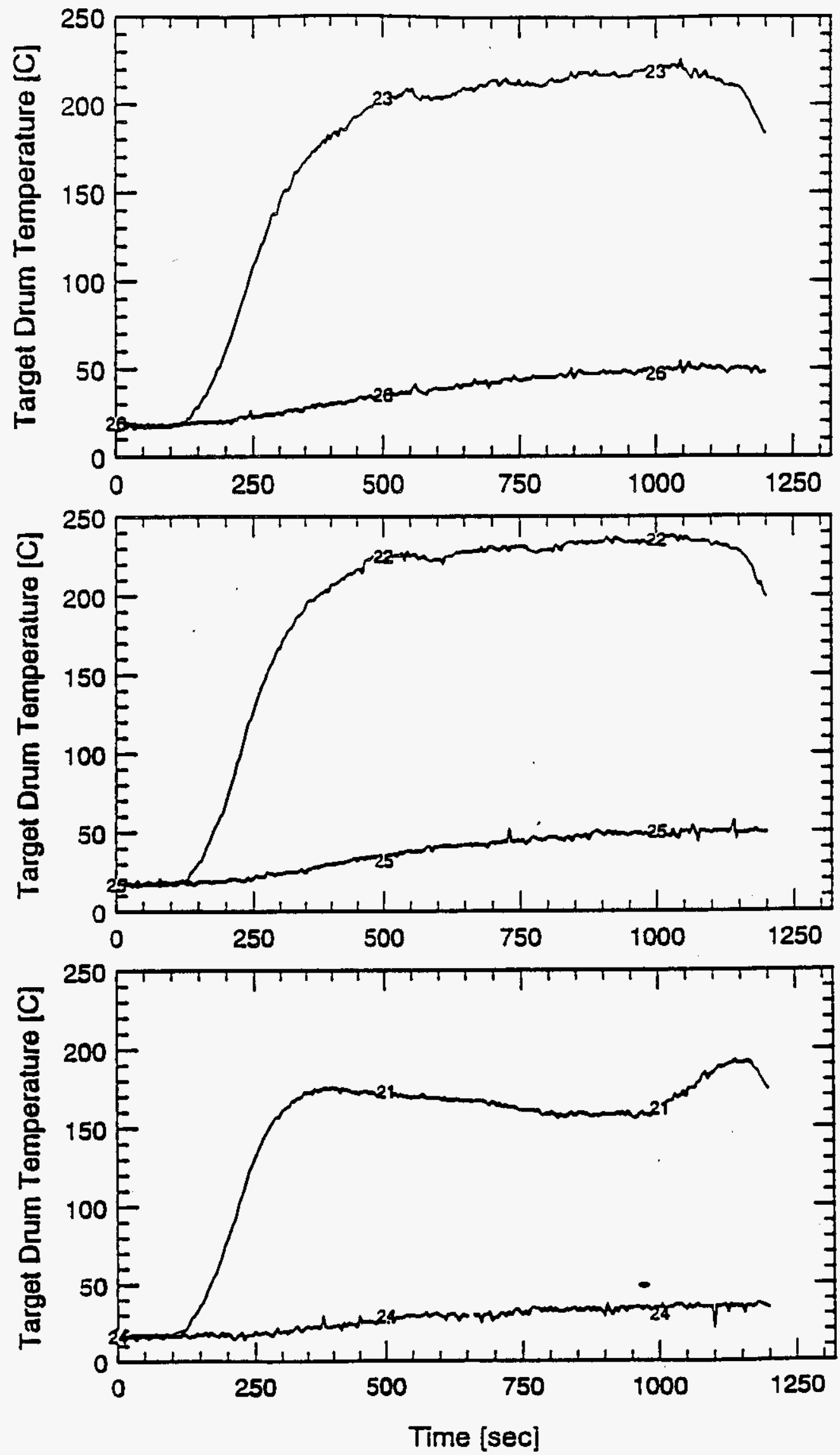
WHC-SD-WM-TRP-233 REV. 0
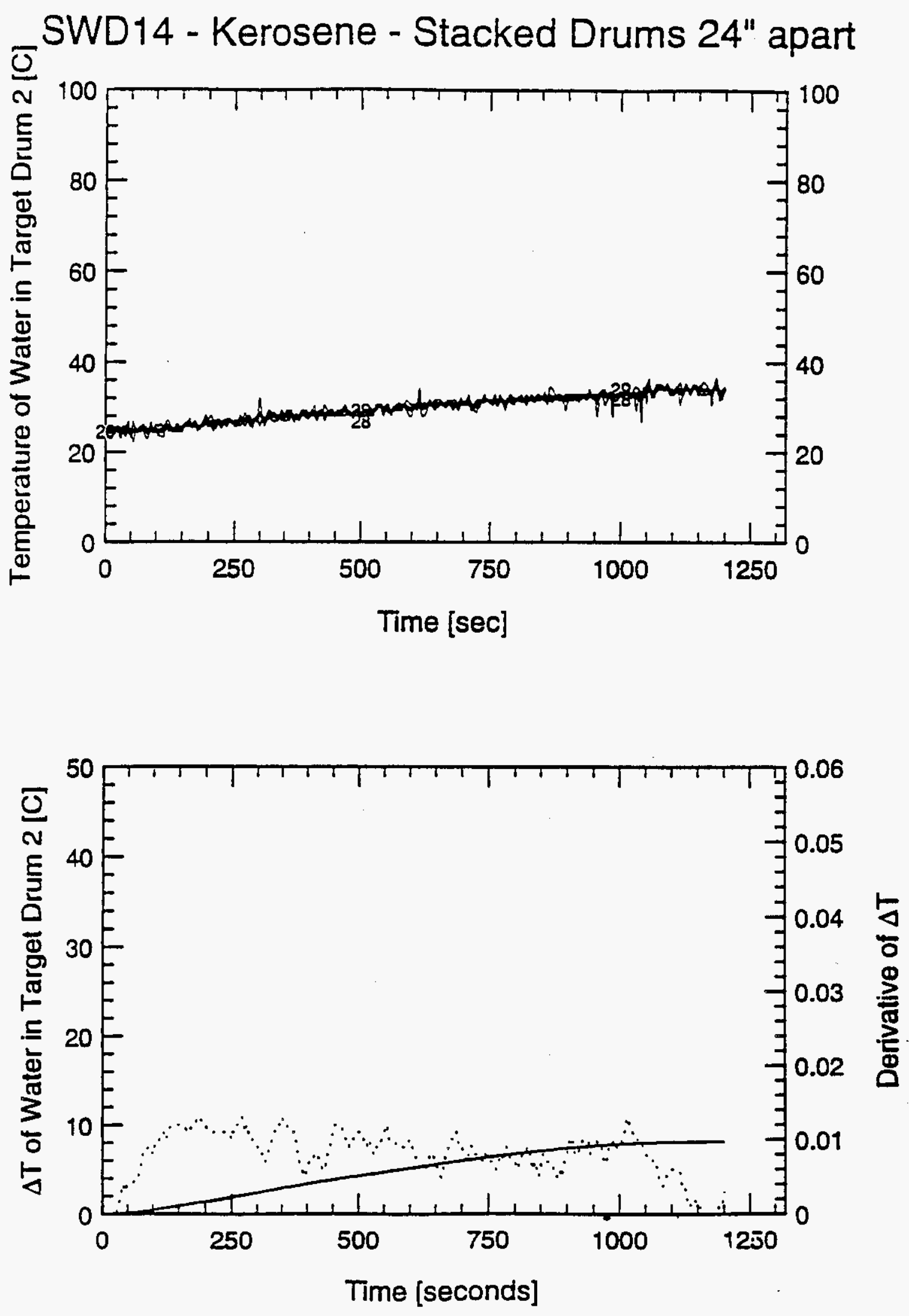
SWD14 - Kerosene - Stacked Drums 24" apart
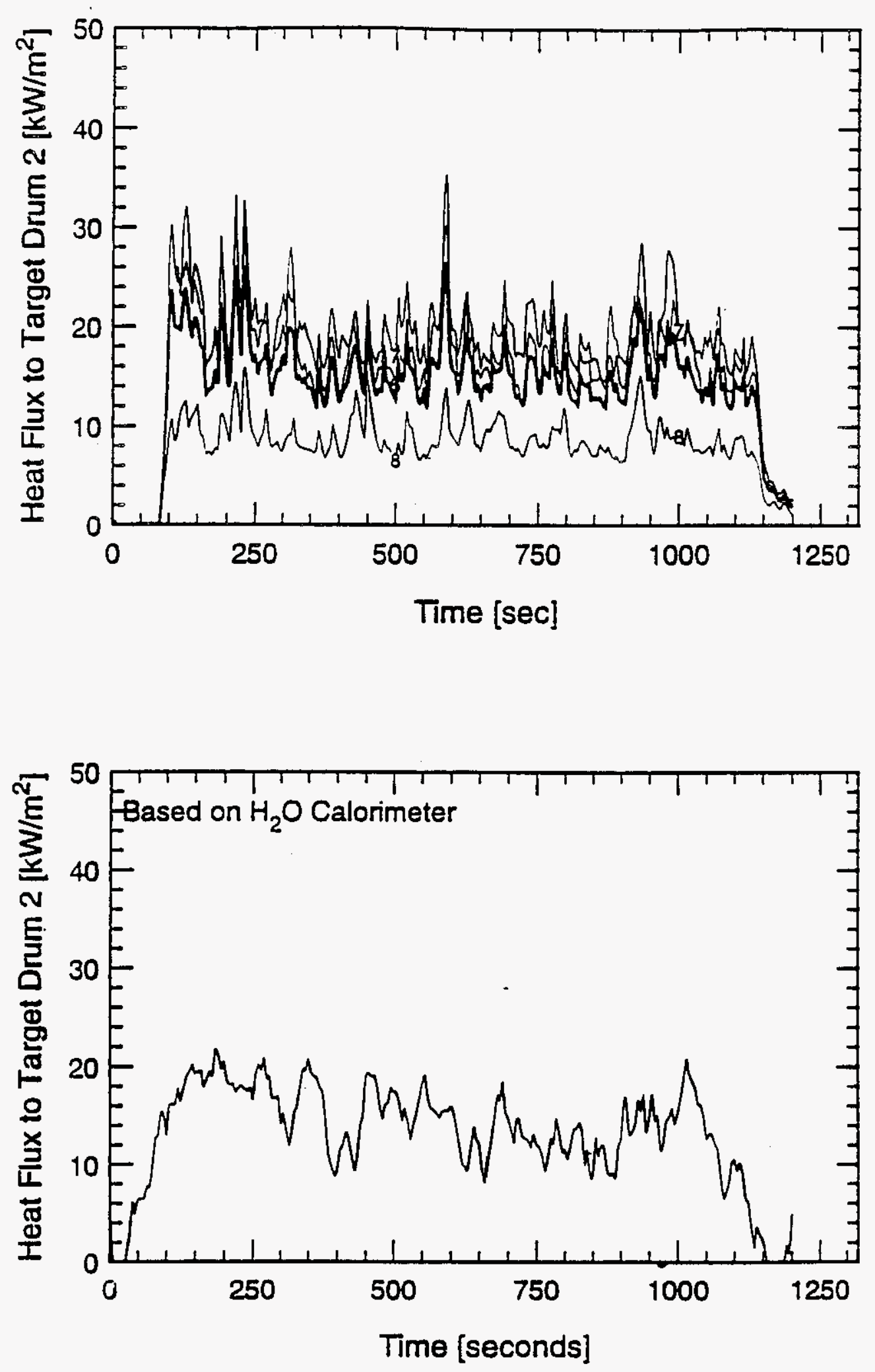
WHC-SD-WM-TRP-233 REV. 0

SWD 15 - DATA

B-94 
SWD15 - 5lb of Plastic - Stacked Drums 6" apart
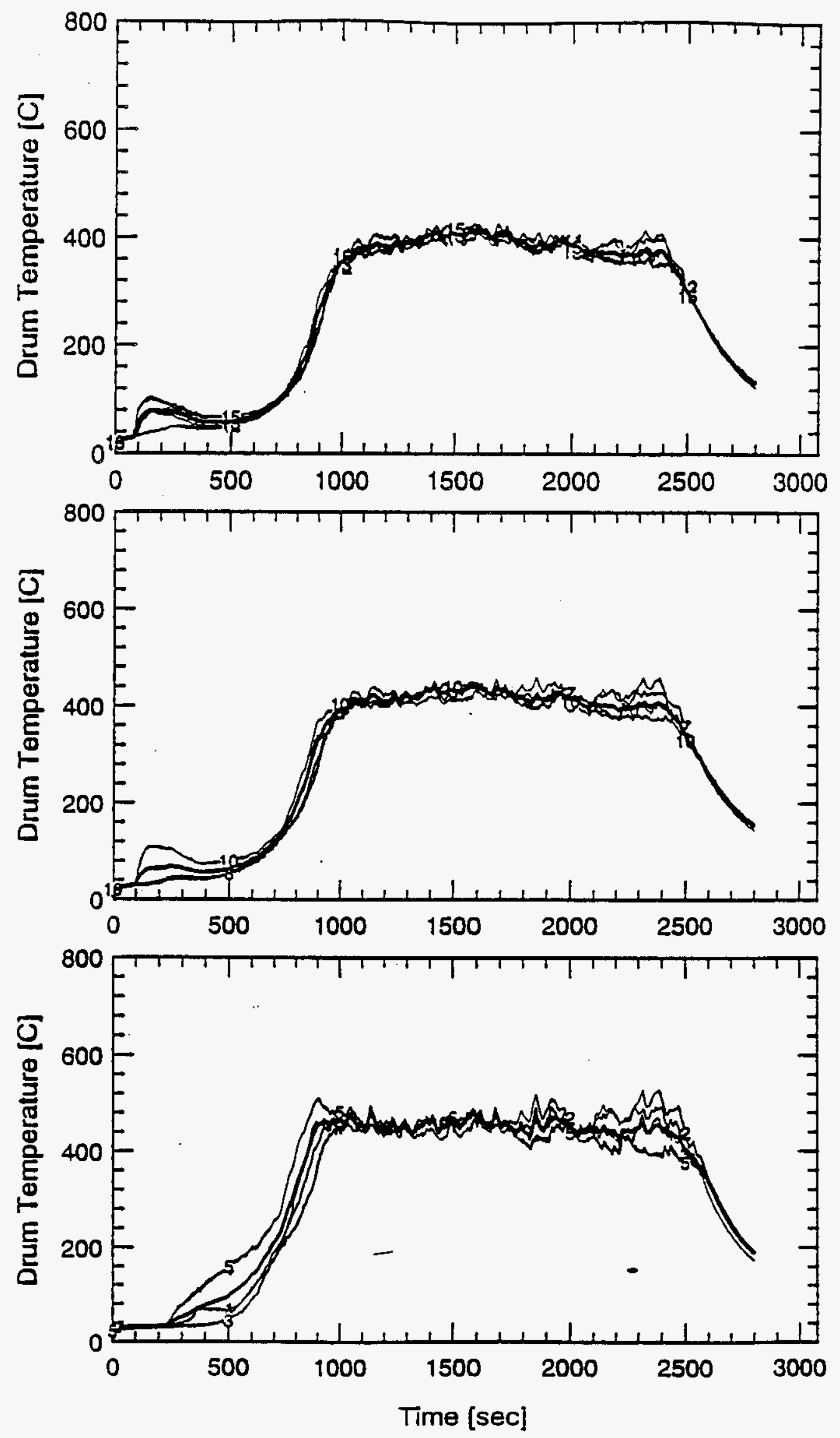
SWD15 - 5lb of Plastic - Stacked Drums 6" apart
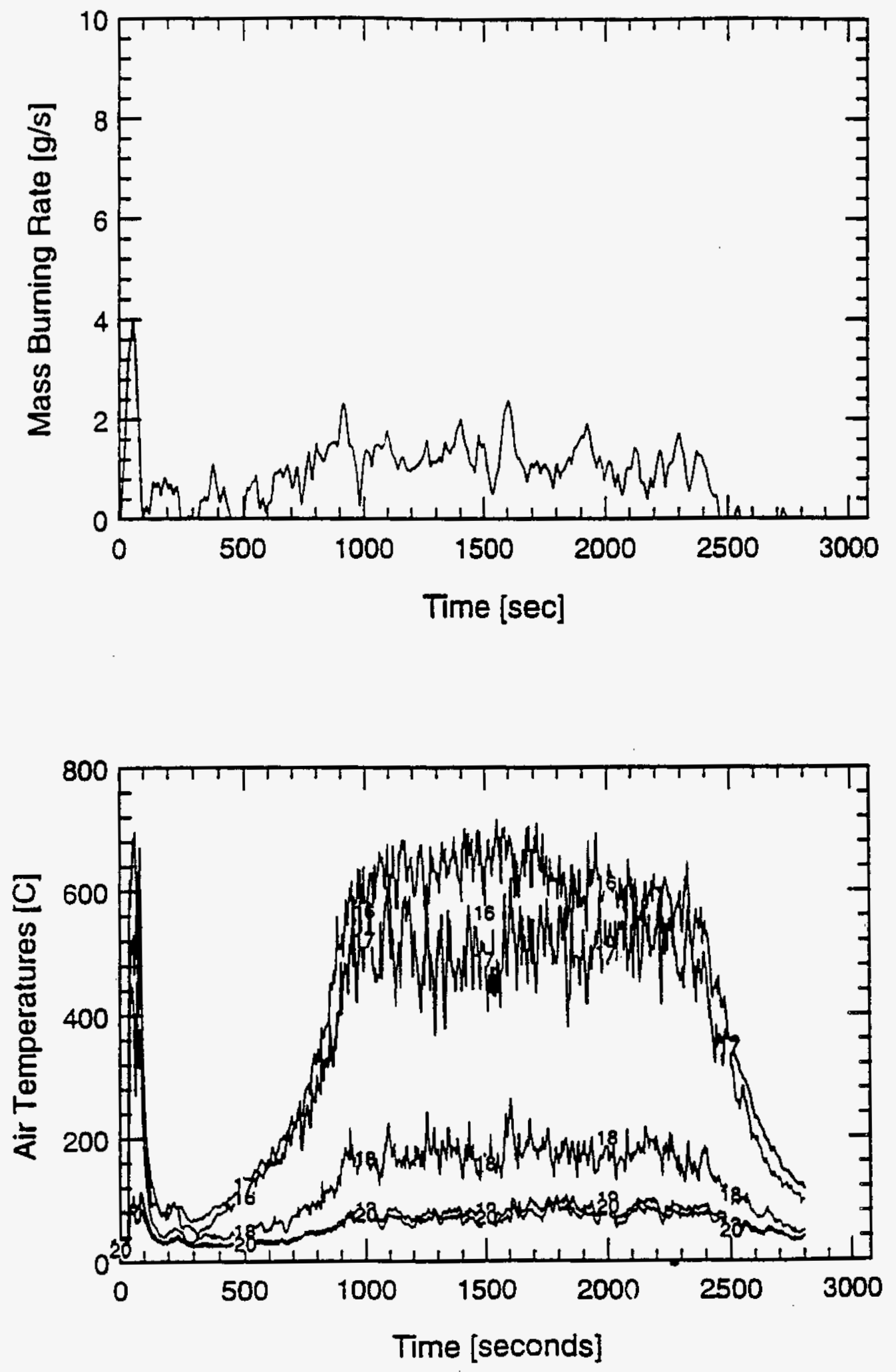
SWD15 - 5lb of Plastic - Stacked Drums 6" apart
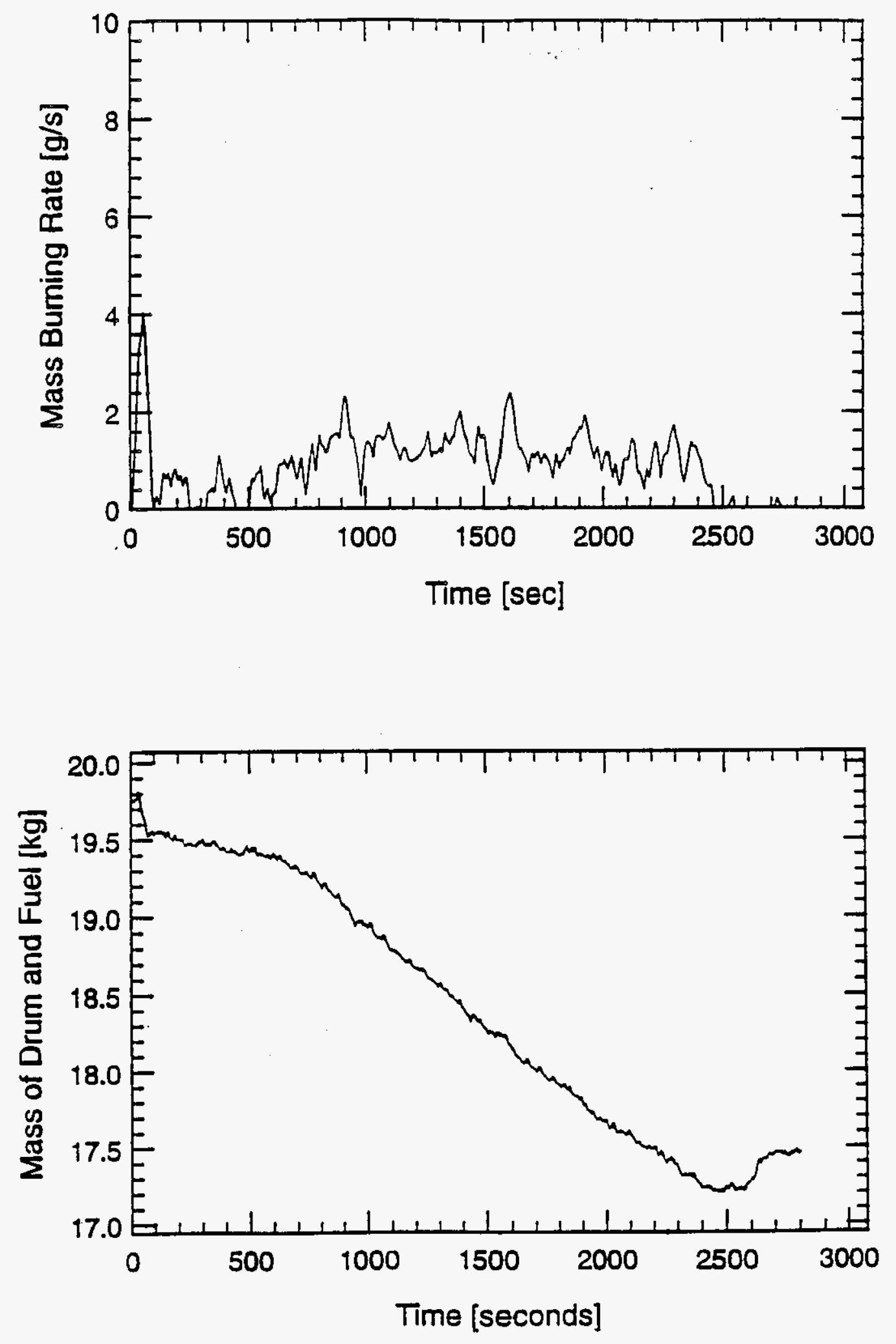
WHC-SD-WM-TRP-233 REV. 0

SWD15 - 5lb of Plastic - Stacked Drums 6" apart
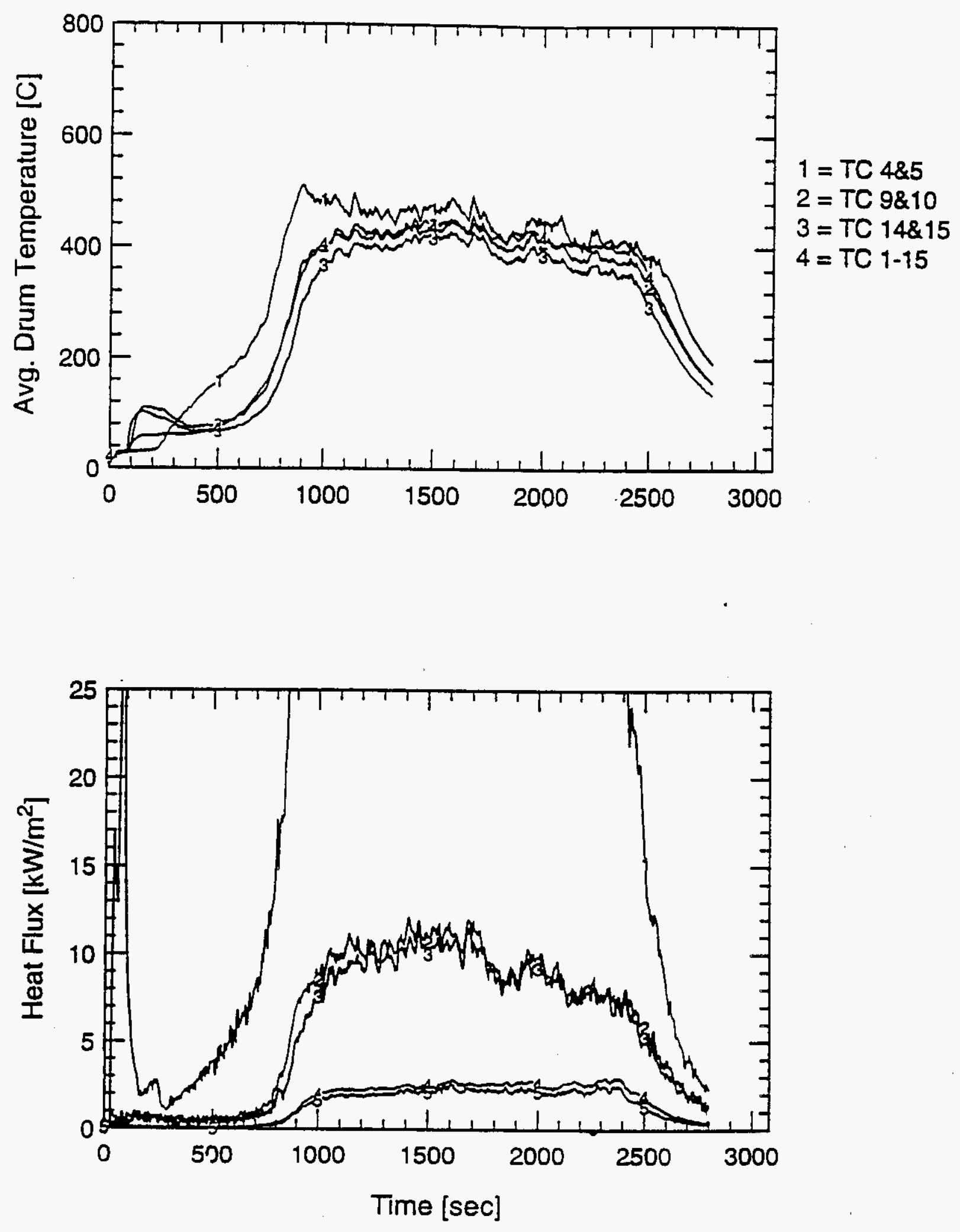


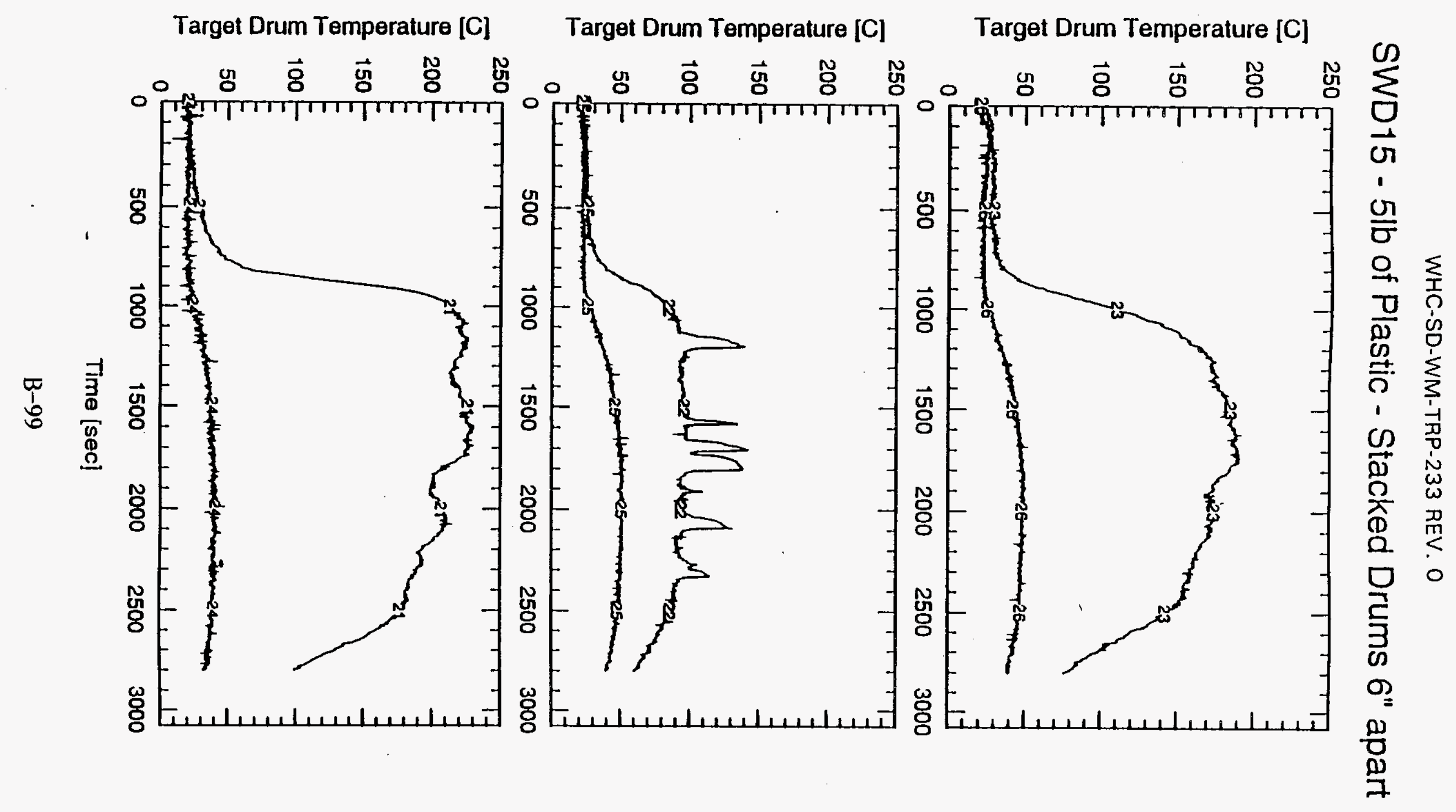



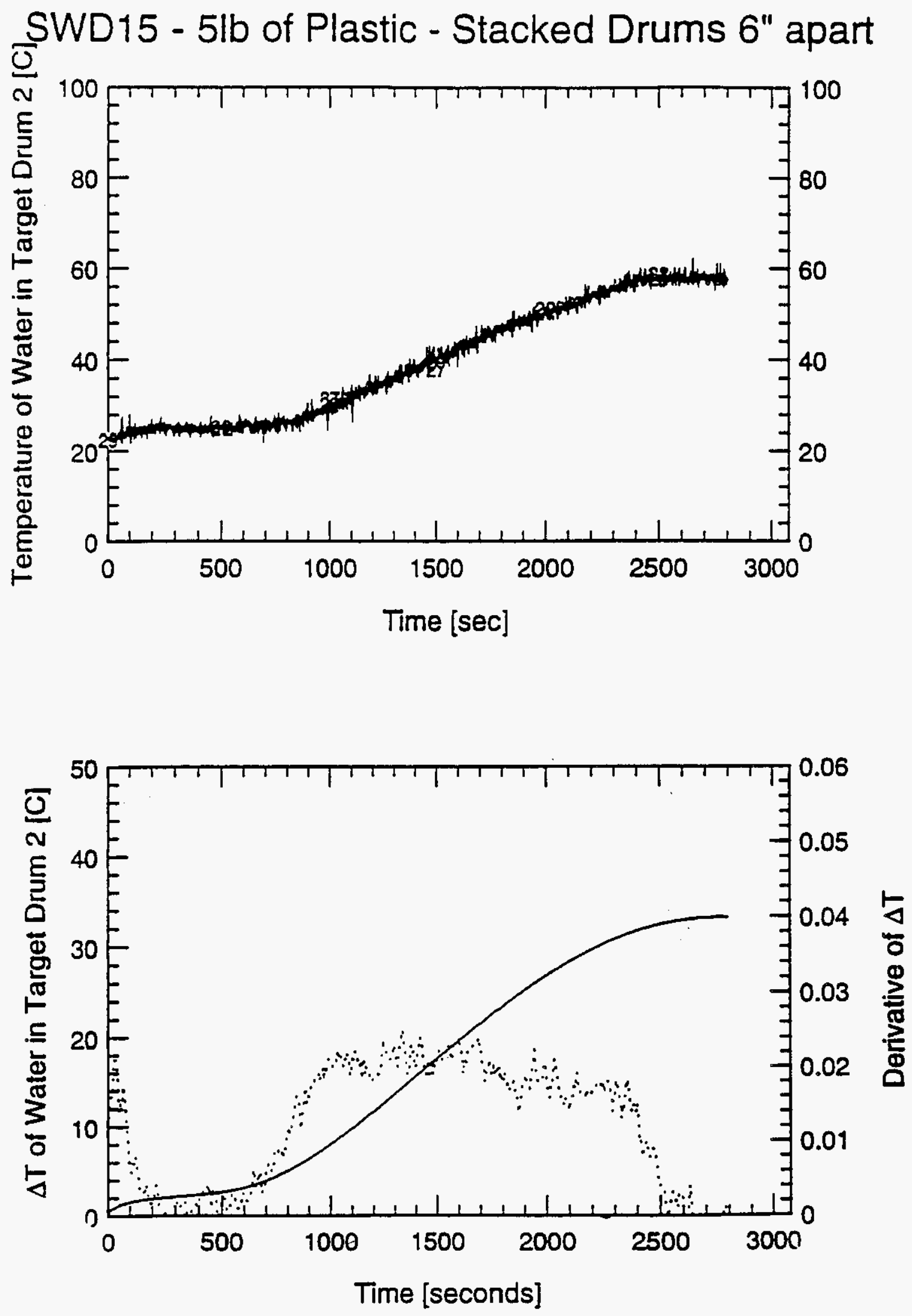
SWD15 - 5lb of Plastic - Stacked Drums 6" apart
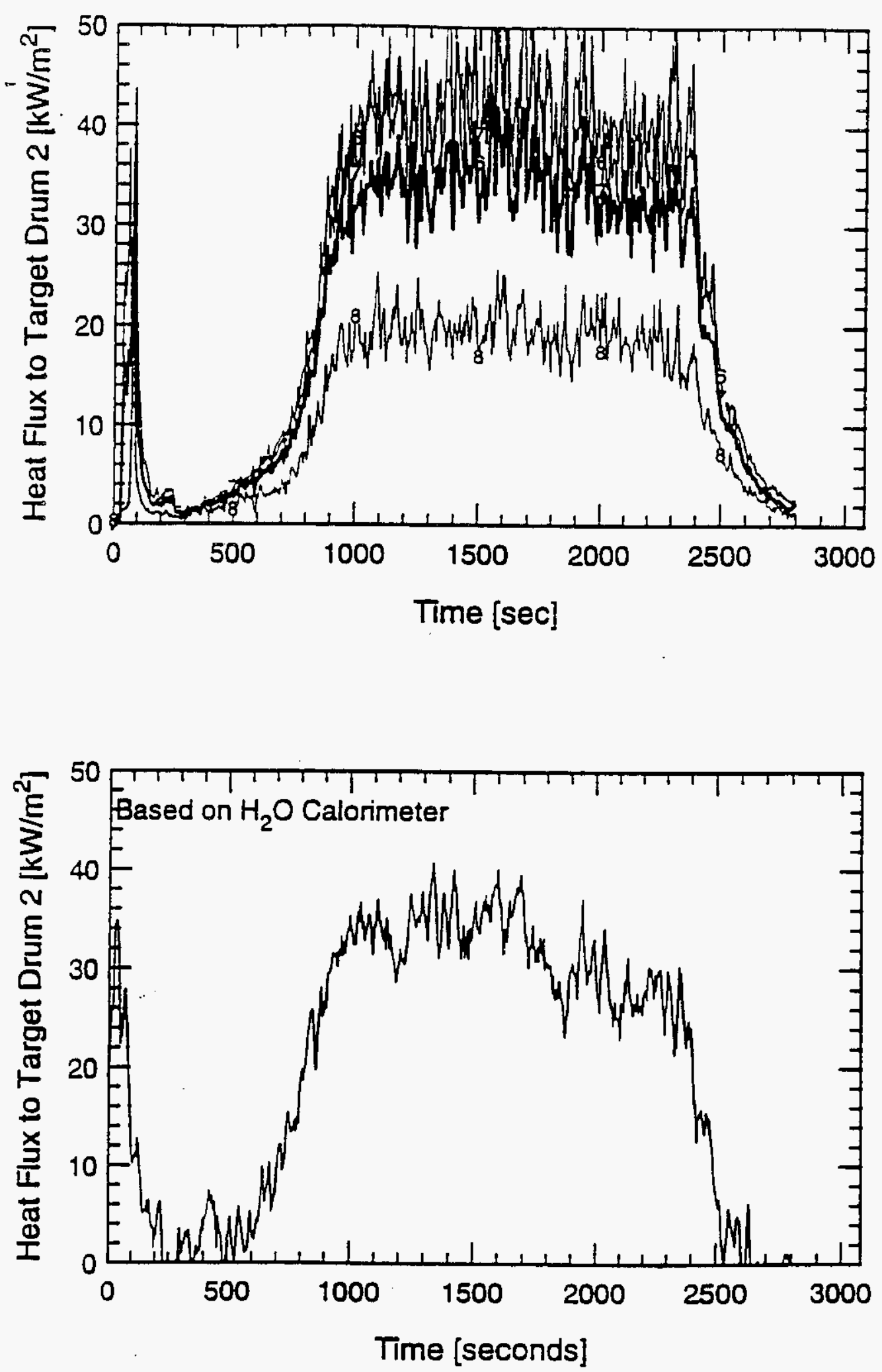
WHC-SD-WM-TRP-233 REV. 0

SWD 16 - DATA

B-102 
SWD16 - 1/3 of Mixed Standard Load - Stacked Drums 6" apart
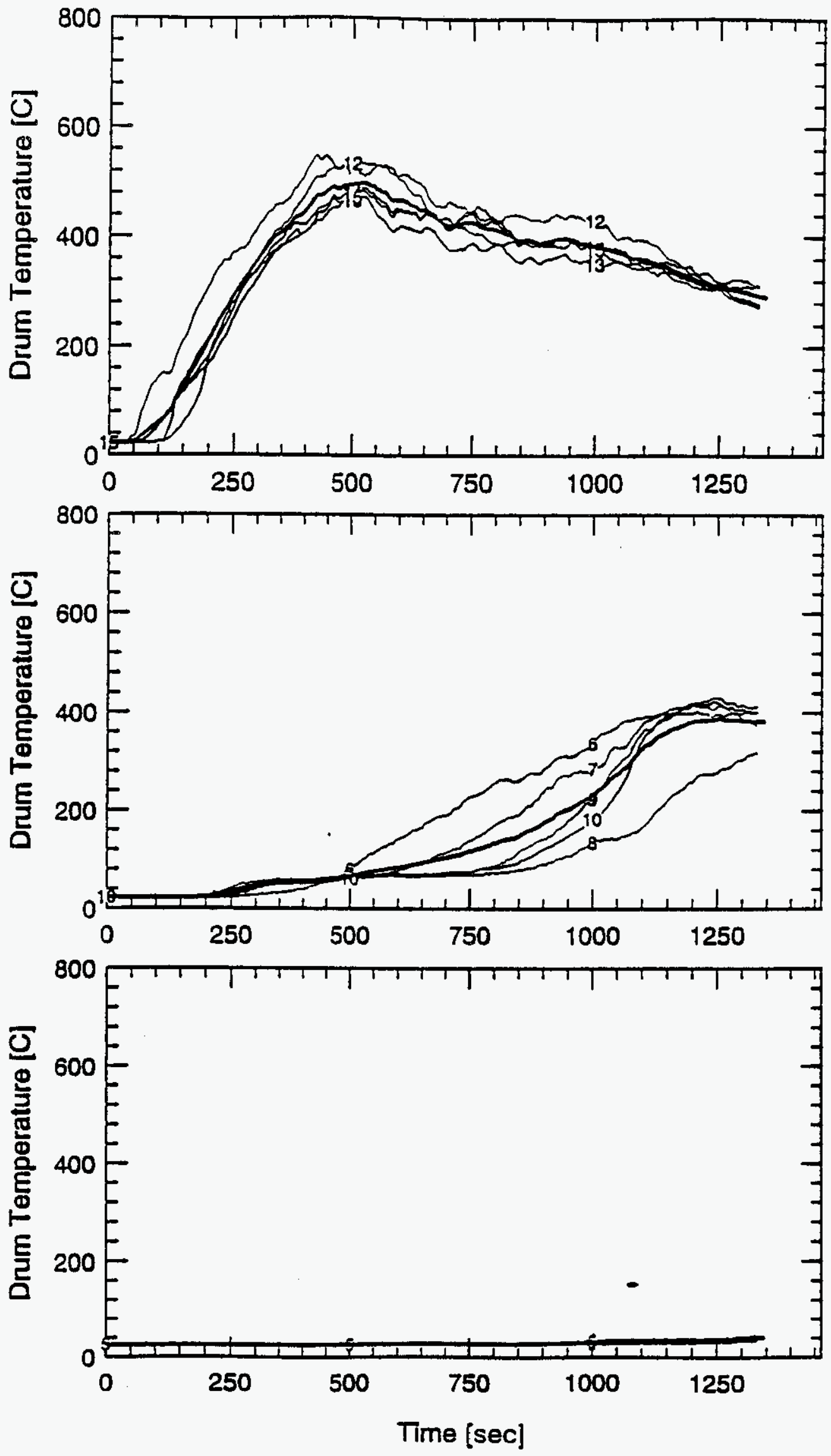
WHC-SD-WM-TRP-233 REV. 0

SWD16 - 1/3 of Mixed Standard Load - Stacked Drums 6" apart
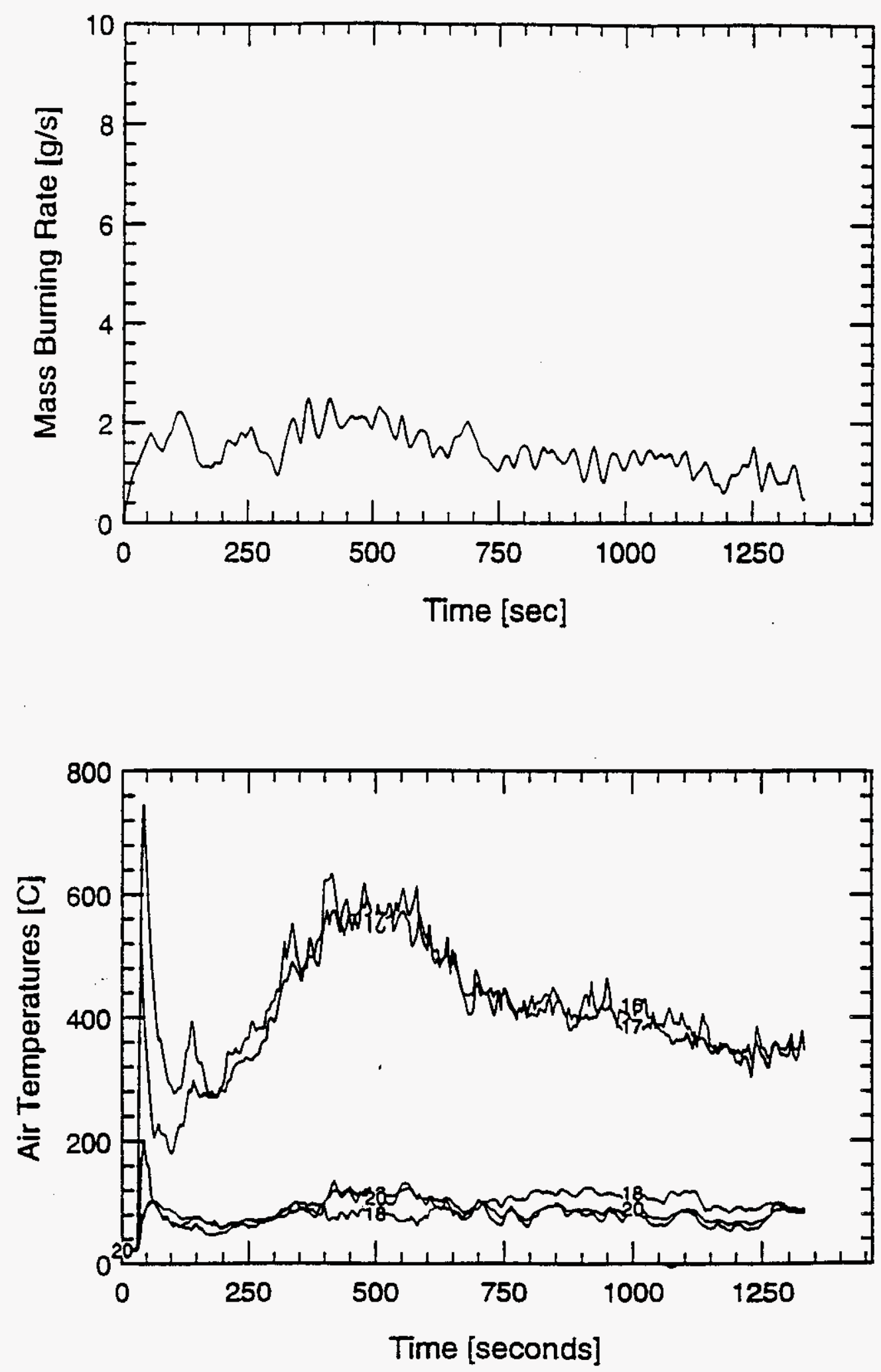
SWD16 - 1/3 of Mixed Standard Load - Stacked Drums 6" apart
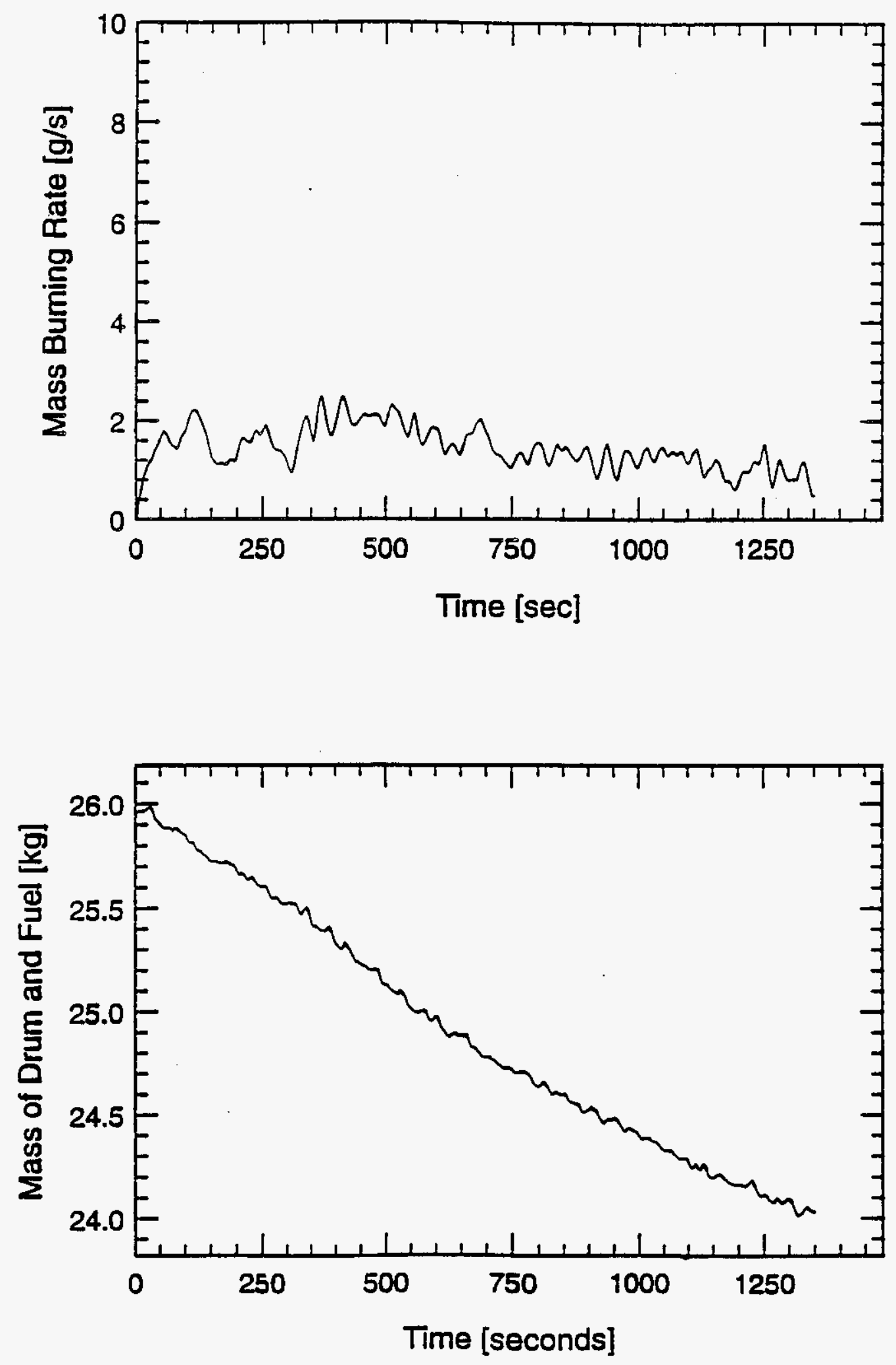
SWD16 - 1/3 of Mixed Standard Load - Stacked Drums 6" apart
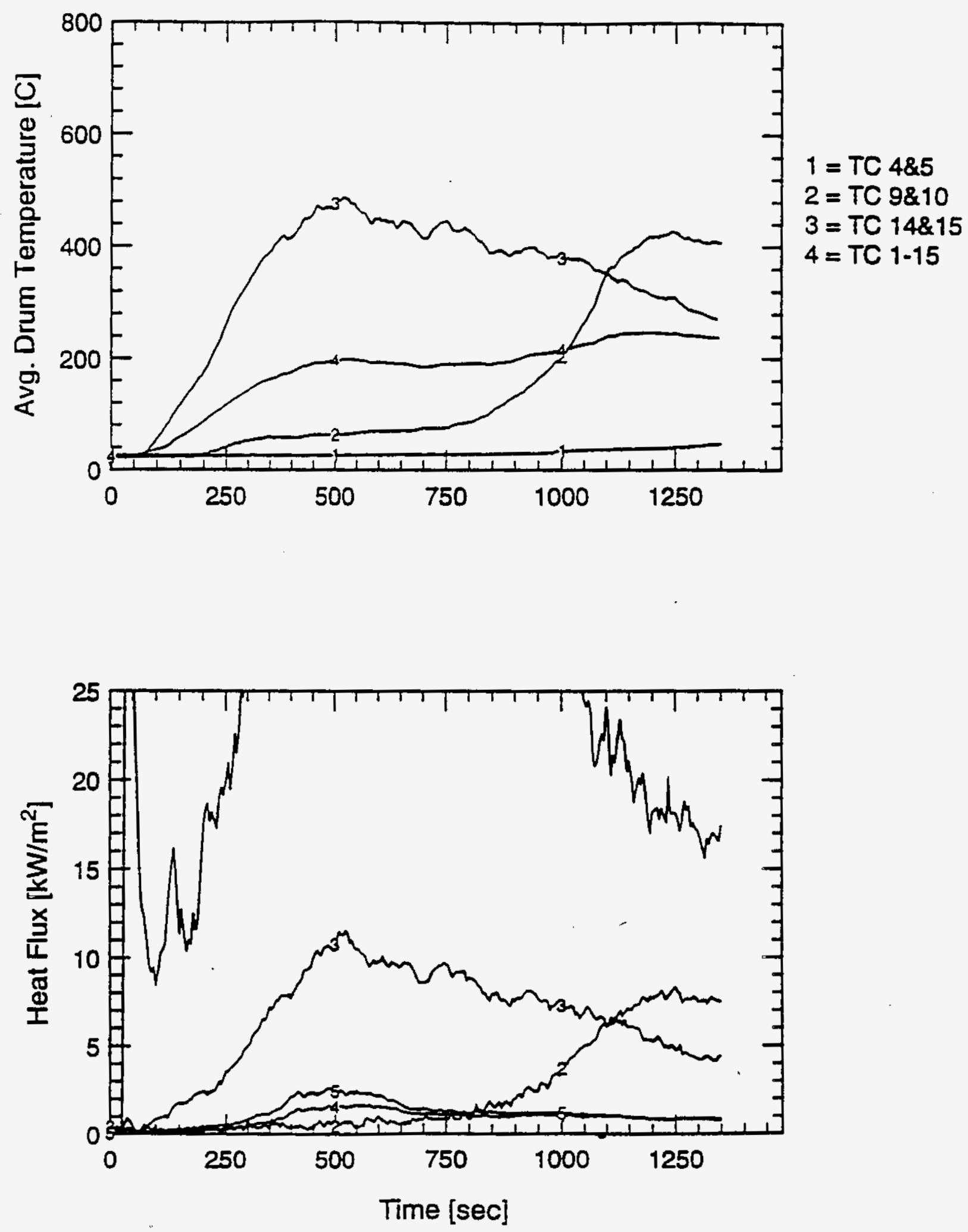
SWD16 - 1/3 of Mixed Standard Load - Stacked Drums 6" apart
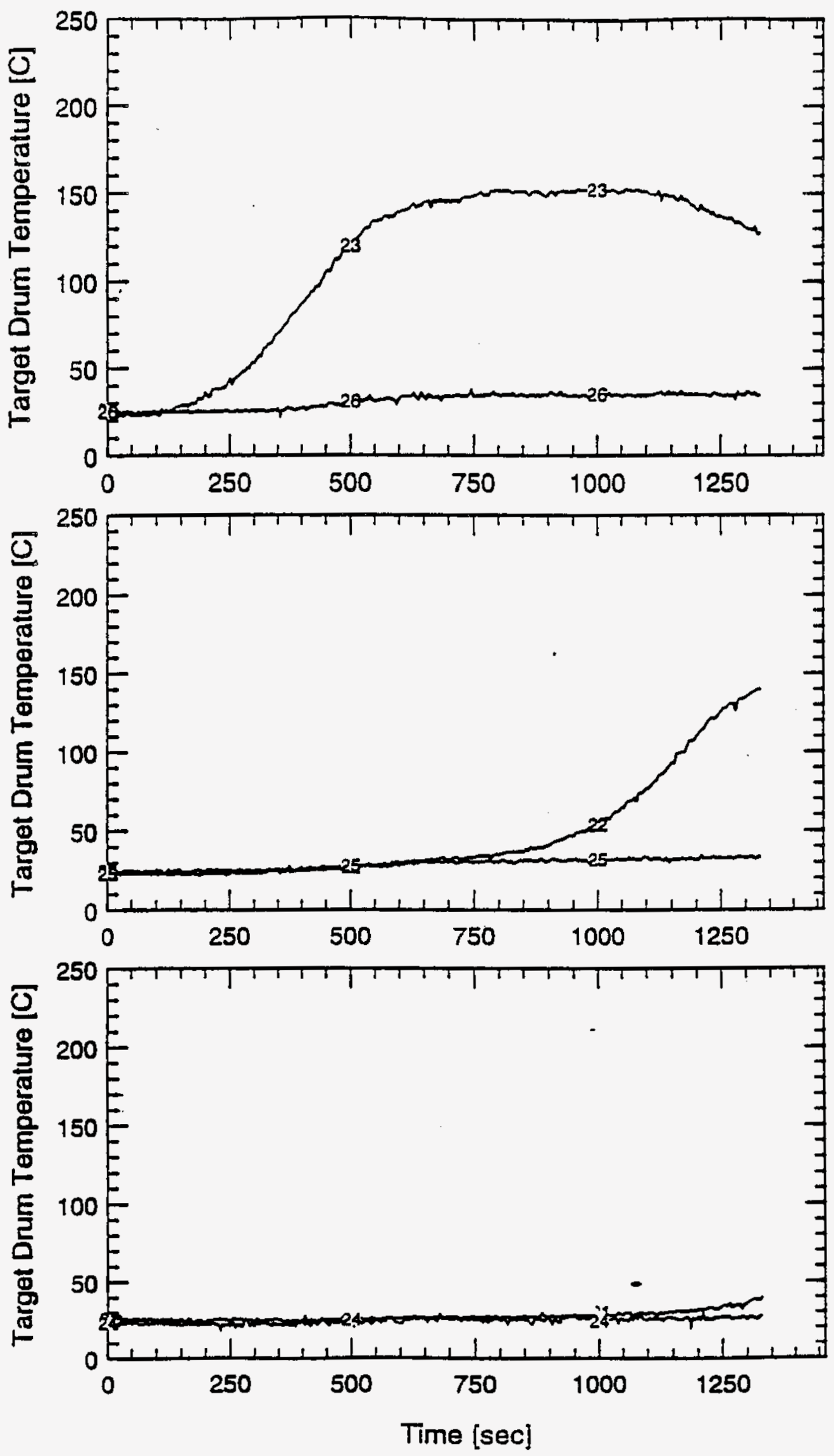
SWD16 - 1/3 of Mixed Standard Load - Stacked Drums 6" apart

次
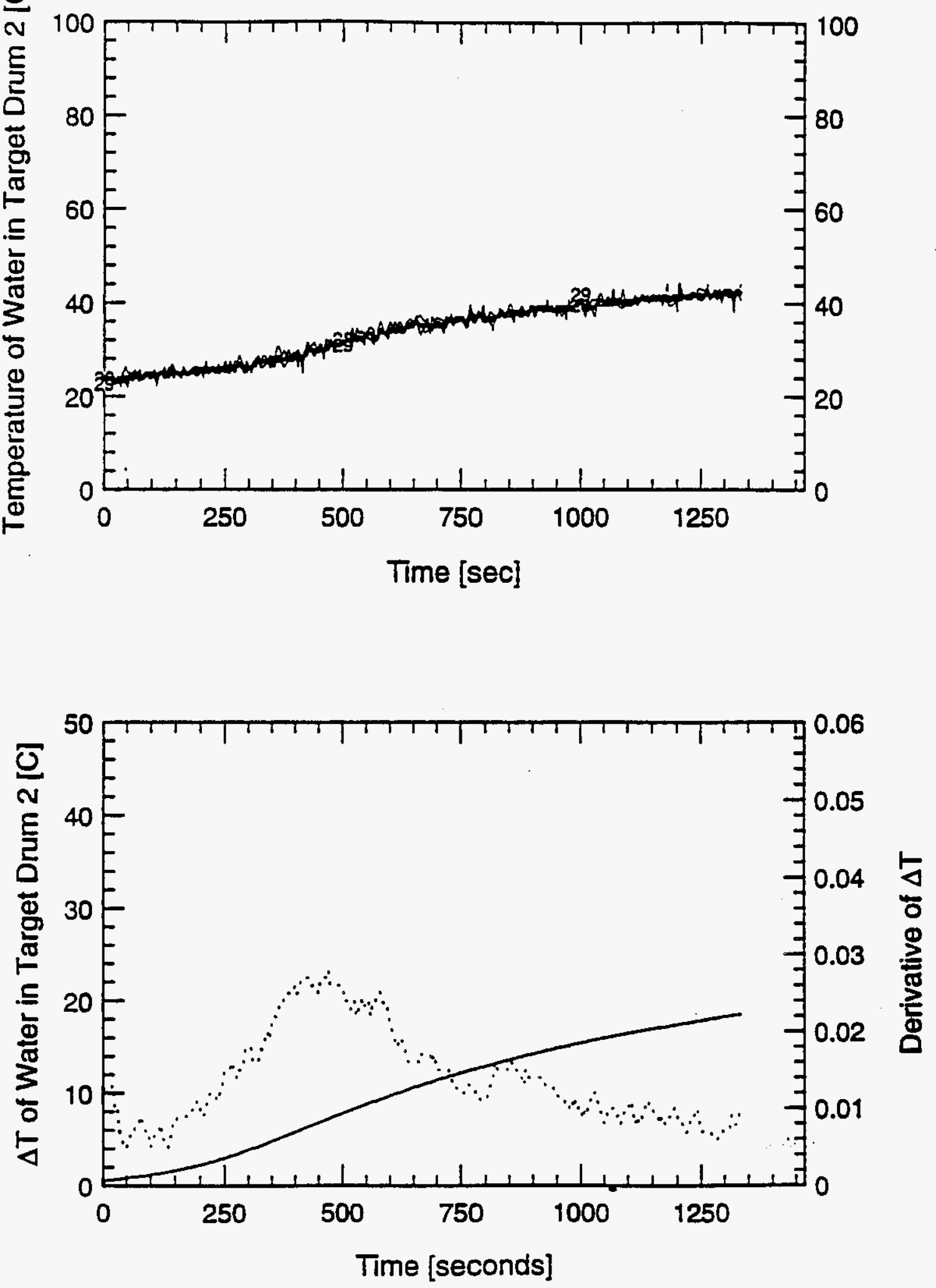
SWD16 - 1/3 of Mixed Standard Load - Stacked Drums 6" apart
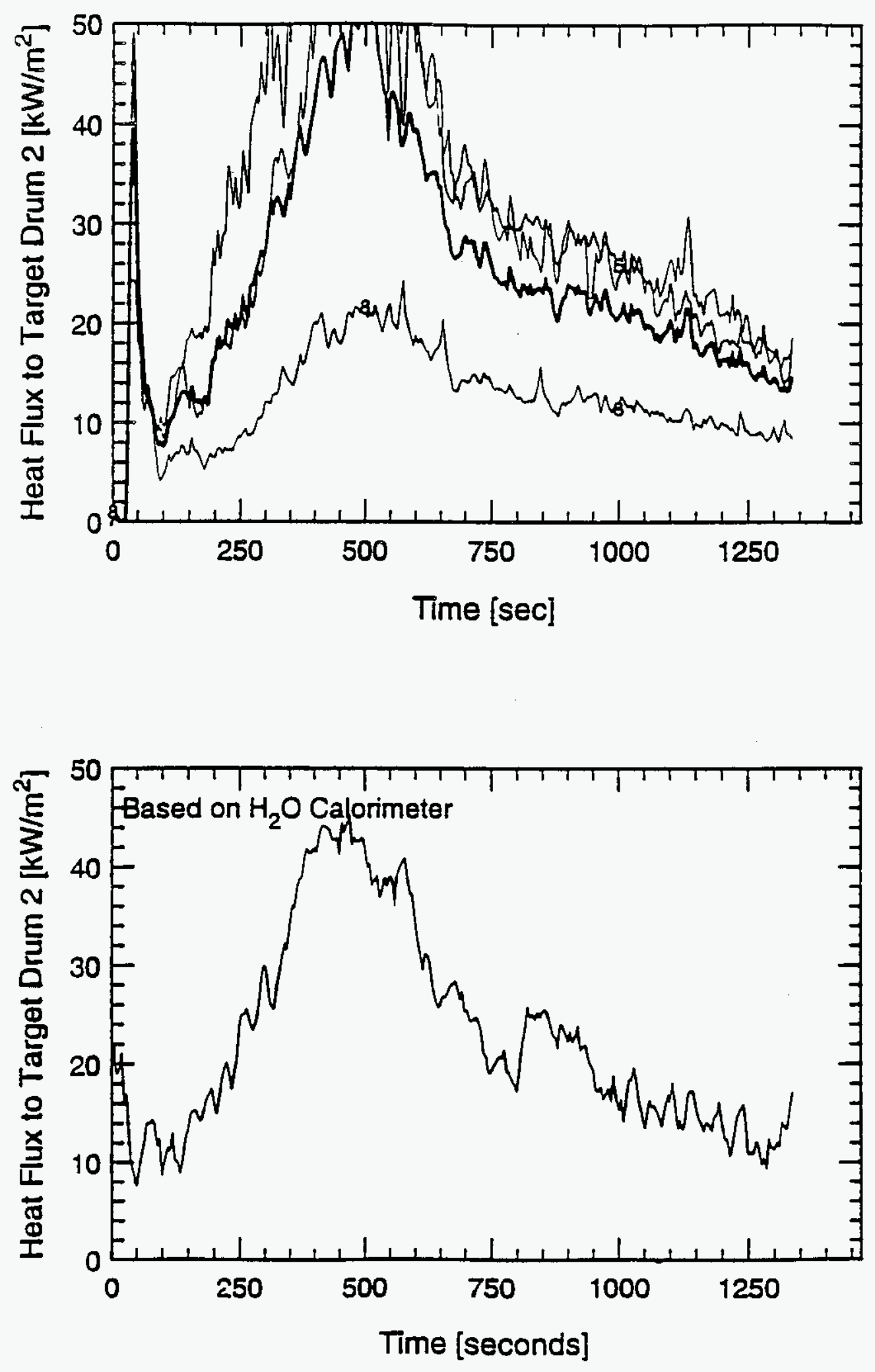
WHC-SD-WM-TRP-233 REV. 0

SWD 17 - DATA 
WHC-SD-WM-TRP-233 REV. 0

SWD17 - Bagged Standard Load - Stacked Drums 6" apart
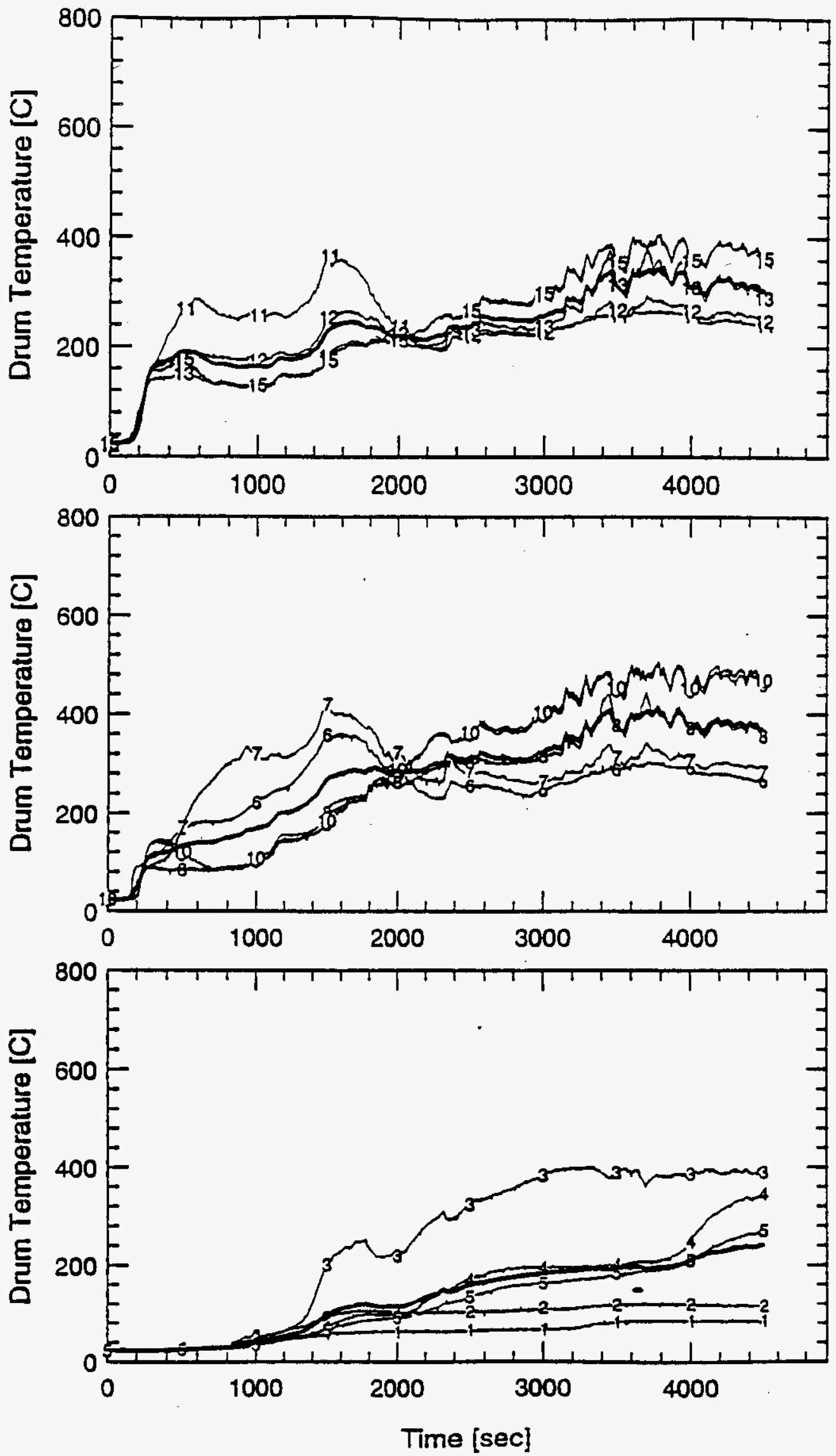
SWD17 - Bagged Standard Load - Stacked Drums 6" apart
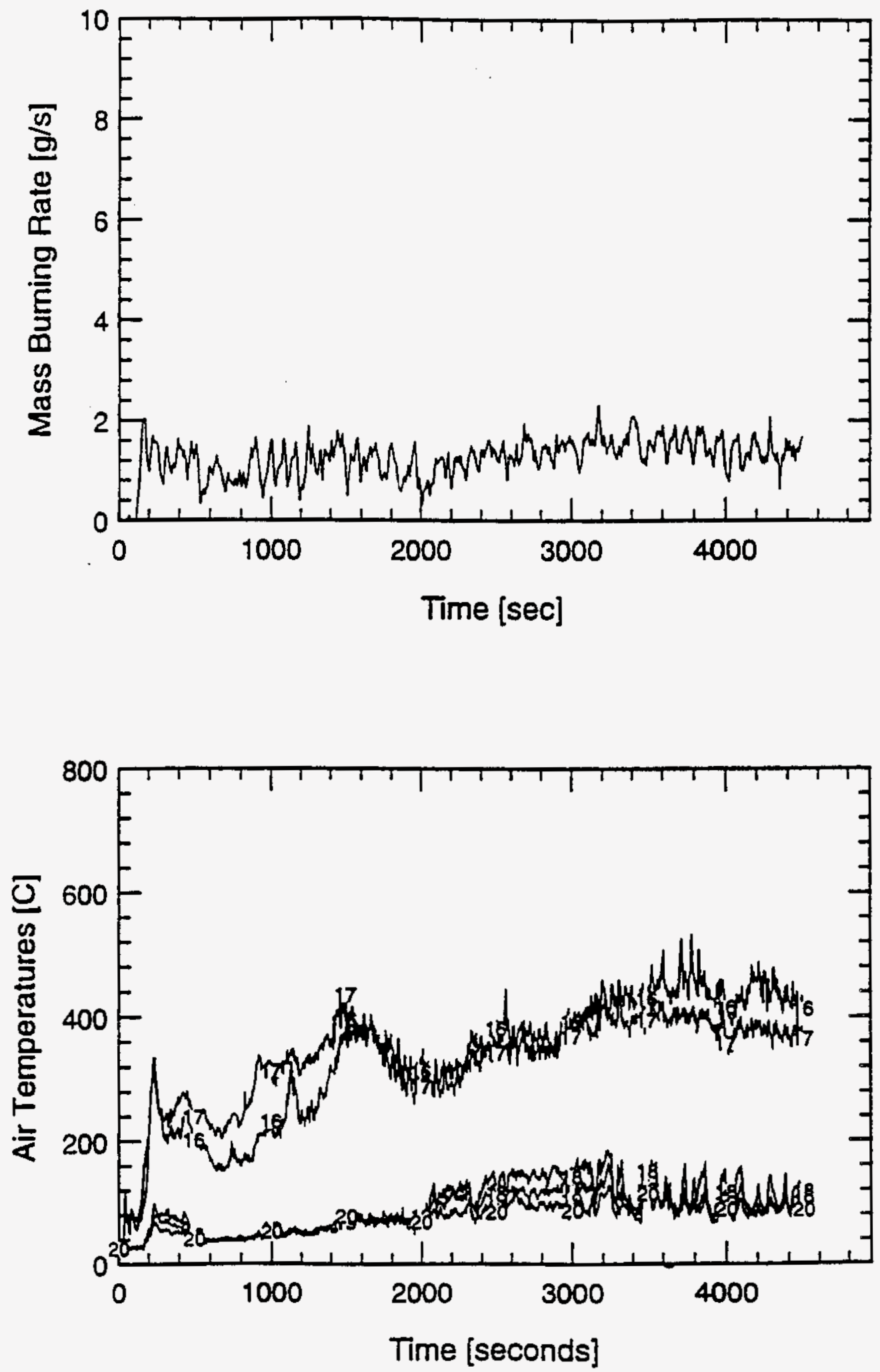
SWD17 - Bagged Standard Load - Stacked Drums 6" apart
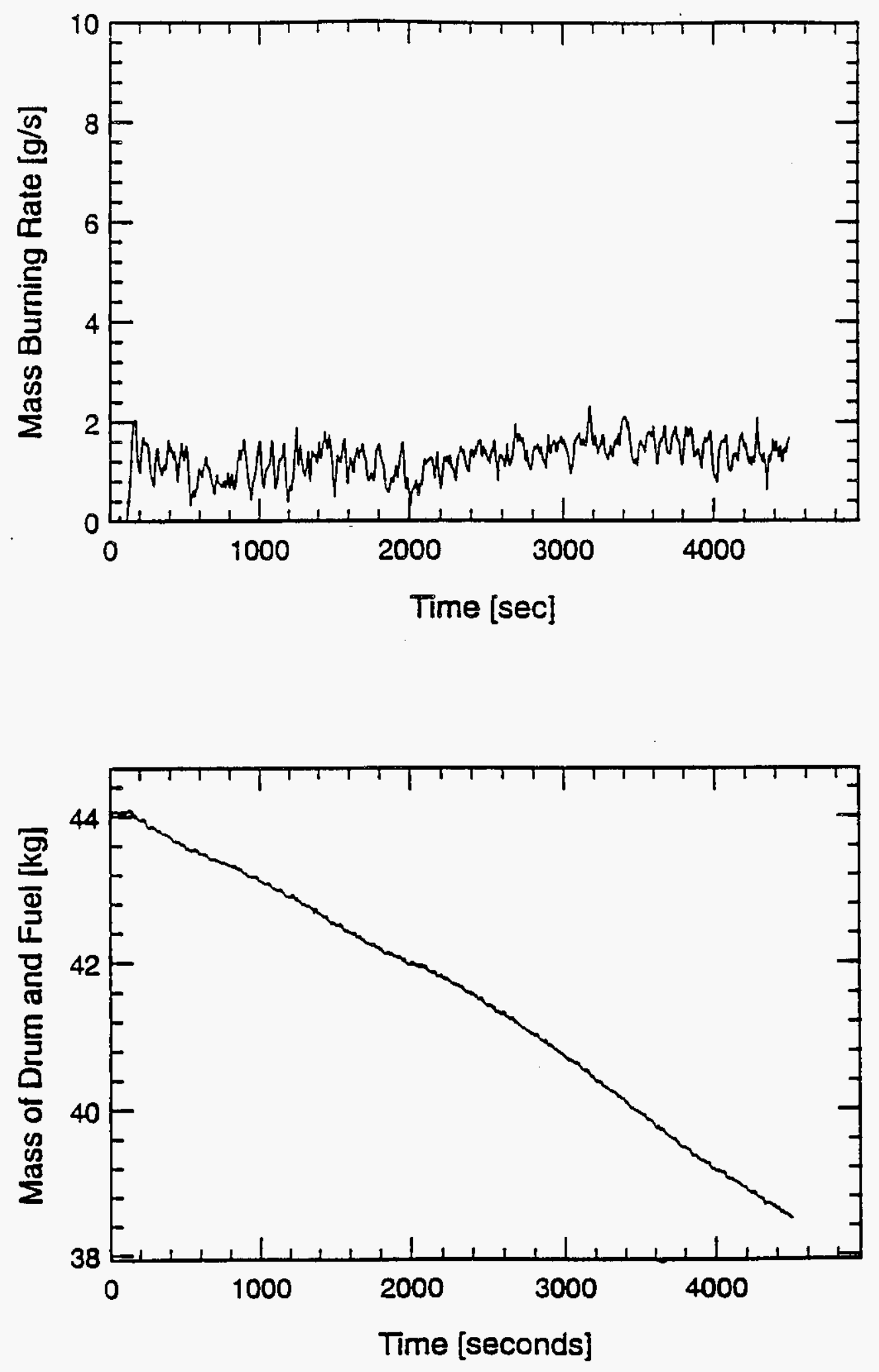
WHC-SD-WM-TRP-233 REV. 0

SWD17 - Bagged Standard Load - Stacked Drums 6" apart
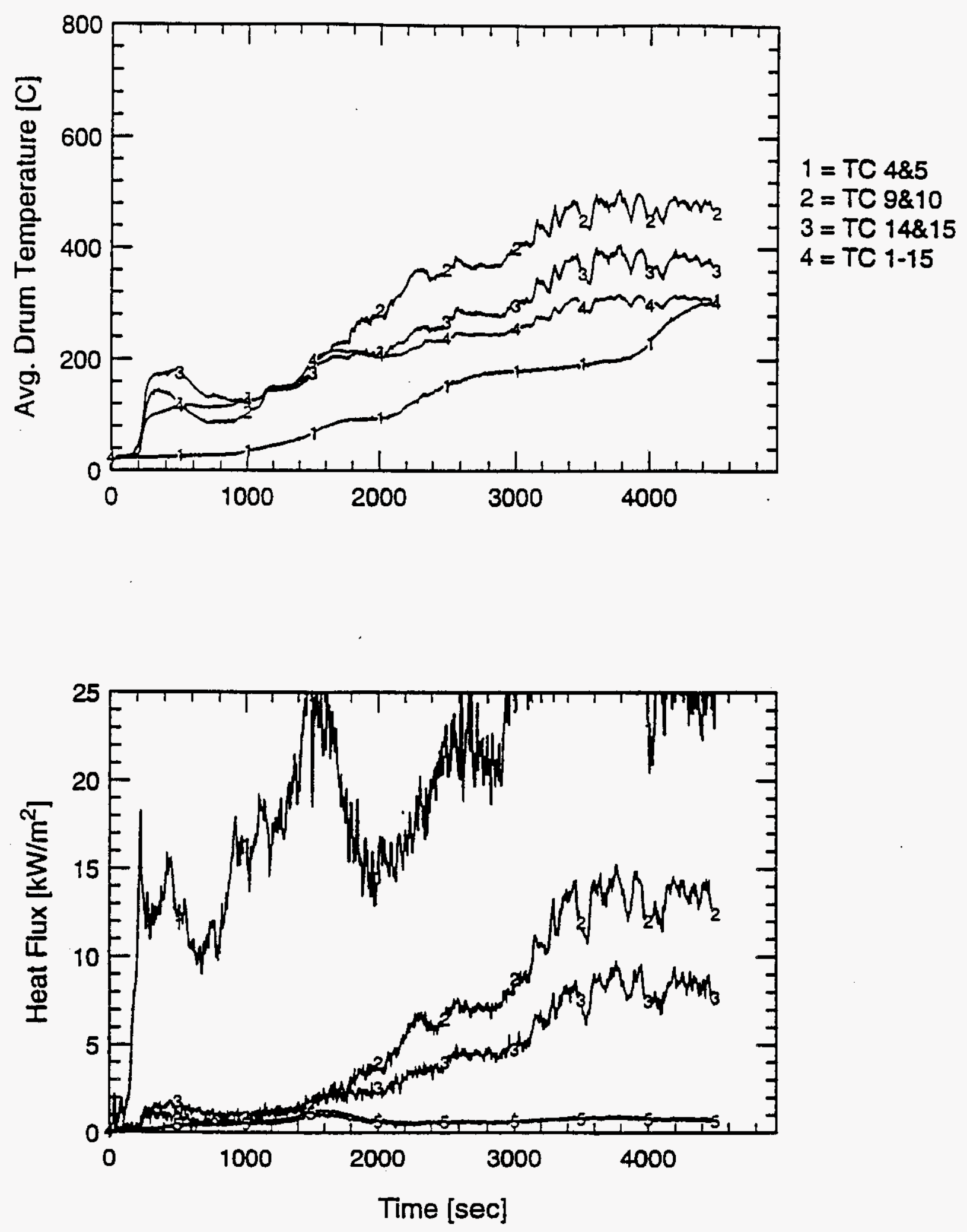
WHC-SD-WM-TRP-233 REV. 0

SWD17 - Bagged Standard Load - Stacked Drums 6" apart
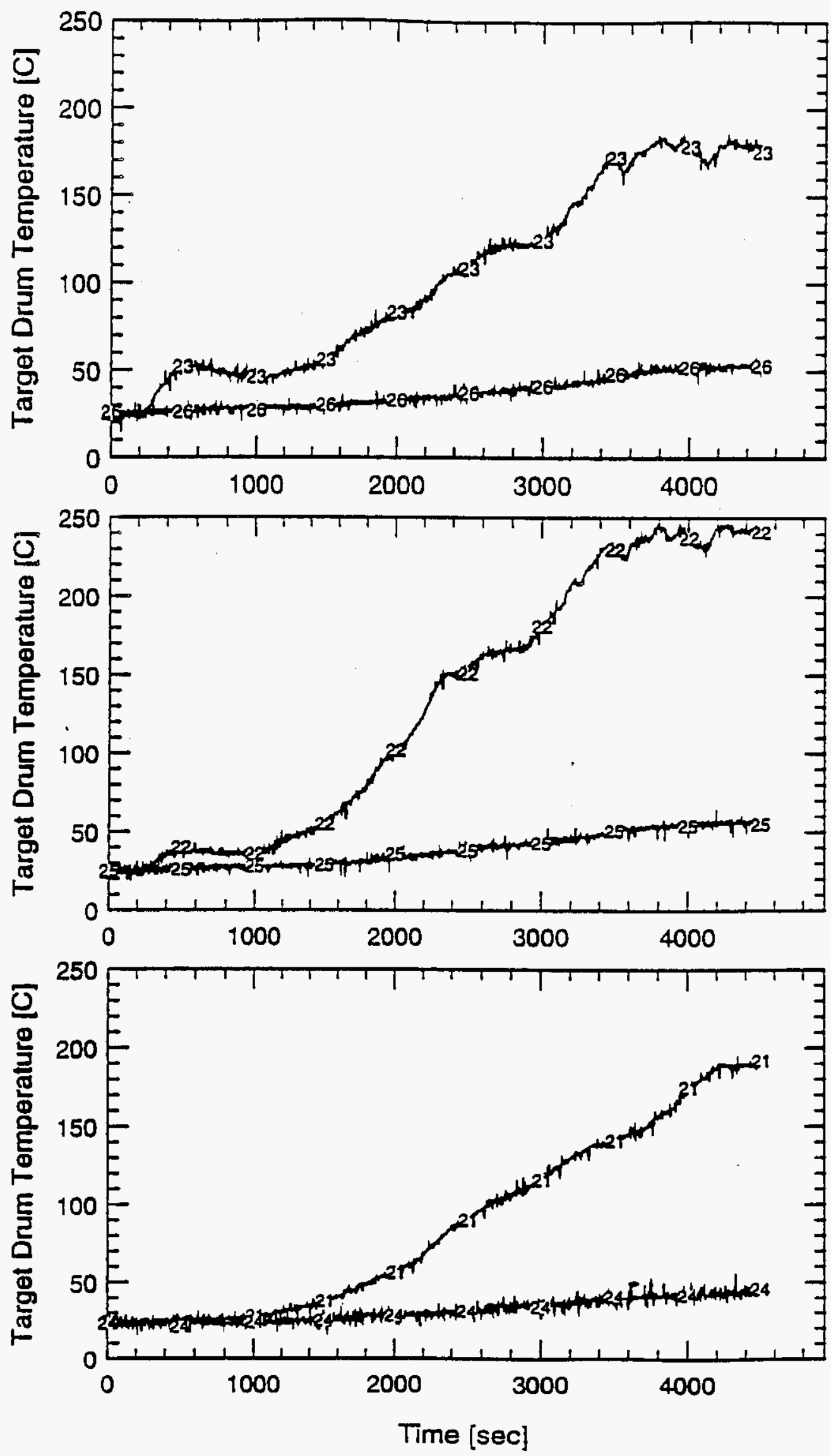
WHC-SD-WM-TRP-233 REV. 0
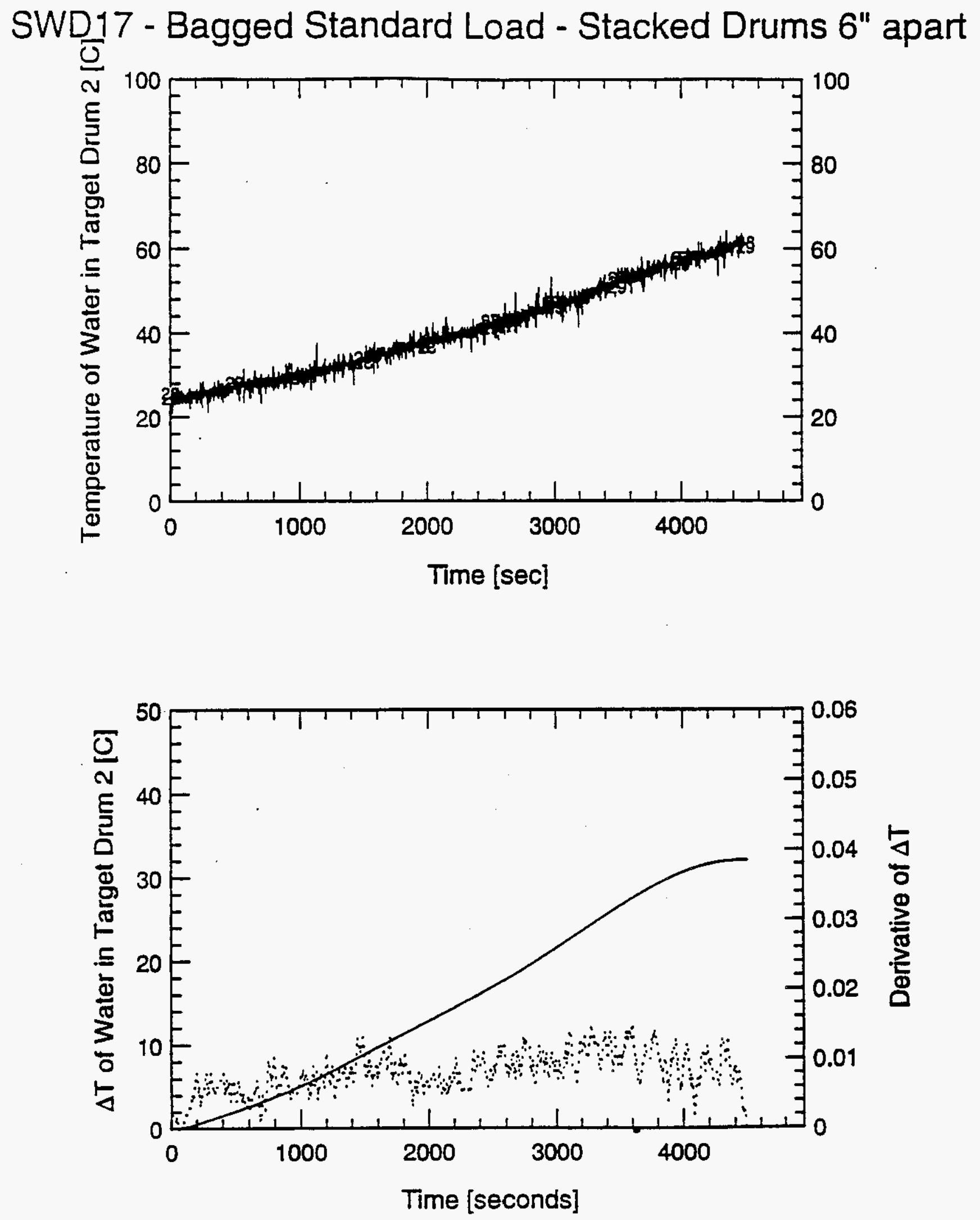
WHC-SD-WM-TRP-233 REV. 0

SWD17 - Bagged Standard Load - Stacked Drums 6" apart
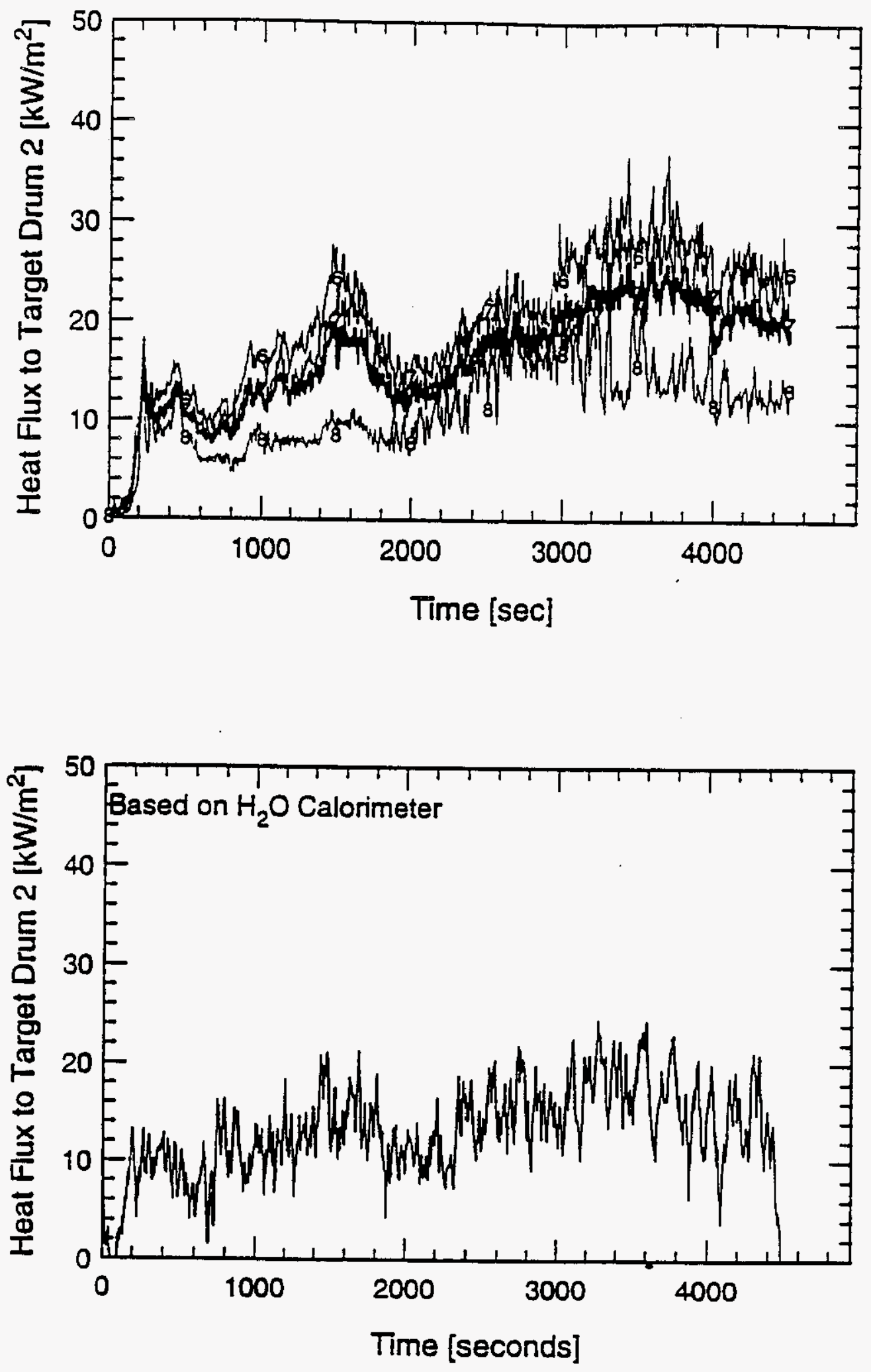
WHC-SD-WM-TRP-233 REV. 0

SWD 18 - DATA 
WHC-SD-WM-TRP-233 REV. 0

SWD18 - Kerosene
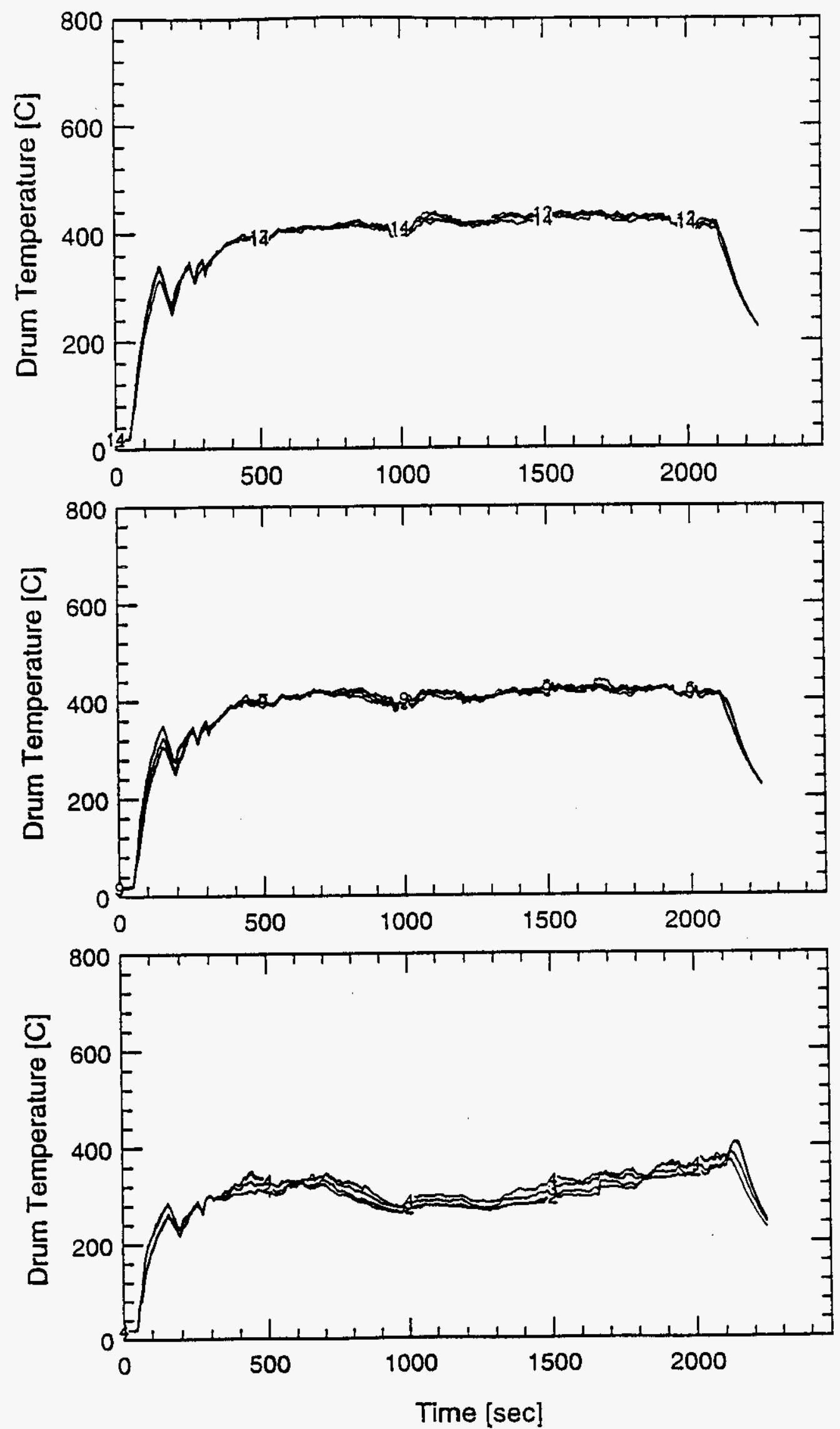

B-119 
WHC-SD-WM-TRP-233 REV. 0

SWD18 - KEROSENE
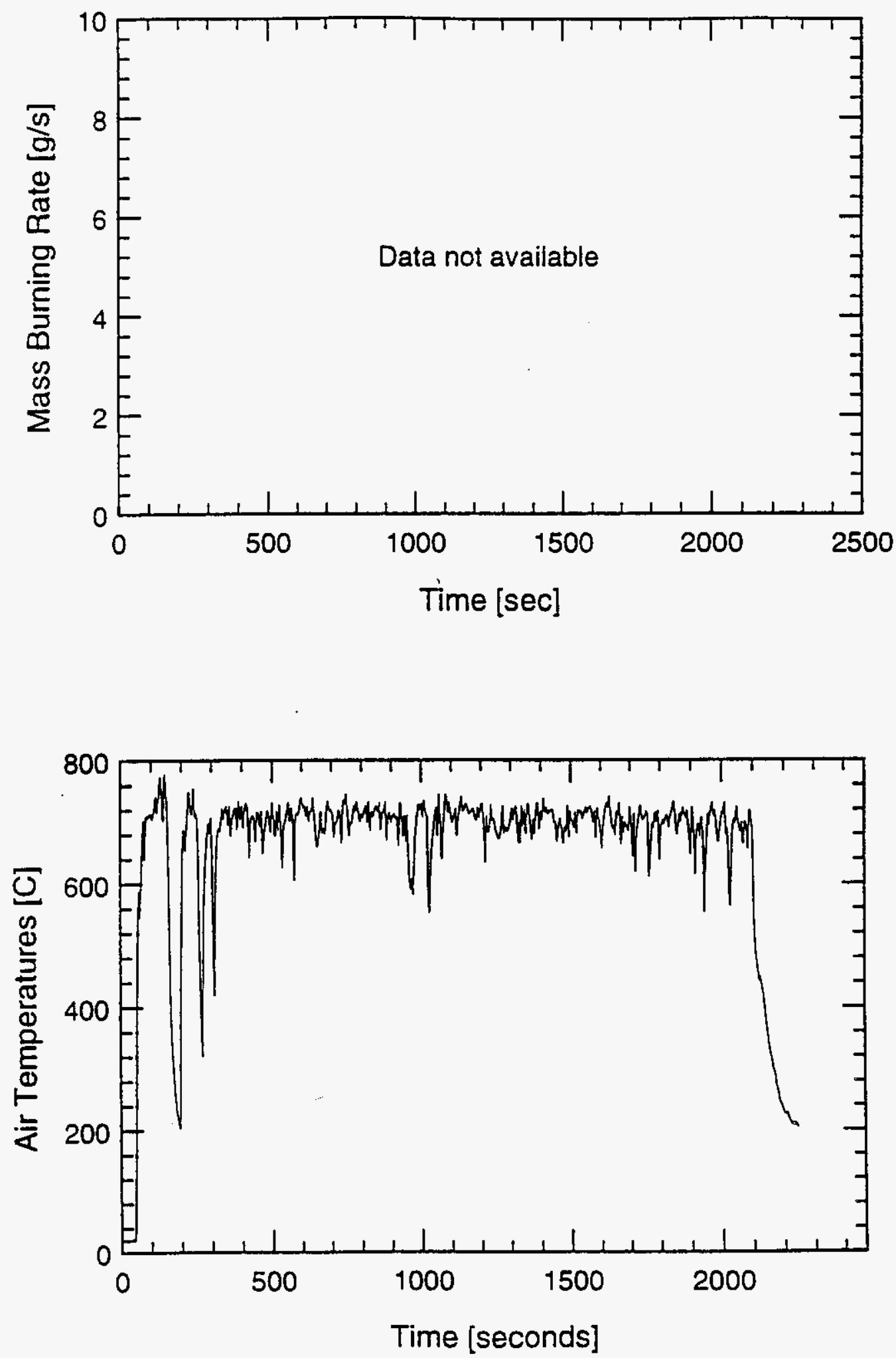
WHC-SD-WM-TRP-233 REV. O

SWD18 - Kerosene
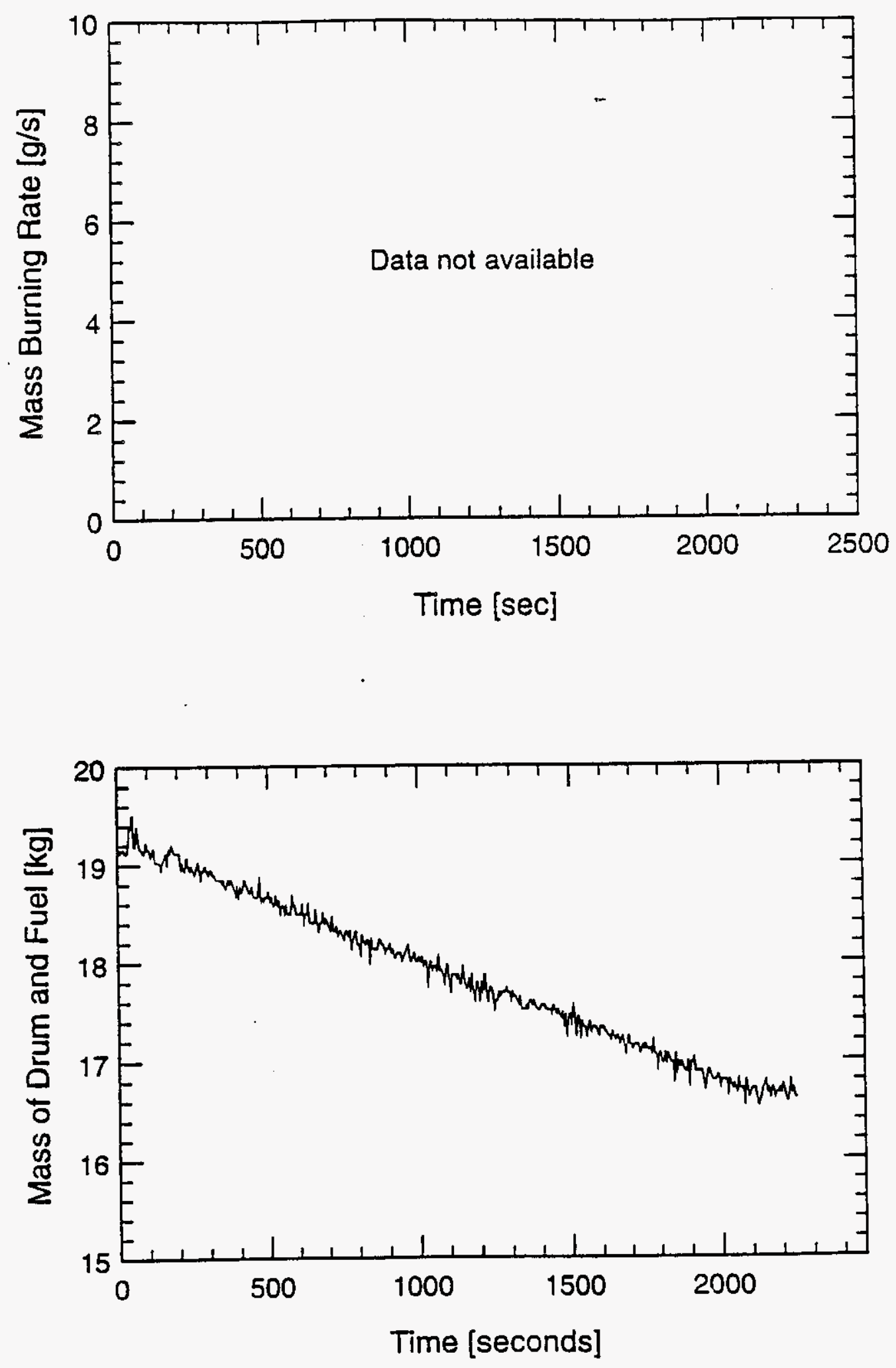


\section{SWD18 Kerosene}
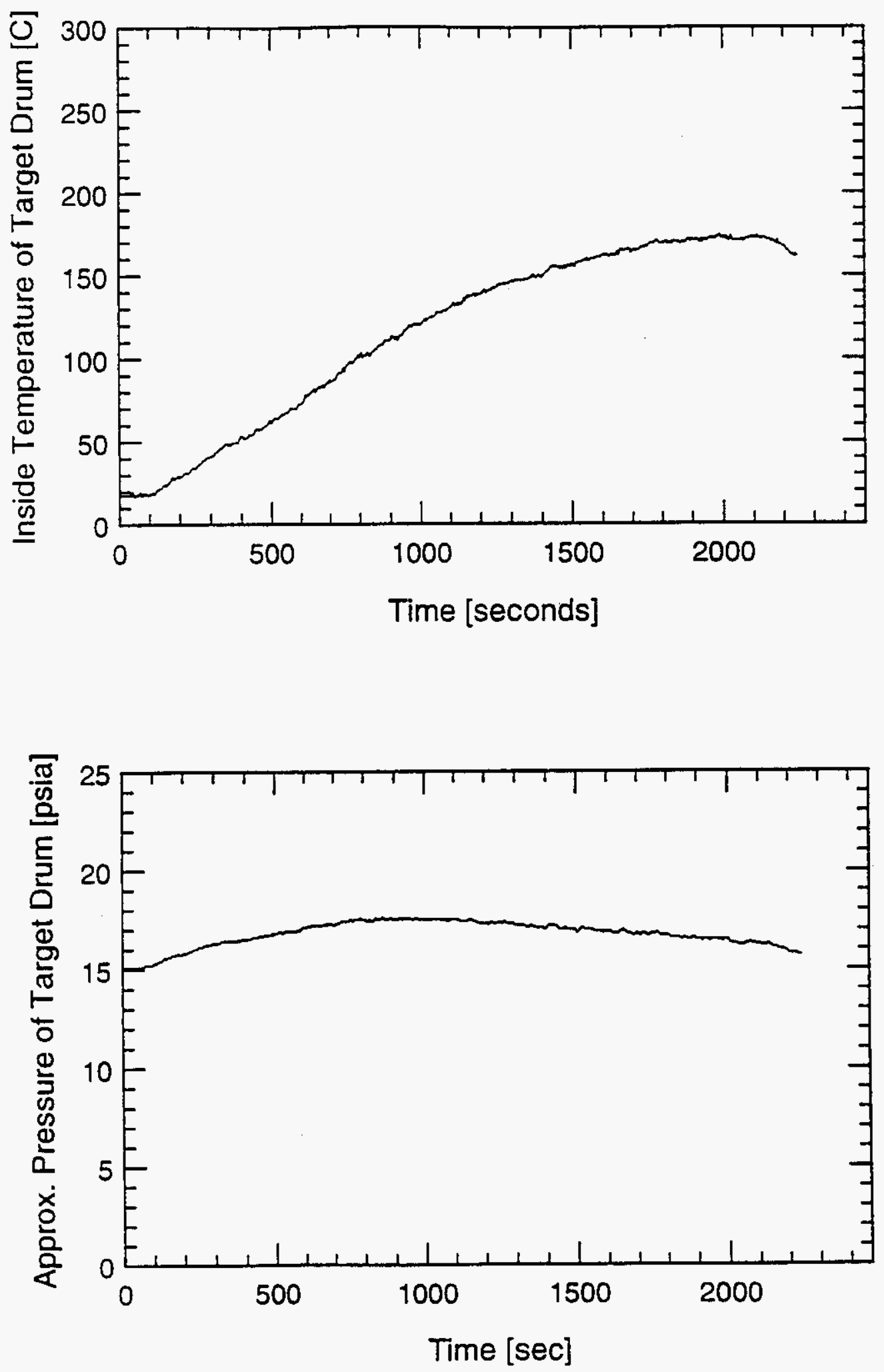
WHC-SD-WM-TRP-233 REV. 0

SWD 19 - DATA 
WHC-SD-WM-TRP-233 REV. 0

SWD19 - Drum 1 - Standard Load
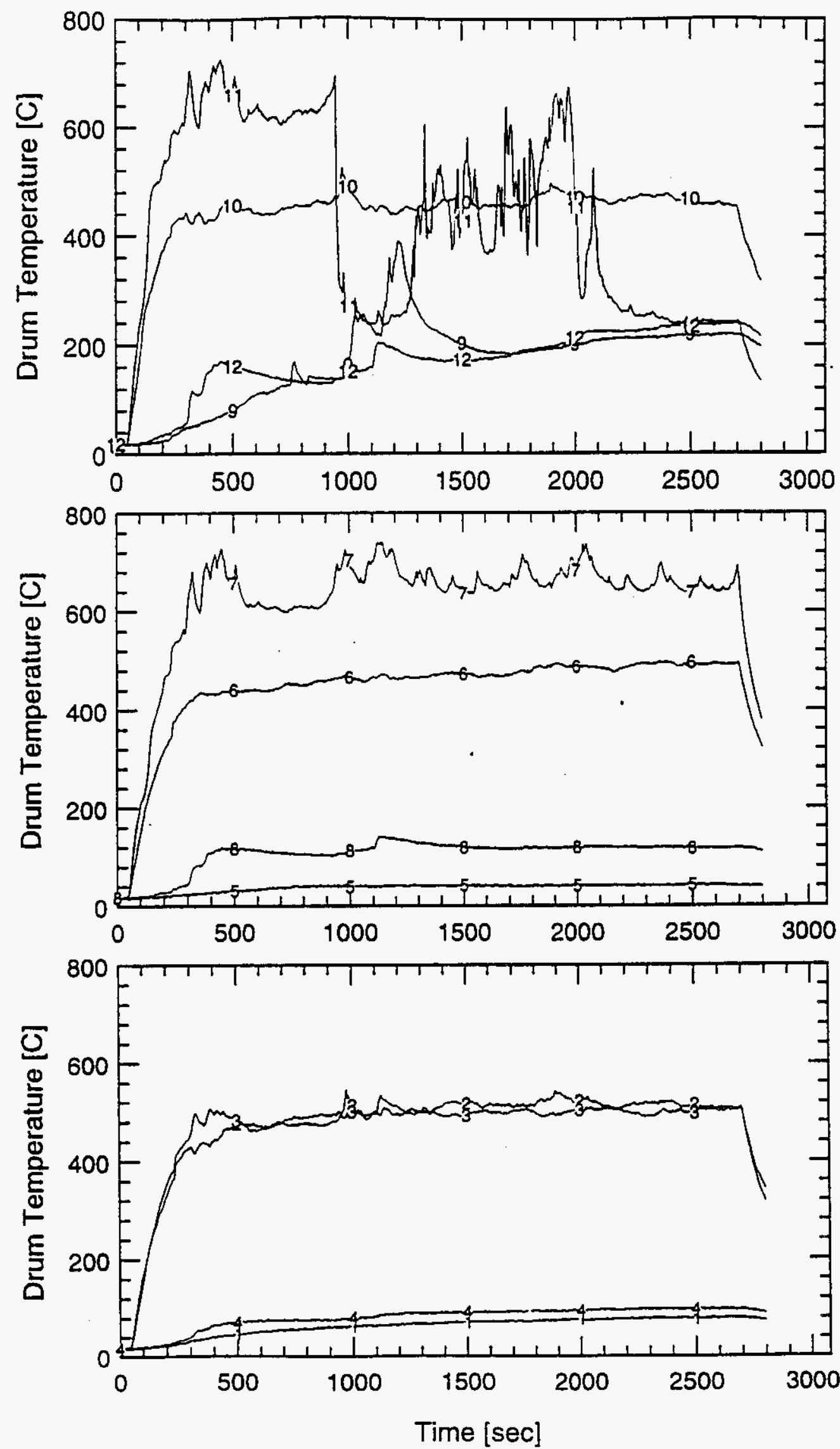


\section{SWD19 - Drum 2}
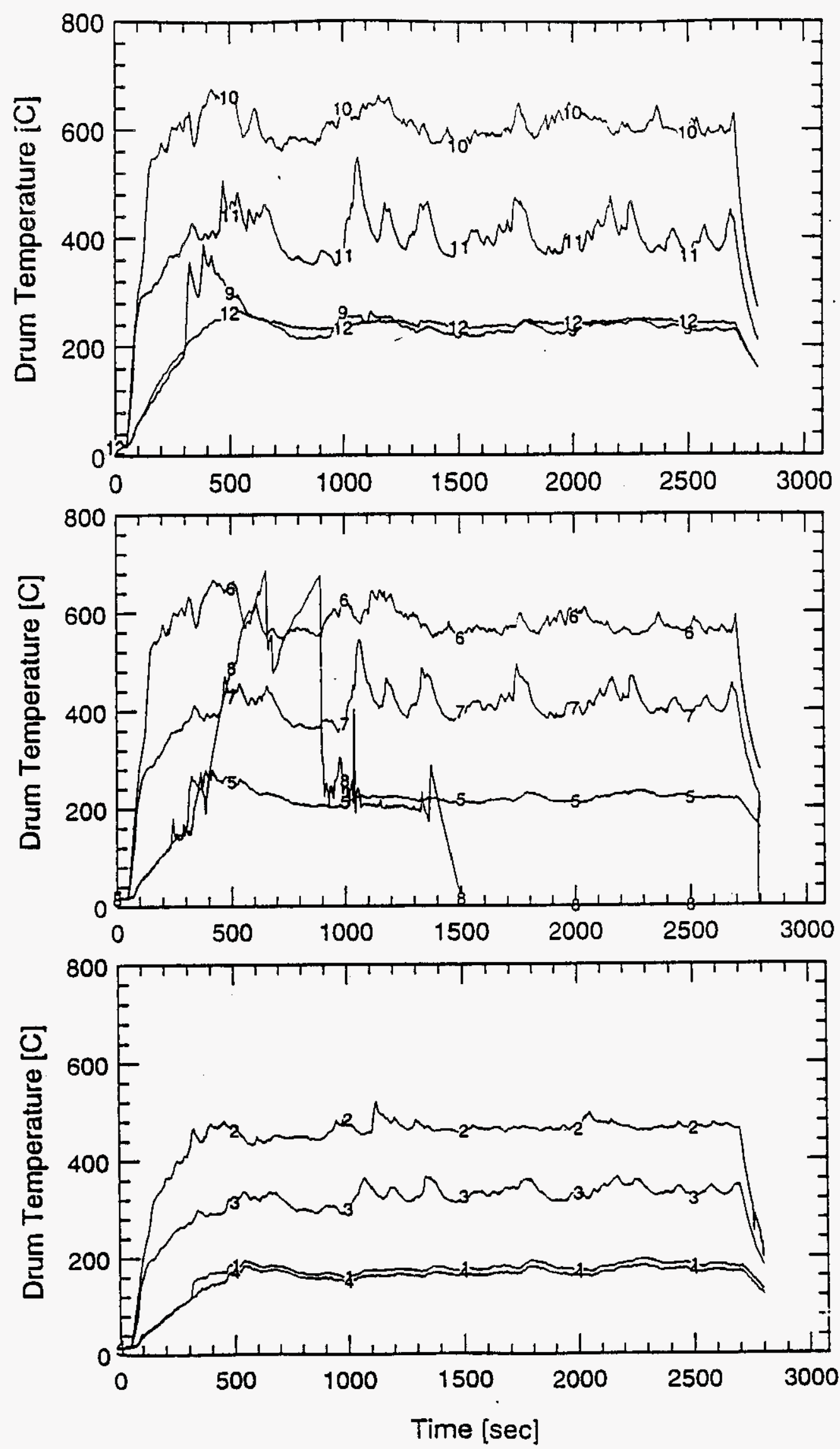
WHC-SD-WM-TRP-233 REV. 0

SWD19 - Drum 3 Water filled drum
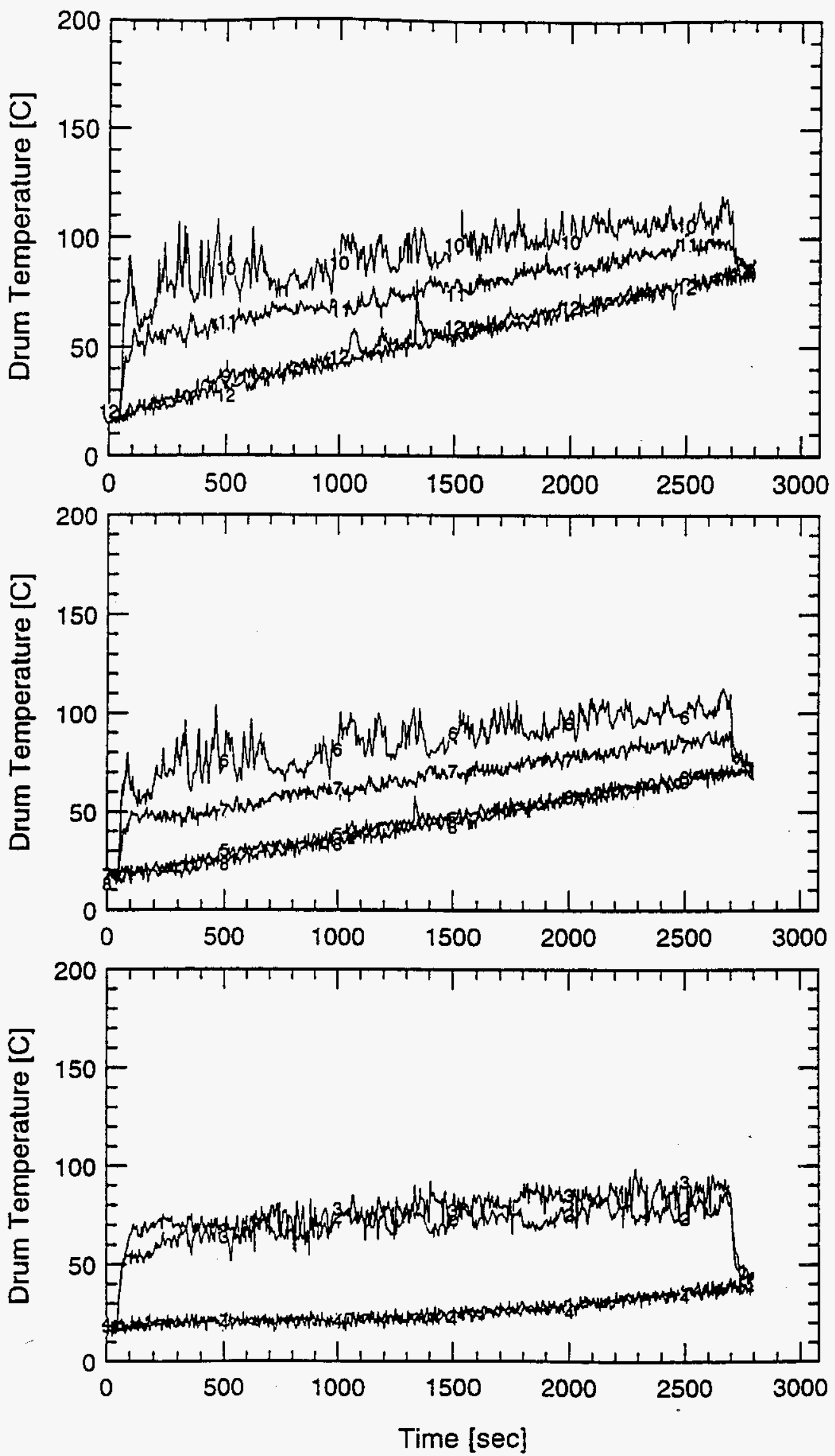

PLOTTC3.PLT 
WHC-SD-WM-TRP-233 REV. 0

\section{SWD19 - Drum 4 - Empty}
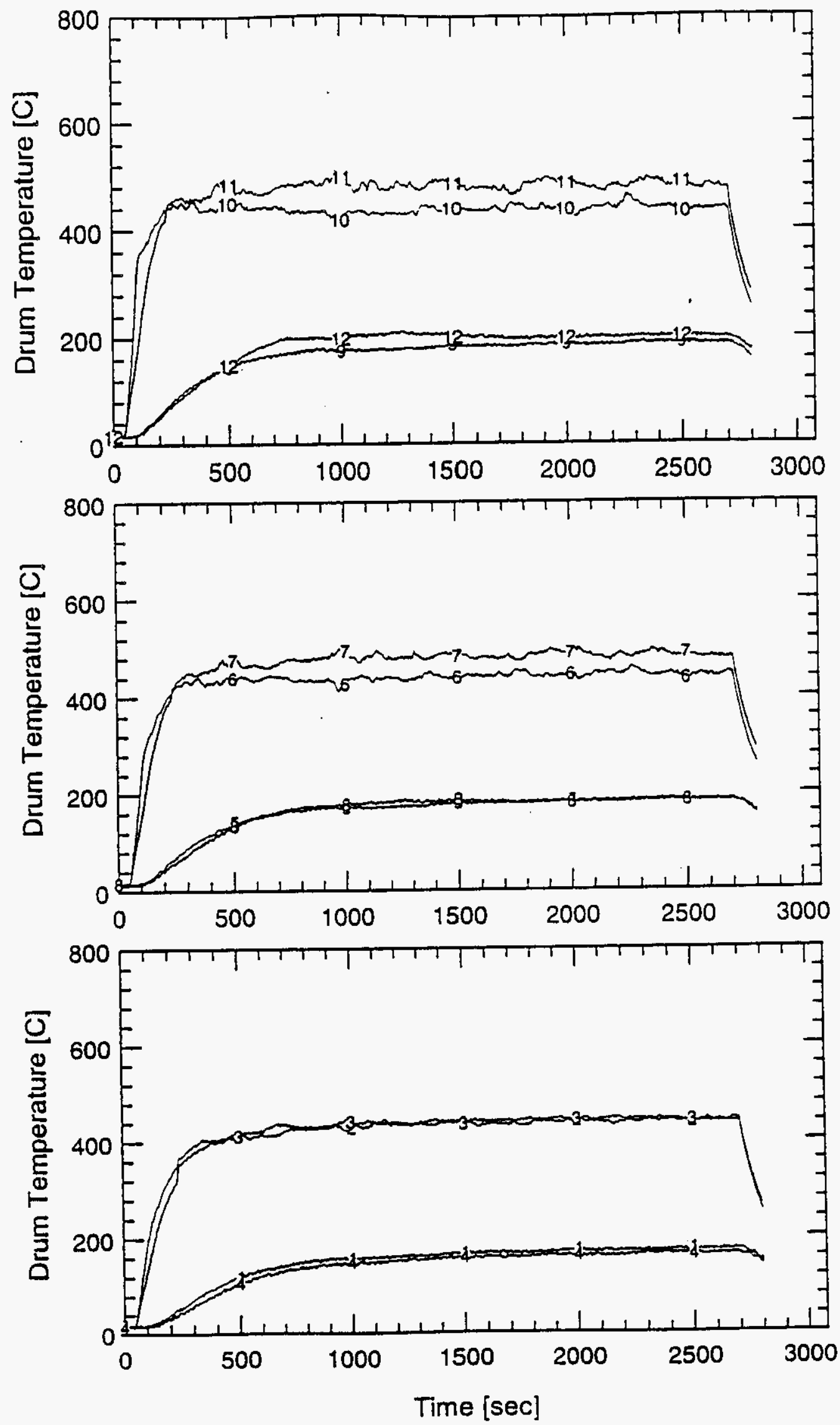


\section{SWD19 19" Burner}
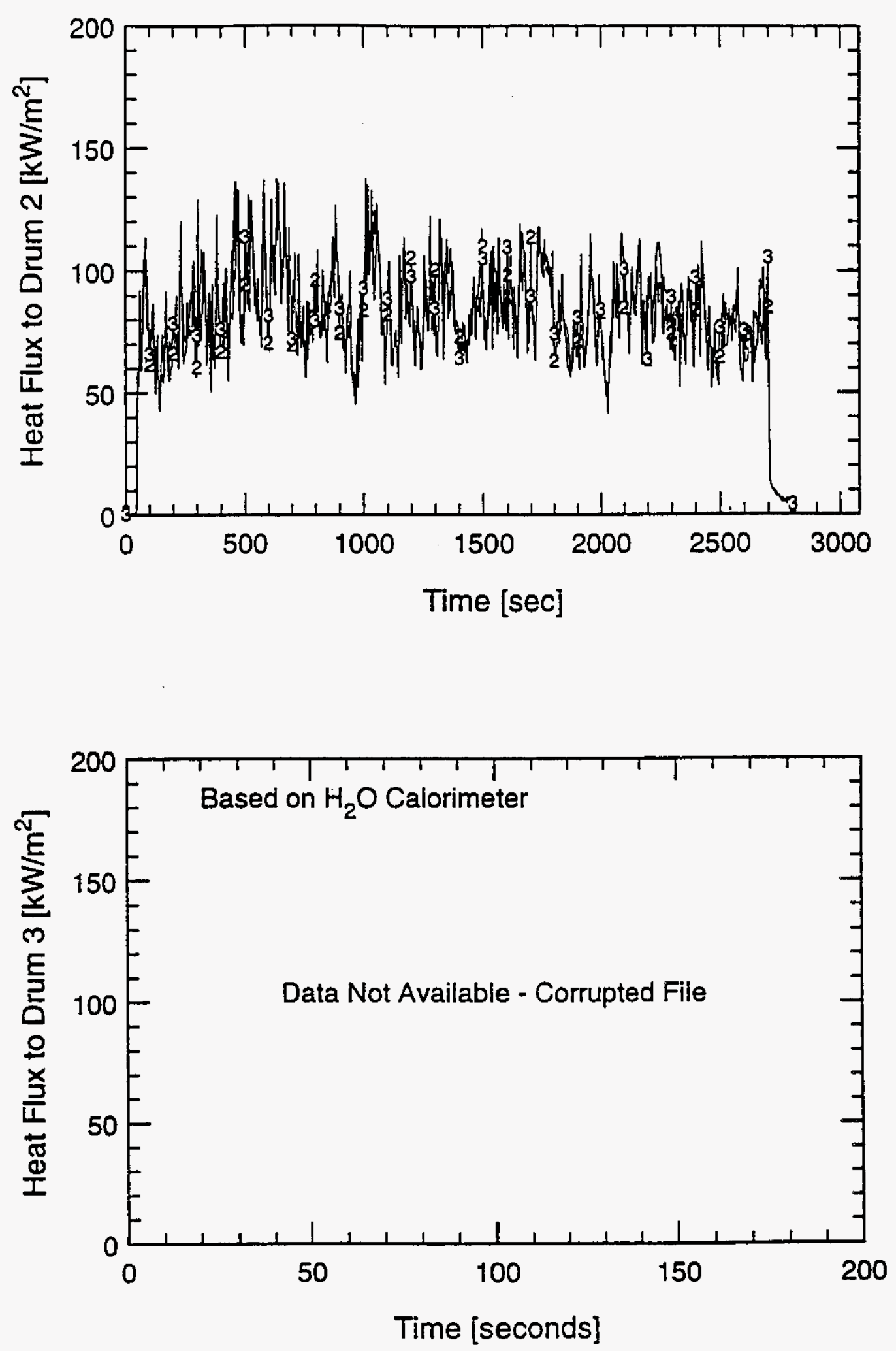
SWD19 DRUM 1 - 19" Burner - Standard Load
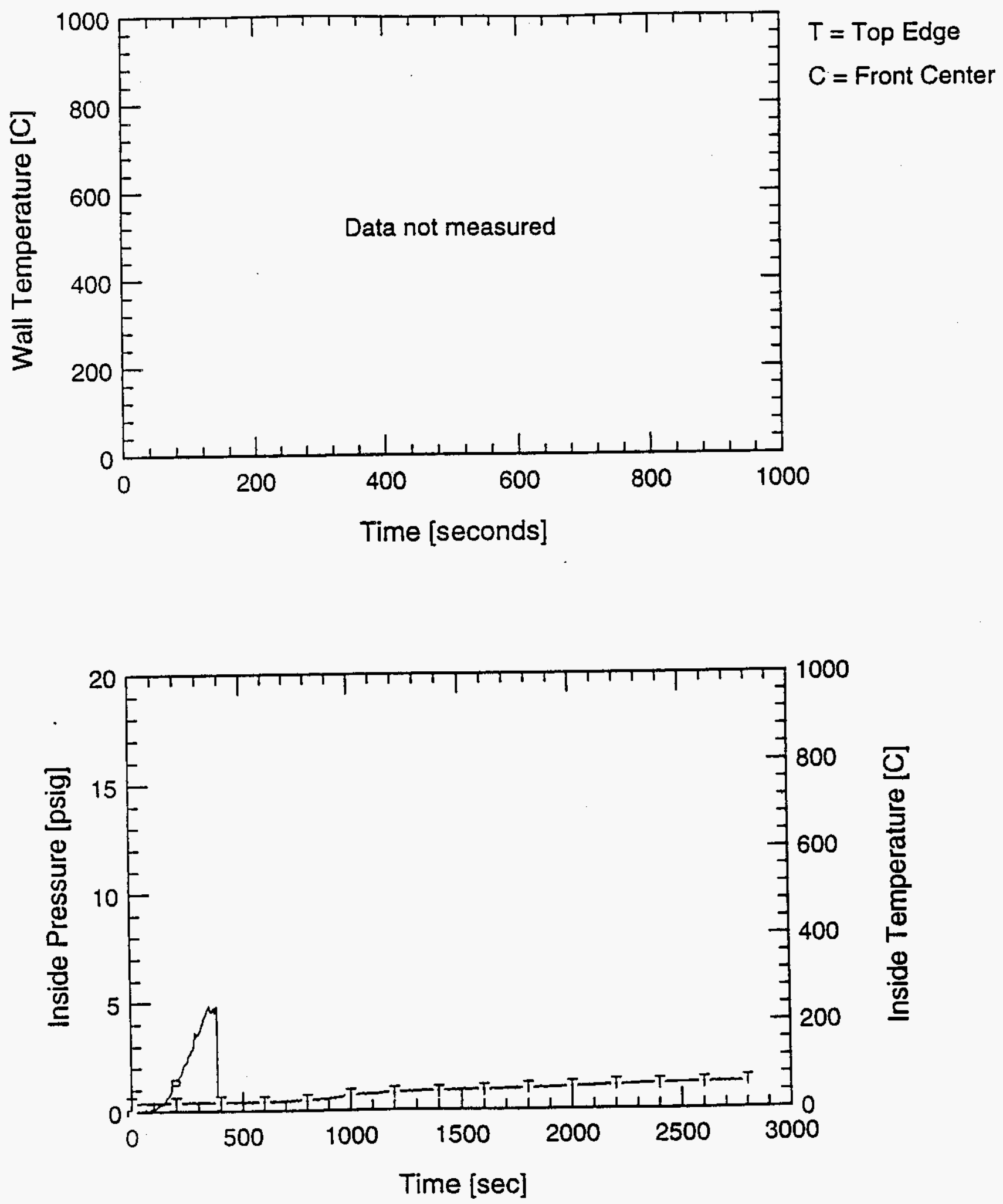
WHC-SD-WM-TRP-233 REV. 0

SWD19 DRUM 4 - 19" Burner - Standard Load
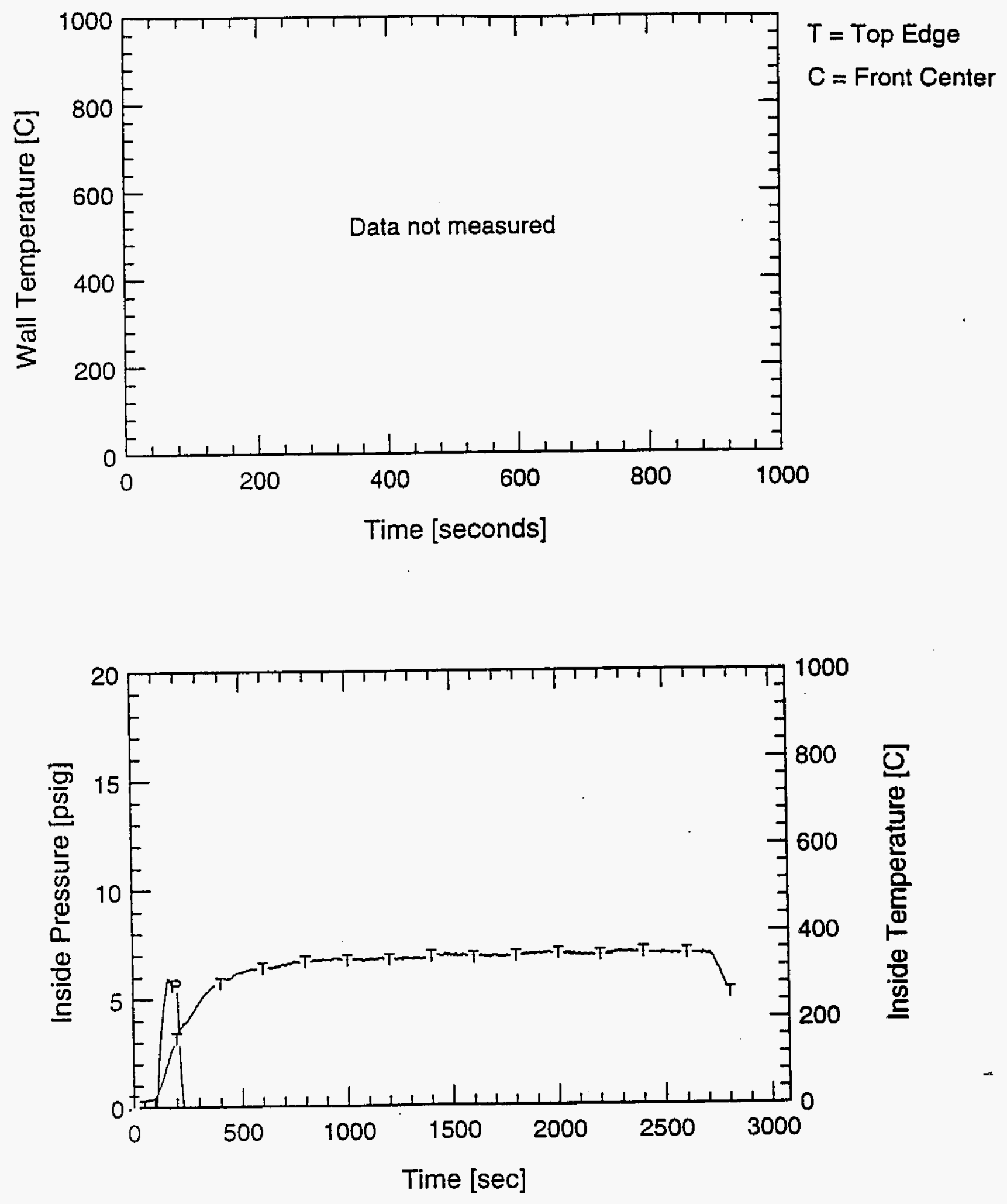
WHC-SD-WM-TRP-233 REV. O

SWD 20 - DATA 


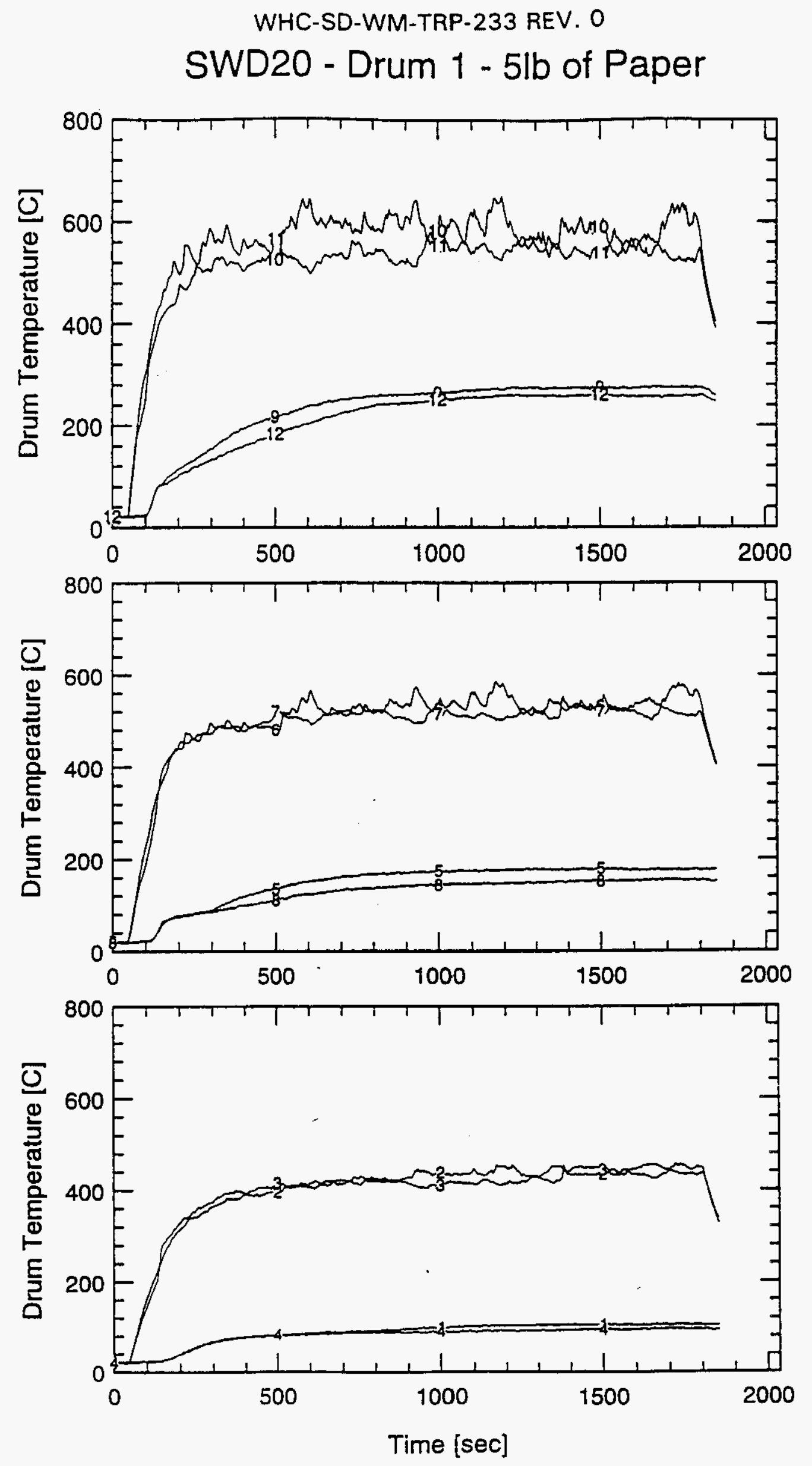

B-132 
WHC-SD-WM-TRP-233 REV. 0

\section{SWD20 - Drum 2}
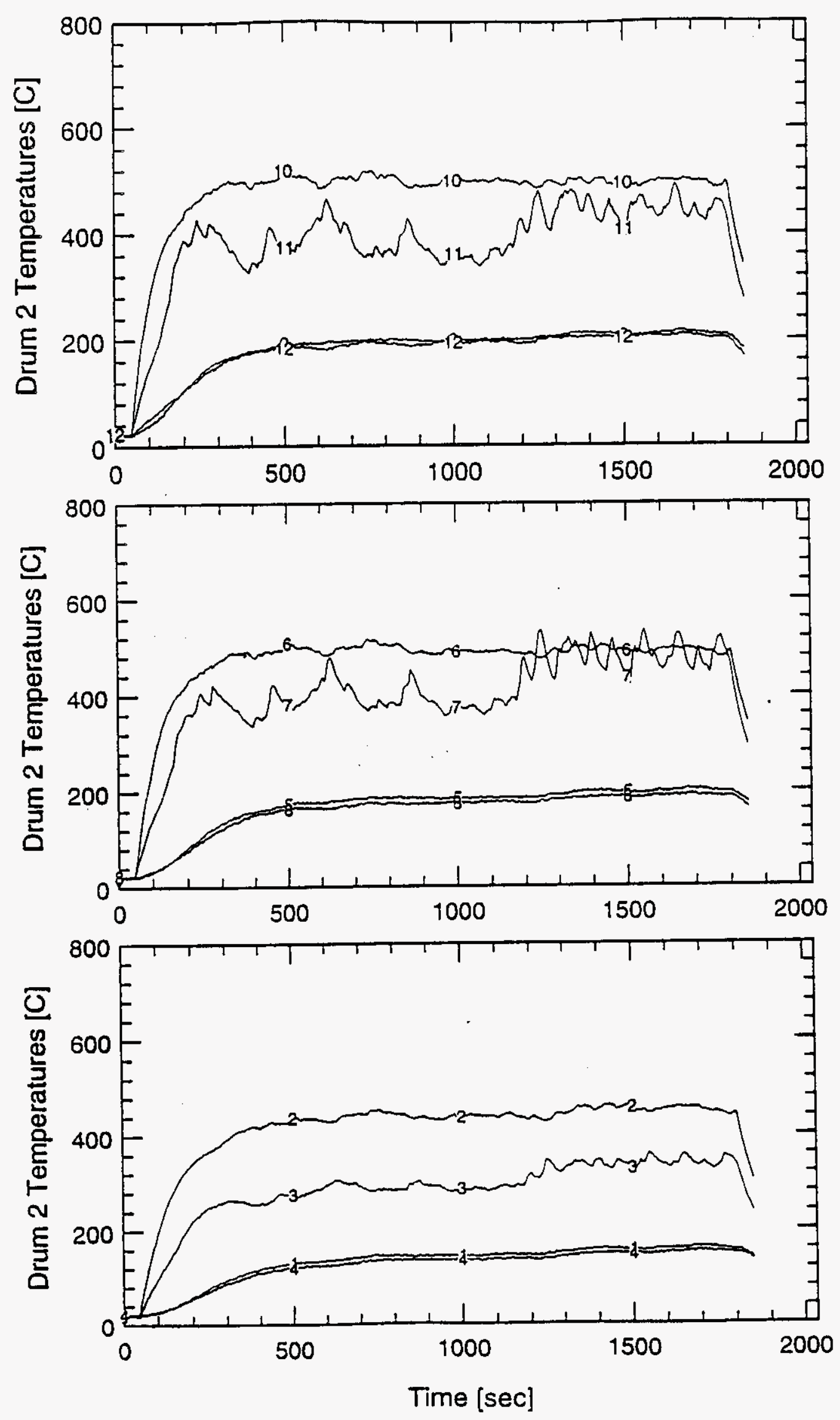
WHC-SD-WM-TRP-233 REV. 0

SWD20 - Drum 3 Water filled drum
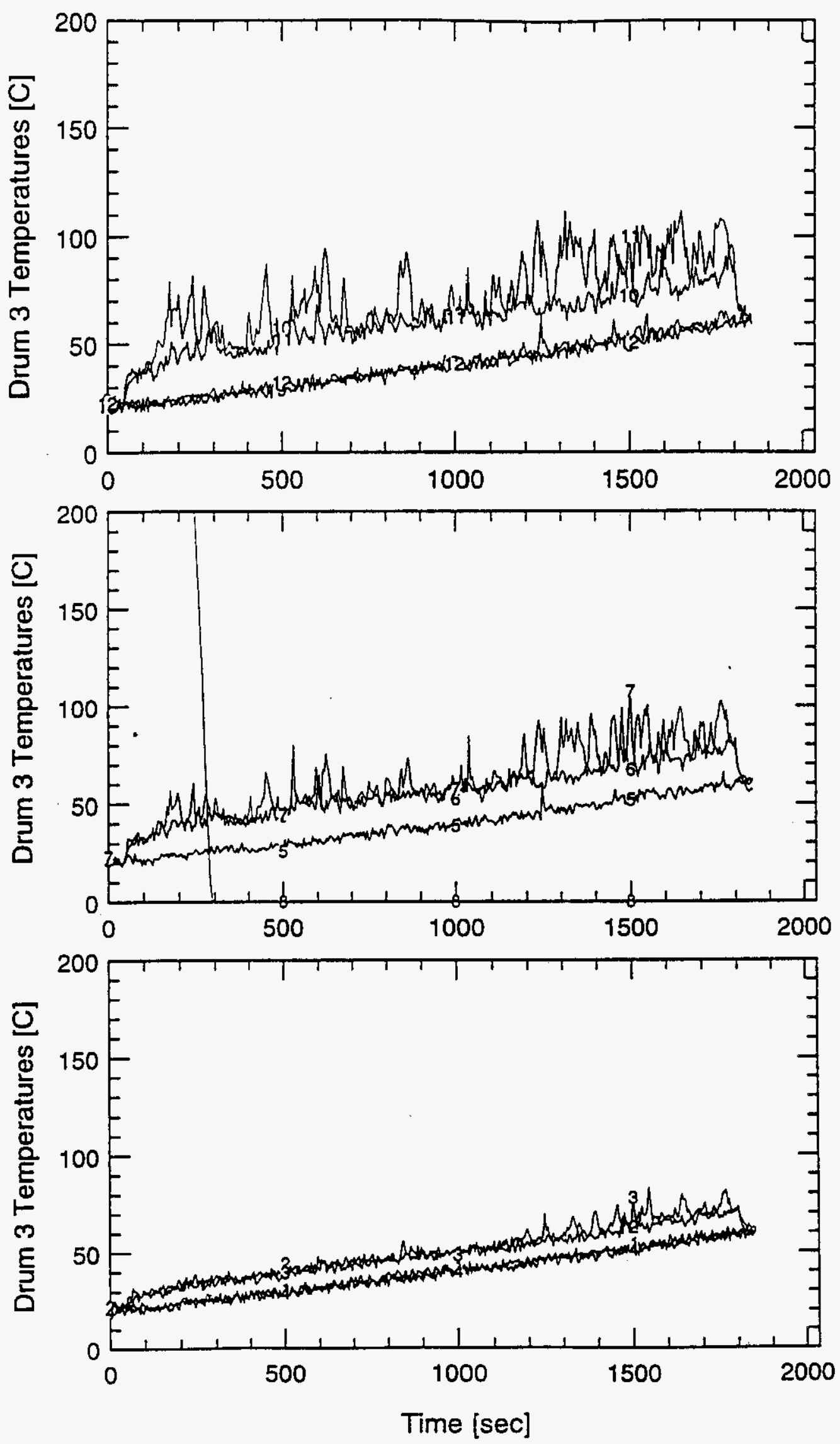
WHC-SD-WM-TRP-233 REV. O

SWD20 - Drum 4 - 5lb of Plastic
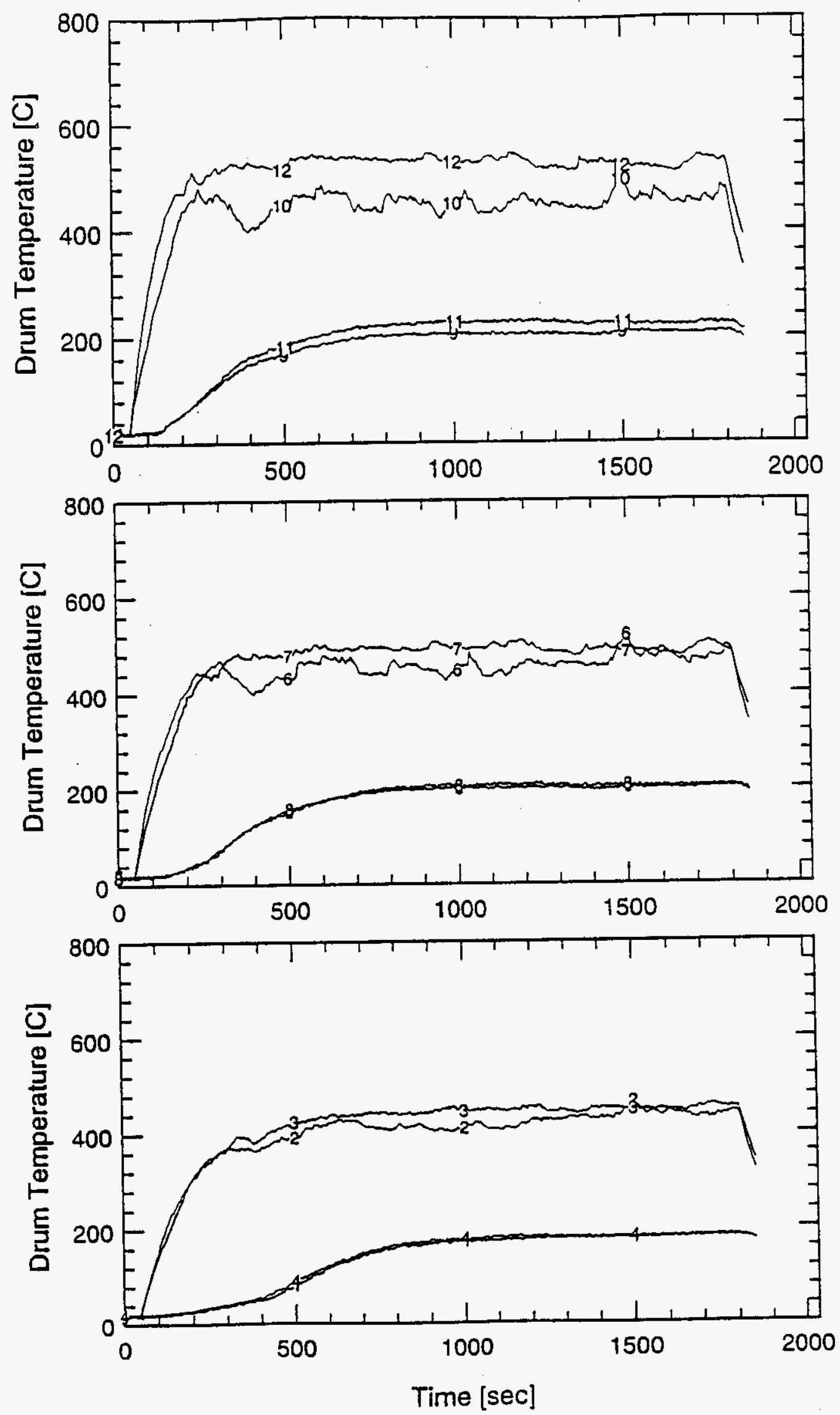


\section{SWD20 19" Burner}
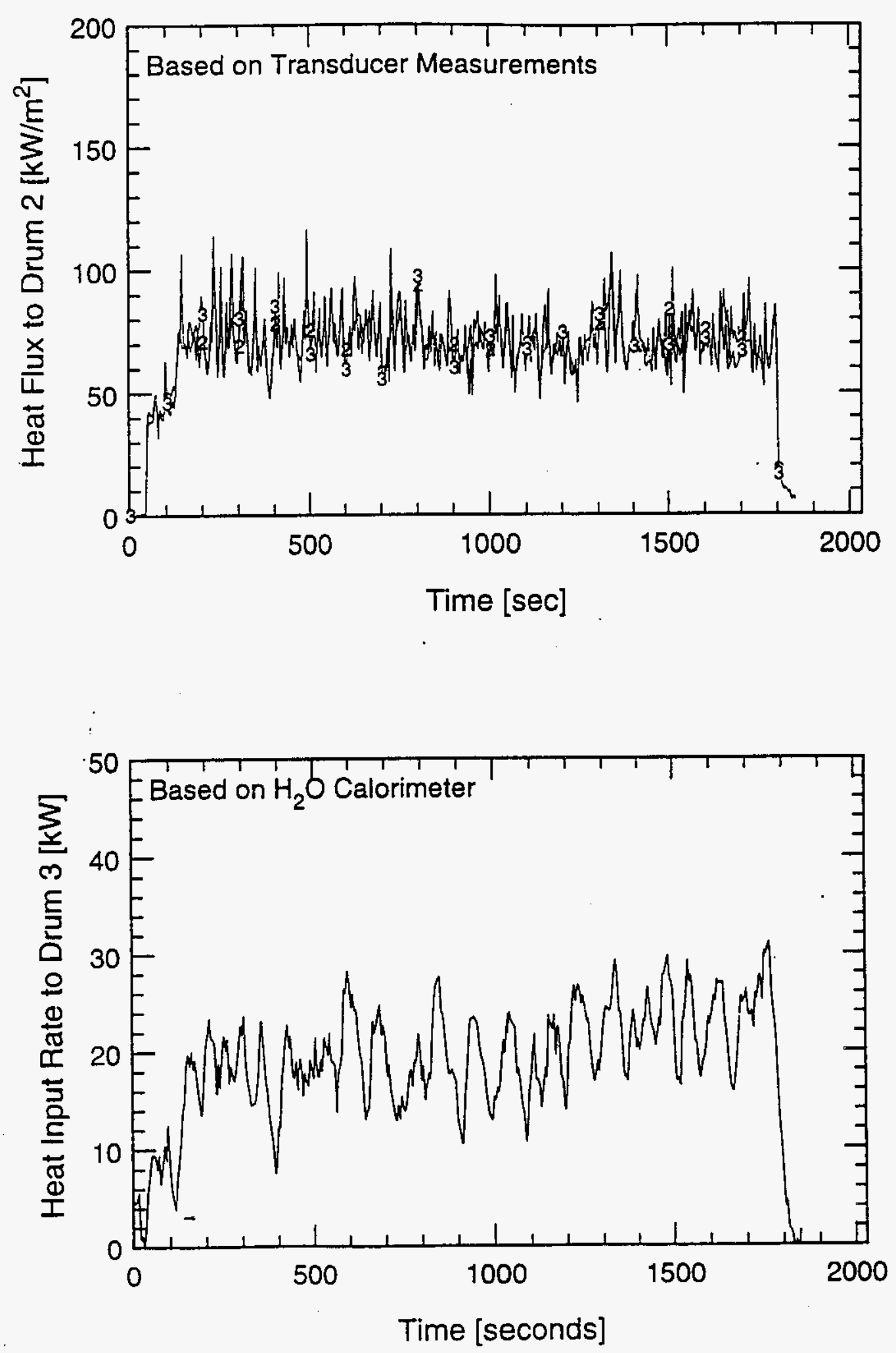
WHC-SD-WM-TRP-233 REV. 0

SWD20 DRUM 1 - 19" Burner - 5lb Paper
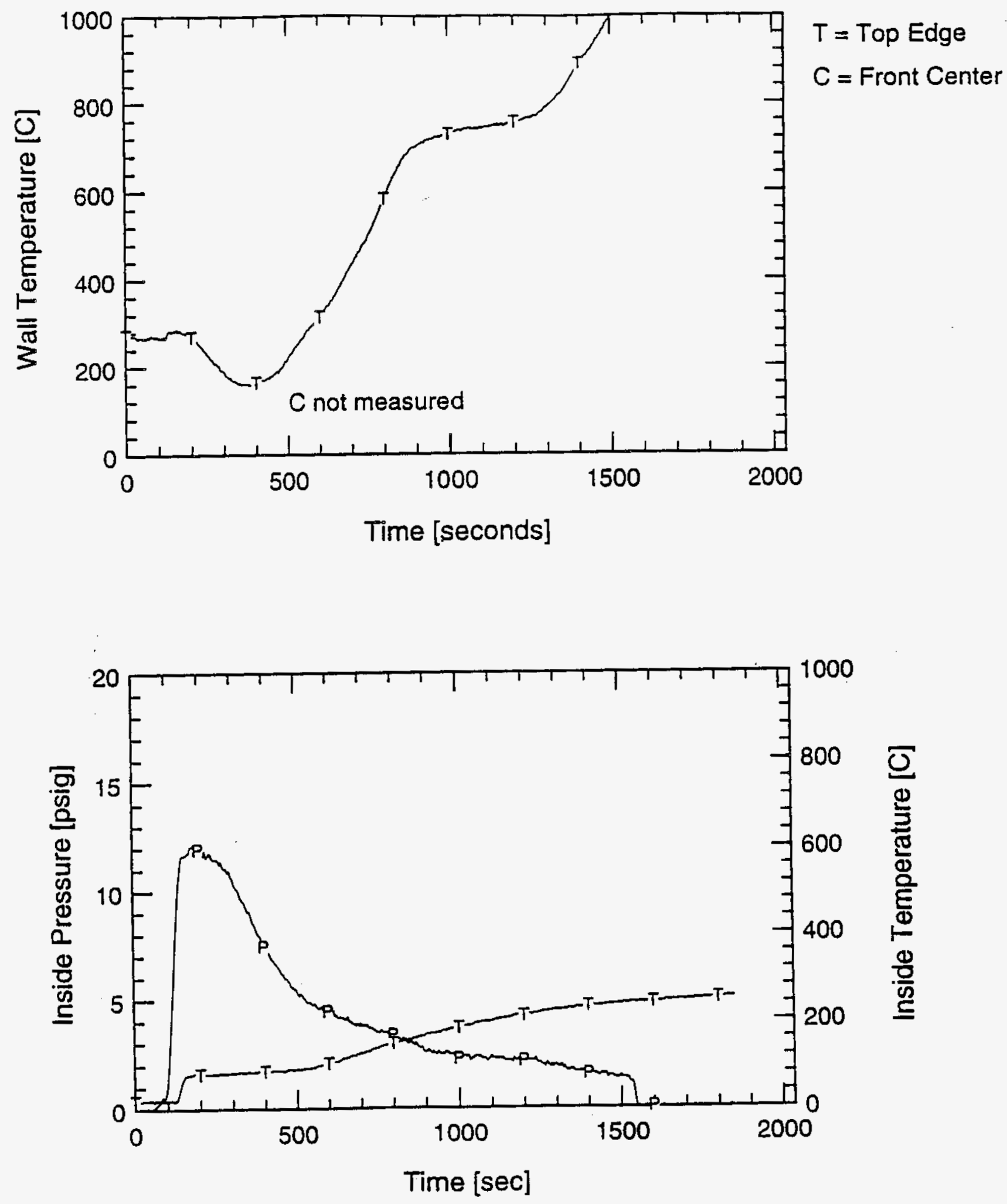
WHC-SD-WM-TRP-233 REV. 0

SWD21 - Drum 1 - $5 \mathrm{lb}$ of Paper - Heptane mid ht/front
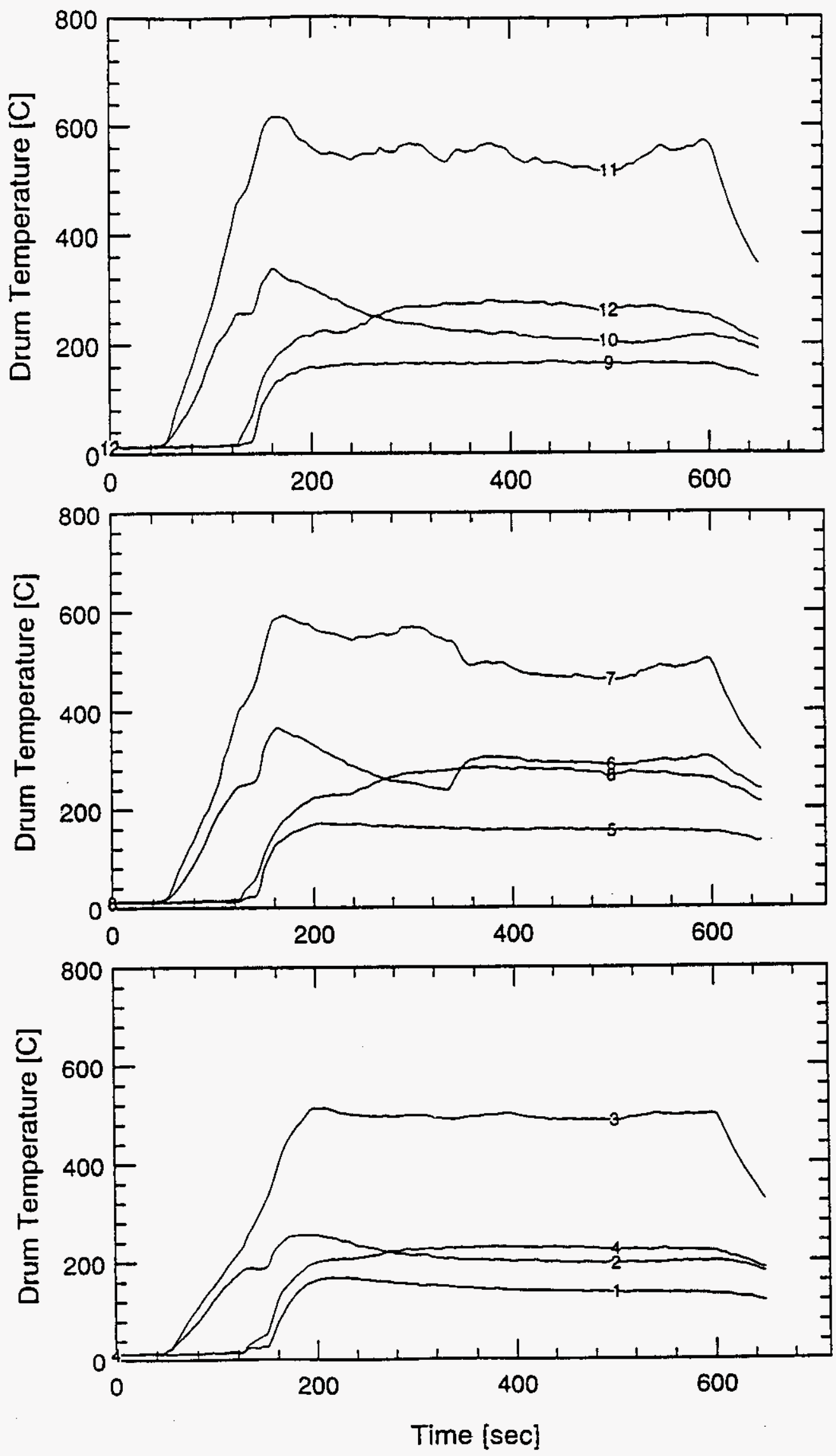

B-138 
SWD20 DRUM 4 - 19" Burner - 5lb Plastic
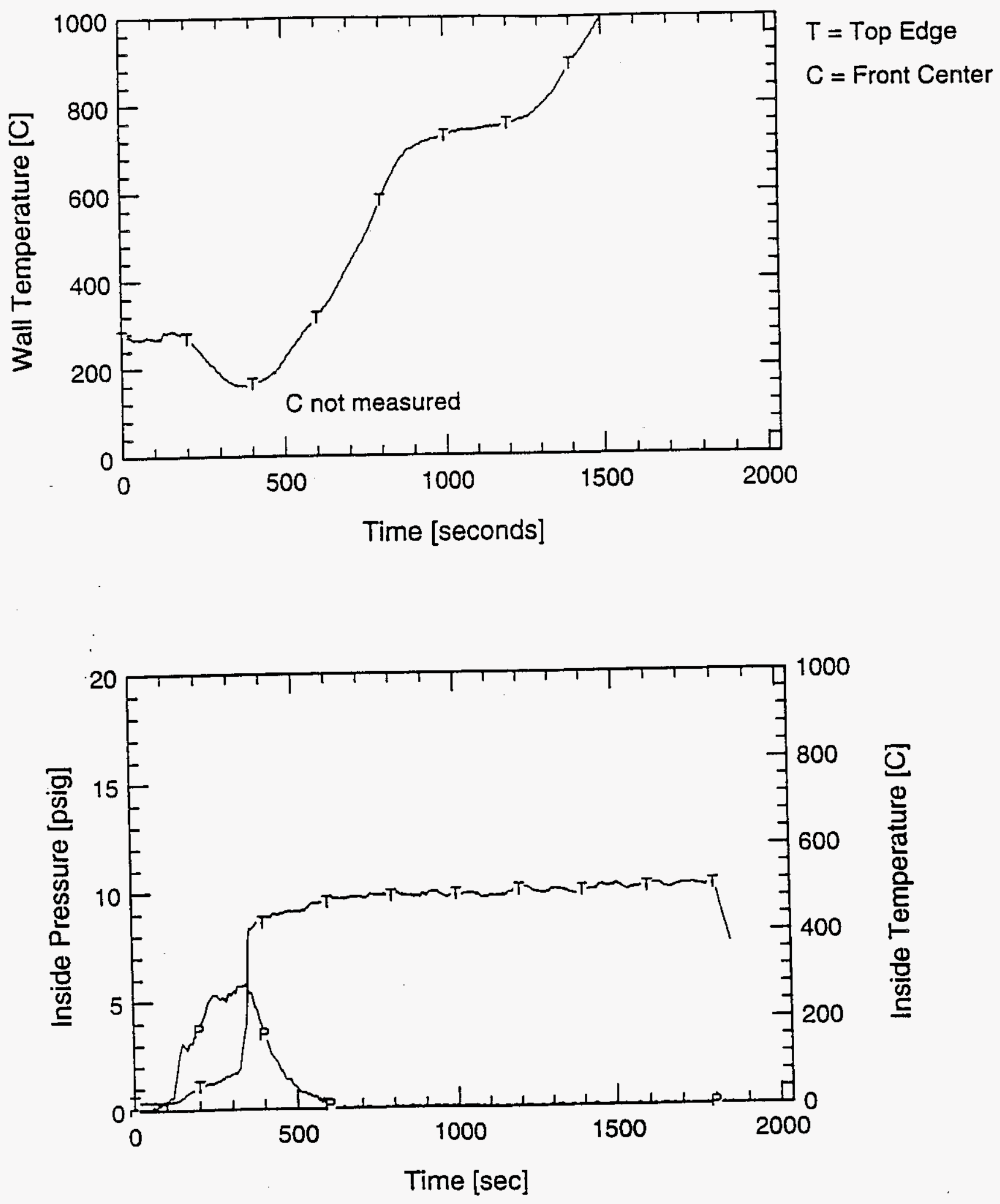
WHC-SD-WM-TRP-233 REV. 0

SWD 21 - DATA 
WHC.SD-WM-TRP-233 REV. O

SWD21 - Drum 2
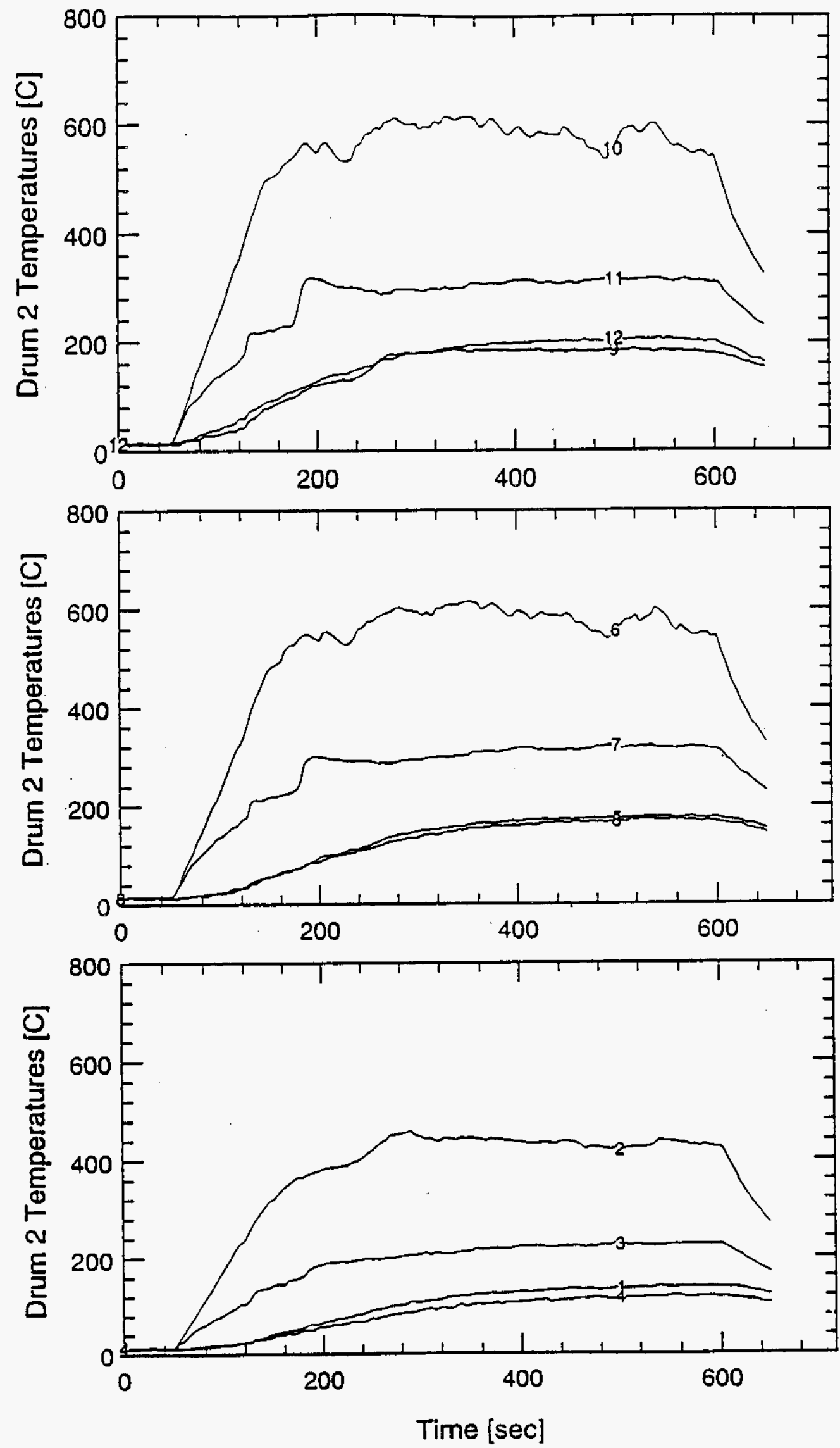
WHC-SD-WM-TRP-233 REV. 0

\section{SWD21 - Drum 3 Water filled drum}
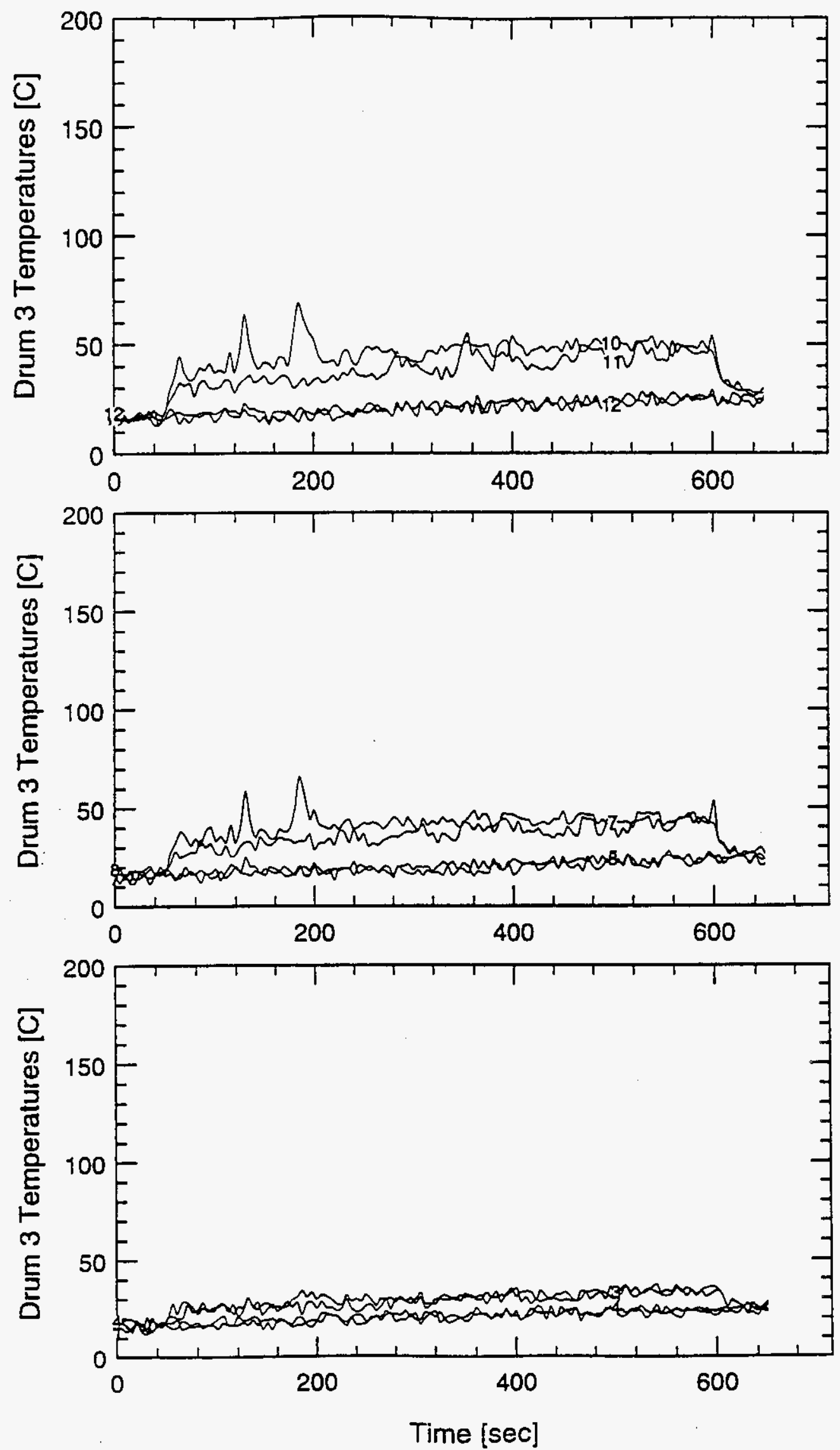
WHC-SD-WM-TRP-233 REV. 0

SWD21 - Drum 4 - Empty
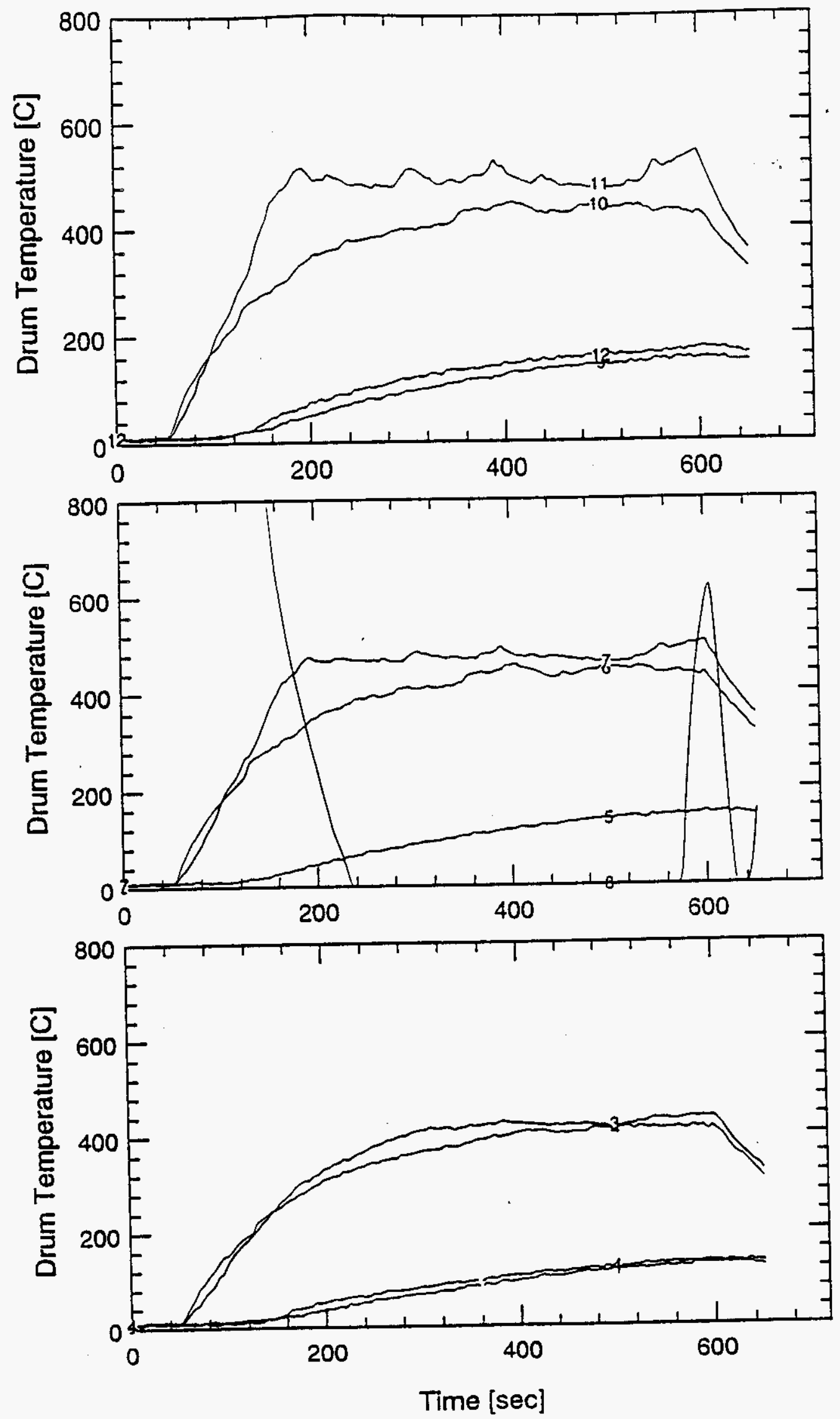
WHC-SD-WM-TRP-233 REV. 0

SWD21 19" Burner
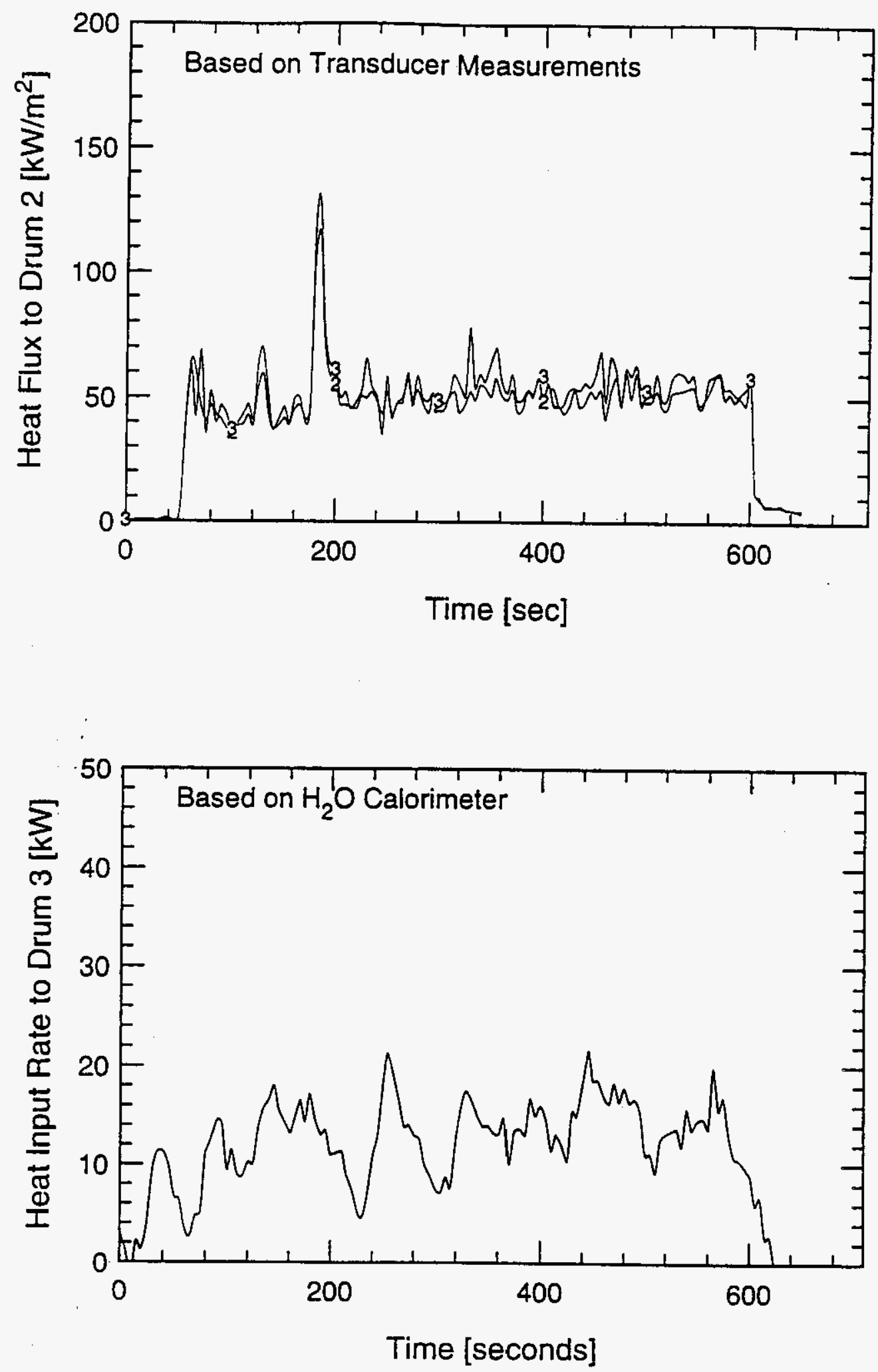
SWD21 DRUM 1 - 19" Burner - 5lb Paper - Heptane
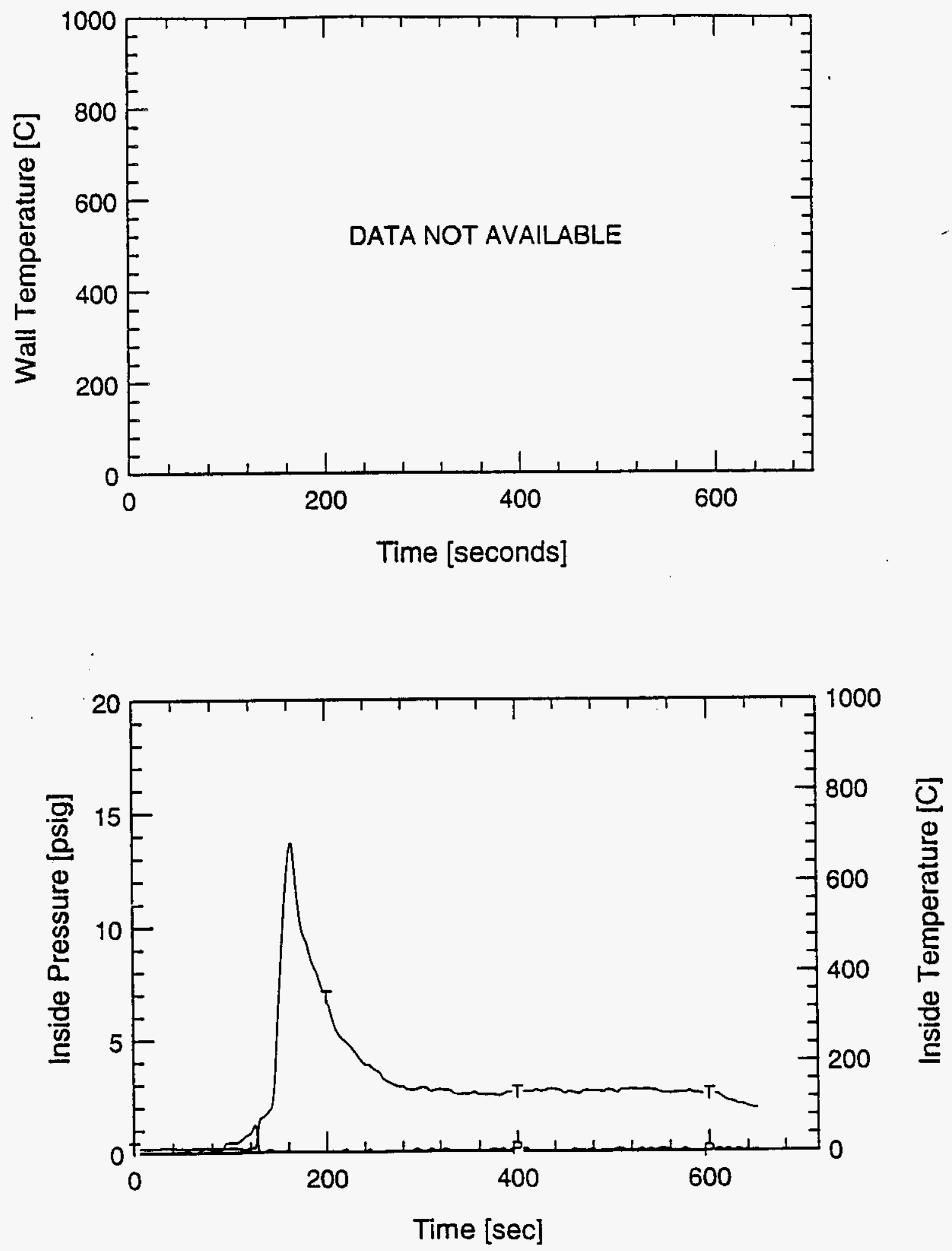
WHC-SD-WM-TRP-233 REV. 0

SWD21 DRUM 4 - 19" Burner - Empty
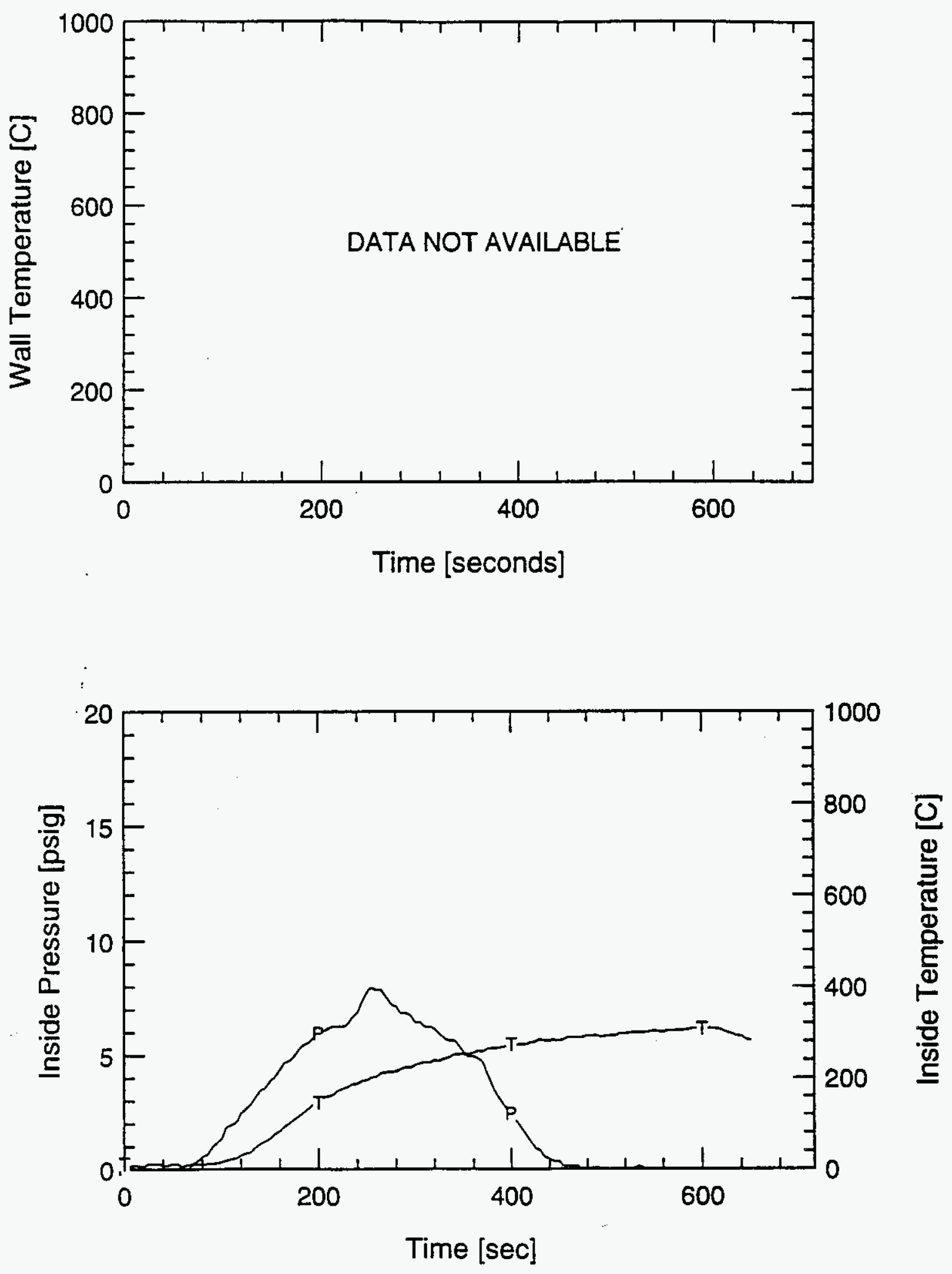
WHC-SD-WM-TRP-233 REV. 0

SWD 22 - DATA 
WHC-SD-WM-TRP-233 REV. 0

SWD22 - 19" Burner - Bagged Std Load - Acetone
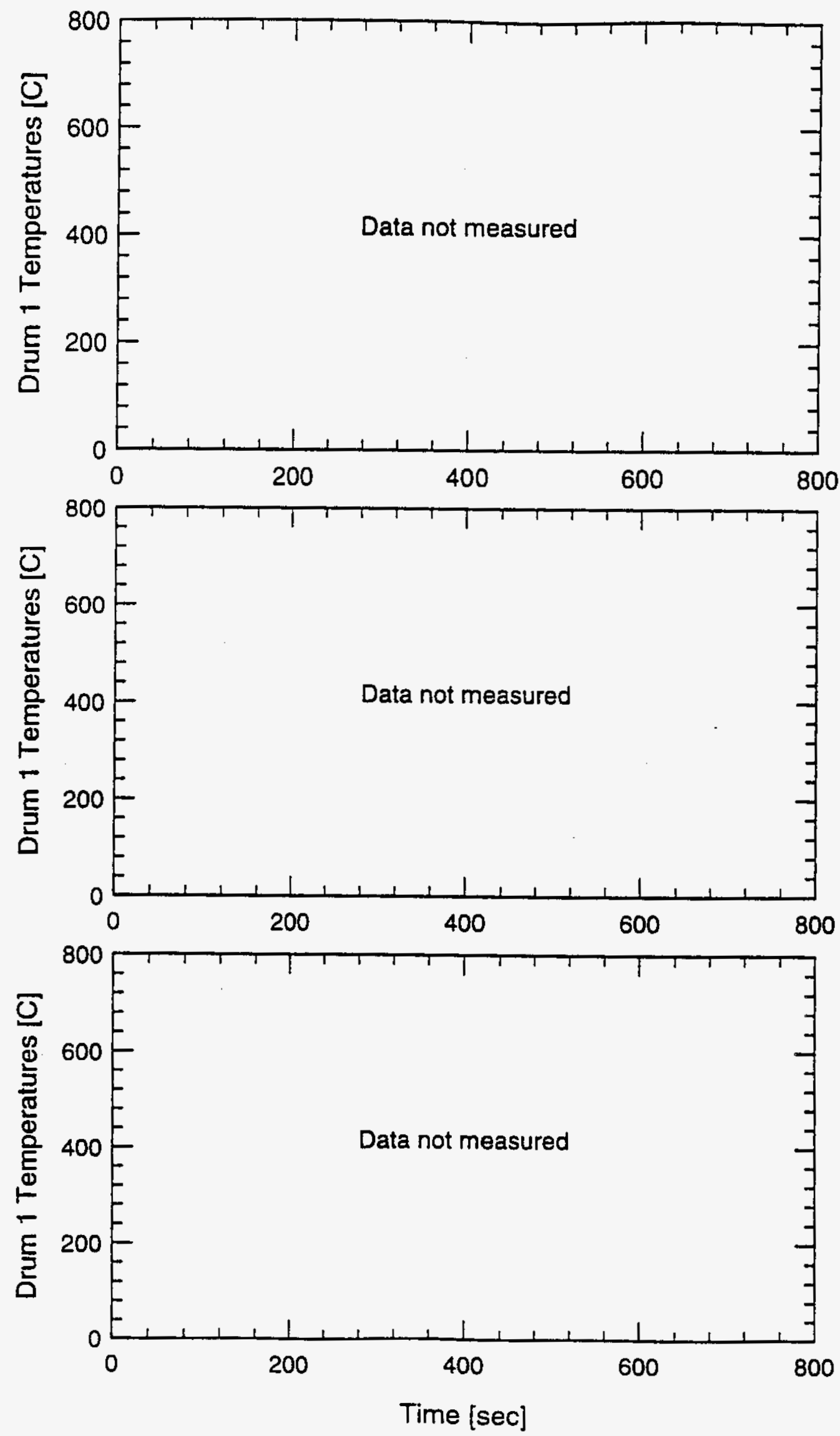
WHC-SD-WM-TRP-233 REV. 0

SWD22 - 19" Burner
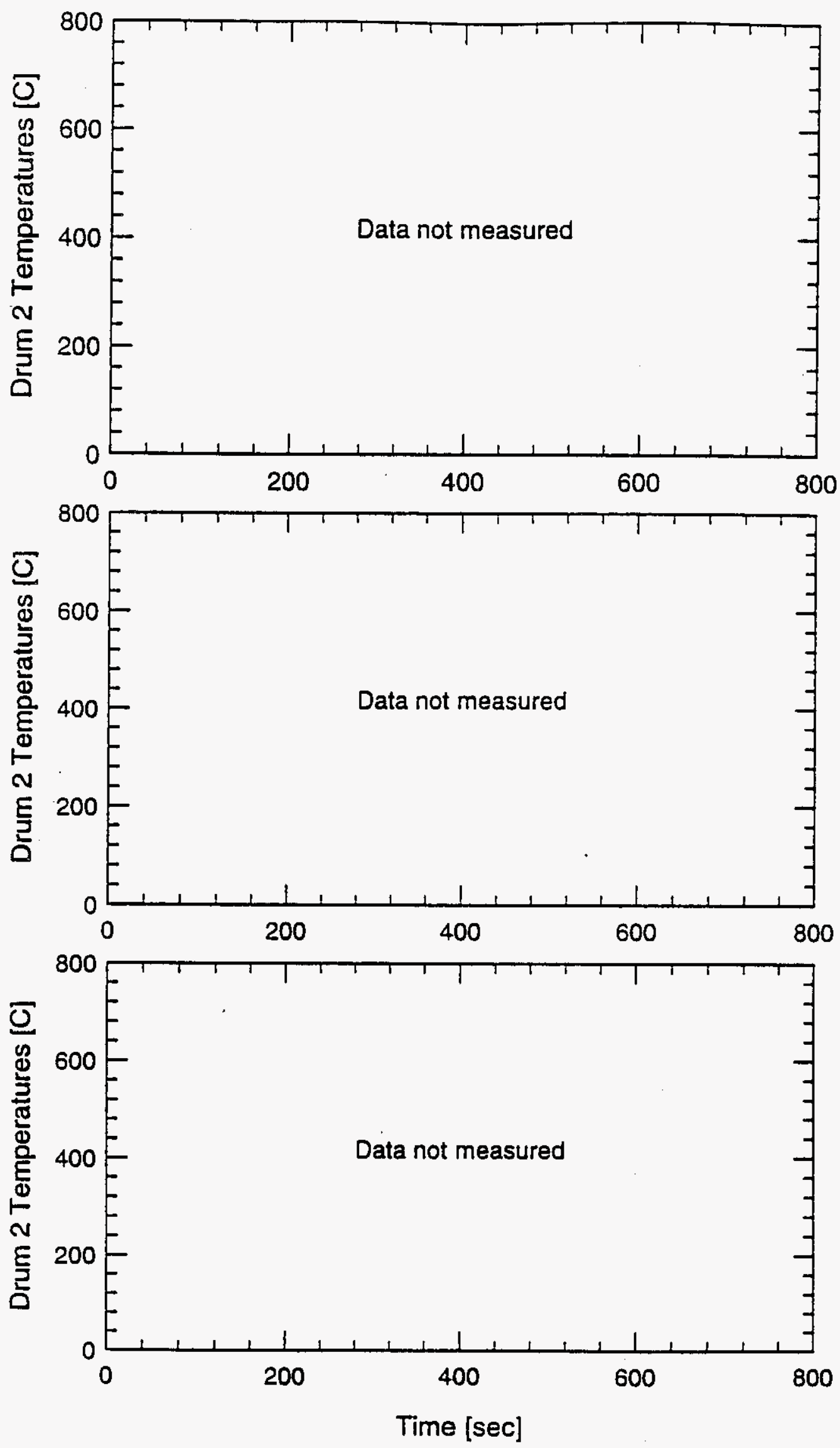
WHC-SD-WM-TRP-233 REV. 0

SWD22 - 19" Burner
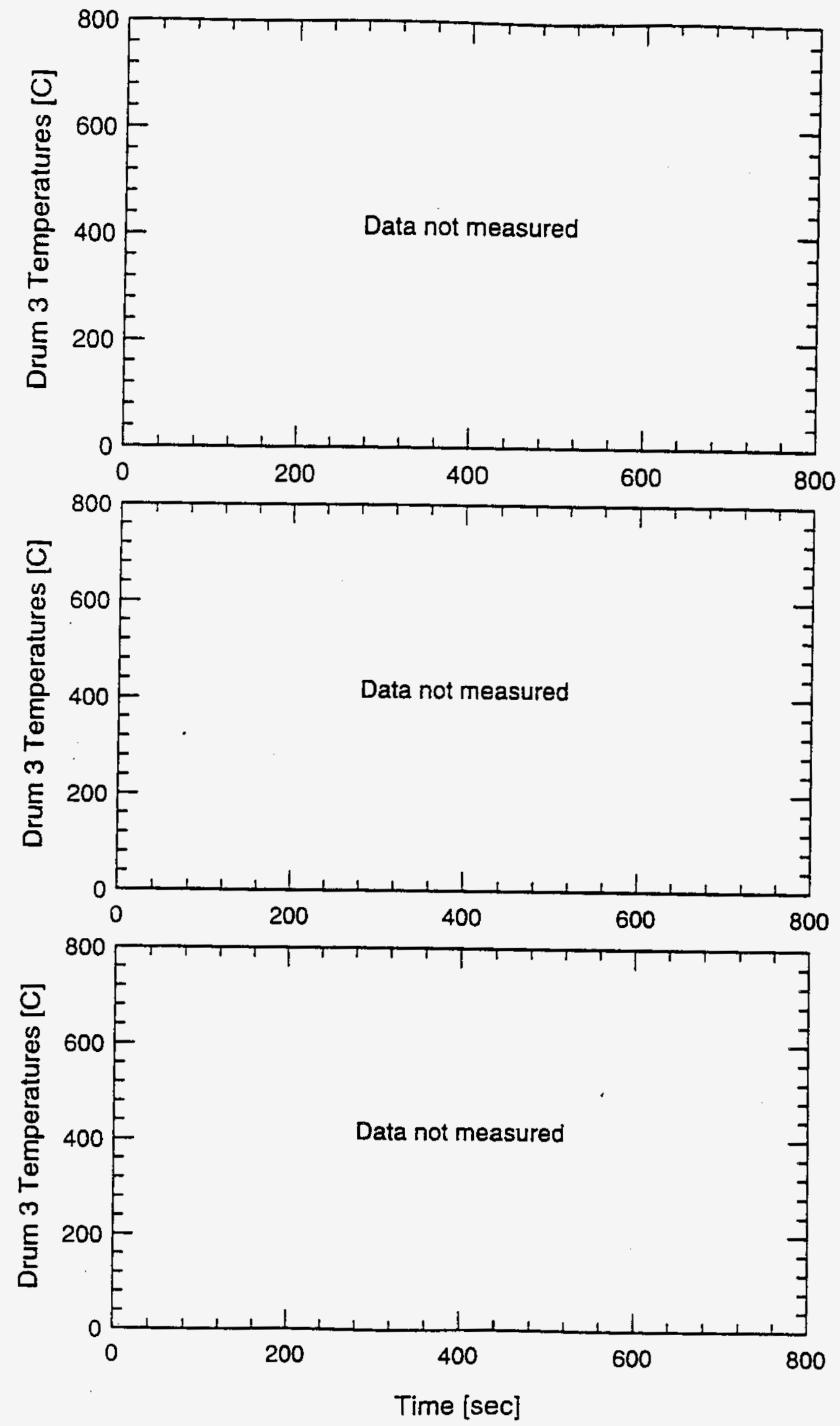
WHC-SD-WM-TRP-233 REV. O

SWD22 - 19" Burner - 5lb Paper - Acetone
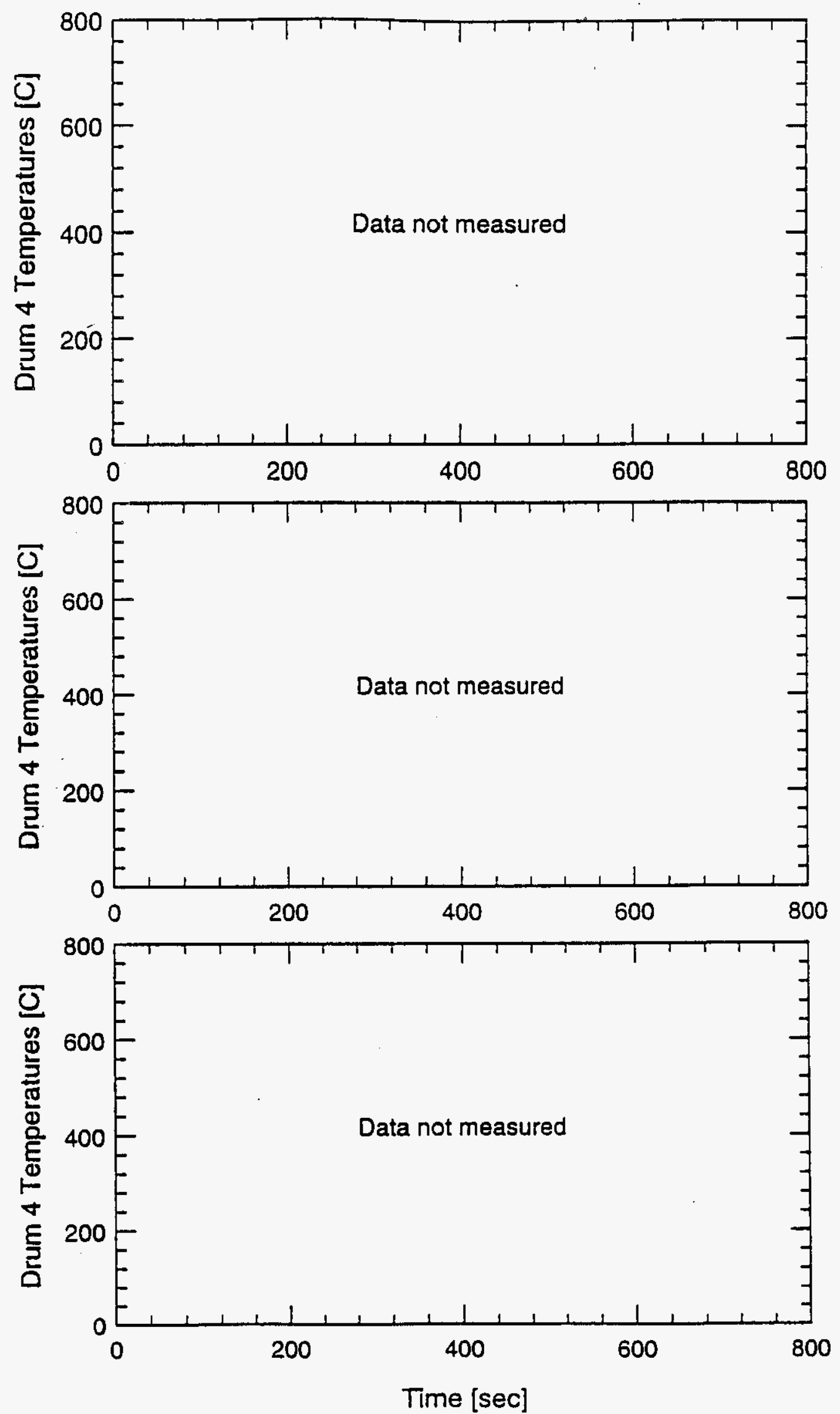
WHC-SD-WM-TRP-233 REV. 0

\section{SWD22 19" Burner}
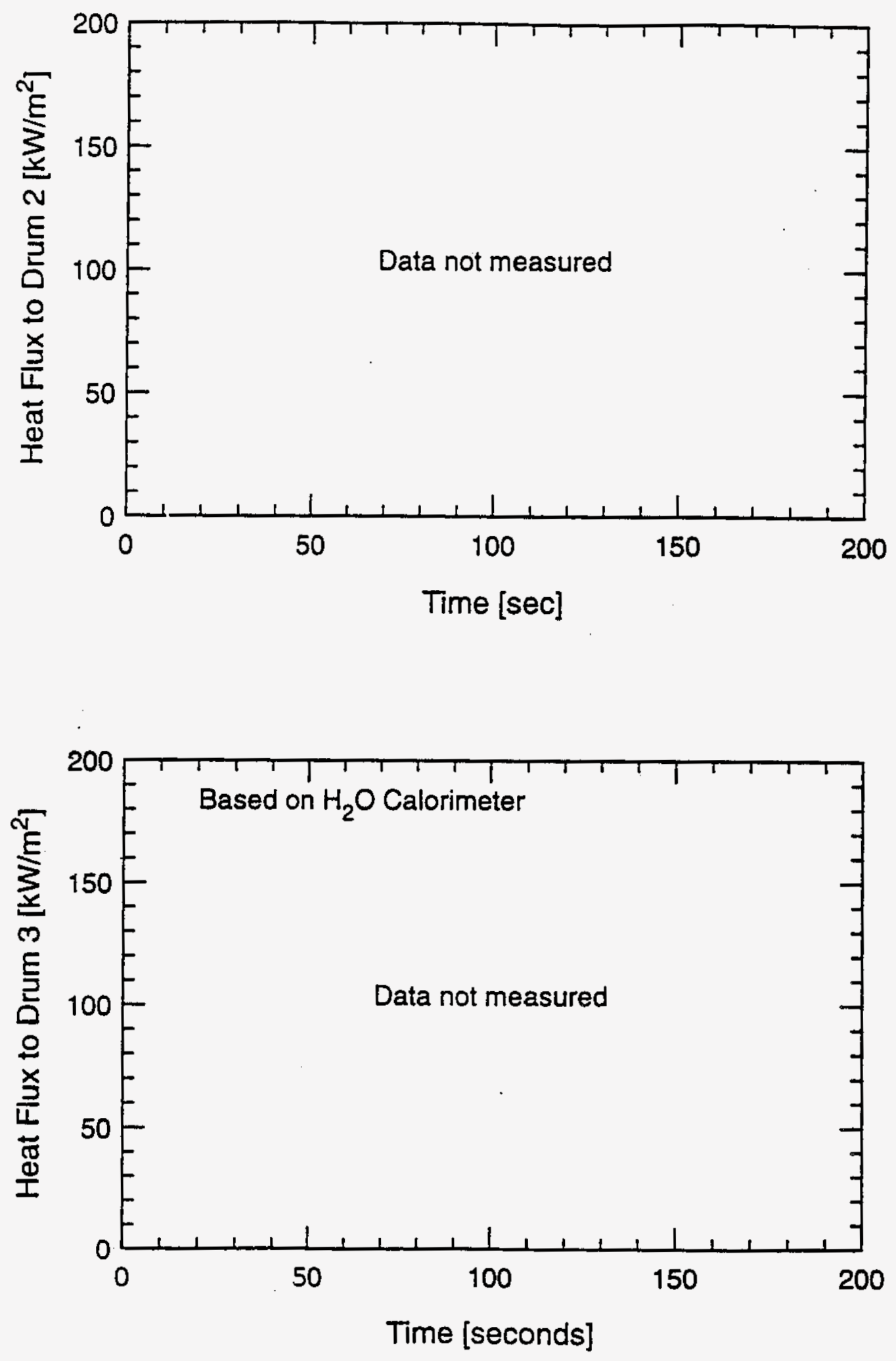
SWD22 DRUM 1 - 19" Burner - Standard Load - Acetone
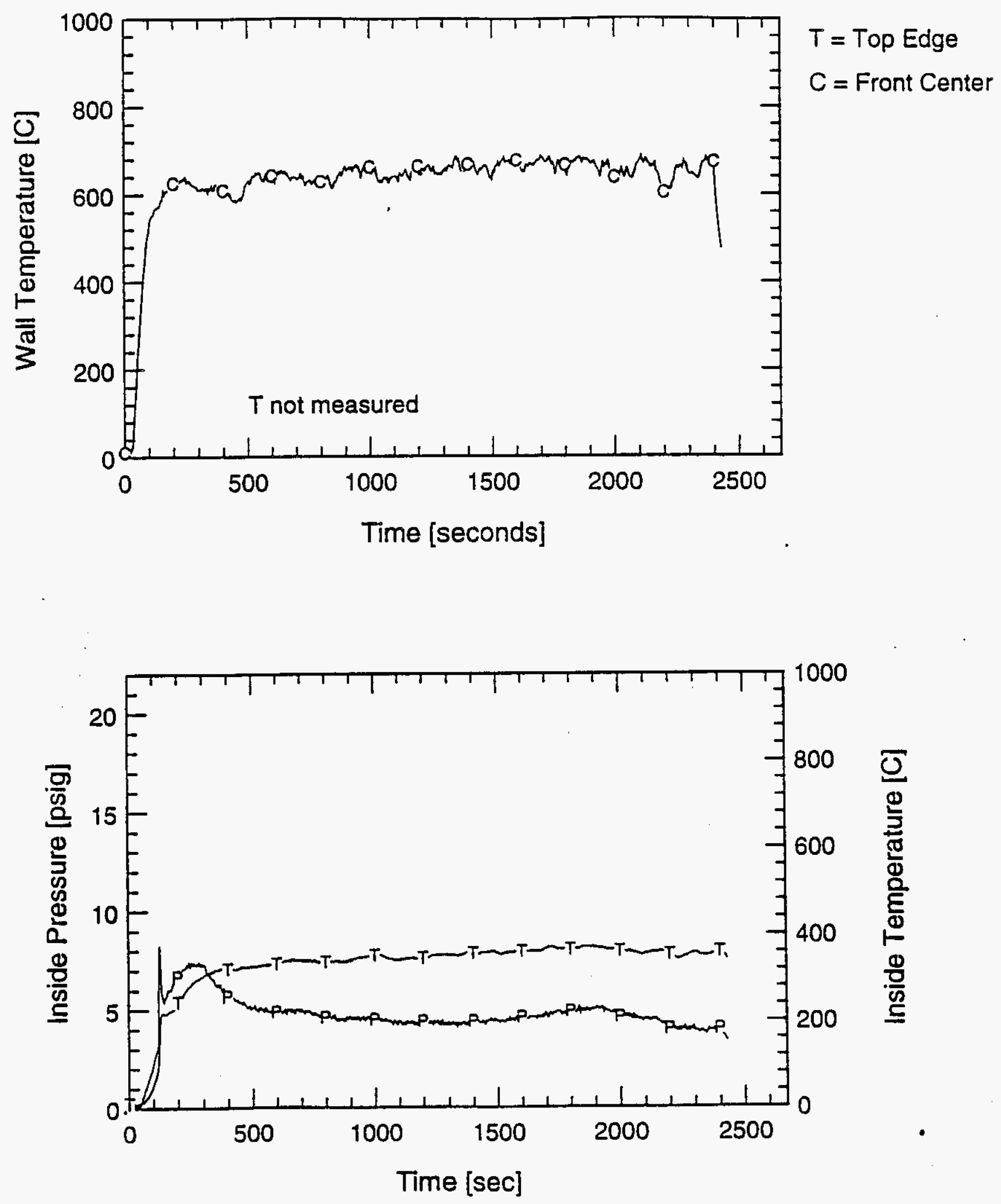
SWD22 DRUM 4 - 19" Burner - 5lb Paper - Acetone
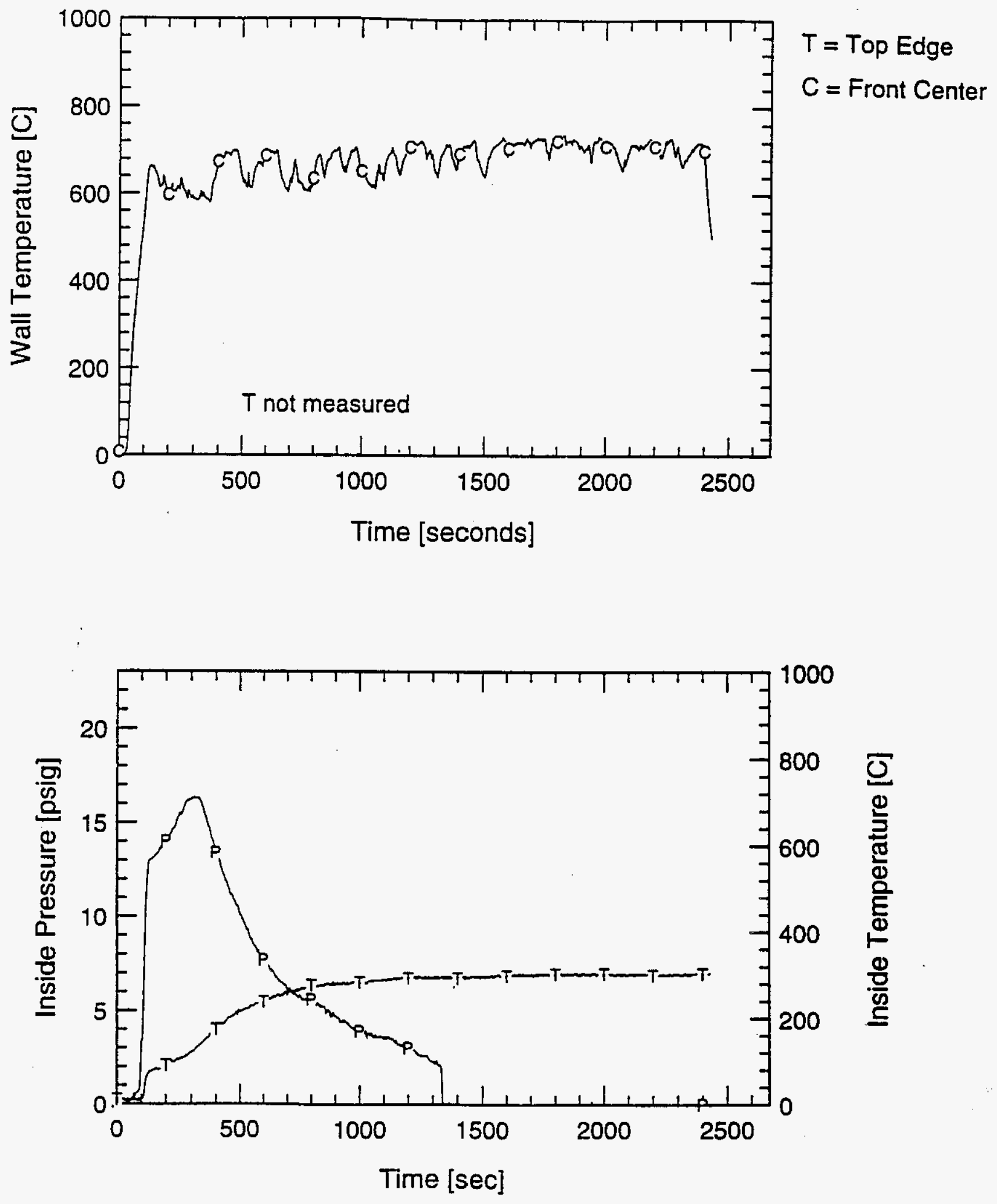
WHC-SD-WM-TRP-233 REV. 0

SWD 23 - DATA 
WHC-SD-WM-TRP-233 REV. 0

SWD23 - 19" Burner - 5lb Paper - Heptane
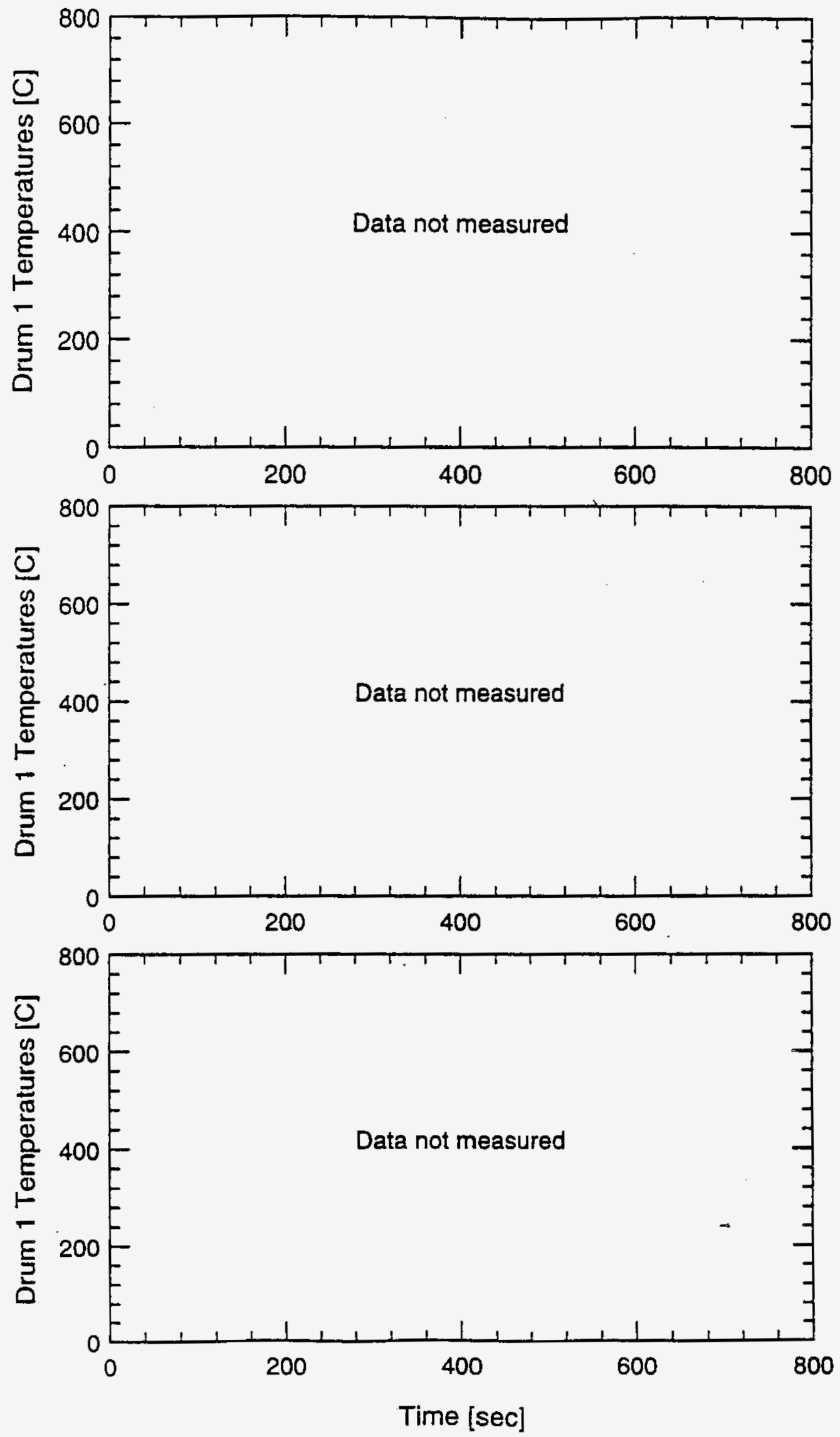

B-156 
WHC-SD-WM-TRP-233 REV. 0

SWD23 - 19" Burner
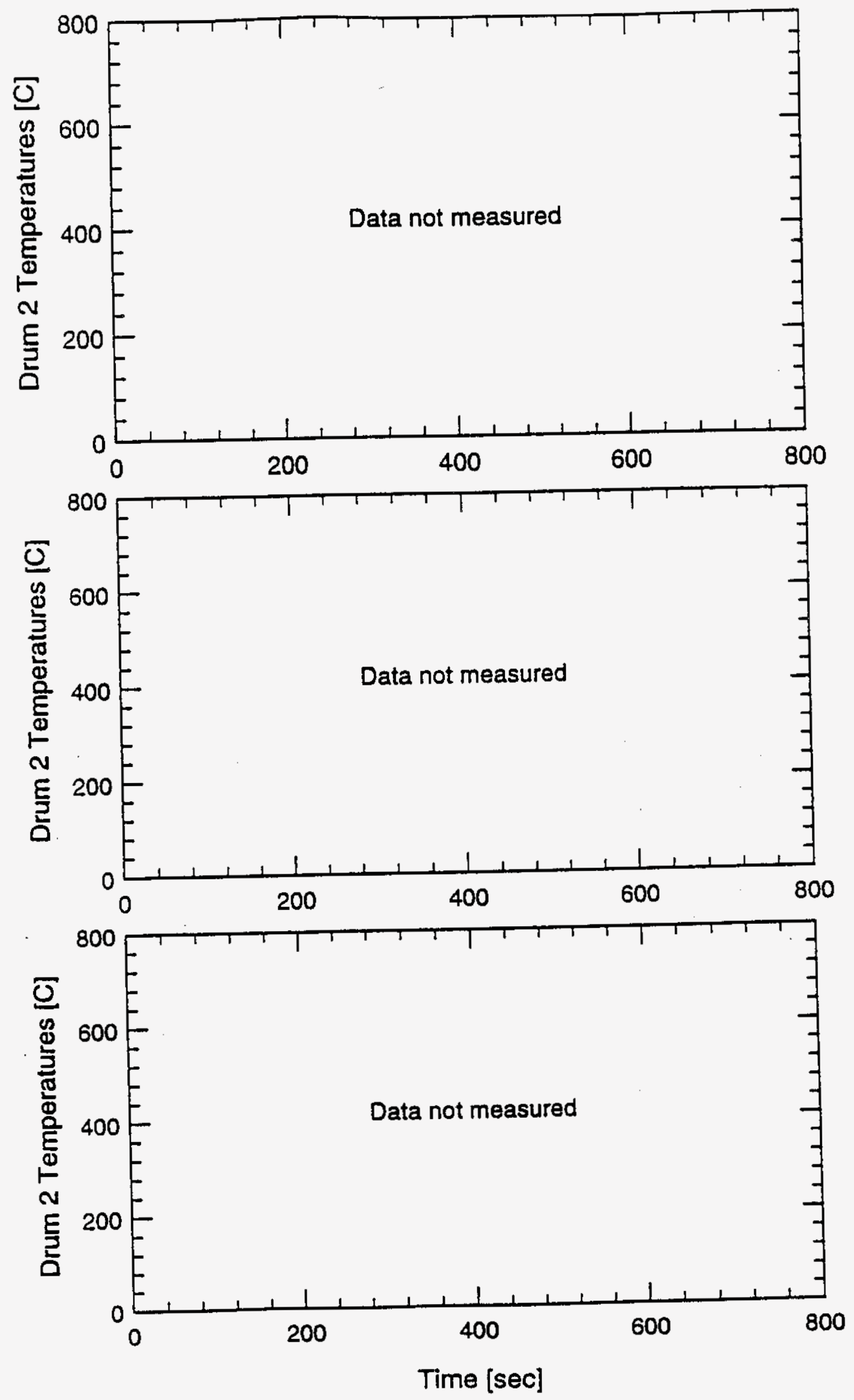
WHC-SD-WM-TRP-233 REV. 0

SWD23 - 19" Burner
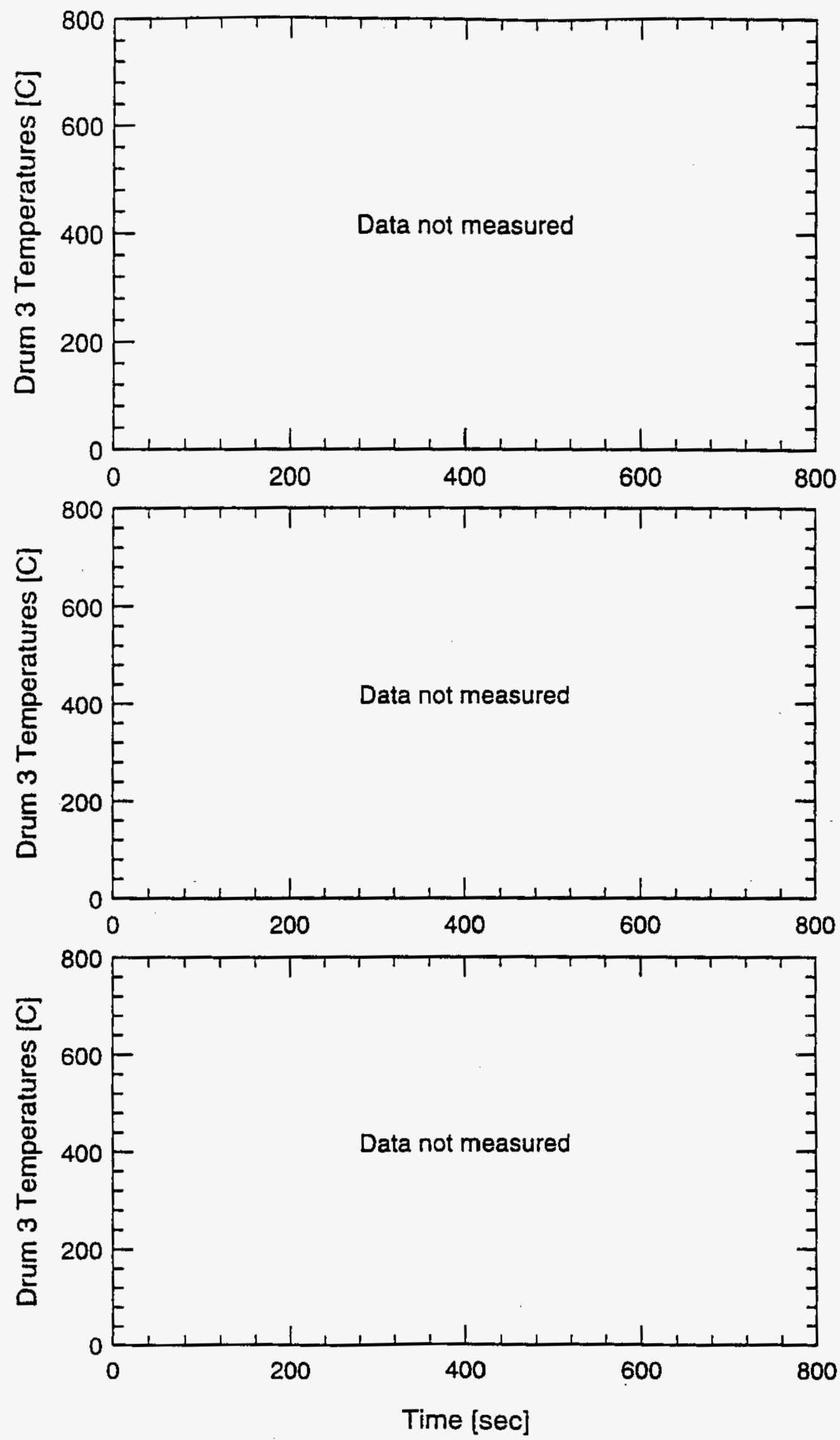

B-158 
WHC-SD-WM-TRP-233 REV. 0

SWD23 DRUM 1 - 19" Burner - 5lb Paper - Heptane
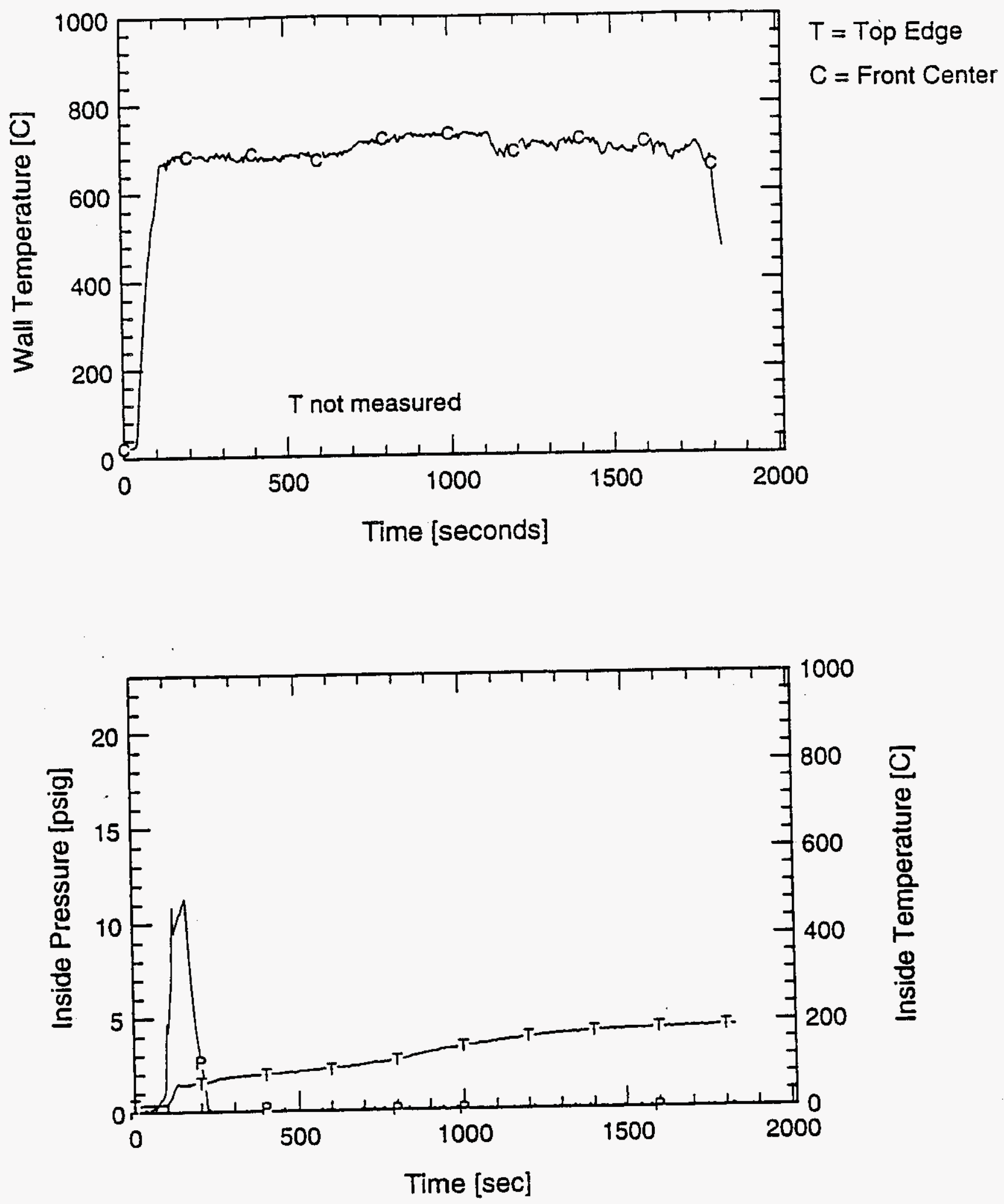
SWD23 DRUM 4 - 19" Burner - 5lb Paper - Acetone
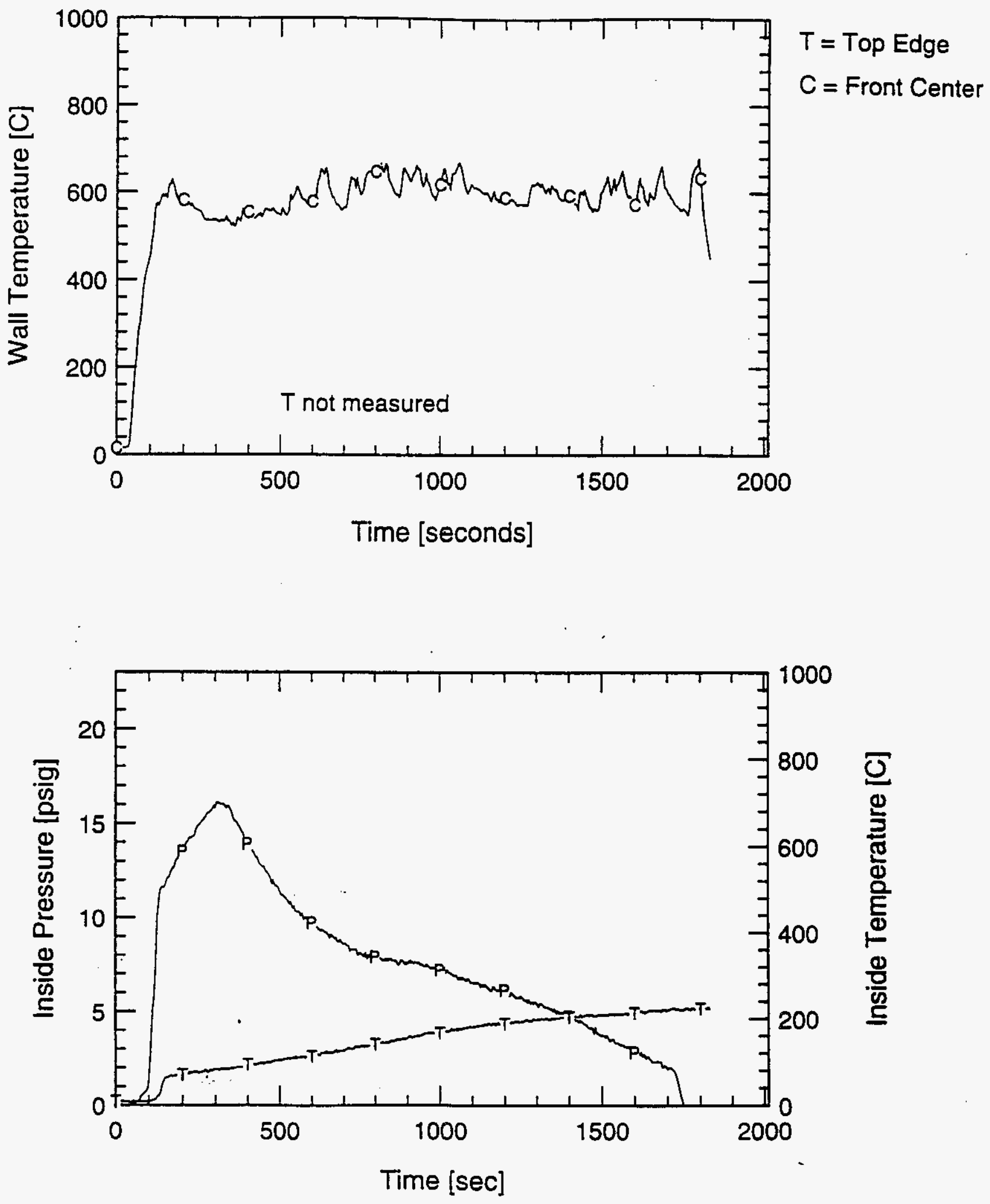
*avis View/Frint Document Cover Sheet

This document was retrieved from the Boeing ISEARCH System.

Accession \#: D196069896

Document \#: SD-WM-TRP-233

Title/Desc:

ANALYTICAL \& EXPERIMENTAL EVALUATION OF SOLID WASTE DRUM FIRE PERFORMANCE [VOL 2] [SEC 3 OF 3] 
This document was too large to scan as a whole document, therefore it required breaking into smaller sections.

DOCUMENT NUMBER: WHC-SD-WM-TRP-233 ReU O

SECTION 3 OF 3

TITLE: Analifical \&' Experimental

Evaluation of Solid Waste Drum Fire Peformance

DATE: $10 / 11 / 1995$

ORIGINATOR: Lovie, RL

CO: WHC

RECIPIENT:

CO:

REFERENCES: EDT-612225 
THIS PAGE INTENTIONALLY LEFT BLANK 
WHC-SD-WM-TRP-233 REV. 0

SWD 24 - DATA

B-163 
WHC-SD-WM-TRP-233 REV. 0

SWD24 19" Burner - Standard Load - EPDM
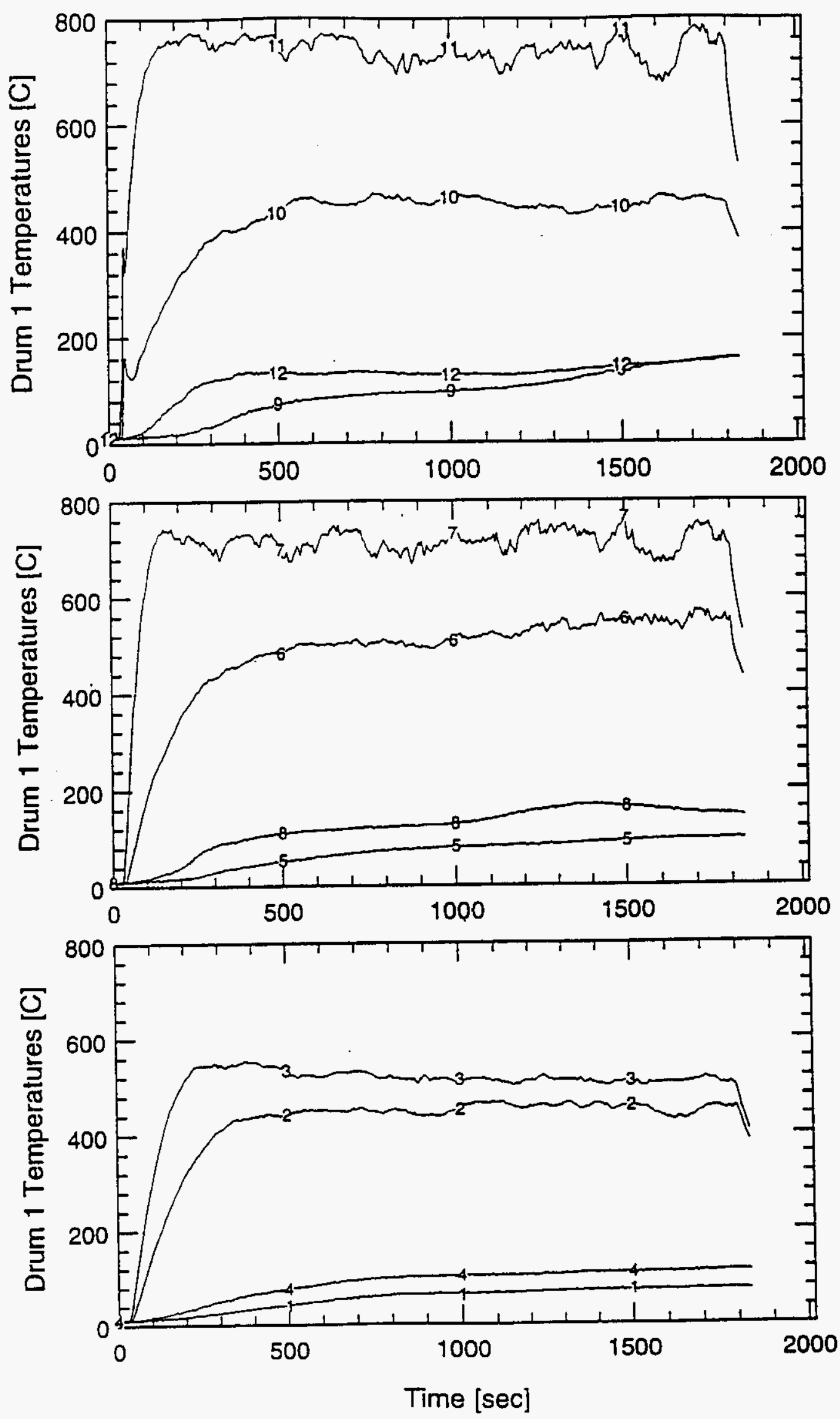

B-164 
WHC-SD-WM-TRP-233 REV. 0

SWD24 - Drum 2
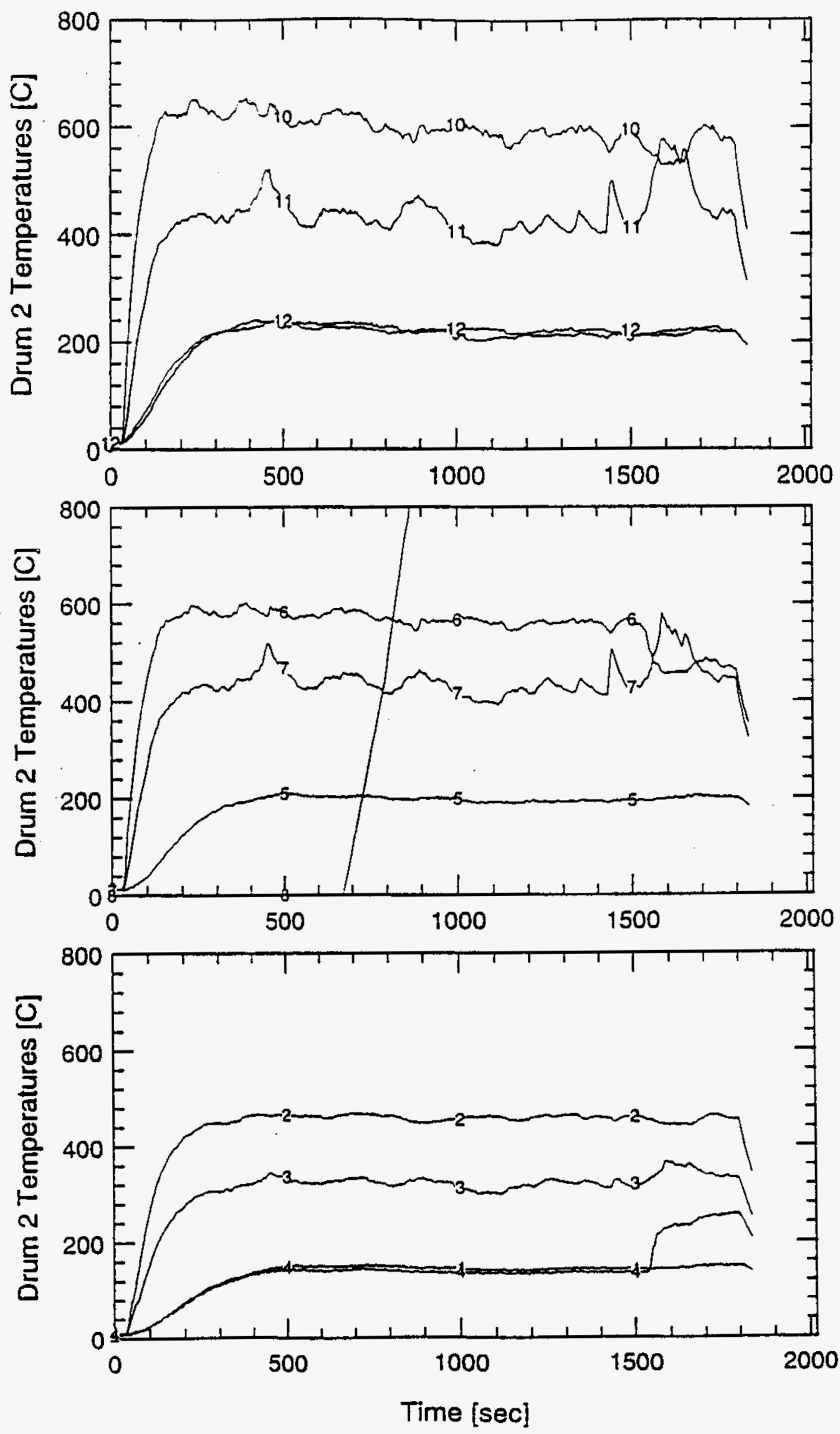

B-165 
WHC-SD-WM-TRP-233 REV. 0

SWD24 - Drum 3 Water filled drum
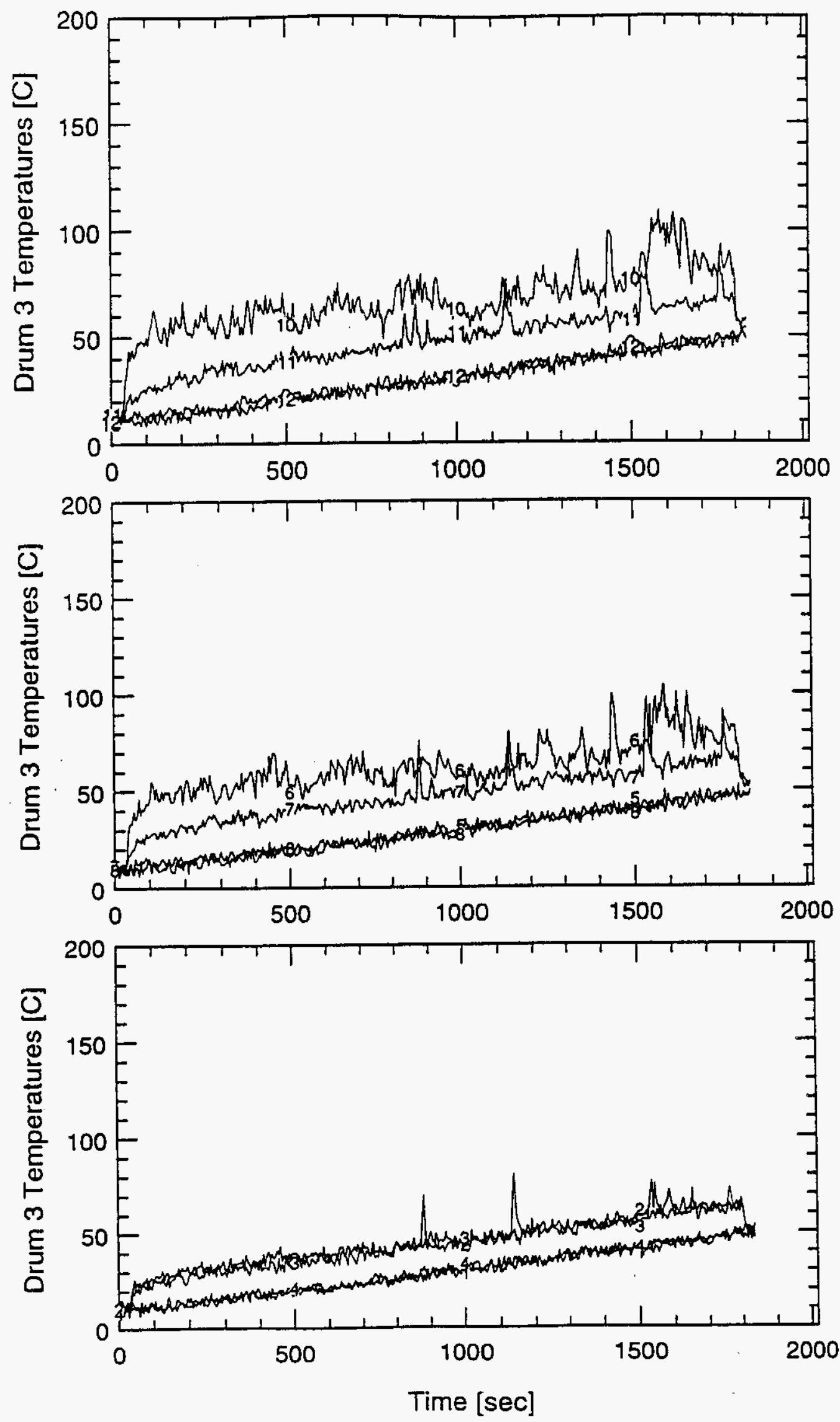
WHC-SD-WM-TRP-233 REV. 0

\section{SWD24 19" Burner - Empty - EPDM}
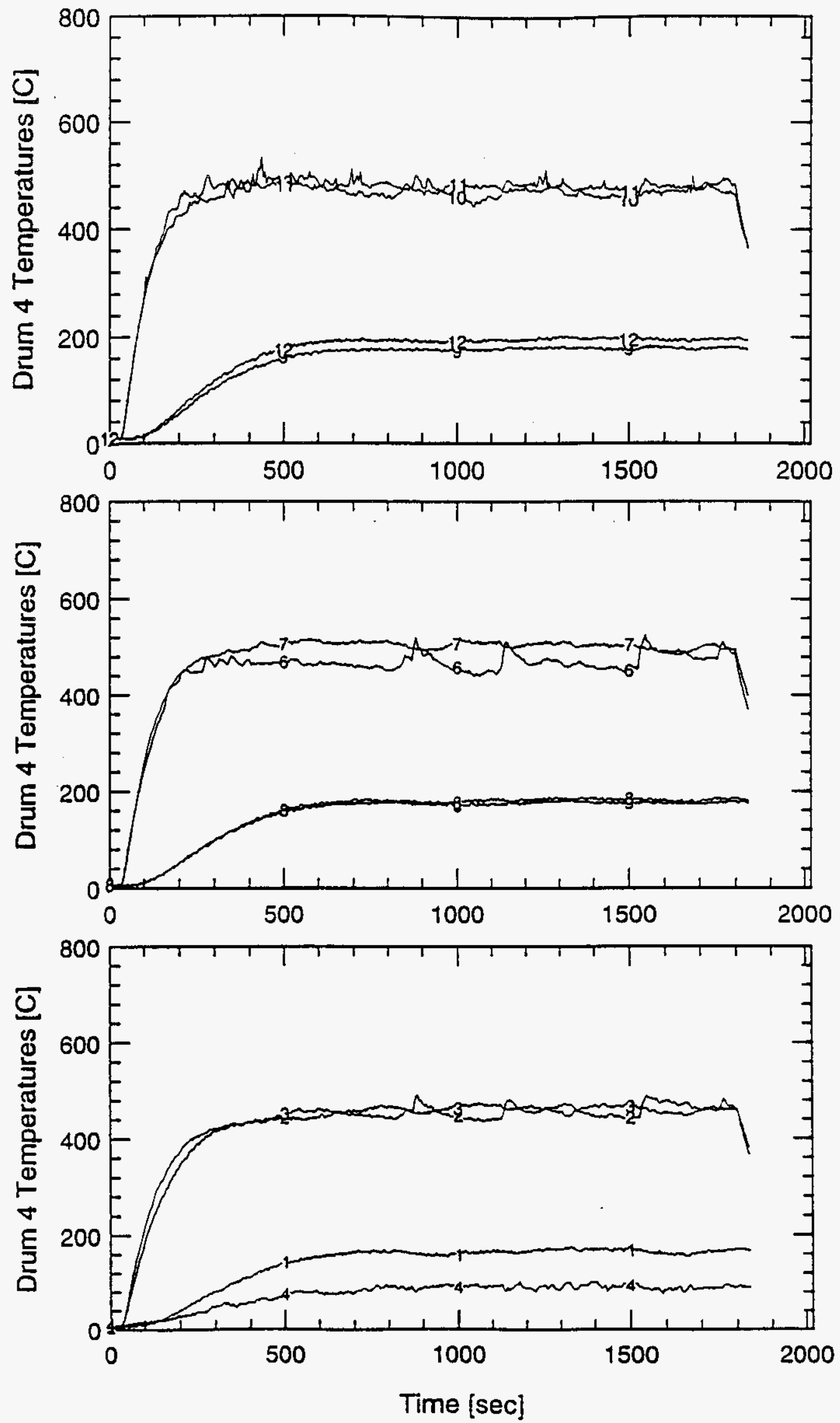
WHC-SD-WM-TRP-233 REV. 0

SWD24 19" Burner
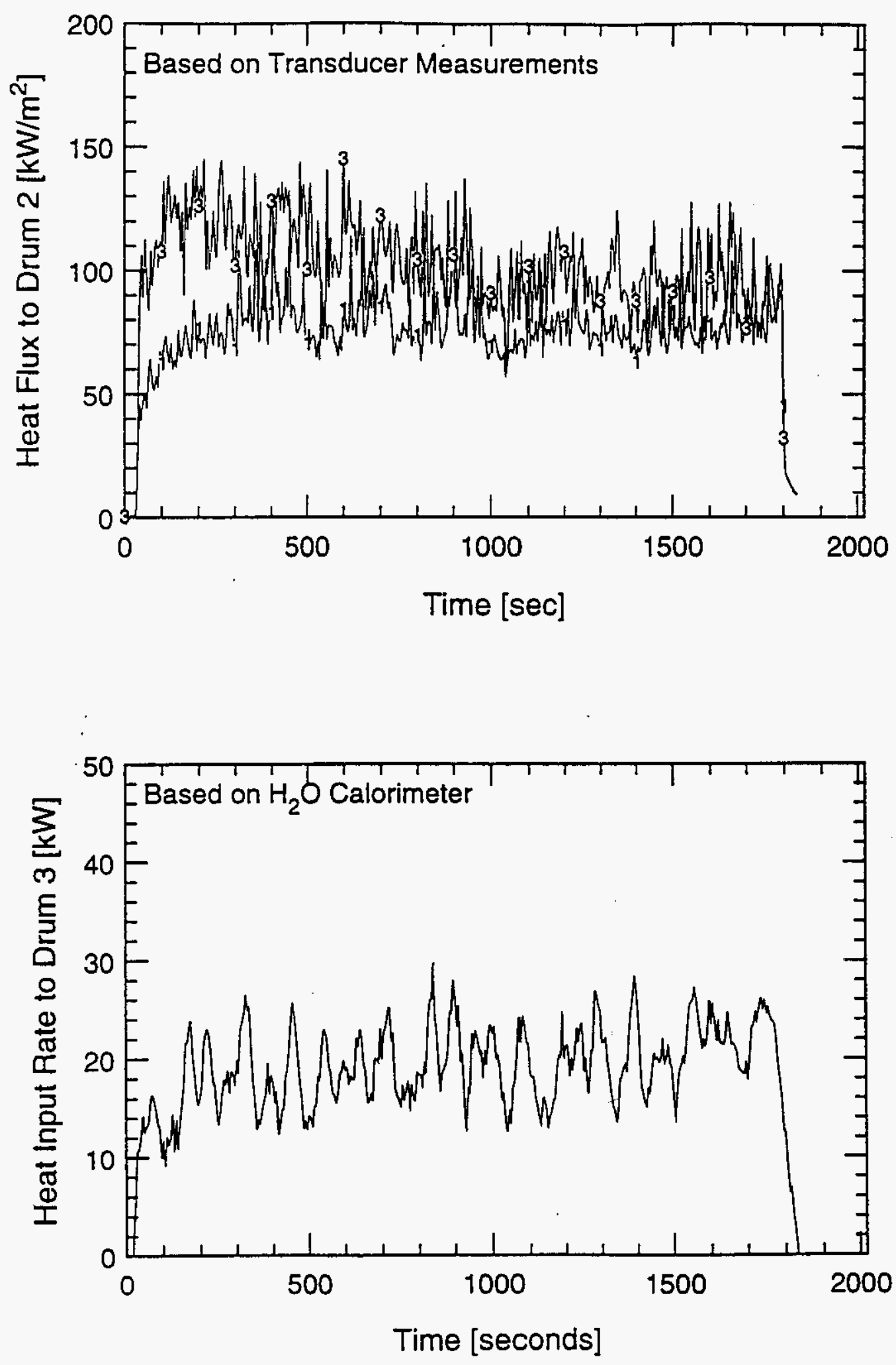
WHC-SD-WM-TRP-233 REV. 0

SWD24 DRUM 1 - 19" Burner - Standard Load - EPDM
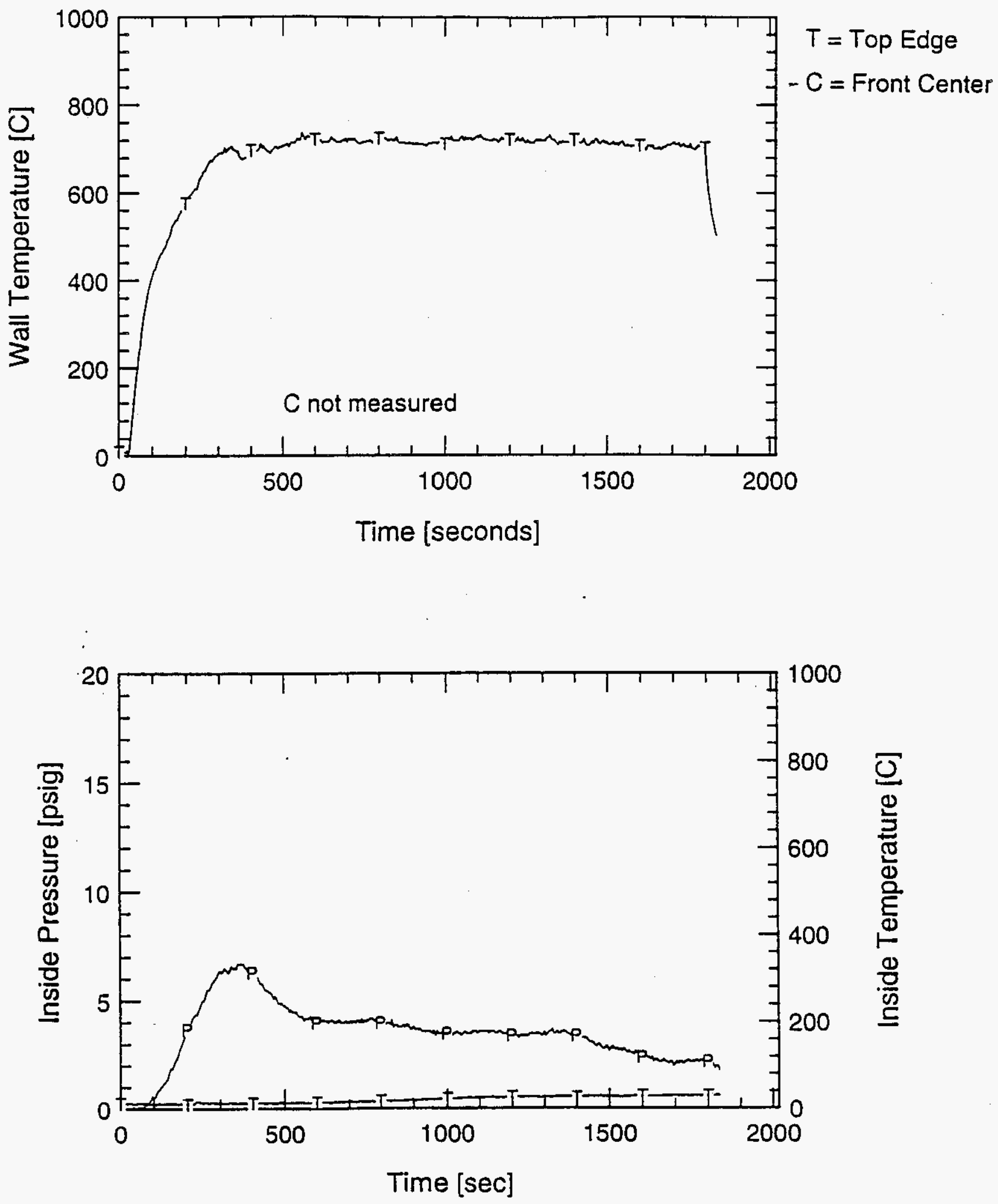
WHC-SD-WM-TRP-233 REV. 0

SWD24 DRUM 4 - 19" Burner - Empty - EPDM
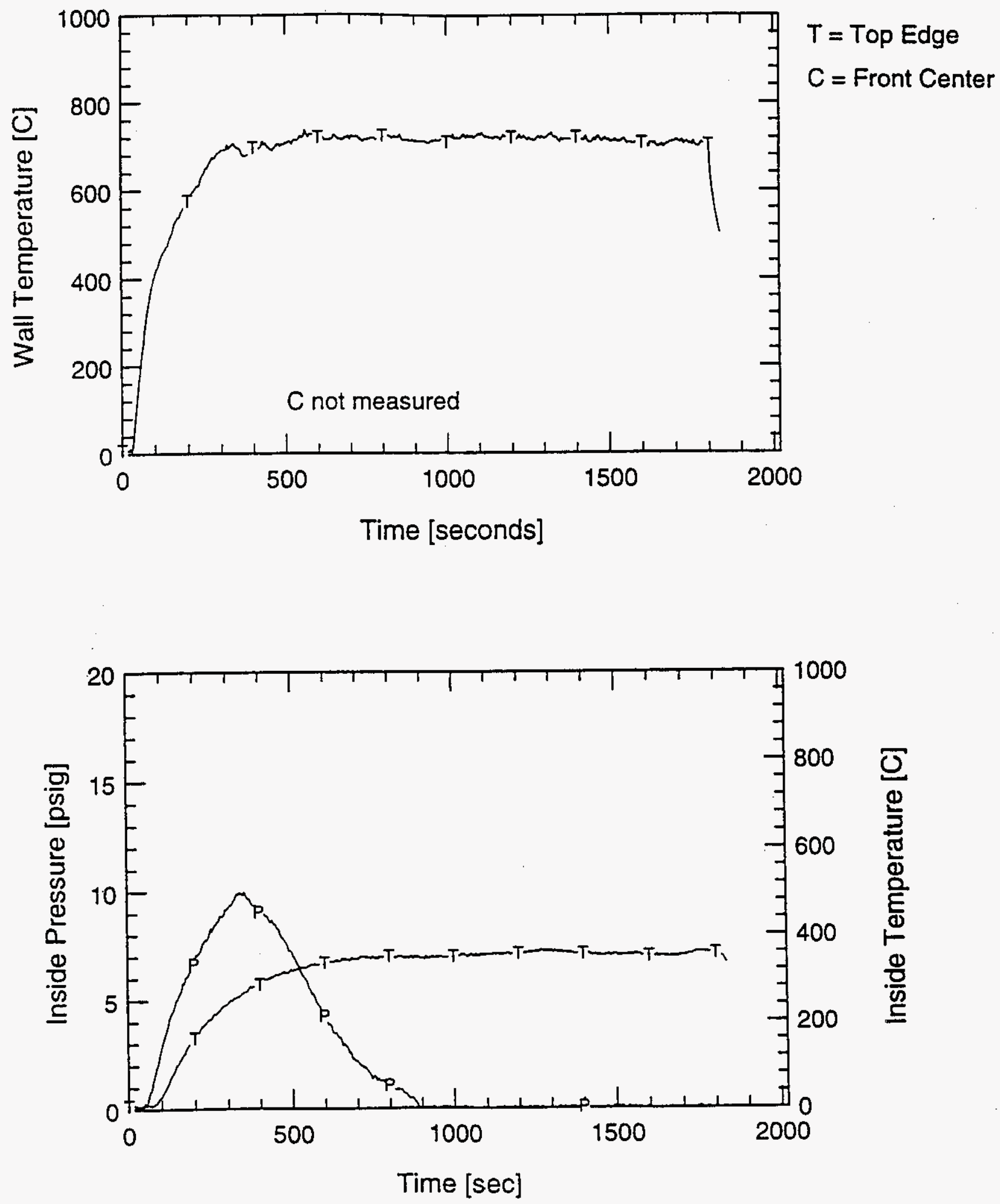
WHC-SD-WM-TRP-233 REV. 0

SWD 25 - DATA 


\section{WHC-SD-WM-TRP-233 REV. 0 \\ SWD25 19" Burner - 5lb Paper - EPDM}
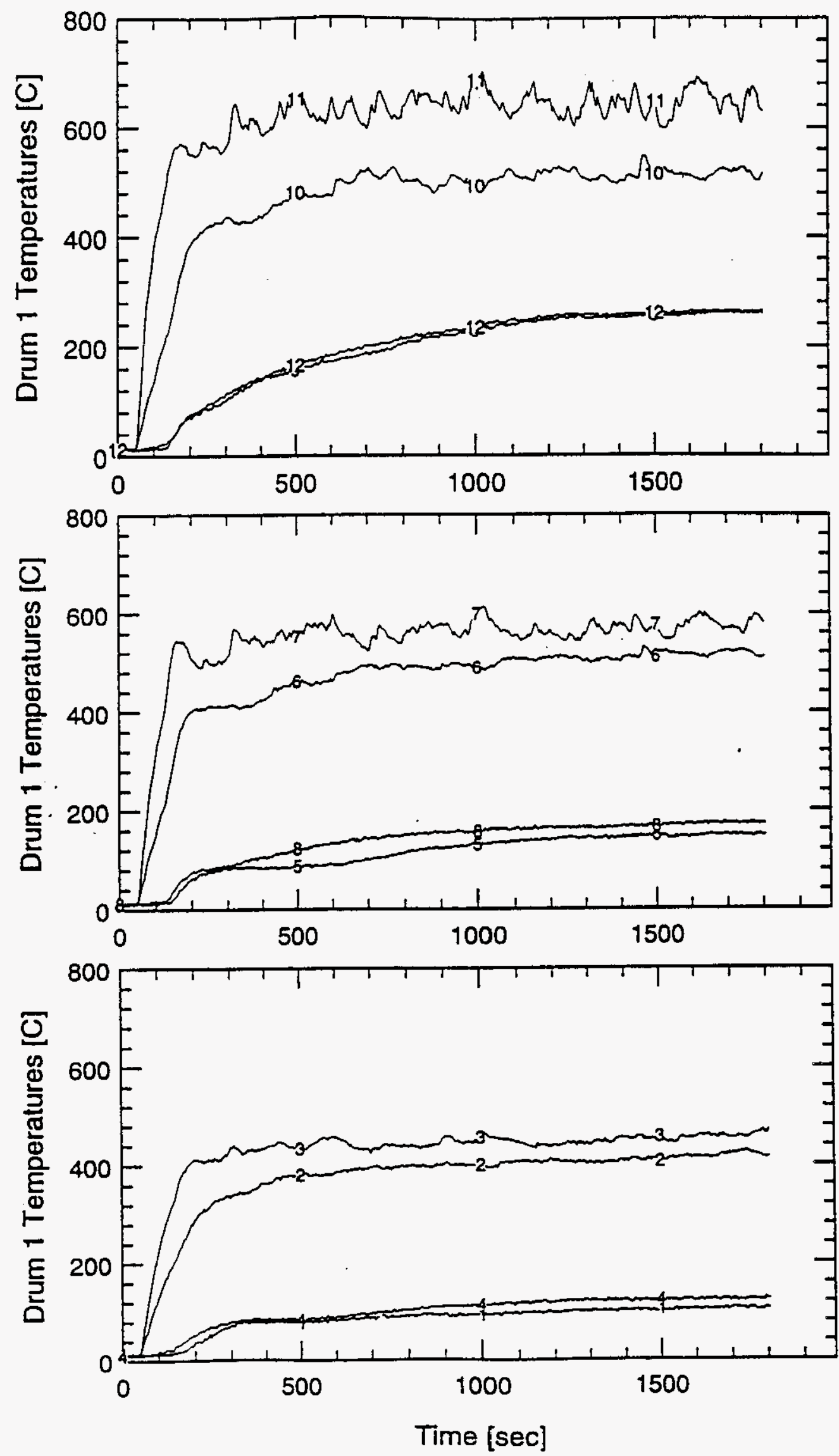
WHC-SD-WM-TRP-233 REV. 0

SWD25 - Drum 2
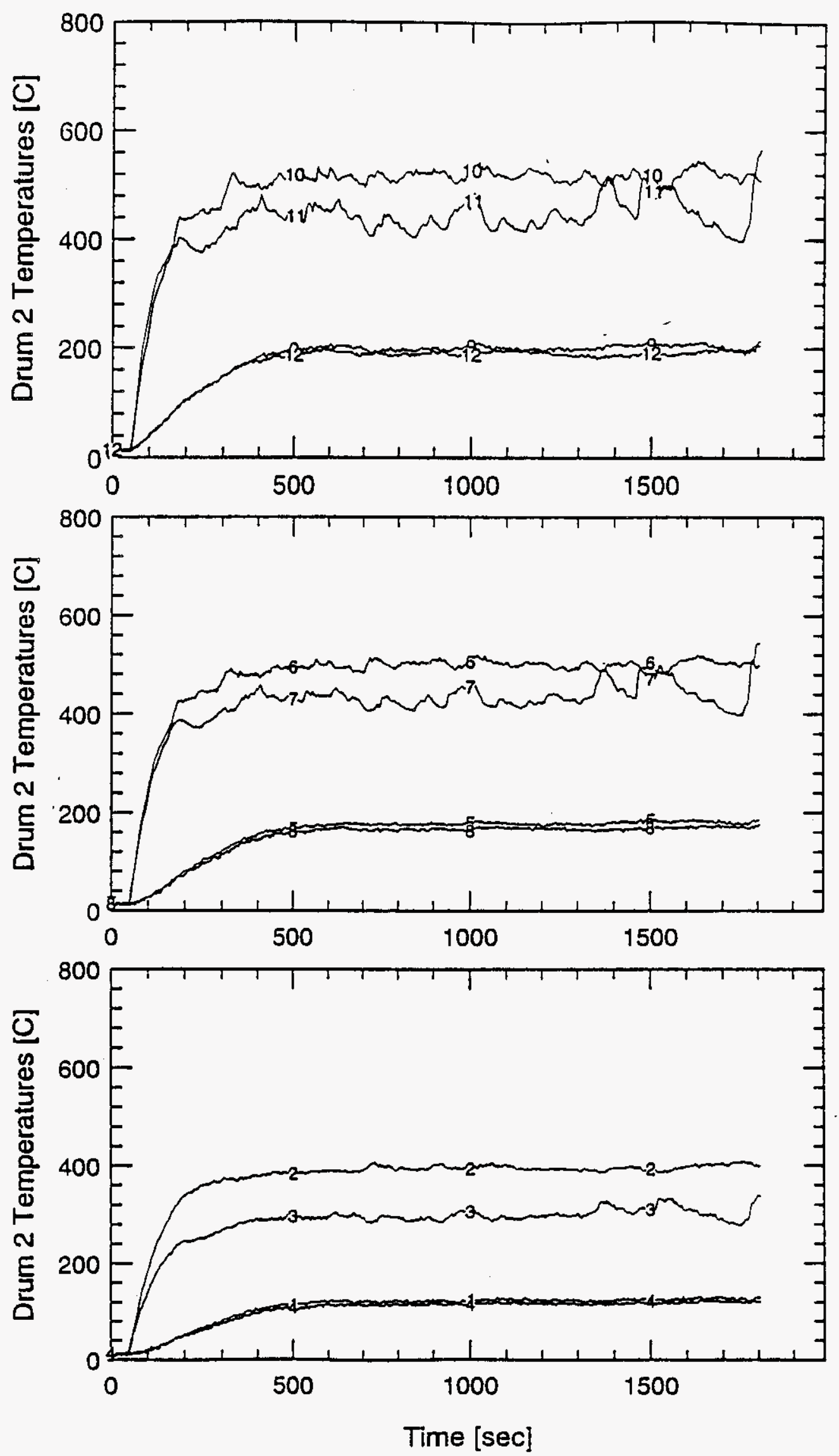
WHC-SD-WM-TRP-233 REV. 0

\section{SWD25 - Drum 3 Water filled drum}
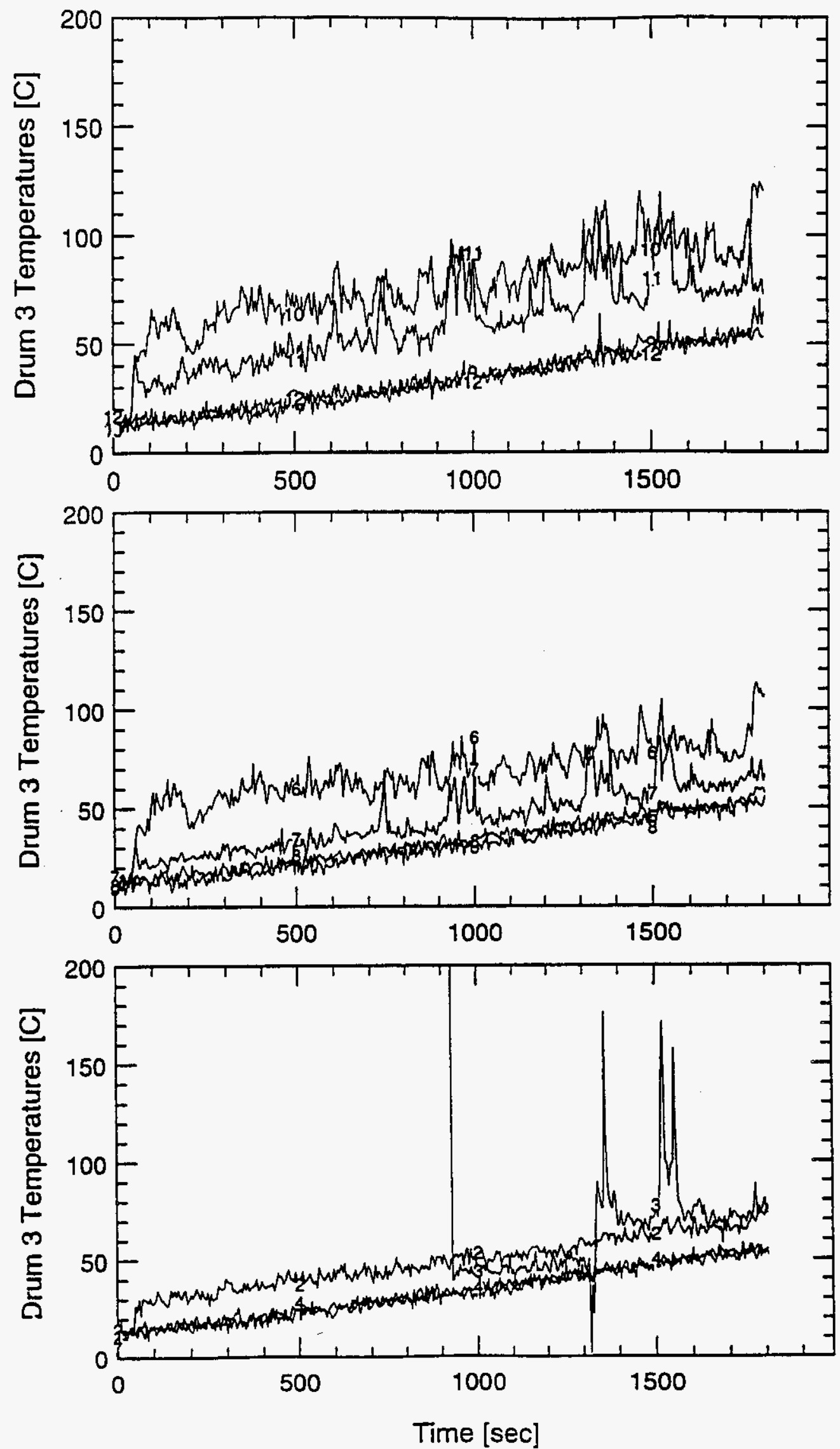
WHC-SD-WM-TRP-233 REV. 0

SWD25 19" Burner - 5lb Plastic - EPDM
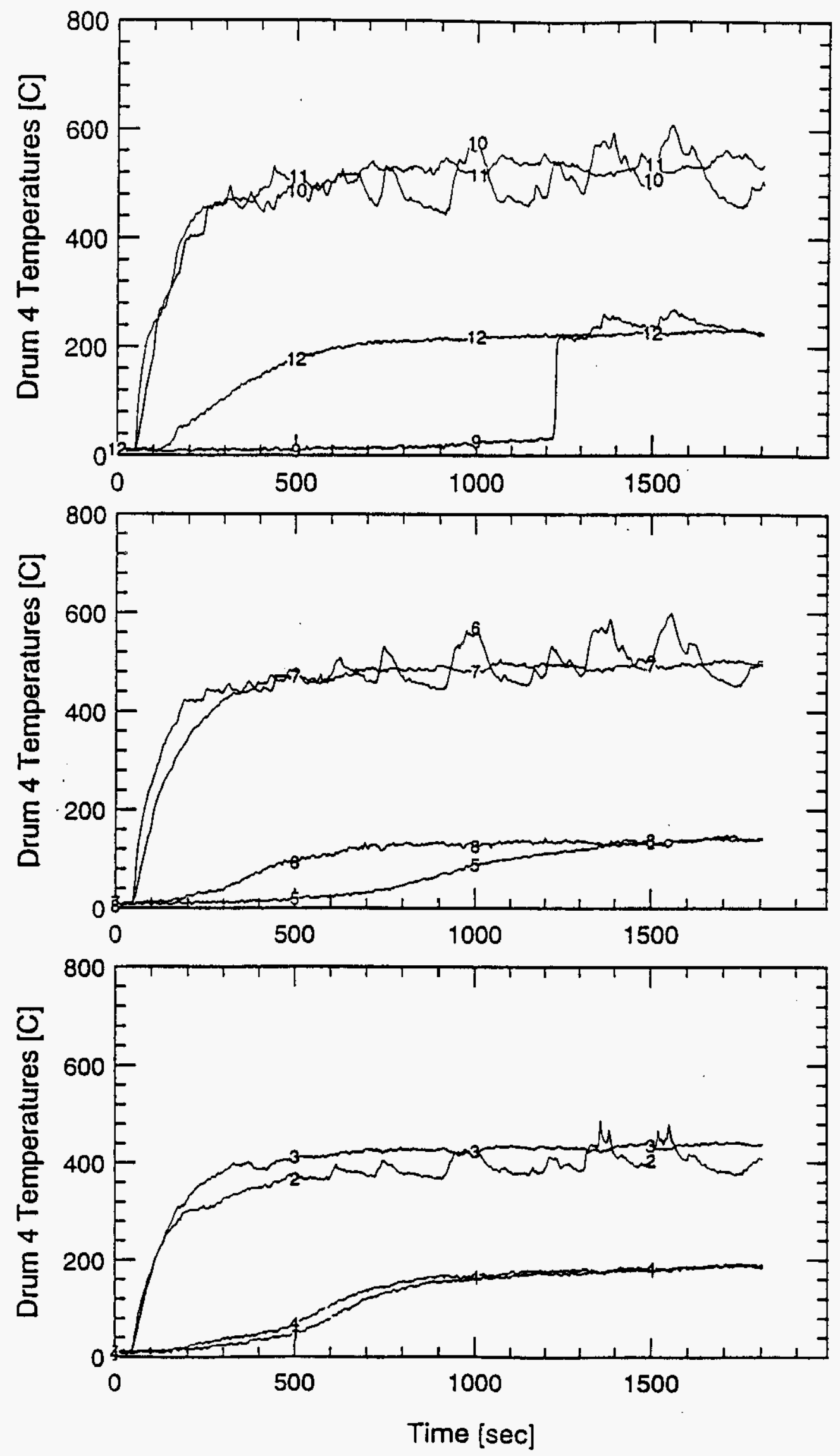


\section{SWD25 19" Burner}
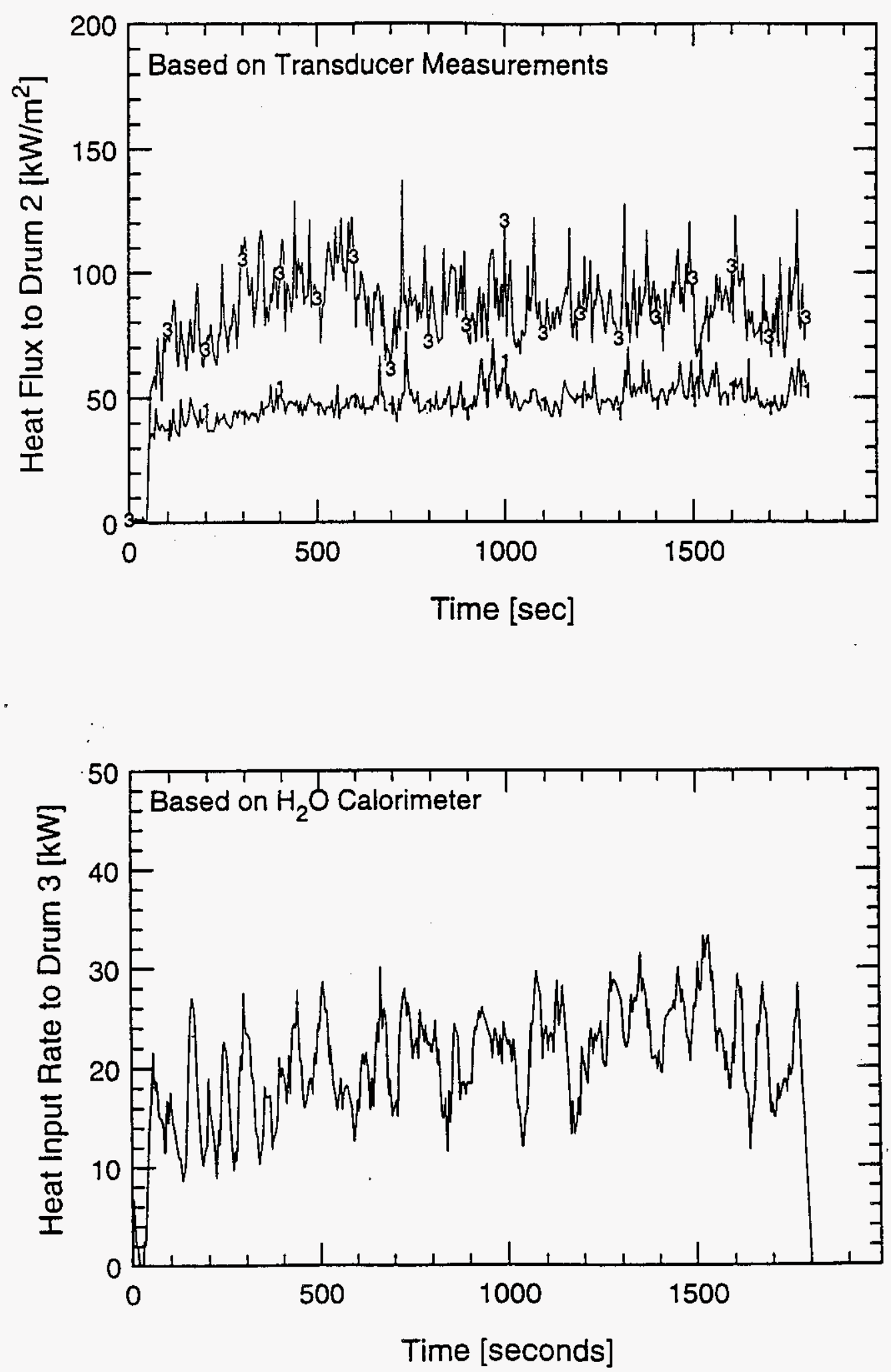
WHC-SD-WM-TRP-233 REV. 0

SWD25 DRUM 1 - 19" Burner - 5lb Paper - EPDM

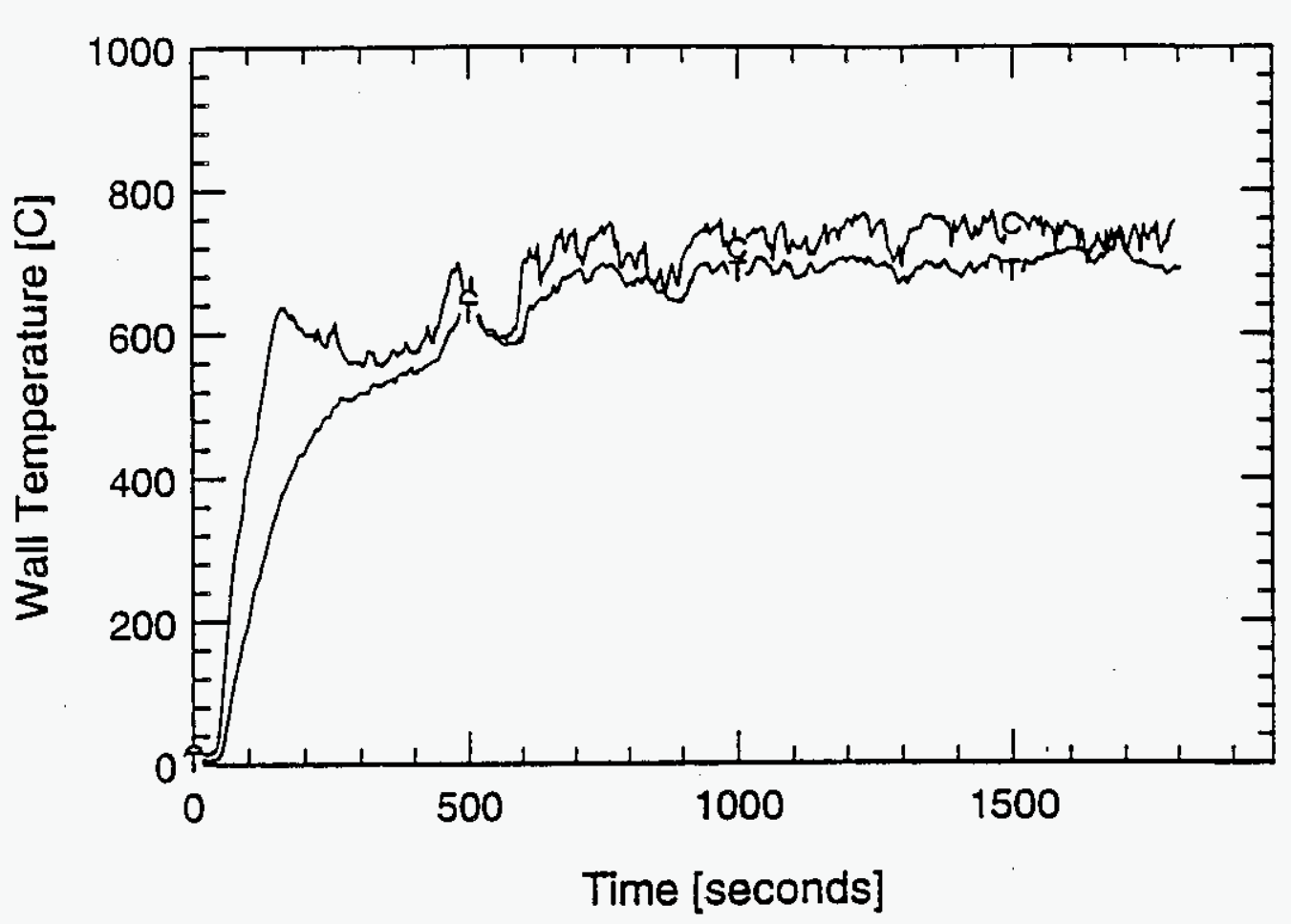

$T=$ Top Edge

$\mathrm{C}=$ Front Center

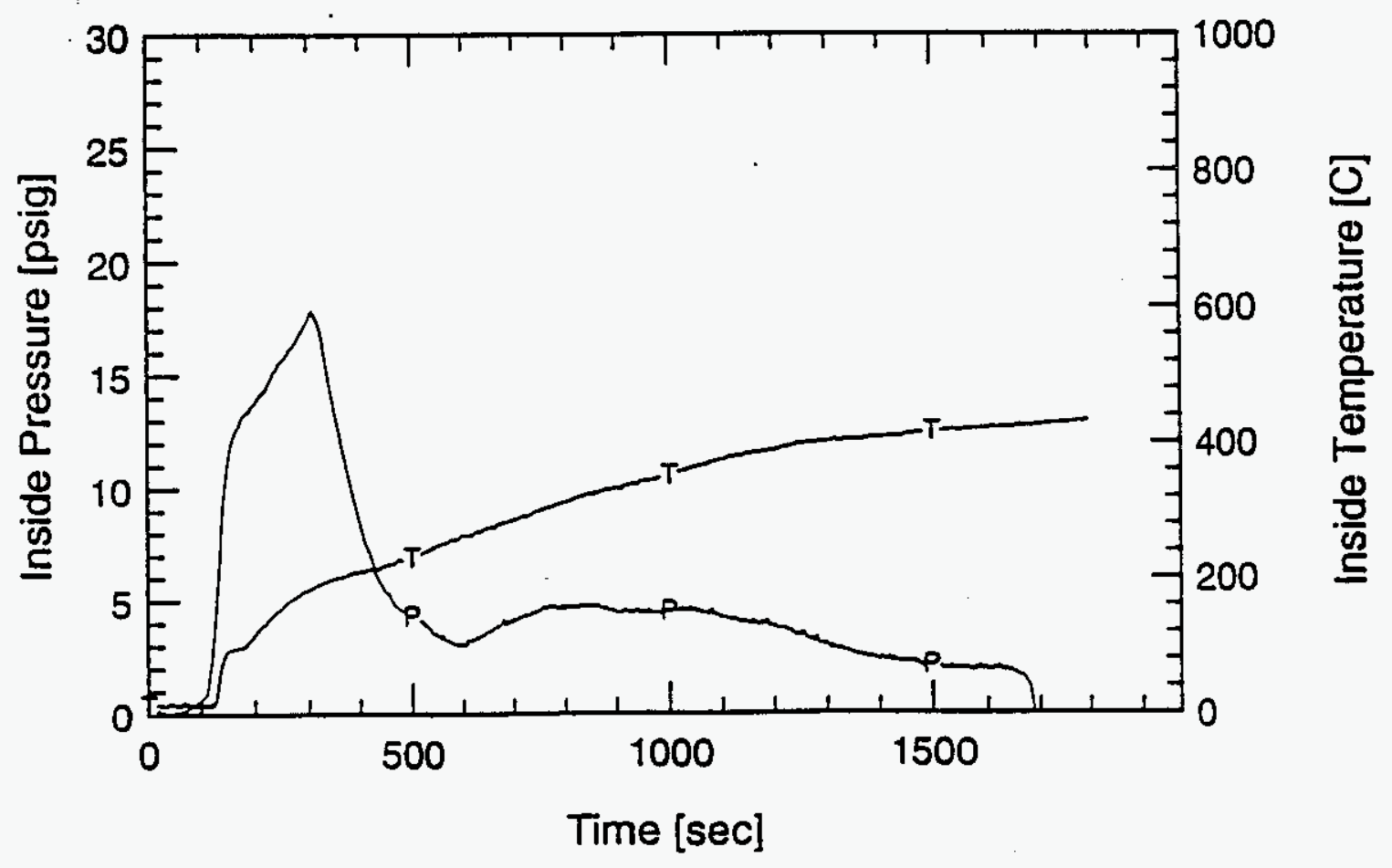


WHC-SD-WM-TRP-233 REV. 0

SWD25 DRUM 4 - 19" Burner - 5lb Plastic - EPDM
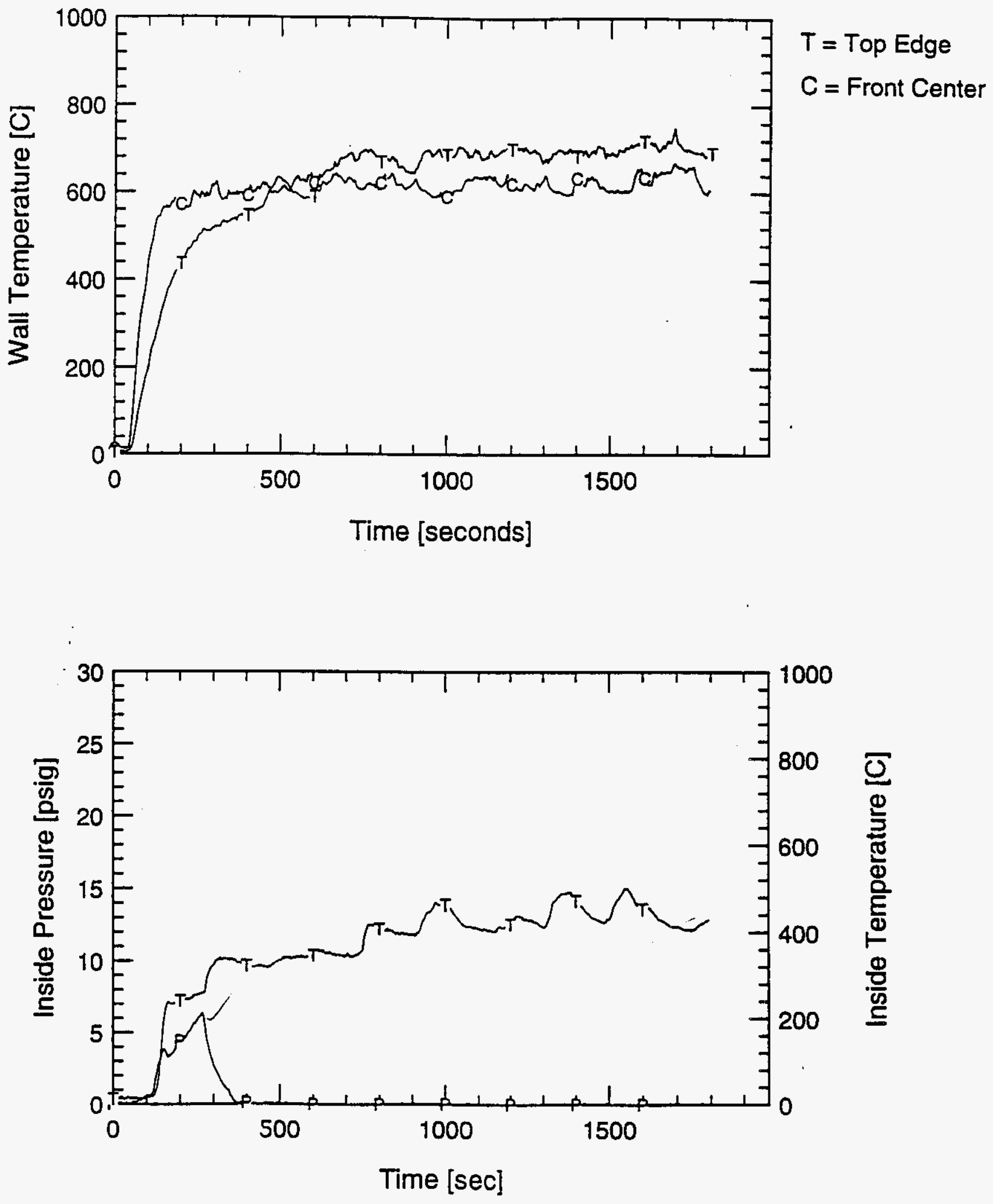
WHC-SD-WM-TRP-233 REV. 0

SWD 26 - DATA 
WHC-SD-WM-TRP-233 REV. 0 SWD26 19" Burner - 5lb Paper - Acetone bottom/back - EPDM
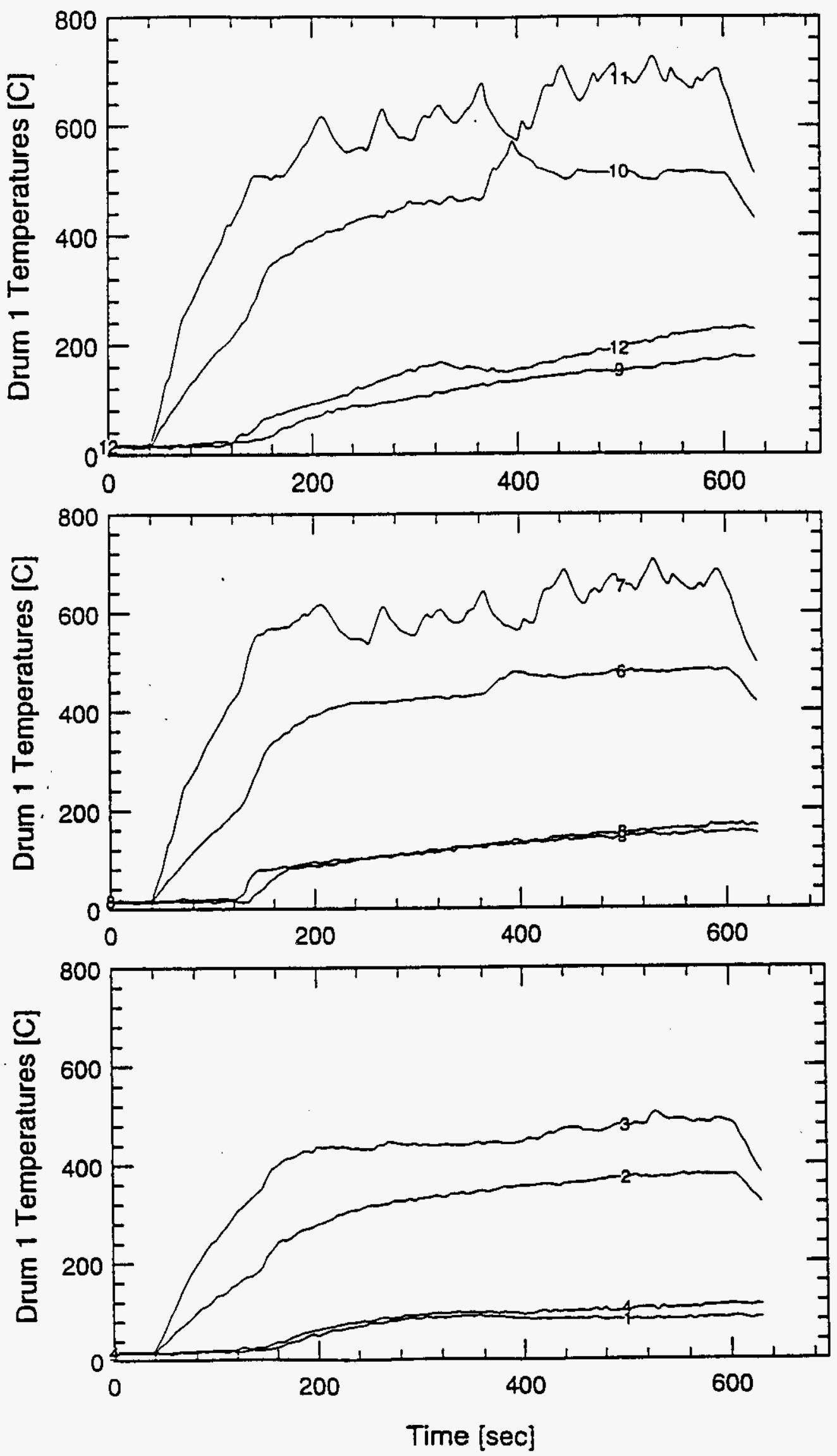
WHC-SD-WM-TRP-233 REV: 0

SWD26 - Drum 2
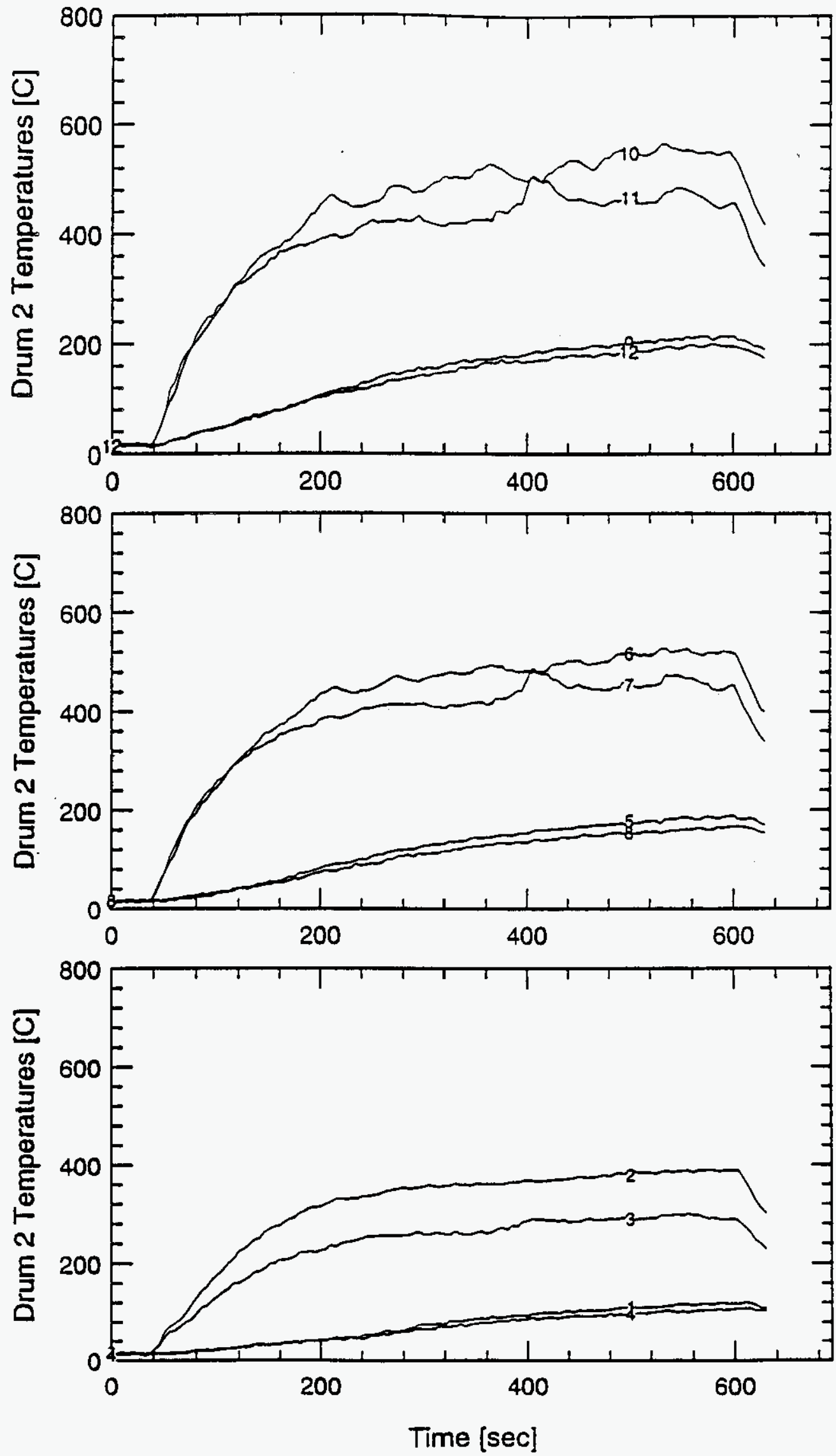
WHC-SD-WM-TRP-233 REV. 0

SWD26 - Drum 3 Water filled drum
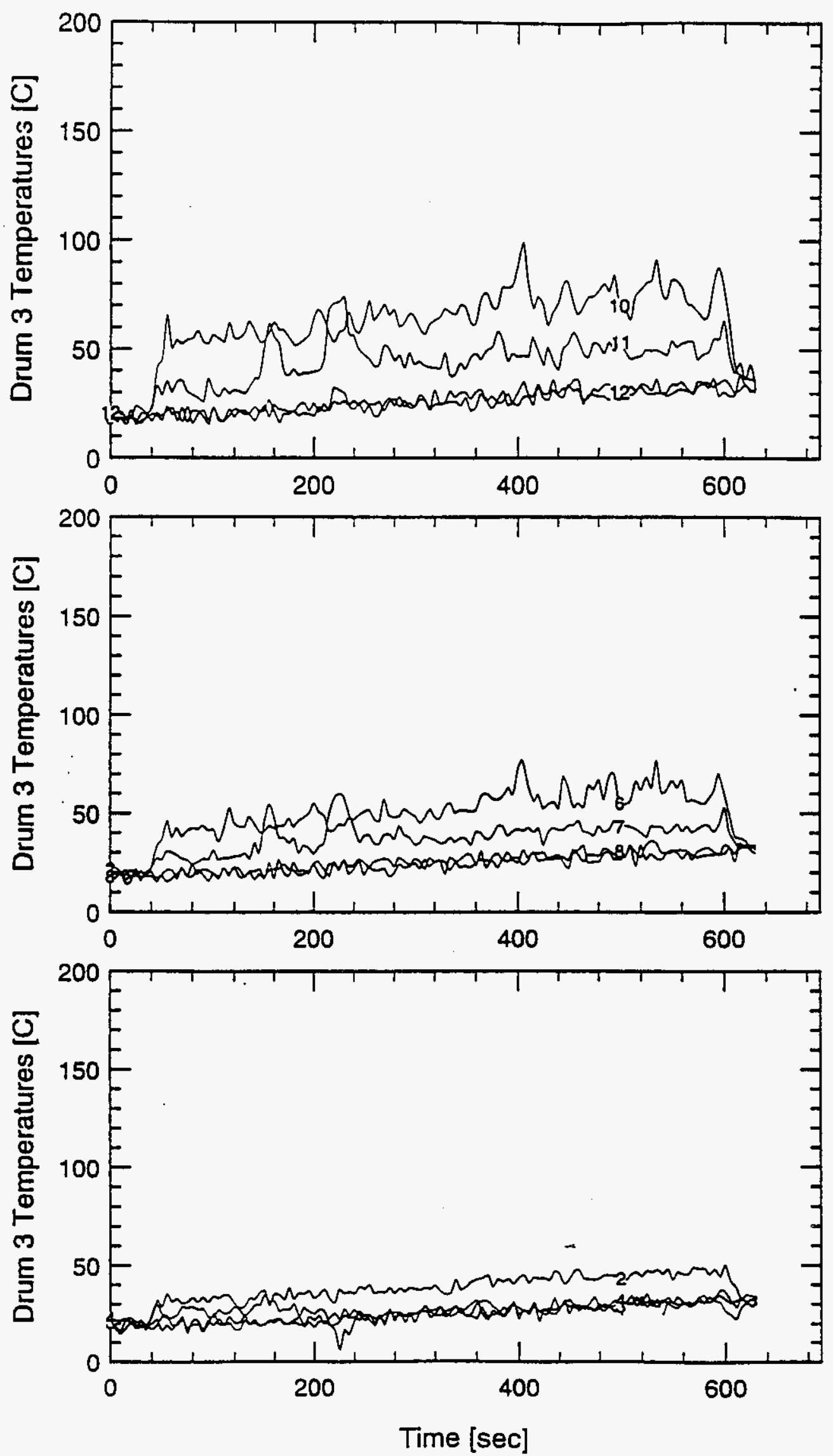
WHC-SD-WM-TRP-233 REV. 0

SWD26 19" Burner - Bag Std Load - Toluene - EPDM - Sqr Rim
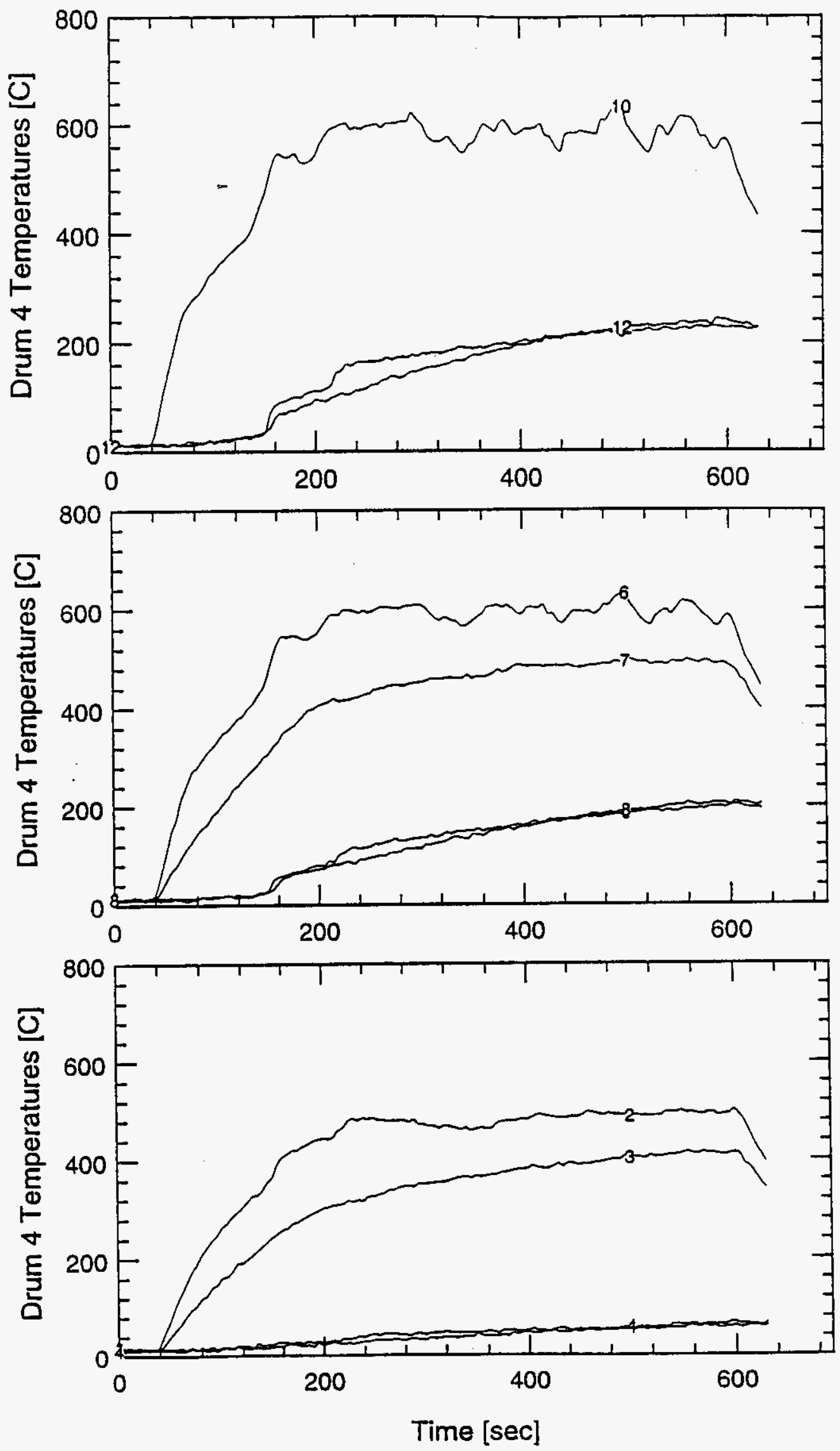
WHC-SD-WM-TRP-233 REV. O

SWD26 19" Burner
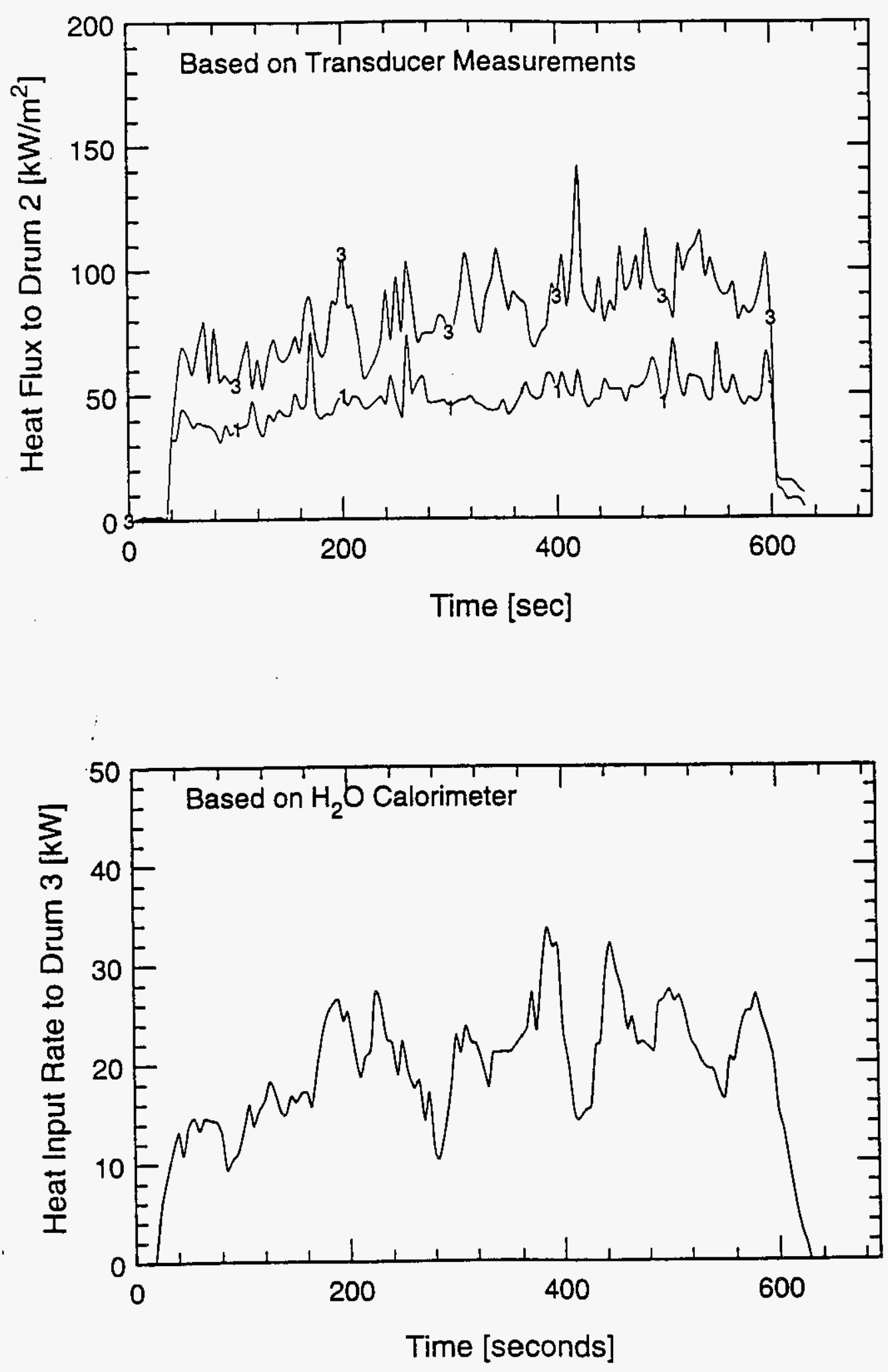


\section{SWD26 DRUM 1 - 19" Burner - 5lb Paper - Toluene - EPDM}
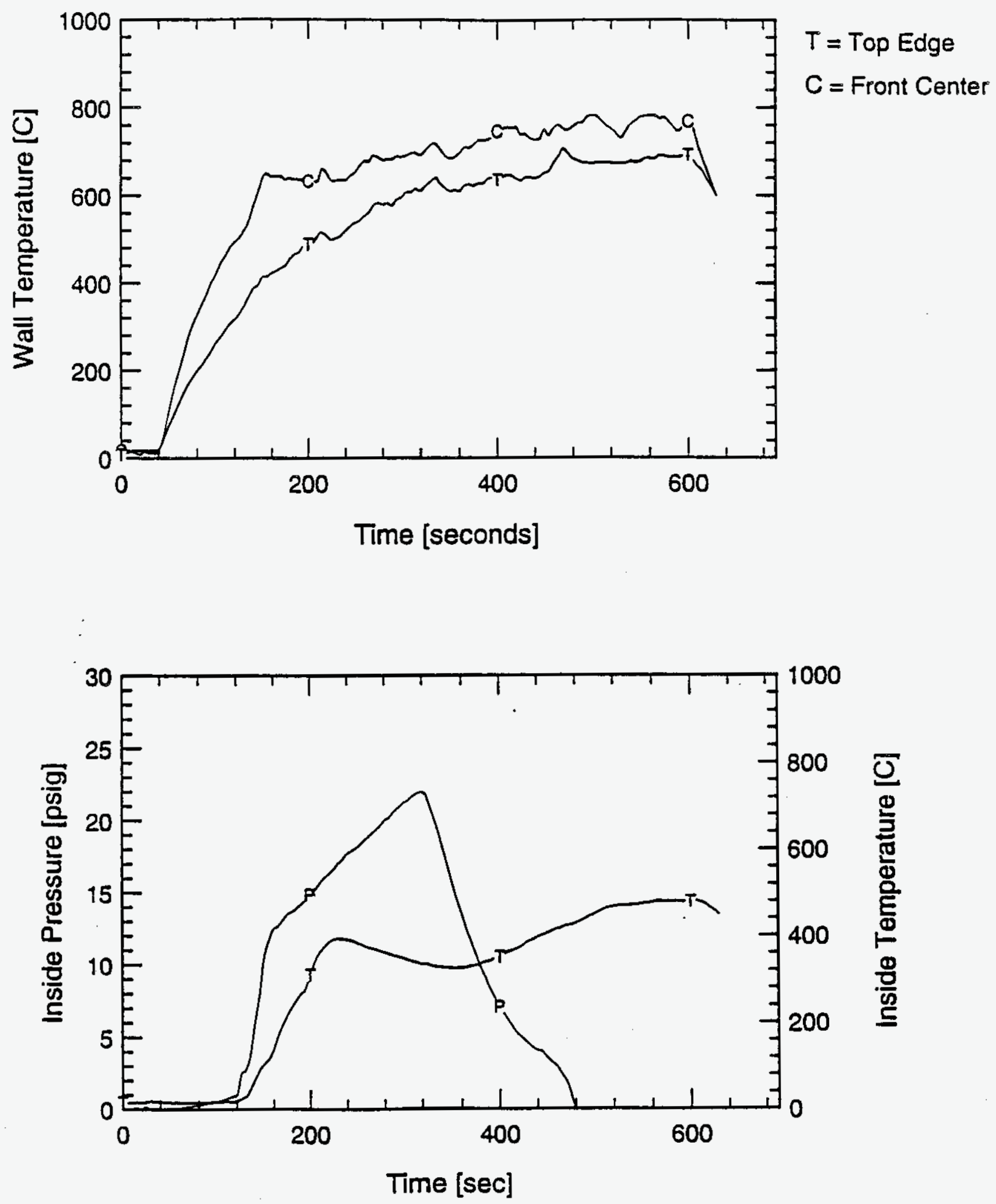
WHC-SD-WM-TRP-233 REV. 0

SWD26 DRUM 4 - 19" Burner - Bagged Std Load - Toluene - EPDM
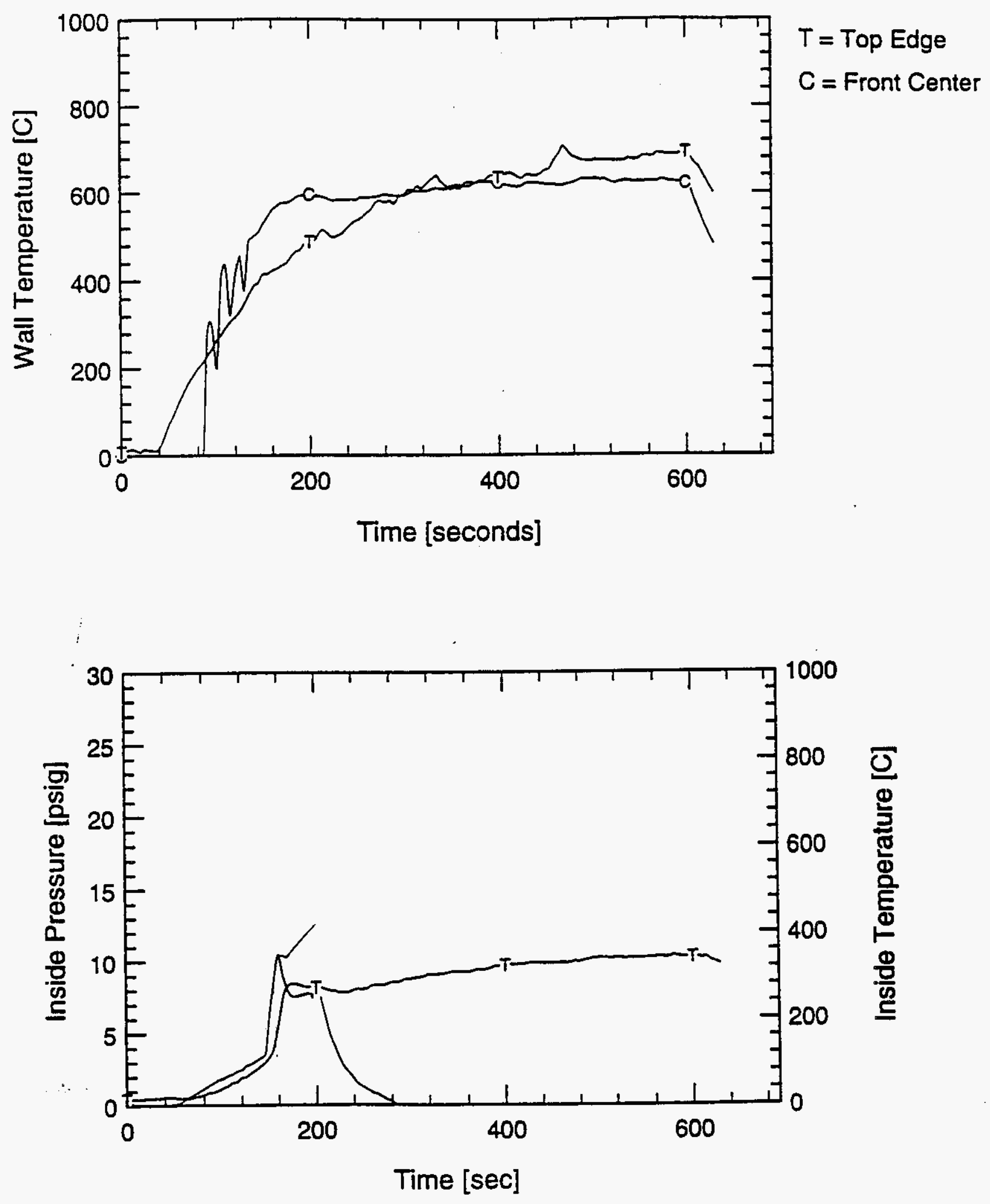
WHC-SD-WM-TRP-233 REV. 0

SWD 27 - DATA 
WHC-SD-WM-TRP-233 REV. 0

\section{SWD27 19" Burner - 5lb Paper - Toluene - EPDM}
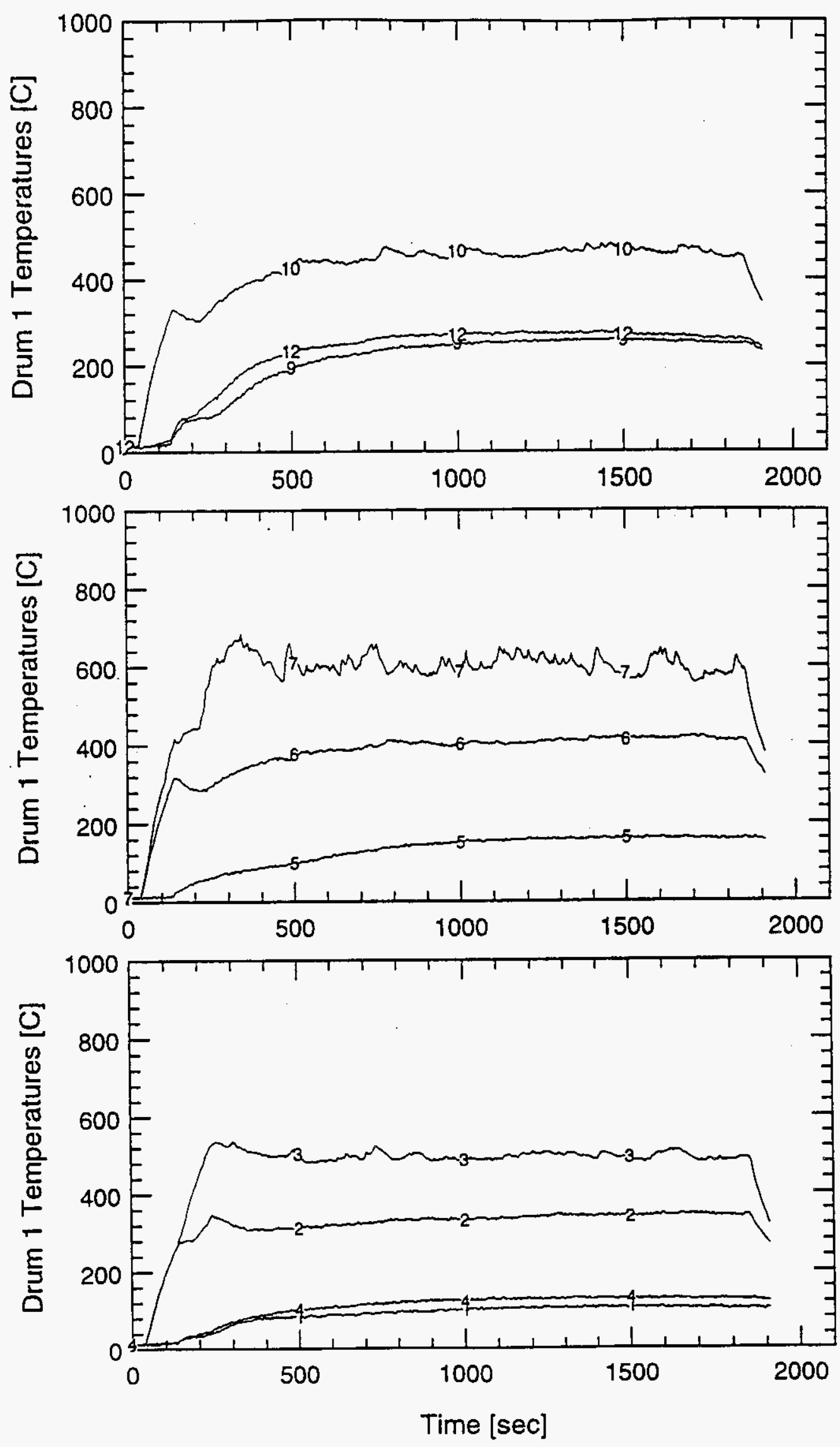
WHC-SD-WM-TRP-233 REV. O

SWD27 - Drum 2
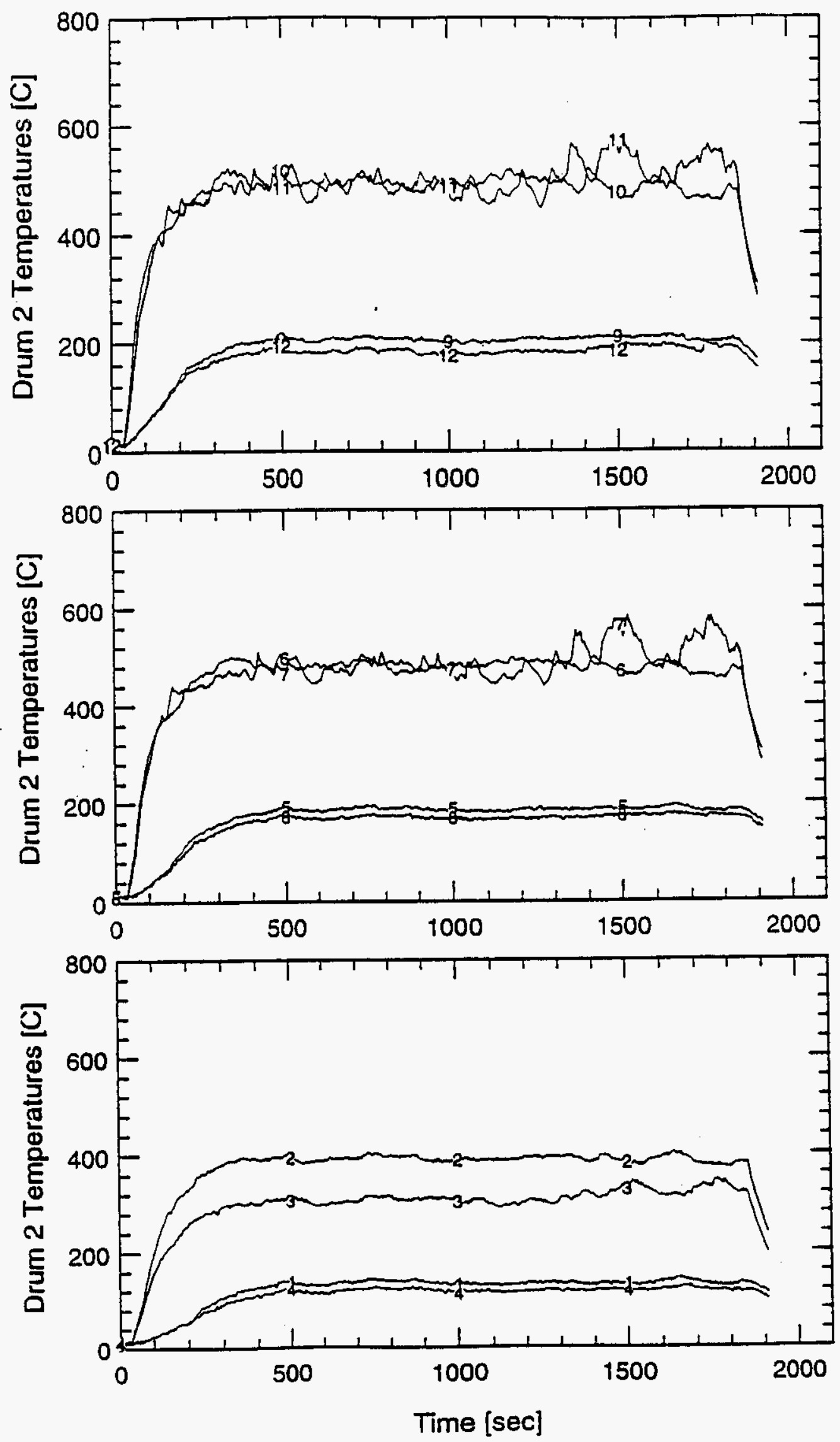

B-189 
WHC-SD-WM-TRP-233 REV. 0

\section{SWD27 - Drum 3 Water filled drum}
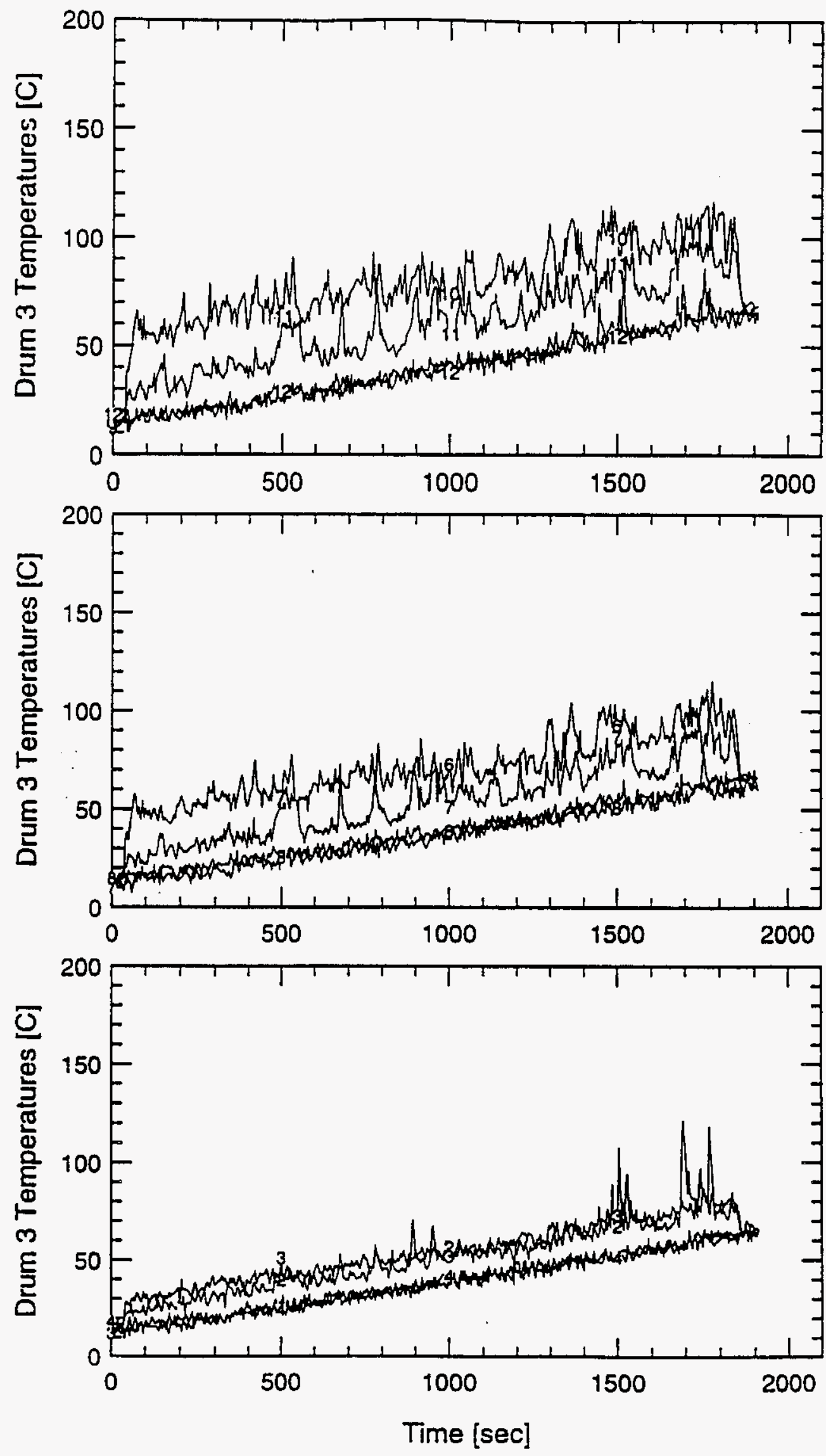
WHC-SD-WM-TRP-233 REV. 0

SWD27 19" Burner - Bagged Standard Load - Toluene - EPDM
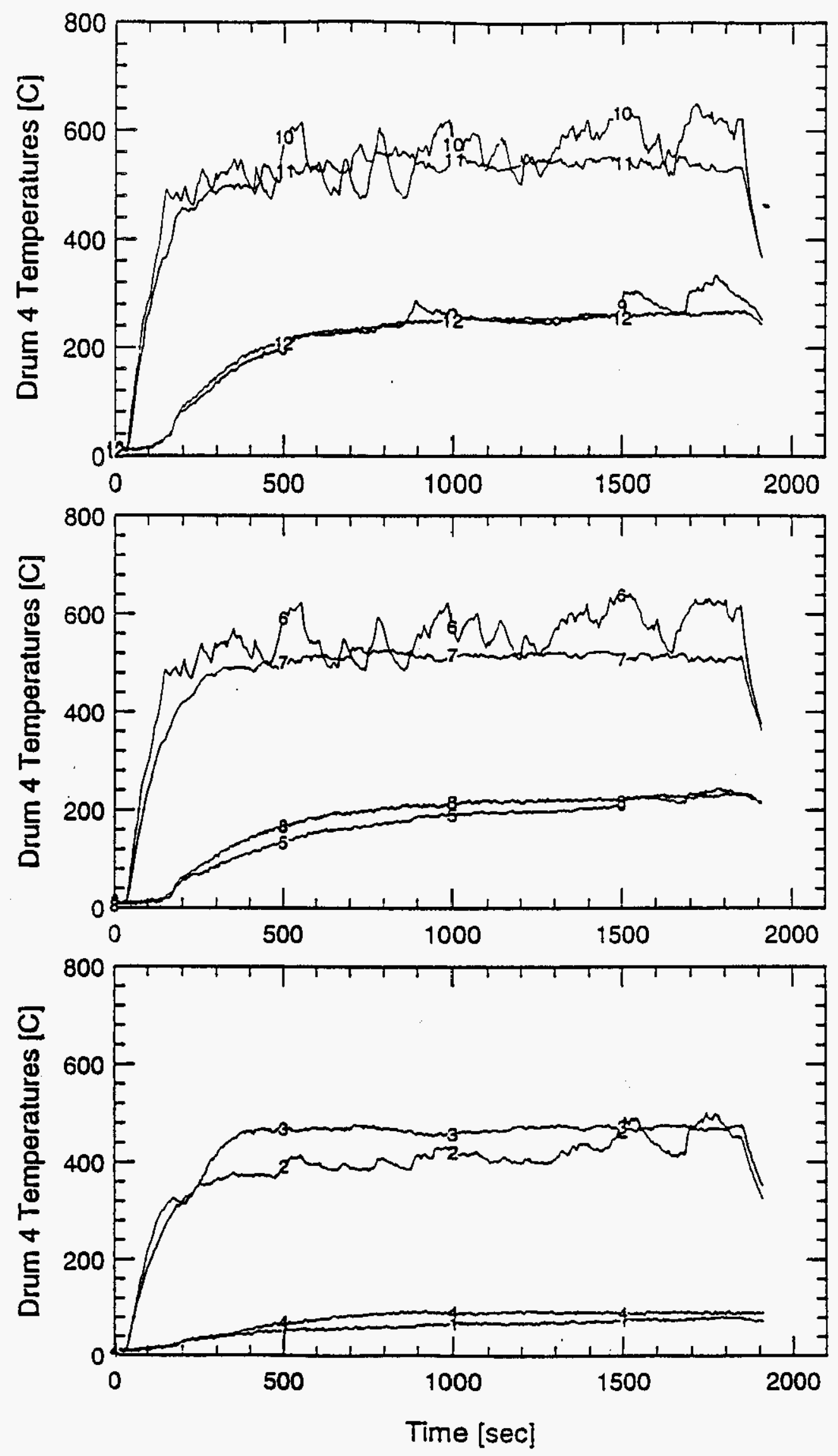
WHC-SD-WM-TRP-233 REV. 0

\section{SWD27 19" Burner}
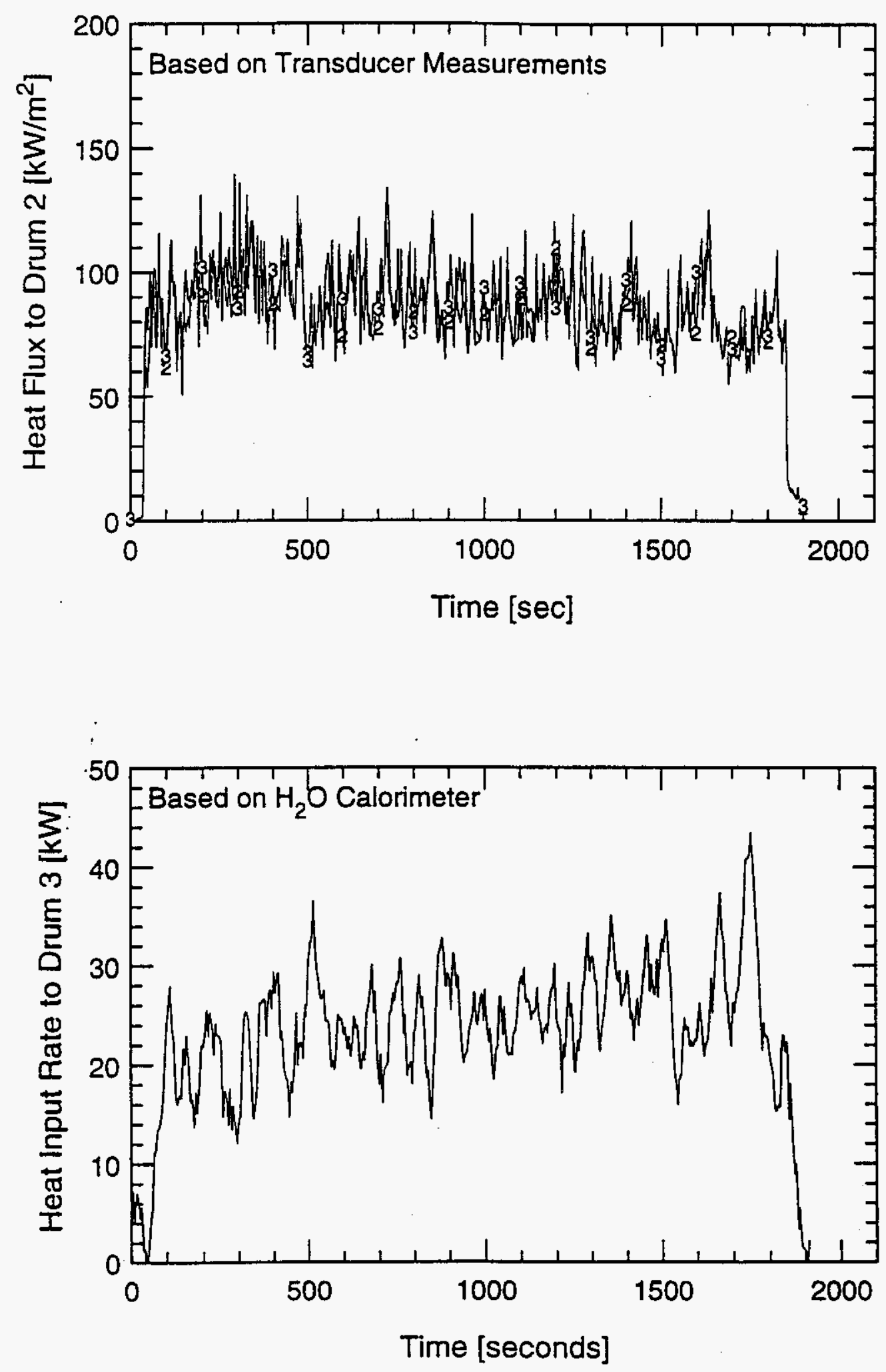
WHC-SD-WM-TRP-233 REV. 0
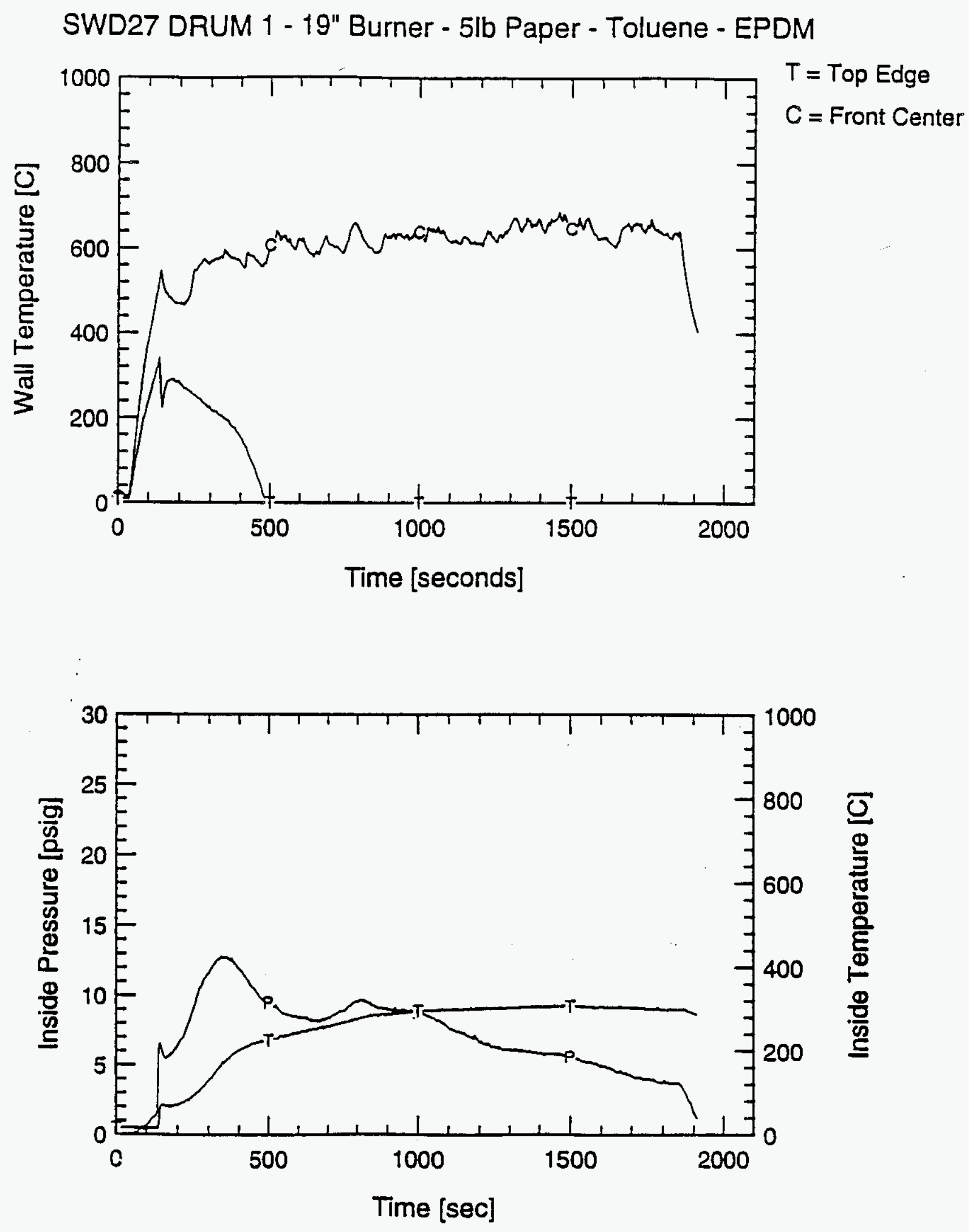
SWD27 DRUM 4 - 19" Burner - Bagged Std Load - Toluene - EPDM
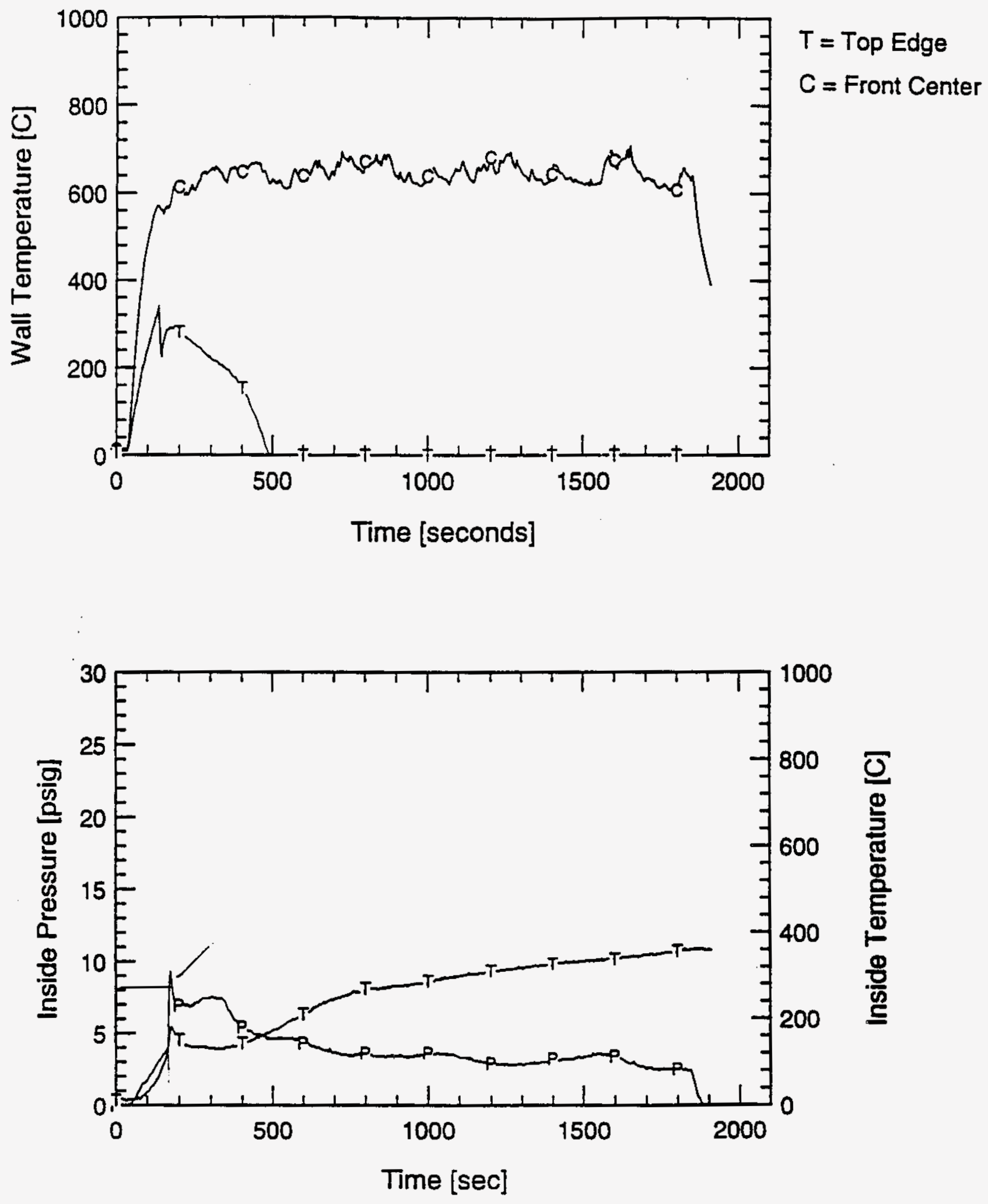
WHC-SD-WM-TRP-233 REV. 0

SWD 28 - DATA 
WHC-SD-WM-TRP-233 REV. 0

\section{SWD28 48" Burner - 5lb Paper}
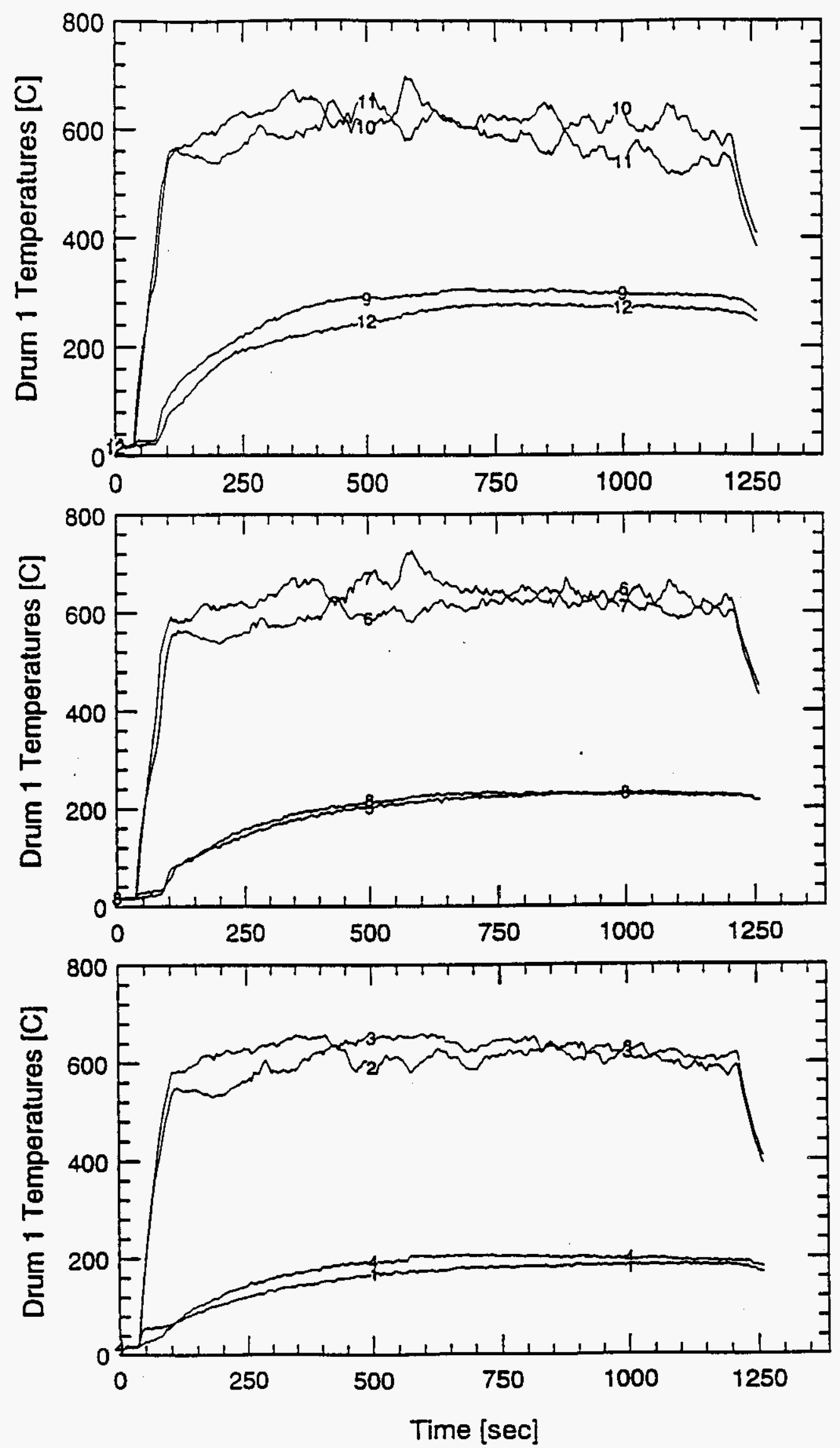
WHC-SD-WM-TRP-233 REV. O

SWD28 - Drum 2
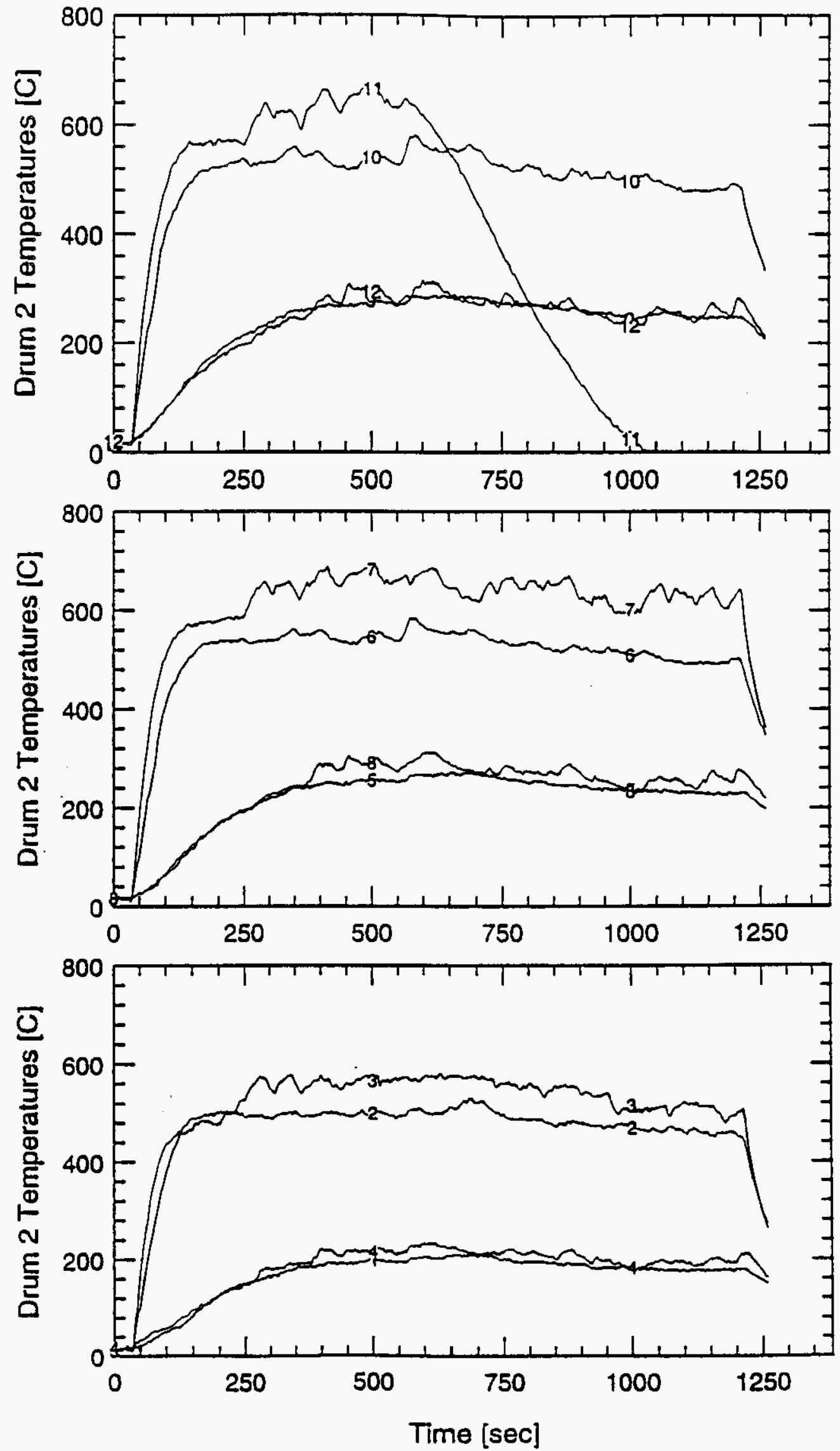
WHC-SD-WM-TRP-233 REV. 0

SWD28 - Drum 3 Water filled drum
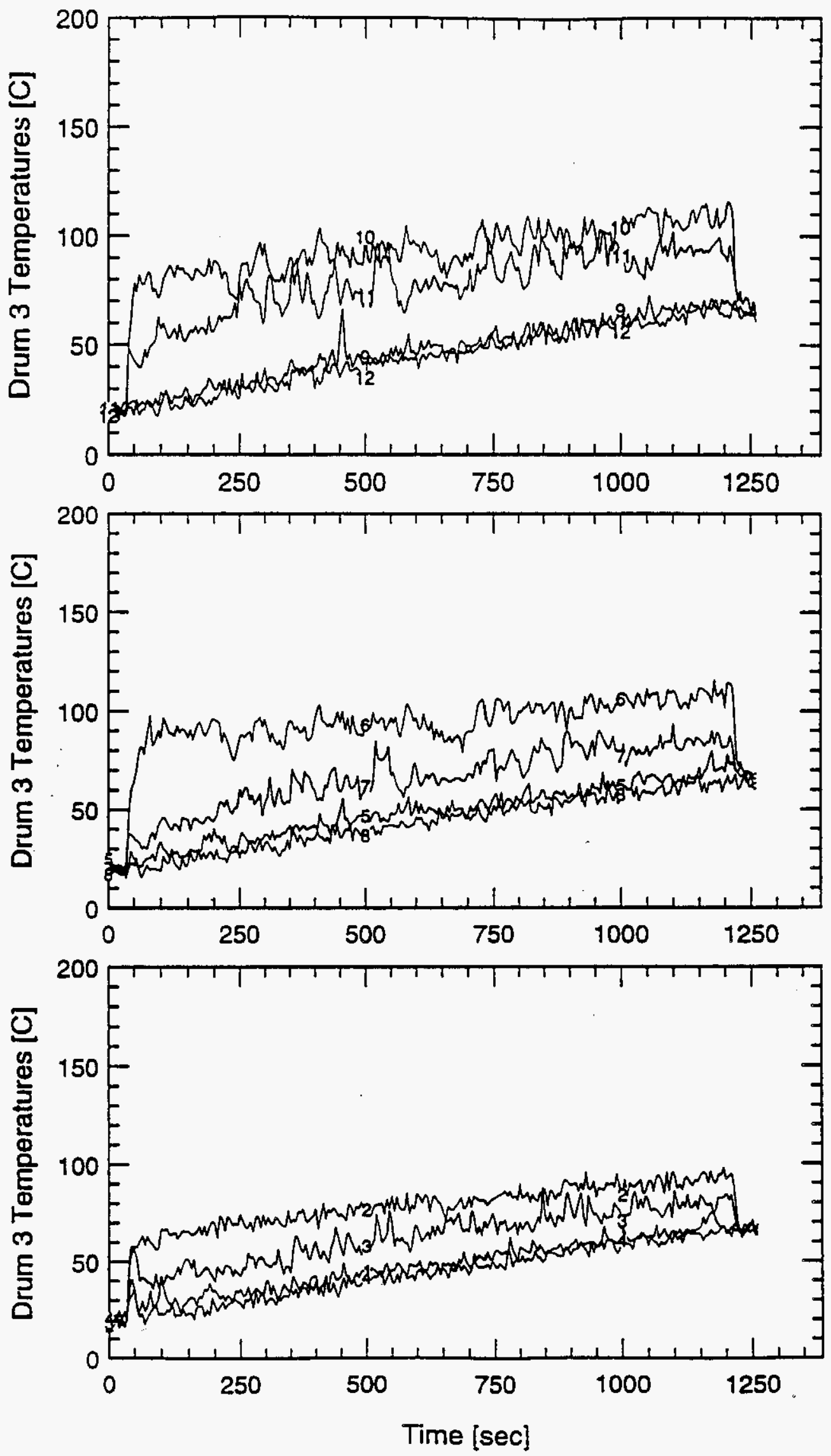
WHC-SD-WM-TRP-233 REV. 0

SWD28 48" Burner - 5lb Plastic
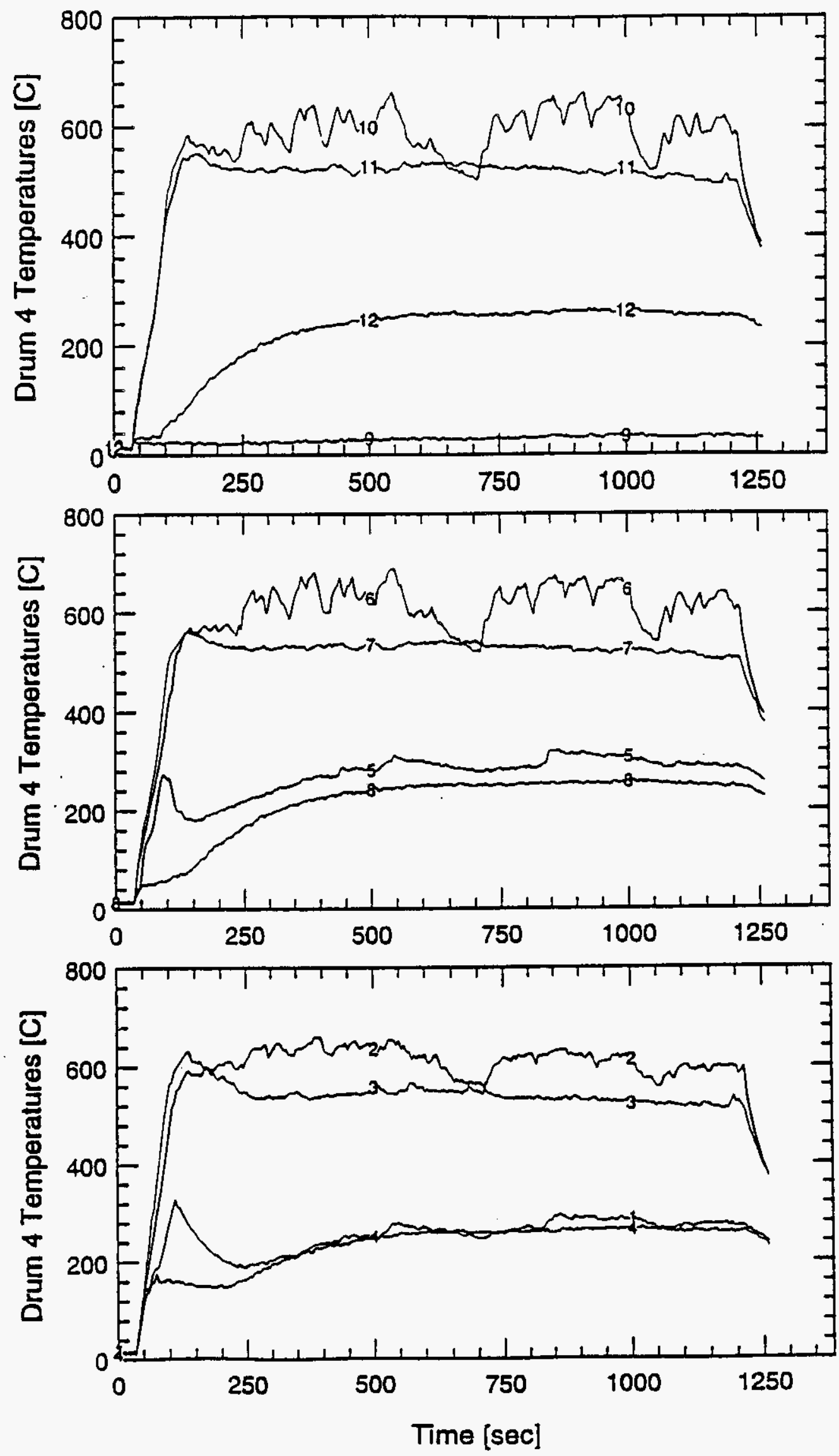
WHC-SD-WM-TRP-233 REV. 0

SWD28 48" Burner
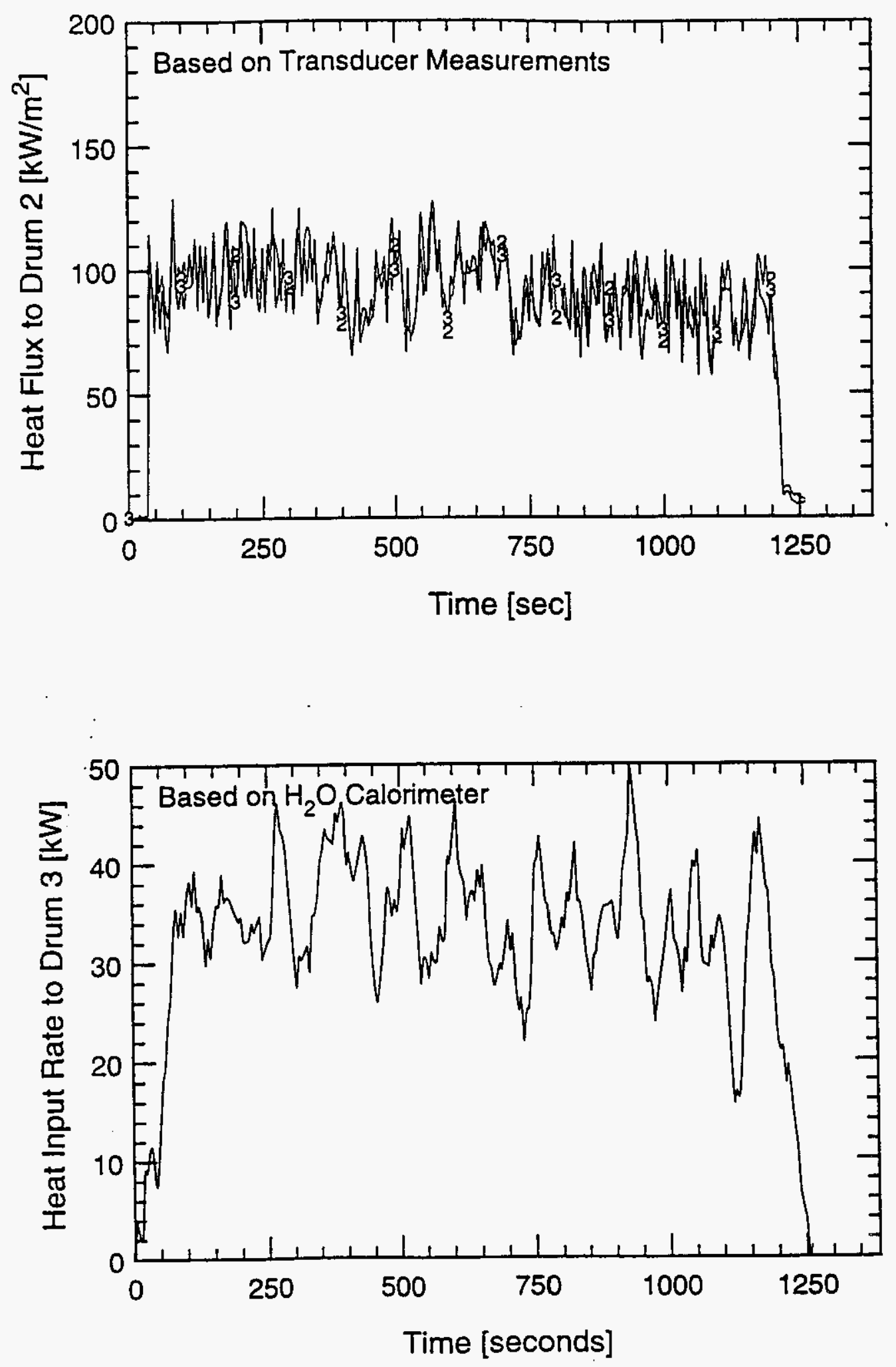
SWD28 DRUM 1 - 48" Burner - 5lb Paper

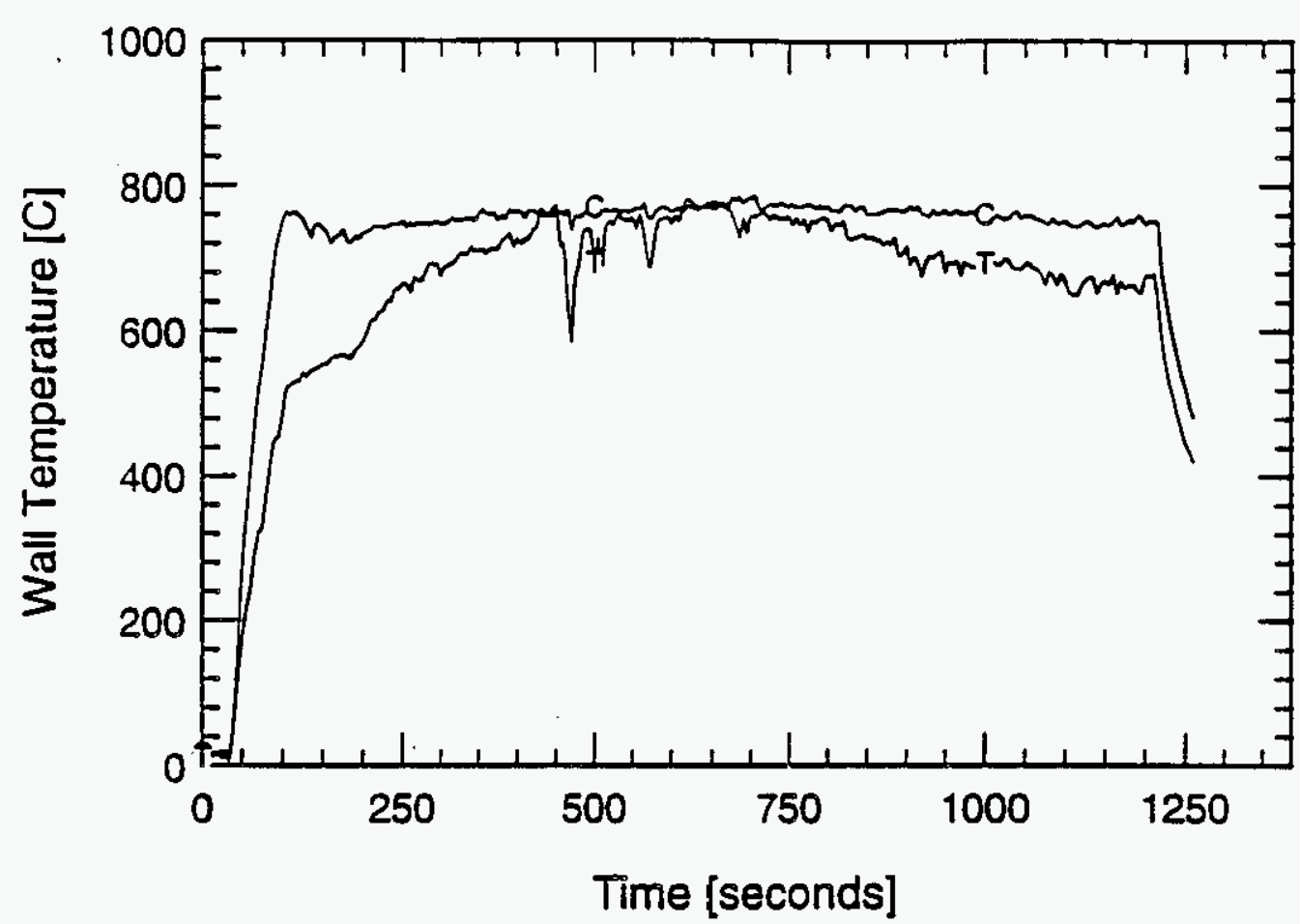

$T=$ Top Edge

$\mathrm{C}=$ Front Center

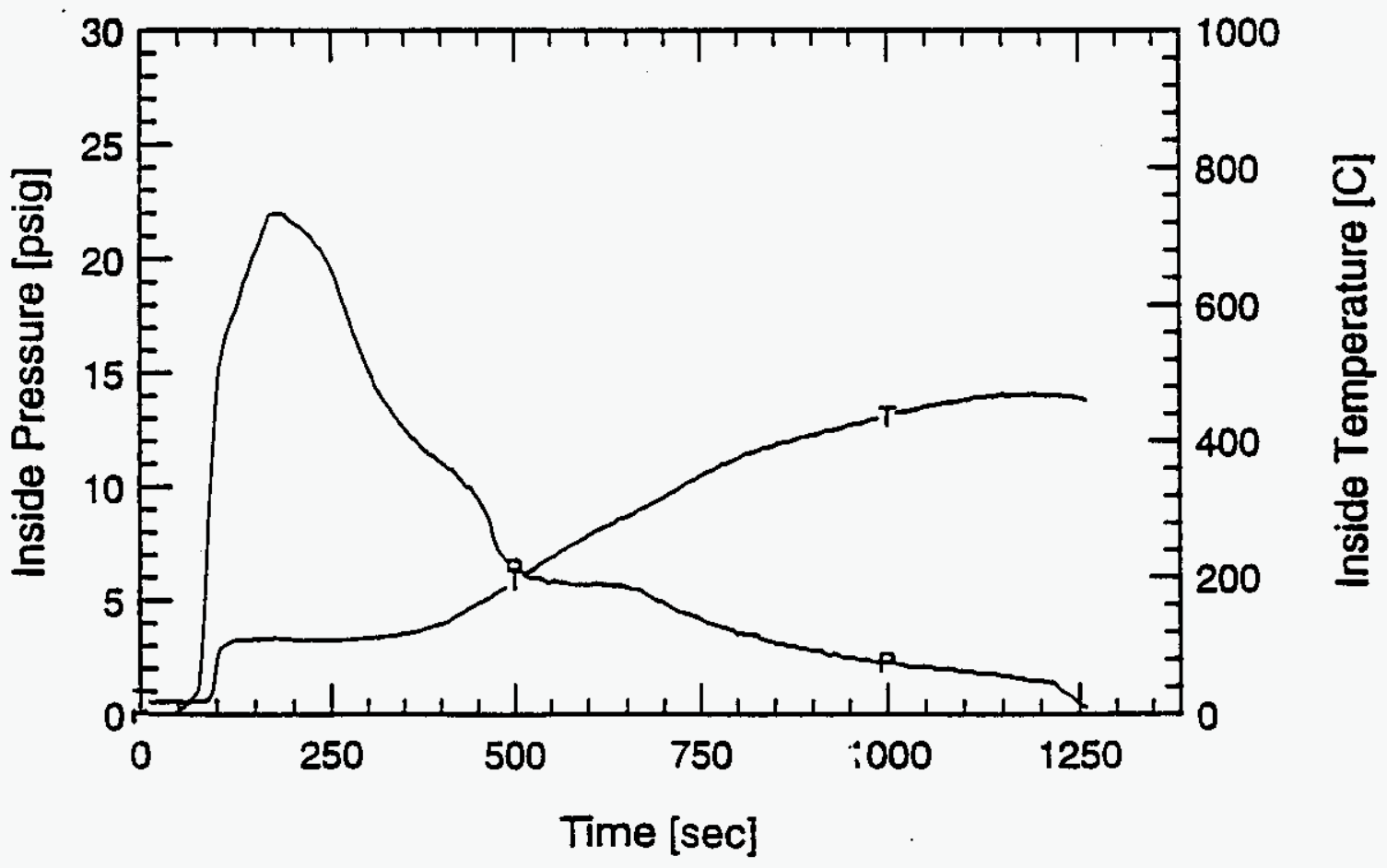


WHC-SD-WM-TRP-233 REV. 0

SWD28 DRUM 4 - 48" Burner - 5lb Plastic
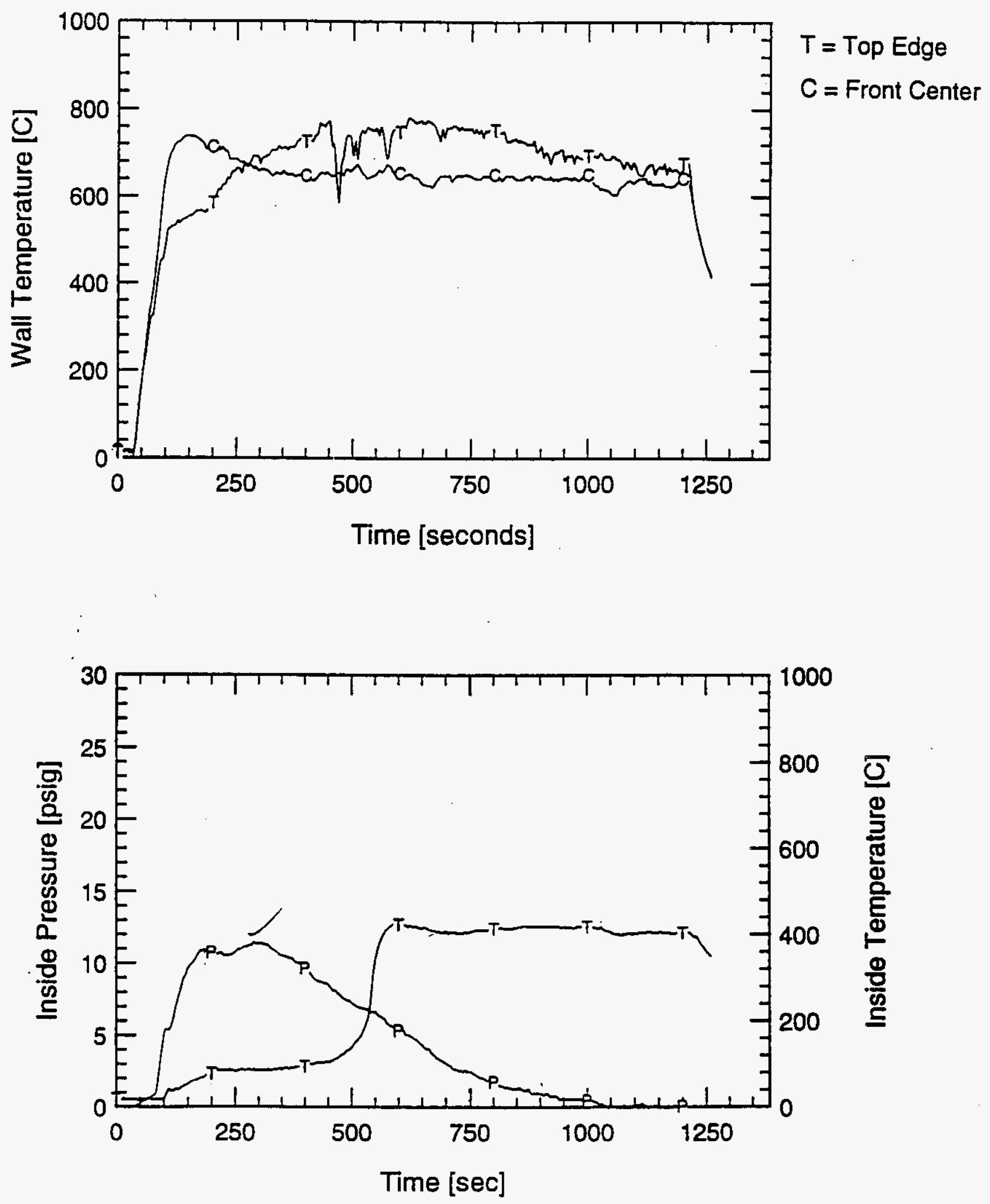
WHC-SD-WM-TRP-233 REV. O

SWD 29-DATA 
WHC-SD-WM-TRP-233 REV. 0

\section{SWD29 Pool Fire - 5lb Paper - Toluene}
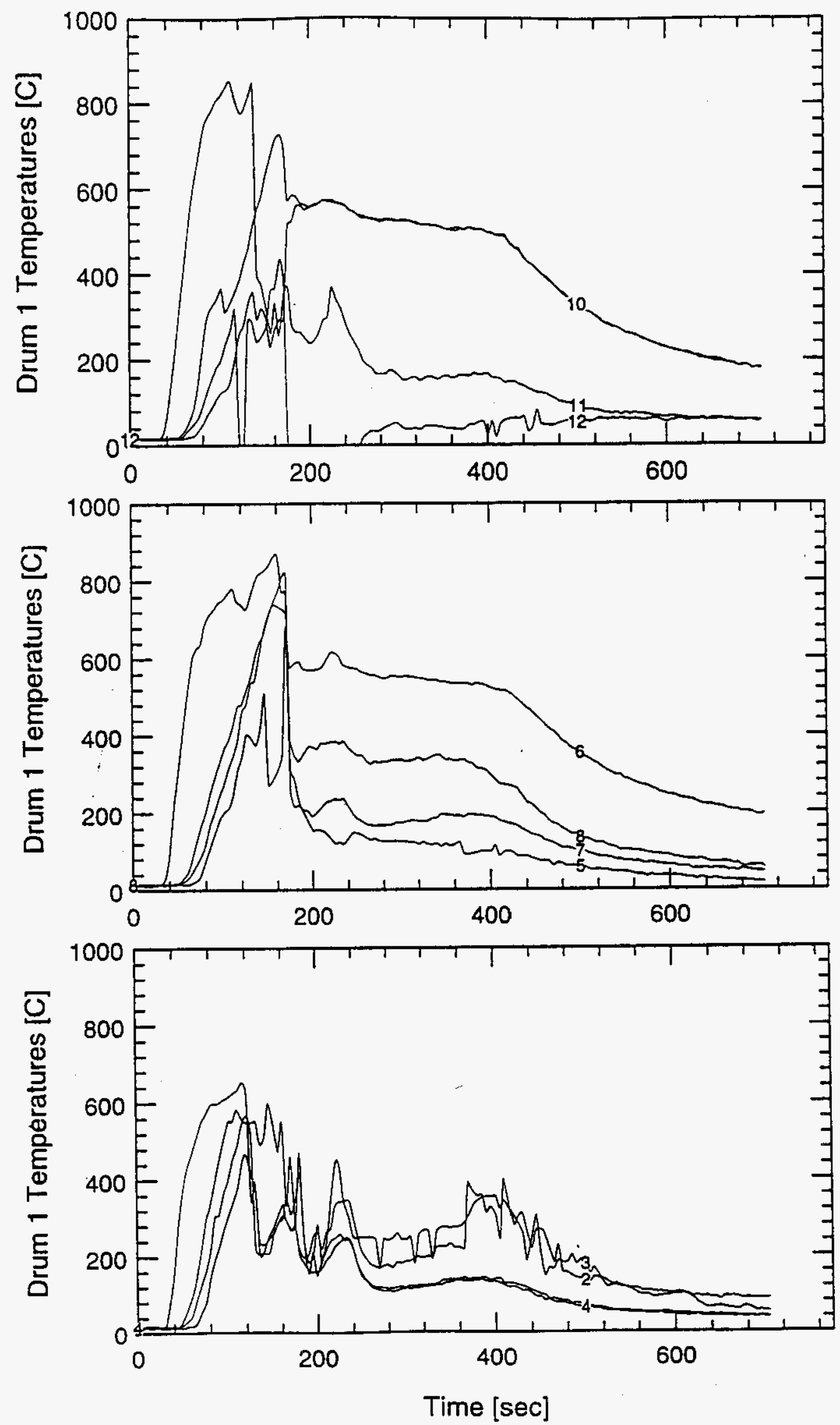
WHC-SD-WM-TRP-233 REV. 0

SWD29 - Drum 2
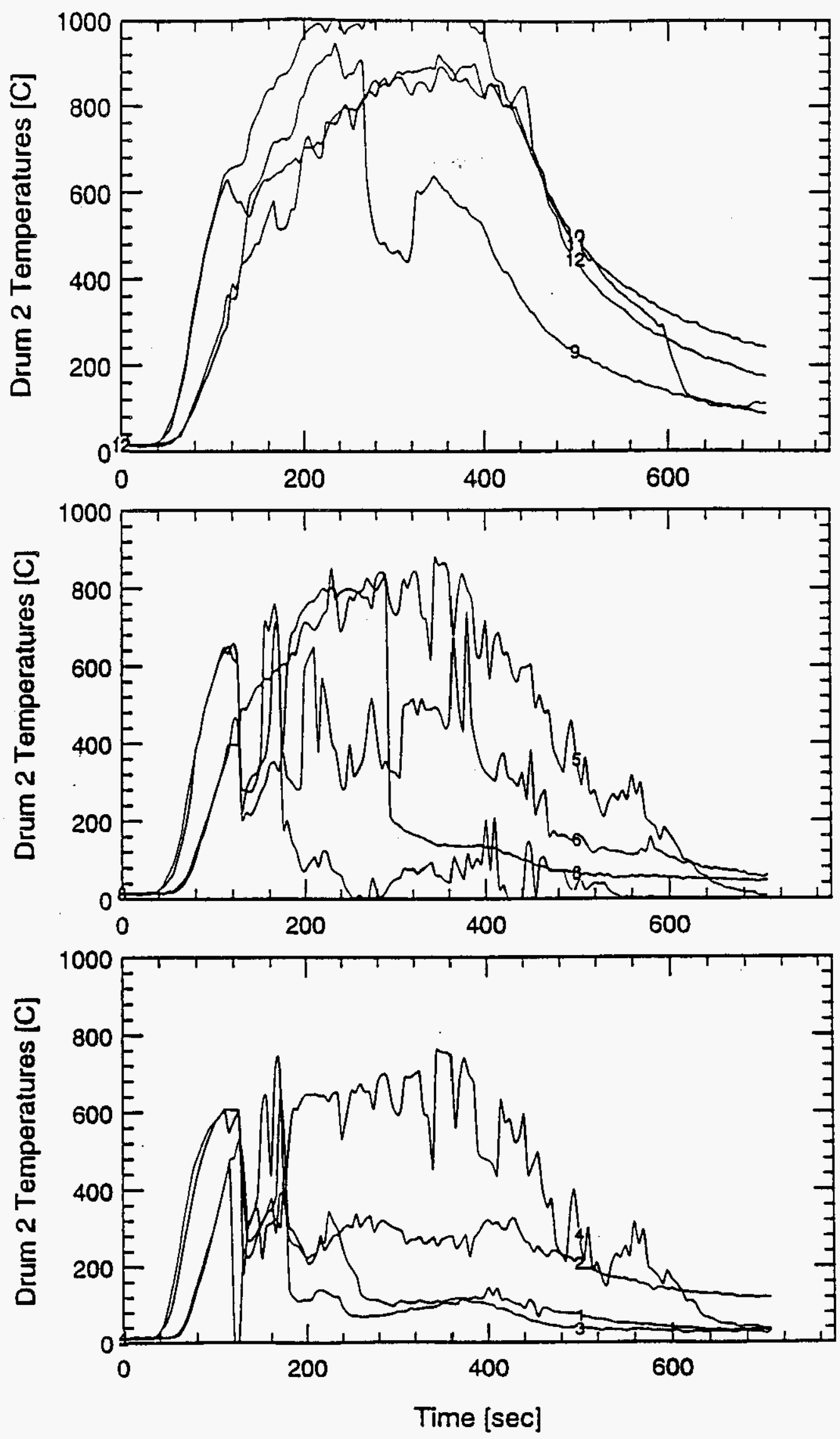

B-205 
WHC-SD-WM-TRP-233 REV. $O$

SWD29 Pool Fire - Bagged Standard Load - Toluene
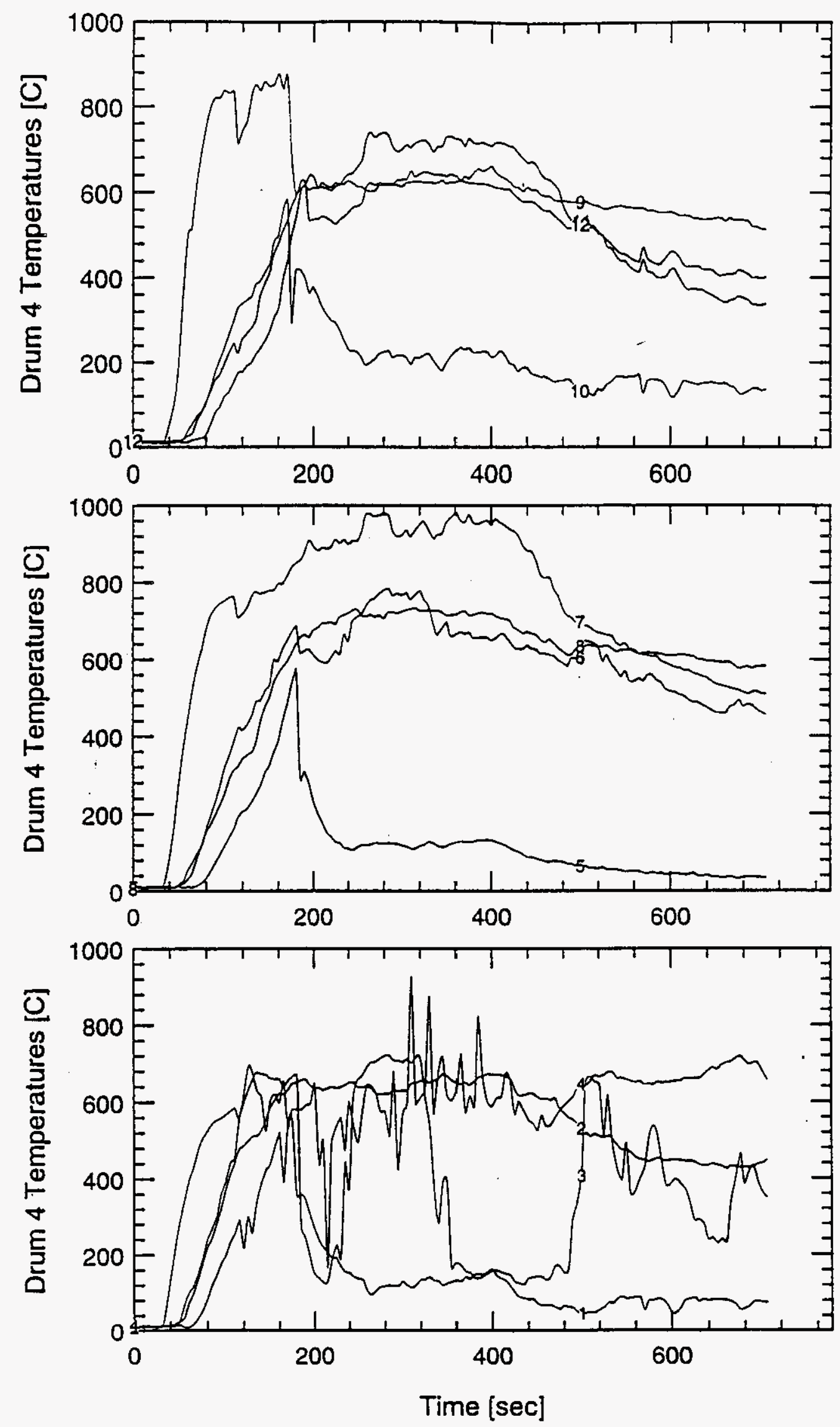

B-207 
SWD29 Pool Fire
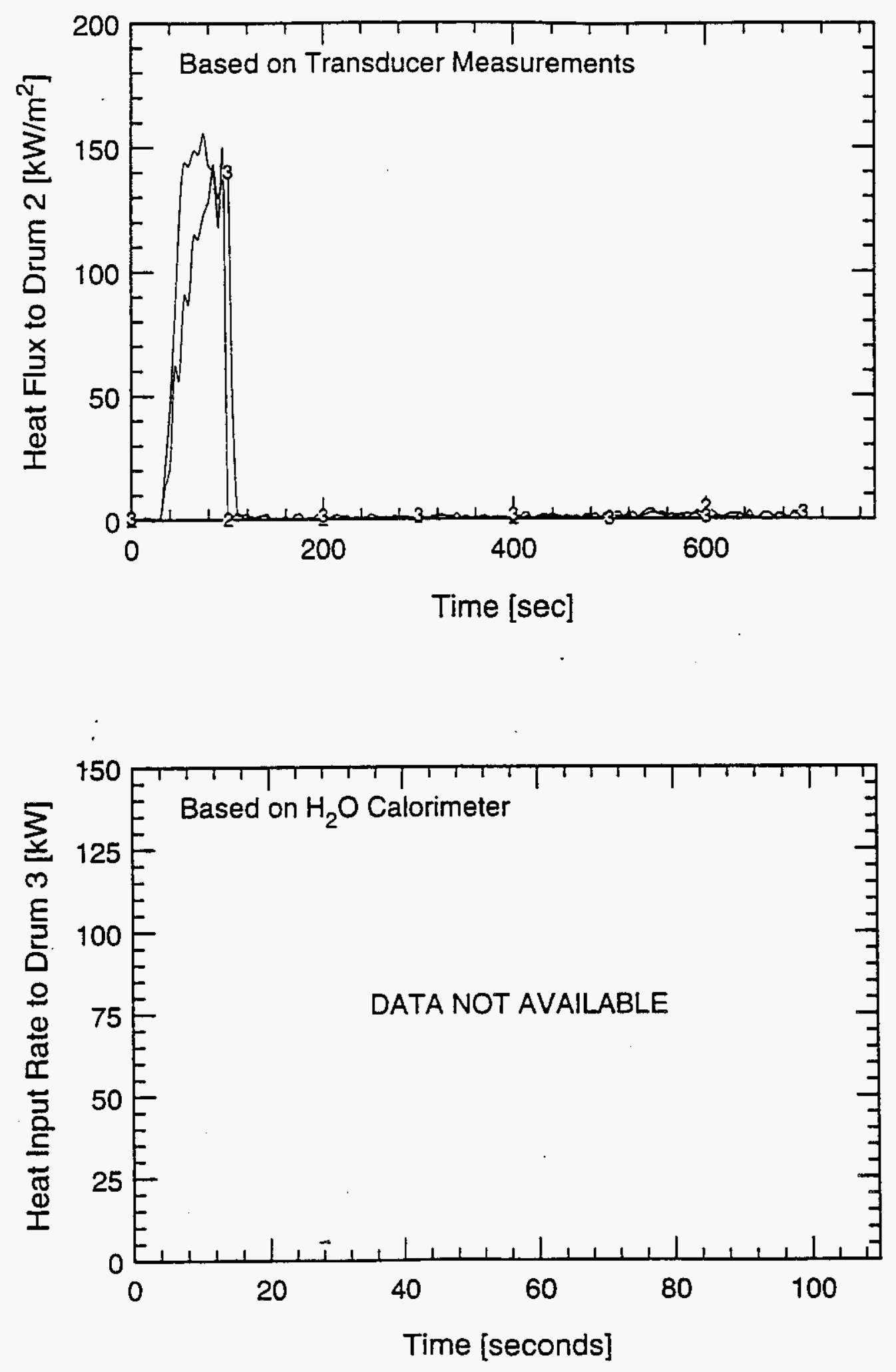


\section{SWD29 DRUM 1 - Pool Fire - 5lb Paper - Toluene}
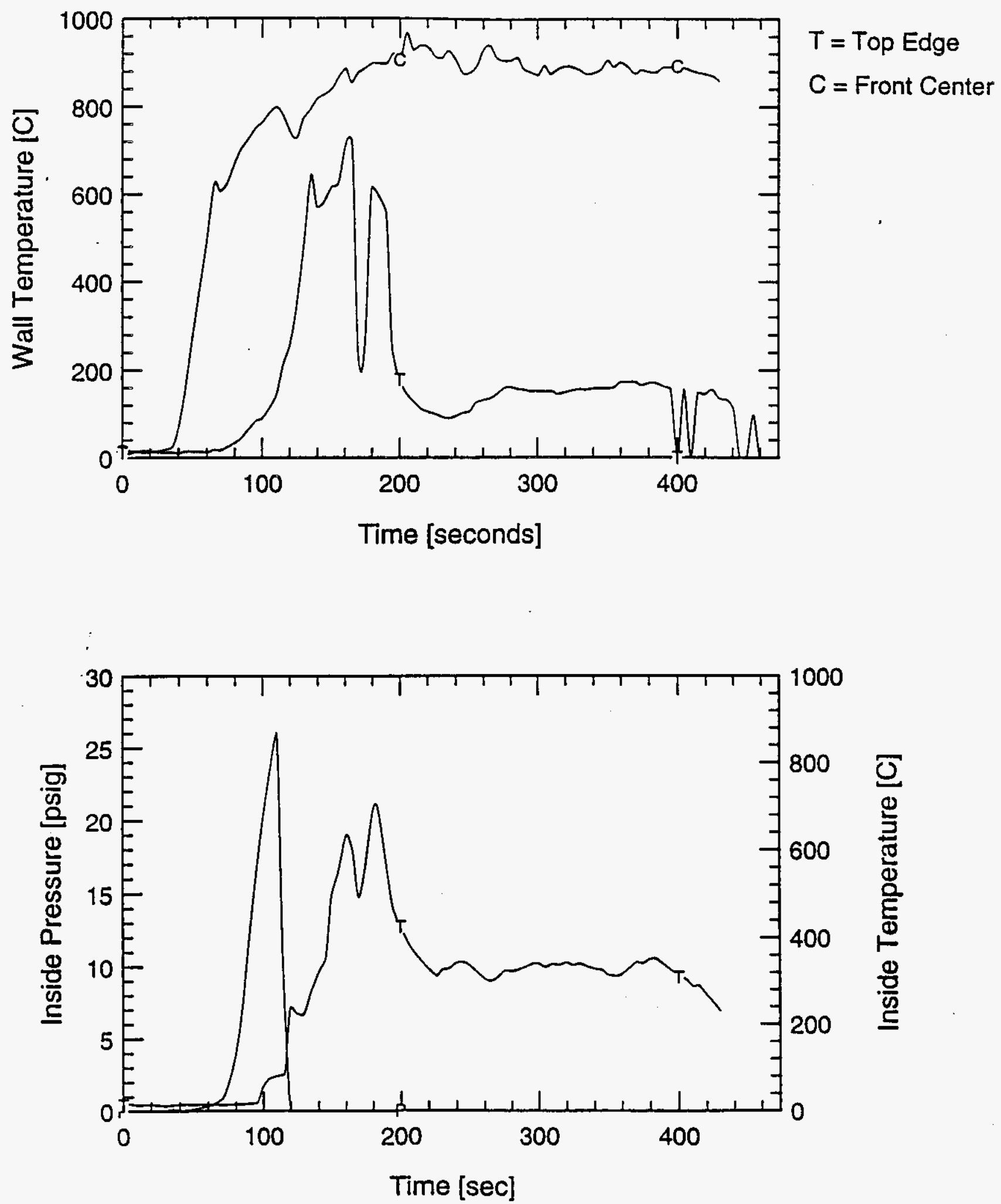
WHC-SD-WM-TRP-233 REV. 0

SWD29 DRUM 4 - Pool Fire - Bagged Std Load - Toluene
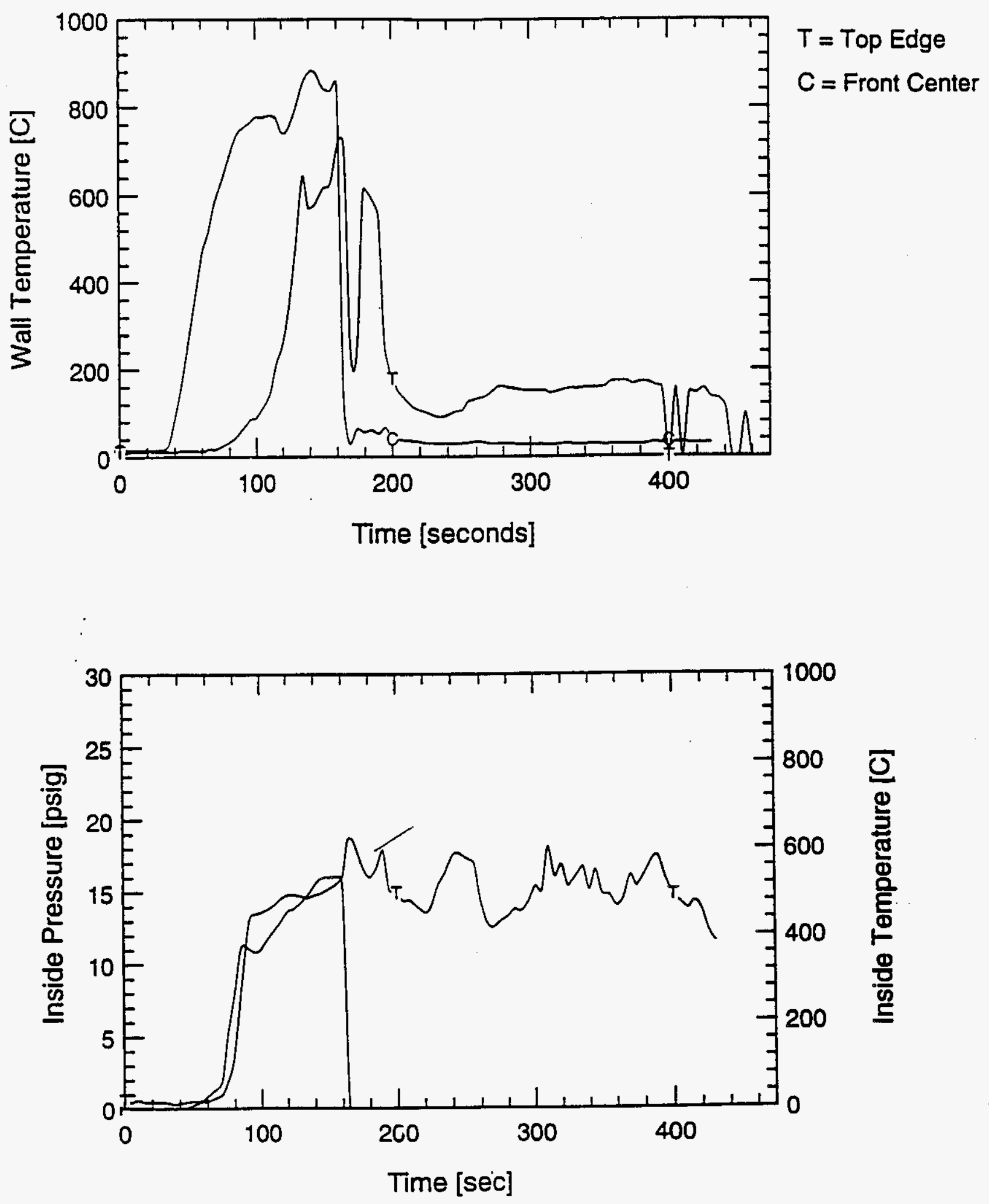
WHC-SD-WM-TRP-233 REV. 0

SWD 30 - DATA 
WHC-SD-WM-TRP-233 REV. 0

\section{SWD30 Pool Fire - 5lb Paper}
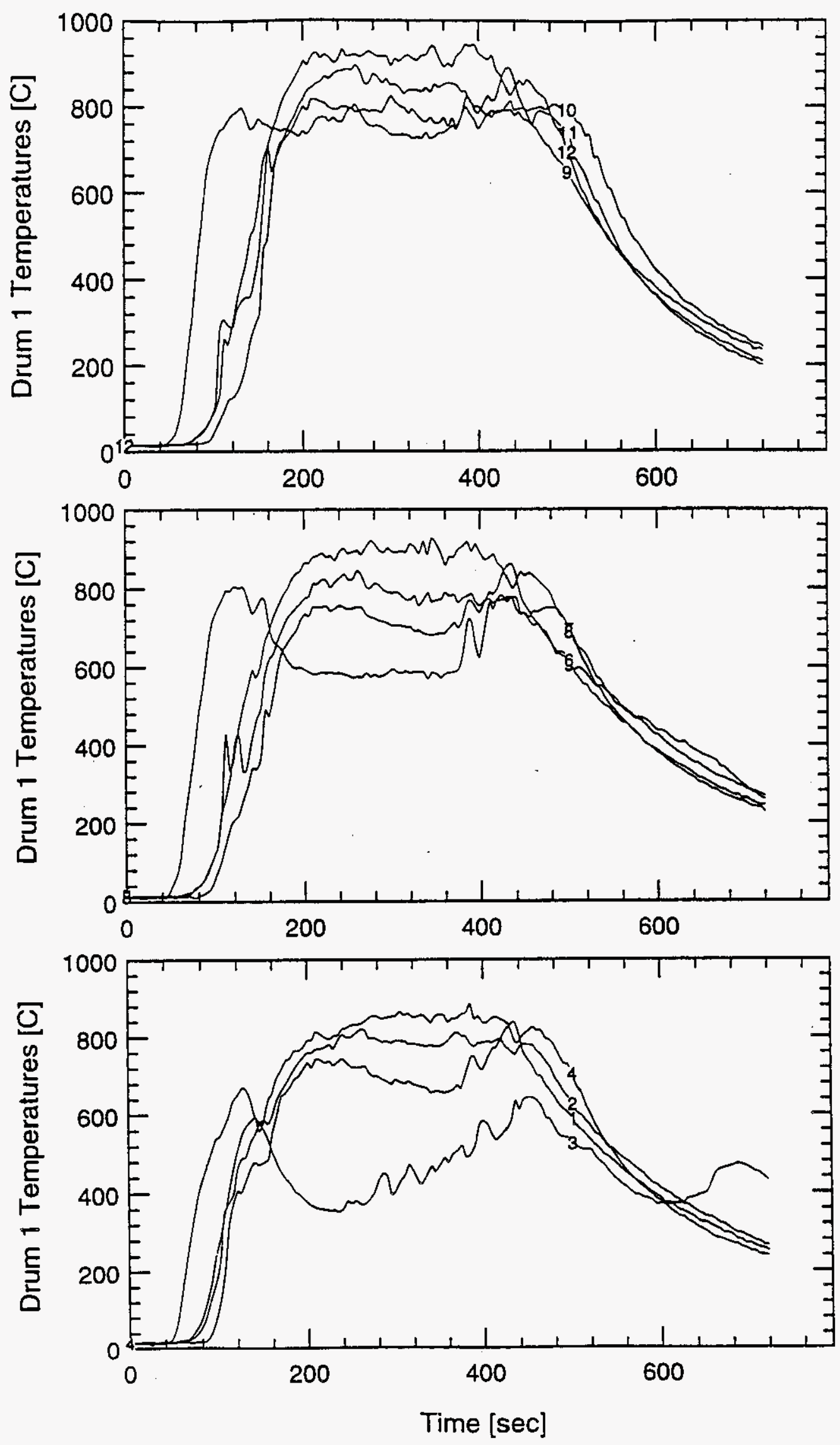

B-212 
WHC-SD-WM-TRP-233 REV. O

SWD30 - Drum 2
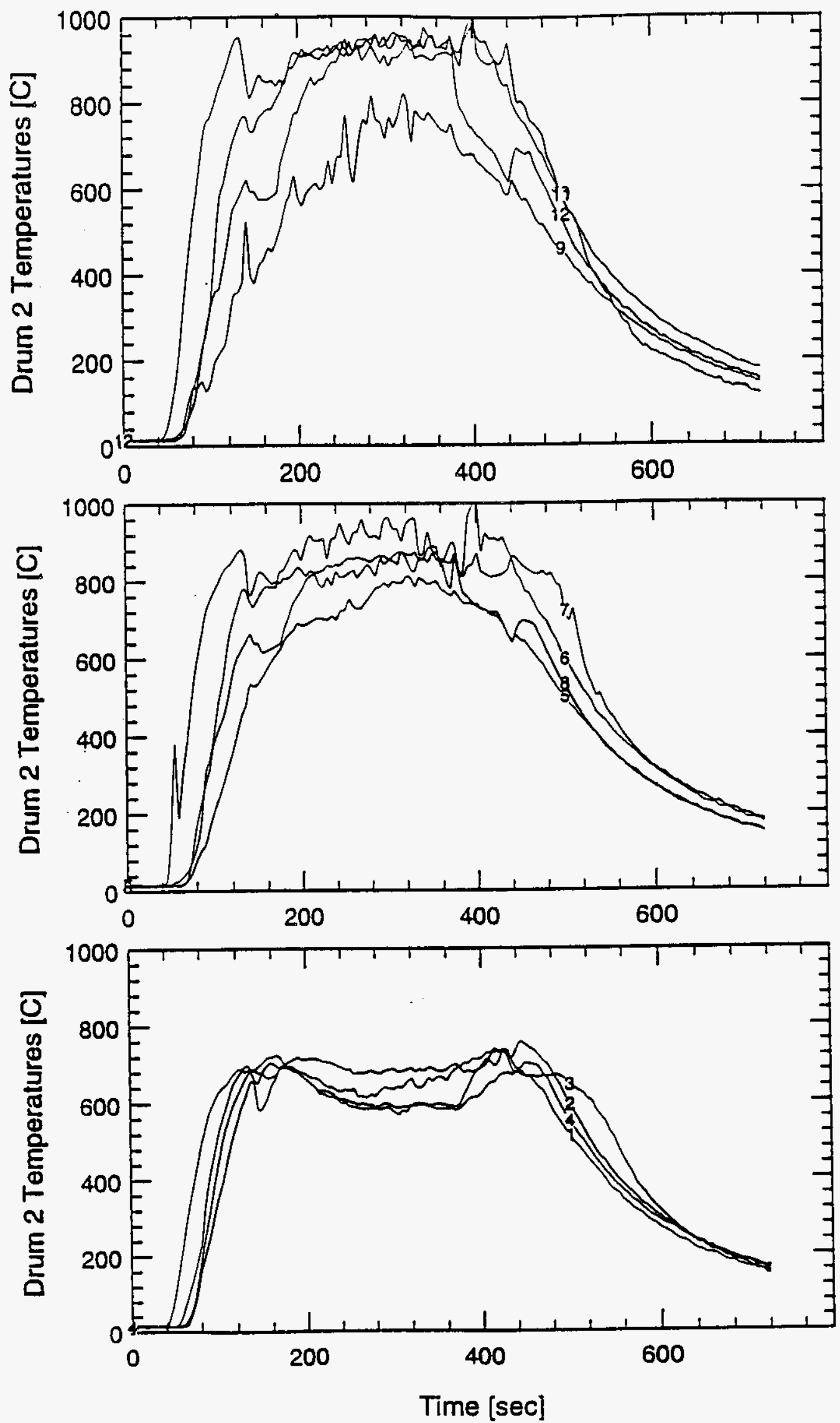

B-213 

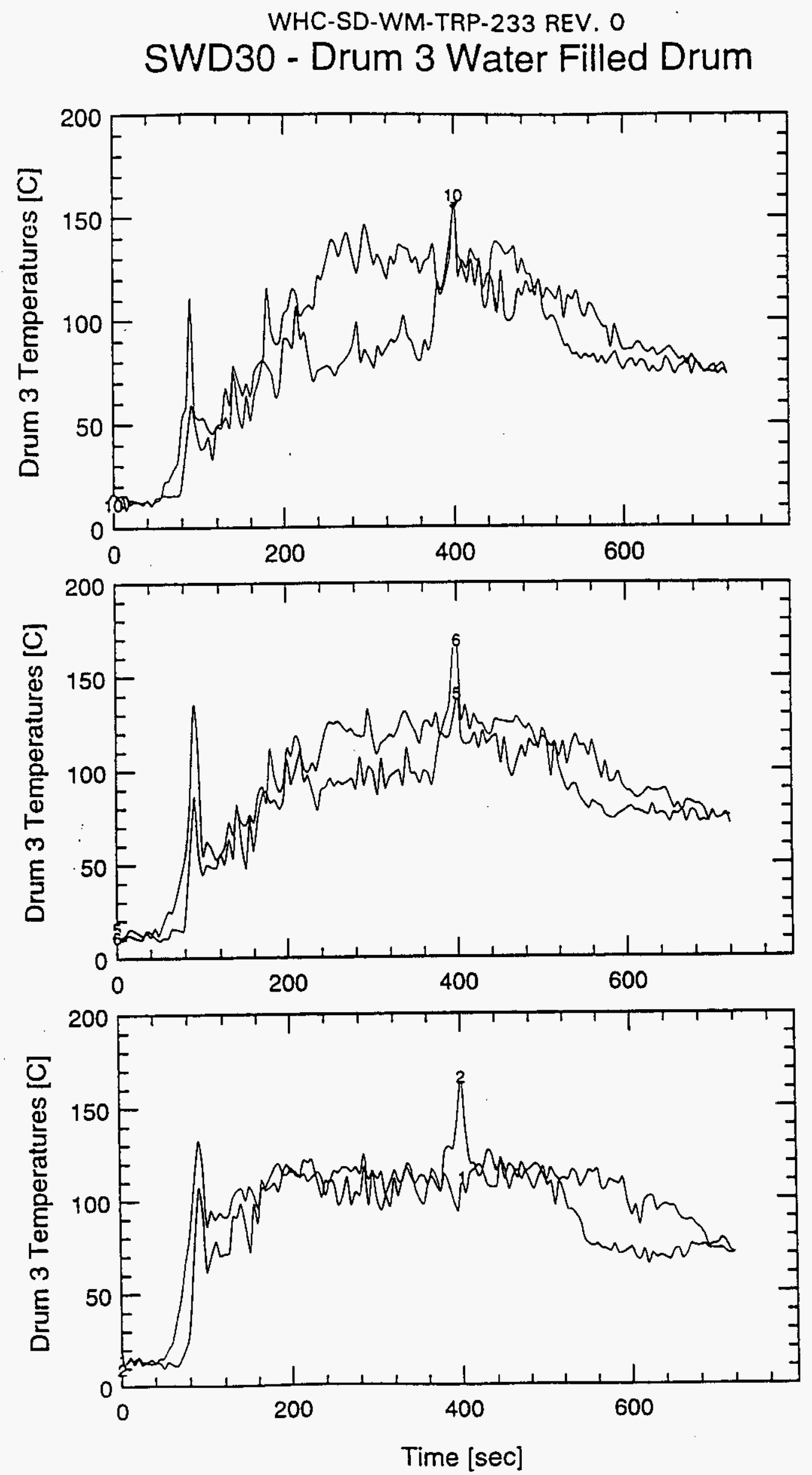
WHC-SD-WM-TRP-233 REV. 0

SWD30 Pool Fire - Empty
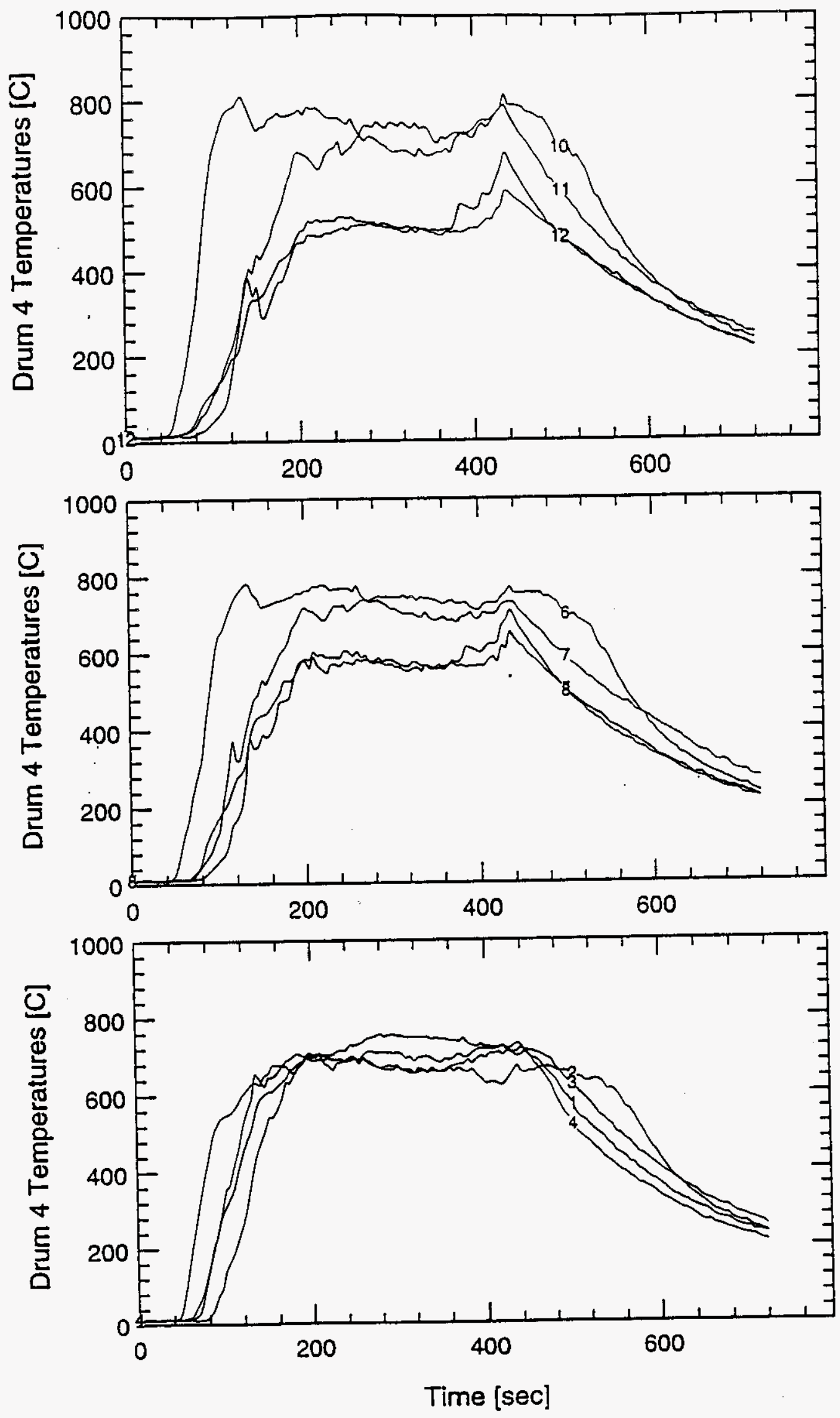

B-215 
WHC-SD-WM-TRP-233 REV. 0

SWD30 Pool Fire
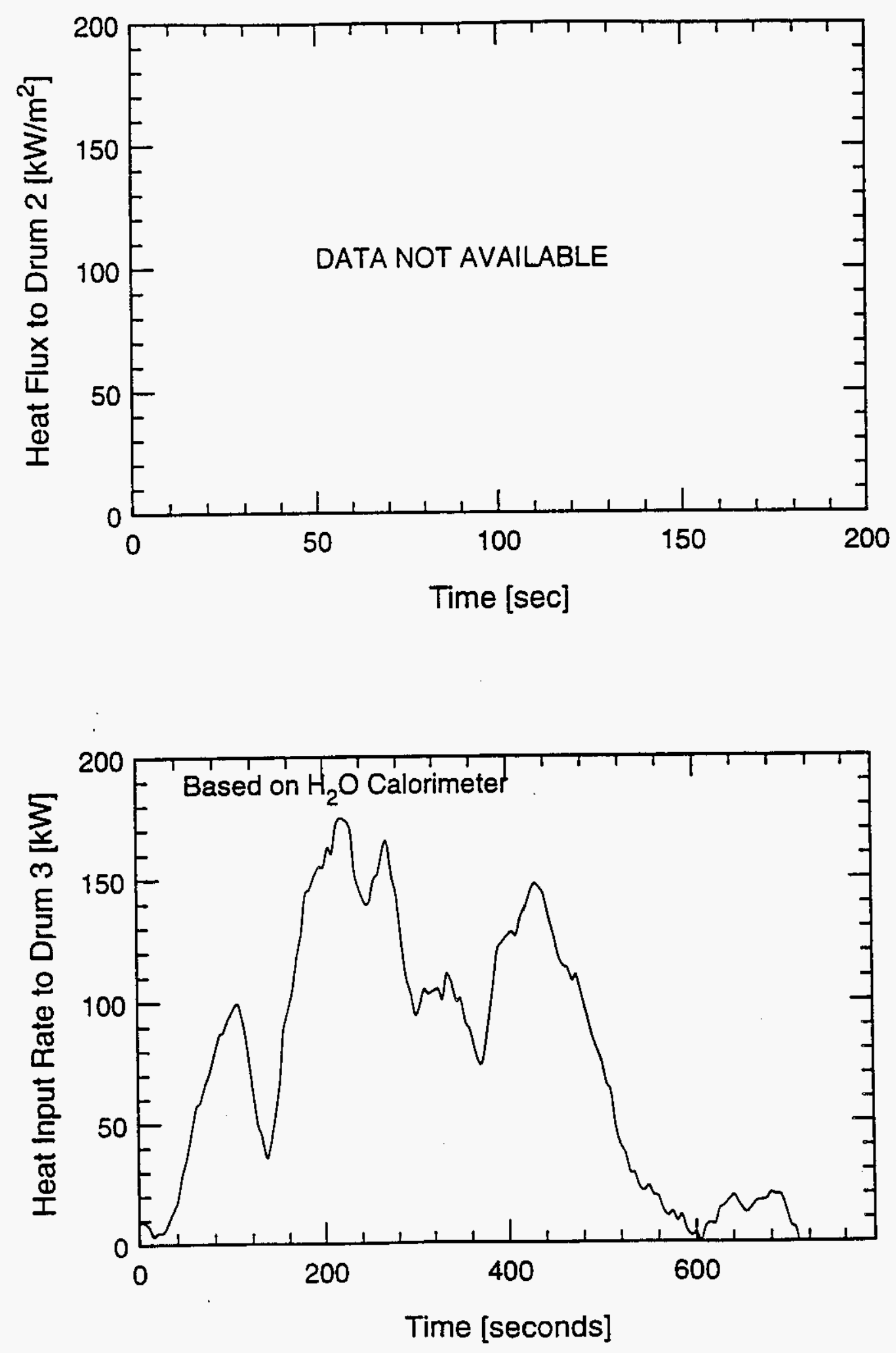
WHC-SD-WM-TRP-233 REV. 0

SWD30 DRUM 1 - Pool Fire - 5lb Paper
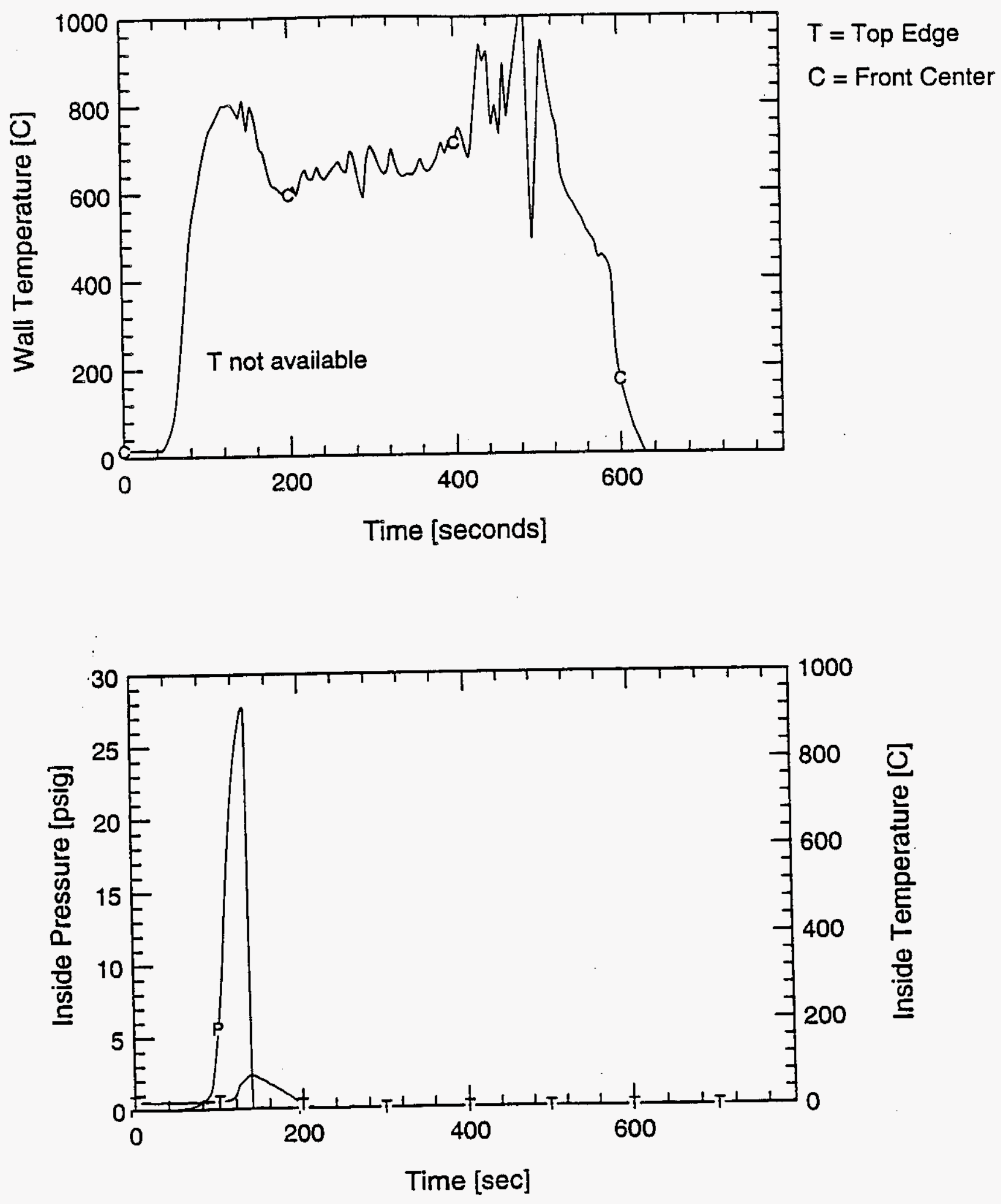
WHC-SD-WM-TRP-233 REV. 0

\section{SWD30 DRUM 4 - Pool Fire - Empty}
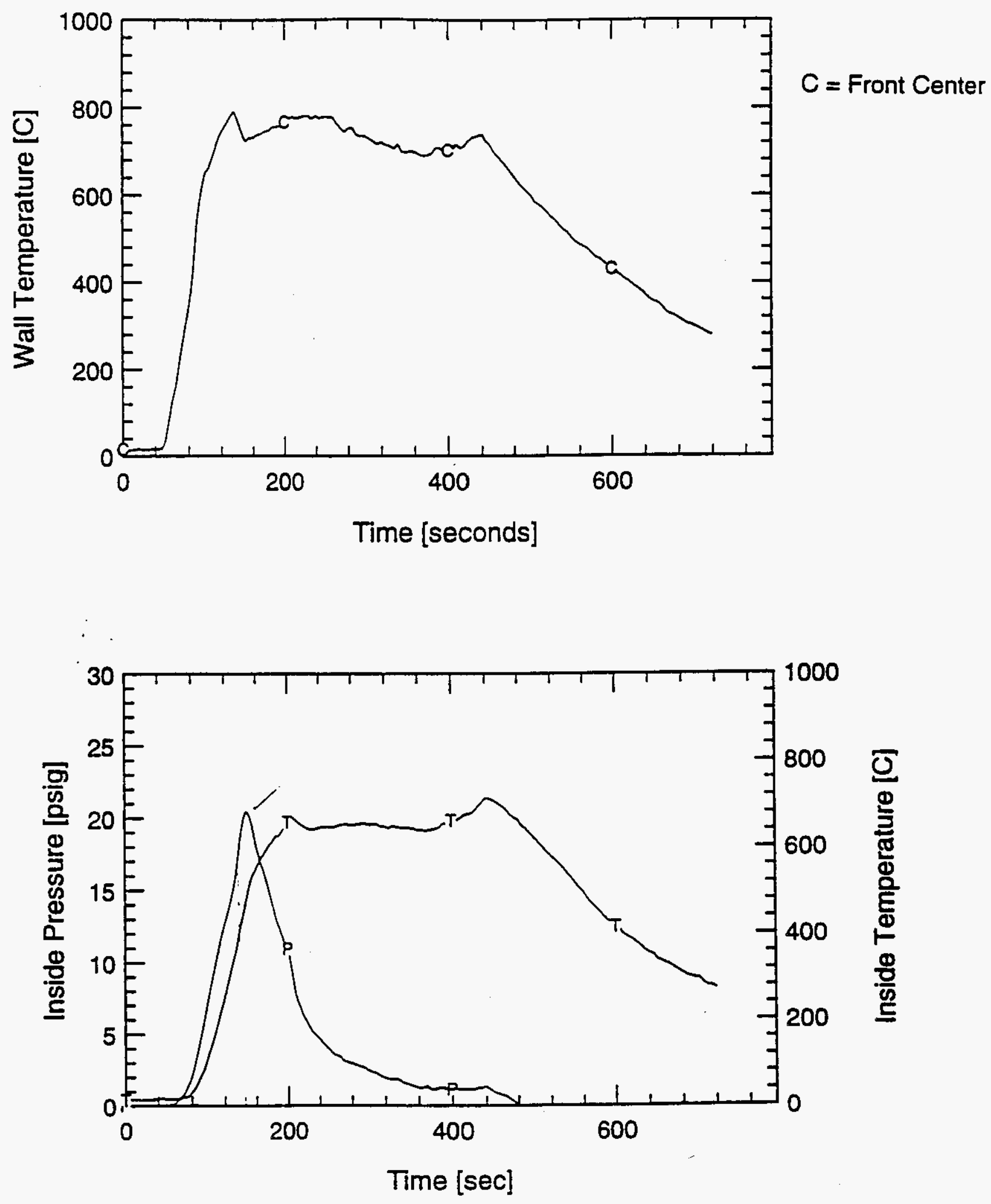
WHC-SD-WM-TRP-233 REV. 0

$$
\text { SWD } 31 \text { - DATA }
$$




\section{SWD31 Pool Fire - 5lb Plastic}
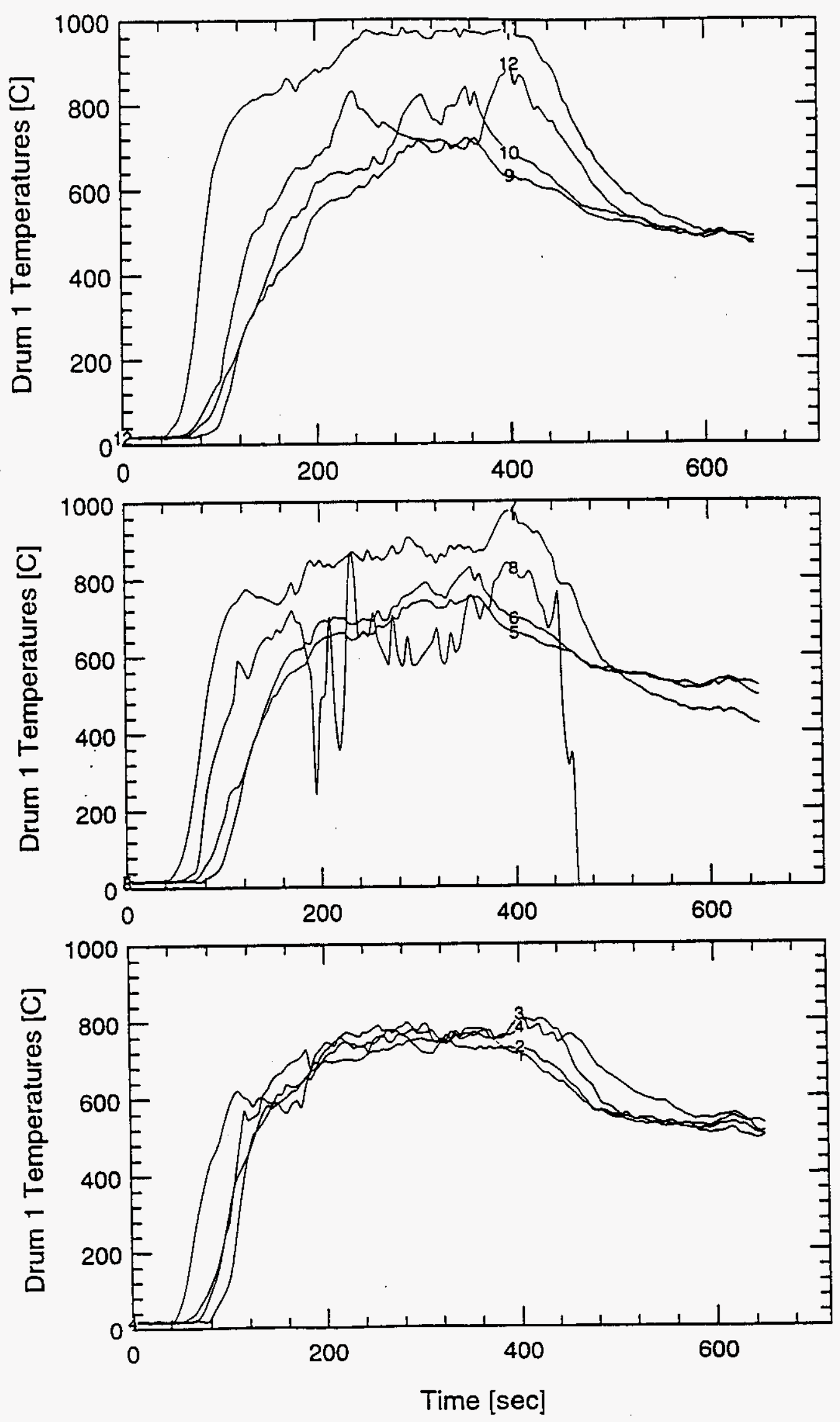
WHC-SD-WM-TRP-233 REV. 0

SWD31 - Pool Fire
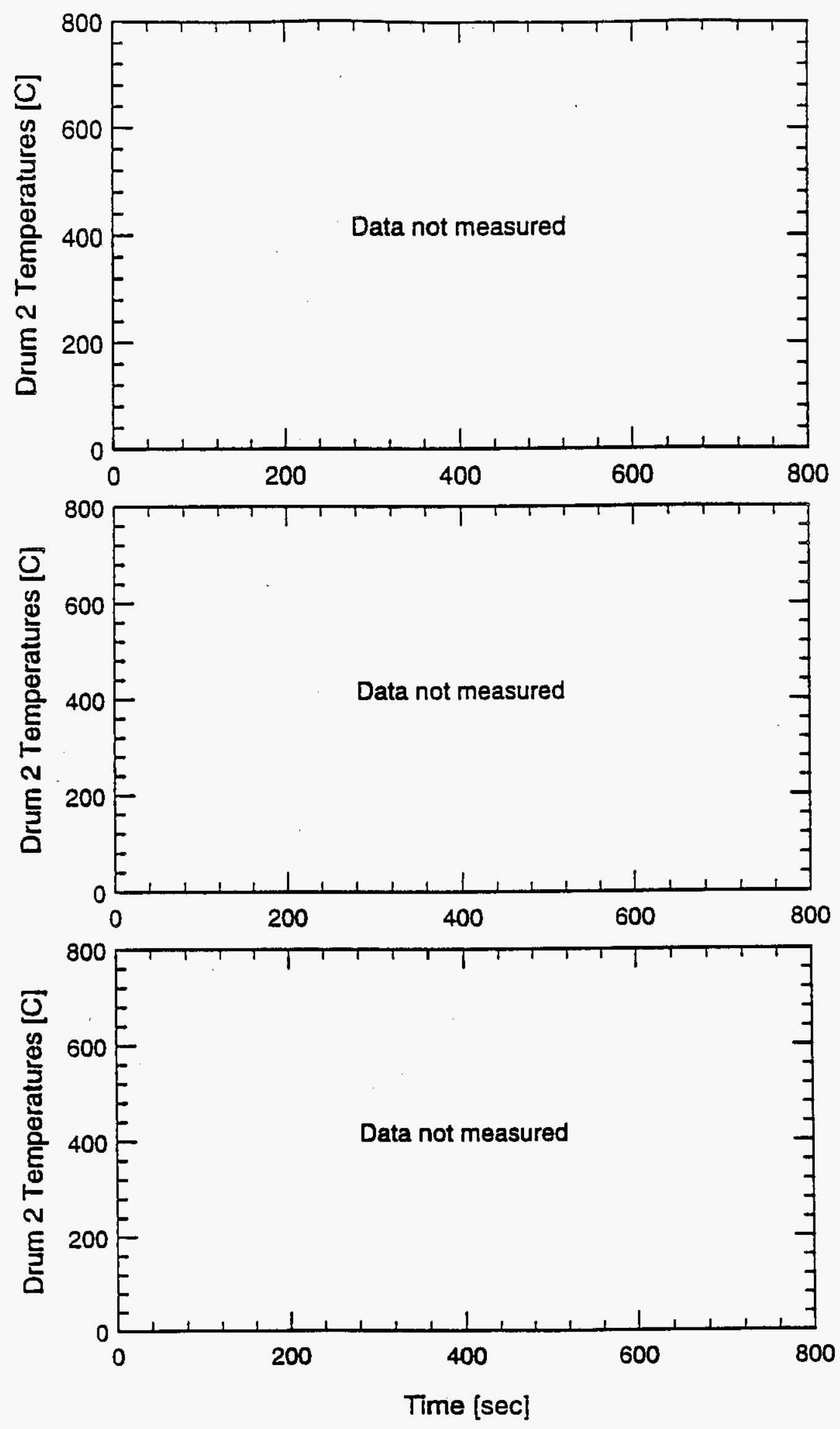

B-221 
WHC-SD-WM-TRP-233 REV. 0

SWD31 - Pool Fire
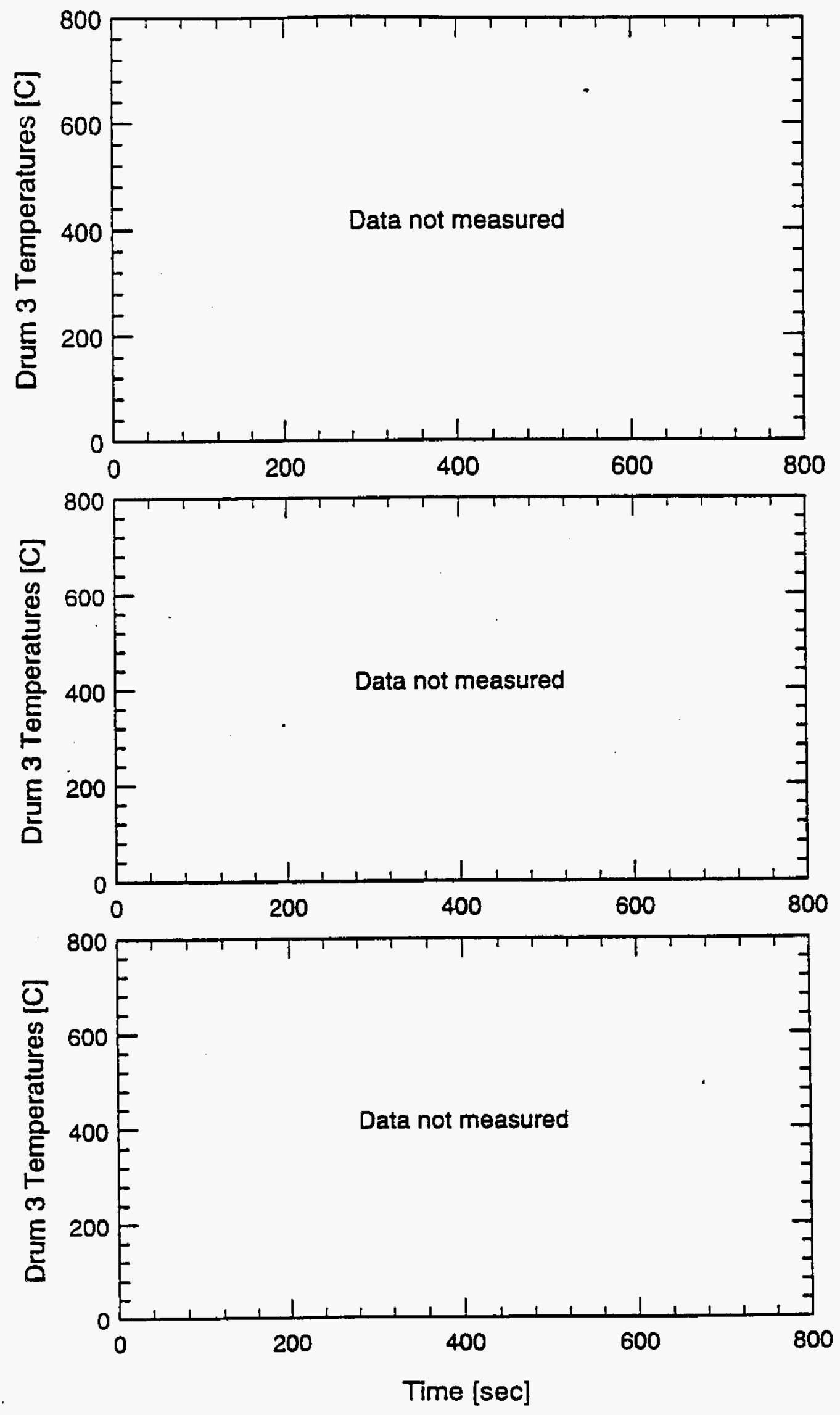

B-222 
WHC-SD-WM-TRP-233 REV. 0

SWD31 Pool Fire - Standard Load
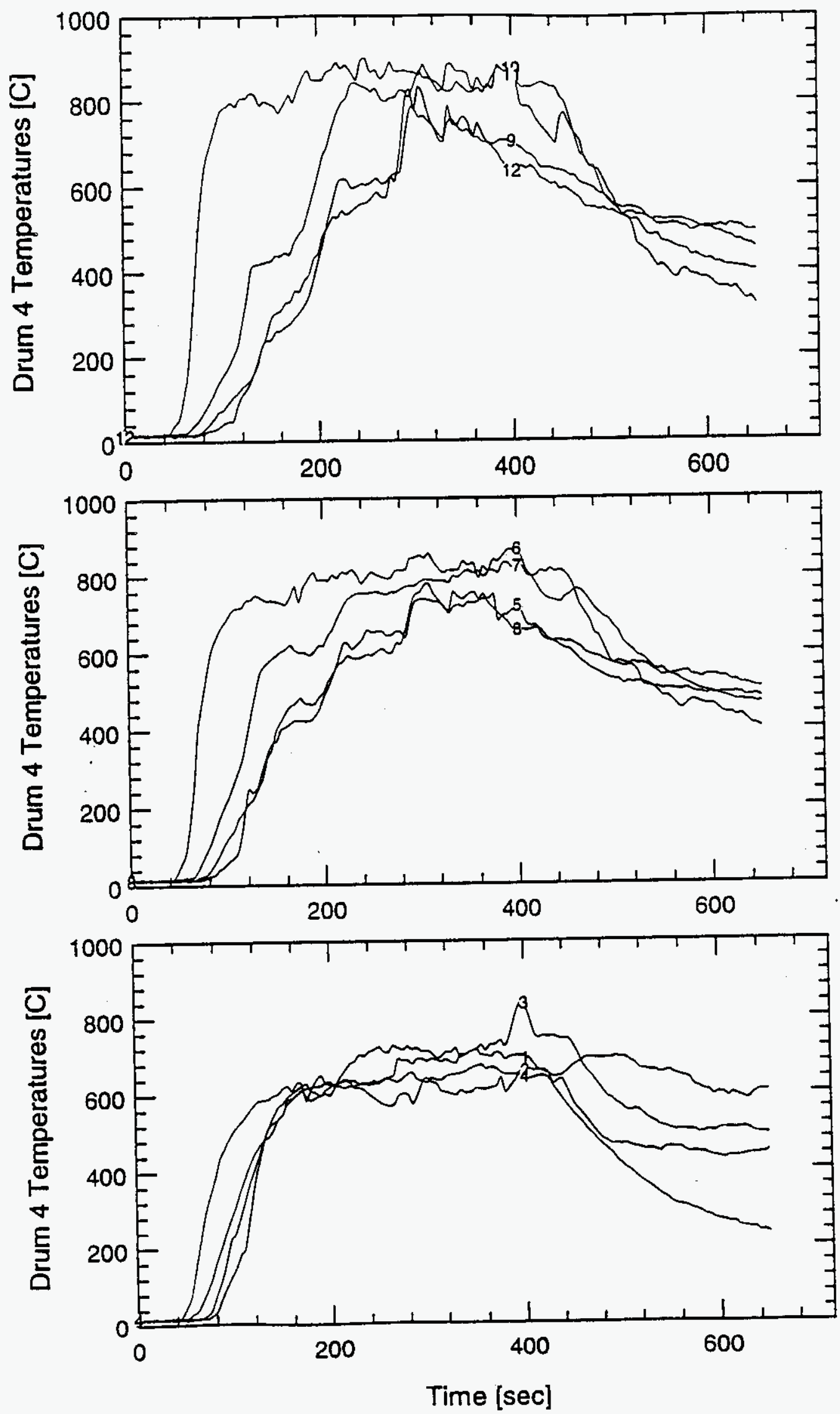

B-223 
WHC-SD-WM-TRP-233 REV. 0

\section{SWD31 Pool Fire}
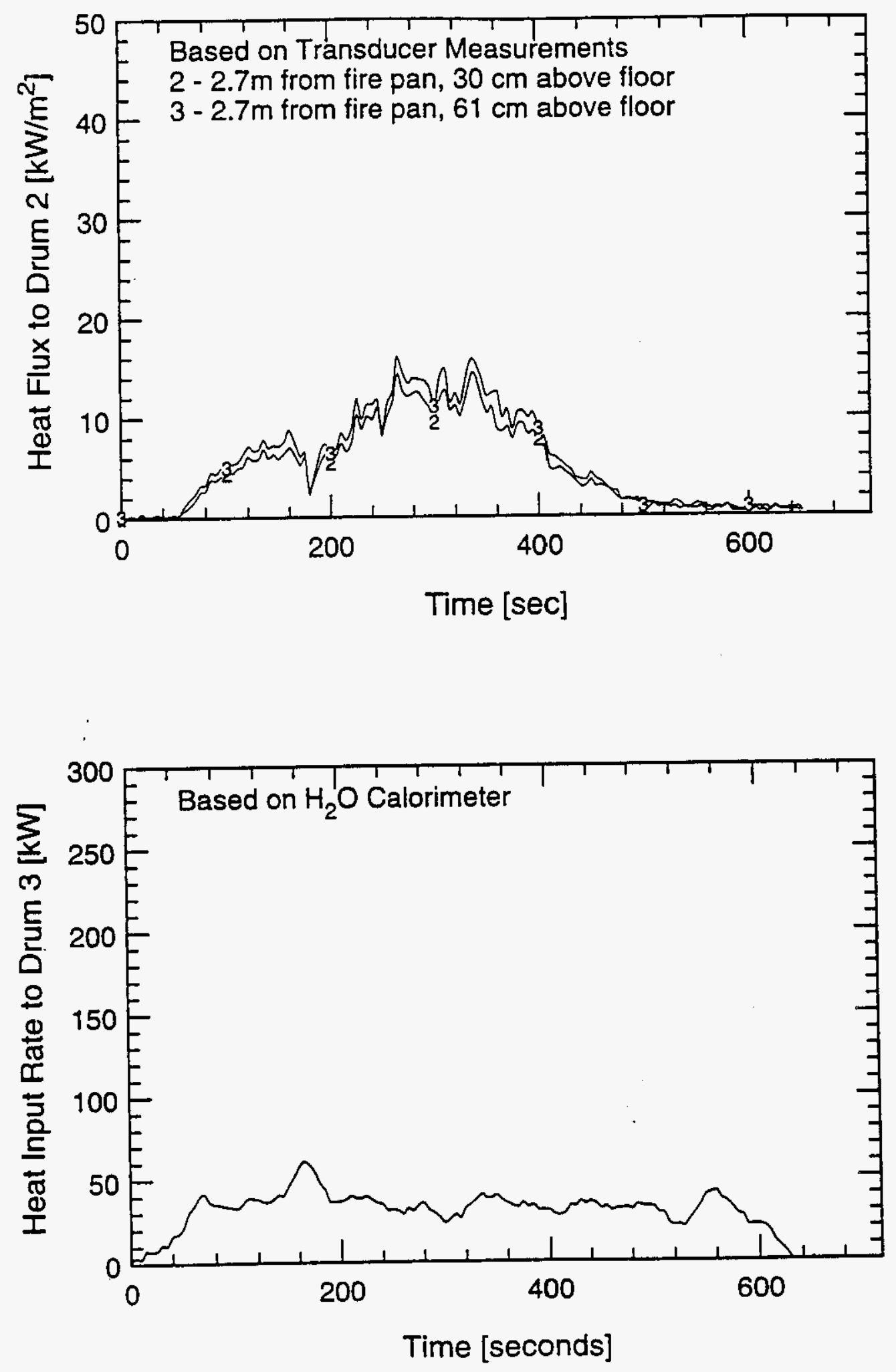


\section{SWD31 DRUM 1 - Pool Fire - 5lb Plastic}
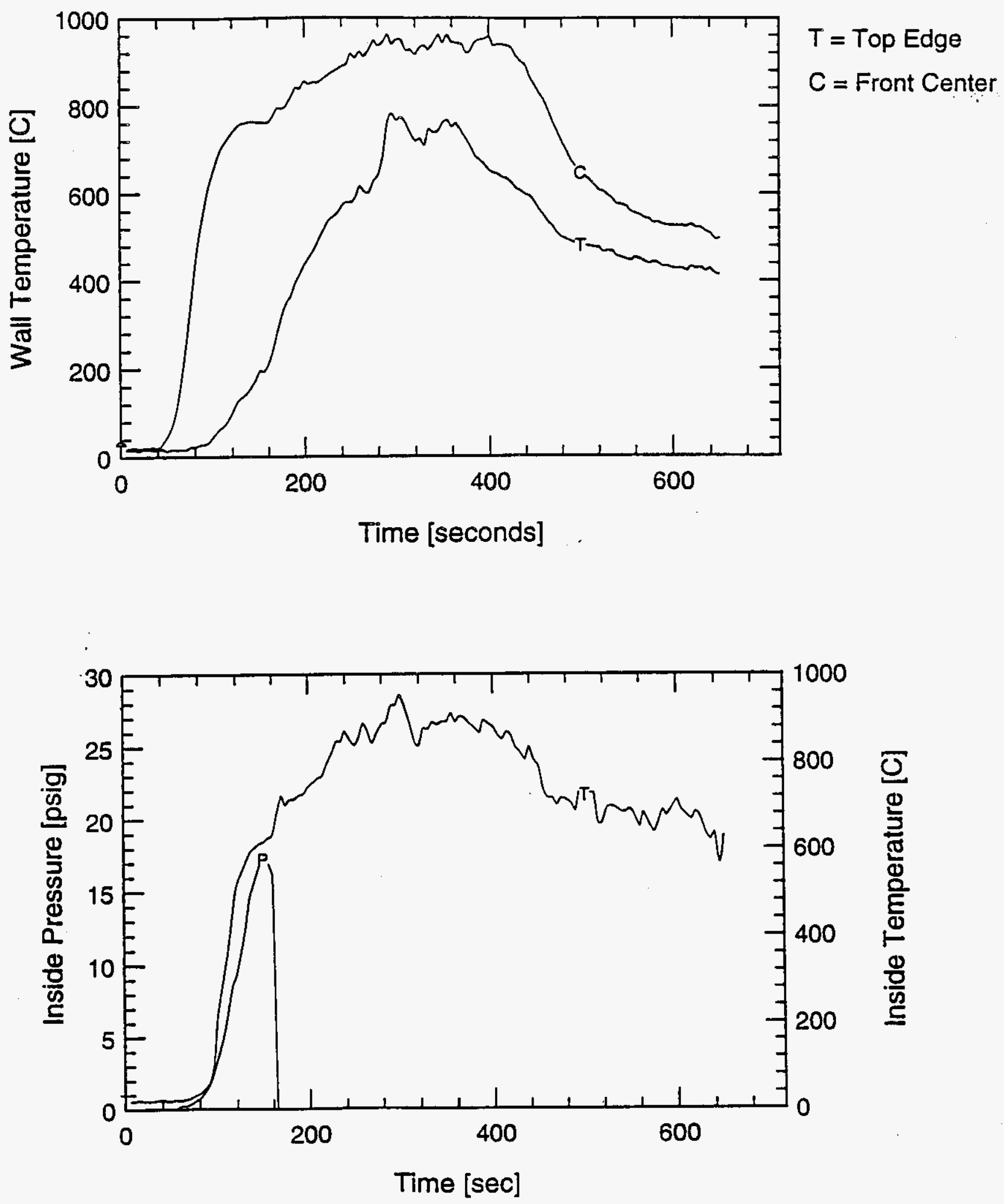

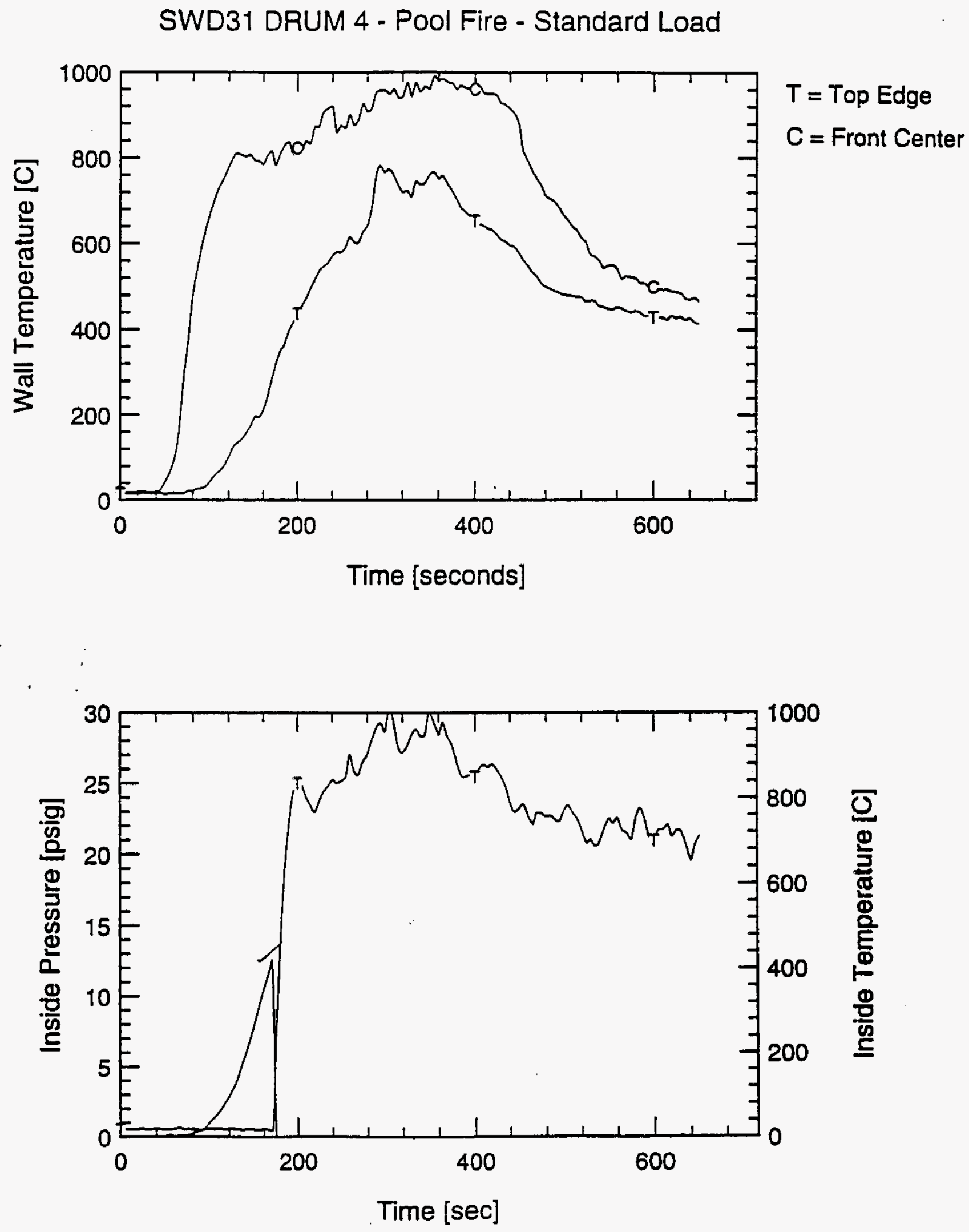
WHC-SD-WM-TRP-233 REV. 0

SWD 32 - DATA

B-227 

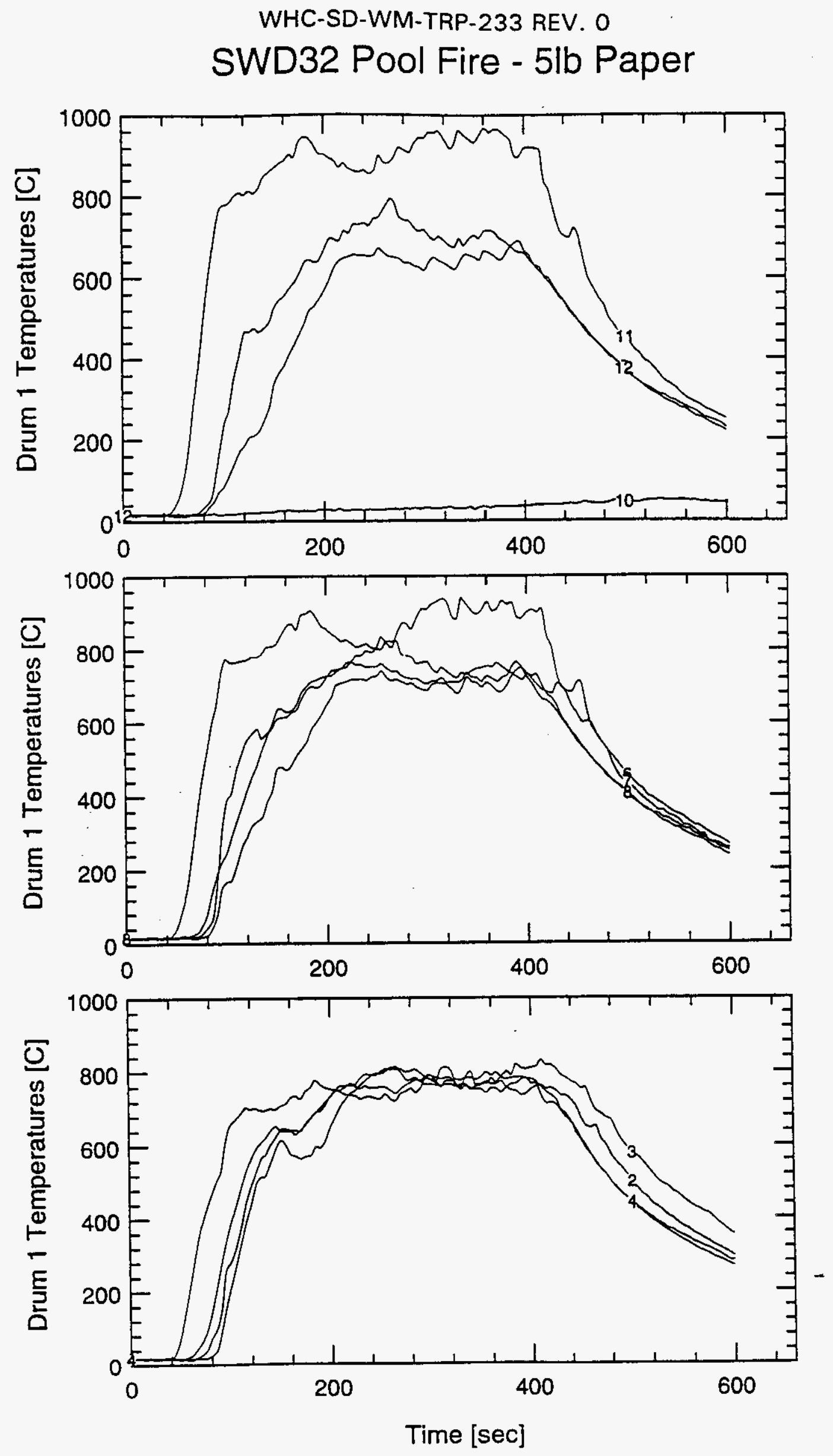

B-228 
WHC-SD-WM-TRP-233 REV. 0

SWD32 - Pool Fire
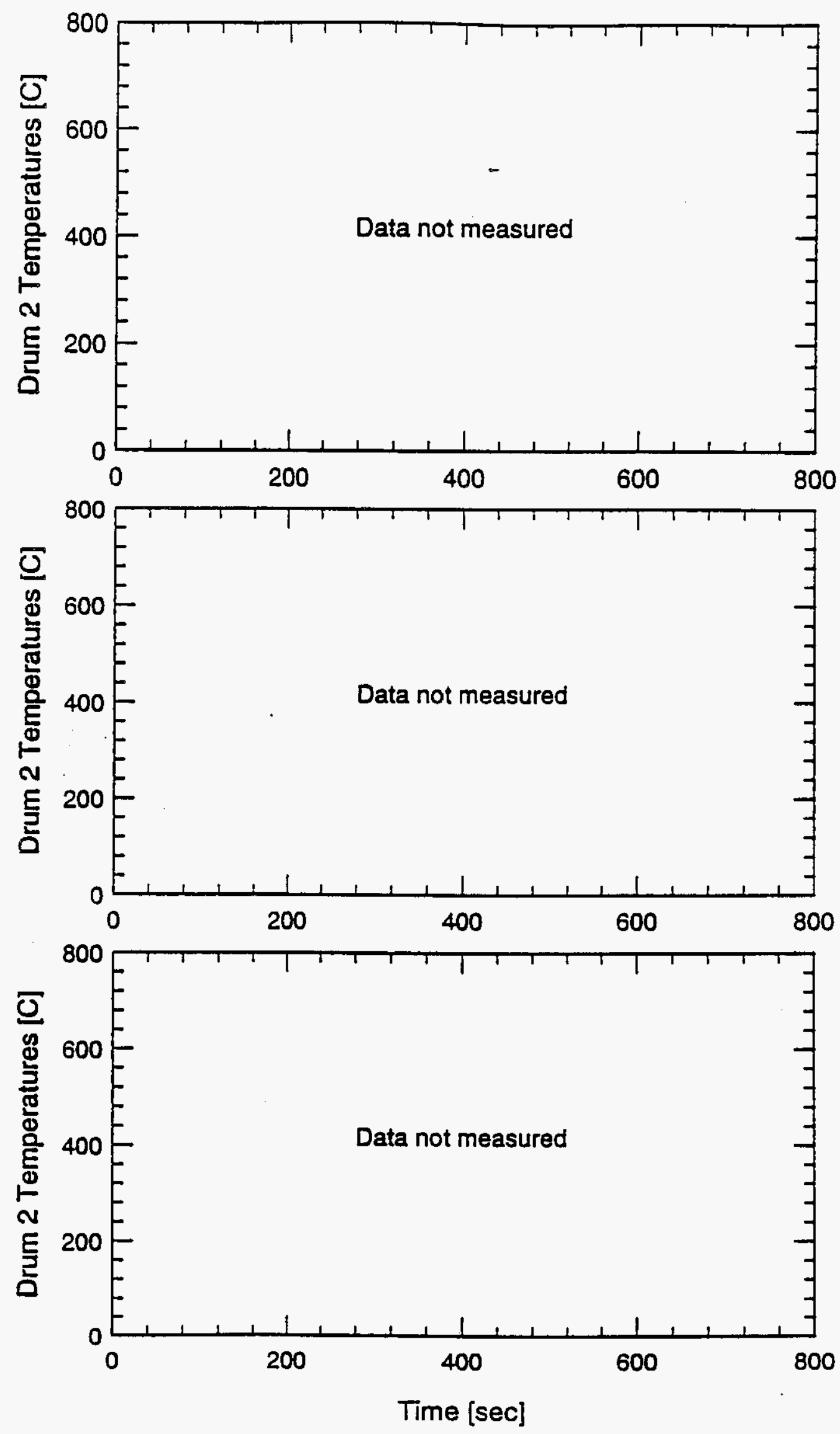

B-229 
WHC-SD-WM-TRP-233 REV. 0

SWD32 - Pool Fire
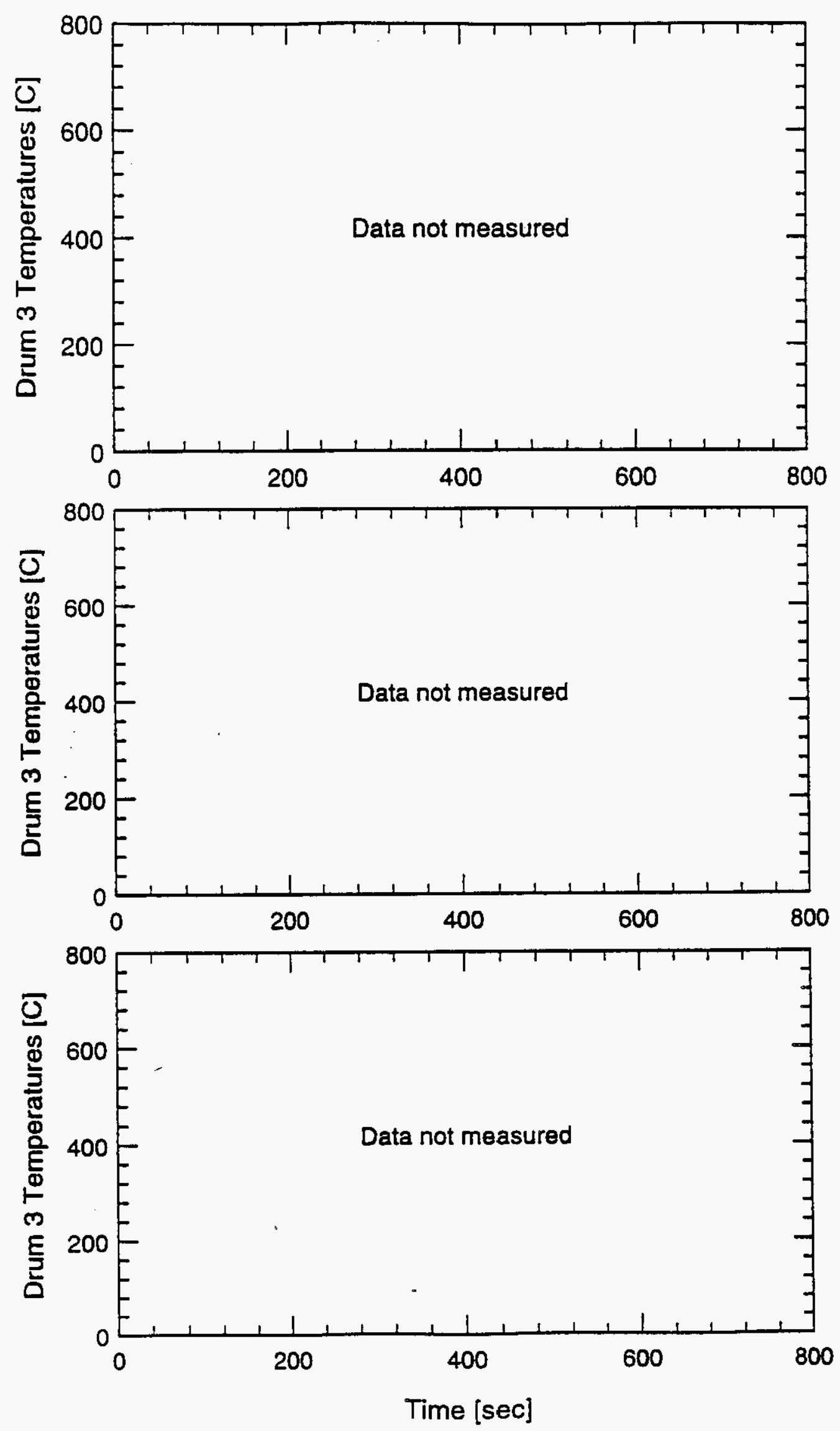
WHC-SD-WM-TRP-233 REV. 0

SWD32 Pool Fire - $5 \mathrm{lb}$ Plastic
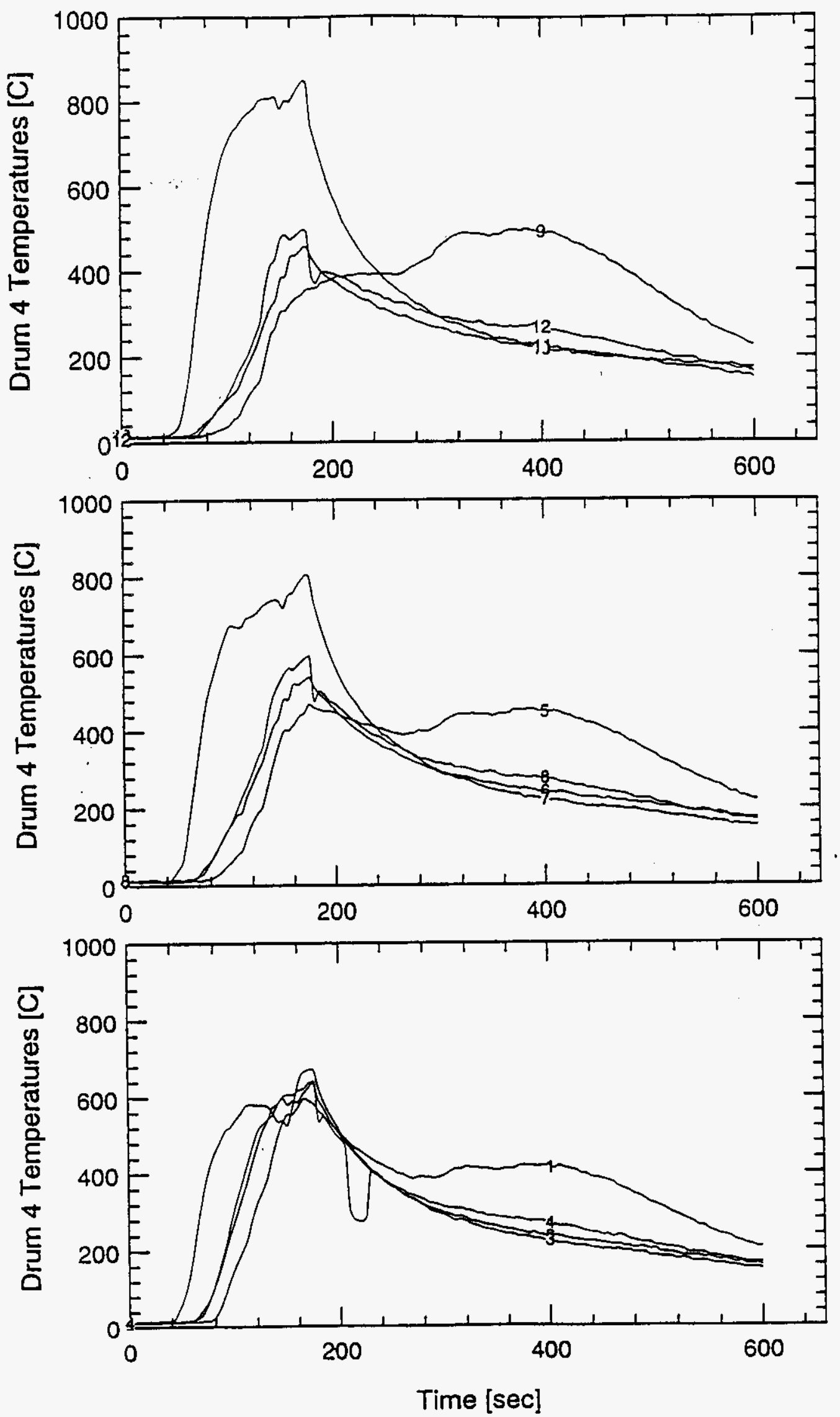
WHC-SD-WM-TRP-233 REV. 0

\section{SWD32 Pool Fire}
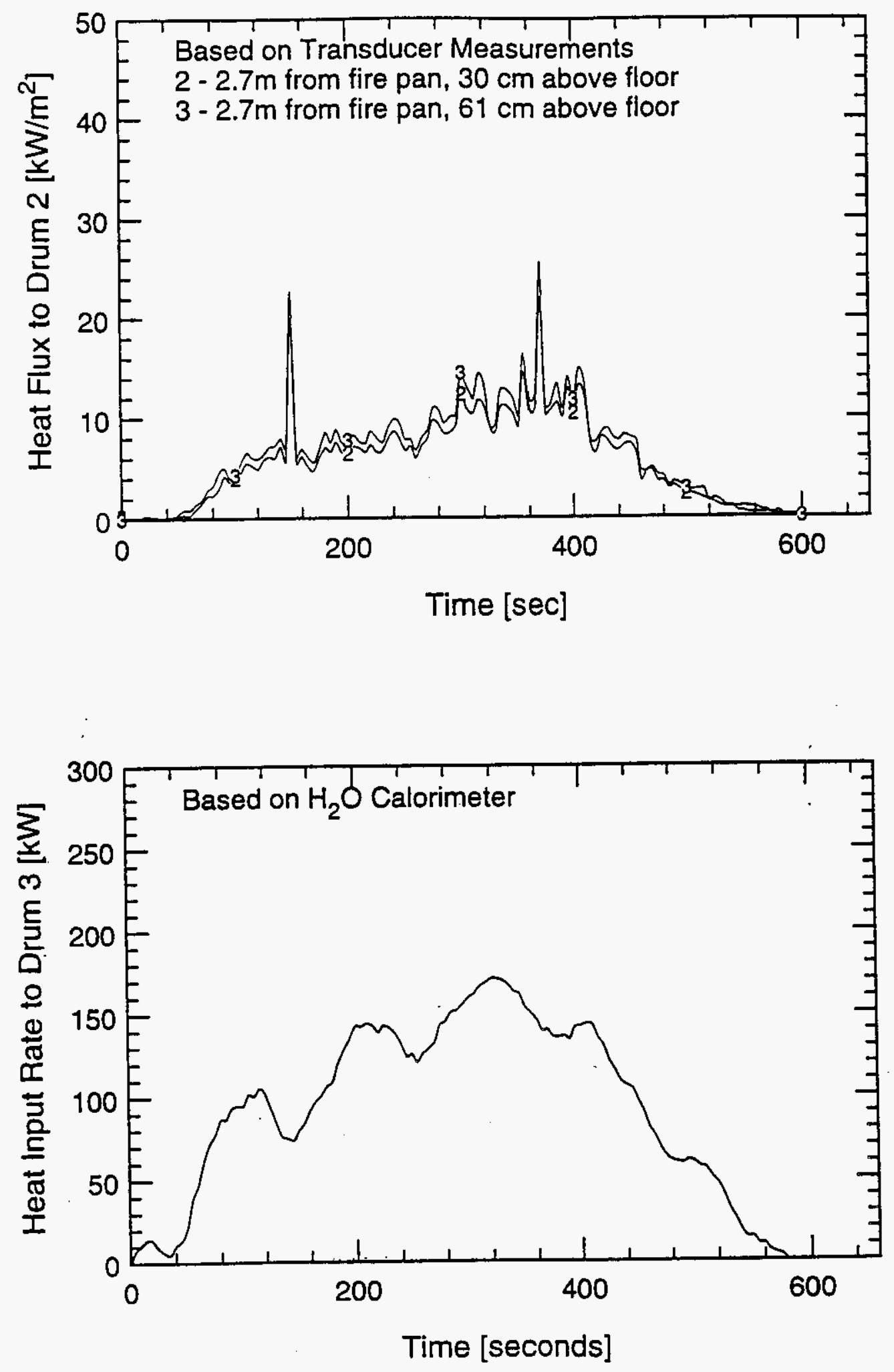

B-232 


\section{SWD32 DRUM 1 - Pool Fire - 5lb Paper}
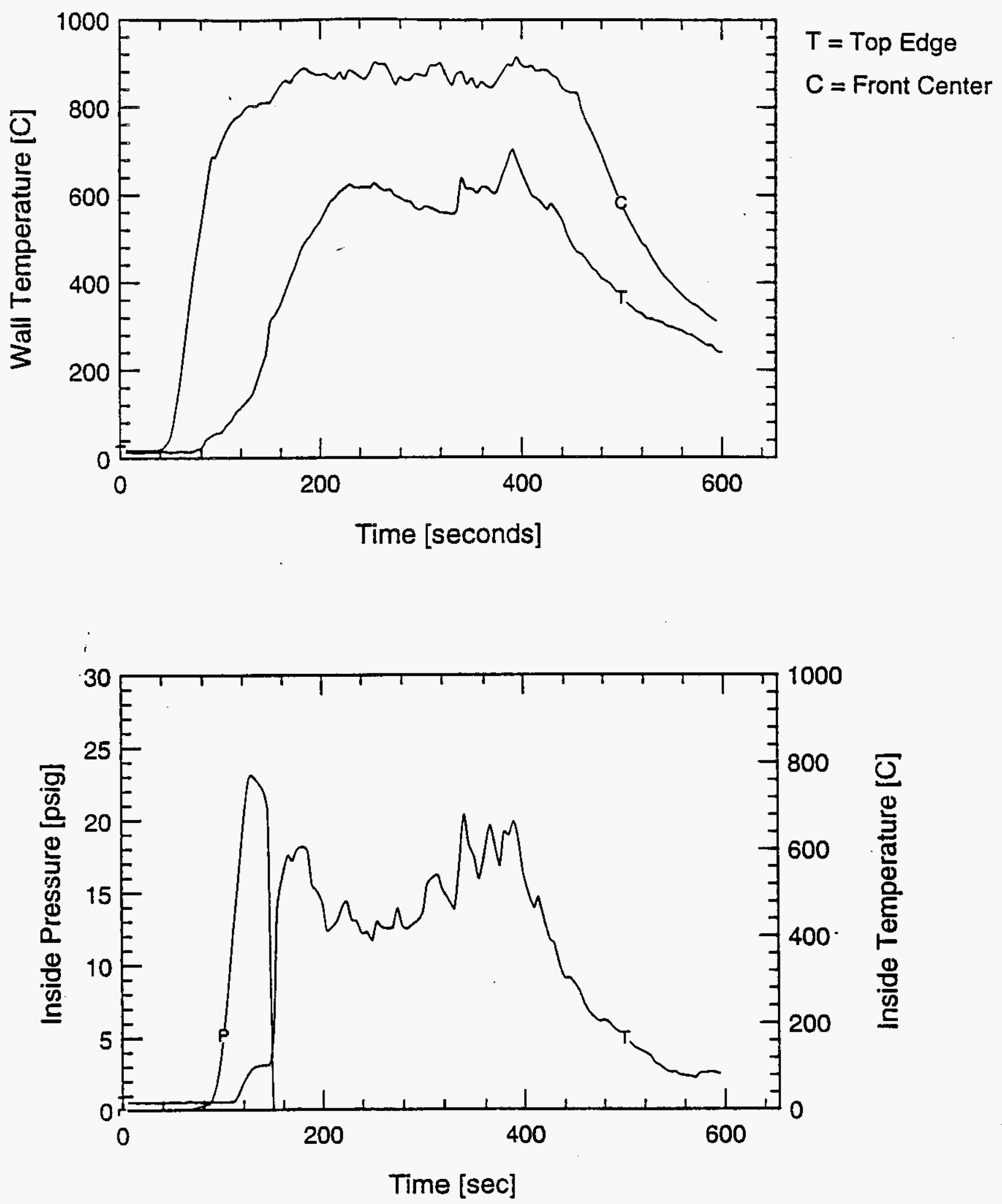
WHC-SD-WM-TRP-233 REV. 0

SWD32 DRUM 4 - Pool Fire - 5lb Plastic
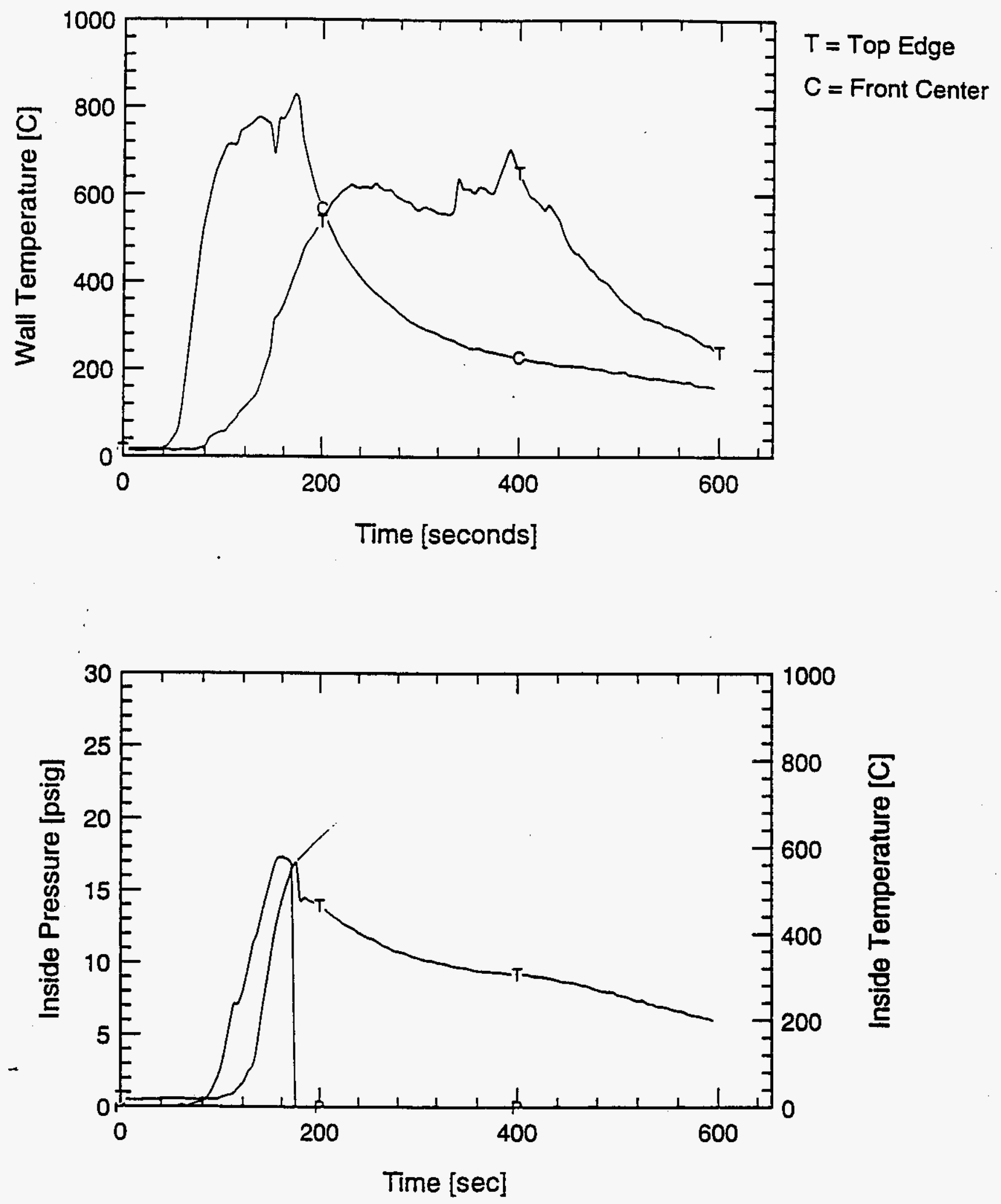
WHC-SD-WM-TRP-233 REV. 0

SWD $33-$ DATA 

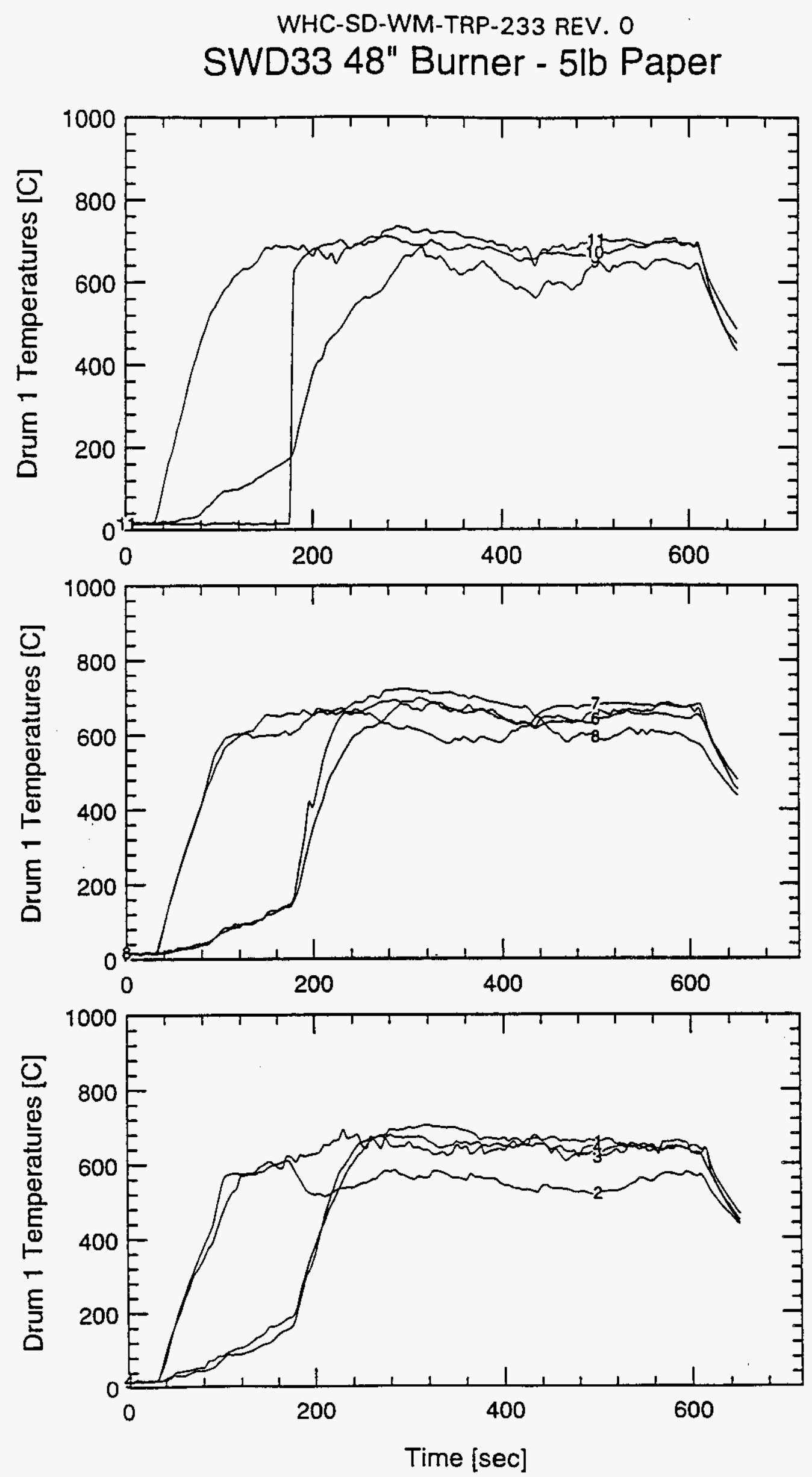

B-236 
WHC-SD-WM-TRP-233 REV. 0

SWD33 - Drum 2
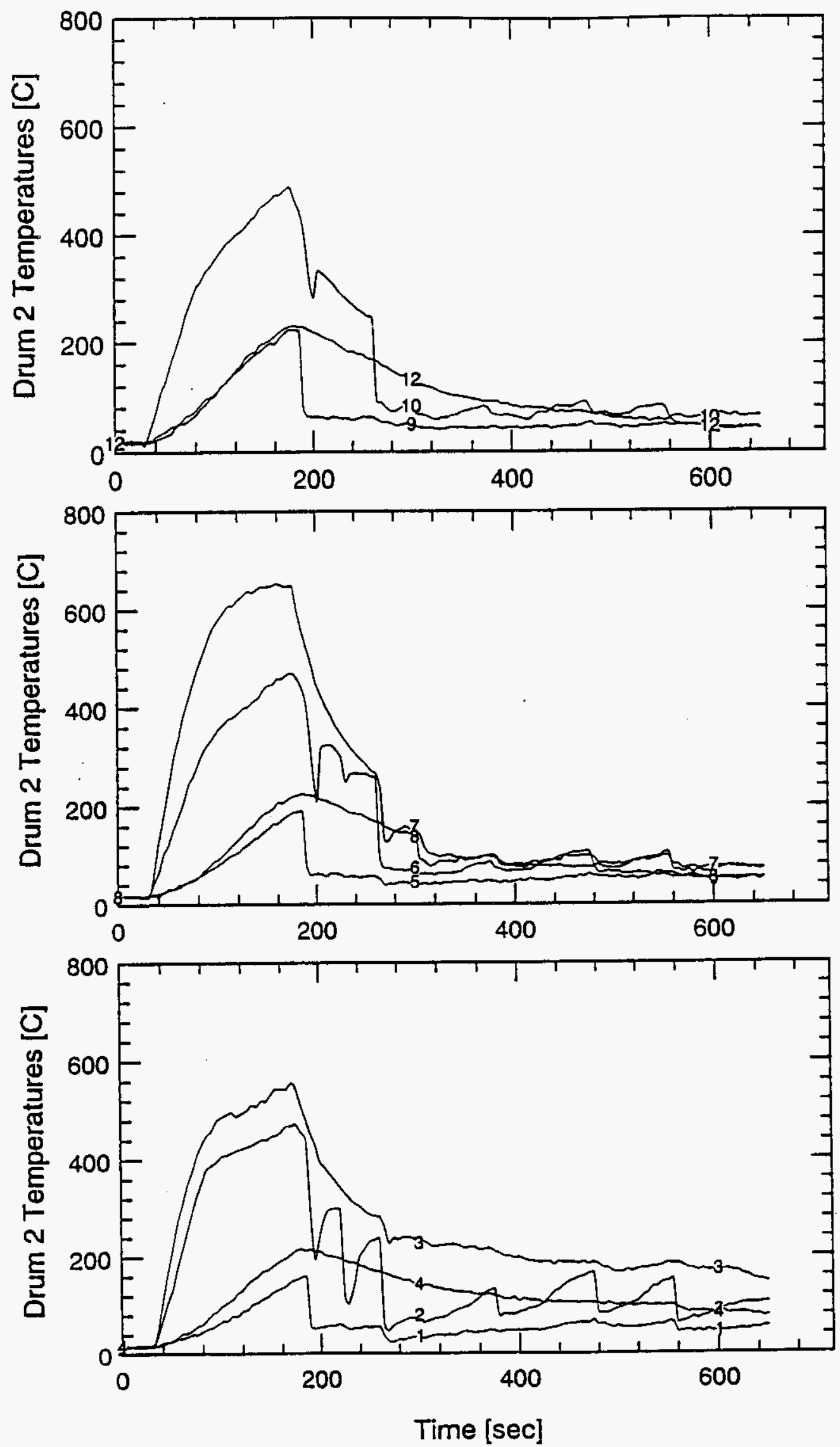

B-237 
WHC-SD-WM-TRP-233 REV. O

SWD33 - Drum 3 Water filled drum
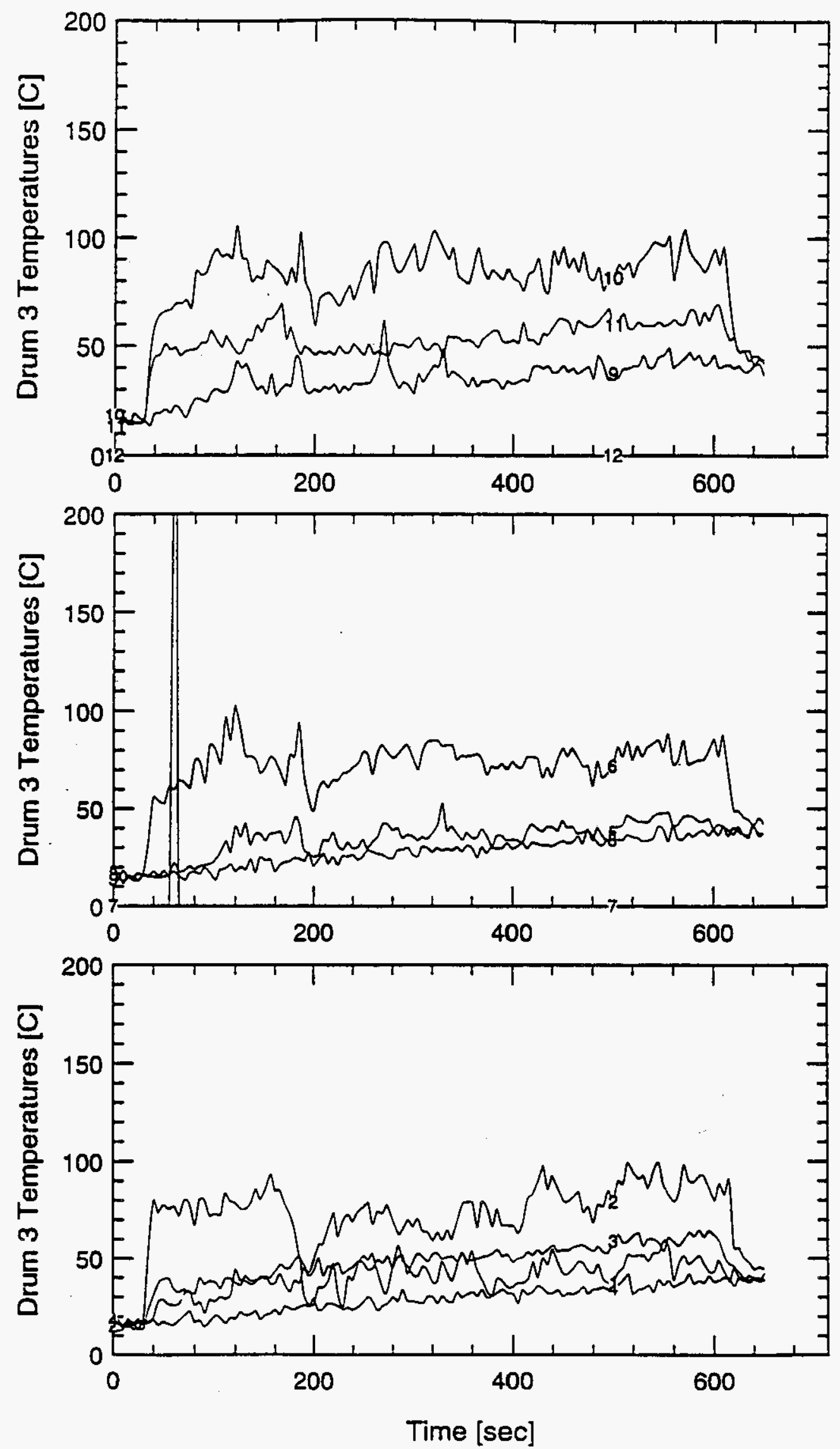
WHC-SD-WM-TRP-233 REV. 0

SWD33 48" Burner - 5lb Plastic
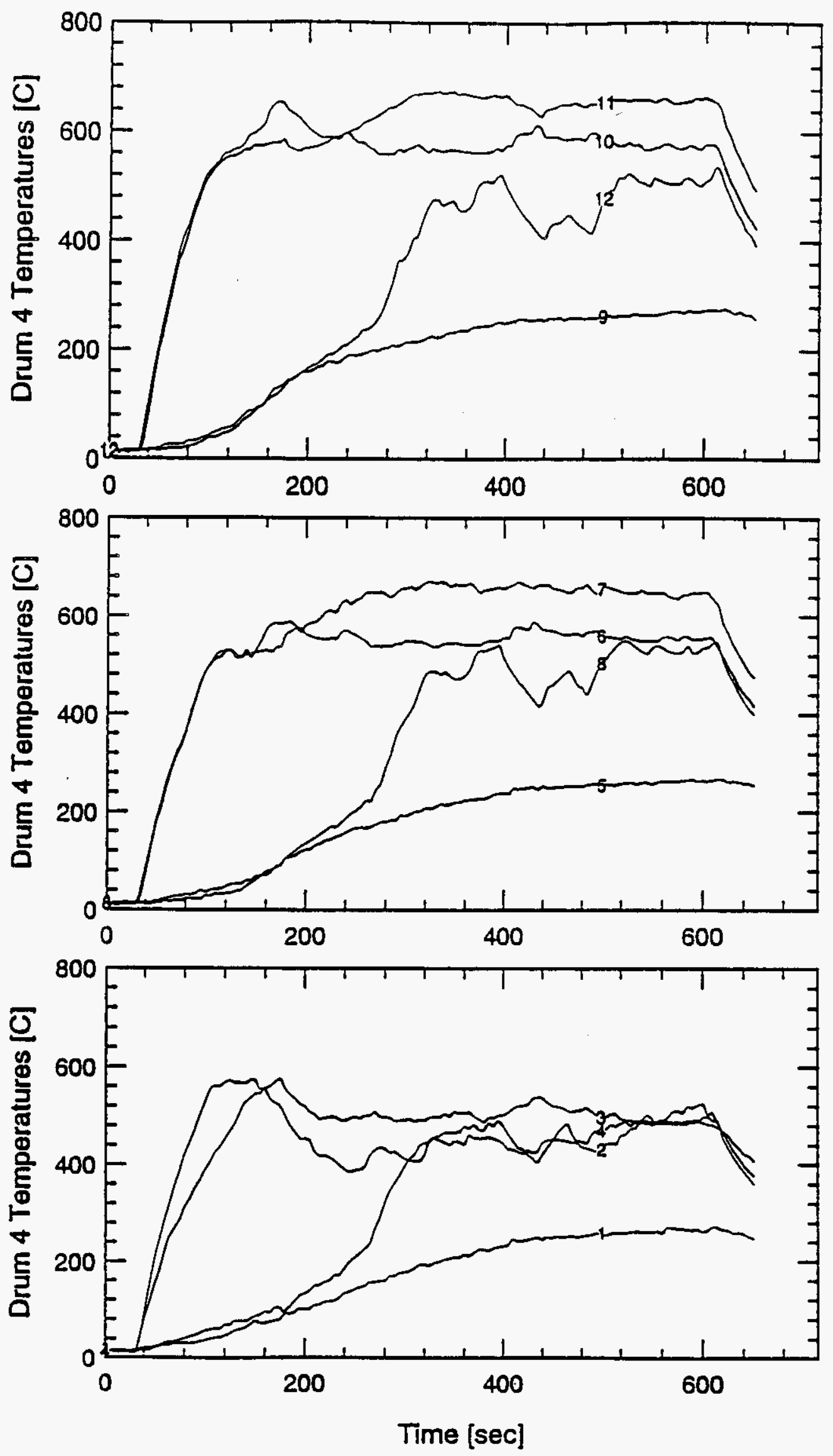
WHC-SD-WM-TRP-233 REV. 0

SWD33 48" Burner
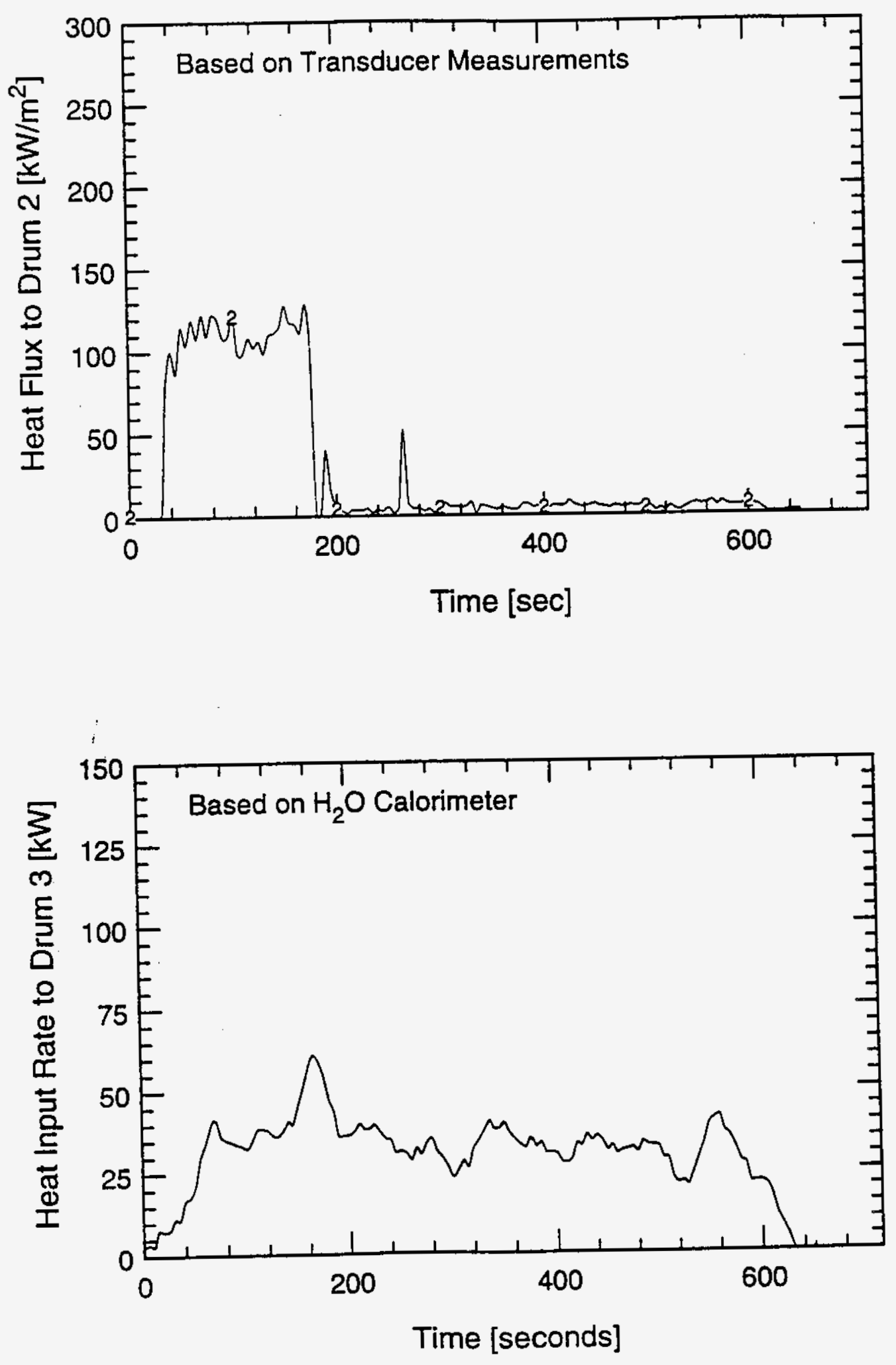

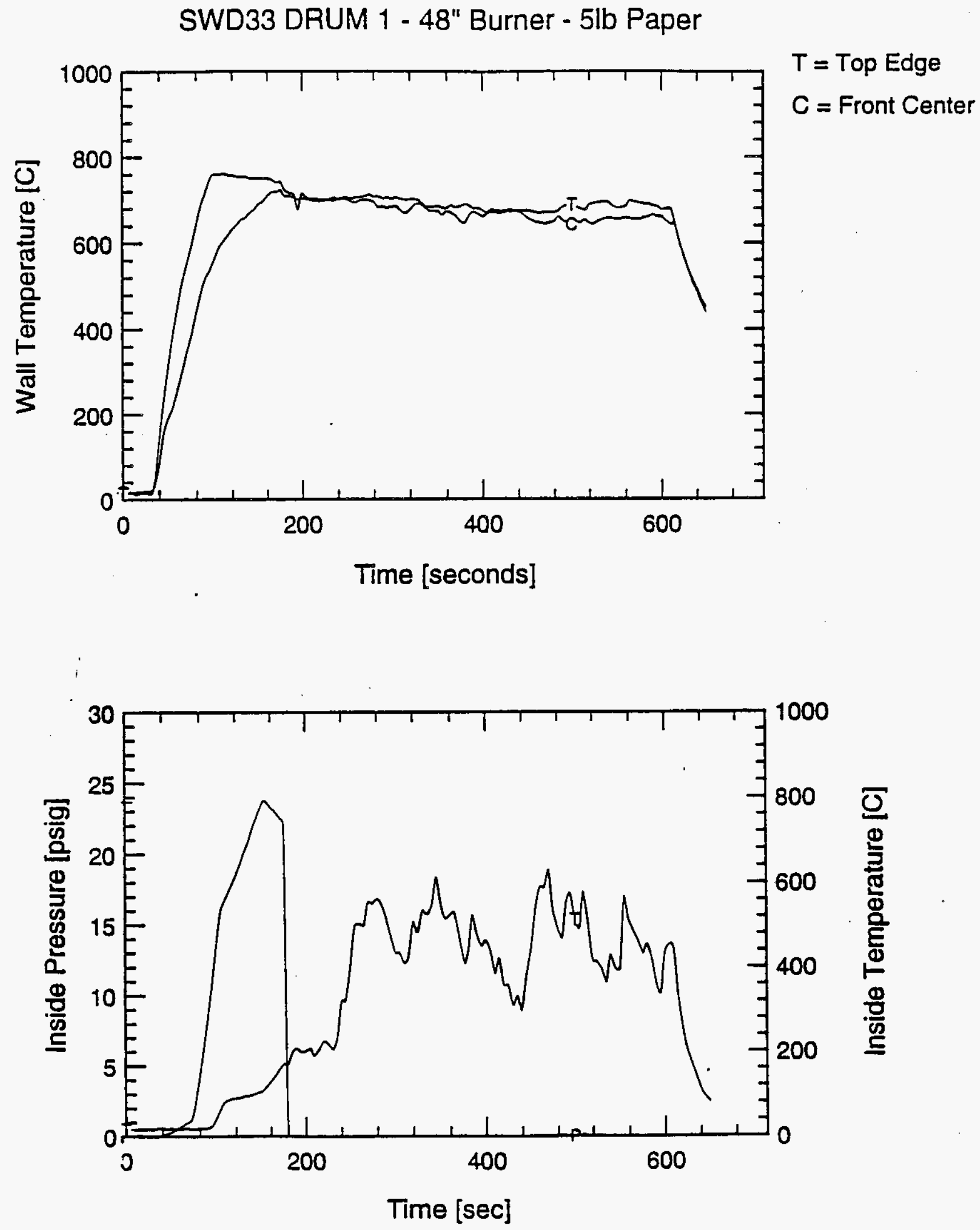
WHC-SD-WM-TRP-233 REV. 0

SWD33 DRUM 4 - 48" Burner - 5lb Plastic
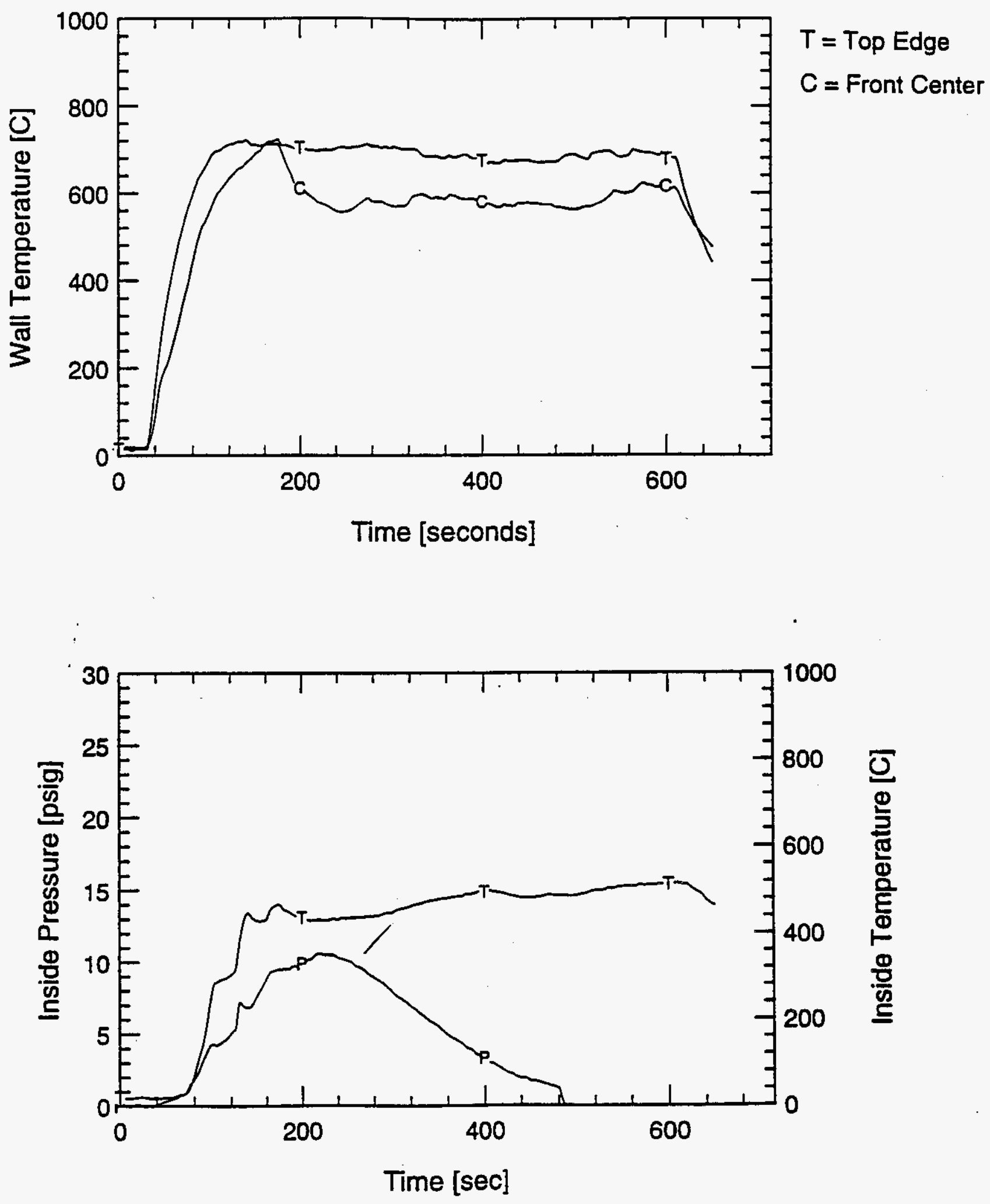
WHC-SD-WM-TRP-233 REV. O

SWD 34 - DATA 
WHC-SD-WM-TRP-233 REV. 0

SWD34 48" Burner - 5lb Paper - Heptane
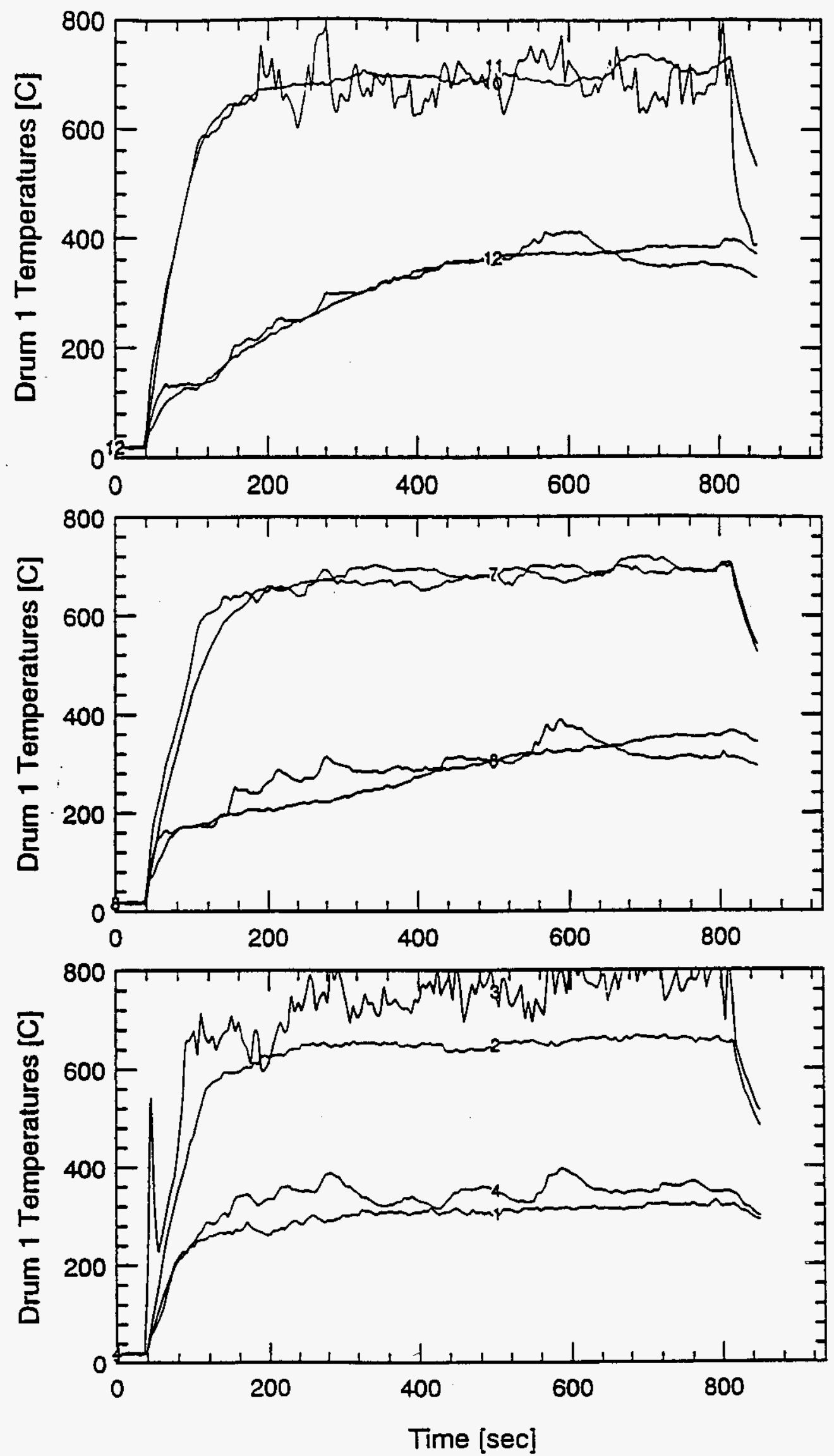
WHC-SD-WM-TRP-233 REV. 0

SWD34 - Drum 2
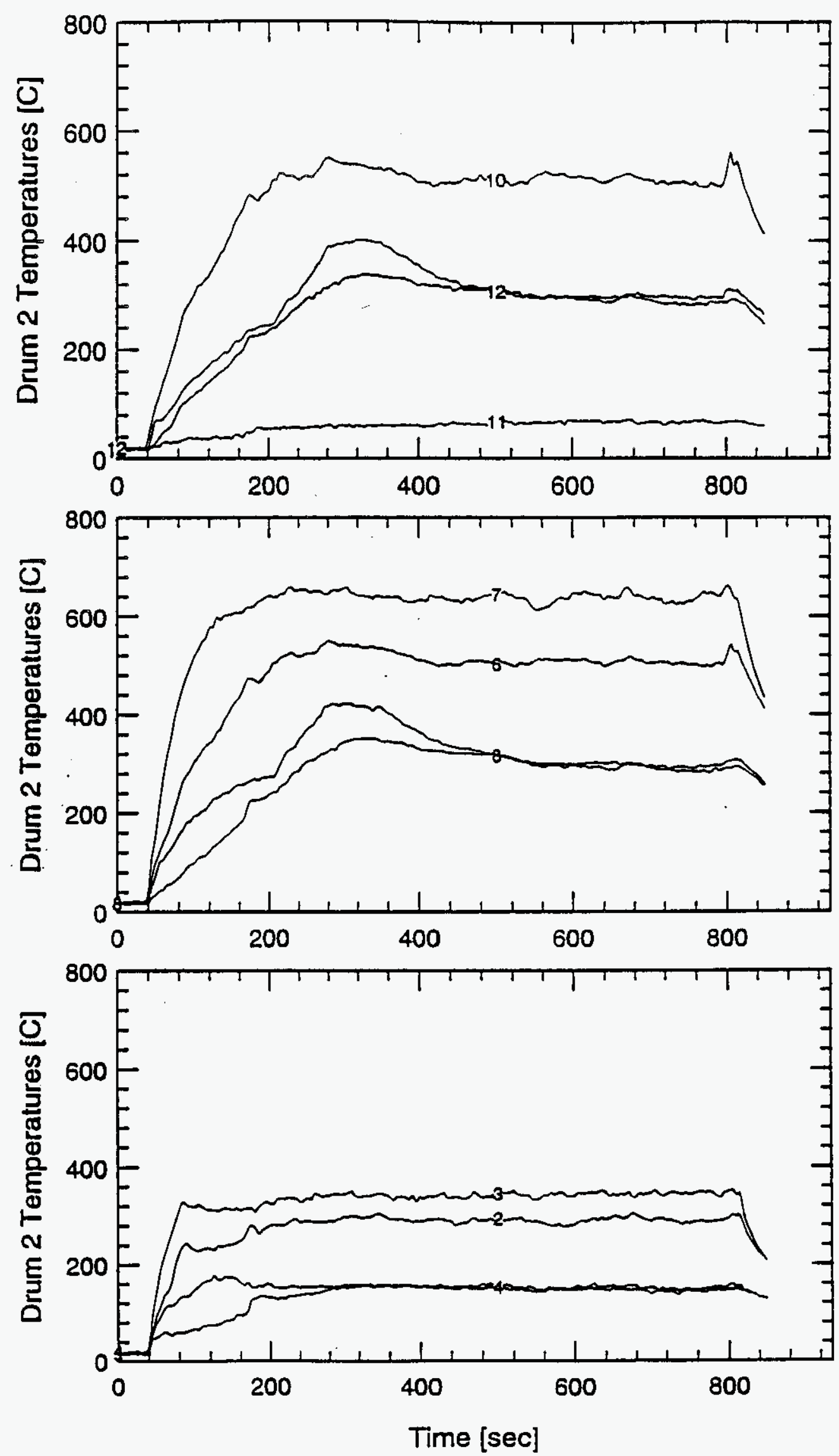
WHC-SD-WM-TRP-233 REV. 0

SWD34 - Drum 3 Water filled drum
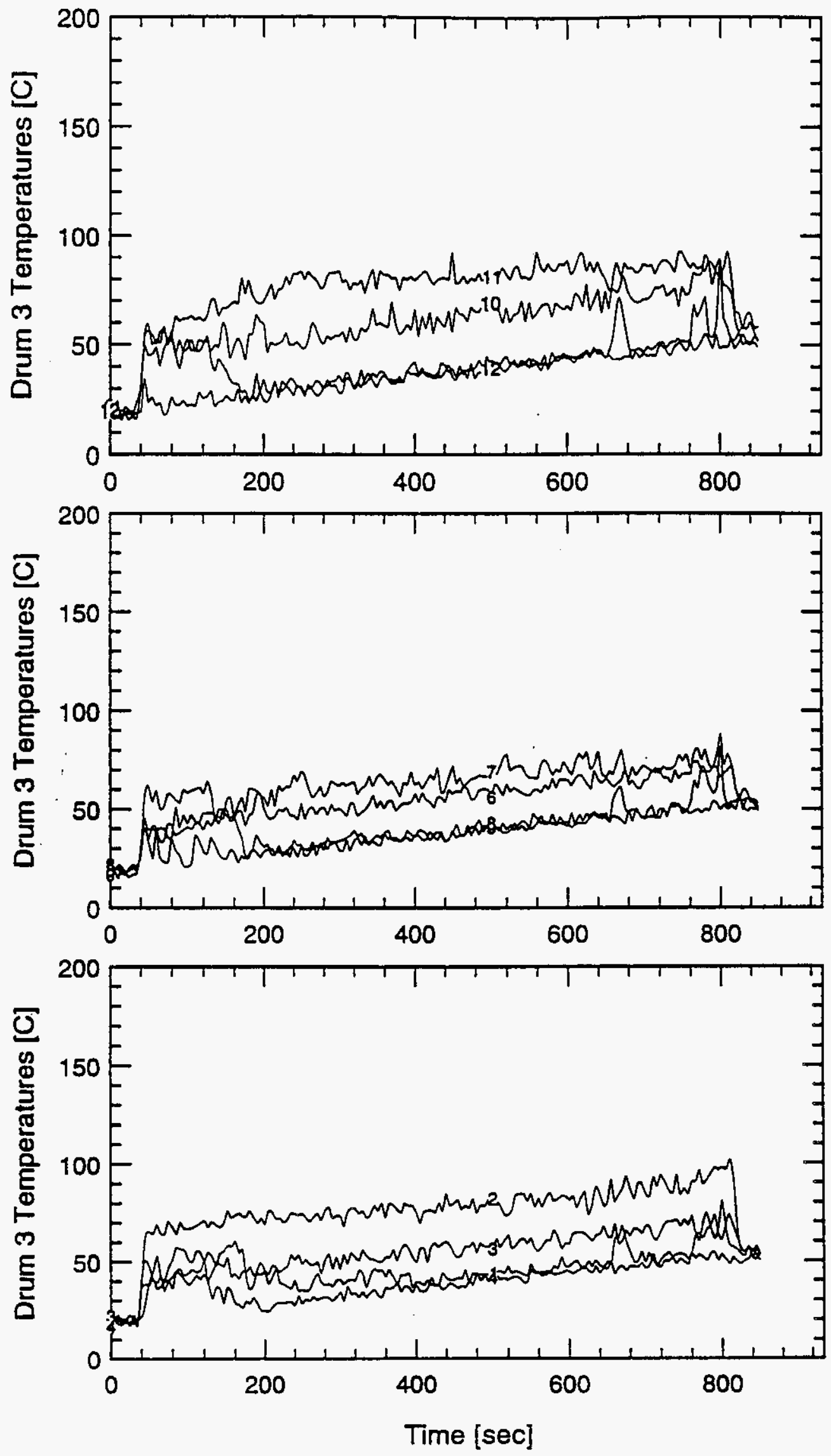
WHC-SD-WM-TRP-233 REV. 0

SWD34 48" Burner - Empty
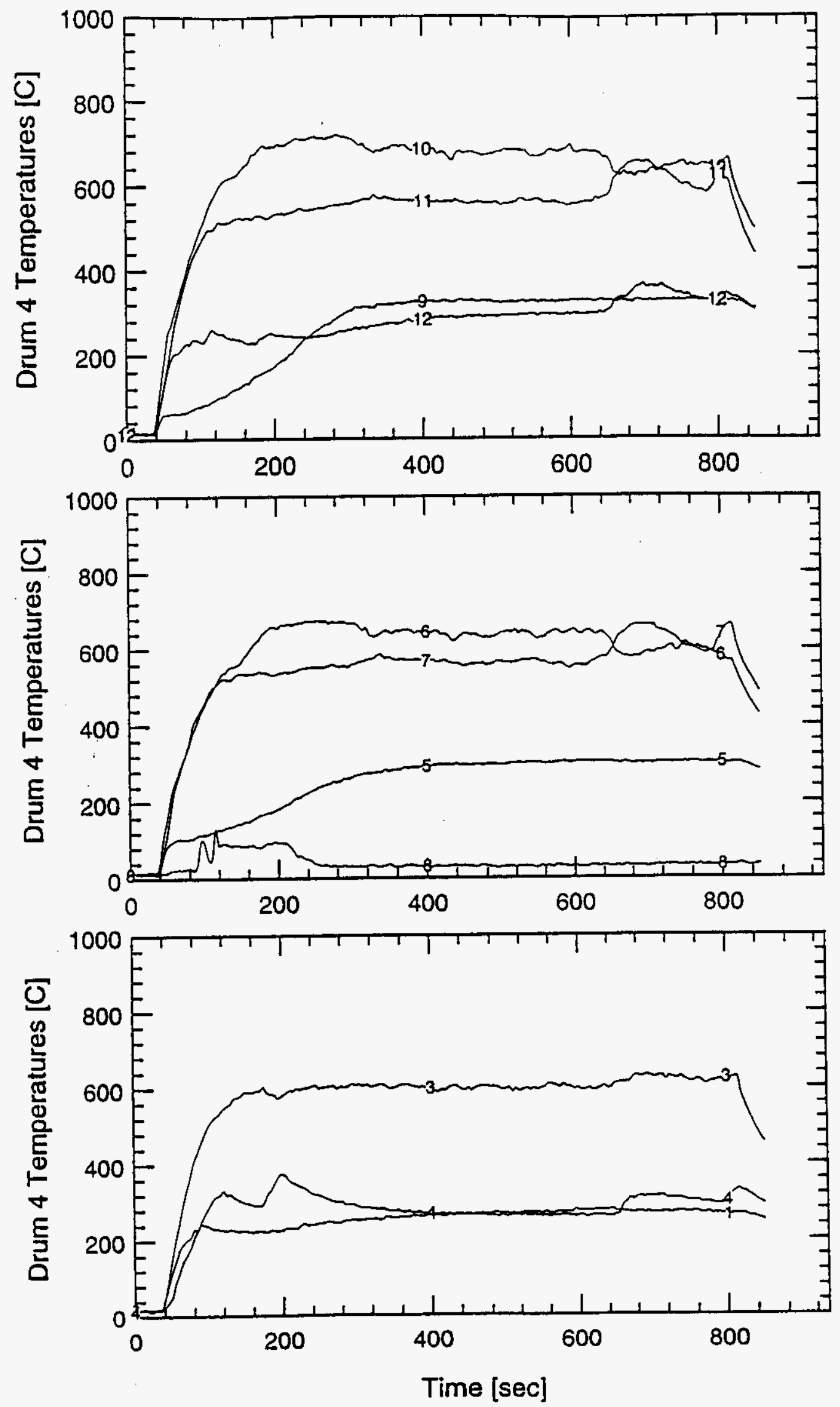

B-247 
WHC-SD-WM-TRP-233 REV. 0

SWD34 48" Burner
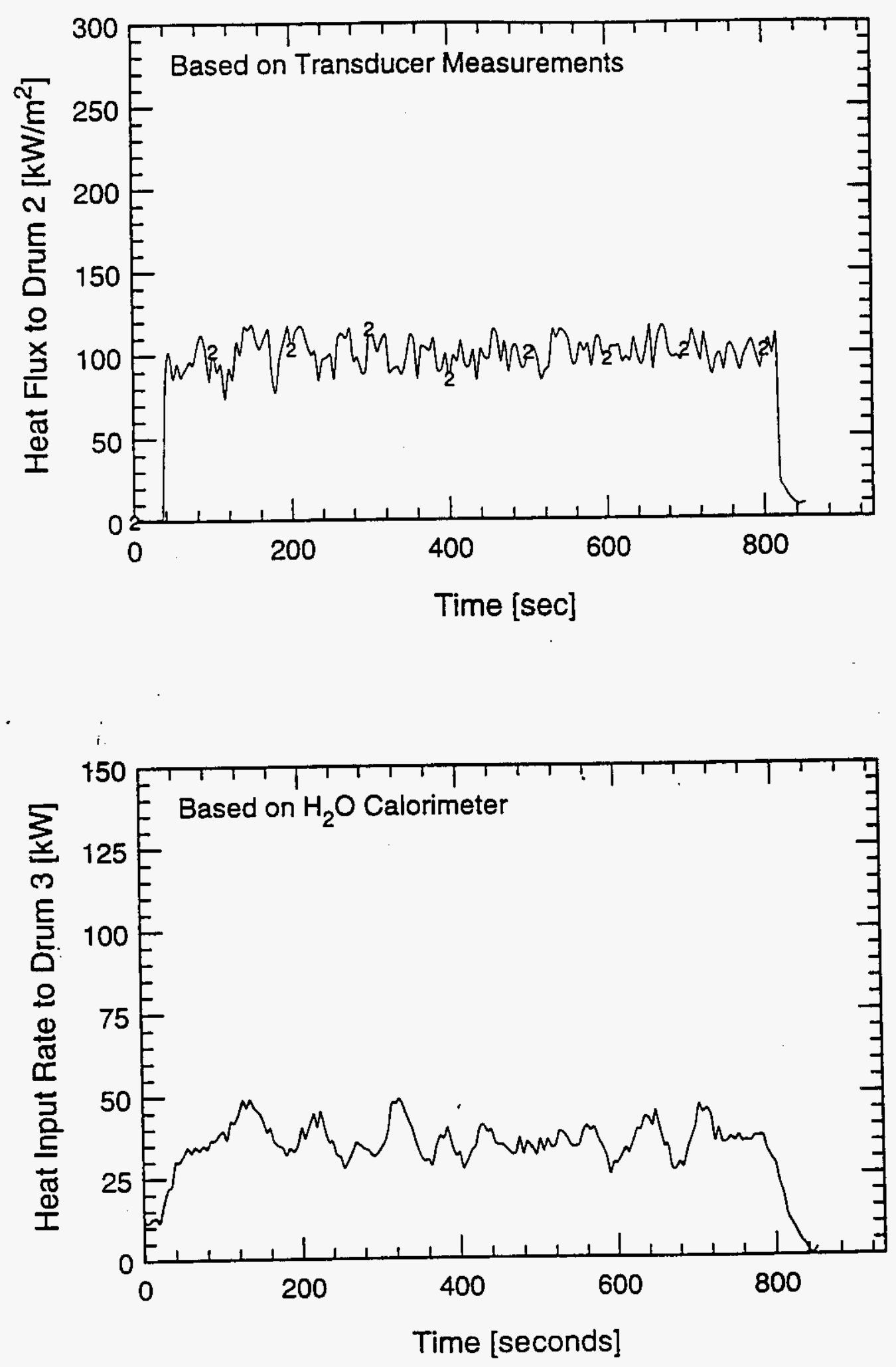
SWD34 DRUM 1 - 48" Burner - 5lb Paper - Heptane
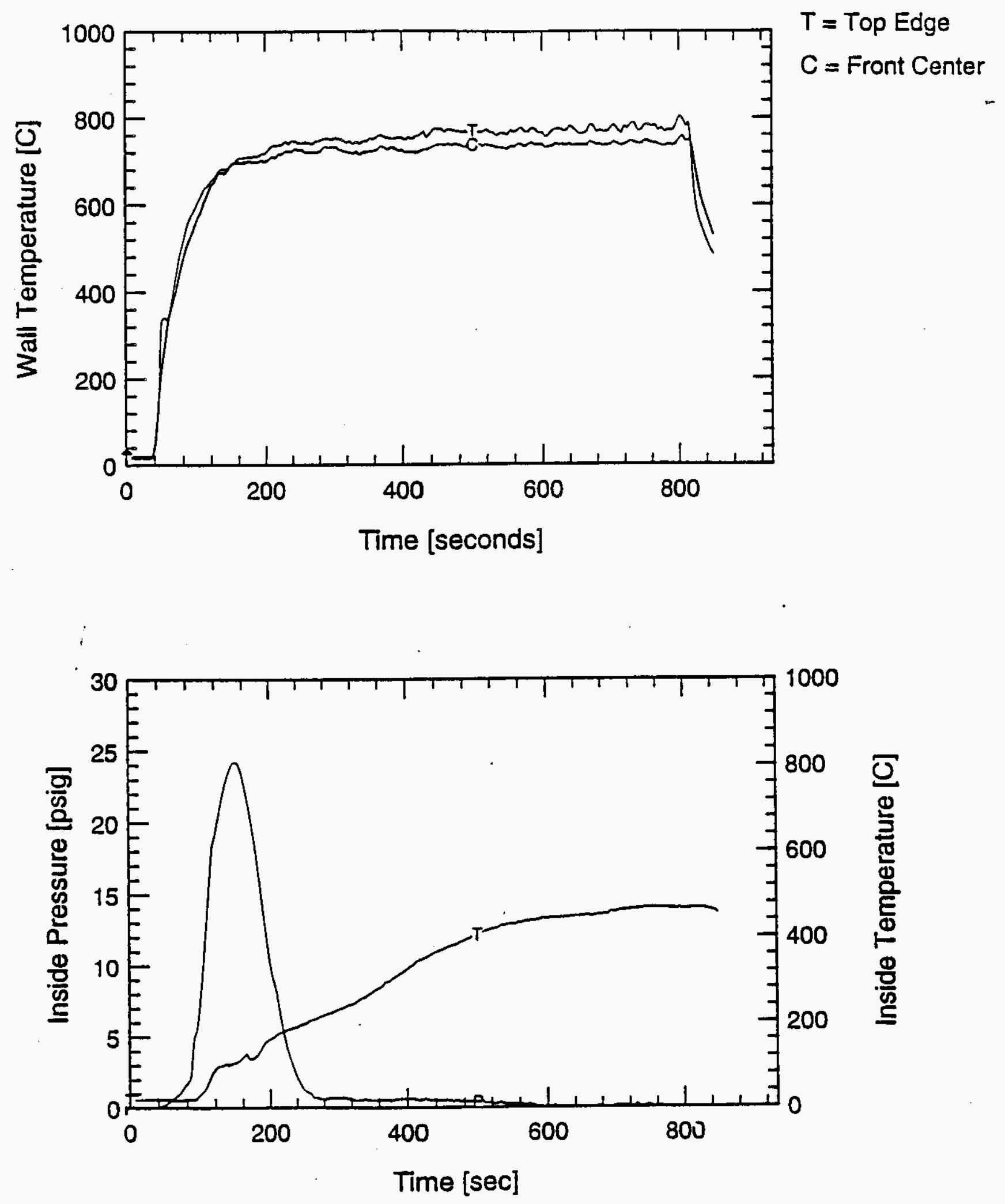
WHC-SD-WM-TRP-233 REV. 0

SWD34 DRUM 4 - 48" Burner - Empty
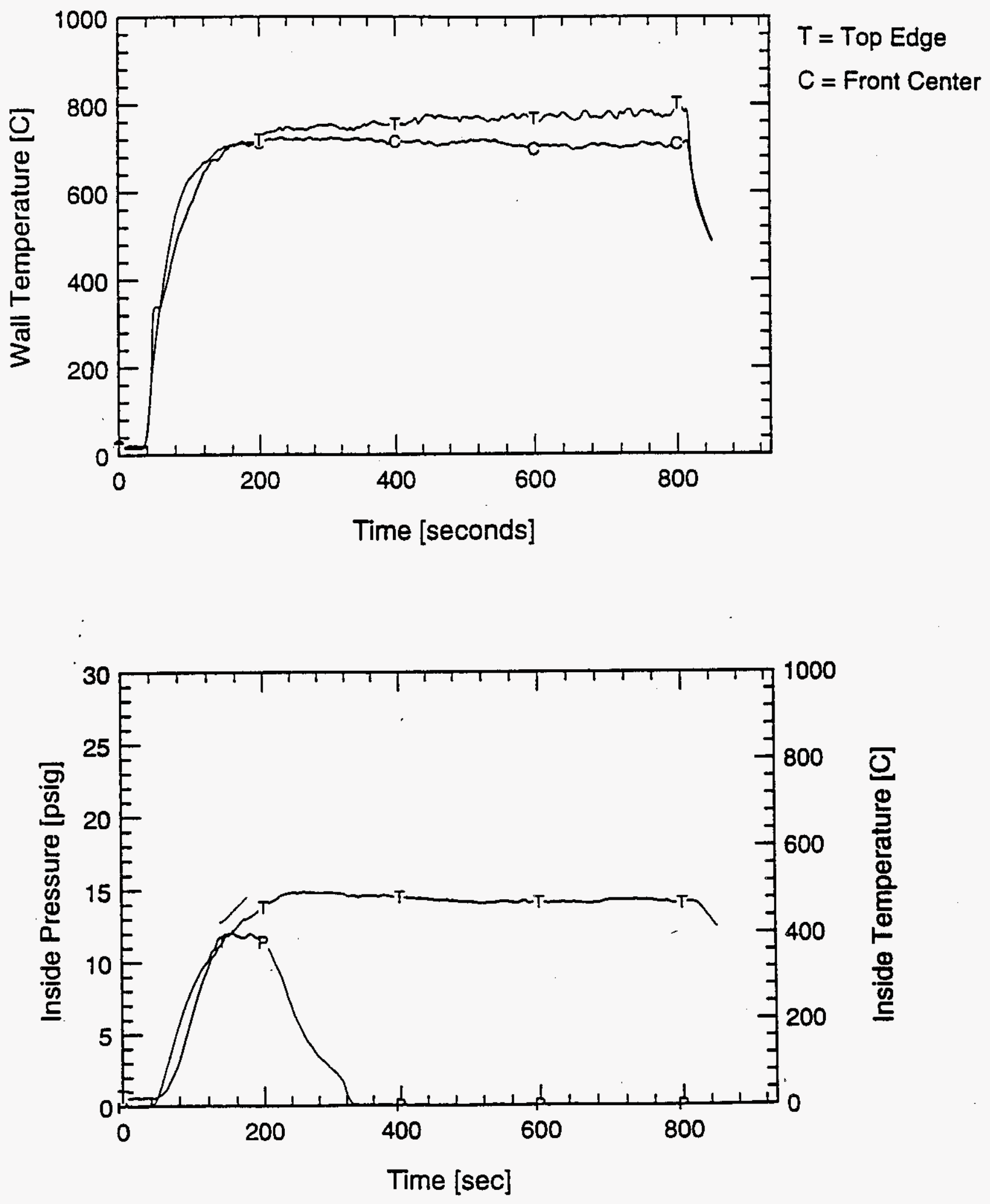
WHC-SD-WM-TRP-233 REV. 0

SWD 35 - DATA 
WHC-SD-WM-TRP-233 REV. 0

SWD35 48" Burner - 5lb Paper - Heptane
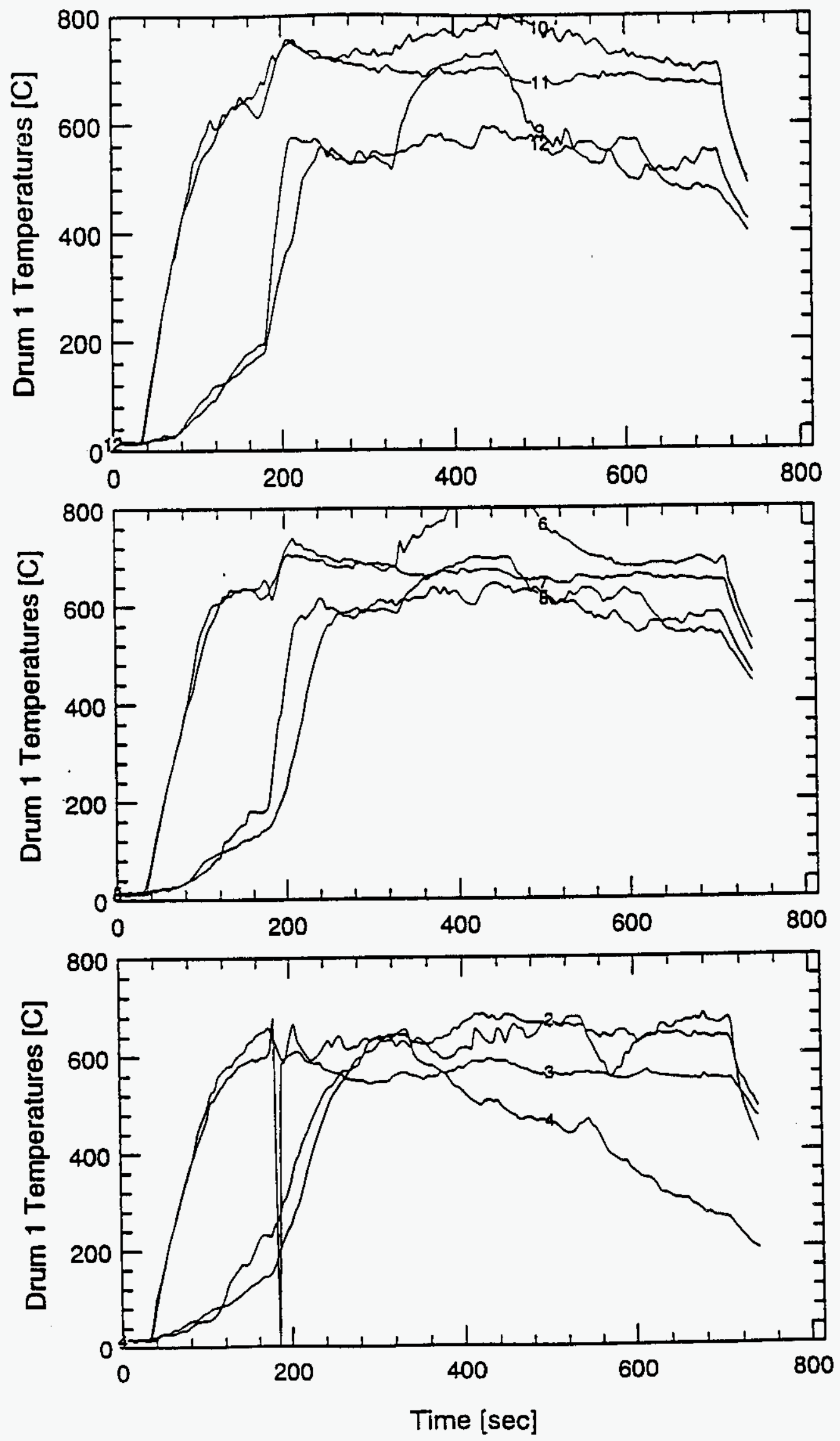

B-252 
WHC-SD-WM-TRP-233 REV. 0

SWD35 - Drum 2
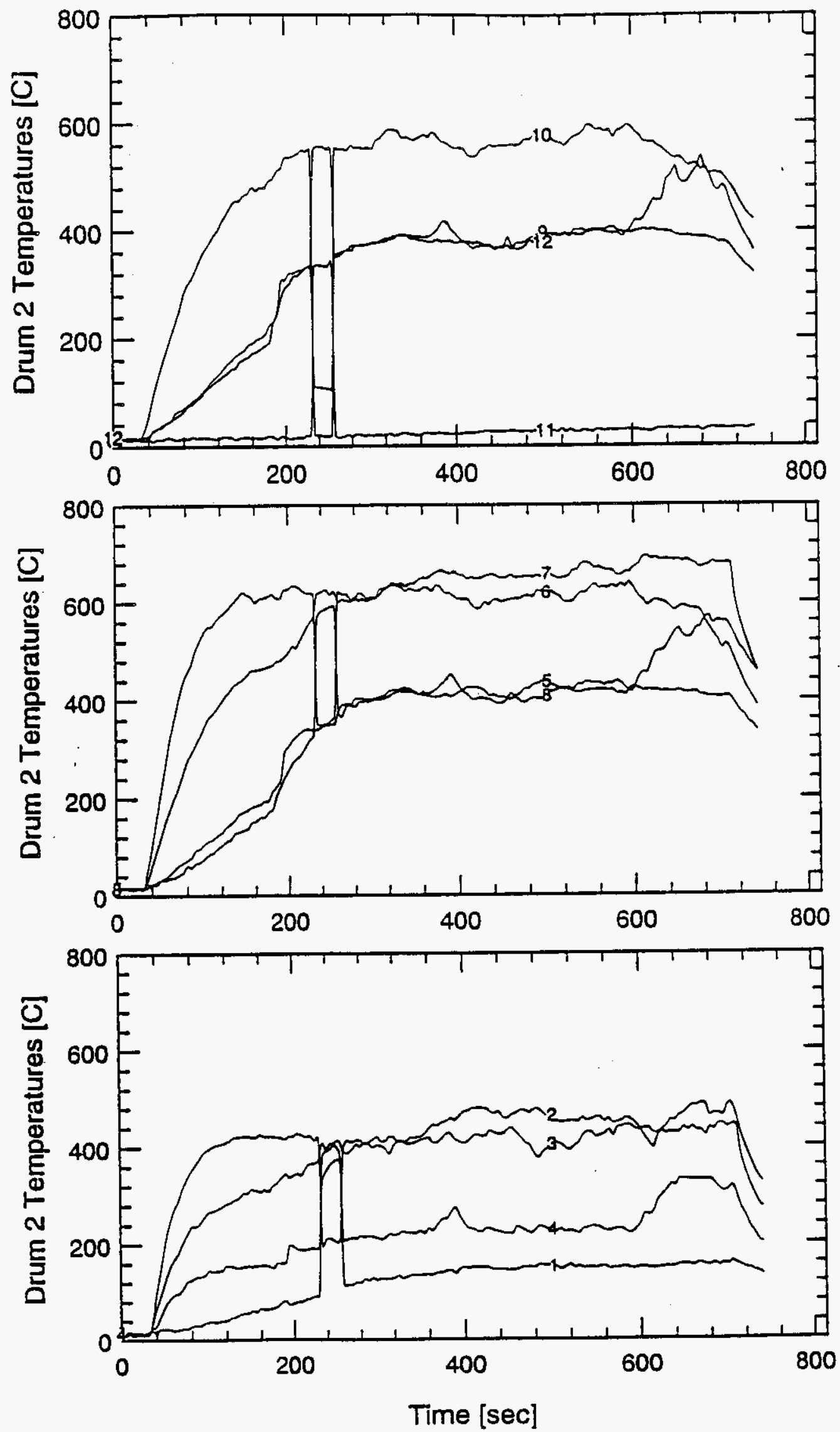

B-253 


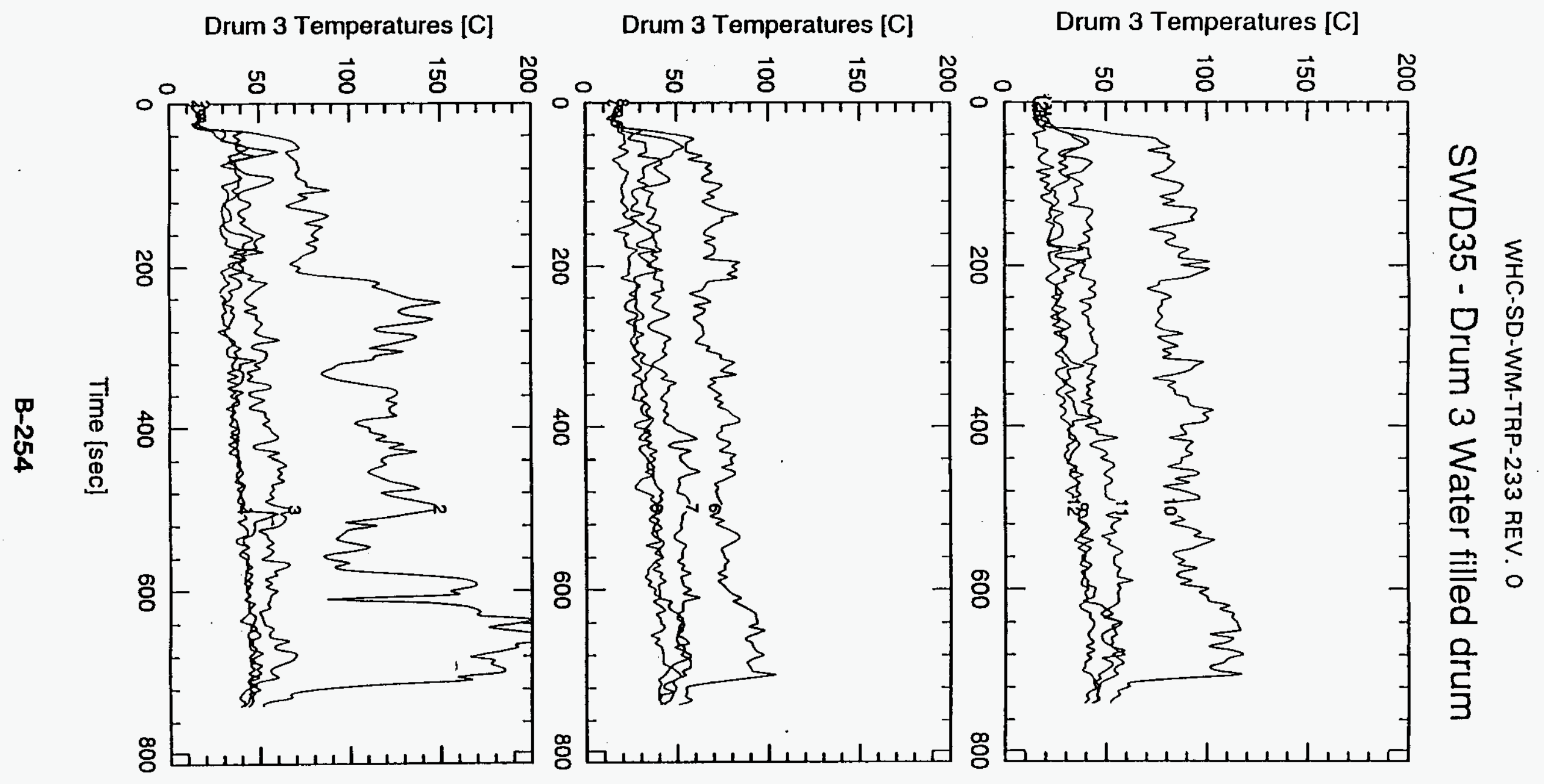

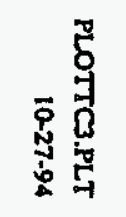



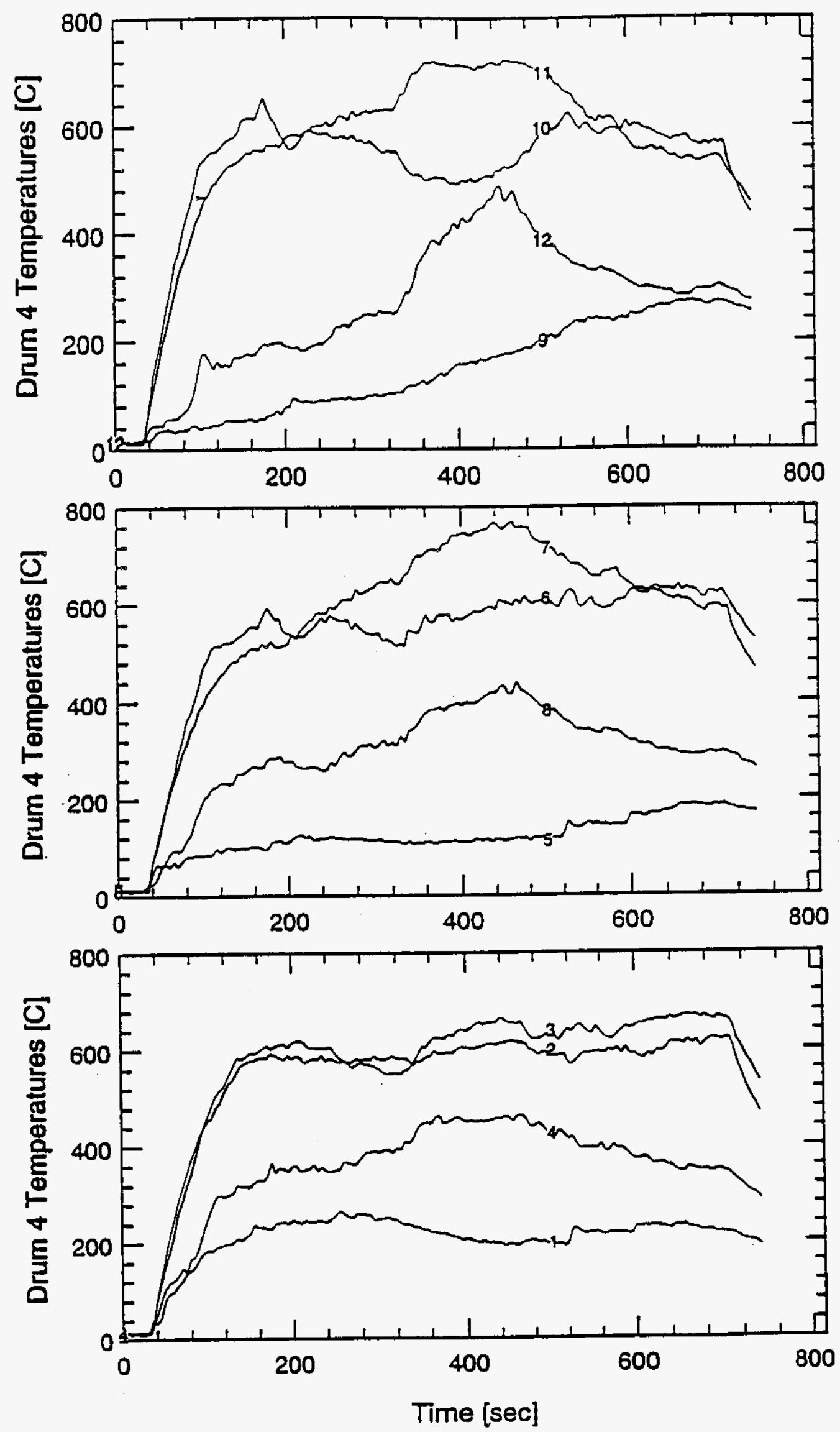
WHC-SD-WM-TRP-233 REV. 0

SWD35 48" Burner
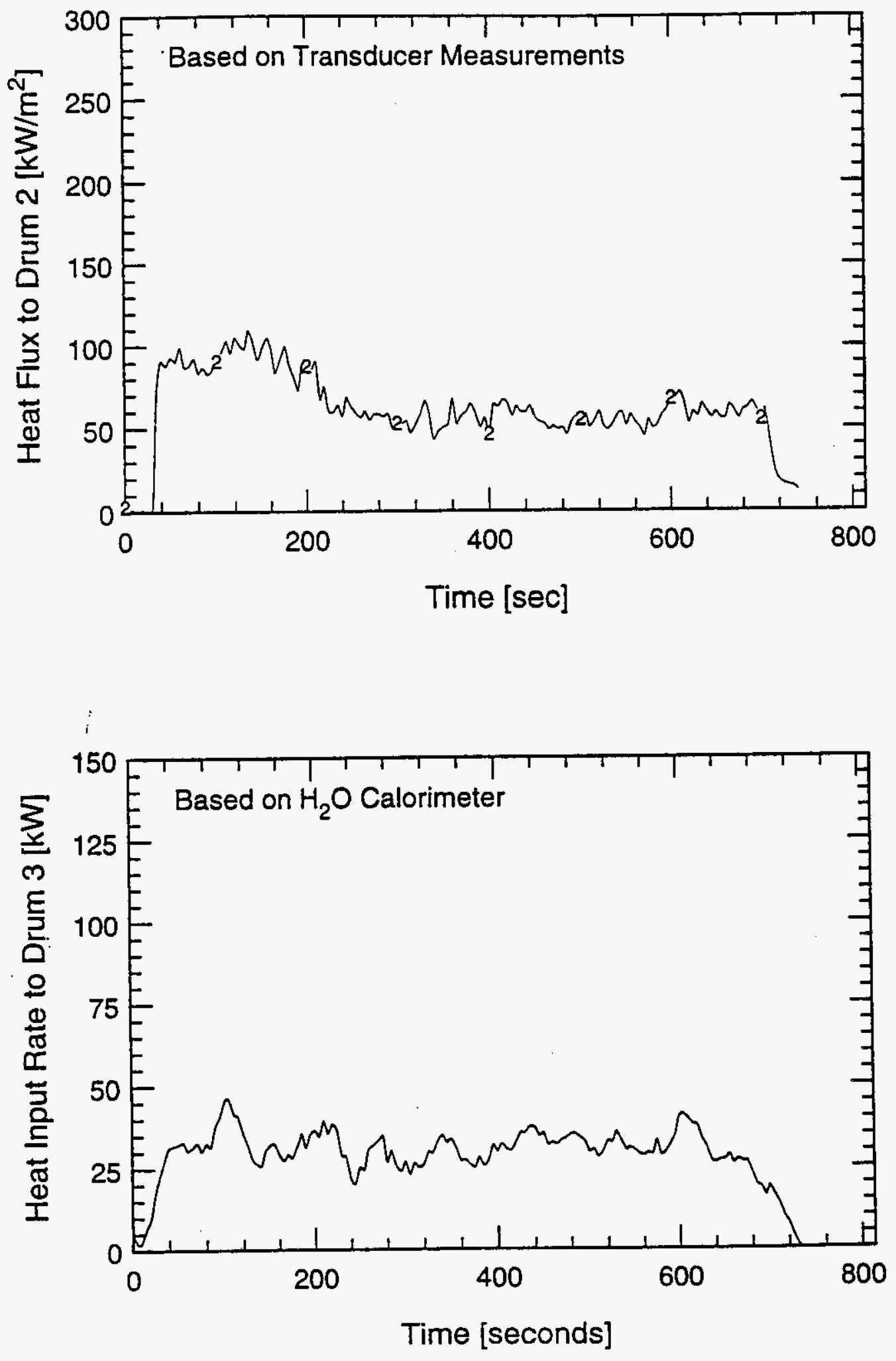
SWD35 DRUM 1 - 48" Burner - 5lb Paper - Heptane
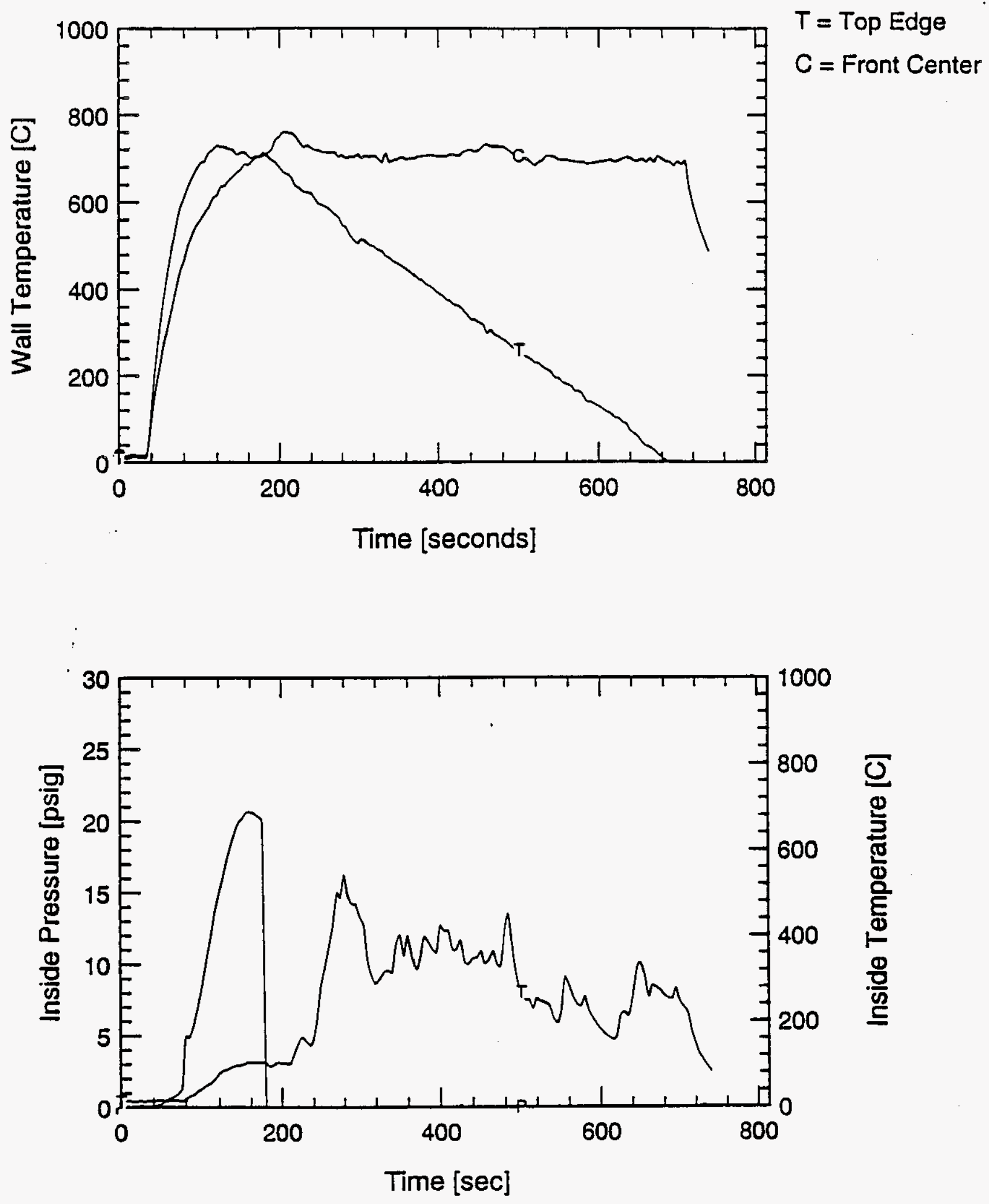
WHC-SD-WM-TRP-233 REV. 0

SWD35 DRUM 4 - 48" Burner - Standard Load
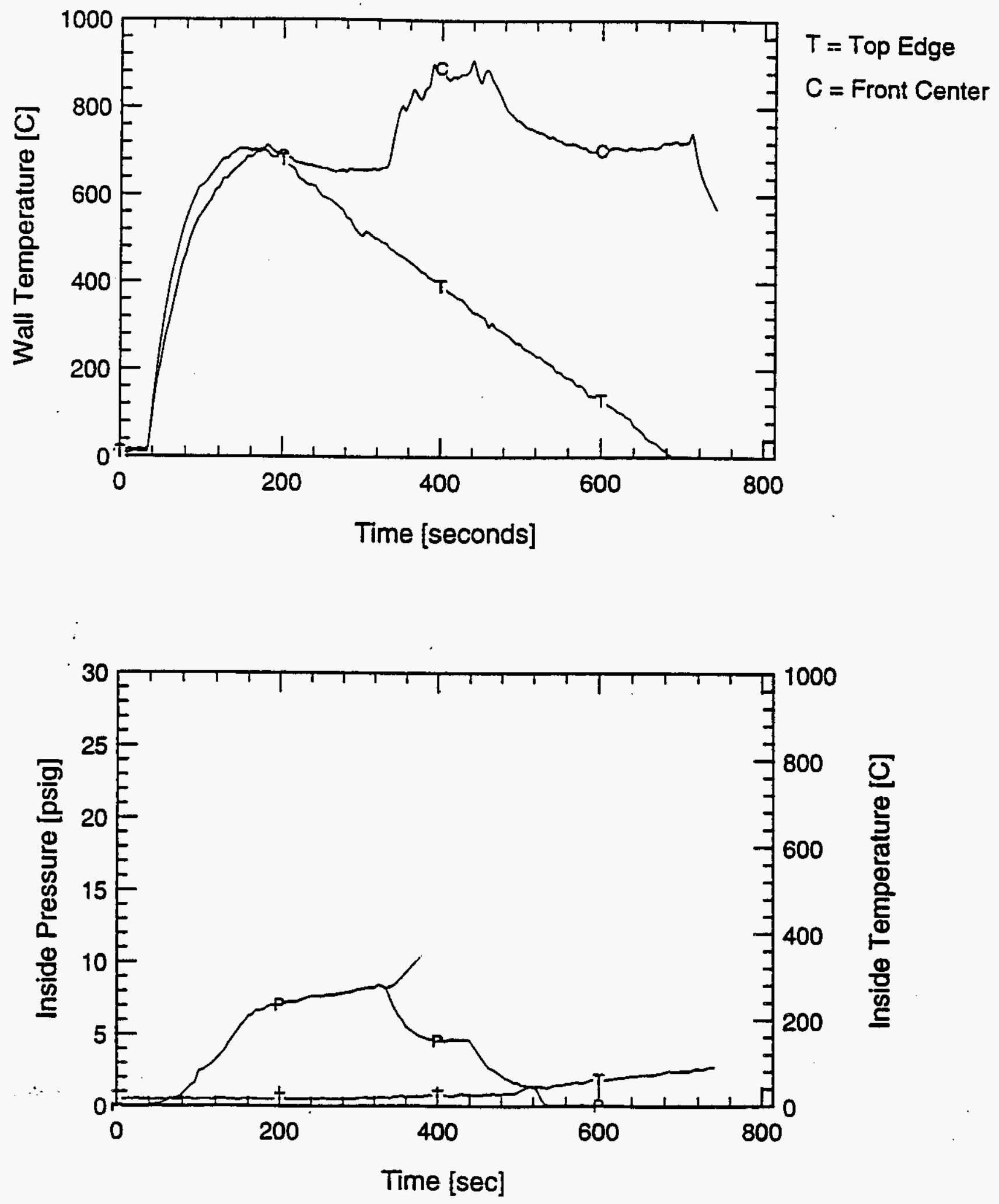
WHC-SD-WM-TRP-233 REV. 0

SWD 36 - DATA 
WHC-SD-WM-TRP-233 REV. 0

SWD36 48" Burner - 5lb Paper - Toluene
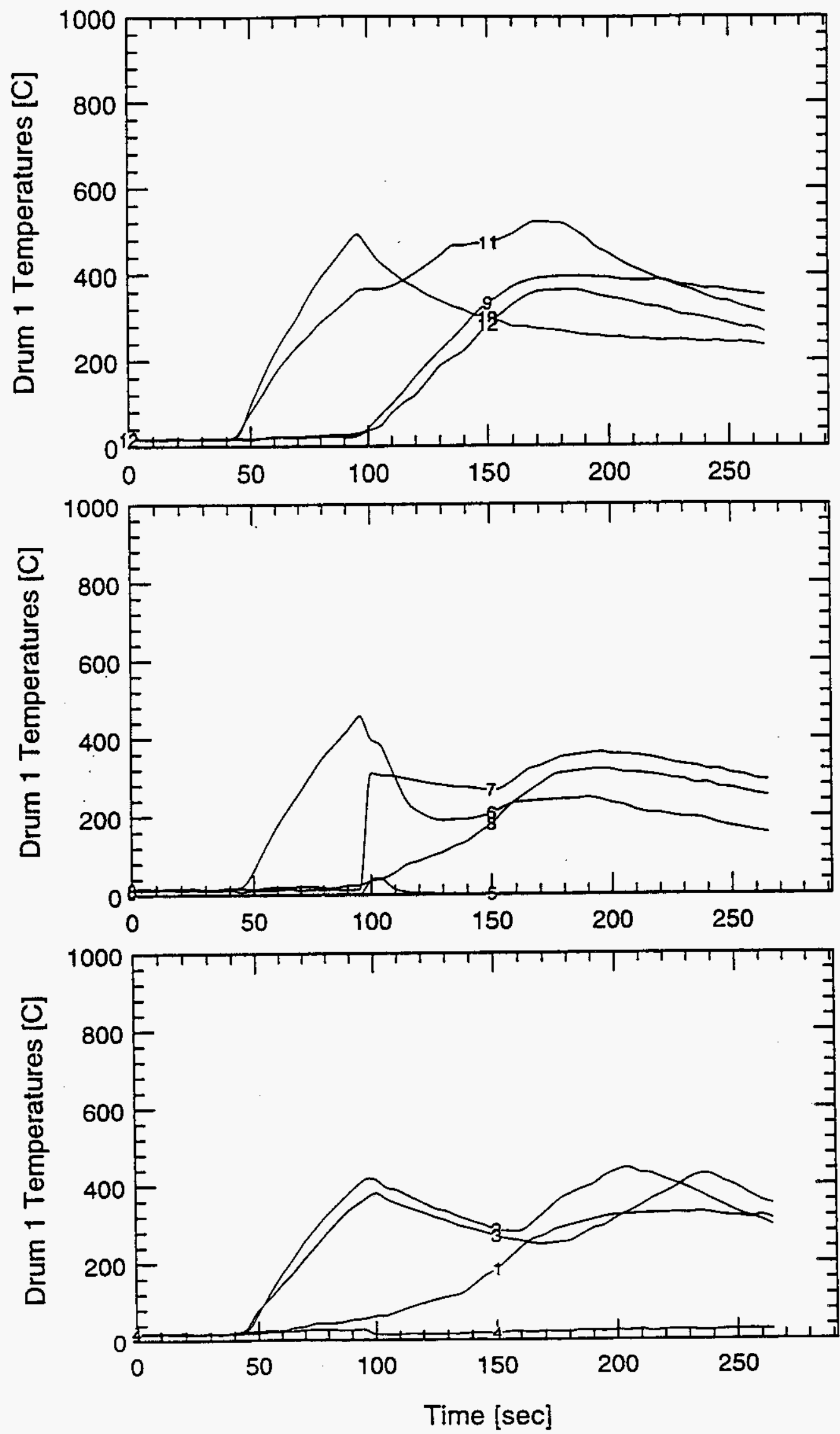

B-260 
WHC-SD-WM-TRP-233 REV. O

SWD36 - Drum 2
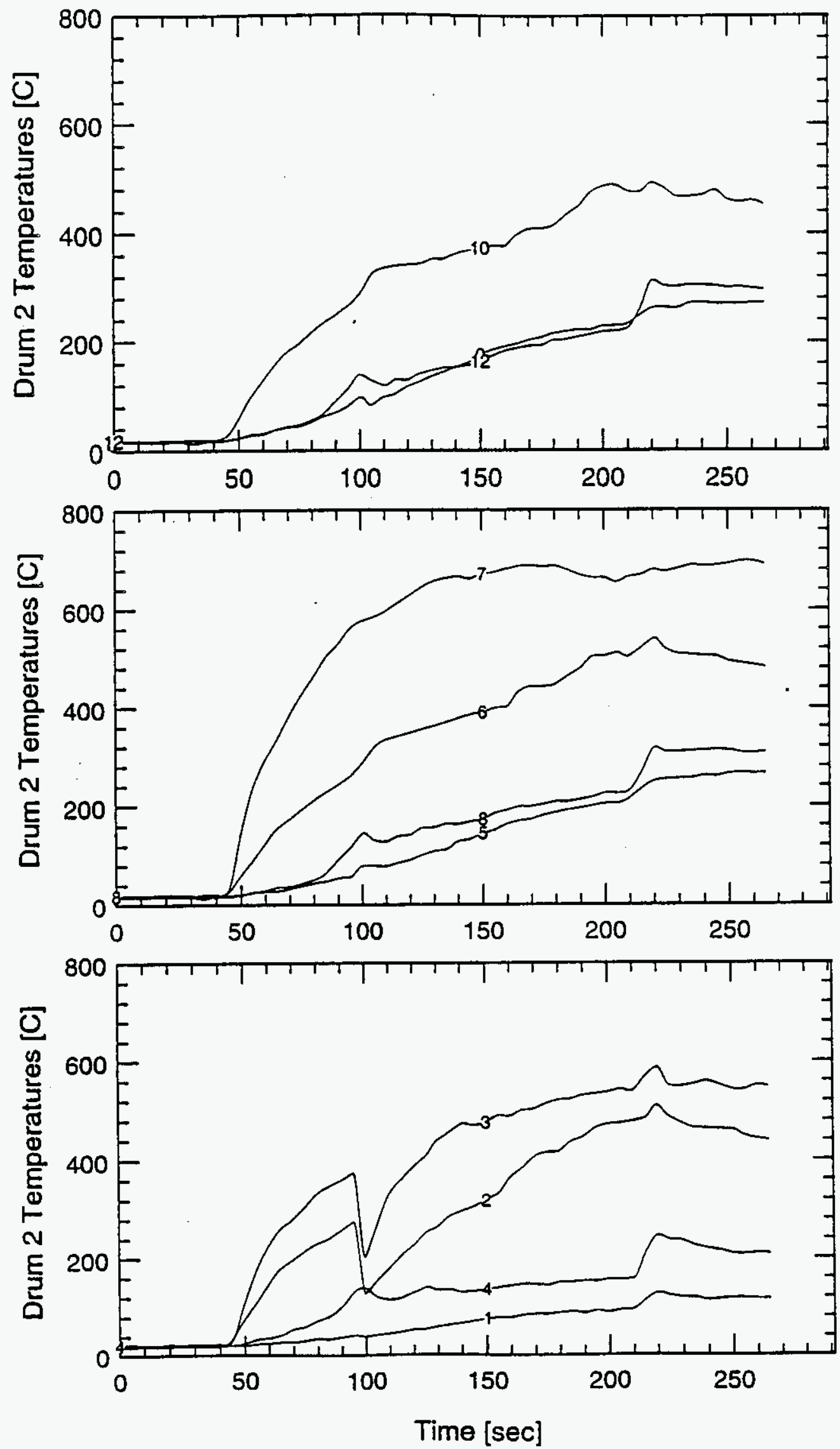
WHC-SD-WM-TRP-233 REV. 0

SWD36 - Drum 3 Water Filled Drum
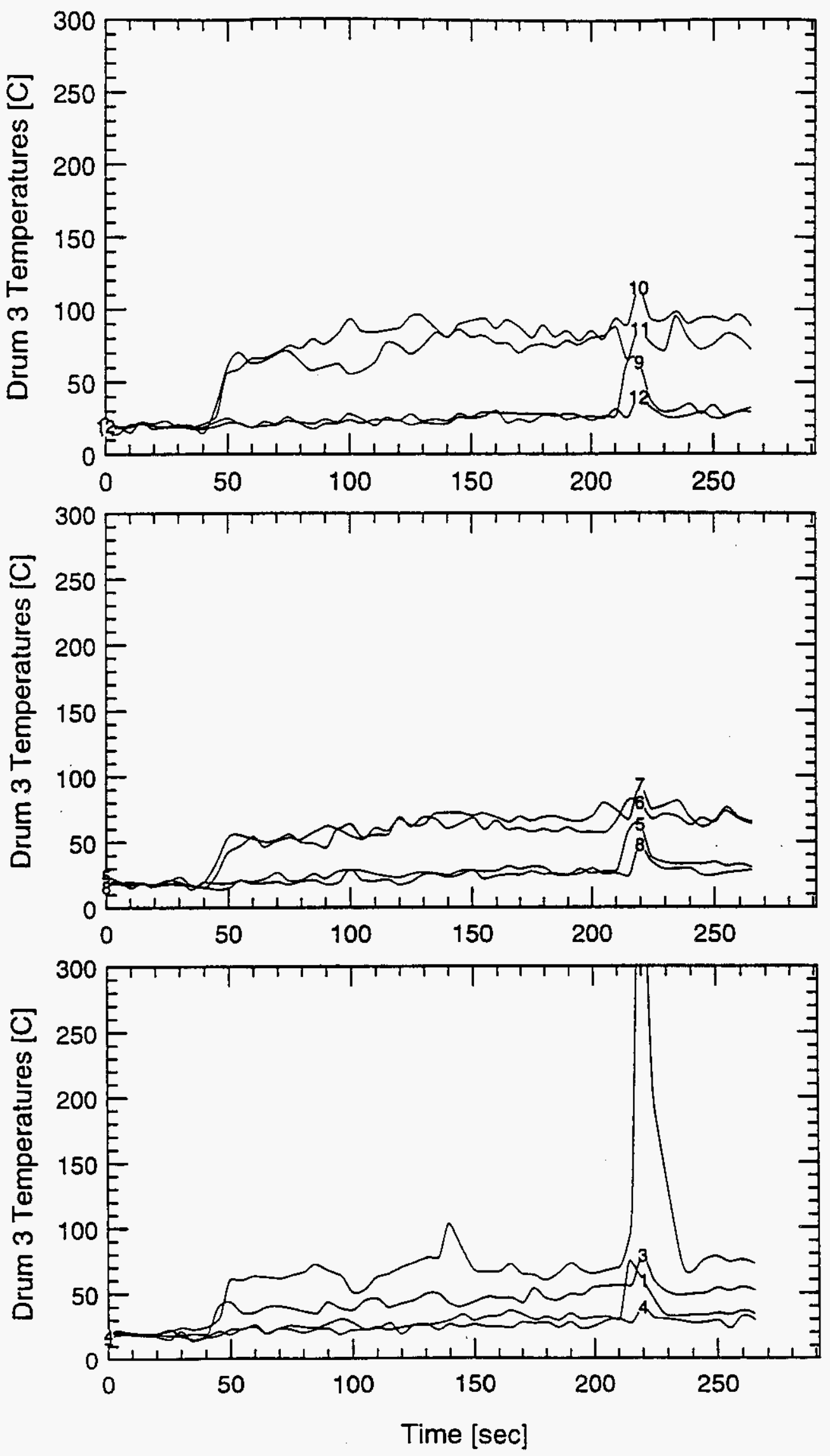
WHC-SD-WM-TRP-233 REV. 0

SWD36 48" Burner - Bagged Standard Load - Toluene
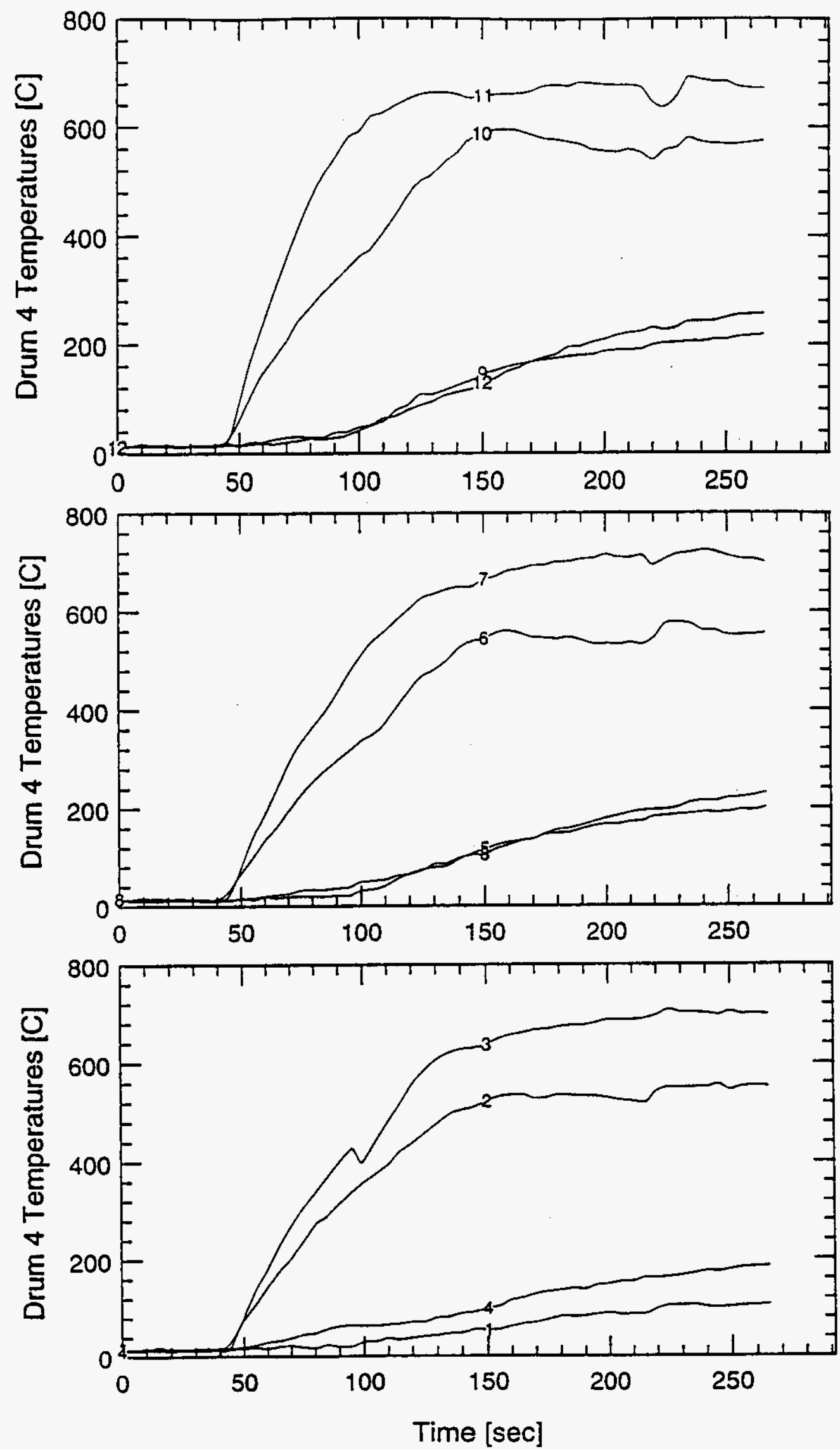
WHC-SD-WM-TRP-233 REV. 0

\section{SWD36 48" Burner}
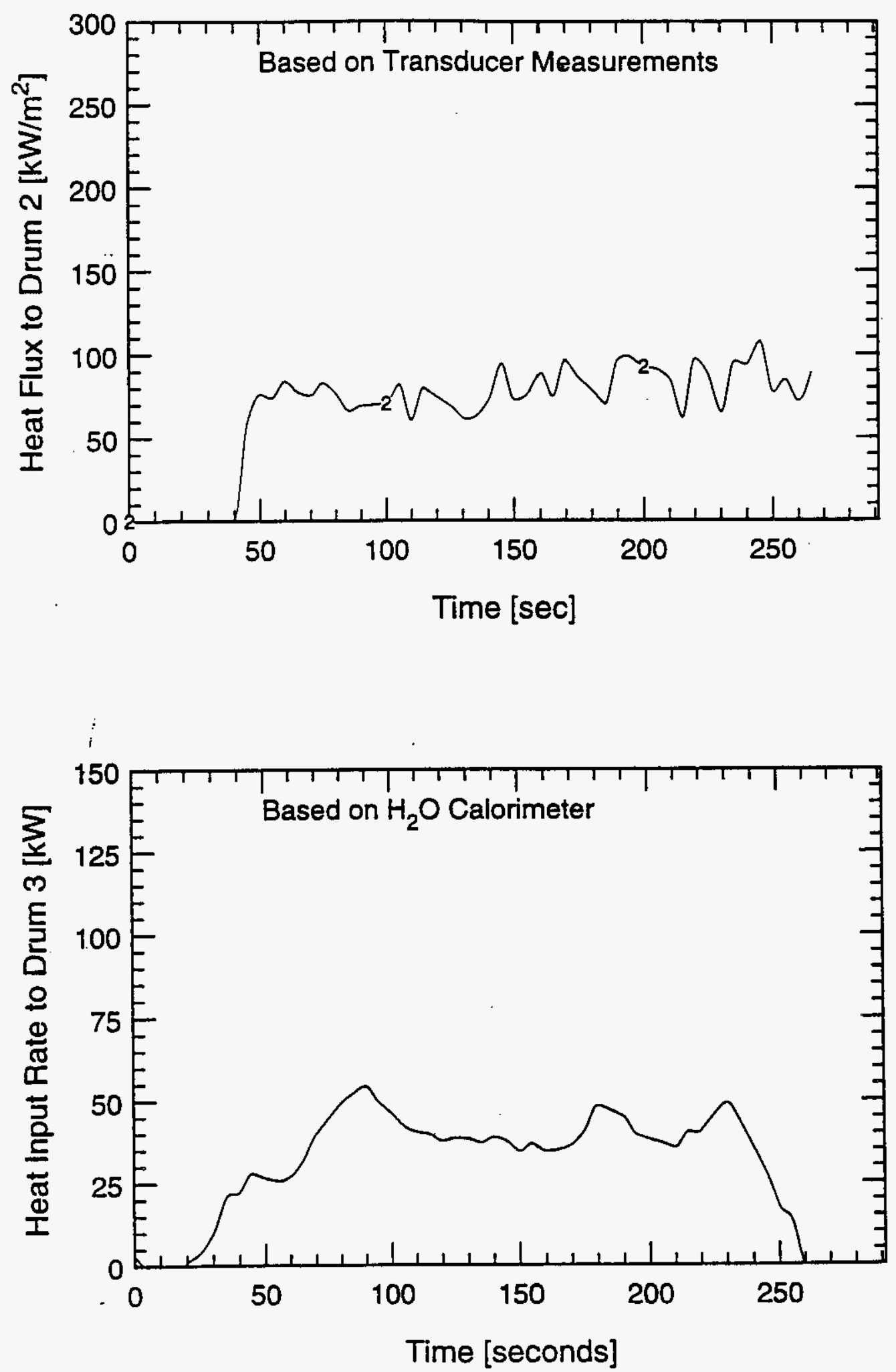
SWD36 DRUM 1 - 48" Burner - 5lb Paper - Toluene
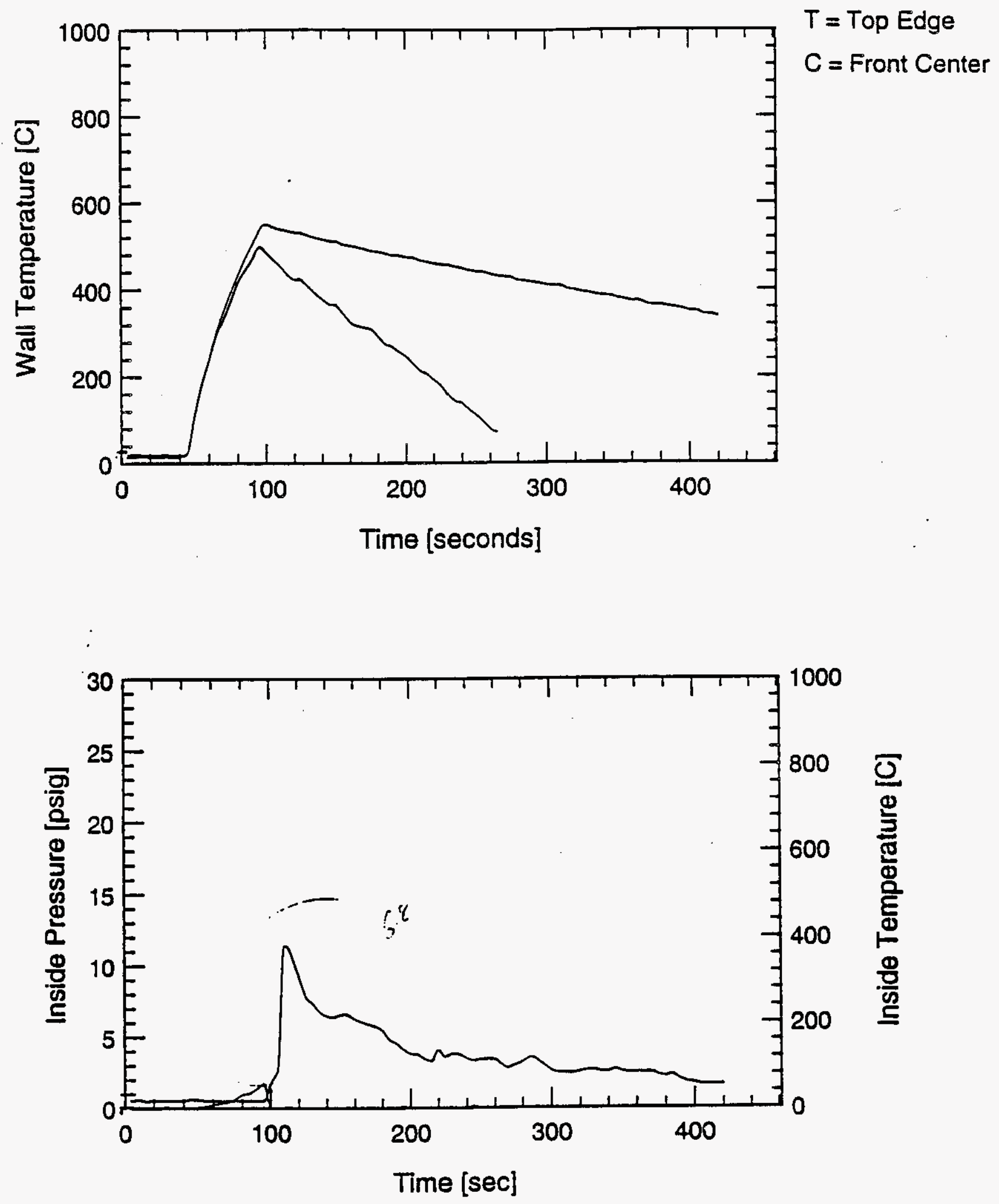
SWD36 DRUM 4 - 48" Burner - Bagged Std Load - Toluene
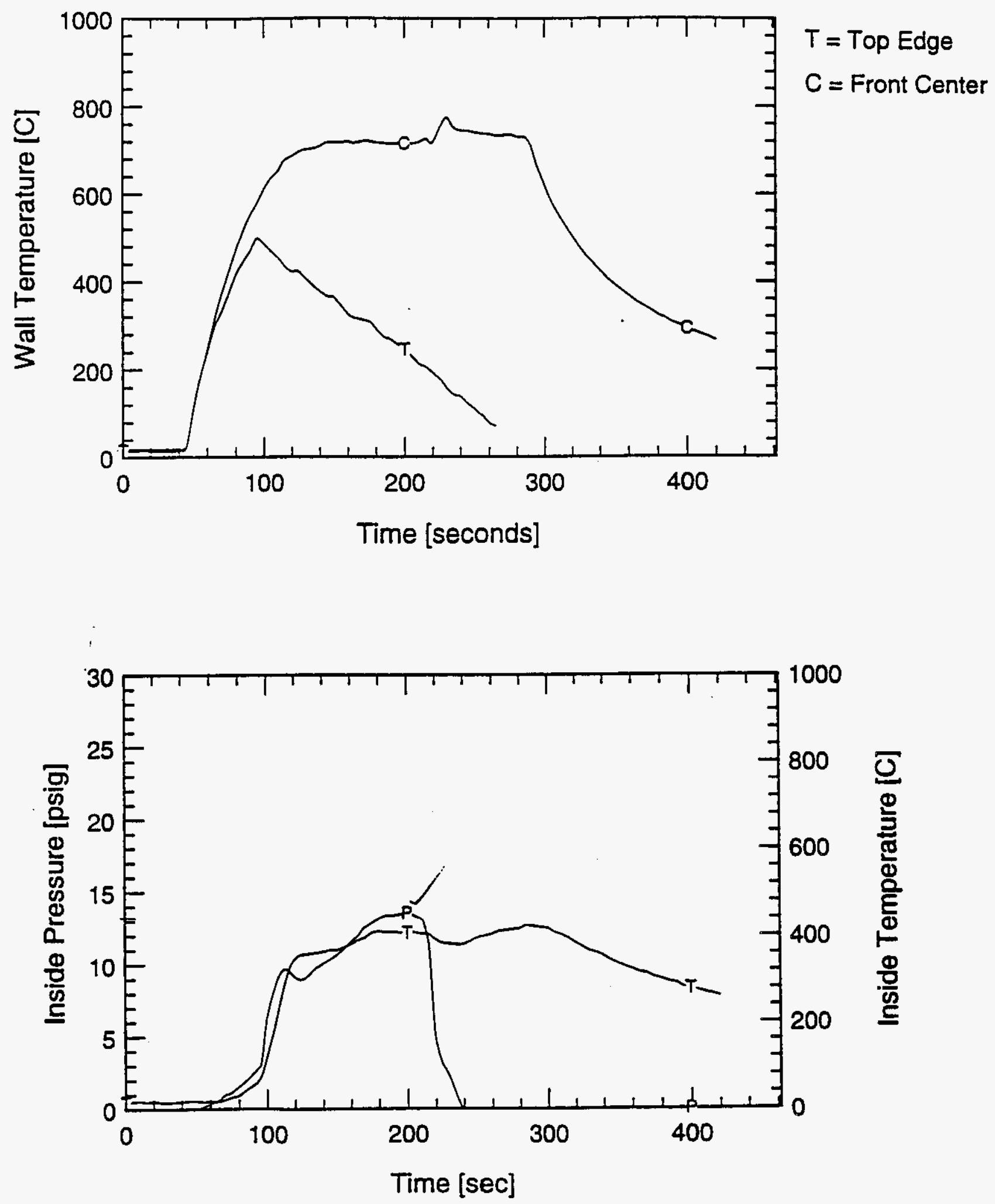
WHC-SD-WM-TRP-233 REV. 0

SWD 37 - DATA 
WHC-SD-WM-TRP-233 REV. 0

SWD37 - Trash Fire - 5lb Paper
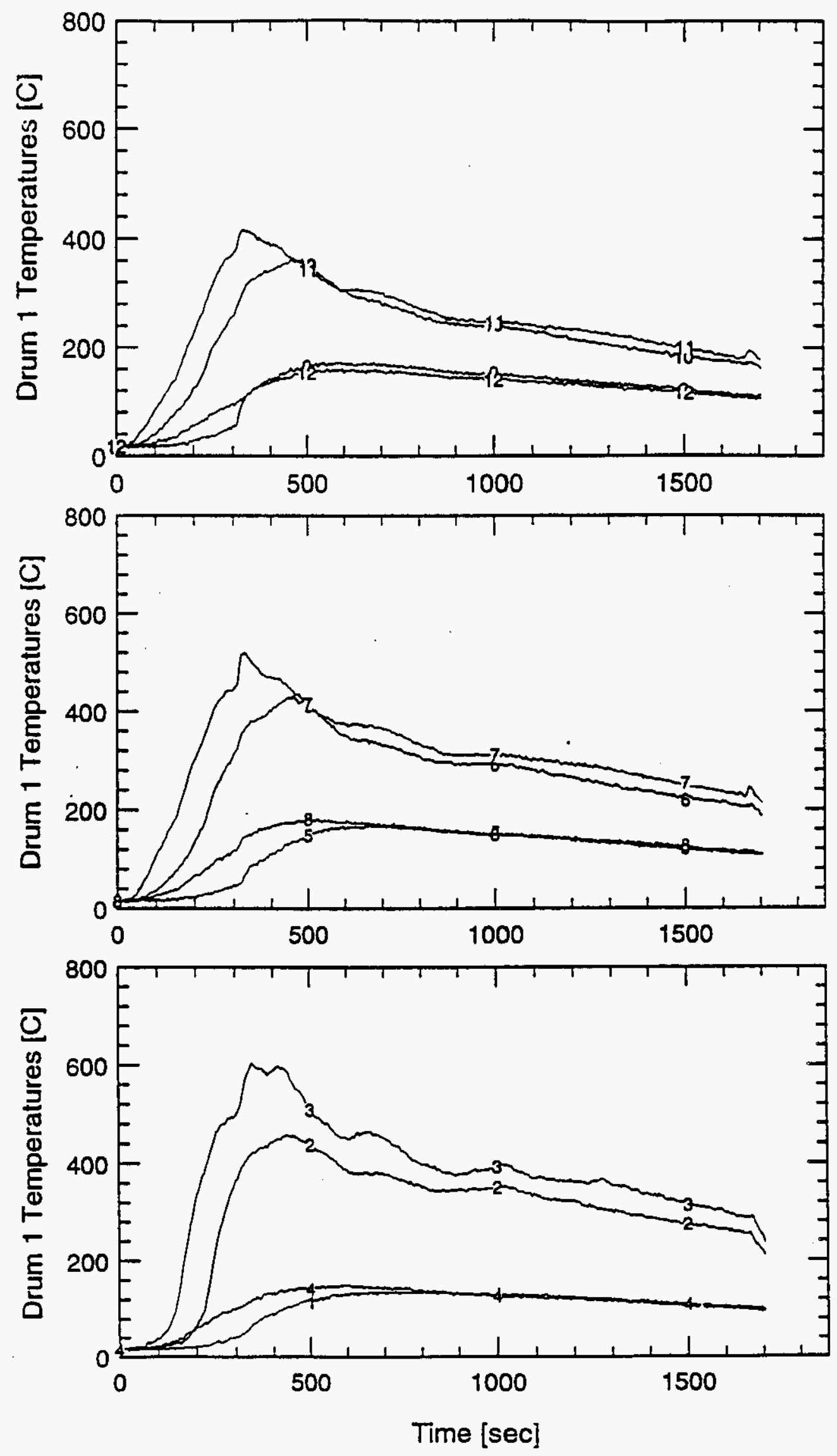

B-268 
WHC-SD-WM-TRP-233 REV. 0

\section{SWD37 - Trash Fire}
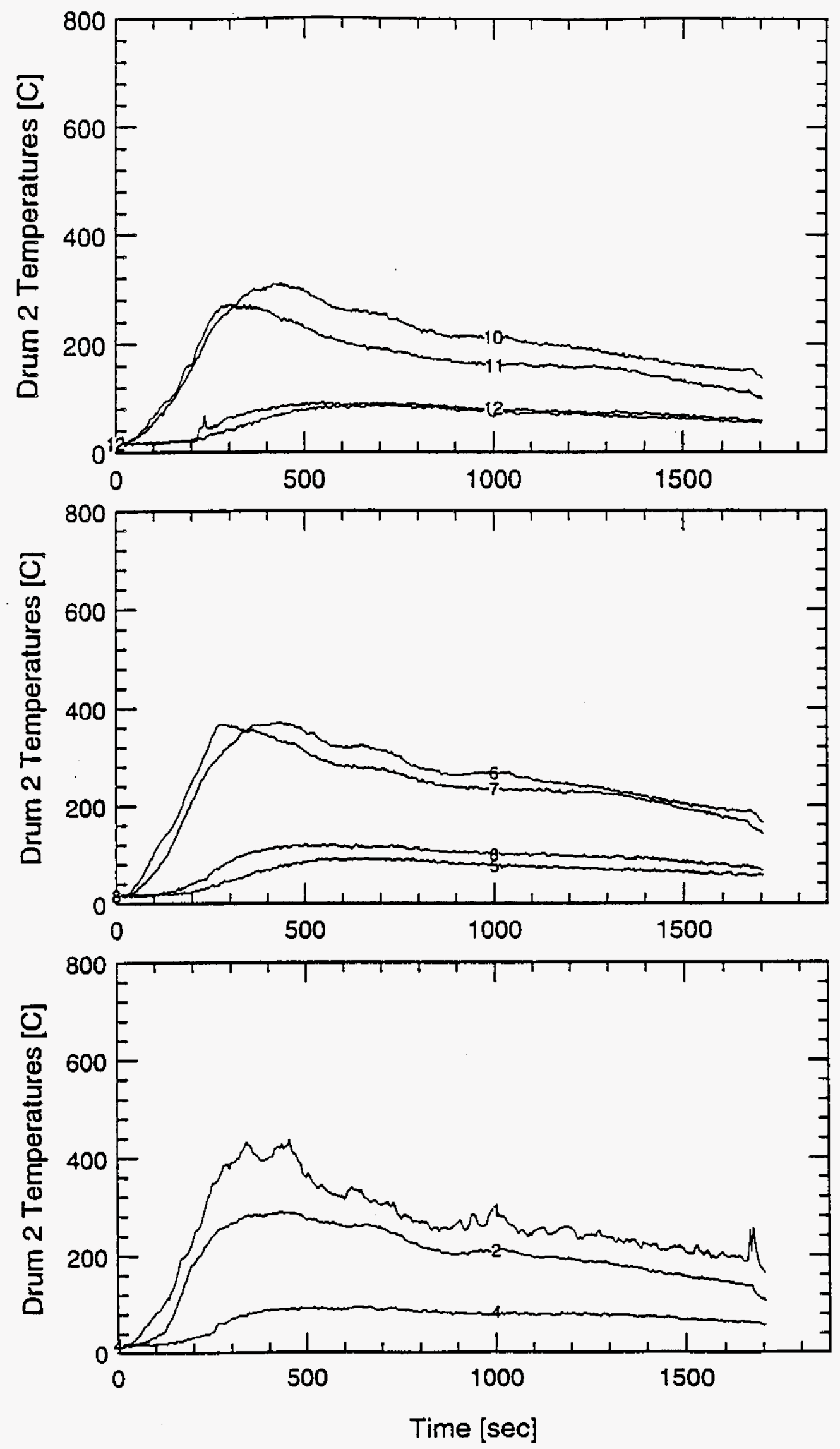
WHC-SD-WM-TRP-233 REV. 0

SWD37 - Trash Fire
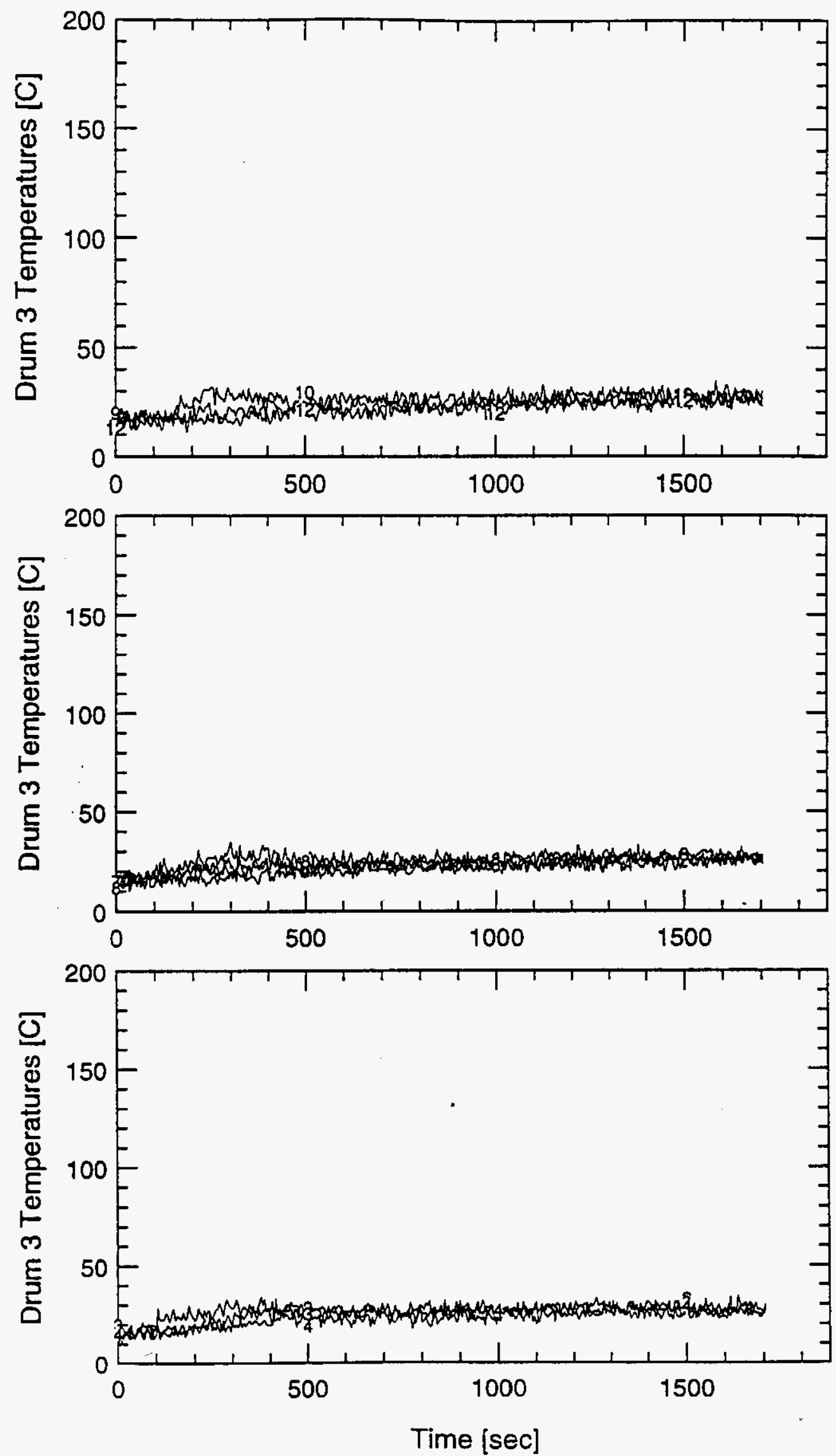
WHC-SD-WM-TRP-233 REV. 0

SWD37 - Trash Fire - 5lb Paper
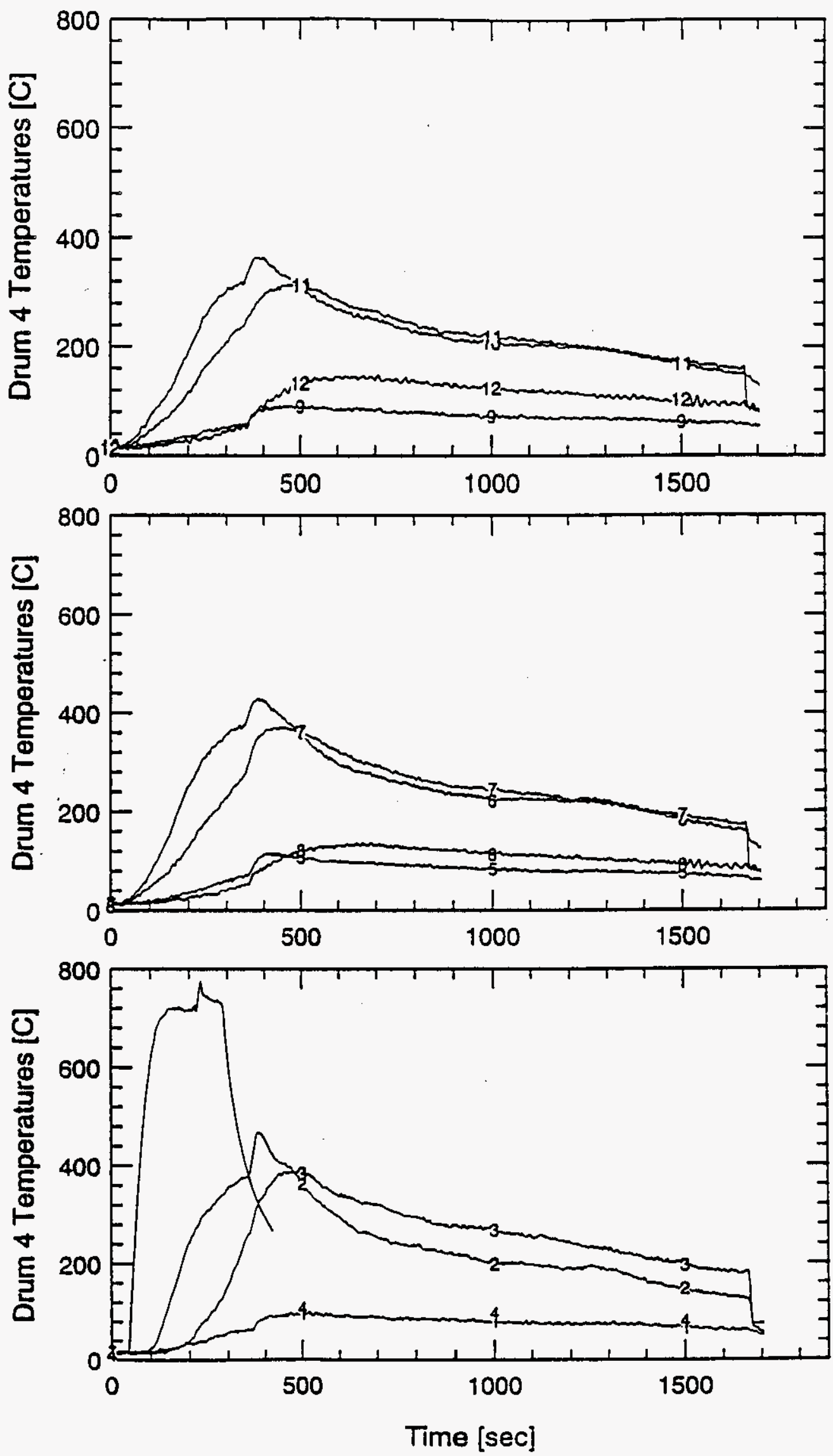
WHC-SD-WM-TRP-233 REV. 0

\section{SWD37 DRUM 1 - Trash Fire - 5lb Paper}
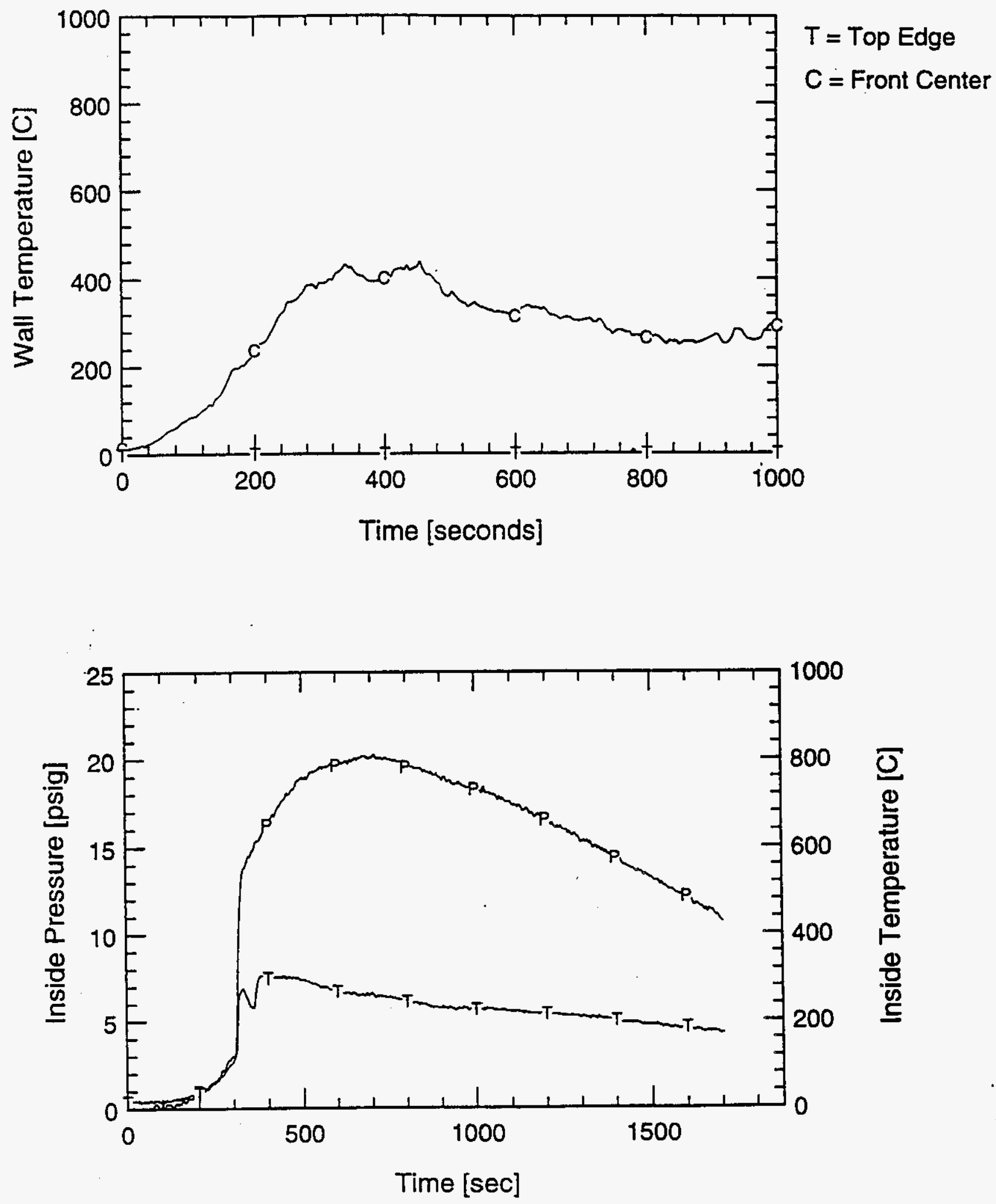
SWD37 DRUM 4 - Trash Fire - 5lb Paper
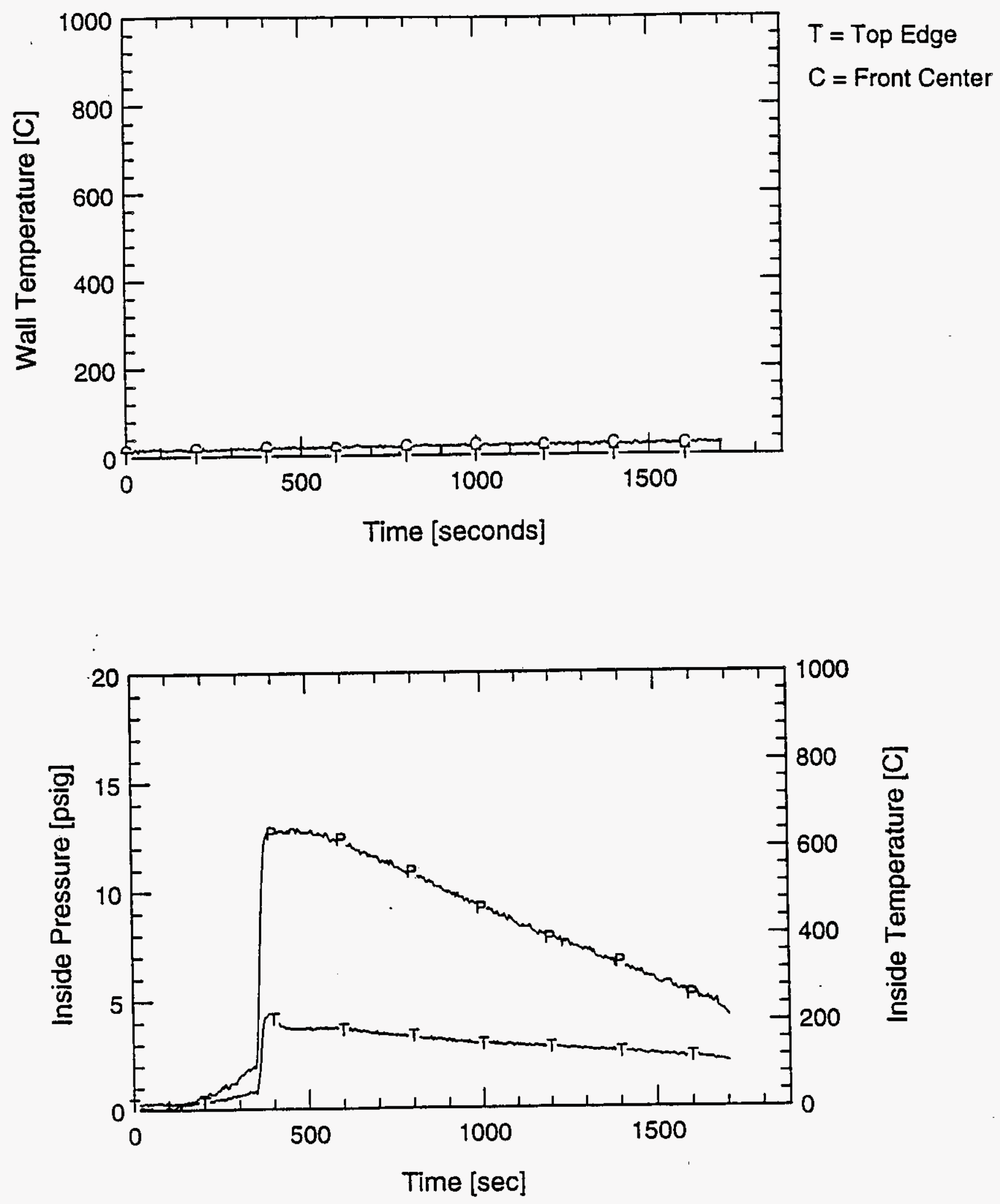
WHC-SD-WM-TRP-233 REV. 0

\section{SWD37 Trash Fire}
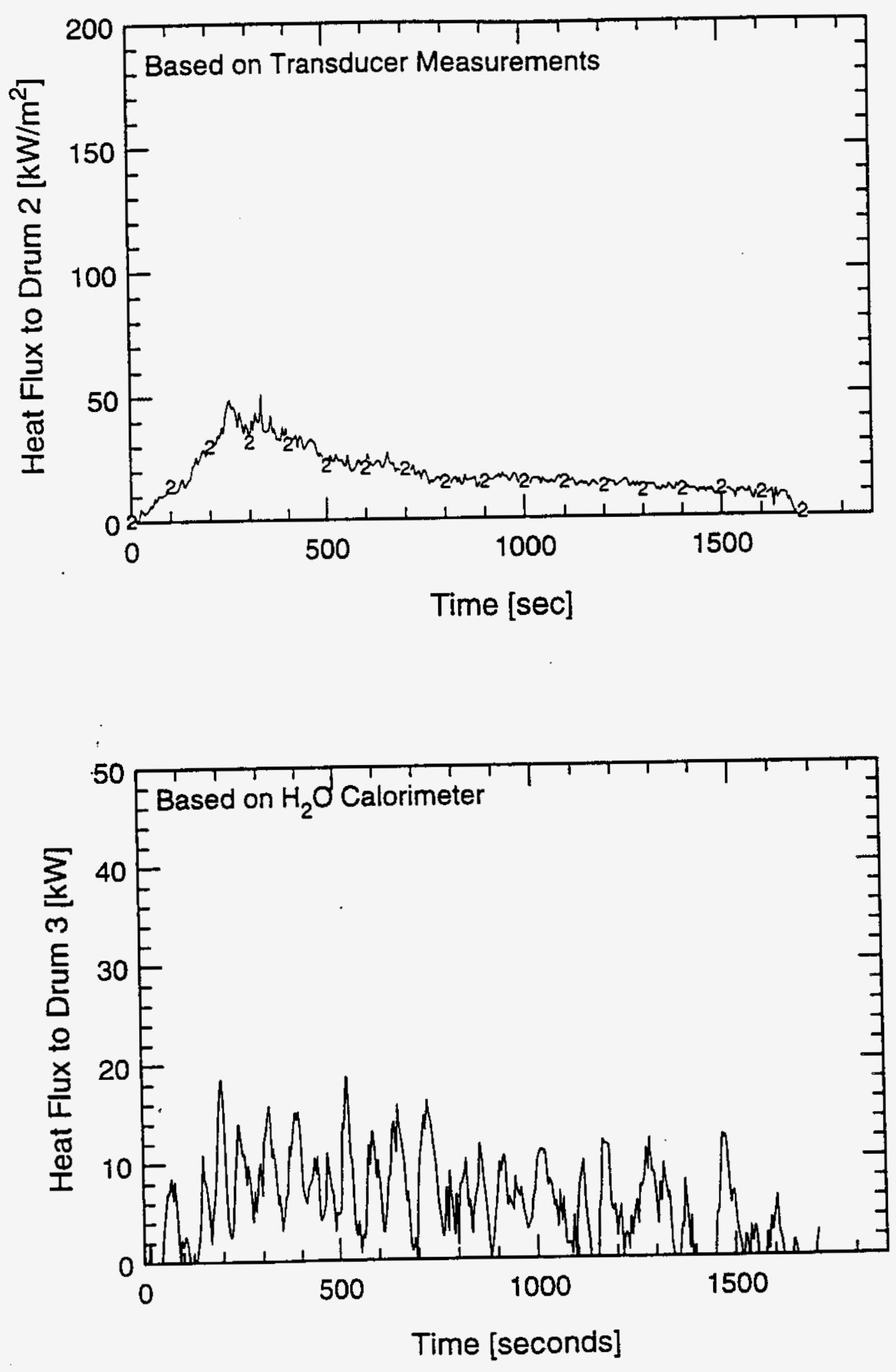
WHC-SD-WM-TRP-233 REV. 0
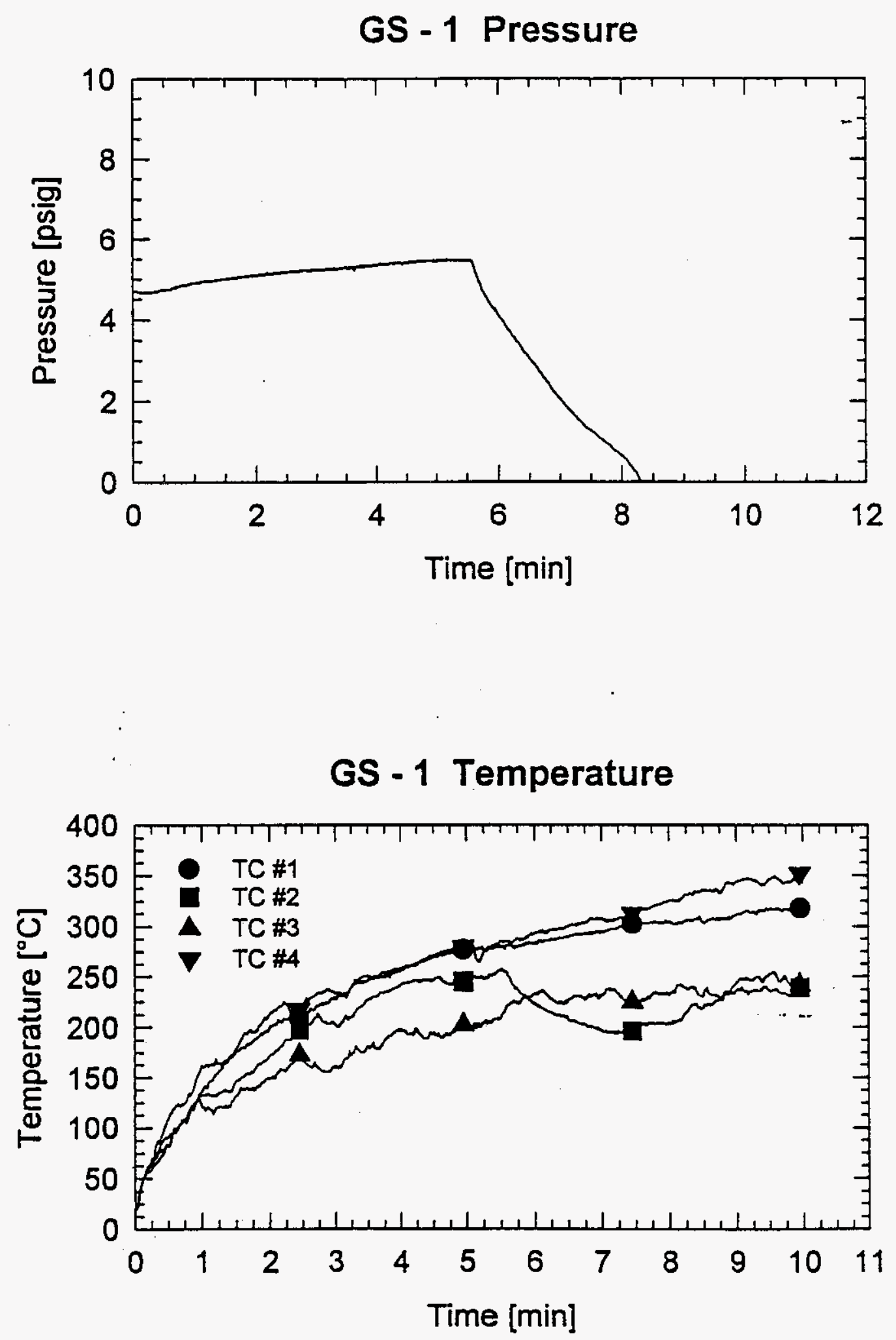
WHC-SD-WM-TRP-233 REV. 0

\section{GS - 2 Pressure}

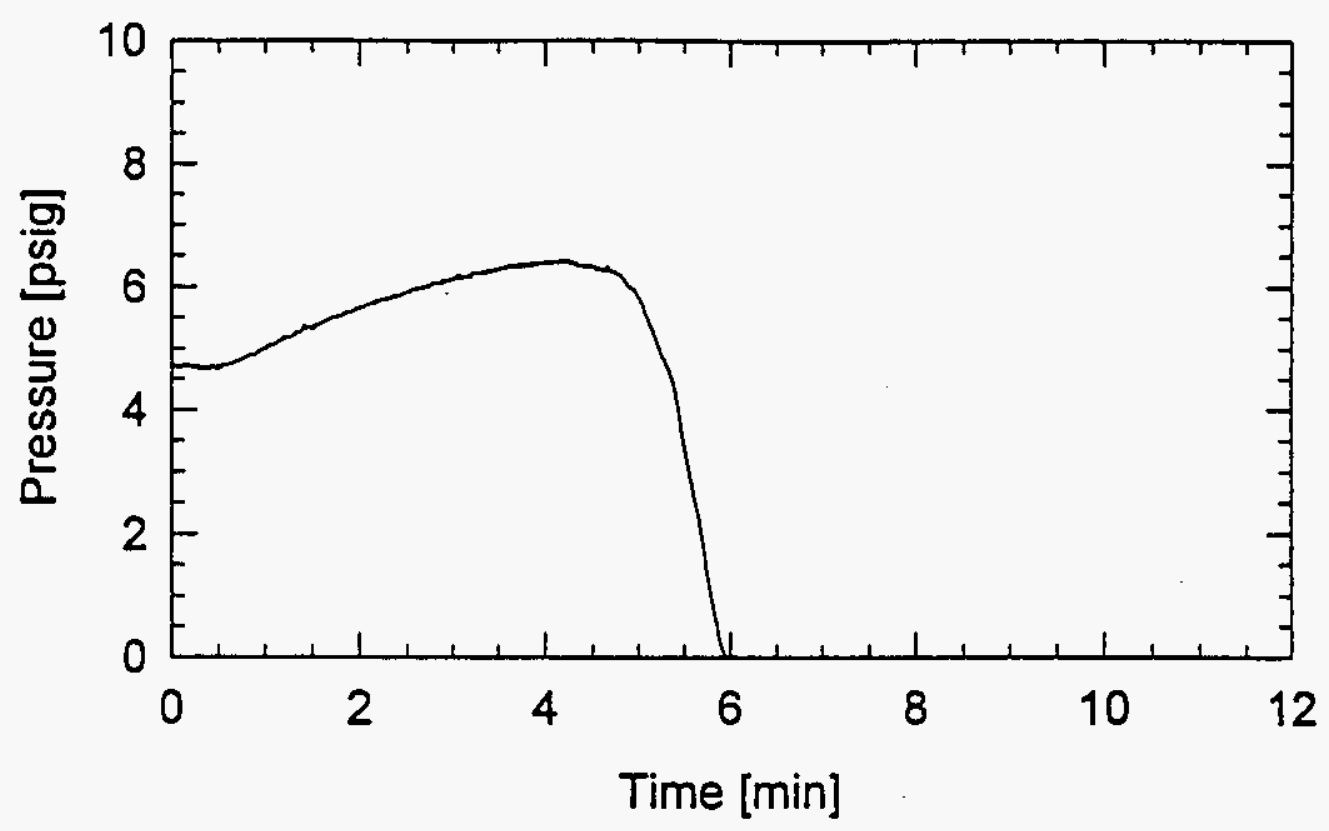

GS - 2 Temperature

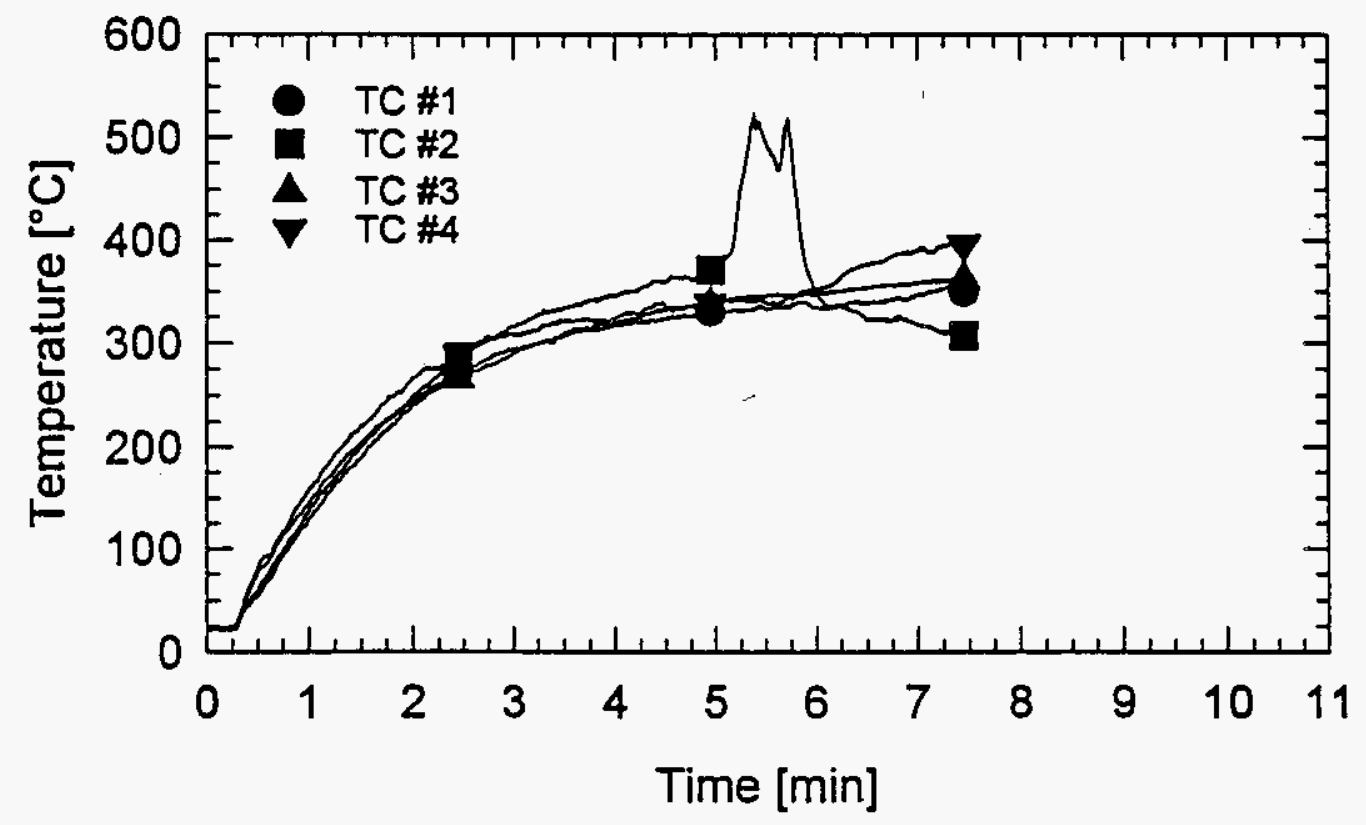


WHC-SD-WM-TRP-233 REV. 0

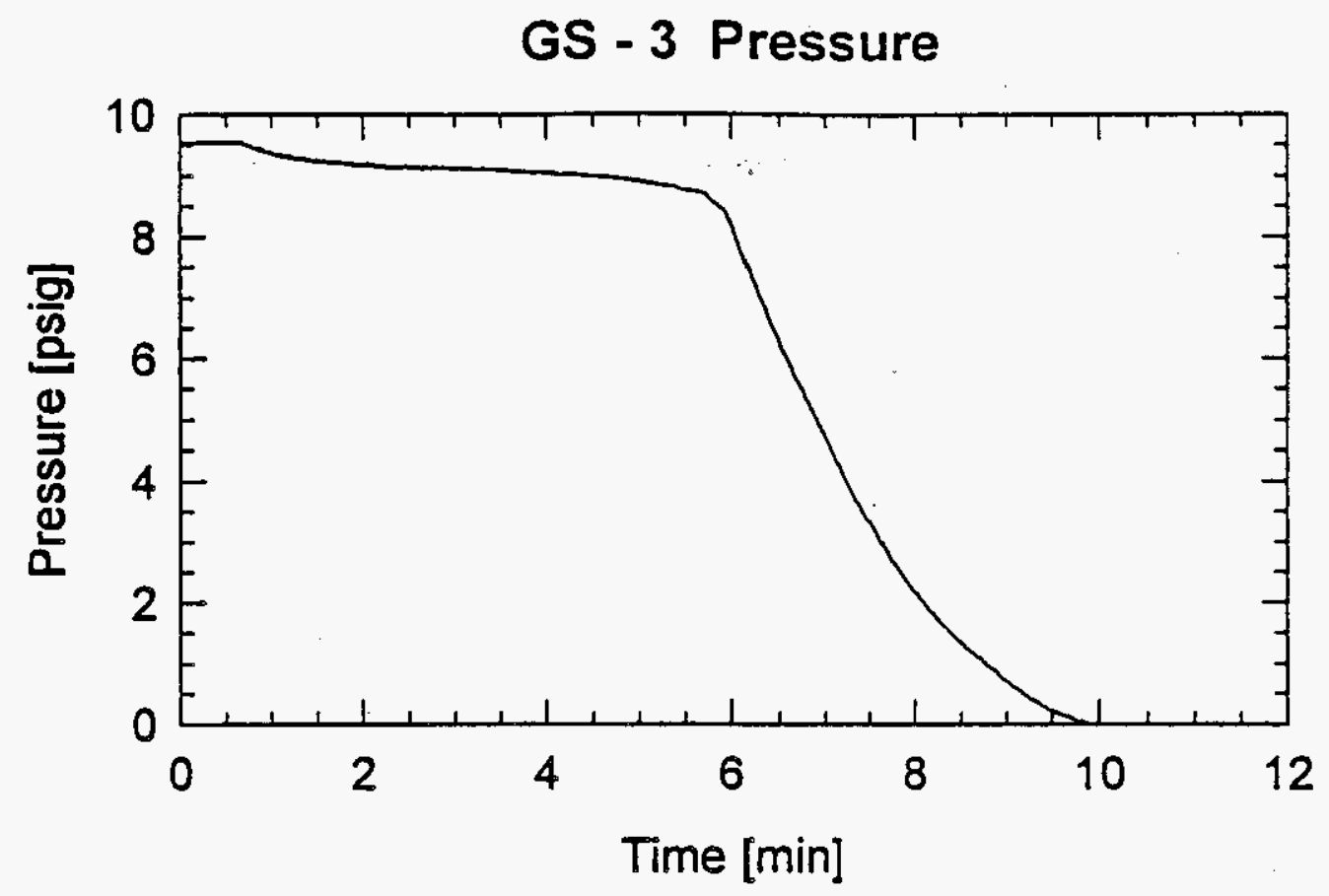

\section{GS -3 Temperature}

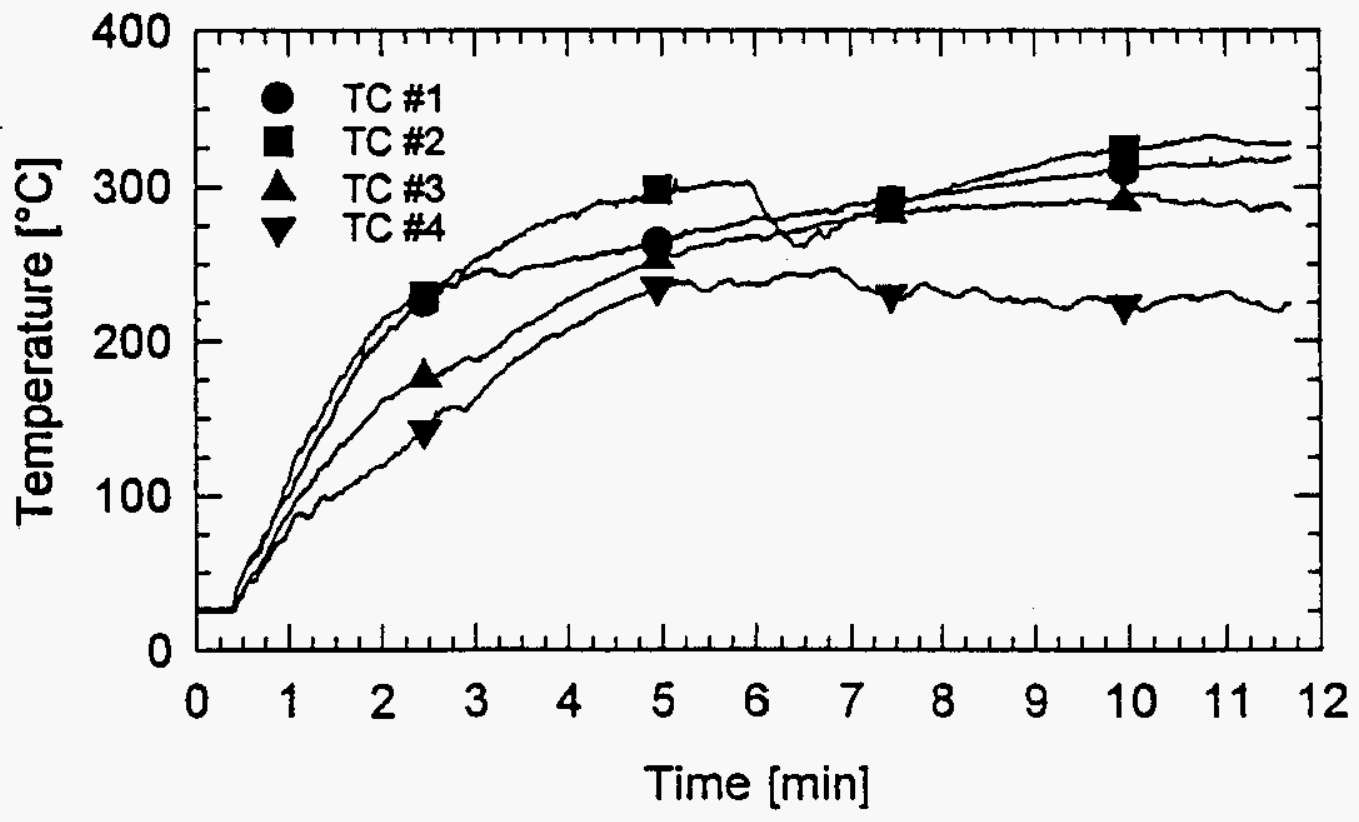


WHC-SD-WM-TRP-233 REV. 0
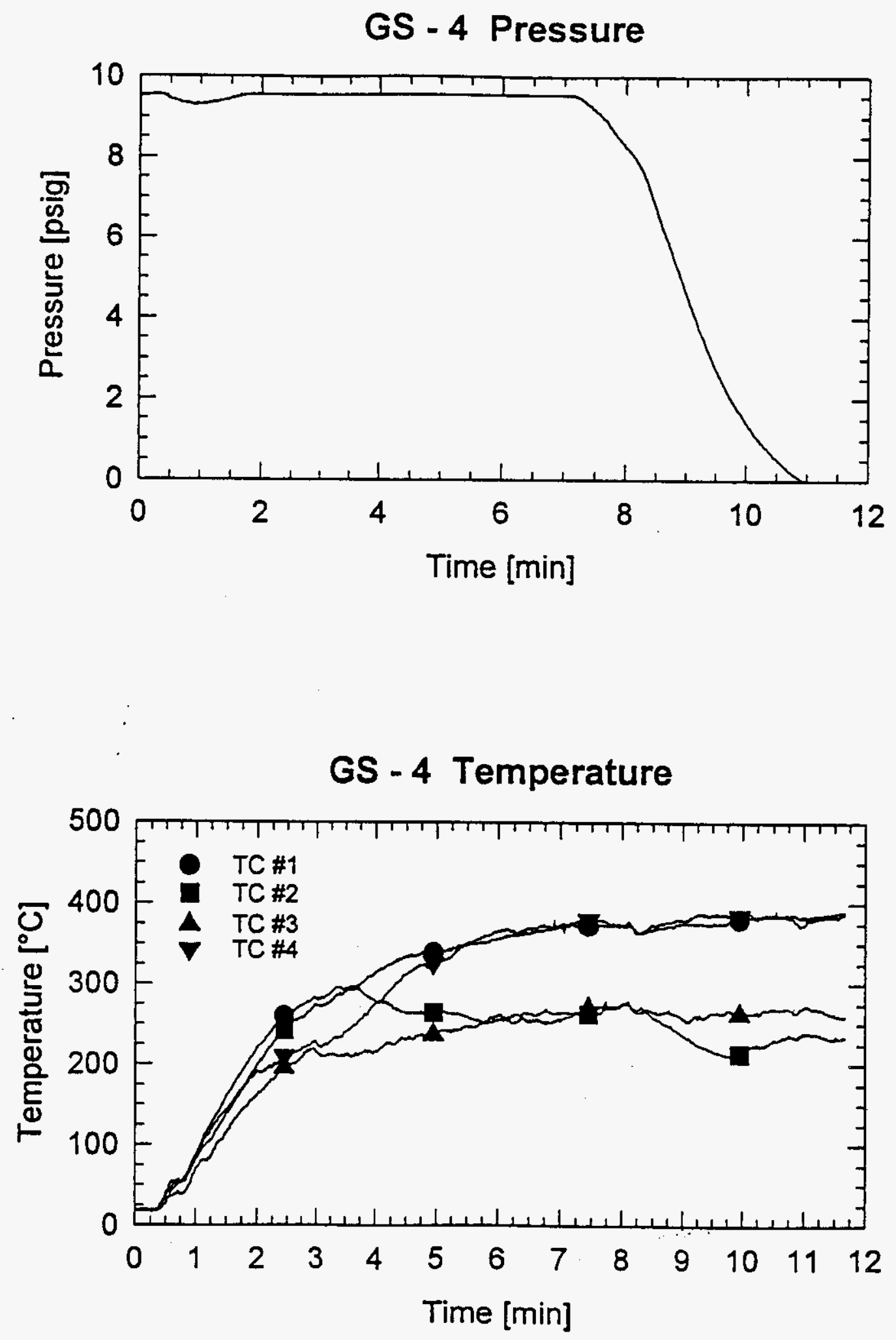

B-278 
WHC-SD-WM-TRP-233 REV. 0

Appendix C

Project Plan for Phase 2

Drum Array Fire Tests of Solid Waste Drums

\section{Prepared for}

Westinghouse Hanford Company

Richland, WA 99352

Prepared by

Chris F. Haecker

Los Alamos Technical Associates, Inc.

Kennewick, WA 99306

Brian T. Rhodes, Jesse J. Beitel, Daniel T. Gottuk

Craig L. Beyler, and Eric R. Rosenbaum

Hughes Associates, Inc.

Columbia, MD 21045

April 28, 1995

C-1 
THIS PAGE INTENTIONALIY LEFT BLANK

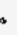


CONTENTS

Page

-1.0 OBJECTIVE ......................... C-1-1

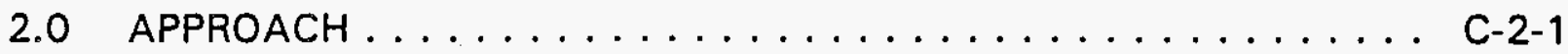

3.0 EXPERIMENTAL RACK STORAGE TEST $\ldots \ldots \ldots \ldots \ldots \ldots$ C-3-1

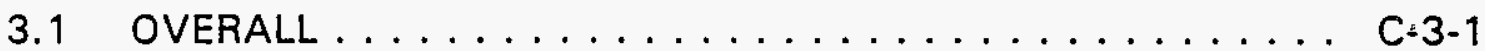

3.2 RACK SYSTEM $\ldots \ldots \ldots \ldots \ldots \ldots \ldots \ldots \ldots \ldots$ C-3-1

3.3 DRUMS ......................... C-3-1

3.3.1 Water-filled Drums . . . . . . . . . . . C-3-7

3.3.2 Standard Load Drums . . . . . . . . . . . . . C-3-9

3.4 INSTRUMENTATION $\ldots \ldots \ldots \ldots \ldots \ldots \ldots \ldots \ldots \ldots$ C-3-10

3.5 FIRE SOURCE $\ldots \ldots \ldots \ldots \ldots \ldots \ldots \ldots \ldots \ldots \ldots \ldots \ldots \ldots$

3.6 TEST TERMINATION $\ldots \ldots \ldots \ldots \ldots \ldots \ldots \ldots \ldots$ C-3-11

3.7 DOCUMENTATION . . . . . . . . . . . . . C-3-11

3.8 TEST PROCEDURES $\ldots \ldots \ldots \ldots \ldots \ldots \ldots \ldots \ldots \ldots$ C-3-11

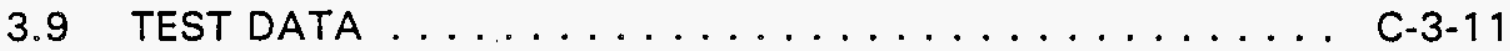

4.0 EXPERIMENTAL PALLET STORAGE TEST $\ldots \ldots \ldots \ldots \ldots \ldots$. . . . . $\ldots$ C

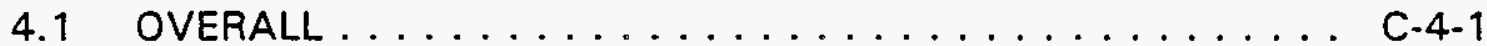

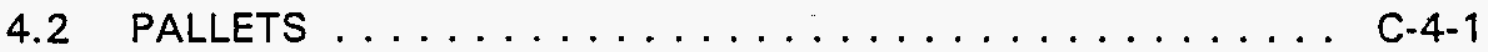

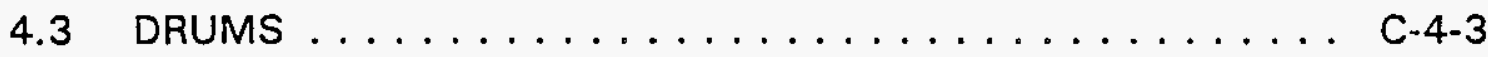

4.4 INSTRUMENTATION $\ldots \ldots \ldots \ldots \ldots \ldots \ldots \ldots \ldots$ C-4-8

4.5 FIRE SOURCE $\ldots \ldots \ldots \ldots \ldots \ldots \ldots \ldots \ldots \ldots \ldots$ C-4-9

4.6 TEST TERMINATION .................. C-4-9

4.7 DOCUMENTATION . . . . . . . . . . . . . C-4-9

4.8 TEST PROCEDURES $\ldots \ldots \ldots \ldots \ldots \ldots \ldots \ldots \ldots$ C-4-10

4.9 TEST DATA $\ldots \ldots \ldots \ldots \ldots \ldots \ldots \ldots \ldots \ldots \ldots \ldots$ C-4-10

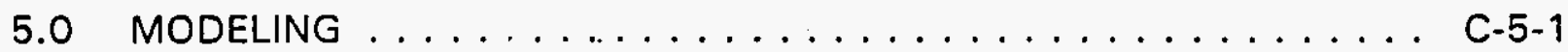

6.0 DATA REPORTING AND ANALYSIS ............. C-6-1

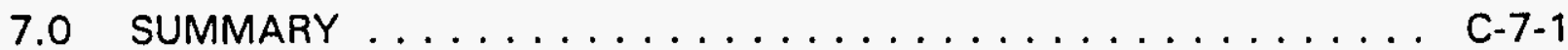

8.0 REFERENCES $\ldots \ldots \ldots \ldots \ldots \ldots \ldots \ldots \ldots \ldots \ldots$ C-8-1

Appendix $A-$ Rack Storage $\ldots \ldots \ldots \ldots \ldots \ldots \ldots$ C-A-1

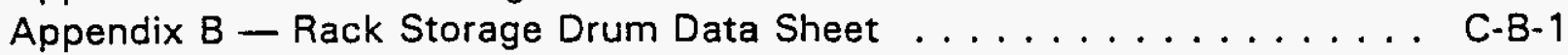

Appendix $\mathrm{C}$ - Rack Storage Pre-test Checklist . . . . . . . . . . . C-C-1

Appendix D - Rack Storage and Pallet Storage Test Procedure Checklist . C-D-1

Appendix E - Rack Storage Post-test Rack Assessment . . . . . . . C C-E-1

Appendix $\mathrm{F}$ - Pallet Storage Pre-test Checklist . . . . . . . . . . . C C-F-1

Appendix G - Pallet Storage Post-test Pallet Assessment . . . . . . . . C-G-1 


\section{FIGURES}

Page

Figure 1. Overall rack storage experimental setup $\ldots \ldots \ldots \ldots \ldots$ C-3-2

Figure 2. Rack storage experimental setup and instrumentation layout . . C C-3-4

Figure 3. Rack storage drum location diagram $\ldots \ldots \ldots \ldots \ldots \ldots$ C-3-5

Figure 4. Water-filled calorimeter drum $\ldots \ldots \ldots \ldots \ldots \ldots$ C-3-6

Figure 5. Overall pallet storage experimental setup $\ldots \ldots \ldots \ldots \ldots$ C-4-2

Figure 6. Pallet storage experimental setup and instrumentation layout . . C-4-4

Figure 7. Pallet storage drum location diagram $\ldots \ldots \ldots \ldots \ldots \ldots$ C-4-5

\section{TABLES}

Page

Table 1. Standard Combustible Loading $\ldots \ldots \ldots \ldots \ldots \ldots \ldots$ C-3-8

Table 2. Standard Loading - Into Drum $\ldots \ldots \ldots \ldots \ldots \ldots \ldots$ C-3-8

Table 3. Standard Combustible Loading $\ldots \ldots \ldots \ldots \ldots \ldots$. . . . . . .

Table 4. Standard Loading - Into Drum . . . . . . . . . . C-4-7 


\section{Project Plan for Phase 2 \\ Drum Array Fire Tests of Solid Waste Drums}

\subsection{OBJECTIVE}

Phase 2 work is intended to assess the validity of the models developed in Phase 1. While the drum testing included in Phase 1 is an essential element of establishing the validity of the models, it is also necessary to assess the models by comparison with drum array tests. Individual drum tests as were performed in Phase 1 can be instrumented thoroughly, the fire environment can be controlled well, and a relatively large number of tests can be performed. Array tests cannot be so thoroughly instrumented or controlled, and a very limited number of tests is possible. Array tests do, however, allow the various component phenomena to interact. This can give rise to phenomena which cannot occur in individual drum tests. An obvious example is drum array stability which inherently cannot be studied in single drum tests. Thus, the drum array testing is needed to validate the models developed. Another benefit of the drum array testing will be to provide a more quantitative measure of the amount of drum contents expelled during the tests. 
THIS PAGE INTENTIONALLY LEFT BLANK 


\subsection{APPROACH}

In Phase 2, drum array tests will be performed and predictions of the tests will be made prior to the conduct of the test. Predictions and test results will be compared and the performance of the models will be assessed. In general, the tests will consist of exposing a section of a drum storage array to an initiating pool fire and documenting via test measurements and observations the drum response and extent of fire propagation beyond the initiating fire.

In Phase 2, one pallet array test and one rack storage array test will be conducted. These represent the two primary storage configurations. The pallets represent storage configurations in which the drums are stacked in arrays. The number of drum levels within the array may vary and the properties of the level separations may vary. In particular, current WHC practice and/or plans include pallets and plywood as level separations. The rack storage configuration may vary in the number of tiers included, the nature of the structure as well as the nature of the drum supports. The configurations which will be used in these tests are reasonable representations of their respective storage configurations.

While the test setups to be used in the tests are intended to be representative of the general storage configurations in use, the initiating fire is designed to maximize the damage potential. As such, pool fire initiating sources are used which are large enough to be optically thick ( $1 \mathrm{~m}$ or more) and to last longer than any drum lid loss failure time in Phase 1 testing. This assures that if a lid loss could occur, it will occur in the testing. This is consistent with a philosophy of "testing to failure." While this philosophy cannot be fully implemented in a single test situation, the use of a very severe loptically thick and long duration) initiating source avoids the concern that a longer duration or larger pool fire might have a more severe effect. Clearly, if a shorter fire duration is expected in practice, the data will be of value up to the pool fire burn time expected.

The size of the storage array is dictated by the need for an optically thick initiating fire exposure as well as the need for the storage array to be sufficiently large relative to the initiating source that fire propagation remote from the initiating source will be geometrically possible in the test. Implied in the test array size criterion is that if the fire propagates to the end of the storage array, it would be expected to propagate indefinitely. While this can only be assured in an indefinitely long storage array, the array length must be long enough to approach this limit credibiy, but practically.

While instrumenting every drum in detail is desirable, this is not an economically feasible option. The level of instrumentation to be used will be sufficient to allow the fire propagation to be understood in terms of the modeling and testing performed in Phase 1. Extensive use of thermocouples will be made, 
since these are relatively inexpensive, very diagnostic, and do not interfere with drum movement. Pressure transducers on the drums will be used to a lesser extent due to cost, data value, and the interference of transducer tubing on drum movement. Heat flux transducers will be used in sufficient numbers to yield a reasonable mapping of the initiating fire heat fluxes. A few drum calorimeters will be used in the tests to provide total heat input to selected locations. Since these drums are filled with water, they could potentially interfere with the outcome of the test. As such their use will be limited in number and will be used in locations which are unlikely to interfere with the test outcome while providing needed data.

The tests will be performed and documented in accordance with the standards employed in the peer reviewed fire science literature. This requires that the tests be described in sufficient detail to allow the tests to be replicated by others (if ever required) and that instrumentation and data collection methods used conform to the accuracy and precision established in the fire science literature. 


\subsection{EXPERIMENTAL RACK STORAGE TEST}

\subsection{OVERALL}

The rack storage test will be conducted "inside" such that the effects of wind, rain, etc. will be negated. The fire test facility to be used is located at Southwest Research Institute in San Antonio, Texas. The building is $12.2 \mathrm{~m}(40$ $\mathrm{ft})$ by $18.3 \mathrm{~m}(60 \mathrm{ft})$ by $9.1 \mathrm{~m}(30 \mathrm{ft}$ high). The building will be naturally ventilated during the test through vents located at floor level along the periphery of the building and exhaust vents in the roof and high on the walls. All doors to the building will be closed during the test. Post test clearing of smoke from the building can be accelerated via an exhaust fan. This fan (which is located high on the south wall) will not be used during the test to avoid nonuniform air flow through the building and, subsequent, deflection of the pool fire.

Figure 1 provides an overall view of the proposed test arrangement for the rack storage configuration. In general, the test configuration will consist of three rows of storage with each row being six tiers high. Two rows will be back-to-back and one row will be separated from the other rows by a $1.5 \mathrm{~m}(5 \mathrm{ft})$ aisle.

\subsection{RACK SYSTEM}

The rack system will be similar to that proposed for use at WHC. WHC/LATA will provide exact specifications and provide the rack system to the test laboratory (Appendix A). The rack system will include a containment pan and mesh arrangement under the drums. Seismic event bars will also be in place for drum containment. All ground level racks will be anchored to the concrete floor of the test facility using Hilti or similar anchors. The single rack will also be secured to the ceiling of the building. The back-to-back racks will be mechanically secured. Each beam pair has a rated capacity of $6000 \mathrm{lb}$ and each upright has a rated capacity of $32000 \mathrm{lb}$. The pans ( 29 inch wide $\times 28$ inch deep $\times 2$ inch high) will be suspended by $S$ hooks from the mesh above. There will be a 6 inch air space between adjacent pans.

\subsection{DRUMS}

A total of 144 drums will be used in this test. The drums will be new $17-\mathrm{H}$, 55-gallon (UN1A1) drums, painted black. No 85-gallon overpacks will be incorporated into the test. The drums will be sealed using removable lids with styrene-butadiene sponge rubber gaskets and metal closure rings, rounded-section type. After loading, the closure rings will be tightened, per WHC specification [Carver, 1993] to $54 \mathrm{~J}(40 \mathrm{ft}-\mathrm{lb})$ torque. 
WHC-SD-WM-TRP-233 REV. 0

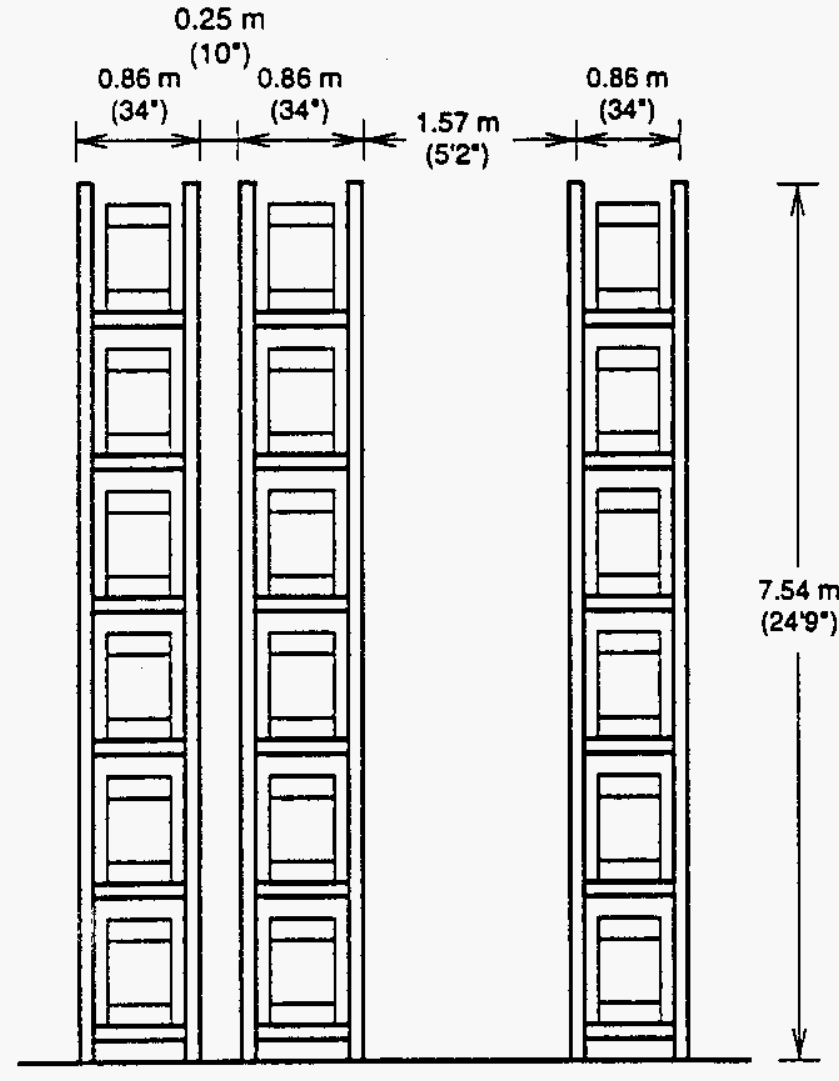

Side View

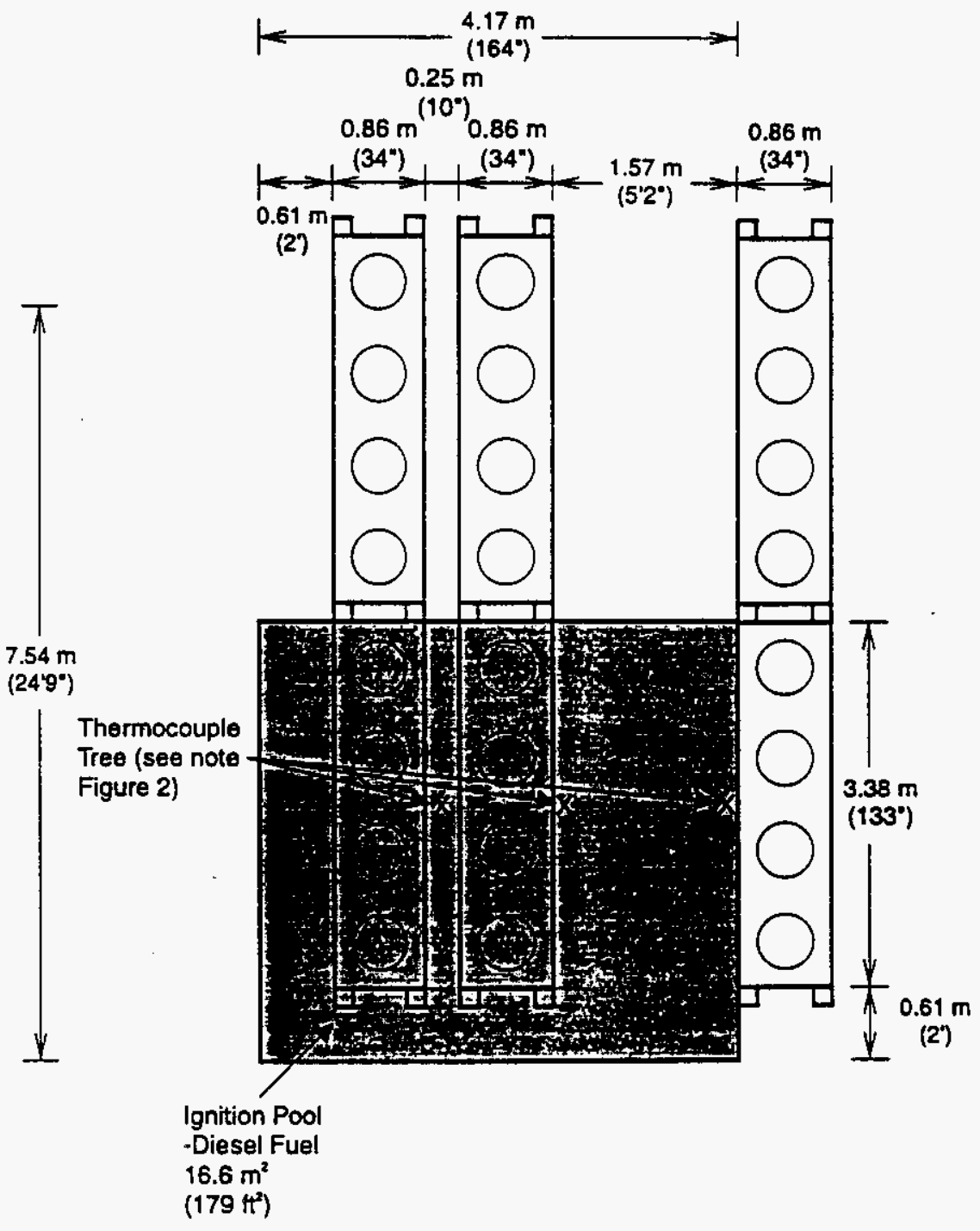

Plan View

Figure 1. Overall rack storage experimental setup 
Close attention must be given to the method of torquing the closure rings. Depending on the torquing method used, a range of tightness can be achieved with the closure ring still measuring $54 \mathrm{~J}(40 \mathrm{ft}-\mathrm{lb})$ torque. For example, if one tightens the closure bolt with a torque wrench, $54 \mathrm{~J}$ can be achieved with a $5 \mathrm{~cm}$ separation between the ends of the closure ring. However, this original torque value is typically from the boit head binding against the closure ring surface rather than the tightness of the ring around the lid and drum. Consequently, when the bolt is backed off and then tightened again, $54 \mathrm{~J}$ is not achieved until the separation between the ends of the closure ring is smaller, such as $0.6 \mathrm{~cm}$. Therefore, depending on the person and method used, a significant range of closure ring tightness can be achieved.

Therefore, all lids are to be secured by the same technician using the method of repetitively backing the bolt out and then tightening to $54 \mathrm{~J}$ torque. This procedure was used in the Phase 1 testing and assures that the rings are torqued to the same specification. As a result of using this method, it is believed that the drums tested will probably be tightened more than the existing drums at WHC. The net effect of this is not clear. The fire exposed drums may require a higher pressure for lid loss or may not lose lids due to the closure ring securing the lids tighter to the drum. On the other hand, the tighter closure ring may result in the lid blowing earlier and more violently. This may occur due to a more rapid buildup of pressure in the drum whereas a looser closure ring may allow sufficient gas to vent from the drum, thus avoiding lid loss.

In this test, 140 of the drums will represent typical WHC loaded drums and contain the standard load [Rhodes et al., 1995] while four drums will serve as water calorimeters. Figure 2 shows the layout of drums in the rack storage system along with the instrumentation. As each drum is prepared, it will be identified with a painted number according to the drum location diagram (Figure 3 ) and all preparation will be documented on Drum Data Sheets (Appendix B).

\subsubsection{Water-filled Drums}

The water-filled drums will act as calorimeters to provide a measure of the integrated heat input to the surrounding target drums. These measurements will provide one measure of the severity of the exposing fire. Heat flux transducers will provide local heat flux measurements.

Each of the water-filled drums will have a submersible pump inside to mix the water, thus, assuring a uniform temperature profile. As shown in Figure 4, the pump is to rest on the bottom of the drum and have a section of 1 inch pvc pipe to deliver the water to the top of the drum. The drums will be filled with water to 0.5-1 in. of its capacity and will be instrumented as described below. The actual placement of the drums in the test array is shown in Figure 2. 


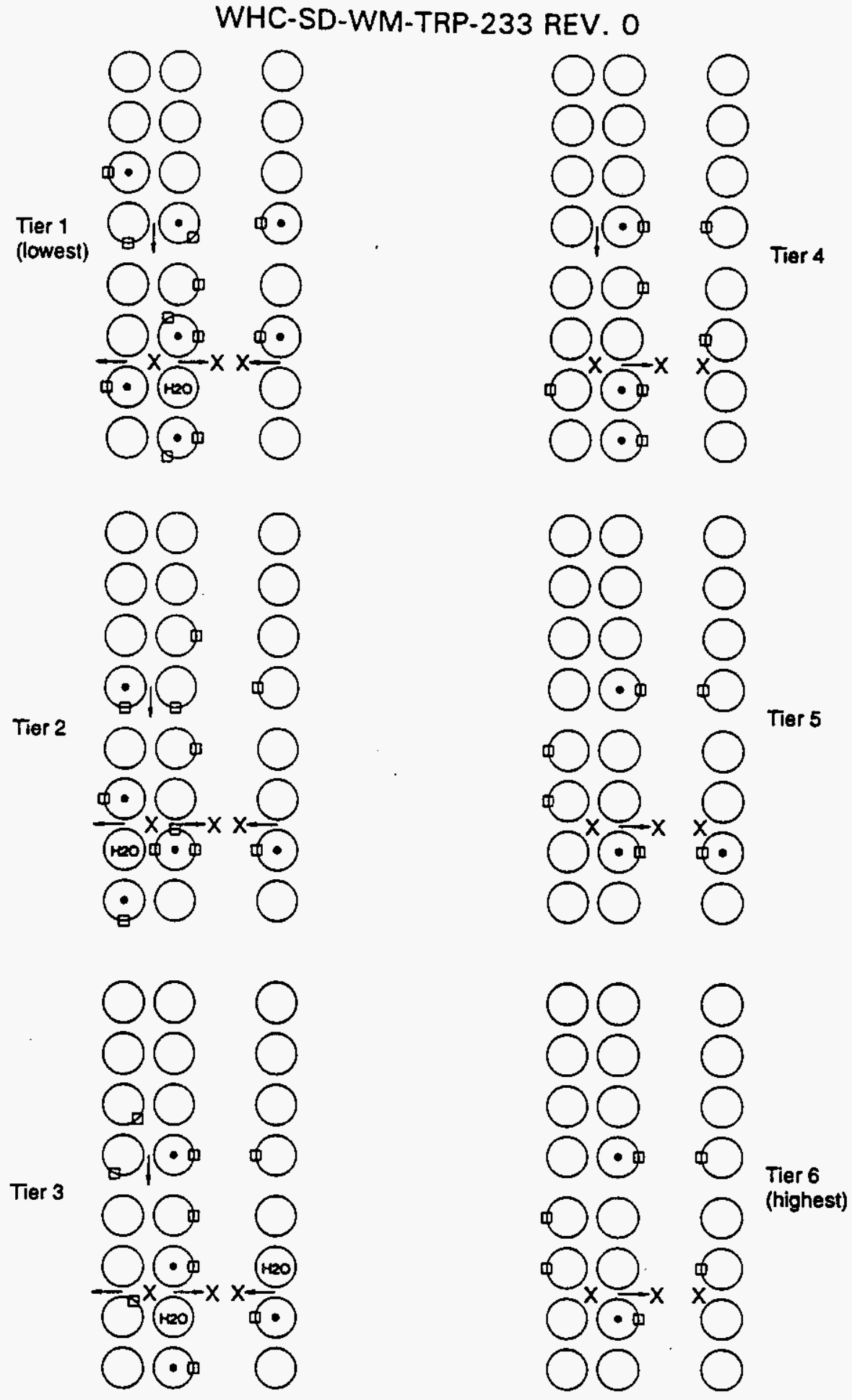

Notes:

Except As Noted, All Drums Standard Load - w/Steel

- = Pressure $=24$ Locations

$\mathrm{Q}=$ Drum Surface -2 Each - Near Top \& $1 / 2 \mathrm{Ht} .=50$ Locations $=100 \mathrm{TC}$ 's

$\mathrm{H} 2 \mathrm{O}=$ Temps - In $\mathrm{H} 2 \mathrm{O}$ (3/Drum) $=12$

Temps On Racks/Pans $=\mathbf{4 0}$

$X=$ Temp in Fire $=$ TC Trees - Each 13 TC's X $3=36$ TC's Total

$\rightarrow=$ Heat Flux Gauges $=16$ Total

Total - 188 TC's

Figure 2. Rack storage experimental setup and instrumentation layout

$$
\text { C-3-4 }
$$


Tier 1

Tier 2
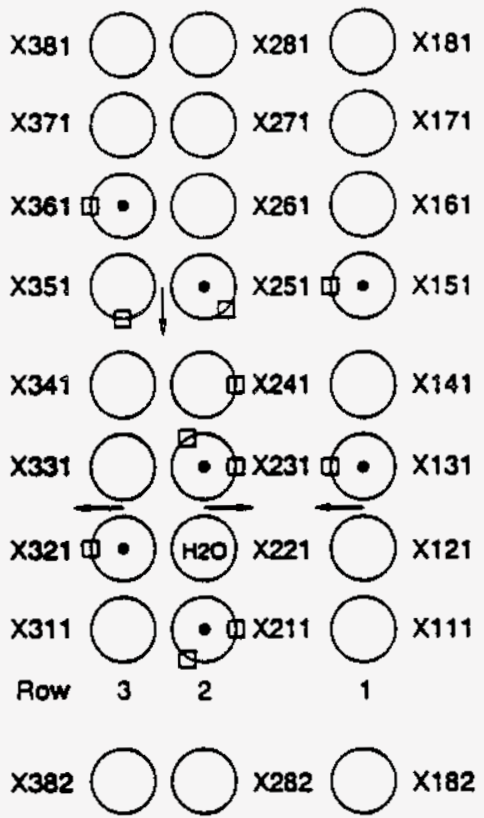

$\times 372 \bigcirc \times 272 \bigcirc \times 172$

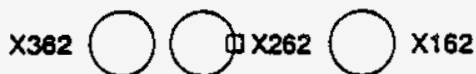

$\times 352 \bigcirc \times 252$ Q
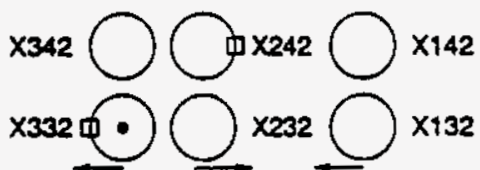

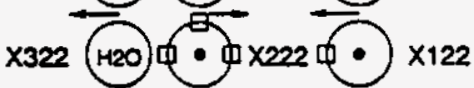
$x 312 \bigcirc \times 212 \bigcirc \times 112$
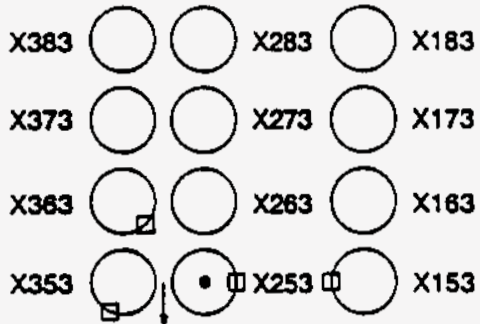

Tier 3

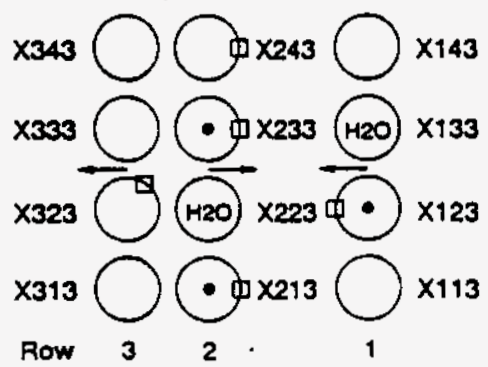

$\times 384 \bigcirc \times 284 \bigcirc \times 184$

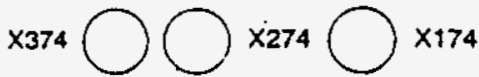

$\times 364 \bigcirc \times 264 \bigcirc \times 164$

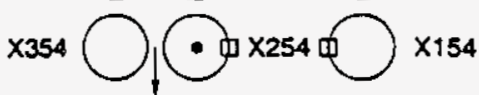

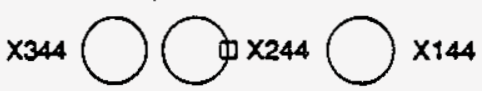

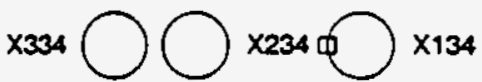

x324巾@ $\bigcirc \times 224 \bigcirc \times 124$

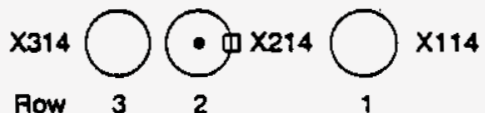

Row 32

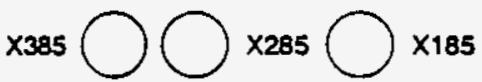

$\times 375 \bigcirc \times 275 \bigcirc \times 175$

x365 $\bigcirc \times 265 \bigcirc \times 165$

x355 ๑中x255中 x155

x345巾 $\bigcirc \times 245 \bigcirc \times 145$

x335中 $\bigcirc \times 235 \bigcirc \times 135$

x325 @ $\bigcirc 225$ 中 $\times 125$

x315 $\bigcirc_{\text {Row }} \times 215 \bigcirc_{1} \times 115$

$\times 386 \bigcirc \times 286 \bigcirc \times 186$

$\times 376 \bigcirc \times 276 \bigcirc \times 176$

$\times 366 \bigcirc \times 266 \bigcirc \times 166$

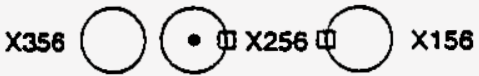

X346吊 $\bigcirc 246 \bigcirc \times 146$

$\times 336$ 乐 $\bigcirc 236$ 田 $\times 136$

$\times 326 \bigcirc \times 126$

$\times 316 \bigcirc_{\text {Row }} \bigcap_{2} \times 216 \bigcirc_{1} \times 116$

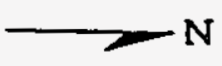

Tier 4

Tier 5

Tier 6

Figure 3. Rack storage chum location cliagram. Drum number indicates Test-Row-Bay-Tier. 
WHC-SD-WM-TRP-233 REV. O Thermocouple Tree
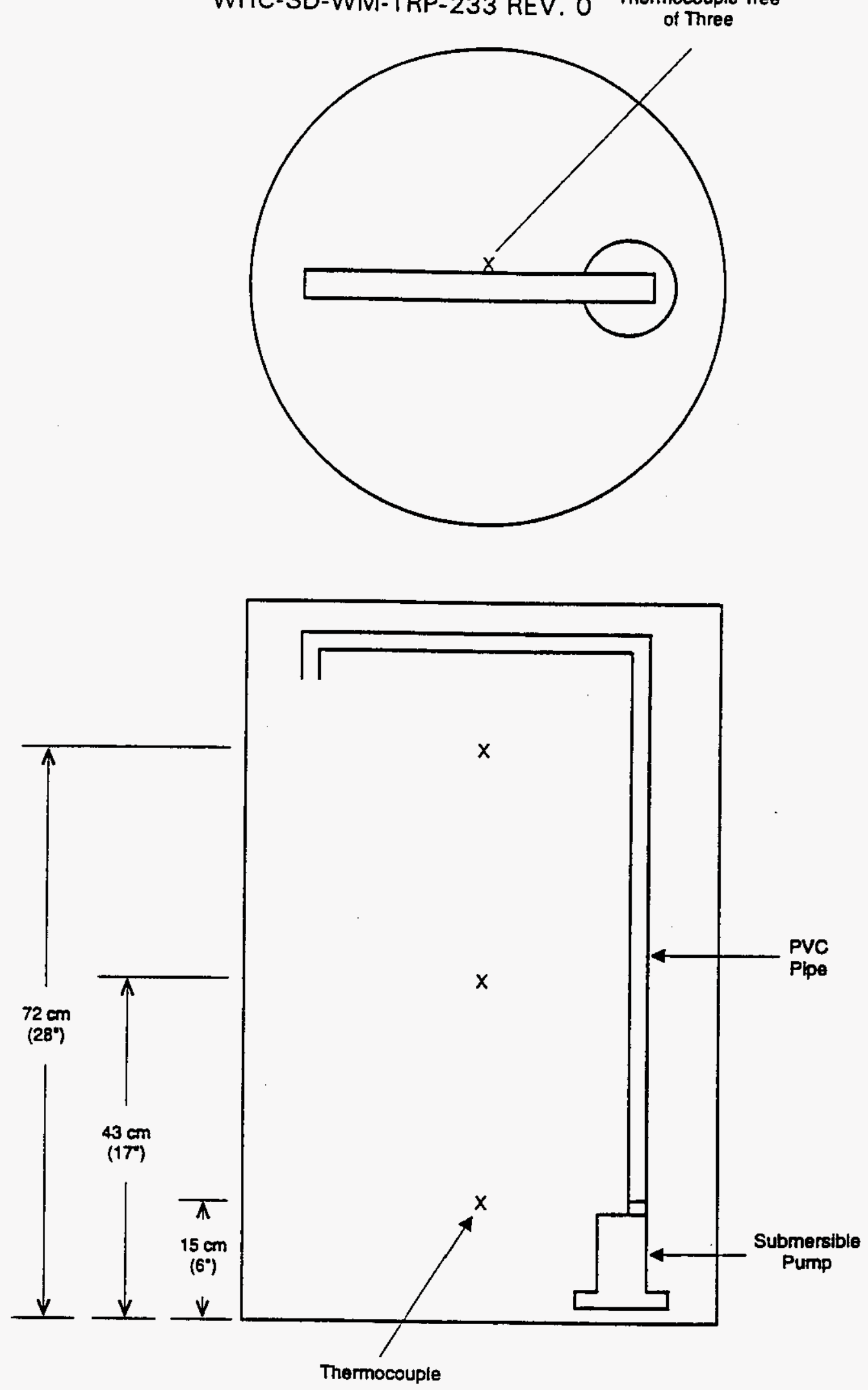

Figure 4. Water-filled calorimeter drum 


\subsubsection{Standard Load Drums}

Each of the 140 drums that contain combustible materials will be loaded in the same manner. Each drum will be loaded with the standard combustible load of $25.9 \mathrm{~kg}(57 \mathrm{lb})$ [Rhodes et al., 1995 ) and a noncombustible load of $31.7 \mathrm{~kg}(70 \mathrm{lb})$ for an overall loading of $57.6 \mathrm{~kg}(127 \mathrm{lb})$ in each drum. Based on these values and the breakdown of the materiais [Boothe, 1994], the average contents will be composed of the following:

\begin{tabular}{lll} 
Rubber & $2.7 \mathrm{~kg}(6.0 \mathrm{lb})$ & 4.7 percent \\
Plastic & $13.4 \mathrm{~kg}(29.5 \mathrm{lb})$ & 23.3 percent \\
Paper & $6.6 \mathrm{~kg}(14.5 \mathrm{lb})$ & 11.5 percent \\
Cotton & $3.2 \mathrm{~kg}(7.0 \mathrm{lb})$ & 5.6 percent \\
Steel & $31.7 \mathrm{~kg}(70 \mathrm{lb})$ & 55.0 percent \\
\hline Total & $57.6 \mathrm{~kg}(127 \mathrm{lb})$ & 100 percent
\end{tabular}

Based on the above described average combustible loading, a "standard" combustible load was developed for use in the Phase 1 program and will be also used for the Phase 2 testing. The combustible drum contents will consist of the following commercially available items which simulate the actual drum contents:

- Rubber - The rubber material is simulated using neoprene rubber sheets that will be approximately $0.32 \mathrm{~cm}(0.125$ in.) thick and approximately $15 \mathrm{~cm}(6 \mathrm{in.}) \times 30 \mathrm{~cm}(12$ in.).

- Plastic - The plastic commodities consist of 50 percent by weight of 6-mil polyethylene plastic bags and 50 percent by weight of 10 -mil polyethylene sheet.

- Paper - The paper consists of $1 / 3$ by weight of brown paper sheet $(61 \mathrm{~cm}$ (24 in.) wide rolls), $1 / 3$ by weight of bond paper $(22 \times 28 \mathrm{~cm}$ $(8.5 \times 11 \mathrm{in})$.$) , and 1 / 3$ by weight of cut-up bond paper $(11 \times 14 \mathrm{~cm}$ $(4.25 \times 5.5$ in. $))$, which simulates filter paper.

- Cotton - The cotton consists of 57 percent by weight of cotton fabric and 43 percent by weight of cotton towels.

Table 1 provides a breakdown of the combustible contents for the "standard" combustible load, and Table 2 provides the procedure for its loading into the drums. The loading procedure requires that materials such as the brown paper, the plastic sheet, and the cotton fabric will be unfolded and placed into the drums. This combustible loading will require compression as it is placed into the drums so that the entire load can be placed in the drum. 
Table 1. Standard Combustible Loading

\begin{tabular}{|c|c|}
\hline \multicolumn{2}{|c|}{ COMBUSTIBLE CONTENTS } \\
\hline MATERIAL & STANDARD LOADING \\
\hline Plastic bags & $6.7 \mathrm{~kg}(14.8 \mathrm{lb})$ \\
\hline 10 -mil plastic sheet & $6.7 \mathrm{~kg}(14.8 \mathrm{lb})$ \\
\hline Rubber, $15 \times 30 \mathrm{~cm}(6 \times 12 \mathrm{in})$. & $2.7 \mathrm{~kg}(6.0 \mathrm{lb})$ \\
\hline Brown paper & $2.2 \mathrm{~kg}(4.8 \mathrm{lb})$ \\
\hline Bond paper, $22 \times 28 \mathrm{~cm}(8.5 \times 11 \mathrm{in})$. & $2.2 \mathrm{~kg}(4.8 \mathrm{lb})$ \\
\hline Cut paper & $2.2 \mathrm{~kg}(4.8 \mathrm{lb})$ \\
\hline Cotton towels & $1.4 \mathrm{~kg}(3.0 \mathrm{lb})$ \\
\hline Cotton fabric & $1.8 \mathrm{~kg}(4.0 \mathrm{lb})$ \\
\hline Total & $25.9 \mathrm{~kg}(57.0 \mathrm{lb})$ \\
\hline
\end{tabular}

Table 2. Standard Loading - Into Drum

\begin{tabular}{|c|c|}
\hline ORDER STARTING AT THE BOTTOM & MATERIAL \\
\hline 1 & $1 / 3$ of the brown paper \\
\hline 2 & $1 / 3$ of the 10 -mil plastic \\
\hline 3 & $1 / 3$ of the bond paper \\
\hline 4 & $1 / 3$ of the towels \\
\hline 5 & $1 / 3$ of the bags \\
\hline 6 & $1 / 3$ of the cut paper \\
\hline 7 & $1 / 3$ of the fabric \\
\hline 8 & $1 / 3$ of the rubber \\
\hline
\end{tabular}

in addition to the "standard" combustible load, the noncombustible contents will be composed of $5.1 \mathrm{~cm}(2 \mathrm{in}$.) round carbon steel stock. Three pieces, each $66 \mathrm{~cm}$ (26 in.) long, will be placed vertically and evenly spaced along the inside wall of the drum. The primary purpose of the steel is to simulate the typical weight 
of the noncombustible contents. This weight constitutes a significant factor in testing rack, pallet, and drum stability.

Each drum will be loaded as follows (see Appendix B for checklist):

(1) Insert three steel bars vertically along the sides of the drums;

(2) Place one-third of the standard load into the drum in the order shown in Table 2;

(3) Place one-third of the standard load into the drum in the order shown in Table 2; and

(4) Place final one-third of the standard load into the drum in the order shown in Table 2.

Upon completion of the loading, the drum will be weighed to the nearest $\pm 0.09 \mathrm{~kg}(0.2 \mathrm{lb})$ accuracy. The drum ID number and weight will be recorded on the Drum Data Sheet. All drums will be positioned on the racks per the drawings of Appendix A. The drums instrumented for internal pressure measurements will be leak tested to ensure the integrity of the drum. The drums will be pressurized to $34.5 \mathrm{kPa}(5 \mathrm{psig})$ for a period of 5 minutes. After 5 minutes the internal pressure shall not be below $31.0 \mathrm{kPa}(4.5 \mathrm{psig})$.

\subsection{INSTRUMENTATION}

Figure 2 provides an overall sketch of the test instrumentation. The instrumentation will consist of the following:

(1) Thirty six (36) type $K$ thermocouples placed on three thermocouple trees. The thermocouples will be spaced $61 \mathrm{~cm}$ (24 inches) apart starting at $122 \mathrm{~cm}$ (48 inches) above the floor. The trees will be placed as shown in Figures 1 and 2 . The thermocouples will provide temperature measurements to the ceiling which can be used for flame height verification;

(2) Approximately 100 type $K$ thermocouples placed on the exterior surfaces of selected drums. This will provide a measure of the drum wall temperatures at specific locations;

(3) Approximately 40 type $K$ thermocouples placed on the surfaces of the racks, containment pans, and mesh. These temperatures will provide data concerning the exposure of the items to the fire;

(4) Internal pressure of 24 selected drums will be monitored using $0-50$ psig transducers (Setra, model no. 206). The pressure measurements will provide a measure of venting or lid loss during the test. Each 
pressure transducer calibration will be verified before the test using a pressure calibrator (Beamex, model no. PG105);

(5) Sixteen (16) heat flux measurements using $200 \mathrm{~kW} / \mathrm{m}^{2}$ total heat flux calorimeters (Medtherm, model no. 64-20-5B-19) will be made. Each instrument, which has a stated accuracy of \pm 3 percent, will be provided with a certified calibration. The heat flux transducers will be placed at various locations per Figure 2 to provide representative local heat flux measurements from the fire; and

(6) Twelve (12) type $K$, sheathed thermocouples ( 3 per drum) will be placed inside the water-filled drums to provide a measure of the temperature increase inside each drum.

The data acquisition system for the thermocouples and heat flux transducers will scan and record data at a 1 scan per second rate. The pressure transducers will be scanned and recorded every 20 milliseconds $(50 \mathrm{~Hz})$ rate. test.

All instrumentation will be calibrated and/or functionally verified prior to the

\subsection{FIRE SOURCE}

The ignition source for the rack storage test will be a $16.6 \mathrm{~m}^{2}\left(179 \mathrm{ft}^{2}\right)$ diesel fuel pool fire positioned under a portion of the rack array as shown in Figure 1. The fuel spill area will consist of a watertight dam approximately $3.7 \times 4.3 \mathrm{~m}$ $(12 \times 14 \mathrm{ft})$ utilizing $10 \times 10 \mathrm{~cm}(4 \times 4 \mathrm{in}$.) angle bolted to the concrete floor. $A$ layer of water, approximately $2.5 \mathrm{~cm}$ (1 in.) deep will be poured into the spill fire area and approximately $208 \mathrm{~L}(55 \mathrm{gal})$ of diesel fuel will then be spilled on top of the water. It is estimated that this quantity of fuel will provide a burn time of approximately five minutes. The 5 minute burn time was chosen based on the Phase 1 work which showed that in the engulfing fires the drums experienced lid loss in less than 3 minutes. Additionally, $201 \mathrm{~L}$ of fuel represents a conservatively high value for a credible fuel spill.

\subsection{TEST TERMINATION}

Data collection and video recording will continue after the completion of the 5-minute diesel fuel fire. During this time the extent of fire propagation will be assessed. The test will be terminated upon the following:

(1) all fires have self extinguished, or

(2) the test director has determined that further fire propagation is unlikely and further drum seal failures are improbable. 
Upon termination of the test, extinguishment of all burning items such as expelled loading materials will be performed.

\subsection{DOCUMENTATION}

The fire test will be documented using two VHS video camera and color photographs. Complete photographic documentation will be taken of pre-fire test arrangement, during the test and post-test arrangement.

\subsection{TEST PROCEDURES}

In general, the following procedures will be followed:

(1) loading, weighing and identification of test drums;

(2) instrumenting test drums as required;

(3) placement of test drums into rack array and development of drum ID versus position diagram;

(4) instrumentation of racks, pans, etc.;

(5) instrumentation and/or connection of pressure transducers, heat flux transducers, and thermocouples;

(6) verification of instrumentation after connection to data acquisition system;

(7) creation of fuel spill;

(8) verification of documentation;

(9) start of test;

(10) extinguishment as required; and

(11) post-test damage assessments.

The Rack Storage Pre-test Check List is included as Appendix C and the Test Procedure Check List is included as Appendix D. All procedures will be carried out by SwRI personnel and overseen by HAl test engineers. The test engineer or appointed designee will verify and initialize all checklists and data sheets. Prior to the test being conducted, the HAl test supervisor will verify that all items have been completed.

\subsection{TEST DATA}

The following data will be obtained:

(1) drum wall surface temperatures;

(2) fire exposure temperatures;

(3) rack, pan, and mesh temperatures;

(4) heat flux measurements; 
(5) pressure measurements;

(6) weight loss of drums (before and after weights);

(7) visual observations with respect to the following:

(a) lid loss (number and location),

(b) explosion of contents (amount and location).

(c) lid venting (lids bent but not expelled) (number and location),

(d) toppled drums (number and location),

(e) structural performance of rack system, i.e., warpage, distortion, collapse, etc., and

(8) estimates of charring/burning of contents of each drum.

Data collection pertaining to drums and fuel content will be recorded on the Drum Data Sheets (Appendix B). There will be a data sheet for each drum used in the test. As drums are removed from the test site, they will be re-painted (as needed) with the appropriate drum identification number. The drums will then be weighed and inspected. Post-test photographs and/or video will be taken of each drum and its contents.

Each section of the rack system will be assessed and documented on a Posttest Rack Assessment Sheet as shown in Appendix E. Deflections in the rack structure due to twisting or bending will be measured with respect to the original position. Post-test photographs and video will be taken of each section of the structure.

All instrument data (converted to physical units via the established calibration constants) will be plotted with respect to time. Tabular listings of all data will be supplied. All data obtained will also be provided on an IBM compatible computer disk. 


\subsection{EXPERIMENTAL PALLET STORAGE TEST}

\subsection{OVERALL}

The pallet storage test will be conducted "inside" such that the effects of wind, rain, etc. will be negated. The fire test facility to be used is located at Southwest Research Institute in San Antonio, Texas. The building is $12.2 \mathrm{~m}$ (40 $\mathrm{ft})$ by $18.3 \mathrm{~m}(60 \mathrm{ft})$ by $9.1 \mathrm{~m}(30 \mathrm{ft}$ high). The building will be naturally ventilated during the test through vents located at floor level along the periphery of the building and exhaust vents in the roof and high on the walls. All doors to the building will be closed during the test. Post test clearing of smoke from the building can be accelerated via an exhaust fan. This fan (which is located high on the south wall) will not be used during the test to avoid nonuniform air flow through the building and, subsequent, deflection of the pool fire.

Figure 5 provides an overall view of the proposed test arrangement for the pallet storage configuration. In general, the test configuration will consist of three rows of storage with each row being three tiers high. There will be four drums per pallet and four pallets per row (i.e., four in each tier). Two rows will consist of wooden pallets and one row will consist of steel pallets. The rows of pallets will be separated by $0.91 \mathrm{~m}(3 \mathrm{ft})$ aisles. Within a row, pallets will be positioned flush with one another.

\subsection{PALLETS}

The pallets will be similar to those proposed for use at WHC. WHC/LATA will provide exact specifications and provide the pailets to the test laboratory. Central Waste Complex practices for banding drums is described as follows:

1) A 0.25 inch by 0.02 inch, high tensile steel strapping (e.g. McMasterCarr, \#2071T61) will be used for banding. There is no specification for band tightness, except that the bands are secured "good and tight."

2) The first band is placed horizontally around the four drums just below the top (i.e., within the top third of the drum).

3) The second band is placed over the tops of two drums and through the pallet.

4) The third band is similarly placed over the adjacent two drums and through the pallet. 
TH Li PAGE INTENTIONALLY
LEFT BLANK 

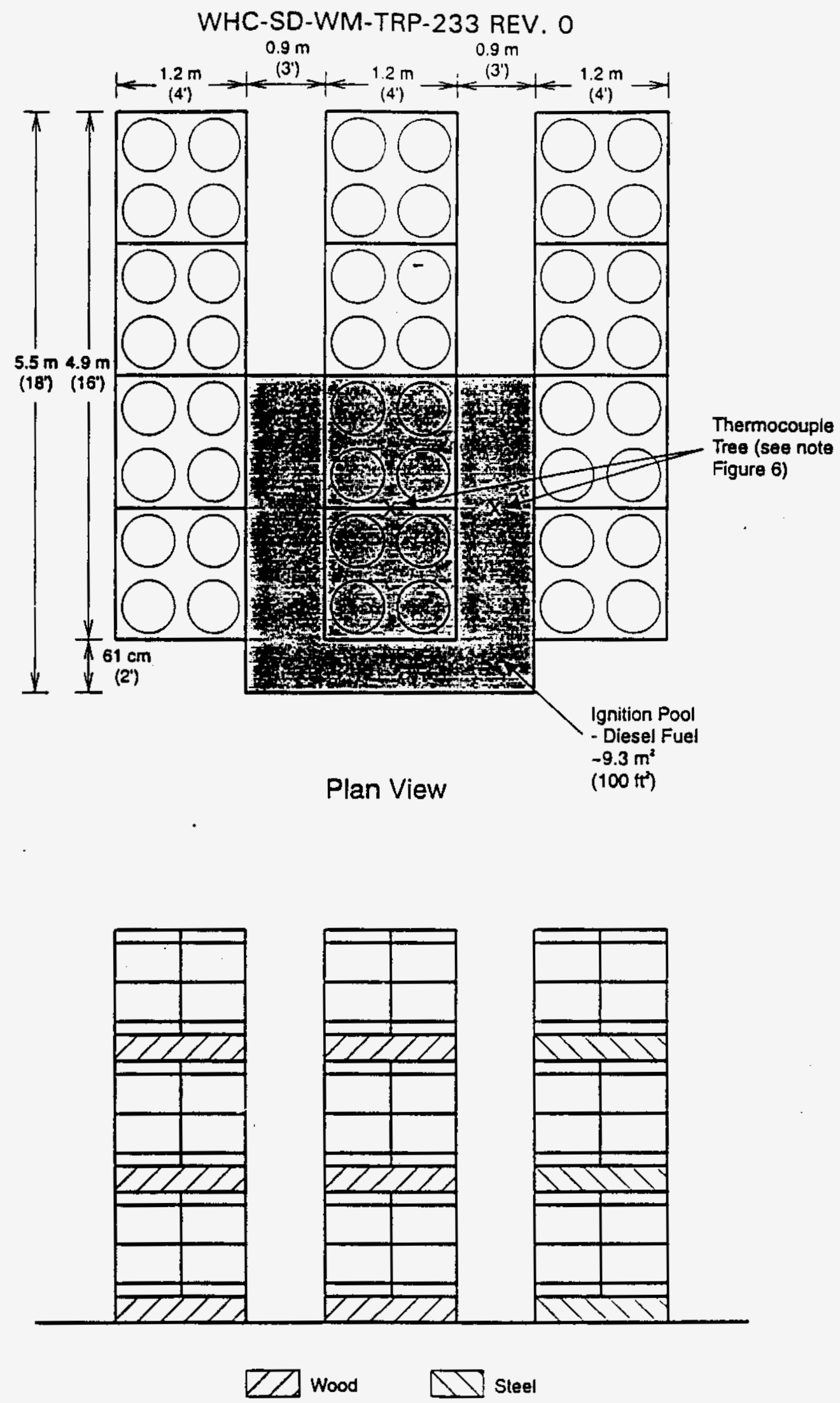

Side View

Figure 5. Overall pallet storage experimental setup

C-4-2 
Although this is the practice at CWC, there are drums stored at WHC in which banding consists of only one band horizontally around the four drums just below the top. Since excluding the two top bands represents a worse case scenario in terms of lid loss and pallet stability, this will be the method used in this test.

\subsection{DRUMS}

A total of 144 drums will be used in this test. The drums will be new $17-\mathrm{H}$, 55-gallon (UN IA1) drums, painted black. The drums will be sealed using removable lids with styrene-butadiene sponge rubber gaskets and metal closure rings, rounded-section type. After loading, the closure rings will be tightened, per WHC specification [Carver, 1993] to $54 \mathrm{~J}(40 \mathrm{ft}-\mathrm{lb})$ torque.

Close attention must be given to the method of torquing the closure rings. Depending on the torquing method used, a range of tightness can be achieved with the closure ring still measuring $54 \mathrm{~J}(40 \mathrm{ft}-(\mathrm{b})$ torque. For example, if one tightens the closure bolt with a torque wrench, $54 \mathrm{~J}$ can be achieved with a $5 \mathrm{~cm}$ separation between the ends of the closure ring. However, this original torque value is typically from the bolt head binding against the closure ring surface rather than the tightness of the ring around the lid and drum. Consequently, when the bolt is backed off and then tightened again, $54 \mathrm{~J}$ is not achieved until the separation between the ends of the closure ring is smaller, such as $0.6 \mathrm{~cm}$. Therefore, depending on the person and method used, a significant range of closure ring tightness can be achieved.

Therefore, all lids are to be secured by the same technician using the method of repetitively backing the bolt out and then tightening to $54 \mathrm{~J}$ torque. This procedure was used in the Phase 1 testing and assures that the rings are torqued to the same specification. As a result of using this method, it is believed that the drums tested will probably be tightened more than the existing drums at WHC. The net effect of this is not clear. The fire exposed drums may require a higher pressure for lid loss or may not lose lids due to the closure ring securing the lids righter to the drum. On the other hand, the tighter closure ring may result in the lid blowing earlier and more violently. This may occur due to a more rapid buildup of pressure in the drum whereas a looser closure ring may allow sufficient gas to vent from the drum, thus avoiding lid loss.

In this test, all 144 of the drums will represent typical WHC loaded drums and contain the standard load [Rhodes et al., 1995]. Figure 6 shows the layout of drums in the rack storage system along with the instrumentation. As each drum is prepared, it will be identified with a painted number according to the drum location diagram (Figure 7) and all preparation will be documented on Drum Data Sheets (Appendix B). 
WHC-SD-WM-TRP-233 REV. 0
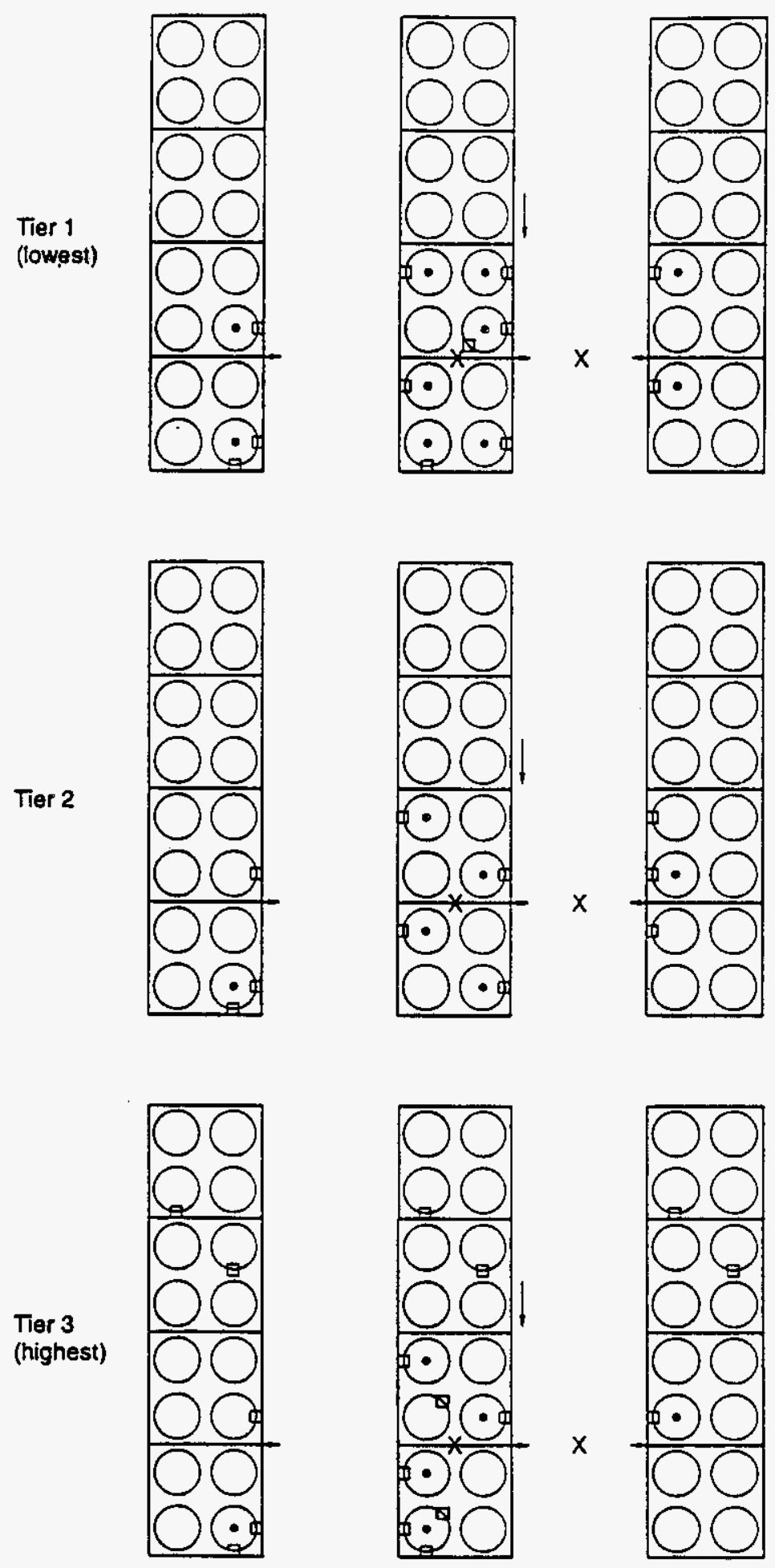

Notes:

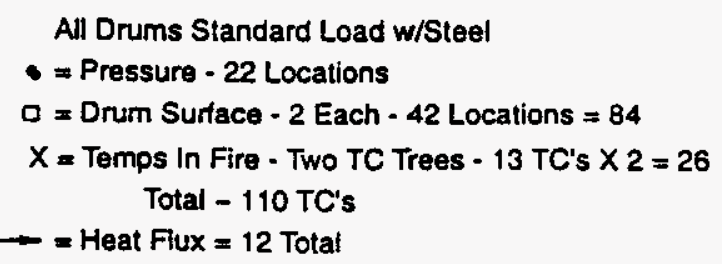

Figure 6. Pallet storage experimental setup and instrumentation layout C- $4-4$ 
WHC-SD-WM-TRP-233 REV. 0
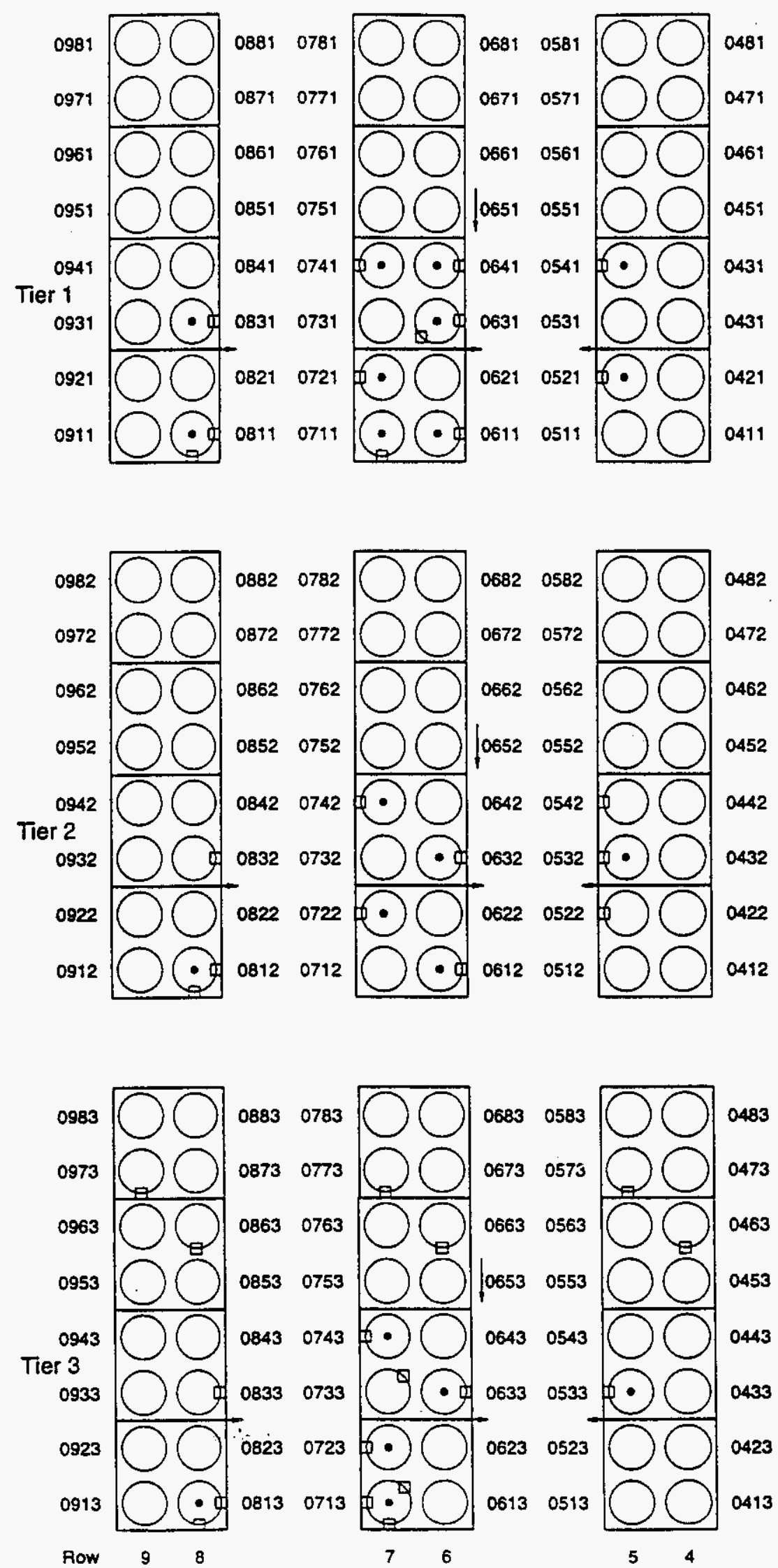

Figure 7. Pallet storage drum location diagram. Drum number indicates Test-Row-Bay-Tier. 
Each drum will be will be loaded in the same manner with the standard combustible load of $25.9 \mathrm{~kg} \mathrm{(57} \mathrm{lb)} \mathrm{[Rhodes} \mathrm{et} \mathrm{al.,} \mathrm{1995]} \mathrm{and} \mathrm{a} \mathrm{noncombustible}$ load of $31.7 \mathrm{~kg}(70 \mathrm{lb})$ for an overall loading of $57.6 \mathrm{~kg}(127 \mathrm{lb})$ in each drum. Based on these values and the breakdown of the materials [Boothe, 1994], the average contents will be composed of the following:

\begin{tabular}{lll} 
Rubber & $2.7 \mathrm{~kg}(6.0 \mathrm{lb})$ & 4.7 percent \\
Plastic & $13.4 \mathrm{~kg}(29.5 \mathrm{lb})$ & 23.3 percent \\
Paper & $6.6 \mathrm{~kg}(14.5 \mathrm{lb})$ & 11.5 percent \\
Cotton & $3.2 \mathrm{~kg}(7.0 \mathrm{lb})$ & 5.6 percent \\
Steel & $-31.7 \mathrm{~kg}(70 \mathrm{lb})$ & 55.0 percent \\
\hline Total & $57.6 \mathrm{~kg}(127 \mathrm{lb})$ & 100 percent
\end{tabular}

Based on the above described average combustible loading, a "standard" combustible load was developed for use in the Phase 1 program and will be also used for the Phase 2 testing. The combustible drum contents will consist of the following commercially available items which simulate the actual drum contents:

- Rubber - The rubber material is simulated using neoprene rubber sheets that will be approximately $0.32 \mathrm{~cm}(0.125 \mathrm{in}$.$) thick and$ approximately $15 \mathrm{~cm}(6 \mathrm{in.}) \times 30 \mathrm{~cm}(12 \mathrm{in.})$.

- Plastic - The plastic commodities consist of 50 percent by weight of 6-mil polyethylene plastic bags and 50 percent by weight of 10 -mil polyethylene sheet.

- Paper - The paper consists of $1 / 3$ by weight of brown paper sheet (61 $\mathrm{cm}$ (24 in.) wide rolls), $1 / 3$ by weight of bond paper $(22 \times 28 \mathrm{~cm}$ $(8.5 \times 11$ in. $))$, and $1 / 3$ by weight of cut-up bond paper $(11 \times 14 \mathrm{~cm}$ $(4.25 \times 5.5$ in.)), which simulates filter paper.

- Cotton - The cotton consists of 57 percent by weight of cotton fabric and 43 percent by weight of cotton towels.

Table 3 provides a breakdown of the combustible contents for the "standard" combustible load, and Table 4 provides the procedure for its loading into the drums. The loading procedure requires that materials such as the brown paper, the plastic sheet, and the cotton fabric will be unfolded and placed into the drums. This combustible loading will require compression as it is placed into the drums so that the entire load can be placed in the drum. 
Table 3. Standard Combustible Loading

\begin{tabular}{|c|c|}
\hline \multicolumn{2}{|c|}{ COMBUSTIBLE CONTENTS } \\
\hline MATERIAL & STANDARD LOADING \\
\hline Plastic bags & $6.7 \mathrm{~kg}(14.8 \mathrm{lb})$ \\
\hline 10 -mil plastic sheet & $6.7 \mathrm{~kg}(14.8 \mathrm{lb})$ \\
\hline Rubber, $15 \times 30 \mathrm{~cm}(6 \times 12 \mathrm{in})$. & $2.7 \mathrm{~kg}(6.0 \mathrm{lb})$ \\
\hline Brown paper & $2.2 \mathrm{~kg}(4.8 \mathrm{lb})$ \\
\hline Bond paper, $22 \times 28 \mathrm{~cm}(8.5 \times 11 \mathrm{in})$. & $2.2 \mathrm{~kg}(4.8 \mathrm{lb})$ \\
\hline Cut paper & $2.2 \mathrm{~kg}(4.8 \mathrm{lb})$ \\
\hline Cotton towels & $1.4 \mathrm{~kg}(3.0 \mathrm{lb})$ \\
\hline Cotton fabric & $1.8 \mathrm{~kg}(4.0 \mathrm{lb})$ \\
\hline Total & $25.9 \mathrm{~kg}(57.0 \mathrm{lb})$ \\
\hline
\end{tabular}

Table 4. Standard Loading - Into Drum

\begin{tabular}{|c|c|}
\hline ORDER STARTING AT THE BOTTOM & MATERIAL \\
\hline 1 & $1 / 3$ of the brown paper \\
\hline 2 & $1 / 3$ of the 10 -mil plastic \\
\hline 3 & $1 / 3$ of the bond paper \\
\hline 4 & $1 / 3$ of the towels \\
\hline 5 & $1 / 3$ of the bags \\
\hline 6 & $1 / 3$ of the cut paper \\
\hline 7 & $1 / 3$ of the fabric \\
\hline 8 & $1 / 3$ of the rubber \\
\hline
\end{tabular}

In addition to the "standard" combustible load, the noncombustible contents will be composed of $5.1 \mathrm{~cm}(2 \mathrm{in}$.) round carbon steel stock. Three pieces, each $66 \mathrm{~cm}$ (26 in.) long, will be placed vertically and evenly spaced along the inside wall of the drum. The primary purpose of the steel is to simulate the typical weight of the noncombustible contents. This weight constitutes a significant factor in testing rack, pallet, and drum stability. 
Each drum will be loaded as follows (see Appendix B for checklist):

(1) Insert three steel bars vertically along the sides of the drums;

(2) Place one-third of the standard load into the drum in the order shown in Table 2;

(3) Place one-third of the standard load into the drum in the order shown in Table 2; and

(4) Place final one-third of the standard load into the drum in the order shown in Table 2.

Upon completion of the loading, the drum will be weighed to the nearest $\pm 0.09 \mathrm{~kg}(0.2 \mathrm{lb})$ accuracy. The drum ID number and weight will be recorded on the Drum Data Sheet. The drums instrumented for internal pressure measurements will be leak tested to ensure the integrity of the drum. The drums will be pressurized to $34.5 \mathrm{kPa}$ ( $5 \mathrm{psig}$ ) for a period of 5 minutes. After 5 minutes the internal pressure shall not be below $31.0 \mathrm{kPa}(4.5 \mathrm{psig})$.

\subsection{INSTRUMENTATION}

Figure 6 provides an overall sketch of the test instrumentation. The instrumentation will consist of the following:

(1) Twenty-six (26) type $K$ thermocouples placed on two thermocouple trees. The thermocouples will be spaced $61 \mathrm{~cm}(24$ inches) apart starting at $122 \mathrm{~cm}$ (48 inches) above the floor. The trees will be placed as shown in Figures 5 and 6 . The thermocouples will provide temperature measurements to the ceiling which can be used for flame height verification;

(2) Eighty four (84) type $K$ thermocouples placed on the exterior surfaces of selected drums. This will provide a measure of the drum wall temperatures at specific locations;

(3) Internal pressure of 22 selected drums will be monitored using 0-50 psig transducers (Setra, model no. 206). The pressure measurements will provide a measure of venting or lid loss during the test. Each pressure transducer calibration will be verified before the test using a pressure calibrator (Beamex, model no. PG 105);

(4) Twelve (12) heat flux measurements using $200 \mathrm{~kW} / \mathrm{m}^{2}$ total heat flux calorimeters (Medtherm, model no. 64-20-5B-19) will be made. Each instrument, which has a stated accuracy of \pm 3 percent, will be provided with a certified calibration. The heat flux transducers will be placed at various locations per Figure 2 to provide representative local heat flux measurements from the fire; and 
The data acquisition system for the thermocouples and heat flux transducers will scan and record data at a 1 scan per second rate. The pressure transducers will be scanned and recorded every 20 milliseconds $(50 \mathrm{~Hz})$ rate. test.

All instrumentation will be calibrated and/or functionally verified prior to the

\subsection{FIRE SOURCE}

The ignition source for the rack storage test will be a $9.3 \mathrm{~m}^{2}\left(100 \mathrm{ft}^{2}\right)$ diesel fuel pool fire positioned under a portion of the rack array as shown in Figure 1.

The fuel spill area will consist of a watertight dam approximately $3.0 \times 3.0 \mathrm{~m}(10 \times$ $10 \mathrm{ft}$ ) utilizing $10 \times 10 \mathrm{~cm}(4 \times 4 \mathrm{in}$.) angle bolted to the concrete floor. A layer of water, approximately $2.5 \mathrm{~cm}$ ( $1 \mathrm{in}$.) deep will be poured into the spill fire area and approximately $135 \mathrm{~L}(35 \mathrm{gal})$ of diesel fuel will then be spilled on top of the water. It is estimated that this quantity of fuel will provide a burn time of approximately five minutes. The 5 minute burn time was chosen based on the Phase 1 work which showed that in the engulfing fires the drums experienced lid loss in less than 3 minutes. Additionaliy, $135 \mathrm{~L}$ of fuel represents a conservatively high value for a credible fuel spill.

\subsection{TEST TERMINATION}

Data collection and video recording will continue after the completion of the 5 -minute diesel fuel fire. During this time the extent of fire propagation will be assessed. The test will be terminated upon the following:

(1) all fires have self extinguished, or

(2) the test director has determined that further fire propagation is unlikely and further drum seal failures are improbable.

Upon termination of the test, extinguishment of all burning items such as expelled loading materials will be performed.

\subsection{DOCUMENTATION}

The fire test will be documented using two VHS video camera and color photographs. Complete photographic documentation will be taken of pre-fire test arrangement, during the test and post-test arrangement. 


\subsection{TEST PROCEDURES}

In general, the following procedures will be followed:

(1) loading, weighing and identification of test drums;

(2) instrumenting test drums as required;

(3) placement of test drums into rack array and development of drum ID versus position diagram;

(4) instrumentation of racks, pans, etc.;

(5) instrumentation and/or connection of pressure transducers, heat flux transducers, and thermocouples;

(6) verification of instrumentation after connection to data acquisition system;

(7) creation of fuel spill;

(8) verification of documentation;

(9) start of test;

(10) extinguishment as required; and

(11) post-test damage assessments.

The Pallet Storage Pre-test Check List is included as Appendix F and the Test Procedure Check List is included as Appendix D. All procedures will be carried out by SwRI personnel and overseen by HAl test engineers. The test engineer or appointed designee will verify and initialize all checklists and data sheets. Prior to the test being conducted, the HAl test supervisor will verify that all items have been completed.

\subsection{TEST DATA}

The following data will be obtained:

(1) drum wall surface temperatures;

(2) fire exposure temperatures;

(3) heat flux measurements;

(4) pressure measurements;

(5) weight loss of drums (before and after weights);

(6) visual observations with respect to the following:

(a) lid loss (number and location),

(b) explosion of contents (amount and location),

(c) lid venting (lids bent but not expelled) (number and location),

(d) toppled drums (number and location),

(e) structural performance of pallets, i.e., warpage, distortion, collapse, etc., and

(7) estimates of charring/burning of contents of each drum. 
Data collection pertaining to drums and fuel content will be recorded on the Drum Data Sheets (Appendix B). There will be a data sheet for each drum used in the test. As drums are removed from the test site, they will be re-painted (as needed) with the appropriate drum identification number. The drums will then be weighed and inspected. Post-test photographs and/or video will be taken of each drum and its contents.

Each section of the pallet system will be assessed and documented on a Post-test Pallet Assessment Sheet as shown in Appendix G. Movement in the pallets due to drum explosions or mechanical failure due to the fire will be measured with respect to the original position. Post-test photographs and video will be taken of each section of the structure. 


\subsection{MODELING}

Prior to the conduct of the testing, the models developed in Phase 1 will be used to predict the outcome of the two array tests. The methodology used to generate the prediction will be documented in sufficient detail to allow an independent analyst to reproduce the results. This will serve as a description of the methodology and an example of its use. 
THIS PAGE INTENTIONALLY LEFT BLANK 


\subsection{DATA REPORTING AND ANALYSIS}

All data collected in these tests (pre-test, test, and post-test data) will be thoroughly analyzed and reported. The following list summarizes the major analyses which are planned at this time. Of course, the test results themselves most often dictate analyses not planned prior to the testing.

1. Conversion of raw data to physical units via the established calibration constants.

2. Plotting of all data channels.

3. Calculation/tabulation of drum damage effects from the test from pre and post test drum data(written and photo/video).

4. Analysis of data, videotape record, test observation records, and post test data to derive a time line of test events (includes lid loss, lid seal failure, contents expulsion, material ignition, flame spread, flame heights, drum movements, etc.).

5. Comparison of overall performance of the drum arrays with the predicted drum array performance.

6. Detailed comparison of individual drum responses in the tests with performance as predicted by the model. These predictions will not be made prior to the test. Calculations will use measured heat fluxes, temperatures, and pressures as appropriate and available from the test itself.

7. Evaluation of the thermal criterion for lid loss with the test data.

8. Evaluate pressures at lid loss and relation to array stability.

9. Evaluate critical temperature of steel for structural failure of rack elements.

10. Evaluate the extent of array fire propagation in the tests against the predicted fire propagation.

11. Evaluation of the degree and distribution of expelled contents from drums. 
THIS PAGE INTENTIONAILY LEFT BLANK 


\subsection{SUMMARY}

Two large scale tests will be conducted consisting of exposing sections of drum storage arrays to an initiating pool fire and documenting results via test measurements and observations of drum responses and the extent of fire propagation beyond the initiating fire. Both a rack storage system and pallet storage arrays will be studied. The tests will be performed and documented in accordance with the standards employed in the peer reviewed fire science literature. The tests will be described in sufficient detail to allow the tests to be replicated by others (if ever required). The instrumentation and data collection methods used will conform to the accuracy and precision established in the fire science literature. Prior to the conduct of the testing, the models developed in Phase 1 will be used to predict the outcome the two array tests. The methodology used to generate the prediction will be documented in sufficient detail to allow an independent analyst to reproduce the results. The Phase 2 work will result in an assessment of the drum array fire propagation model developed in Phase 1, such that it can be used in support of safety analyses in WHC facilities. 
THIS PAGE INTENTIONALLY LEFT BLANK 


\subsection{REFERENCES}

Boothe, G.F. (1994), "Radiological and Chemical Source Terms for Solid Waste Operations, Complex," WHC-SD-W306-TI-001 REV 1, 1994.

Carver, M.R. (1993), "Drums, DOT UN1A2, Solid Material, 114/208/322 Liter Salvage, Galvanized, Steel," HS-V-P0010, Impact Level 3-SQ, Westinghouse Hanford Company, January 20, 1993.

Rhodes, B.T., Beitel, J.J., Gottuk, D.T., Beyler, C.L., and Rosenbaum, E.R. (1995), "Analytical and Experimental Evaluation of Solid Waste Drum Fire Performance," prepared for Westinghouse Hanford Company, Hughes Associates, Inc., Columbia, MD, April 1995. 
THIS PAGE INTENTIONALLY

LEFT BLANK

\section{4}


WHC-SD-WM-TRP-233 REV. 0

Appendix A

Rack Storage

C-A-1 
THIS PAGE INTENTIONALLY LEFT BLANK

\section{4}


LATA DWG-001

DRAWING B: BAY DIMENSIONS

LOCATE gATRET CONivEGTORS

THS SOS

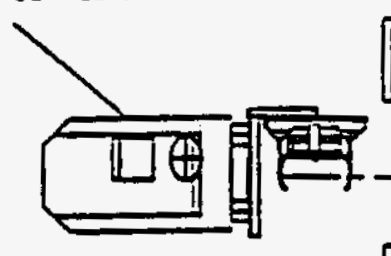

0000

0000

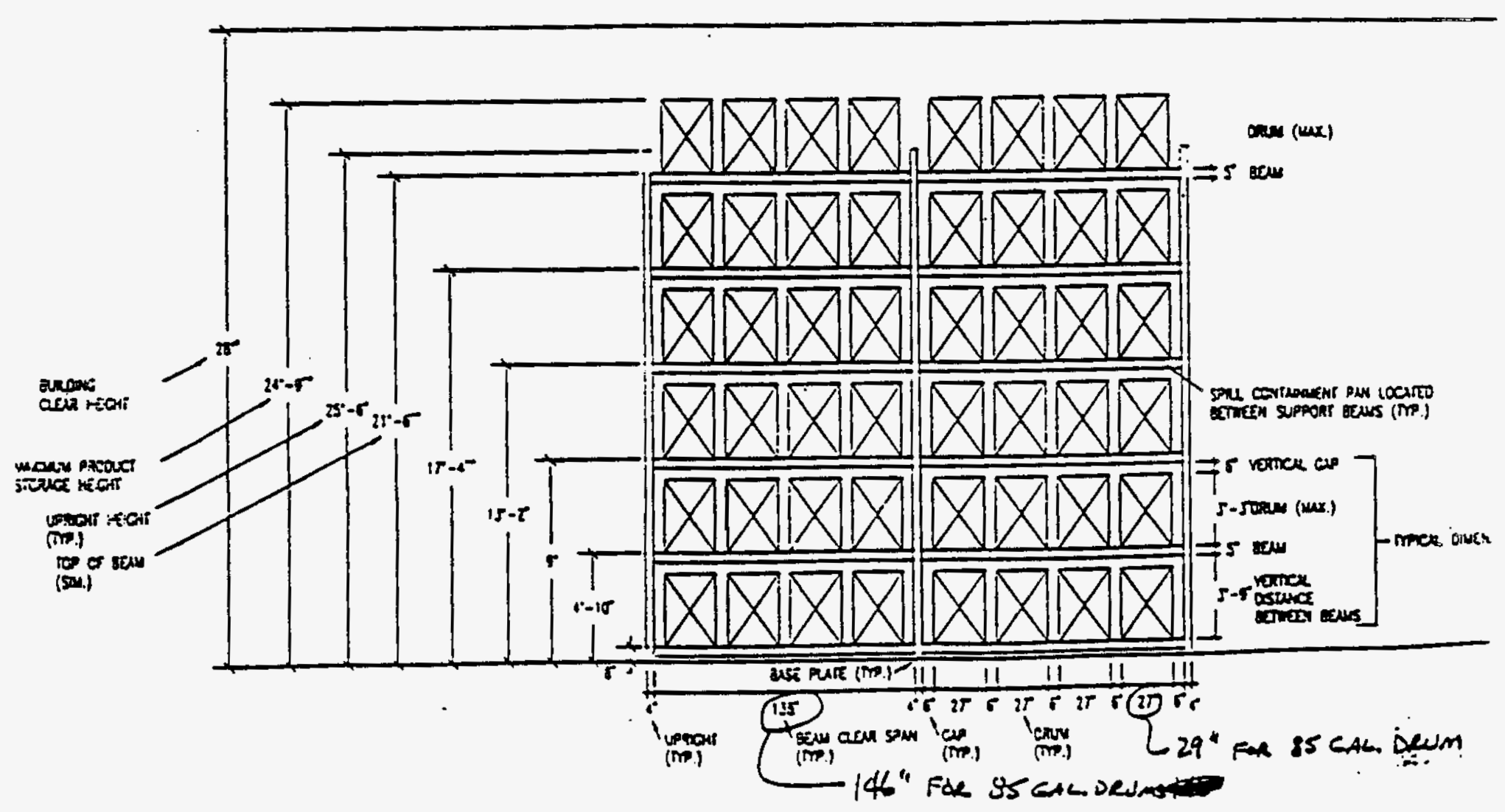


LATA DWG-002

DRAWING C: ROW DIMENSIONS

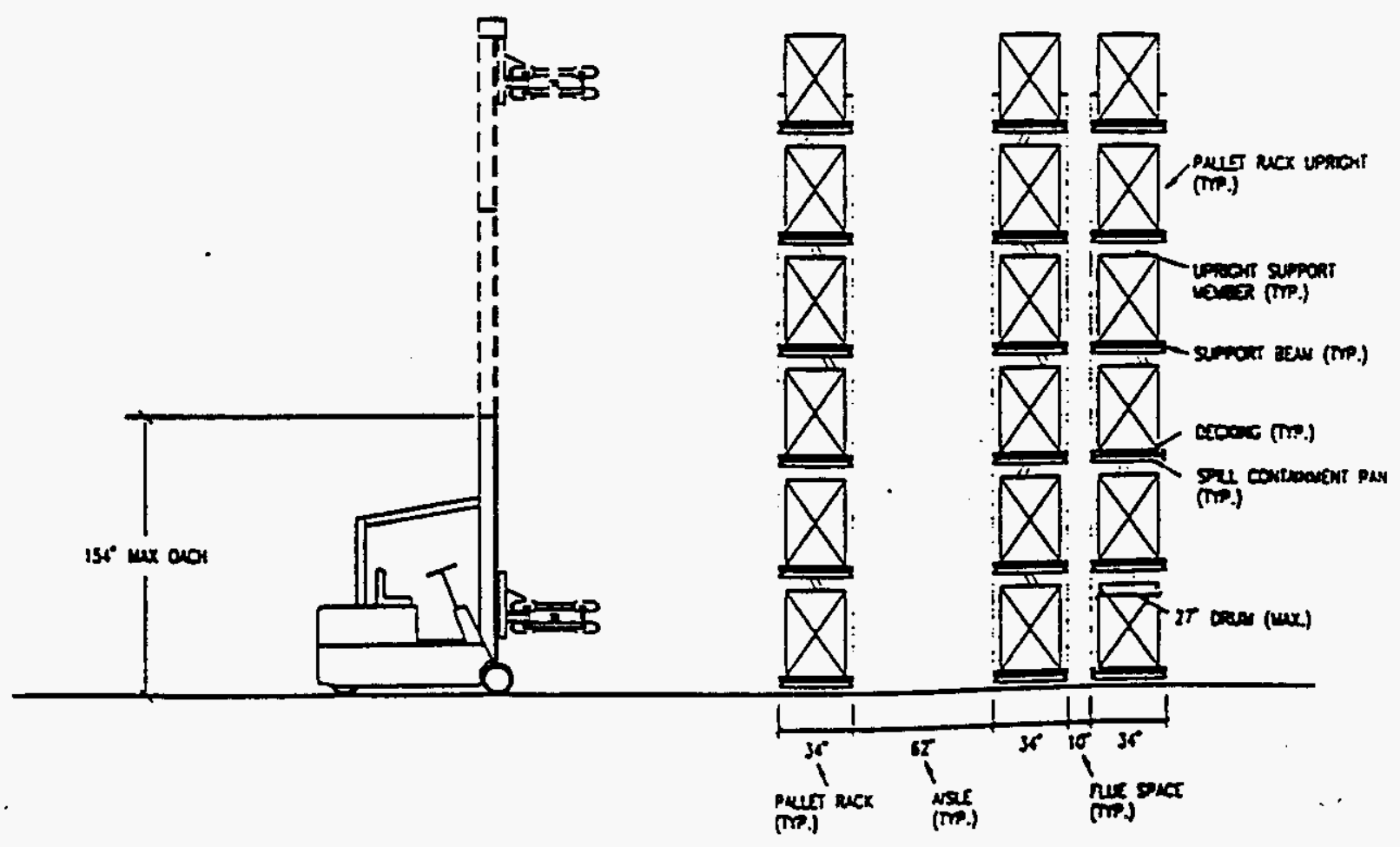


RAYTHEON

HANFORD WASTE STORAGE - WII2

CNA COMPANIES, INC.

MARCH 16, 1995

REV I - 3/17/95

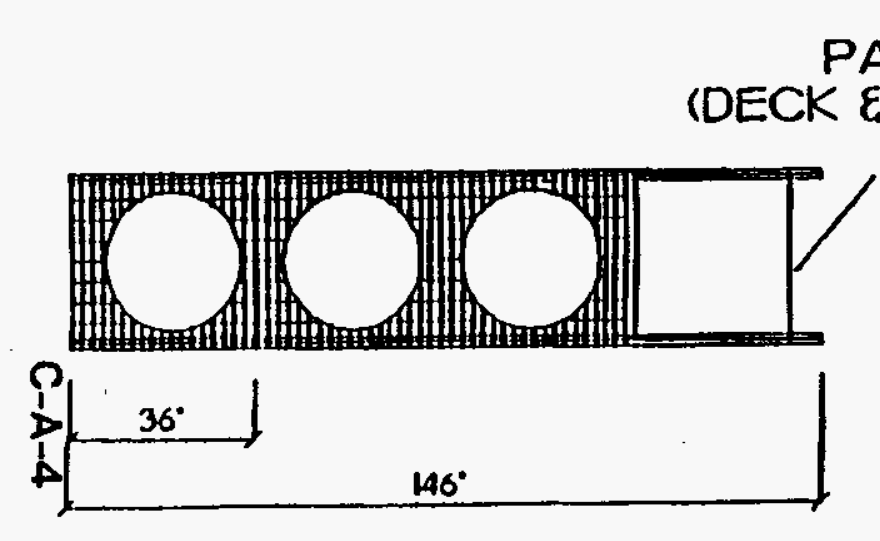

PAN (DECK \& DRUM REMOVED)

(RESTRANTS REMOVED)

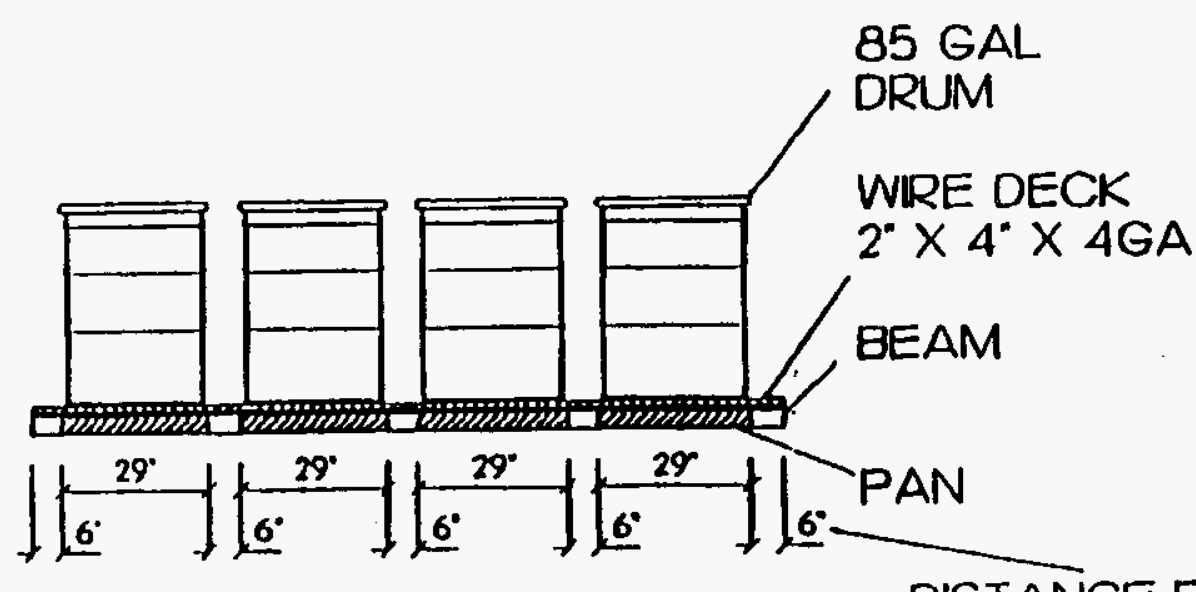

DISTANCE BETWEEN PANS
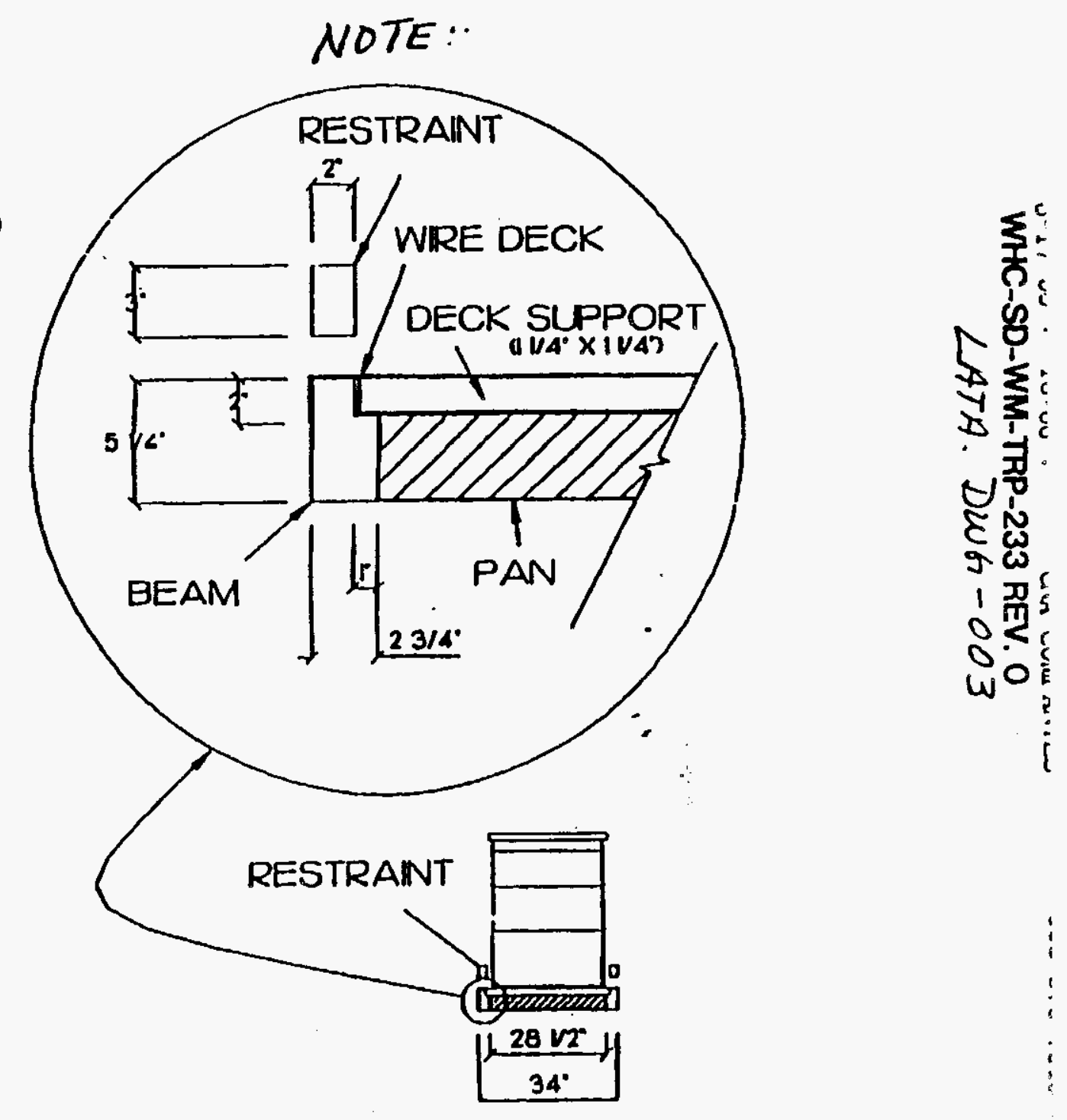


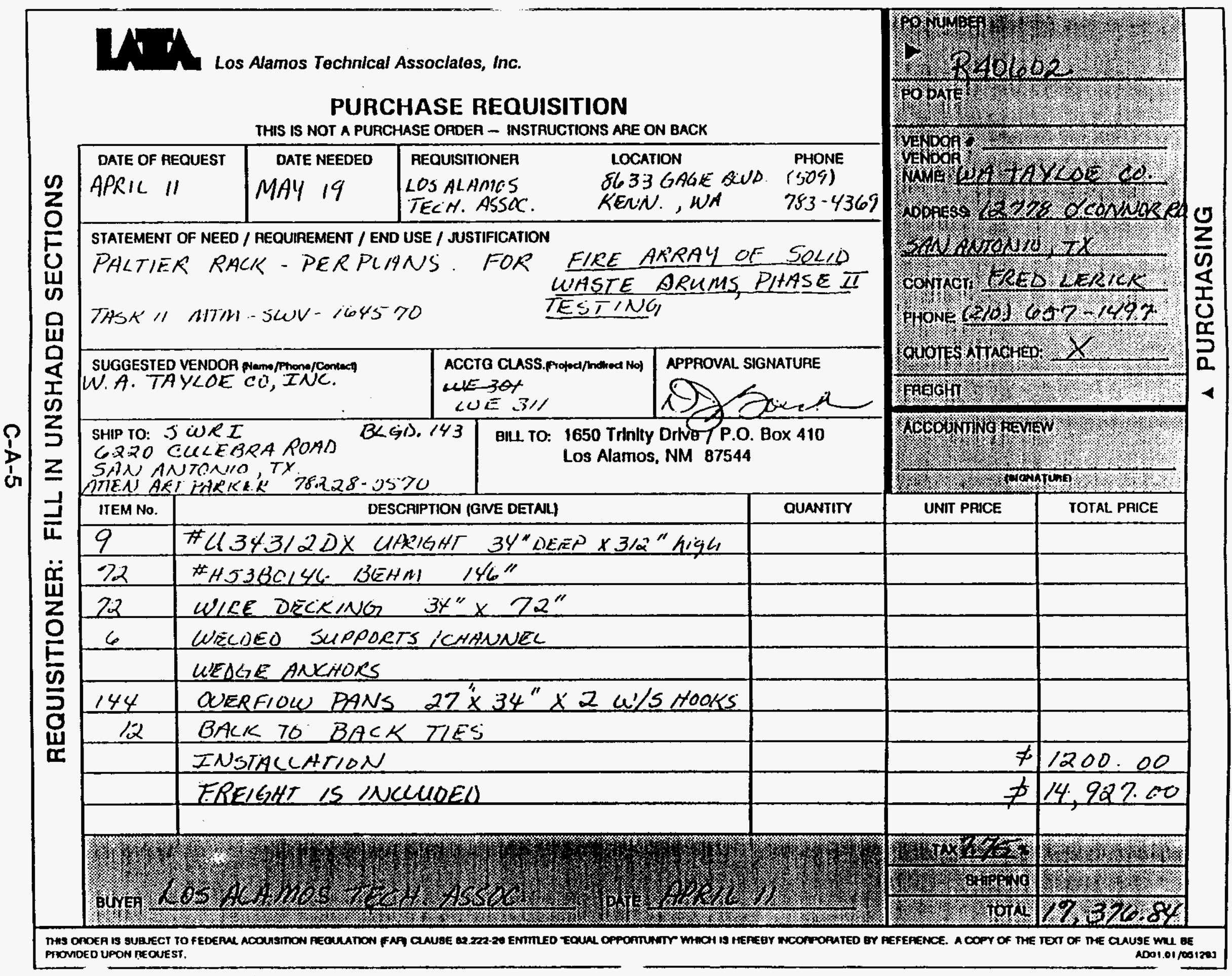


WHC-SD-WM-TRP-233 REV. 0

\section{W.A.TAYLOE CO., INC. MATERIALS HANDLING EQUIPMENT DALLAS - FORT WORTH . HOUSTON - SAN ANTONIO}

April 10, 1995

Los Alamos

Attention: Ms. Julie Dewberry

Dear Julle:

We are pleased to:quote as follows:

PALTIER RACK - Per your plans. To be delivered and installed at Southwest Research, San Antonio, TX.

9 Each - "034312DX Upright. 34" deep X 312" high.

72 Each - "H5380146 Beam. 146".

72 Each - Wire Decking, $34^{\prime \prime}$ deep $x 72^{\prime \prime}$ wide.

6 Welded supports per channel.

144 Each - Overflow pans. 27" wide $x 34$ " deep x 1 ./." high, with $S$ hooks.

12 Each - Back to back ties.

Your deltvered price for materials is $\$ 14,927.00$.

Your price for installation is $\$ 1200.00$, which includes unloading material and furnishing a fork truck.

Prices do not inelude any applicable taxes.

Quotation Supplement $A$ is attached and made an integral part of this quotation.

Lead time on this project is approximately 6 to 8 weeks after order, plus in transit time.

We appreciate this opportunity to quote and look forward to speaking with you soon.

EL/ab

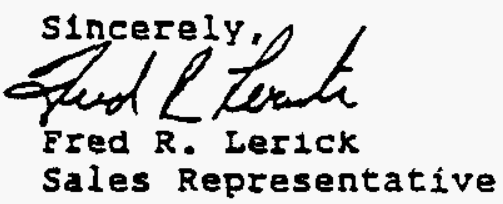

Terms: Net 10 days with approved credit.

12778 O'CONNOR RD. • SAN ANTONIO. TEXAS 78233 • (210) 657-1497 • FAX (210) 655-3302 
WHC-SD-WM-TRP-233 REV. O

W. A. TAYLOE CO., INC.

QUOTATION SUPPLEMENT A

\section{INSTALLATION RESPONSTBILITLES OR TRE BUYEB}

DNLESS OTHERKISE SPECIEIED IN WRITIKG

CLQUSE 1. OWLOAD ALL. EOUIPMENT FROM TRANSPORT VEBICLES AND PLACE \# 1 is Waivad. cost. ADJACENT TO INSTALIATION SITE. ASSUME ANY DEMURRAGE Qum Blactiman- w. a. Tayboe C, Lue. - 4-6-95

2. OBTAIN BUILDING, INSTALLATION AND OPERATING PERMITS AS REQUIRED BY ALI APPLICABLE CODES AND LAWS.

3. PROVIDE A CLEAR, CLEAN AND UNOBSTROCTED SITE FOR INSTALLATION, AS WELL AS CLEAR ACCESS FROM POINT OF ENTRY TO INSTALLATION SITE.

4. TURNISE TO TEE SITE SUITABLE ELECTRIC CURRENT, ALSO, DRY FILTERED AND RSGULATED COMPRESSED AIR IF REQUIRED FOR INSTALLATION AND TESTING OF EQUIPMENT.

5. PROVIDE SAFE, DRY AND CONVENIENT STORAGE SPACE FOR INSTALLATION EQUIPMENT, MATERIAI, AND TOOLS WITE SUITABLE PROTECTION EROM THE ELEMENTS.

6. PROVIDE SOFYICIENT LIGET, HEAT AND/OR VENTILATION TO MEET NORMAL WORKING REQUIRDMENTS.

7. PROVIDE SUFFICIENT SPACE ADJACENT TO THE INSTALLATION SITE FOR TEE LAYOUT, INSPECIION AND ASSEHBLY OF THE EQUIPMENT AS REQUIRED.

B. VERIFY STRUCTURAL CAPACITY AND IIMITATIONS OF BUILDINGS AND MARE NECESSARY ALTERATIONS TO BUILDING(S), FIXTURES. OR EXISTING EQUIRMENT.

9. FURNISE ANY IIFT EQUIPMENT NEEDED.

10. COORDINATION OF TRADES AND A COOPERATIVE ATHOSPHERE IS EXPECTED. UNLESS SPECIFICALIY STATED OTHERWISE, ALL INSTALLATION IS WITE NON-ONION LABOR.

11. PAYMENT FOR MATERIAL IS DUE UPON SHIPMENT AND PAYMENT FOR INSTALLATION IS DUE UTON COMPLETION OR IN PROGRESS PAYMENTS: AS MAY BE SPECIFIED. 
WHC-SD-WM-TRP-233 REV. 0

Appendix 8

Rack Storage Drum Data Sheet

C-B-1 
THIS PAGE INTENTIONALLY LEFT BLANK 
Rack Storage Drum Data Sheet

Drum No.

Description (check):

Standard load Instrumented for pressure Instrumented for temperature $\mathrm{H}_{2} \mathrm{O}$ filled drum

\begin{tabular}{|c|l|l|l|}
\hline Loading Starting at Bottom & Layer 1 & Layer 2 & Layer 3 \\
\hline \hline 3 pieces of steel & & & \\
\hline $1 / 3$ of the brown paper & & & \\
\hline $1 / 3$ of the $10-$ mil plastic & & & \\
\hline $1 / 3$ of the bond paper & & & \\
\hline $1 / 3$ of the towels & & & \\
\hline $1 / 3$ of the bags & & & \\
\hline $1 / 3$ of the cut paper & & & \\
\hline $1 / 3$ of the fabric & & & \\
\hline $1 / 3$ of the rubber & & & \\
\hline \hline & Go to Layer 2 & Go to Layer 3 & \\
\hline
\end{tabular}

Drum Filled:

Date

Initial

Lid sealed and torqued:

Date

Initial

Leak tested:

N/A or Pressure (psig)

Pressure (psig)

Date

\begin{tabular}{lll} 
Time $(\min )$ & 0 \\
\hline & Time $(\min )$
\end{tabular}

Initial

Pre-test weight $(\mathrm{kg})$

Post-test weight $(\mathrm{kg})$ 
WHC-SD-WM-TRP-233 REV. 0

Rack Storage Drum Data Sheet

Drum No.

Post-test Assessment

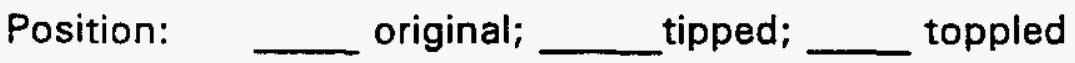

Lid on drum:

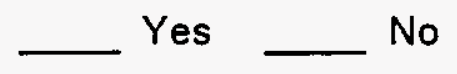

Gasket missing $(\mathrm{cm})$

Contents expelled:

Y Yes __ No

Seal failure:

Yes

Not visible

Burn pattern:

Photo taken:

Yes __ No

Leak tested:

N/A or Pressure (psig)

\begin{tabular}{lll} 
Time $(\min )$ & 0 \\
\hline & Time $(\min )$
\end{tabular}

Pressure (psig)

Date

$\overline{\text { Initial }}$

Interior assessment:

Photo taken:

Yes

No

Comments: 
WHC-SD-WM-TRP-233 REV. 0

Appendix C

Rack Storage Pre-test Checklist

C.C-1 
THIS PAGE INTENTIONALLY LEFT BLANK

$+$ 
Rack Storage Pre-test Checklist

Racks:

3 rows of 6 tiers installed per test plan

Bottom rack units anchored to floor

Single rack secured to ceiling

Back-to-back rows fastened together

All hardware secure

Containment pans and mesh installed

Structures plumb and level

Seismic event bars installed

Verify dimensions of rack storage system

Drums:

144 drums labeled per Drum Location Diagram (DDS)

All drums identified correctly on DDS (initialize description)

All (except $\mathrm{H}_{2} \mathrm{O}$ filled drums) loaded with standard load and steel

Drums sealed, torqued, and documented on DDS

Leak test drums with pressure measurements and document of DDS

All drums weighed and recorded on DDS

Thermocouples installed on appropriate drums

Photograph typical setup of each drum type

Videotape typical setup of each drum type

Drums positioned per Drum Location Diagram

Verify correct spacing between drums as installed in racks

Instrumentation:

Check each pressure transducer calibration

Catalog all heat flux transducer, certified calibration sheets

Verify correct calibrations for instruments in data acquisition

Ambient pressure measurements verified

$\overline{\text { heat) }}$

Verify operation and location of thermocouples through computer lapply

Verify operation and location of heat flux transducers through computer

Verify operation of $\mathrm{H}_{2} \mathrm{O}$ calorimeter pumps

Fuel:

$14.7 \mathrm{~m}^{2}\left(162 \mathrm{ft}^{2}\right)$ pool area fabricated per test plan

Pool area filled with $\sim 2.5 \mathrm{~cm}$ (1 in.) of water and inspected for leaks

$208 \mathrm{~L}(55 \mathrm{gal})$ of diesel fuel staged

$8 \mathrm{~L}(2 \mathrm{gal})$ of gasoline staged

Foam extinguishing system in place and tested 
Rack Storage Pre-test Checklist (Continued)

Data Acquisition:

Data acquisition system ready

Pre-test video and still photographs complete

Video cameras positioned per test plan and operable

Camera 1 tape set

Camera 2 tape set

Photographer positioned and ready

Remote video monitors setup

Remote monitors setup for data acquisition if required

Observation structure in place under overhead door

Tape recorders loaded and ready 
WHC-SD-WM-TRP-233 REV. 0

\section{Appendix D}

Rack Storage and Pallet Storage Test Procedure Checklist

C-D-1 


\section{Rack Storage and Pallet Storage \\ Test Procedure Checklist}

Pre-test checklist complete

Verify all vents in building open

All observers in designated locations

Firefighters suited and standing by

Foam extinguishing system charged

Start tape recorders

Start video tapes

Start data acquisition and synchronize with video

Pour diesel fuel

Pour gasoline

Ignite fuel (data acquisition time recorded)

Test termination criteria 
WHC-SD-WM-TRP-233 REV. 0

\author{
Appendix E \\ Rack Storage \\ Post-test Rack Assessment
}

C-E-1 
WHC-SD-WM-TRP-233 REV. 0

Rack Storage Post-test Rack Assessment

Row

Bay

Tier

Mechanical failure of welds or connections:

Rack warping (include measurements and location):

Overall structural stability: 
WHC-SD-WM-TRP-233 REV. 0

Appendix $\mathrm{F}$

Pallet Storage Pre-test Checklist

C.F-1 
THIS PAGE INTENTIONALUY LEFT BLANK 
Pallet Storage Pre-test Checklist

Pallets:

Banded together

Properly oriented per test plan

Drums:

144 drums labeled per Drum Location Diagram (DDS)

All drums identified correctly on DDS (QA: initialize description)

Drums sealed, torqued, and documented on DDS

Leak test drums with pressure measurements and document of DDS

All drums weighed and recorded on DDS

Thermocouples installed on appropriate drums

Photograph typical setup of each drum type

Videotape typical setup of each drum type

Drums positioned per Drum Location Diagram

Instrumentation:

Check each pressure transducer calibration

Catalog all heat flux transducer, certified calibration sheets

Verify correct calibrations for instruments in data acquisition

Heat flux transducers positioned per test plan

Thermocouples positions per test plan

Ambient pressure measurements verified

heat)

Verify operation and location of thermocouples through computer (apply

Verify operation and location of heat flux transducers through computer

Fuel:

$9.3 \mathrm{~m}^{2}\left(100 \mathrm{ft}^{2}\right)$ pool area fabricated per test plan

Pool area filled with $-2.5 \mathrm{~cm}$ ( 1 in.) of water and inspected for leaks

$135 \mathrm{~L}$ ( $35 \mathrm{gal}$ ) of diesel fuel staged

$8 \mathrm{~L}$ ( 2 gal) of gasoline staged

Foam extinguishing system in place and tested 


\section{Pallet Storage Pre-test Checklist (Continued)}

Data Acquisition:

Data acquisition system ready

Pre-test video and still photographs complete

Video cameras positioned per test plan and operable

Camera 1 tape set

Camera 2 tape set

Still photography camera positioned and ready

Remote video monitors setup if required

Remote monitors setup for data acquisition if required Observation structure in place under overhead door

Tape recorders loaded and ready 
WHC-SD-WM-TRP-233 REV. 0

Appendix G

Pallet Storage

Post-test Pallet Assessment

C-G-1 
WHC-SD-WM-TRP-233 REV. 0

Pallet Storage Post-test Pallet Assessment

Row

Bays

Tier

Mechanical failure or damage:

Pallet movement:

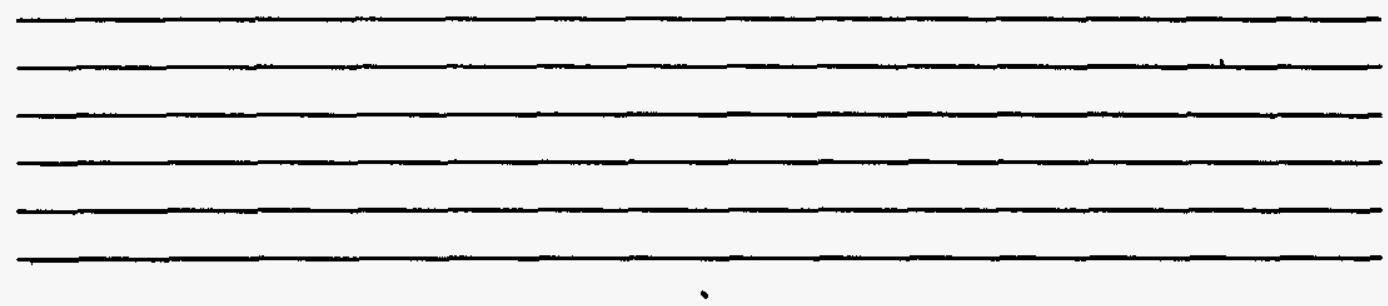

Overall structural stability:

C-G-2 